\title{
Bell violation in the sky
}

\author{
Sayantan Choudhury ${ }^{1,5, a}$, Sudhakar Panda ${ }^{2,3, b}$, Rajeev Singh ${ }^{4, c}$ \\ ${ }^{1}$ Department of Theoretical Physics, Tata Institute of Fundamental Research, Colaba, Mumbai 400005, India \\ ${ }^{2}$ Institute of Physics, Sachivalaya Marg, Bhubaneswar, Odisha 751005, India \\ ${ }^{3}$ Homi Bhabha National Institute, Training School Complex, Anushakti Nagar, Mumbai 400085, India \\ ${ }^{4}$ Department of Physics, Savitribai Phule Pune University, Pune 411007, India \\ ${ }^{5}$ Present Address: DTP, TIFR, Mumbai, India
}

\begin{abstract}
In this work, we have studied the possibility of setting up Bell's inequality violating experiment in the context of cosmology, based on the basic principles of quantum mechanics. First we start with the physical motivation of implementing the Bell inequality violation in the context of cosmology. Then to set up the cosmological Bell violating test experiment we introduce a model independent theoretical framework using which we have studied the creation of new massive particles by implementing the WKB approximation method for the scalar fluctuations in the presence of additional time-dependent mass contribution in the cosmological perturbation theory. Here for completeness we compute the total number density and the energy density of the newly created particles in terms of the Bogoliubov coefficients using the WKB approximation method. Next using the background scalar fluctuation in the presence of a new time-dependent mass contribution, we explicitly compute the expression for the one point and two point correlation functions. Furthermore, using the results for a one point function we introduce a new theoretical cosmological parameter which can be expressed in terms of the other known inflationary observables and can also be treated as a future theoretical probe to break the degeneracy amongst various models of inflation. Additionally, we also fix the scale of inflation in a model-independent way without any prior knowledge of primordial gravitational waves. Also using the input from a newly introduced cosmological parameter, we finally give a theoretical estimate for the tensor-to-scalar ratio in a modelindependent way. Next, we also comment on the technicalities of measurements from isospin breaking interactions and the future prospects of newly introduced massive particles in a cosmological Bell violating test experiment. Further, we
\end{abstract}

\footnotetext{
a e-mails: sayantan@theory.tifr.res.in; sayanphysicsisi@gmail.com

be-mail: panda@iopb.res.in

c e-mail: rajeevsingh240291@gmail.com
}

cite a precise example of this setup applicable in the context of string theory motivated axion monodromy model. Then we comment on the explicit role of the decoherence effect and high spin on cosmological Bell violating test experiment. Finally, we provide a theoretical bound on the heavy particle mass parameter for scalar fields, gravitons and other high spin fields from our proposed setup.

\section{Contents}

1 Introduction ................ 2

2 Bell test experiment in quantum mechanics . . . . 8

2.1 What Bell's inequality tells us? . . . . . . . . 8

2.2 1st Example on Bell's inequality . . . . . . . 9 9

2.3 2nd Example on Bell's inequality . . . . . . . 9

2.4 Review on the proof of Bell's inequality . . . . 10

2.5 Bell's inequality in a spin system . . . . . . . . 10

2.6 Violation of Bell inequality . . . . . . . . . . 11

2.6.1 Case I: Explanation from earlier experiments . . . . . . . . . 11

2.6.2 Case II: Explanation from recent experiments ........... 11

2.6.3 Case III: Explanation for entanglement . 12

3 Bell test experiment in primordial cosmology . . . . 12

3.1 Setup for the cosmological Bell violating experiment ............ 12

3.2 Creation of new massive particle . . . . . . 14

3.2 .1 Case I: $m \approx H \ldots \ldots \ldots . \ldots 23$

3.2 .2 Case II: $m>>$ H. . . . . . . . 35

3.2.3 Case III: $m<<H \quad \ldots \ldots$. . . . . . 51

3.3 Cosmological scalar curvature fluctuations from new massive particles . . . . . . . . . . . 67

3.3.1 Case I: $m \approx H \quad \ldots \ldots . . . . .84$

3.3.2 Case II: $m>>$ H . . . . . . . . . 89

3.3.3 Case III: $m<<H \quad \ldots \ldots$. . . . . . 91 
4 Specific example: analogy with axion fluctuations in string theory . . . . . . . . . . . . . . . 92

4.1 Axion monodromy model . . . . . . . . . . 92

4.2 Axion effective interaction . . . . . . . . . . . 94

4.3 Axion creation from quantum fluctuation . . . . 95

4.3.1 Case I: $m_{\text {axion }} / f_{a} \approx H \ldots 103$

4.3.2 Case II: $m_{\text {axion }} / f_{a}<<H$. . . . . . . 115

4.3.3 Case III: $m_{\text {axion }} / f_{a}>>H$. . . . . . . 125

4.4 Axion-massive particle correspondence . . . . 136

4.5 Cosmological implication from axion fluctuation . . . . . . . . . . . . 145

4.6 Role of isospin breaking interaction . . . . . 155

5 Conclusion ................. 160

6 Appendix .................. 162

6.1 Role of quantum decoherence in Bell violating cosmological setup . . . . . . . . . . 162

6.2 Time dependent mass profile for heavy field . . 165 6.2.1 Profile A: $m=\sqrt{\gamma\left(\frac{\eta}{\eta_{0}}-1\right)^{2}+\delta} H \quad .165$

6.2.2 Profile B: $m=\frac{m_{0}}{\sqrt{2}} \sqrt{\left[1-\tanh \left(\frac{\rho}{H} \ln (-H \eta)\right)\right]} \cdot .172$

6.2.3 Profile C: $m=m_{0} \operatorname{sech}\left(\frac{\rho}{H} \ln (-H \eta)\right)$. . 174

6.3 Role of spin for heavy field . . . . . . . . . . 175

6.4 More on Bell inequalities . . . . . . . . . . . . 179

6.4.1 CHSH inequality . . . . . . . . . . 179

6.4.2 Consequences of Bell inequality violation . . . . . . . . . 179

References ............... 179

"That one body may act upon another at a distance through a vacuum without the mediation of anything else....is to me so great an absurdity, that I believe no man, who has in philosophical matters a competent faculty for thinking, can ever fall into" -Sir Isaac Newton

\section{Introduction}

In the year 1935, Einstein, Podolsky and Rosen (EPR) in Ref. [1] mentioned that, if, without in any way disturbing a system, we can predict with certainty (i.e., with probability equal to unity) the value of a physical quantity, then there exists an element of physical reality corresponding to this physical quantity. This work also claimed that quantum mechanics cannot be a complete theoretical framework, therefore there has to be some element in existence using which it is not possible to describe within the basic principles of quantum mechanics. Furthermore the authors also added that, while we have thus shown that the wave function does not provide a complete description of the physical reality, we left open the question of whether or not such a description exists. We believe, however, that such a theory is possible. Based on all such statements one can ask a question regarding the existence of all such missing elements in quantum physics theory.

Later Bell introduced the existence of "hidden" variables which directly implies that in spin correlation measurements the measurable probabilities must satisfy the proposed Bell's inequality [2] within the framework of quantum mechanics. For completeness here we also mention some of the remarkable works in the area of quantum mechanics proposed up to the Bell test experiment:

- 1927 Copenhagen interpretation of quantum mechanics (Bohr, Heisenberg),

- 1935 Einstein-Podolsky-Rosen (EPR) paradox,

- 1952 De Broglie-Bohm nonlocal hidden-variable theory (Bohmian mechanics),

- 1964 Bell's theorem on local hidden variables,

- 1972 First experimental Bell test(Freedman and Clauser).

Later the actual version of the Bell inequality has been proved incorrect by many experiments performed till date, which in turn proves that nature is nonlocal and hence all the particles can interact with each other without bothering about the underlying interaction scale and the corresponding distance (length scale) between all of them. This underlying principle of violation of Bell's inequality is thoroughly used in our work to set up the cosmological experiment and to study some of the unexplored important features in the context of the early universe.

It is a very well-known fact that our present understanding of the large scale structure formation of universe is that it actually originates from the small scale perturbations and once the universe became matter dominated then gravitational effects mimic its role in cosmological evolution, which we observe today through various cosmological observations. For the formation of the structure due to gravitational instability of what we observe today, there have to be preexisting small fluctuations on physical length scales. In the model of the Big Bang it is almost impossible to produce fluctuations in any arbitrary length scale, so in such a case we put these small perturbations by hand. The proper physical explanation for these small scale perturbations is that these perturbations arise due to density fluctuations in the inflationary epoch [3-8], which have a quantum mechanical origin.

In the context of modern cosmology, as is well known, one of the main basic idea is that there occurred an event namely an epoch in the very early time of the universe where the universe is vacuum dominated matter or radiation. Therefore during this era the scale factor grew almost exponentially in time. We can also understand why the observable universe is homogeneous and isotropic if this quasi exponential 
expansion occurred in the very early age of universe. This epoch is commonly known as inflation. This theory was first introduced by Guth in Ref. [9]. A primordial density perturbation is actually a vacuum fluctuation which survived after the period of inflation which may be the best possible reason for the large scale structure formation of our universe and CMB anisotropy. In the present context we are primarily interested in the specific type of inflation theory which removes the shortcomings of standard Big Bang theory, which also helps us to get the most favoured possible explanation of the homogeneity and isotropy of CMB and to construct a Bell inequality violating cosmological setup. Therefore the inflationary paradigm predicts that the origin of large scale structure, which we actually observe, is nothing but the outcome of quantum mechanical fluctuations after the inflationary period. Such quantum fluctuations make the inflationary paradigm consistent with various cosmological observations compared to the other classical statistical fluctuations appearing in the present context by following the same epoch [4-9]. Here it is important to note that, in the case of a classical statistical approach, the frictional force acts as an external source using which inflaton energy is converted to the other forms of energy and finally produces fluctuations. Now further using this information one can compute, and also compare and constrain two and three point correlation functions from quantum fluctuations and classical statistical fluctuations and check the consistency relations from any higher point correlation functions. Here additionally it is important to note that, in the quantum mechanical interpretation of the required fluctuations, the highly entangled quantum mechanical wave function of the universe plays a significant role. Due to this fact, quantum fluctuations can be theoretically demonstrated as well as implemented in the context of primordial cosmology, iff we can perform a Bell inequality violating cosmological experiment using the highly quantum mechanical entangled wave function of the universe defined in the inflationary period. Throughout this paper we will develop a theoretical setup to address various fundamental questions related to general aspects of Bell's inequality violation and also study the various unexplored physical consequences from cosmological Bell's inequality violating experiment. Now to describe the theoretical framework and background methodology in detail it is important to mention that, in the context of quantum mechanics, the Bell test experiment is described by the measurement of two non-commutating physical operators which are associated with two distinctive locations in the space-time. Using a similar analogy in the context of primordial cosmology, one can also perform similar cosmological observations on two spatially separated as well as causally disconnected places up to the epoch of reheating. In the case of cosmological observations this may enable one to measure the numerical values of various cosmological observables (along with cosmic variance), which can be computed from a scalar curvature fluctuation. Apart from the observational success it is important to point out that for all such observations it is impossible to measure the value of associated canonically conjugate momentum. Consequently, for these observables it is impossible to measure the imprints of two non-commuting operators in the context of primordial cosmology. This directly implies that due to this serious drawback in the underlying structural setup it is not at all possible to set up a Bell inequality violating experimental setup in the context of cosmology. But to make a further strong conclusive statement regarding this issue one needs to investigate the decoherence effect and its impact in cosmological observation [10-19]. If the cosmological observables satisfy the basic requirements of the decoherence effect then it is possible to perform measurements from two exactly commuting cosmological observables and this can enable one to design a Bell inequality violating cosmological experimental setup. In the context of quantum mechanics, to design such an experimental setup one needs to perform repeated measurements on the same object (here it is the same quantum state) and in such a physical situation one can justify the appearance of each and every measurement through a single quantum state. Using the same idea one can also design a cosmological experimental setup in the present context. In the context of cosmology, one can similarly consider two spatially separated portions in the full sky which exactly mimics the role of performing a repeated cosmological Bell inequality violating experiment via the same quantum mechanical state. Due to this here one can choose the appropriate and required properties of two spatially separated portions in the full sky to set up a Bell inequality violating experimental setup in cosmology. Most importantly it is important to mention here that if it is possible to connect direct a link between these mentioned non-commuting cosmological observables and a classical probability distribution function originating from the inflationary paradigm then it is surely possible to have a Bell inequality violating cosmological experimental setup.

In this work we have addressed the following important points through which it is possible to understand the underlying framework and consequences from the proposed Bell inequality violating experimental setup in the context of cosmology. These issues are:

- Setting up a cosmological Bell inequality violating experiment in the presence of new heavy fields within the framework of inflation where these heavy fields are the additional field content, appearing along with the inflaton field. We have shown that the time-dependent mass profile for such heavy fields plays a significant role to set up a Bell inequality violating experiment.

- The explicit role of one point and two point correlation functions, which play a significant role to quantify the 
effect of Bell's inequality violation in the presence of a significant heavy field mass profile.

- Particle creation mechanism of all such heavy fields for different time-dependent mass profiles which are responsible for Bell's inequality violation in a cosmological setup.

- The exact connection between all such heavy fields and axion fields as appearing in the context of a monodromy model in string theory.

- The specific role of isospin breaking phenomenological interactions for heavy fields during the Bell inequality violating experimental measurement.

- The exact role of high spin for heavy particles to determine the particle creation and quantify the amount of Bell's inequality violation in a cosmological setup.

- To give a generic mass bound on the scalar heavy fields and high spin heavy fields within a model-independent framework of inflationary paradigm. For this purpose we use the Effective Field Theory (EFT) framework for inflation [20-26] in the present context.

- To identify the connection between scale of inflation or more precisely the exact theory of inflation and amount of Bell's inequality violation in proposed cosmological experimental setup.

- To give a model-independent quantification for primordial gravitational waves through tensor-to-scalar ratio from inflation with the help of the amount of Bell's inequality violation in cosmology. If we have any prior knowledge of the amount of Bell's inequality violation in the cosmological setup then using this model independent relation we can put a stringent constraint on various inflationary models. If it is not possible to quantify the amount of Bell's inequality violation from any other experimental probe and if this can enable one to measure the value of tensor-to-scalar from future observational probes, subsequently it is possible to quantify the amount of Bell's inequality violation in cosmology with the help of this proposed model-independent relation.

- To study the exact role of initial conditions or choice of inflationary vacuum to violate Bell's inequality in the context of the de Sitter and the quasi de Sitter cosmological setup.

- The proposed specific form of cosmological observable within the framework of inflationary paradigm through which the effect of Bell's inequality violation can be explicitly quantified. ${ }^{1}$ Also one expressed various known

\footnotetext{
1 In Ref. [3] one also mentioned this possibility in the context of a baroque inflationary model where one can perform the cosmological Bell inequality violating experiment. In this paper we explore other possibilities in detail by proposing various time-dependent mass profiles for the heavy fields for arbitrary choice of initial conditions or choice of vacuum. Hence we will quote the results for a Bunch-Davies vacuum and $\alpha$ vacuum for the sake of completeness. Also in our paper we provide
}

inflationary observables in terms of this newly proposed observable. Here it is important to note that this conversion is only possible if the heavy fields are massive compared to the Hubble scale and follow a profile as mentioned earlier.

Now before going to the further details let us mention the underlying assumptions clearly to understand the background setup for this paper:

1. UV cut-off of the effective theory is given by the scale $\Lambda_{U V}$. For our purpose we fix $\Lambda_{U V}=M_{p}$, where $M_{p}$ is the reduced Planck mass.

2. Inflaton and the heavy fields are minimally coupled to the Einstein gravity sector.

3. Effective sound speed $c_{S} \neq 1$. Within EFT it is always $c_{S} \leq 1$. For canonical slow-roll models $c_{S}=1$ and for other cases $c_{S}<1$.

4. Various choices for initial conditions are taken into account during our computation. We first derive the results for arbitrary choice of vacuum and then quote the results for Bunch-Davies, $\alpha$ and special type of vacuum.

5. To express the scale of inflation in terms of the amount of Bell's inequality violation in cosmological experimental setup we assume that slow-roll prescription perfectly holds good in the EFT sector. Consequently we have used the consistency conditions which are applicable to the slow-roll case to find the expression for tensor-to-scalar ratio in terms of the Bell inequality violating observable. For example, we use here $r=16 \epsilon c_{S}$. But without assuming any slow roll one can find the expression for the first Hubble slow-roll parameter $\epsilon=-\dot{H} / H^{2}$ in terms of the Bell inequality violating observable within the framework of EFT.

6. For the computation of the Bogoliubov coefficients we have introduced a cut-off in a conformal time scale to collect the regularized finite analytical contribution for different time-dependent mass profile. Consequently the rest of the parameters derived from Bogoliubov coefficients i.e. reflection and transmission coefficients, number density and energy density follow the same approximation during massive particle creation.

7. To use the analogy with the axion monodromy model in the context of string theory we neglect the effect of backreaction and of being restricted up to the mass term in the effective potential. This helps us to perfectly identify the analogy between heavy fields and axion.

\footnotetext{
Footnote 1 continued

an explicit form of the new inflationary observable through which one can quantify the effect of Bell's inequality violation in cosmological setup.
} 
Table 1 Table showing the connection between relativistic quantum theory and cosmology in the context to Bell's inequality violation

\begin{tabular}{|c|c|c|}
\hline Properties & Relativistic quantum theory & Cosmology \\
\hline Importance & Theory of entanglement came into picture & $\begin{array}{l}\text { Important hidden features in the context } \\
\text { of the early universe can be known }\end{array}$ \\
\hline Fluctuation & $\begin{array}{l}\text { Helps to produce virtual particles (pairs of } \\
\text { particle and antiparticle) }\end{array}$ & $\begin{array}{l}\text { Helps to produce hot and cold spots in } \\
\text { CMB }\end{array}$ \\
\hline Assumptions & Concepts of locality and reality & Slow-roll prescription \\
\hline Decoherence & $\begin{array}{l}\text { Provides reasons for the collapse of wave } \\
\text { function }\end{array}$ & $\begin{array}{l}\text { Primordial non gaussianity can be } \\
\text { enhanced }\end{array}$ \\
\hline Applications & $\begin{array}{l}\text { Quantum information, computing and } \\
\text { many more }\end{array}$ & Origin of large scale structure formation \\
\hline
\end{tabular}

8. We use approximated WKB solutions to quantify the particle creation for different arbitrary time-dependent mass profile for heavy fields as it is not always possible to compute the exact mode functions for the heavy fields in Fourier space by exactly solving the equation of motion for the heavy fields. In some of the cases we provide an exact solution where the time dependence in the mass parameter is slowly varying. We use these results to compute the one point and two point correlation functions in the present context.

9. To study the role of arbitrary spin fields with $\operatorname{spin} \mathcal{S}>2$ in Bell's inequality violation we assume that the dynamics of all such fields is similar to the scalar field and graviton.

In Table 1, we show the connection between relativistic quantum theory and cosmology in the context of Bell's inequality violation. In Figs. 1 and 2, we have schematically shown the flow chart of the Bell inequality violating cosmological setup and basic structural setup of the present paper which we have discussed in detail as follows:

- Section 2: Here we review Bell's inequality in quantum mechanics and its implications. For this we review the proof of Bell's inequality followed by an example of Bell's inequality with spin system. Further we discuss briefly the violation of Bell's inequality in quantum mechanics. Hence we provide the explanation for such violation and the consequences which finally give rise to new physical concepts like quantum entanglement.

- Section 3: Here in Sect. 3.1 we briefly discuss the setup for Bell's inequality violating test experiment in the context of primordial cosmology. Then we study creation of new massive particles as introduced in the context of inflationary paradigm for various choice of timedependent mass profile in Sect. 3.2. We also present the calculation for the three limiting situations: (1) $m \approx H$, (2) $m>>H$ and (3) $m<<H$. Now to describe a very small fraction of particle creation after inflation we need to find the Bogoliubov coefficient $\beta$ in FLRW spacetime, which characterizes the amount of mixing between the two types of WKB solutions. Therefore we provide detailed mathematical calculations to find the Bogoliubov coefficient $\beta$ for each of the different cases. Using the results for the Bogoliubov coefficients we further calculated reflection and transmission coefficients, number density and energy density of the created particles for various mass profiles for two equivalent representations. Since the exact analytical expression for the integrals involved in all of these parameters are not always computable, we use the approximation in three physical sub regions. Here we provide the results for three specific cases:-

1. $\left|k c_{S} \eta\right|=c_{S} k / a H<<1$ (super horizon),

2. $\left|k c_{S} \eta\right|=c_{S} k / a H \approx 1$ (horizon crossing),

3. $\left|k c_{S} \eta\right|=c_{S} k / a H>>1$ (sub horizon).

Further in Sect. 3.3 we study cosmological scalar curvature fluctuations in the presence of new massive particles for arbitrary choice of initial condition and also for any arbitrary time-dependent mass profile. Here we explicitly derive the expression for the one point and the two point correlation functions using in-in formalism. Then we quote the results for the three limiting situations: (1) $m \approx H$, (2) $m>>H$ and (3) $m<<H$ in super horizon, sub horizon and horizon crossing. Here we introduce a new cosmological observable which captures the effect of Bell's inequality violation in cosmology. Further we express the scale of inflation in terms of the amount of Bell's inequality violation in cosmology experimental setup. Additionally we derive a modelindependent expression for first Hubble slow-roll parameter $\epsilon=-\dot{H} / H^{2}$ and tensor-to-scalar ratio in terms of the Bell inequality violating observable within the framework of EFT. Additionally, in subsection 3.3 we give an estimate of inflaton mass parameter $m_{\text {inf }} / H .^{2}$ Further we consider a very special phenomenological case, where the inflaton mass is comparable with the new particle mass parameter $m_{\mathrm{inf}} \approx m$ and using this we provide an esti-

\footnotetext{
${ }^{2}$ Here $m_{\text {inf }}$ is the mass of inflaton field and $H$ is the Hubble scale.
} 


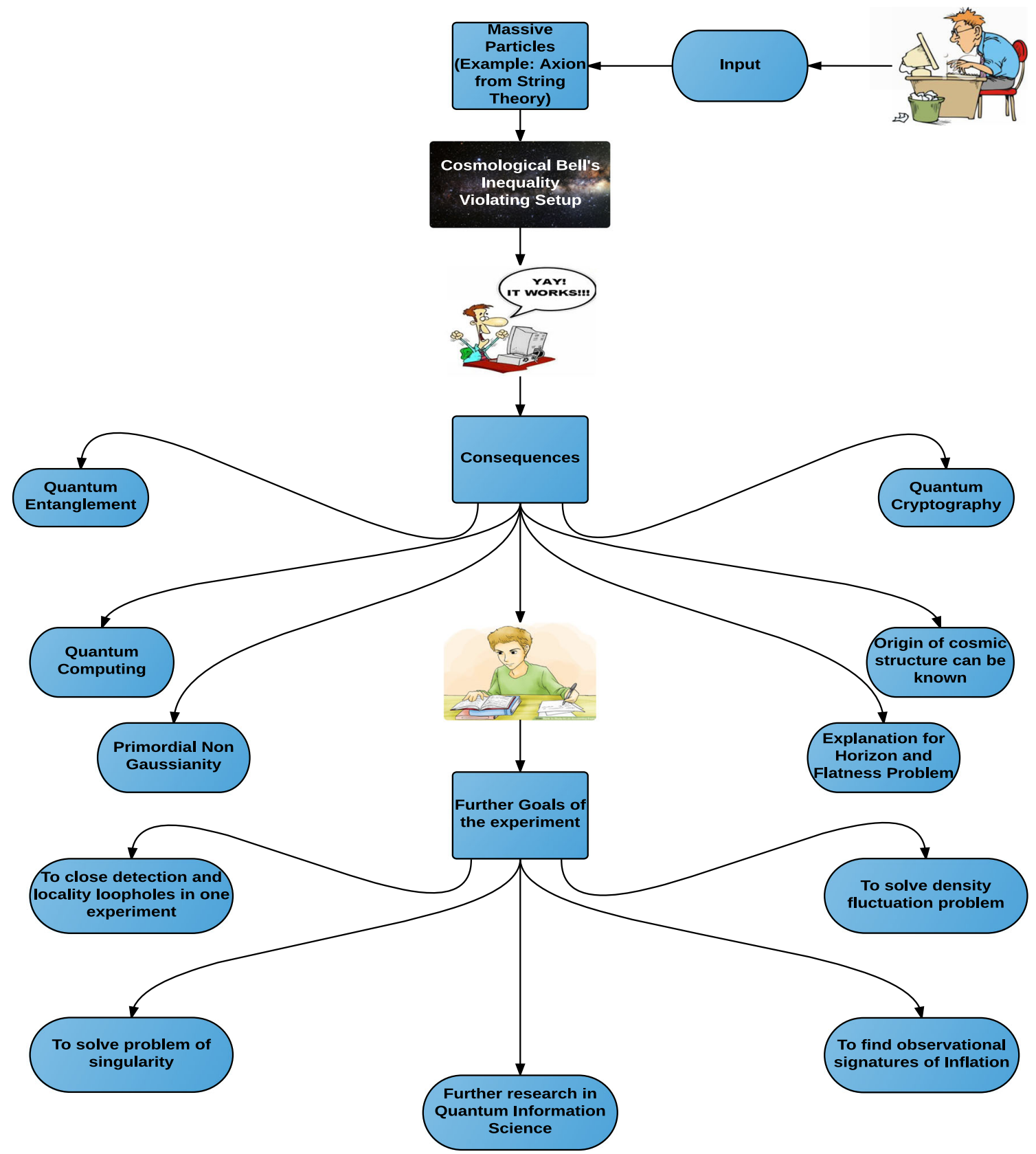

Fig. 1 Flow chart of the Bell inequality violating cosmological setup

mate of heavy field mass parameter $m / H^{3}$ which is an important ingredient to violate Bell's inequality within the cosmological setup.

- Section 4: In Sect. 4.1 we give an example of an axion model with time-dependent decay constant as appearing in the context of string theory. Hence in the next Sect. 4.2 we mention the effective axion interaction of axion fields. Now to give an analogy between the newly intro-

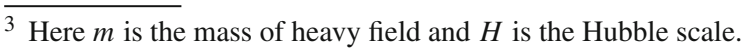

duced massive particle and the axion we further discuss the creation of axion in early universe in Sect. 4.3. Further in Sects. 4.4 and 4.5 we establish the one to one correspondence between heavy field and axion by comparing the particle creation mechanism, one and two point correlation functions. Additionally, in Sect. 4.5 we give an estimate of axion mass parameter $m_{\text {axion }} / f_{a} H^{4}$ which

\footnotetext{
${ }^{4}$ Here $m_{\text {axion }}$ is the axion mass, $f_{a}$ is the time-dependent decay constant for axion and $H$ is the Hubble scale.
} 


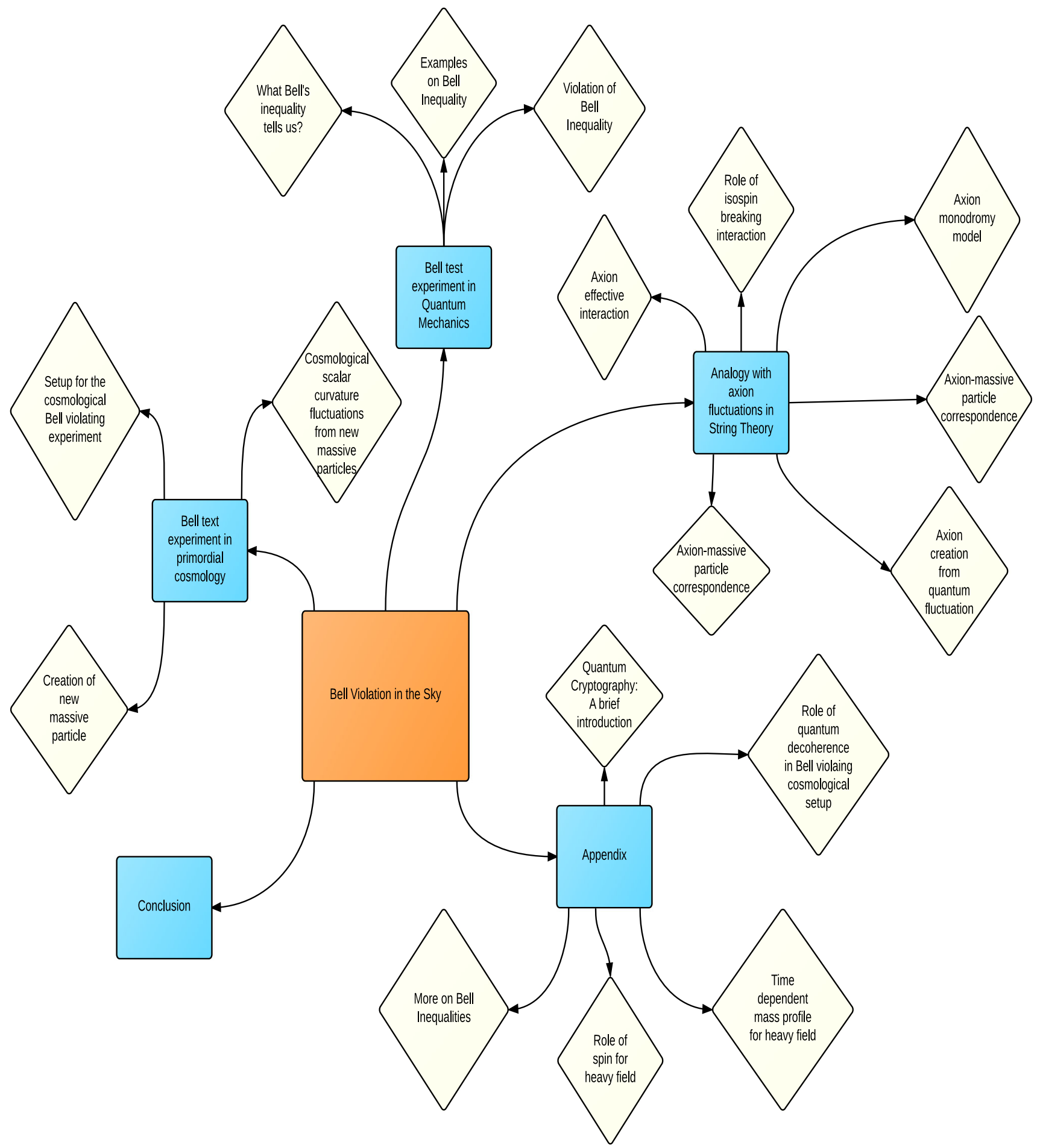

Fig. 2 Flow chart of the basic structural setup of this paper

is an important ingredient to violate Bell's inequality within cosmological setup. Finally, in Sect. 4.6 we discuss the specific role of isospin breaking phenomenological interaction for axion type of heavy fields to measure the effect of Bell's inequality violation in primordial cosmology.
- Section 5: Here we conclude with future prospects from this present work.

- Appendix 6: In Appendix 6.1 we explicitly show the role of quantum decoherence in cosmological setup to violate Bell's inequality. Additionally here we also mention a possibility to enhance the value of primordial non- 
Gaussianity from Bell's inequality violating setup in the presence of massive time-dependent field profile. Furthermore, in Appendix 6.2 we discuss the role of three specific time-dependent mass profile for producing massive particles and to generate quantum fluctuations. Furthermore, in Appendix 6.2 we discuss the role of arbitrary spin heavy field to violate Bell's inequality. Here we provide a bound on the mass parameter for massive scalar with spin $\mathcal{S}=0$, axion with spin $\mathcal{S}=0$, graviton with spin $\mathcal{S}=2$ and for particles with high spin $\mathcal{S}>2$ in horizon crossing, super horizon and sub horizon regime. Then we provide the extended class of Bell's inequality, called CHSH inequality. Finally, we give a very brief discussion of quantum cryptography related to the present topic of the paper.

\section{Bell test experiment in quantum mechanics}

\subsection{What Bell's inequality tells us?}

In Ref. [1] authors first demonstrated that quantum theory is incomplete with the help of EPR (Einstein Podolsky Rosen) paradox. According to Einstein's theory of special relativity, we know that speed of light is the fastest that we can get. Indeed it was the discrepancy between the predictions of relativity and quantum theory concerning the correlations between events in space-like separated regions that led Albert Einstein, Boris Podolsky and Nathan Rosen to point out an effect, known as EPR, where one part of entangled quantum systems appears to influence another at the same instant. To Einstein, Podolsky and Rosen quantum theory gave only an incomplete account of physical reality. As special theory of relativity $[27,28]$ says that nothing can go faster than light or in other words speed of light is the fastest we can get, they believed that the correlations in measurement outcomes of experiments which measures both members of particles which are highly separated and entangled could be explained by hypothesizing that separated particles are not entangled rather had fixed values of all their measurable attributes from the outset. Hence the outcomes of experiment must be determined by "hidden variables".

Later in Ref. [2], John Stewart Bell showed that "In a theory in which parameters are added to quantum mechanics to determine the results of individual measurements, without changing the statistical predictions, there must be a mechanism whereby the setting of one measuring device can influence the reading of another instrument, however remote. (Bell 1987, p. 20.)"'. He also showed that there was the difference between the predictions of any hidden-variable theory and predictions of quantum theory. Bell's article refers to Ref. [1] that challenged the completeness of quantum theory.
In that paper, Bell started his theory with two assumptions which were:

1. concept of reality (real properties of microscopic objects determine the results of quantum mechanical experiments),

2. concept of locality (reality in one place is not affected by experiments done at the same time at a distant place).

Using these two assumptions Bell derived an important result, which is known as 'Bell's inequality'. Bell proved with his inequality that "no local hidden-variable theory is compatible with quantum mechanics". The following example will develop some physical intuition for Bell inequality which is clearly explained in the following steps:

- What would we observe if an experiment is performed on a set of pairs of polarization measurements? For simplicity let us say that the pair of photons exist in an entangled state such that both polarizations are the same but are otherwise unknown when they are measured.

- Let us call our experimentalists Aace and Bace, and let us say that they agree to place their polarizers in the same direction. Thus the angle between their polarizers is $0^{\circ}$. What would they see? Since the entangled particles are correlated, every time Alice observes 'vertical', Bace also observes the same i.e. 'vertical'. And every time Aace sees 'horizontal', Bace sees 'horizontal'. The percentage that they agree mutually on the result is 100 .

- Now let us rotate the polarizer of Bace by $90^{\circ}$. Now when Bace sees 'vertical', Aace observes 'horizontal'. And when they perform polarization measurements on respective pairs of correlated photons, their results will be anti-correlated. Every time Bace sees 'vertical', Aace sees 'horizontal' and vice versa. The percentage they agree on the results is 0 .

- Now suppose Bace rotates his polarizer back towards Aace's vertical so that polarizer of the Bace makes an angle to Aace's vertical. Now Aace measures her photon to be 'vertical'. Thus the twin photon will also be 'vertical' (in Aace frame). To Bace, the photon he receives will appear to be in a superposition of his 'horizontal' and 'vertical' orientations.

- Hence the result of Bace's polarization measurement is uncertain, sometimes when Bace measures a photon that Aace observes as 'vertical' Bace will also sees 'vertical' too. But at other times when Bace measures a photon Aace sees as 'vertical', but Bace sees 'horizontal'. As a result the percentage they agree is between 0 and 100 . The exact percentage depends on the angle between their polarizers. 


\subsection{1st Example on Bell's inequality}

- Chace's idea was to test the theory of locality of Einstein by using the properties of the correlations between measurement outcomes obtained by experimenters Aace and Bace. Now suppose Aace is in Mumbai with three coins with its head or tails facing upwards, but Aace cannot tell which side is up as he is blind folded and also there is a black cloth on each of the coin.

- When Aace uncover one coin, suddenly other two coins disappear. Therefore probability of getting either head or tails is same.

- Similarly his friend Bace (in Calcutta) has same type of coins and does the same experiment. He too have the same probability of getting either head or tails.

- Both of them repeats their experiments again and again to find out the correlation between their coins. Therefore they found that whenever they uncover their coins with the same label, that is, first, second or third, they both got head $(\mathrm{H})$ or tail $(\mathrm{T})$.

- They did their experiments number of times to be sure but they got their coins correlated each time. But Aace wants to find two coins in one turn, but he cannot as when he uncovers one coin the other coin suddenly disappears.

- So when he talked to Bace, he told that if he (Bace) uncovers second coin and tell him what he got, then Aace will certainly know what he will get if he (Aace) uncovers second coin without uncovering it. Then he can uncover first coin and hence in this way he will get to know the results of two coins.

- But Aace got one doubt which is, if Bace uncovers second coin, his first and third coins disappeared and he himself uncovers first coin and remaining two coin disappeared, but there is no way to find out when they actually uncover the second coin. When Bace uncovers his coin, it does not have any influence on Aace's coin. In fact what Bace finds by uncovering his coin, it reveals some information about the coin of Aace.

- They went to their friend Chace to clarify their doubt. He told Bace to uncover his one coin and assume to know for sure what Aace will find when he uncovers his own coin without Aace disturbing his coin. Therefore there has to be some variables which are hidden that specify the condition of Aace's coins. And if we can anyhow know those hidden variables, then we will be able to find the value of Aace's coins.

- Chace told that there has to be some probability distribution that specify the condition on the three coins of Aace and it must not be negative and its sum is one. Aace cannot uncover all his coins, therefore he will not

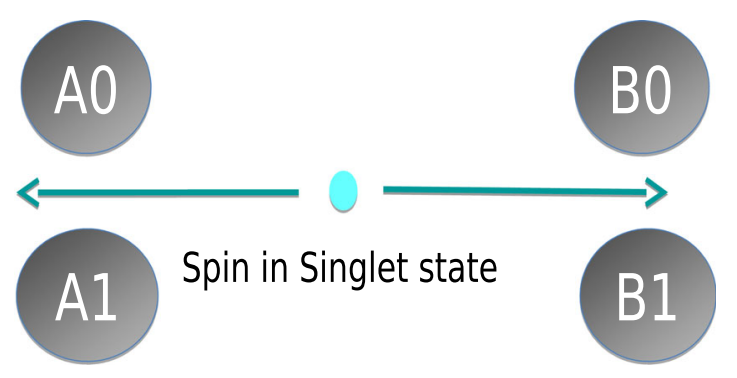

\section{The eigen values of $\mathrm{A} 0, \mathrm{~B} 0, \mathrm{~A} 1$ and $\mathrm{B} 1$ is +1 or -1 .}

Fig. 3 Schematic diagram of Bell's inequality example for a spin system

be able to measure the probability distribution. But with the help of Bace, he can uncover any two coins as Bace suggested.

- After doing the Bell experiment they found that the correlations found by them violate Bell's inequality.

\subsection{2nd Example on Bell's inequality}

See Fig. 3 for the representative setup for the spin system. Here the operators which are $A_{0}, B_{0}, A_{1}$ and $B_{1}$ correspond to measuring the spin and their eigenvalues are \pm 1 , we have to choose the value of operators as

$A_{0}=\mathbf{n}_{\mathbf{0}} . \sigma$,

$A_{1}=\mathbf{n}_{\mathbf{1}} \cdot \sigma$,

$B_{0}=\mathbf{n}_{\mathbf{0}} . \sigma$,

$B_{1}=\mathbf{n}_{\mathbf{1}} \cdot \sigma$.

Therefore assuming the other variable which is

$\langle R\rangle=\left\langle A_{0} B_{0}\right\rangle+\left\langle A_{1} B_{0}\right\rangle+\left\langle A_{0} B_{1}\right\rangle-\left\langle A_{1} B_{1}\right\rangle$.

According to classical theory of hidden variable,

$|\langle R\rangle| \leq 2$.

But in quantum mechanics, the expectation value of $\mathrm{R}$ can be found bigger. By squaring Eq. (2.5) one can show that

$R^{2}=4+\left[A_{1}, A_{0}\right]\left[B_{1}, B_{0}\right] \Rightarrow|\langle R\rangle|>2$,

making $|\langle R\rangle|$ larger than 2, which violates Bell's inequality. The question now arises is how to draw above conclusion, choosing:

$$
\begin{aligned}
& A_{0}=\mathbf{x} \cdot \sigma \\
& A_{1}=\mathbf{y} \cdot \sigma \\
& B_{0}=\sin \theta(\mathbf{x} \cdot \sigma)+\cos \theta(\mathbf{y} \cdot \sigma), \\
& B_{1}=\cos \theta(\mathbf{x} \cdot \sigma)-\sin \theta(\mathbf{y} \cdot \sigma),
\end{aligned}
$$


we get the extra $\sqrt{2}$ factor for the maximal violation i.e.

$|\langle R\rangle|>2 \sqrt{2}$.

\subsection{Review on the proof of Bell's inequality}

Bell's inequality gives a general condition, which holds for any local deterministic hidden-variable theory. Let us consider two spin half particles and we define two functions, which are $\mathrm{P}(a, \lambda)$ and $\mathrm{Q}(b, \lambda)$, which give results of the spin measurements on particle 1 in the direction of ' $a$ ' and on particle 2 in the direction of ' $b$ ', respectively. Here these functions depend on the parameter $\lambda$, which is a hidden variable.

Therefore we have

$P(a, \lambda)= \pm 1$,

$Q(b, \lambda)= \pm 1$.

Now we want to calculate average value of the product of two components $P(a, \lambda)$ and $Q(b, \lambda)$

$A(a, b)=\int \rho(\lambda) P(a, \lambda) Q(b, \lambda) \mathrm{d} \lambda$

where $\rho(\lambda)$ is the probability distribution of $\lambda$. Since

$A(d, d)=-1 \forall \mathrm{d}$

where the detectors are perfectly aligned and the results are perfectly anti-correlated. It means

$P(\mathrm{~d}, \lambda)=-Q(\mathrm{~d}, \lambda) \forall \lambda$.

Therefore

$$
\begin{aligned}
& A(a, b)=-\int \rho(\lambda) P(a, \lambda) Q(b, \lambda) \mathrm{d} \lambda, \\
& A(a, b)-A(a, c) \\
& \quad=-\int[\rho(\lambda) P(a, \lambda) P(b, \lambda)-P(a, \lambda) P(c, \lambda)],
\end{aligned}
$$

where ' $\mathrm{c}$ ' is taken as the unit vector.

Since

$$
(P(b, \lambda))^{2}=1 .
$$

Then

$A(a, b)-A(a, c)=-\int \rho(\lambda)[1-P(b, \lambda) P(c, \lambda)]$

$P(a, \lambda) P(b, \lambda) \mathrm{d} \lambda$.

Now we know that

$P(a, \lambda)= \pm 1$.
Therefore we can write

$-1 \leqslant P(a, \lambda) P(b, \lambda) \leqslant 1$,

$\rho(\lambda)[1-P(b, \lambda) P(c, \lambda)] \geqslant 0$.

Hence,

$|A(a, b)-A(a, c)| \leqslant \int \rho(\lambda)[1-P(b, \lambda) P(c, \lambda)] d \lambda$

or equivalently

$|A(a, b)-A(a, c)| \leqslant 1+A(b, c)$.

The above equation is the Bell inequality.

2.5 Bell's inequality in a spin system

In accordance to Chace, there has to be a variable $\lambda$, which is hidden to both Aace and Bace, and $\lambda$ describes spins of particles. Chace sets

$S(\hat{\mathbf{p}}, \lambda)= \pm 1$,

indicating the sign of the projection of the spin in $\hat{\mathbf{p}}$ direction. We know that the total spin is zero, therefore ' $S$ ' will give opposite spins of particles 1 and 2, due to the conservation of angular momentum. For Chace, $\lambda$ can take any value, but it will be fixed if the initial state is set up. We can find the value of $\lambda$, from the probability distribution $P(\lambda)$ where

$\int \mathrm{d} \lambda P(\lambda)=1$

Aace and Bace can measure the projection of spin in $\hat{\mathbf{d}}_{\mathbf{1}}$ and $\hat{\mathbf{d}_{2}}$ direction, respectively. While doing measurements consecutively, correlation function is

$$
\begin{aligned}
\left\langle\left(\mathbf{s}_{\mathbf{1}} \cdot \hat{\mathbf{d}_{\mathbf{1}}}\right)\left(\mathbf{s}_{\mathbf{2}} \cdot \hat{\mathbf{d}_{\mathbf{2}}}\right)\right\rangle & =-\left\langle\left(\mathbf{s}_{\mathbf{1}} \cdot \hat{\mathbf{d}_{\mathbf{1}}}\right)\left(\mathbf{s}_{\mathbf{1}} \cdot \hat{\mathbf{d}_{\mathbf{2}}}\right)\right\rangle \\
& =-\frac{1}{4} \int \mathrm{d} \lambda P(\lambda) S\left(\hat{\mathbf{d}_{\mathbf{1}}}, \lambda\right) \mathbf{S}(\hat{\mathbf{d}} \mathbf{2}, \lambda) \\
& =-\frac{1}{4} \hat{\mathbf{d}_{\mathbf{1}}} \cdot \hat{\mathbf{d}_{\mathbf{2}}}+\frac{i}{2}\left(\hat{\mathbf{d}_{\mathbf{1}}} \times \hat{\mathbf{d}_{2}}\right) \cdot\left\langle\mathbf{s}_{\mathbf{1}}\right\rangle .
\end{aligned}
$$

Now since here

$$
\left\langle\mathbf{s}_{\mathbf{1}}\right\rangle=\mathbf{0}
$$

one can finally write the following expression:

$$
\begin{aligned}
& \left\langle\left(\mathbf{s}_{\mathbf{1}} \cdot \hat{\mathbf{d}_{\mathbf{1}}}\right)\left(\mathbf{s}_{\mathbf{2}} \cdot \hat{\mathbf{d}_{2}}\right)\right\rangle=-\frac{1}{4} \hat{\mathbf{d}_{\mathbf{1}}} \cdot \hat{\mathbf{d}_{\mathbf{2}}} \\
& =\left\langle\left(\mathbf{s}_{\mathbf{1}} \cdot \hat{\mathbf{d}_{2}}\right)\left(\mathbf{s}_{\mathbf{2}} \cdot \hat{\mathbf{d}_{\mathbf{3}}}\right)\right\rangle+\frac{1}{4} \int \mathrm{d} \lambda P(\lambda) S\left(\hat{\mathbf{d}_{\mathbf{2}}}, \lambda\right) \\
& \quad \times\left[S\left(\hat{\mathbf{d}_{3}}, \lambda\right)-S\left(\hat{\mathbf{d}_{\mathbf{1}}}, \lambda\right)\right] .
\end{aligned}
$$


Now here we use the following constraint:

$$
S^{2}\left(\hat{\mathbf{d}_{3}}, \lambda\right)=1,
$$

and consequently we get

$$
\begin{gathered}
\left\langle\left(\mathbf{s}_{\mathbf{1}} \cdot \hat{\mathbf{d}_{\mathbf{1}}}\right)\left(\mathbf{s}_{\mathbf{2}} \cdot \hat{\mathbf{d}_{2}}\right)\right\rangle-\left\langle\left(\mathbf{s}_{\mathbf{1}} \cdot \hat{\mathbf{d}_{2}}\right)\left(\mathbf{s}_{\mathbf{2}} \cdot \hat{\mathbf{d}_{3}}\right)\right\rangle \\
=\frac{1}{4} \int d \lambda P(\lambda) S\left(\hat{\mathbf{d}_{2}}, \lambda\right) S\left(\hat{\mathbf{d}_{3}}, \lambda\right) \\
\quad \times\left[1-S\left(\hat{\mathbf{d}_{1}}, \lambda\right) S\left(\hat{\mathbf{d}_{3}}, \lambda\right)\right] .
\end{gathered}
$$

This directly implies that

$$
\begin{aligned}
& \left|\left\langle\left(\mathbf{s}_{\mathbf{1}} \cdot \hat{\mathbf{d}_{\mathbf{1}}}\right)\left(\mathbf{s}_{\mathbf{2}} \cdot \hat{\mathbf{d}_{2}}\right)\right\rangle-\left\langle\left(\mathbf{s}_{\mathbf{1}} \cdot \hat{\mathbf{d}_{\mathbf{2}}}\right)\left(\mathbf{s}_{\mathbf{2}} \cdot \hat{\mathbf{d}_{3}}\right)\right\rangle\right| \\
& \quad \leq \frac{1}{4} \int d \lambda P(\lambda)\left[1-S\left(\hat{\mathbf{d}_{1}}, \lambda\right) S\left(\hat{\mathbf{d}_{3}}, \lambda\right)\right] .
\end{aligned}
$$

Hence Bell's inequality follows from the theory of hidden variable and one can write

$\left|\left\langle\left(\mathbf{s}_{\mathbf{1}} \cdot \hat{\mathbf{d}_{\mathbf{1}}}\right)\left(\mathbf{s}_{\mathbf{2}} \cdot \hat{\mathbf{d}_{\mathbf{2}}}\right)\right\rangle-\left\langle\left(\mathbf{s}_{\mathbf{1}} \cdot \hat{\mathbf{d}_{\mathbf{2}}}\right)\left(\mathbf{s}_{\mathbf{2}} \cdot \hat{\mathbf{d}_{\mathbf{3}}}\right)\right\rangle\right| \leq \frac{1}{4}+\left\langle\left(\mathbf{s}_{\mathbf{1}} \cdot \hat{\mathbf{d}_{\mathbf{1}}}\right)\left(\mathbf{s}_{\mathbf{2}} \cdot \hat{\mathbf{d}_{\mathbf{3}}}\right)\right\rangle$

If we choose

$$
\begin{aligned}
\hat{\mathbf{d}_{1}} \cdot \hat{\mathbf{d}_{2}} & =0, \\
\hat{\mathbf{d}_{3}} & =\hat{\mathbf{d}_{\mathbf{1}}} \cos \theta+\hat{\mathbf{\mathbf { d } _ { 2 }}} \sin \theta,
\end{aligned}
$$

then one can write the following expression:

$$
\begin{aligned}
& \left\langle\left(\mathbf{s}_{\mathbf{1}} \cdot \hat{\mathbf{d}_{\mathbf{2}}}\right)\left(\mathbf{s}_{\mathbf{2}} \cdot \hat{\mathbf{d}_{3}}\right)\right\rangle=-\frac{1}{4} \sin \theta, \\
& \left\langle\left(\mathbf{s}_{\mathbf{1}} \cdot \hat{\mathbf{d}_{\mathbf{1}}}\right)\left(\mathbf{s}_{\mathbf{2}} \cdot \hat{\mathbf{d}} \mathbf{3}\right)\right\rangle=-\frac{1}{4} \cos \theta
\end{aligned}
$$

Hence we get

$$
\begin{aligned}
& \left|\left\langle\left(\mathbf{s}_{\mathbf{1}} \cdot \hat{\mathbf{d}_{\mathbf{1}}}\right)\left(\mathbf{s}_{\mathbf{2}} \cdot \hat{\mathbf{d}_{2}}\right)\right\rangle-\left\langle\left(\mathbf{s}_{\mathbf{1}} \cdot \hat{\mathbf{d}_{\mathbf{2}}}\right)\left(\mathbf{s}_{\mathbf{2}} \cdot \hat{\mathbf{d}_{3}}\right)\right\rangle\right|=\frac{1}{4}|\sin \theta|, \\
& \frac{1}{4}+\left\langle\left(\mathbf{s}_{\mathbf{1}} \cdot \hat{\mathbf{d}_{\mathbf{1}}}\right)\left(\mathbf{s}_{\mathbf{2}} \cdot \hat{\mathbf{d}_{3}}\right)\right\rangle=\frac{1}{4}(1-\cos \theta) .
\end{aligned}
$$

Here it is important to note that, according to Bell's inequality, the following quantity $I(\theta)$ is negative i.e.

$$
\begin{aligned}
I(\theta)= & \left|\left\langle\left(\mathbf{s}_{\mathbf{1}} \cdot \hat{\mathbf{d}_{\mathbf{1}}}\right)\left(\mathbf{s}_{\mathbf{2}} \cdot \hat{\mathbf{d}_{2}}\right)\right\rangle-\left\langle\left(\mathbf{s}_{\mathbf{1}} \cdot \hat{\mathbf{d}_{2}}\right)\left(\mathbf{s}_{\mathbf{2}} \cdot \hat{\mathbf{d}_{3}}\right)\right\rangle\right| \\
& +\frac{1}{4}+\left\langle\left(\mathbf{s}_{\mathbf{1}} \cdot \hat{\mathbf{d}_{\mathbf{1}}}\right)\left(\mathbf{s}_{\mathbf{2}} \cdot \hat{\mathbf{d}_{3}}\right)\right\rangle \\
= & \frac{1}{4}[|\sin \theta|+\cos \theta-1]<0 .
\end{aligned}
$$

But as an exception for the range $\theta<|\theta|<\frac{\pi}{2}$, the quantity $I(\theta)>0$.

\subsection{Violation of Bell inequality}

If reality is local then Bell's inequality must hold regardless of the angles at which polarization detectors are set. The first actual Bell test was done using Freedman's inequality in Ref. [29]. The delay in experiment was due to the inability to build perfect polarization detectors and to coordinate closed timed measurements that no speed of light could make it from one photon to the other within the duration of pair of measurements. The results of the above experiment confirmed the violation of Bell's inequality. Hence the inequality is wrong. However, the only assumption we used was the concept of locality.

\subsubsection{Case I: Explanation from earlier experiments}

After the proof presented in Ref. [29], many experiments were done such as

- Aspect (1982) [30,31], Tittel and Geneva group (1998) [32], Rowe (2001) [33]: These experiments are performed to close the detection loophole,

- Groblacher (2007) [34]: Test of Leggett-type nonlocal realist theories,

- Salart (2008) [35]: Separation in a Bell Test,

- Ansmann (2009) [36]: Overcoming the detection loophole in solid state,

- Christensen (2013) [37]: Overcoming the detection loophole for photons,

- Hensen (2015) [38]: A loophole-free Bell test and many others,

- Giustina (2015) [39], Shalm (2015) [40]: Recently performed Loophole-free Bell tests with photons which provide strong experimental proof for nonlocal reality.

\subsubsection{Case II: Explanation from recent experiments}

According to the local realism concept, physical properties of objects exist independently of measurement and physical influences cannot exceed the speed of light as we already know. Even though the previous experiments supported the predictions of quantum theory, yet every experiment requires assumptions which will provide loopholes for a local realist explanation. Therefore in this experiment they reported a Bell test that closes the most significant of these loopholes at the same instant of time. They used photons which are entangled in nature, rapid setting generation, and superconducting detectors with very efficiency and then observed violation of Bell inequality. Every time particles interact with one another their quantum states tend to entangle. Hence when one mem- 
ber of the pair is being measured then the other member behaves as if it is also being measured, and thus acquires a definite state.

\subsubsection{Case III: Explanation for entanglement}

We deduced from the violation of Bell's inequality that hidden variables theory is incorrect, therefore let us consider an experiment where large number of measurements are done on the spin of particles then the outcome should follow Bell's inequality but that does not happen. There are many experimental evidence which proves the violation of Bell's inequality, but those experiments have loophole problems that is the results of the measurements are correlated with each other which means we cannot measure properties simultaneously.

The following example will explain the entanglement in simple way in some steps:

- Let us say we have two-particle states having same mass, spin and also no forces acting on both particles.

- Let $\mathbf{p}_{\mathbf{1}}$ and $\mathbf{m}_{\mathbf{1}}$ and $\mathbf{p}_{\mathbf{2}}$ and $\mathbf{m}_{\mathbf{2}}$ be the position and momentum of first and second particle respectively. Therefore for the two particle system the basis states will be $\left.\left|\mathbf{p}_{\mathbf{1}}\right\rangle \otimes \mathbf{p}_{\mathbf{2}}\right\rangle$. But we should have states labeled by center of mass momentum, i.e.

$$
\begin{aligned}
\mathbf{M} & =\mathbf{m}_{\mathbf{1}}+\mathbf{m}_{\mathbf{2}}, \\
\mathbf{p} & =\mathbf{p}_{\mathbf{1}}+\mathbf{p}_{\mathbf{2}},
\end{aligned}
$$

therefore, unitary transformation to the basis is $|\mathbf{M}, \mathbf{p}\rangle$

- Now for instance Aace and Bace set up the two-particle system where initially $\mathbf{M}$ is 0 i.e. $\left|0, \mathbf{p}_{\mathbf{0}}\right\rangle$.

- Now Aace makes a measurement on the momentum of first particle and found the accurate outcome to be $\mathbf{m}_{\mathbf{1}}$, then

$\mathbf{m}_{2}=-\mathbf{m}_{1}$

There are large uncertainties in the positions of the two particles but

$\mathbf{p}=\mathbf{p}_{\mathbf{0}}+2 t \mathbf{m}_{\mathbf{1}} 1 /$ mass.

- When the two particles are very far from each other, then Bace accurately measures the position of the second particle which is $\mathbf{p}_{2}$. But we do not have any idea about the accurate values of position and momentum of both particles. When Bace measures $\mathbf{p}_{2}$, it makes momentum of the second particle $\mathbf{m}_{\mathbf{2}}$ uncertain, making $\mathbf{m}_{\mathbf{1}}$ uncertain instantaneously, this will occur even if the distance is very large.

- Hence this spooky action at a distance is known as quantum entanglement.

\section{Bell test experiment in primordial cosmology}

3.1 Setup for the cosmological Bell violating experiment

Metric of a uniform, spatially flat $(k=0)$, FLRW space-time is given by

$\mathrm{d} s^{2}=-\mathrm{d} t^{2}+a(t)^{2} \mathrm{~d} \mathbf{x}^{2}=a^{2}(\eta)\left[-\mathrm{d} \eta^{2}+\mathrm{d} \mathbf{x}^{2}\right]$

where $\mathrm{t}$ is proper time and $\eta$ is conformal time defined as

$\eta=\int \frac{\mathrm{d} t}{a(t)}$.

The conformal time described here is negative (so that we can make scale factor positive) and ranges from $-\infty$ to 0 . Here $a(t)$ is the scale factor which characterize the Hubble parameter $\frac{\dot{a}}{a} \approx H(t)$. During the inflationary period the scale factor grows exponentially $\left(a(t) \propto e^{H t}\right)$, just like de Sitter and quasi de Sitter space and consequently the Hubble parameter $H(t)$ varying slowly. Using this fact in Eq. (3.2), during inflation the scale factor can be expressed in terms of the conformal time $\eta$ as

$a(\eta)= \begin{cases}-\frac{1}{H \eta} & \text { for dS, } \\ -\frac{1}{H \eta}(1+\epsilon) & \text { for qdS, }\end{cases}$

where $\epsilon$ is the Hubble slow-roll parameter defined in Eq. (3.20). But for simplicity one can neglect the contribution from $\epsilon$ in the leading order for quasi de Sitter case as it is sufficiently small in the slow-roll regime. For our computation henceforth we will make this assumption. Additionally it is important to note that for the de Sitter case and the quasi de Sitter case the relation between conformal time $\eta$ and physical time $t$ can be expressed through the following expression:

$t=-\frac{1}{H} \ln (-H \eta)$,

which we will use throughout the paper. Within this setup inflation ends when the conformal time $\eta \sim 0$, as clearly depicted in Fig. 4.

Here it can easily be shown that quantum mechanics plays a very significant role producing spatially dependent fluctuations in terms of the scalar fields. We know that according to the theory of inflation, in the early universe, quantum mechanical effects are responsible for primordial fluctuations. But it is interesting to know that the fluctuations we have observed today is completely classical in nature. It is a very well-known fact that in the context of inflation all such fluctuations become classical as they exit the horizon and inside the horizon all of them are quantum. In this discussion the fluctuations are characterized by the following quantity, 


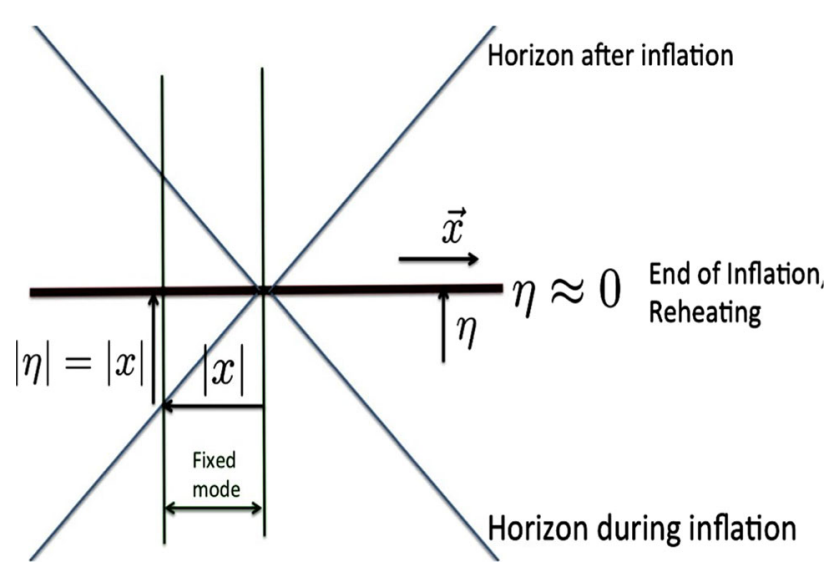

Fig. 4 Schematic diagram of the evolution of the universe

known as the curvature perturbation:

$$
\zeta=-\frac{H}{\dot{\bar{\rho}}} \delta \rho \quad \stackrel{\text { Fourier transform }}{\longrightarrow} \zeta_{k}=-\frac{H}{\dot{\overline{\phi_{0}}} M_{p}} \phi_{k}
$$

where for each value of $k$ in Fourier space it represents a harmonic oscillator. Now in FLRW background one can compute the following commutator:

$[\zeta, \dot{\zeta}] \propto a^{-3}$

where $\dot{\zeta}$ is the canonically conjugate variable of $\zeta$. Further this expression can be re-expressed after Fourier transformation as

$$
\begin{gathered}
\left|k^{3}\left[\zeta_{\mathbf{k}}, \dot{\zeta}_{\mathbf{k}}\right]\right| \sim k^{3}\left|\left[\phi_{\mathbf{k}}(\eta), \eta \partial_{\eta} \phi_{\mathbf{k}}\right]\right| \\
\approx \begin{cases}H^{3}(\eta k)^{3} & \text { for dS, } \\
H^{3}(\eta k)^{3}(1-3 \epsilon) & \text { for qdS, }\end{cases}
\end{gathered}
$$

which becomes zero as $\eta k \rightarrow 0$, at the end of inflation. This is the signature of Bell inequality violation in the context of primordial cosmological setup. Most importantly, after inflation when reheating occurs one can write down a classical measure or more precisely a classical probability distribution function of fluctuation $\zeta(x)$ as

$\rho[\zeta(x)]=|\Psi[\zeta(x)]|^{2} \Rightarrow \rho[\phi(x)]=\mu[\phi(x)]$.

Here $|\Psi[\zeta(x)]|^{2}$ or equivalently $\mu[\phi(x)]$ represent the classical probability distribution, ${ }^{5}$ which is nothing but the state

\footnotetext{
${ }^{5}$ For multifield case one needs to take the contribution from the isocurvature fluctuation as well. In such a physical situation the classical probability distribution function of curvature fluctuation $\zeta(x)$ and isocurvature fluctuation $\chi(x)$ can be written as

$$
\begin{aligned}
\rho[\zeta(x), \chi(x)] & =|\Psi[\zeta(x), \chi(x)]|^{2} \Rightarrow \rho\left[\phi_{i}(x), \phi_{j}(x)\right] \\
& =\mu\left[\phi_{i}(x), \phi_{j}(x)\right],
\end{aligned}
$$

where $i$ and $j$ stand for number field contents in multifield scenario.
}

of the universe at the spatial hyper surface where reheating occurs. In the present context, all the fluctuations can be treated as distribution of classical random variables. Additionally it is important to note that here, due to commutativity of $\zeta$ and $\dot{\zeta}$ at the end of inflation for the above mentioned non-commutative observables, it is not at all possible to distinguish $|\Psi[\zeta(x)]|^{2}$ from classical probability distribution function $\rho[\zeta(x)]$. Now here one can also calculate spread in the canonically conjugate variable $\dot{\zeta}_{\mathbf{k}}$ of scalar curvature fluctuation $\zeta_{\mathbf{k}}$ in Fourier space as

$\sqrt{\left\langle\left|\dot{\zeta}_{\mathbf{k}}\right|^{2}\right\rangle} \propto \tilde{c}_{S}^{2}(\eta k)^{2}$

where in the present context the effective sound speed $c_{S}$ is defined as

$c_{S}=\tilde{c}_{S} \sqrt{1+\frac{\bar{M}_{1}^{3}}{\epsilon H M_{p}^{2}}}$,

where $\tilde{c}_{S}$ is the actual sound speed in the absence of all effective interactions and in our discussion it is defined as

$\tilde{c}_{S}=\frac{1}{\sqrt{1-\frac{2 M_{2}^{4}}{\dot{H} M_{p}^{2}}}}$.

Here $\bar{M}_{1}^{3}$ and $M_{2}$ are the time-dependent coefficients of specific type of effective interactions introduced in Ref. [25]. In the slow-roll regime, $\frac{\bar{M}_{1}^{3}}{\epsilon H M_{p}^{2}}$ is a very slowly varying function with respect to time and can be treated as a constant for our discussion. Here it is important to note that, after horizon crossing, the modes become classical in nature and in that case the spread becomes zero as $\eta k \rightarrow 0$, at the end of inflation. As a result we are not able to measure the canonically conjugate variable through various cosmological observations. But in the present context of discussion from the computed classical probability distribution function $\rho[\zeta(x)]$ one cannot comment on the exact measurement procedure on a quantum state. This type of phenomena is commonly studied in the context of quantum mechanical decoherence in which to set up a measuring device one needs to introduce a coupling between additional environment and long wavelength cosmological perturbations in the present context of discussion. Decoherence in quantum mechanics is guided by the dynamical behaviour of the phase factor appearing in the expression for the quantum mechanical wave function $\Psi[\zeta(x)]$. On the other hand, here one can say that $|\Psi[\zeta(x)]|^{2}$ is connected with the correlation functions in cosmological perturbation theory. Now as $|\Psi[\zeta(x)]|^{2}$ cannot be distinguishable from classical probability distribution function $\rho[\zeta(x)]$, one can easily identify this quantity 
with the post inflationary correlations functions in the context of primordial cosmology. Let us mention few possibilities in the following for system-environment interactions and associated couplings which are commonly used to study the phenomena of quantum decoherence during inflationary epoch:

1. Gravitational waves $[7-10,12-19,41-58]$,

2. Effects of multifield components and associated isocurvature perturbation [7-10,12-19,41-58],

3. Interaction between short and long wavelength fluctuations in cosmological perturbations $[7-10,12-19,41-$ $58]$,

4. Contribution from the self interaction between inflatons [7-10,12-19,41-58].

More generically, such interactions with the additional environment can be expressed in FLRW background as

$$
\begin{aligned}
H_{\text {int }} & =\int \mathrm{d}^{3} x a^{3} \zeta(\mathbf{x}) G(\mathbf{x}) \\
& = \begin{cases}-\int \mathrm{d}^{3} x \frac{\zeta(\mathbf{x}) G(\mathbf{x})}{H^{3} \eta^{3}} & \text { for } \mathrm{dS}, \\
-\int \mathrm{d}^{3} x \frac{\zeta(\mathbf{x}) G(\mathbf{x})}{H^{3} \eta^{3}}(1+2 \epsilon) & \text { for qdS, }\end{cases}
\end{aligned}
$$

where $\zeta(\mathbf{x})$ signifies the scalar curvature fluctuation and $G(\mathbf{x})$ characterizes source function for high frequency fluctuation in real position space. Additionally it is important to note that, as the approximate time translational symmetry and a nearly scale invariant feature is maintained in the primordial power spectrum for the scalar modes the dynamical behaviour of the decoherence phenomena is the same in all momentum scales. For more details on this crucial aspect, see Appendix 6.1. Additionally, in the present one can interpret $\zeta(\mathbf{x})$ as the Goldstone modes that is appearing from the breaking of time translational symmetry in the de Sitter and quasi de Sitter cosmological background. This is exactly equivalent to spontaneous symmetry breaking mechanism applicable in the context of gauge theory [25].

\subsection{Creation of new massive particle}

The classical time dependence of the inflation leads to a timedependent mass $m(\eta)$. The equation of motion for the massive field is 6

$h_{k}^{\prime \prime}+\left\{c_{S}^{2} k^{2}+\left(\frac{m^{2}}{H^{2}}-2\right) \frac{1}{\eta^{2}}\right\} h_{k}=0 \quad$ for dS,

\footnotetext{
${ }^{6}$ In case of scalar curvature fluctuation equation of motion for inflaton field looks like exactly similar to the heavy field case and in that case we need to replace heavy particle mass term $m(\eta)$ with the inflaton mass term $m_{\text {inf }}$. In most of the computations one assumes that $m_{\text {inf }}<<$ $H$. But we keep this term intact and will explicitly show that this
}

$h_{k}^{\prime \prime}+\left\{c_{S}^{2} k^{2}+\left(\frac{m^{2}}{H^{2}}-\left[v^{2}-\frac{1}{4}\right]\right) \frac{1}{\eta^{2}}\right\} h_{k}=0$ for qdS,

where in the quasi de Sitter case the parameter $v$ can be written as

$v=\frac{3}{2}+\epsilon+\frac{\eta}{2}+\frac{s}{2}$

where $\epsilon$ and $\eta$ are the Hubble slow-roll parameters defined as

$$
\begin{aligned}
\epsilon & =-\frac{\dot{H}}{H^{2}}, \\
\eta & =\frac{\dot{\epsilon}}{H \epsilon}, \\
s & =\frac{\dot{c_{S}}}{H c_{S}} .
\end{aligned}
$$

In the slow-roll regime of inflation $\epsilon<<1$ and $|\eta|<<1$ and at the end of inflation sow-roll condition breaks when any of the criteria satisfy: (1) $\epsilon=1$ or $|\eta|=1,(2) \epsilon=1=|\eta|$.

The most general solution of the mode function for de Sitter case and the quasi de Sitter case can be written as

$$
\begin{aligned}
& h_{k}(\eta)
\end{aligned}
$$

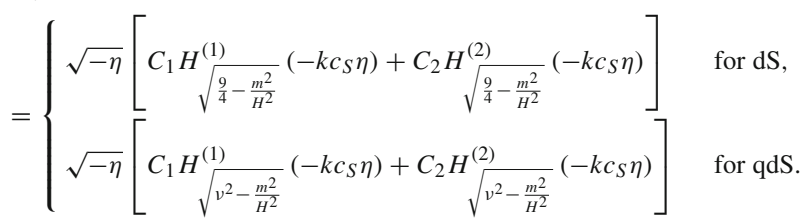

Footnote 6 continued

contribution is necessarily required to explain the observed data for inflation. Here for scalar curvature fluctuation equation of motion for inflaton field can be written as

$h_{k}^{\prime \prime}+\left\{c_{S}^{2} k^{2}+\left(\frac{m_{\mathrm{inf}}^{2}}{H^{2}}-2\right) \frac{1}{\eta^{2}}\right\} h_{k}=0$ for dS,

$h_{k}^{\prime \prime}+\left\{c_{S}^{2} k^{2}+\left(\frac{m_{\mathrm{inf}}^{2}}{H^{2}}-\left[v^{2}-\frac{1}{4}\right]\right) \frac{1}{\eta^{2}}\right\} h_{k}=0$ for qdS.

The most general solution of the mode function for the de Sitter case and the quasi de Sitter case can be written as

$=\left\{\begin{array}{c}\sqrt{-\eta}\left[C_{1} H_{\sqrt{\frac{9}{4}-\frac{m_{\text {inf }}^{2}}{H^{2}}}}^{(1)}\left(-k c_{S} \eta\right)+C_{2} H^{(2)}\right. \\ \left.\sqrt{\frac{9}{4}-\frac{m_{\text {inf }}^{2}}{H^{2}}}\left(-k c_{S} \eta\right)\right] \\ \sqrt{-\eta}\left[C_{1} H_{\sqrt{v^{2}-\frac{m_{\text {inf }}^{2}}{H^{2}}}}^{(1)}\left(-k c_{S} \eta\right)+C_{2} H^{(2)}\right. \\ \left.\sqrt{v^{2}-\frac{m_{\text {inf }}^{2}}{H^{2}}}\left(-k c_{S} \eta\right)\right]\end{array} \quad\right.$ for dS,

Here $C_{1}$ and $C_{2}$ are the arbitrary integration constants and the numerical value depend on the choice of the initial condition or more precisely the vacuum. 
Here $C_{1}$ and $C_{2}$ are the arbitrary integration constants and the numerical value depend on the choice of the initial condition or more precisely the vacuum. In the present context apart from the arbitrary vacuum we consider the following choice of the vacuum for the computation:

1. Bunch-Davies vacuum In this case we choose $C_{1}=\sqrt{\frac{\pi}{2}}$ and $C_{2}=0$.

2. $\alpha$ vacuum Type-IIn this case we choose $C_{1}=\cosh \alpha$ and $C_{2}=e^{i \delta} \sinh \alpha$. Here $\delta$ is a phase factor.

3. $\alpha$ vacuum Type-II In this case we choose $C_{1}=N_{\alpha}$ and $C_{2}=N_{\alpha} e^{\alpha}$. Here $N_{\alpha}=\frac{1}{\sqrt{1-e^{\alpha+\alpha^{*}}}}$.

4. Special vacuum In this case we choose $C_{1}=C_{2}=C$.

Here it is important to mention that the argument in the Hankel function for the solution of the $h_{k}$ takes the following values in different regime: ${ }^{7}$

For dS: $\quad \sqrt{\frac{9}{4}-\frac{m^{2}}{H^{2}}} \approx \begin{cases}\frac{\sqrt{5}}{2} & \text { for } m \approx H, \\ \frac{3}{2} & \text { for } m<<H, \\ i \sqrt{\Upsilon^{2}-\frac{9}{4}} & \text { for } m>>H,\end{cases}$

For qdS: $\quad \sqrt{v^{2}-\frac{m^{2}}{H^{2}}} \approx \begin{cases}\sqrt{v^{2}-1} & \text { for } m \approx H, \\ v & \text { for } m<<H, \\ i \sqrt{\Upsilon^{2}-v^{2}} & \text { for } m>>H .\end{cases}$

Here we set $m=\Upsilon H$, where the parameter $\Upsilon>>1$ for $m>>$. In the present context we are interested in the following cases for both de Sitter and quasi de Sitter solutions which we will follow throughout the rest of the discussion in this paper:

1. Case $I m \approx H$, in which we treat the mass scale of the heavy fields is comparable with the inflationary scale.

$\overline{7}$ In case of inflaton field, argument of the Hankel function involves the inflaton mass $m_{\text {inf }}$ term as given by

For dS: $\quad \sqrt{\frac{9}{4}-\frac{m_{\mathrm{inf}}^{2}}{H^{2}}} \approx \begin{cases}\frac{\sqrt{5}}{2} & \text { for } m_{\mathrm{inf}} \approx H, \\ \frac{3}{2} & \text { for } m_{\mathrm{inf}}<<H,\end{cases}$

For qdS: $\quad \sqrt{v^{2}-\frac{m_{\mathrm{inf}}^{2}}{H^{2}}} \approx \begin{cases}\sqrt{v^{2}-1} & \text { for } m_{\mathrm{inf}} \approx H, \\ v & \text { for } m_{\mathrm{inf}}<<H .\end{cases}$

Here the inflation mass is always $m_{\mathrm{inf}}<<H$ or $m_{\mathrm{inf}} \approx H$, as the mass scale of the inflaton cannot be larger the scale of inflation itself.
This is a special case where we treat $m / H$ is a constant parameter for the sake of simplicity. In this case the particle production of heavy fields deal with nonlocal effects. But only changing the structure of effective Lagrangian it is not at all possible to explain the characteristic of nonlocal effects in the present context.

2. Case II $m>>H$, in which we treat the mass scale of the heavy fields is much higher compared to the the inflationary scale. This is another special case where we treat $m / H$ is a constant parameter for the sake of simplicity. In this case one can interpret that such heavy fields belongs to the hidden sector. In this case we can integrate them from the theory and finally they generate an effective field theory of light inflaton fields. As we do not know anything about the UV complete theory of inflation it is not possible to detect all such heavy contributions.

3. Case III $m<<H$, in which we treat the mass scale of the heavy fields is much smaller compared to the inflationary scale. In this case one can neglect the contributions from all such fields in the mode equation for scalar fluctuations. This situation is exactly similar to the inflationary framework as the mode function for the scalar fluctuation are exactly same and in such a physical situation these extra dynamical fields serves the purpose of inflaton. One can interpret this situation by using the two-field scenario or the inflaton-curvaton scenario in the present context. Here it is important to mention that this specific scenario does not give rise to the violation of cosmological Bell's inequality. We have quoted the results for completeness, which gives the information as regards the particle production during inflation, where the effect of the heavy particle mass is negligibly small compared to the scale of inflation or background cosmological Hubble scale.

4. Case IV We also take the following phenomenological cases for the conformal time-dependent parametrization on mass parameter:

A. $m=\sqrt{\gamma\left(\frac{\eta}{\eta_{0}}-1\right)^{2}+\delta} H$, where $\gamma, \delta$ and $\eta_{0}$ are fixed parameters of the model. This is very special model using which one can explicitly study the specific amount and significant signatures of Bell violation in primordial cosmological setup. In Ref. [3] it is first proposed to study the Baroque model of the universe to study the violation of cosmological Bell inequalities.

B. $m=\frac{m_{0}}{\sqrt{2}} \sqrt{\left[1-\tanh \left(\frac{\rho}{H} \ln (-H \eta)\right)\right]}$, where $\rho$ and $m_{0}$ are fixed parameters of the model. This is a model for the heavy particle mass which was earlier used to study the phenomena of quantum critical quench and thermalization in the context of Conformal Field Theory (CFT). In case of quantum quench $m_{0}$ is known 


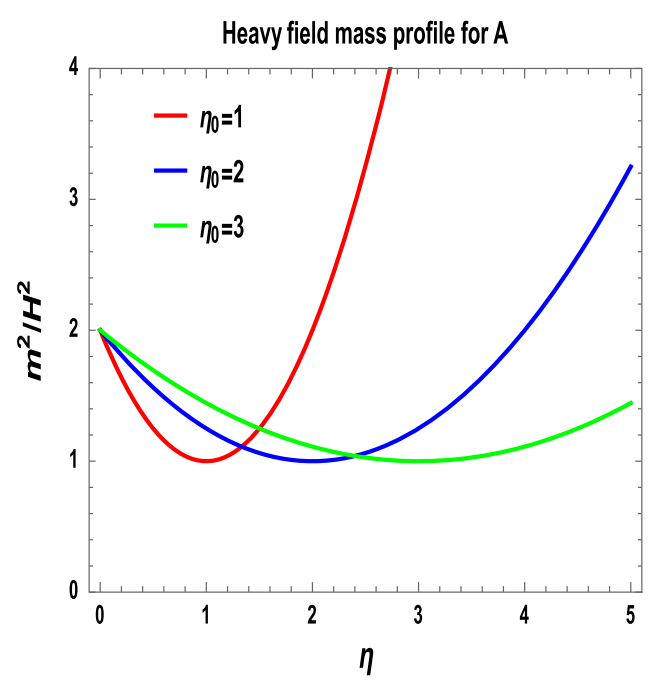

(a) Heavy field mass profile for A with $\gamma=1$ and $\delta=1$.

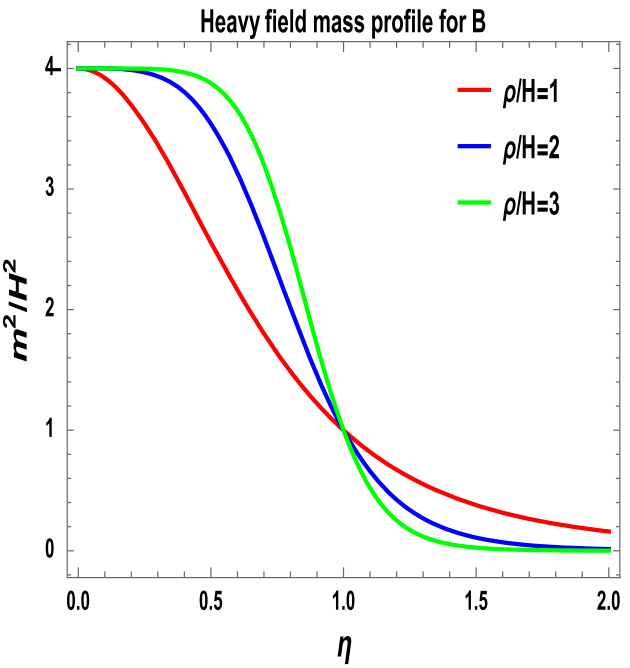

(b) Heavy field mass profile for B with $\frac{m_{0}^{2}}{2 H^{2}}=1$.

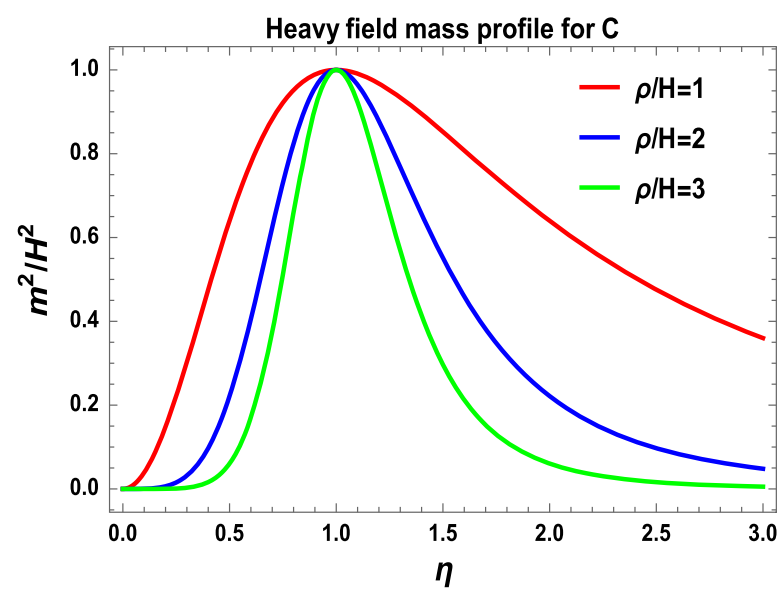

(c) Heavy field mass profile for $\mathrm{C}$ with $\frac{m_{0}^{2}}{H^{2}}=1$.

Fig. 5 Behaviour of the heavy field mass profile with $\eta$

as the quench parameter. See Refs. [59-66] for more details in this direction. In this context we are interested in this specific type of mass parametrization as the corresponding equivalent version of Schrödinger quantum mechanical problem can easily solvable. Here this can be treated as another model to explain the parametrization of heavy particle mass parameter.

C. $m=m_{0} \operatorname{sech}\left(\frac{\rho}{H} \ln (-H \eta)\right)$, where $\rho$ and $m_{0}$ are fixed parameters of the model. This is another model for the heavy particle mass which was also earlier used to study the phenomena of quantum critical quench and thermalization in the context of Conformal Field Theory (CFT). As mentioned earlier in the case of quantum quench $m_{0}$ is known as the quench parameter. See Refs. [59-66] for more details in this direction. Here this can be treated as another model to explain the parametrization of heavy particle mass parameter. In the last part of this paper we have shown that the axion decay constant profile in string theory is exactly mimics the same behaviour as presented in this context (Figs. 5 and 6).

For the most general solution as stated in Eq. (6.85) one can consider the following limiting physical situations:

1. Superhorizon regime: $\left|k c_{S} \eta\right|<<1$ or equivalently $\left|k c_{S} \eta\right| \rightarrow 0$,

2. Horizon crossing: $\left|k c_{S} \eta\right|=1$ or equivalently $\left|k c_{S} \eta\right| \approx$ $1-\Delta$ with $\Delta \rightarrow 0$, 


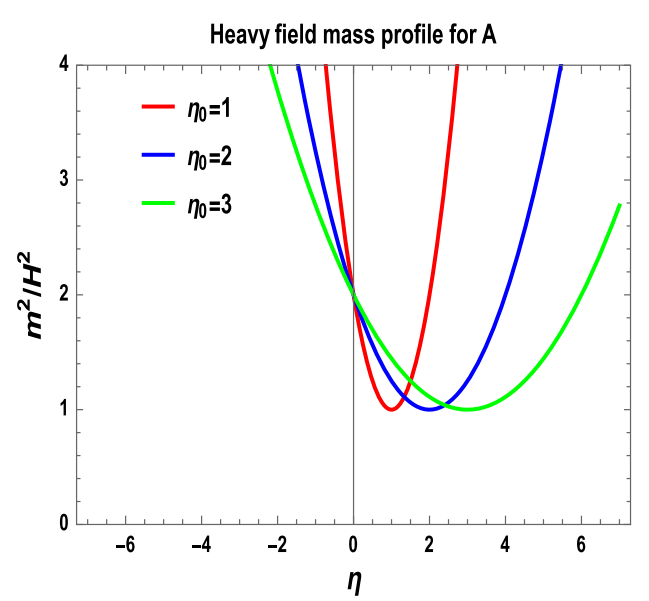

(a) Heavy field mass profile for A with $\gamma=1$ and $\delta=1$.

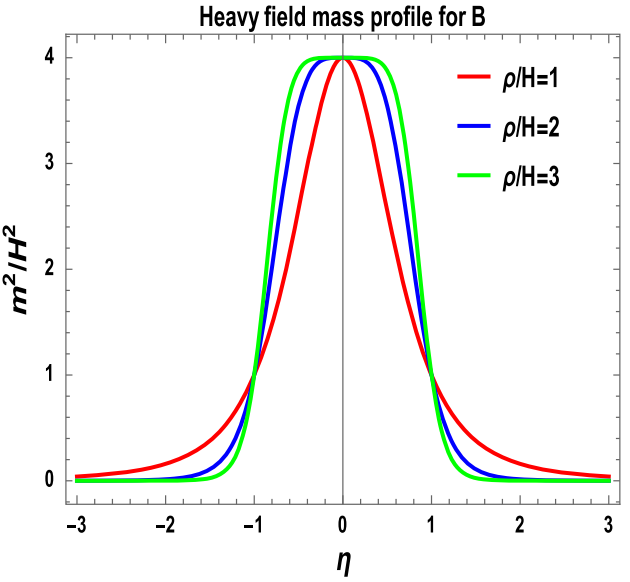

(b) Heavy field mass profile for B with $\frac{m_{0}^{2}}{2 H^{2}}=1$.

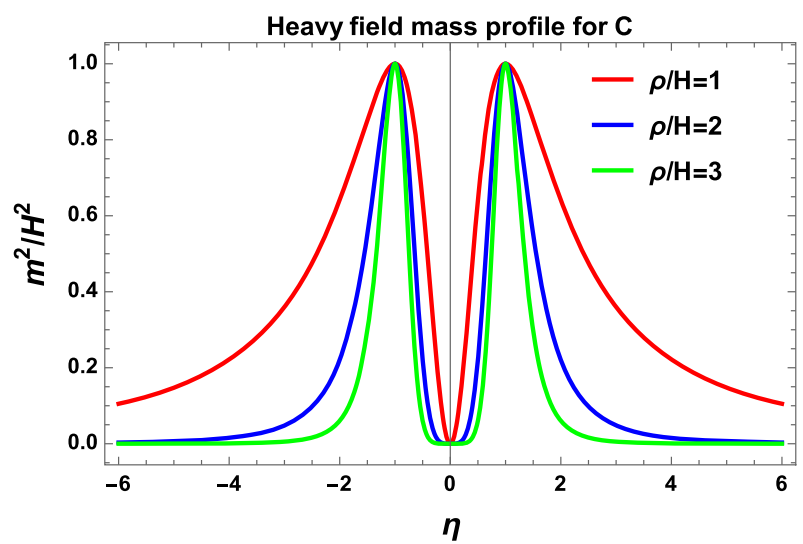

(c) Heavy field mass profile for C with $\frac{m_{0}^{2}}{H^{2}}=1$.

Fig. 6 Behaviour of the heavy field mass profile for the whole range of $\eta$

3. Subhorizon regime: $\left|k c_{S} \eta\right|>>1$ or equivalently $\left|k c_{S} \eta\right| \rightarrow-\infty$.

Consequently for the arbitrary choice of the initial condition or vacuum we get the following results:

$$
\begin{aligned}
& \lim _{k c_{S} \eta \rightarrow-\infty} H_{\Lambda}^{(1,2)}\left(-k c_{S} \eta\right) \\
& = \pm \sqrt{\frac{2}{\pi}} \frac{1}{\sqrt{-k c_{S} \eta}} e^{\mp i k c_{S} \eta} e^{\mp \frac{i \pi}{2}\left(\Lambda+\frac{1}{2}\right)} \\
& \lim _{k c_{S} \eta \rightarrow 0} H_{\Lambda}^{(1,2)}\left(-k c_{S} \eta\right)= \pm \frac{i}{\pi} \Gamma(\Lambda)\left(-\frac{k c_{S} \eta}{2}\right)^{-\Lambda} .
\end{aligned}
$$

where the parameter $\Lambda$ is defined as ${ }^{8}$

$\Lambda= \begin{cases}\sqrt{\frac{9}{4}-\frac{m^{2}}{H^{2}}} & \text { for } \mathrm{dS}, \\ \sqrt{v^{2}-\frac{m^{2}}{H^{2}}} & \text { for qdS. }\end{cases}$

One can also consider the following approximations to simplify the final derived form of the solution for arbitrary vacuum with $\left|k c_{S} \eta\right|=1$ or equivalently $\left|k c_{S} \eta\right| \approx 1-\Delta$ with $\Delta \rightarrow 0$ :

${ }^{8}$ For inflaton field we haveL
$\Lambda= \begin{cases}\sqrt{\frac{9}{4}-\frac{m_{\text {inf }}^{2}}{H^{2}}} & \text { for dS } \\ \sqrt{v^{2}-\frac{m_{\text {inf }}^{2}}{H^{2}}} & \text { for qdS, }\end{cases}$ 
1. We start with the Laurent expansion of the Gamma function:

$$
\begin{aligned}
& \Gamma(\Lambda)=\frac{1}{\Lambda}-\gamma+\frac{1}{2}\left(\gamma^{2}+\frac{\pi^{2}}{6}\right) \Lambda-\frac{1}{6}\left(\gamma^{3}+\frac{\gamma \pi^{2}}{2}+2 \zeta(3)\right) \Lambda^{2}+\mathcal{O}\left(\Lambda^{3}\right)
\end{aligned}
$$

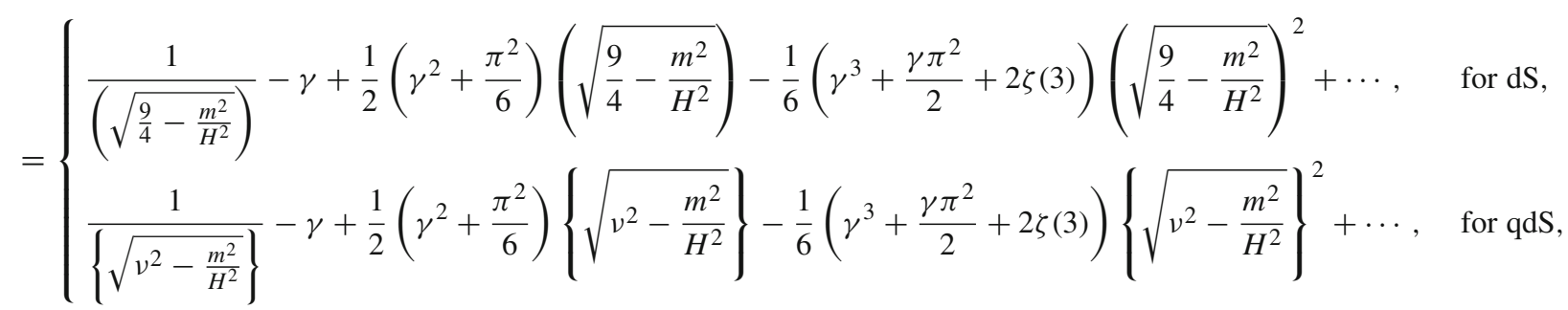

where $\gamma$ is known as the Euler-Mascheroni constant and $\zeta(3)$ characterizing the Reimann zeta function of order 3 originating in the expansion of the gamma function.

2. In this case the solution Hankel functions of first and second kind can be re-expressed in the following simplified form:

$$
\begin{aligned}
\lim _{\left|k c_{S} \eta\right| \approx 1-\Delta(\rightarrow 0)} H_{\Lambda}^{(1,2)}\left(-k c_{S} \eta\right)= & \pm \frac{i}{\pi}\left[\frac{1}{\Lambda}-\gamma+\frac{1}{2}\left(\gamma^{2}+\frac{\pi^{2}}{6}\right) \Lambda\right. \\
& \left.-\frac{1}{6}\left(\gamma^{3}+\frac{\gamma \pi^{2}}{2}+2 \zeta(3)\right) \Lambda^{2}+\cdots\right]\left(\frac{1+\Delta}{2}\right)^{-\Lambda} .
\end{aligned}
$$

After taking the $k c_{S} \eta \rightarrow-\infty, k c_{S} \eta \rightarrow 0$ and $\left|k c_{S} \eta\right| \approx 1-\Delta(\rightarrow 0)$ limits the most general solution as stated in Eq. (6.85) can be recast as

$$
\begin{aligned}
& h_{k}(\eta) \stackrel{\left|k c_{S} \eta\right| \rightarrow-\infty}{=} \sqrt{\frac{2}{\pi k c_{S}}}\left[C_{1} e^{-i k c_{S} \eta} e^{-\frac{i \pi}{2}\left(\Lambda+\frac{1}{2}\right)}+C_{2} e^{i k c_{S} \eta} e^{\frac{i \pi}{2}\left(\Lambda+\frac{1}{2}\right)}\right]
\end{aligned}
$$

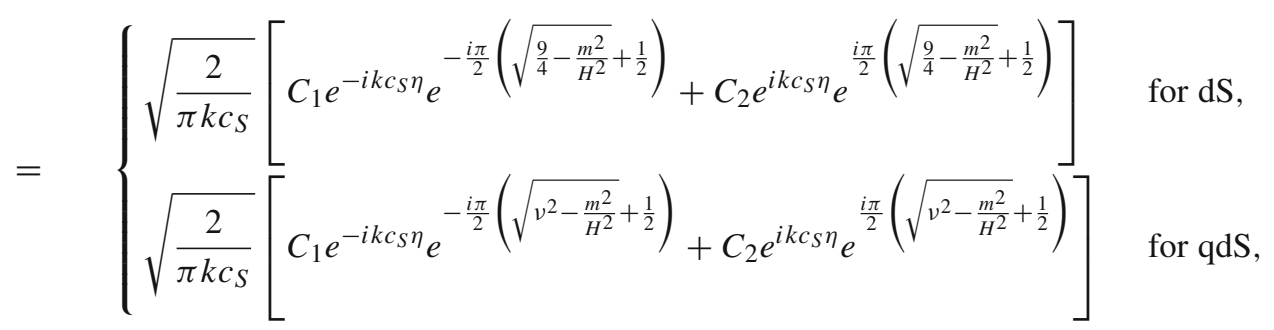

$$
\begin{aligned}
& h_{k}(\eta) \stackrel{\left|k c_{S} \eta\right| \rightarrow 0}{=} \frac{i}{\pi} \sqrt{-\eta} \Gamma(\Lambda)\left(-\frac{k c_{S} \eta}{2}\right)^{-\Lambda}\left[C_{1}-C_{2}\right] \\
& =\left\{\begin{array}{l}
\frac{i \sqrt{-\eta}}{\pi} \Gamma\left(\sqrt{\frac{9}{4}-\frac{m^{2}}{H^{2}}}\right)\left(-\frac{k c_{S} \eta}{2}\right)^{-\sqrt{\frac{9}{4}-\frac{m^{2}}{H^{2}}}}\left[C_{1}-C_{2}\right] \text { for } \mathrm{d} S, \\
\frac{i \sqrt{-\eta}}{\pi} \Gamma\left(\sqrt{v^{2}-\frac{m^{2}}{H^{2}}}\right)\left(-\frac{k c_{S} \eta}{2}\right)^{-\sqrt{v^{2}-\frac{m^{2}}{H^{2}}}}\left[C_{1}-C_{2}\right] \text { for qdS, }
\end{array}\right.
\end{aligned}
$$




$$
\begin{aligned}
& h_{k}(\eta) \stackrel{|k c s \eta| \approx 1-\Delta(\rightarrow 0)}{=} \frac{i}{\pi} \sqrt{-\eta}\left[\frac{1}{\Lambda}-\gamma+\frac{1}{2}\left(\gamma^{2}+\frac{\pi^{2}}{6}\right) \Lambda-\frac{1}{6}\left(\gamma^{3}+\frac{\gamma \pi^{2}}{2}+2 \zeta(3)\right) \Lambda^{2}+\cdots\right]\left(\frac{1+\Delta}{2}\right)^{-\Lambda}\left[C_{1}-C_{2}\right] \\
& =\left\{\begin{array}{l}
\frac{i}{\pi} \sqrt{-\eta}\left[\frac{1}{\left(\sqrt{\frac{9}{4}-\frac{m^{2}}{H^{2}}}\right)}-\gamma+\frac{1}{2}\left(\gamma^{2}+\frac{\pi^{2}}{6}\right)\left(\sqrt{\frac{9}{4}-\frac{m^{2}}{H^{2}}}\right)\right. \\
-\frac{1}{6}\left(\gamma^{3}+\frac{\gamma \pi^{2}}{2}+2 \zeta(3)\right)\left(\sqrt{\left.\left.\frac{9}{4}-\frac{m^{2}}{H^{2}}\right)^{2}+\cdots\right]}\right. \\
\times\left(\frac{1+\Delta}{2}\right)^{-\sqrt{\frac{9}{4}-\frac{m^{2}}{H^{2}}}}\left[C_{1}-C_{2}\right] \\
\frac{i}{\pi} \sqrt{-\eta}\left[\frac{1}{\left\{\sqrt{v^{2}-\frac{m^{2}}{H^{2}}}\right\}}-\gamma+\frac{1}{2}\left(\gamma^{2}+\frac{\pi^{2}}{6}\right)\left\{\sqrt{v^{2}-\frac{m^{2}}{H^{2}}}\right\}\right. \\
\left.\quad-\frac{1}{6}\left(\gamma^{3}+\frac{\gamma \pi^{2}}{2}+2 \zeta(3)\right)\left\{\sqrt{v^{2}-\frac{m^{2}}{H^{2}}}\right\}^{2}+\cdots\right] \\
\quad\left(\frac{1+\Delta}{2}\right)^{-\sqrt{v^{2}-\frac{m^{2}}{H^{2}}}}\left[C_{1}-C_{2}\right]
\end{array}\right.
\end{aligned}
$$

In the next subsections we use all these limiting results for the cases previously mentioned: (1) $m \approx H$, (2) $m>>H$, (3) $m<<H$. Here we can think of a physical condition where the WKB approximation is valid (approximately) for the solution for the mode function $h_{k}$. Here we provide the solution for the fluctuations by exactly solving the equation of motion for the heavy fields, where we assume that time variation in heavy field mass parameter is very slow. For arbitrary time dependence case it is only possible depending on the complexity of the mathematical structure of the heavy field mass parameter $m(\eta)$. In the standard WKB approximation the total solution can be recast in the following form:

$$
h_{k}(\eta)=\left[D_{1} u_{k}(\eta)+D_{2} \bar{u}_{k}(\eta)\right],
$$

where $D_{1}$ and $D_{2}$ are two arbitrary integration constants, which depend on the choice of the initial condition on making the WKB approximation at early and late time scale. In our discussion two arbitrary integration constants $D_{1}$ and $D_{2}$ can be identified with the Bogoliubov coefficient in momentum space: ${ }^{9}$

\footnotetext{
$\overline{9}$ Here one can choose another convention for the Bogoliubov coefficient in momentum space as given by

$D_{1}=\alpha(k)$,

$D_{2}=\beta(k)$.

But for our computation we will follow other convention for the Bogoliubov coefficient in momentum space stated in Eq. (3.40).
}

$D_{1}=\beta(k)$,

$D_{2}=\alpha(k)$.

In the present context $u_{k}(\eta)$ and $\bar{u}_{k}(\eta)$ are defined as

$$
\begin{aligned}
& u_{k}(\eta)=\frac{1}{\sqrt{2 p(\eta)}} \exp \left[i \int^{\eta} \mathrm{d} \eta^{\prime} p\left(\eta^{\prime}\right)\right] \\
& \bar{u}_{k}(\eta)=\frac{1}{\sqrt{2 p(\eta)}} \exp \left[-i \int^{\eta} \mathrm{d} \eta^{\prime} p\left(\eta^{\prime}\right)\right]
\end{aligned}
$$

where we have written the total solution for the mode $h_{k}$ in terms of two linearly independent solutions. Here in the most general situation the new conformal time-dependent factor $p(\eta)$ is defined as

$$
p(\eta)= \begin{cases}\sqrt{\left\{c_{S}^{2} k^{2}+\left(\frac{m^{2}}{H^{2}}-2\right) \frac{1}{\eta^{2}}\right\}} & \text { for dS, } \\ \sqrt{\left\{c_{S}^{2} k^{2}+\left(\frac{m^{2}}{H^{2}}-\left[v^{2}-\frac{1}{4}\right]\right) \frac{1}{\eta^{2}}\right\}} & \text { for qdS. }\end{cases}
$$

which we use thoroughly in our computation. Here it is important to mention the expressions for the controlling factor $p(\eta)$ in different regime of solution: 


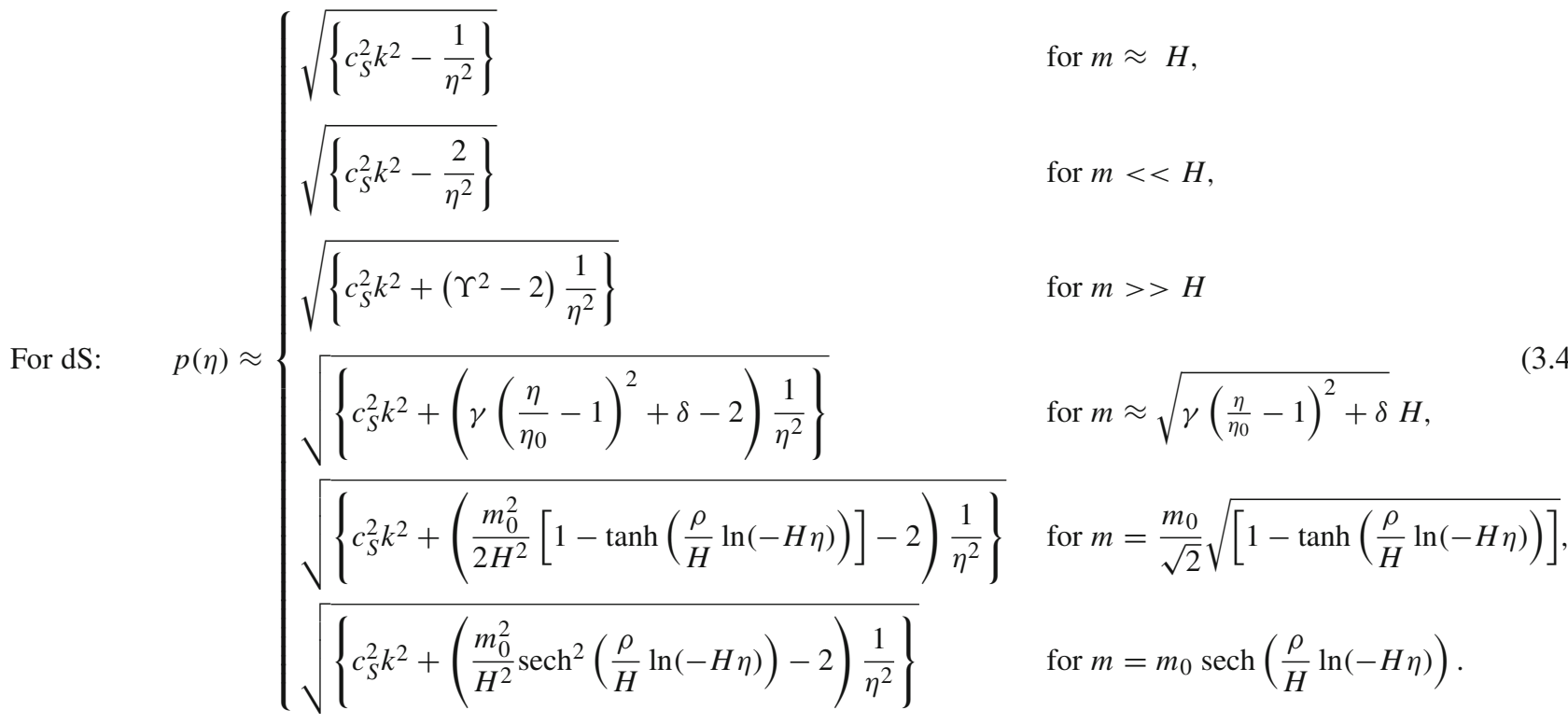

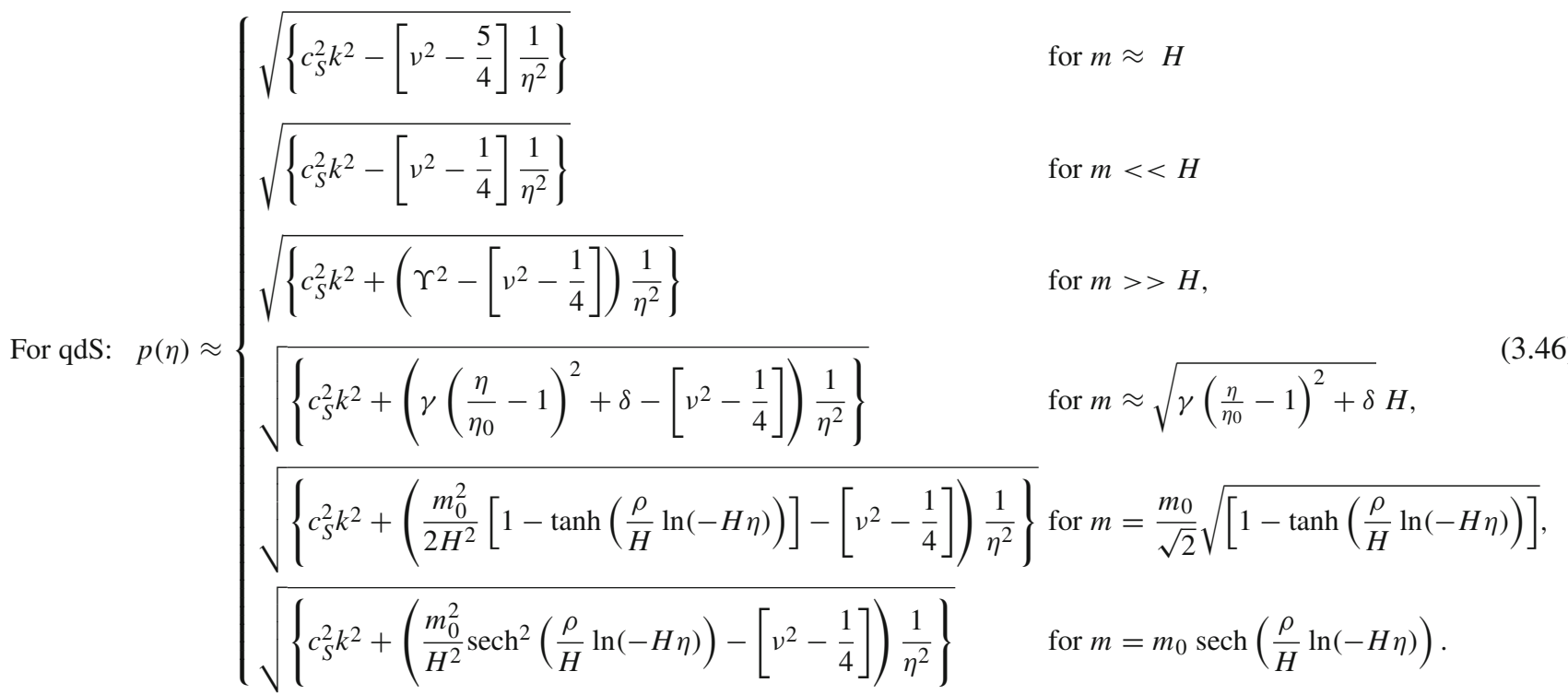

It is important to note that, if in the present discussion the WKB approximation were exactly valid, then for the EFT driven present cosmological setup no particle creation occur. Now to describe a very small fraction of particle creation after inflation in the present context we start with a Bogoliubov coefficient $\beta$ in FLRW space-time, which characterizes the amount of mixing between the two types of WKB approximated solutions. Here it is important to mention that, in the sub Hubble region $\left(\left|k c_{S} \eta\right|>>1\right)$ the Bogoliubov coefficient $\beta$ is small and consequently the representative probability distribution $P(x)$ for the relative comoving distance $x$ between the two pairs peaks at the comoving length scale given by $x \sim\left|\eta_{\text {pair }}\right|$ i.e. $\left.\frac{\mathrm{d} P(x)}{\mathrm{d} x}\right|_{x \sim\left|\eta_{\text {pair }}\right|}=0$, $\left.\frac{\mathrm{d}^{2} P(x)}{\mathrm{d} x^{2}}\right|_{x \sim\left|\eta_{\text {pair }}\right|}<0$ and $P\left(x \sim\left|\eta_{\text {pair }}\right|\right)=P_{\max }$. When the typical comoving distance $x$ is of the order of the time $\eta_{\text {pair }}$, all the pair is created within the present EFT setup. It is important to mention that the general formula for the Bogoliubov coefficient $\beta$ in Fourier space is given by the following approximation:

$\beta(k)=\int_{-\infty}^{0} \mathrm{~d} \eta \frac{\left(p^{\prime}(\eta)\right)^{2}}{4 p^{3}(\eta)} \exp \left[2 i \int_{-\infty}^{\eta} \mathrm{d} \eta^{\prime} p\left(\eta^{\prime}\right)\right]$. 
One can use another equivalent way to define the Bogoliubov coefficient $\beta$ in Fourier space by implementing an instantaneous Hamiltonian diagonalization method in the present context [20-26]. Using this diagonalized representation the regularized Bogoliubov coefficient $\beta$ in Fourier space can be written as

$\beta_{\text {diag }}\left(k ; \tau, \tau^{\prime}\right)=\int_{\tau^{\prime}}^{\tau} \mathrm{d} \eta \frac{p^{\prime}(\eta)}{2 p(\eta)} \exp \left[-2 i \int^{\eta} \mathrm{d} \eta^{\prime} p\left(\eta^{\prime}\right)\right]$

where $\tau$ and $\tau^{\prime}$ are introduced as the conformal time regulator in the present context. We will also derive the expressions using Eq. (3.48) in the next three subsections. In the next three subsection we will explicitly discuss three physical possibilities which captures the effect of massive particles in our computation.

In this context one can compare the dynamical equations for scalar mode fluctuations with the well-known Schrödinger scattering problem in one spatial dimension as given by: ${ }^{10}$

$\left[-\frac{1}{2 m} \frac{\mathrm{d}^{2}}{\mathrm{~d} x^{2}}+V(x)-E\right] \Psi(x)=0$,

where the following identification exists between quantum mechanical Schrödinger equation and cosmological dynamical equations for the scalar mode fluctuations:

$$
\begin{aligned}
& \eta \Longrightarrow t=-\frac{1}{H} \ln (-H \eta) \Longleftrightarrow x, \\
& h_{k}(\eta) \Longrightarrow h_{k}(t) \Longleftrightarrow \Psi(x) \text {, } \\
& p^{2}(\eta) \Longrightarrow p^{2}(t) \\
& = \begin{cases}\left\{c_{S}^{2} k^{2}+\left(\frac{m^{2}}{H^{2}}-2\right) H^{2} e^{2 H t}\right\} & \text { for dS, } \\
\left\{c_{S}^{2} k^{2}+\left(\frac{m^{2}}{H^{2}}-\left[v^{2}-\frac{1}{4}\right]\right) H^{2} e^{2 H t}\right\} & \text { for qdS. }\end{cases} \\
& \Longleftrightarrow 2 m[E-V(t)] \text {. }
\end{aligned}
$$

Here the signature of $p^{2}(t)$ in Schrödinger quantum mechanics signify the following physical situations:

- If $p^{2}(t)>0$ then it corresponds to the propagation over the barrier for $E>V(t)$.

- If $p^{2}(t)<0$ then it corresponds to tunneling solution for $E<V(t)$.

Most importantly if we use the analogy between Schrödinger quantum mechanics and cosmology then one can write

$V(t)= \begin{cases}-\frac{1}{2 m}\left(\frac{m^{2}}{H^{2}}-2\right) H^{2} e^{2 H t} & \text { for dS, } \\ -\frac{1}{2 m}\left(\frac{m^{2}}{H^{2}}-\left[v^{2}-\frac{1}{4}\right]\right) H^{2} e^{2 H t} & \text { for qdS. }\end{cases}$

$$
E=\frac{1}{2 m} c_{S}^{2} k^{2}
$$

$$
V(t)= \begin{cases}\frac{H^{2}}{2 m} e^{2 H t} & \text { for } m \approx H, \\ -\frac{1}{2 m}\left(\Upsilon^{2}-2\right) H^{2} e^{2 H t} & \text { for } m>>H, \\ \frac{H^{2}}{m} e^{2 H t} & \text { for } m<<H, \\ -\frac{1}{2 m}\left[\gamma\left(\frac{e^{-H t}}{H \eta \eta}+1\right)^{2}+\delta-2\right] H^{2} e^{2 H t} & \text { for } m \approx \sqrt{\gamma\left(\frac{\eta}{\eta_{0}}-1\right)^{2}+\delta H}, \\ -\frac{1}{2 m}\left[\frac{m_{0}^{2}}{2 H^{2}}[1+\tanh (\rho t)]-2\right] H^{2} e^{2 H t} & \text { for } m=\frac{m_{0}}{\sqrt{2}} \sqrt{\left[1-\tanh \left(\frac{\rho}{H} \ln (-H \eta)\right)\right]} \\ -\frac{1}{2 m}\left[\frac{m_{0}^{2}}{H^{2}} \operatorname{sech}^{2}(\rho t)-2\right] H^{2} e^{2 H t} & \text { for } m=m_{0} \operatorname{sech}\left(\frac{\rho}{H} \ln (-H \eta)\right),\end{cases}
$$

$\overline{10 \text { Here we set }} h / 2 \pi=1$. 


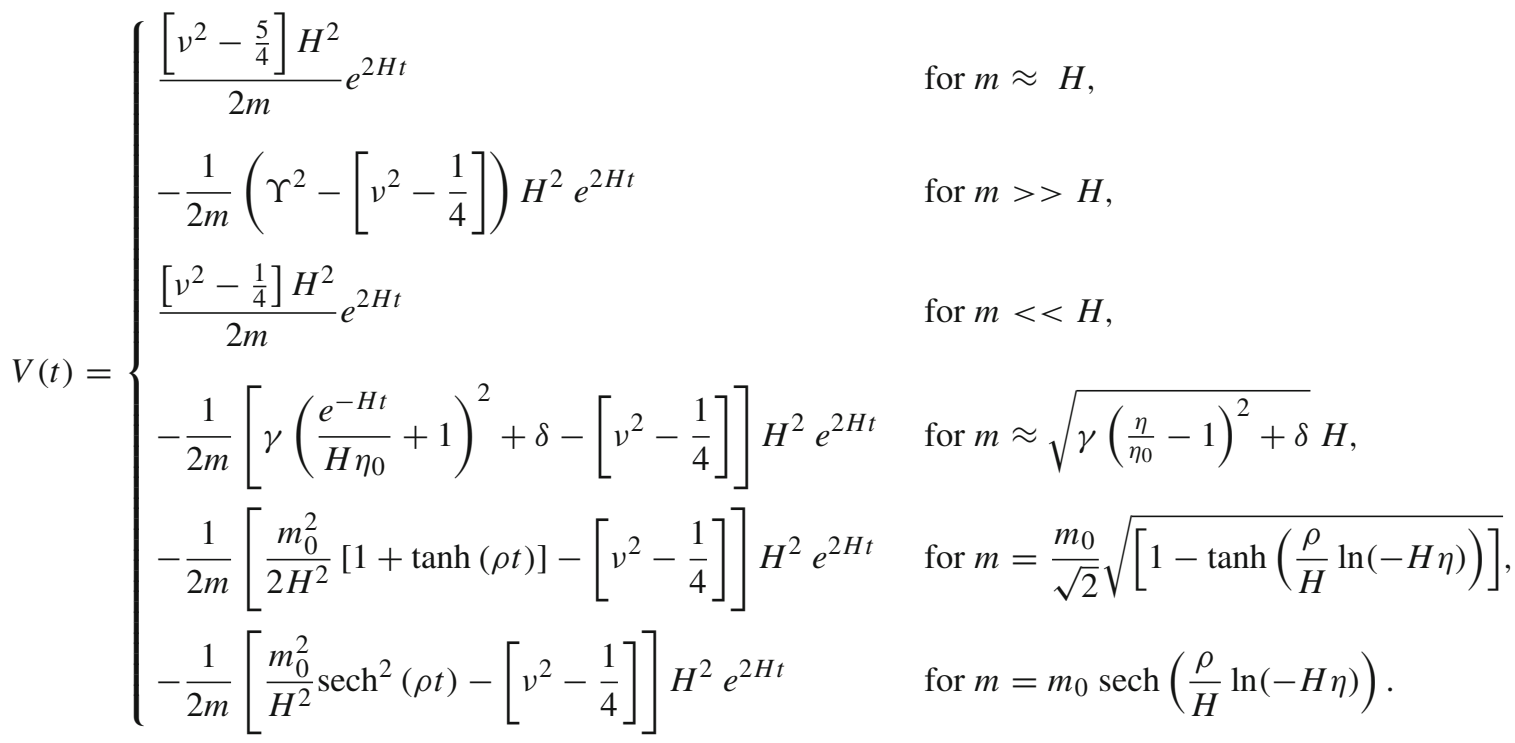

Now if we assume that in the past field has the structure $\Psi_{\text {past }}(t)=e^{i p(t) t}$, in the future the solution is given by $\Psi_{\text {future }}(t)=\alpha e^{i p(t) t}+\beta e^{-i p(t) t}$, due to tunneling. Here $\alpha$ and $\beta$ are the Bogoliubov coefficients in the present context of discussion. This correspond to the particle creation with probability $P \propto|\beta|^{2}$. $^{11}$

In the context of primordial cosmology one can also study the particle creation mechanism following the same prescription in Schrödinger quantum mechanics. In case of cosmology the past field has the pseudo-nomr structure and this could be identified with the left-moving wave $\Psi_{L}=e^{-i p(t) t}$ and in the future the solution is given by $\Psi_{L R}=\alpha e^{-i p(t) t}+\beta e^{i p(t) t}$, which can be interpreted as the mixture of left-moving and right-moving wave. Consequently, the Bogoliubov coefficients $\alpha$ and $\beta$ are related to the refection and transmission coefficients $\mathcal{R}$ and $\mathcal{T}$ via the following identifications:

$\alpha=\frac{1}{\mathcal{T}}$,

$\beta=\frac{\mathcal{R}}{\mathcal{T}}$.

In this context the Bogoliubov coefficients $\alpha$ and $\beta$ satisfies the normalization condition:

$|\alpha|^{2}-|\beta|^{2}=1$,

which implies the following well-known conservation law:

$|\mathcal{R}|^{2}+|\mathcal{T}|^{2}=1$

applicable in the context of Schrödinger quantum mechanics.

Further using the expressions for the Bogoliubov coefficient $\beta$ in two different representations as mentioned in

\footnotetext{
${ }^{11}$ Here it is important to note that for $p^{2}(t)>0$ we also get some amount of scattering over the barrier.
}

Eqs. (3.47) and (3.48), and substituting them in Eq. (3.57) we get the following expressions for the Bogoliubov coefficient $\alpha$ in two different representations as given by

$\alpha(k)$

$$
=\sqrt{\left[1+\left|\int_{-\infty}^{0} \mathrm{~d} \eta \frac{\left(p^{\prime}(\eta)\right)^{2}}{4 p^{3}(\eta)} \exp \left[2 i \int_{-\infty}^{\eta} \mathrm{d} \eta^{\prime} p\left(\eta^{\prime}\right)\right]\right|^{2}\right]} e^{i \phi},
$$

$$
\begin{aligned}
& \alpha_{\text {diag }}\left(k ; \tau, \tau^{\prime}\right) \\
& =\sqrt{\left[1+\left|\int_{\tau^{\prime}}^{\tau} \mathrm{d} \eta \frac{p^{\prime}(\eta)}{2 p(\eta)} \exp \left[-2 i \int^{\eta} \mathrm{d} \eta^{\prime} p\left(\eta^{\prime}\right)\right]\right|^{2}\right]} e^{i \phi_{\text {diag }}}
\end{aligned}
$$

where $\phi$ and $\phi_{\text {diag }}$ are the associated phase factors in two different representations. Further using the expressions for Bogoliubov coefficient $\alpha$ in two different representations as mentioned in Eqs. (3.59) and (3.60), and substituting them in Eq. (3.58) we get the following expressions for the reflection and transmission coefficient in two different representations as given by

$$
\begin{aligned}
\mathcal{R} & =\frac{\beta}{\alpha} \\
& =\frac{\int_{-\infty}^{0} \mathrm{~d} \eta \frac{\left(p^{\prime}(\eta)\right)^{2}}{4 p^{3}(\eta)} \exp \left[2 i \int_{-\infty}^{\eta} \mathrm{d} \eta^{\prime} p\left(\eta^{\prime}\right)\right]}{\sqrt{\left[1+\left|\int_{-\infty}^{0} \mathrm{~d} \eta \frac{\left(p^{\prime}(\eta)\right)^{2}}{4 p^{3}(\eta)} \exp \left[2 i \int_{-\infty}^{\eta} \mathrm{d} \eta^{\prime} p\left(\eta^{\prime}\right)\right]\right|^{2}\right]}} e^{-i \phi},
\end{aligned}
$$




$$
\begin{aligned}
& \mathcal{T}=\frac{1}{\alpha} \\
& =\frac{e^{-i \phi}}{\sqrt{\left[1+\left|\int_{-\infty}^{0} \mathrm{~d} \eta \frac{\left(p^{\prime}(\eta)\right)^{2}}{4 p^{3}(\eta)} \exp \left[2 i \int_{-\infty}^{\eta} \mathrm{d} \eta^{\prime} p\left(\eta^{\prime}\right)\right]\right|^{2}\right]}},
\end{aligned}
$$

and

$$
\begin{aligned}
& \mathcal{R}_{\text {diag }}\left(k ; \tau, \tau^{\prime}\right)=\frac{\beta_{\text {diag }}\left(k ; \tau, \tau^{\prime}\right)}{\alpha_{\text {diag }}\left(k ; \tau, \tau^{\prime}\right)} \\
& =\frac{\int_{\tau^{\prime}}^{\tau} \mathrm{d} \eta \frac{p^{\prime}(\eta)}{2 p(\eta)} \exp \left[-2 i \int^{\eta} \mathrm{d} \eta^{\prime} p\left(\eta^{\prime}\right)\right]}{\sqrt{\left[1+\left|\int_{\tau^{\prime}}^{\tau} \mathrm{d} \eta \frac{p^{\prime}(\eta)}{2 p(\eta)} \exp \left[-2 i \int^{\eta} \mathrm{d} \eta^{\prime} p\left(\eta^{\prime}\right)\right]\right|^{2}\right]}} e^{-i \phi_{\mathrm{diag}}}
\end{aligned}
$$

$$
\begin{aligned}
& \mathcal{T}_{\text {diag }}\left(k ; \tau, \tau^{\prime}\right)=\frac{1}{\alpha_{\text {diag }}\left(k ; \tau, \tau^{\prime}\right)} \\
& =\frac{e^{-i \phi_{\text {diag }}}}{\sqrt{\left[1+\left|\int_{\tau^{\prime}}^{\tau} \mathrm{d} \eta \frac{p^{\prime}(\eta)}{2 p(\eta)} \exp \left[-2 i \int^{\eta} \mathrm{d} \eta^{\prime} p\left(\eta^{\prime}\right)\right]\right|^{2}\right]}} .
\end{aligned}
$$

Next the expression for the number of produced particles at time $\tau$ can be calculated in the two representations using from the following formula as

$$
\begin{aligned}
\mathcal{N}\left(\tau, \tau^{\prime}\right)= & \frac{1}{(2 \pi a)^{3}} \int \mathrm{d}^{3} \mathbf{k}\left|\beta\left(k, \tau, \tau^{\prime}\right)\right|^{2} \\
= & \frac{1}{(2 \pi a)^{3}} \int \mathrm{d}^{3} \mathbf{k} \mid \int_{-\infty}^{0} \mathrm{~d} \eta \frac{\left(p^{\prime}(\eta)\right)^{2}}{4 p^{3}(\eta)} \\
& \times\left.\exp \left[2 i \int_{-\infty}^{\eta} \mathrm{d} \eta^{\prime} p\left(\eta^{\prime}\right)\right]\right|^{2}, \\
\mathcal{N}_{\text {diag }}\left(\tau, \tau^{\prime}\right)= & \frac{1}{(2 \pi a)^{3}} \int \mathrm{d}^{3} \mathbf{k}\left|\beta_{\text {diag }}\left(\tau, \tau^{\prime}\right)\right|^{2} \\
= & \frac{1}{(2 \pi a)^{3}} \int \mathrm{d}^{3} \mathbf{k} \mid \int_{\tau^{\prime}}^{\tau} \mathrm{d} \eta \frac{p^{\prime}(\eta)}{2 p(\eta)} \\
& \quad \times\left.\exp \left[-2 i \int^{\eta} \mathrm{d} \eta^{\prime} p\left(\eta^{\prime}\right)\right]\right|^{2} .
\end{aligned}
$$

Finally, one can define the total energy density of the produced particles using the following expression:

$$
\begin{aligned}
\rho\left(\tau, \tau^{\prime}\right)= & \frac{1}{(2 \pi a)^{3} a} \int \mathrm{d}^{3} \mathbf{k} p(\tau)\left|\beta\left(k, \tau, \tau^{\prime}\right)\right|^{2} \\
= & \frac{1}{(2 \pi a)^{3} a} \int \mathrm{d}^{3} \mathbf{k} p(\tau) \\
& \times\left|\int_{-\infty}^{0} \mathrm{~d} \eta \frac{\left(p^{\prime}(\eta)\right)^{2}}{4 p^{3}(\eta)} \exp \left[2 i \int_{-\infty}^{\eta} \mathrm{d} \eta^{\prime} p\left(\eta^{\prime}\right)\right]\right|^{2},
\end{aligned}
$$

$$
\begin{aligned}
\rho_{\text {diag }}\left(\tau, \tau^{\prime}, \eta^{\prime}\right)= & \frac{1}{(2 \pi a)^{3} a} \int \mathrm{d}^{3} \mathbf{k} p(\tau)\left|\beta_{\text {diag }}\left(k, \tau, \tau^{\prime}\right)\right|^{2} \\
= & \frac{1}{(2 \pi a)^{3} a} \int \mathrm{d}^{3} \mathbf{k} p(\tau) \\
& \times\left|\int_{\tau^{\prime}}^{\tau} \mathrm{d} \eta \frac{p^{\prime}(\eta)}{2 p(\eta)} \exp \left[-2 i \int^{\eta} \mathrm{d} \eta^{\prime} p\left(\eta^{\prime}\right)\right]\right|^{2} .
\end{aligned}
$$

\subsubsection{Case I: $m \approx H$}

The equation of motion for the massive field is

$h_{k}^{\prime \prime}+\left\{c_{S}^{2} k^{2}-\frac{1}{\eta^{2}}\right\} h_{k}=0 \quad$ for dS

$h_{k}^{\prime \prime}+\left\{c_{S}^{2} k^{2}-\left[v^{2}-\frac{5}{4}\right] \frac{1}{\eta^{2}}\right\} h_{k}=0 \quad$ for qdS.

The solution for the mode function for the de Sitter and the quasi de Sitter space can be expressed as

$h_{k}(\eta)$

$= \begin{cases}\sqrt{-\eta}\left[C_{1} H_{\sqrt{5} / 2}^{(1)}\left(-k c_{S} \eta\right)+C_{2} H_{\sqrt{5} / 2}^{(2)}\left(-k c_{S} \eta\right)\right] & \text { for dS, } \\ \sqrt{-\eta}\left[C_{1} H_{\sqrt{v^{2}-1}}^{(1)}\left(-k c_{S} \eta\right)+C_{2} H_{\sqrt{v^{2}-1}}^{(2)}\left(-k c_{S} \eta\right)\right] & \text { for qdS, }\end{cases}$

where $C_{1}$ and $C_{2}$ are two arbitrary integration constants, which depend on the choice of the initial condition.

After taking the $k c_{S} \eta \rightarrow-\infty, k c_{S} \eta \rightarrow 0$ and $\left|k c_{S} \eta\right| \approx$ $1-\Delta(\rightarrow 0)$ limits the most general solution as stated in Eq. (3.71) can be recast as

$$
\begin{aligned}
& h_{k}(\eta) \stackrel{|k c s \eta| \rightarrow-\infty}{=} \\
& \begin{cases}\sqrt{\frac{2}{\pi k c_{S}}}\left[C_{1} e^{-i k c_{S} \eta} e^{-\frac{i \pi}{2}\left(\frac{\sqrt{5}+1}{2}\right)}+C_{2} e^{i k c_{S} \eta} e^{\left.\frac{i \pi}{2}\left(\frac{\sqrt{5}+1}{2}\right)\right]}\right. & \text { for dS, } \\
\sqrt{\frac{2}{\pi k c_{S}}}\left[C_{1} e^{-i k c_{s} \eta} e^{-\frac{i \pi}{2}\left(\sqrt{v^{2}-1}+\frac{1}{2}\right)}+C_{2} e^{i k c c \eta} e^{\frac{i \pi}{2}\left(\sqrt{v^{2}-1}+\frac{1}{2}\right)}\right] & \text { for qdS, }\end{cases}
\end{aligned}
$$




$$
\begin{aligned}
& h_{k}(\eta) \stackrel{\left|k c_{S} \eta\right| \rightarrow 0}{=} \begin{cases}\frac{i \sqrt{-\eta}}{\pi} \Gamma\left(\frac{\sqrt{5}}{2}\right)\left(-\frac{k c_{S} \eta}{2}\right)^{-\frac{\sqrt{5}}{2}}\left[C_{1}-C_{2}\right] & \text { for } \mathrm{dS}, \\
\frac{i \sqrt{-\eta}}{\pi} \Gamma\left(\sqrt{v^{2}-1}\right)\left(-\frac{k c_{S} \eta}{2}\right)^{-\sqrt{v^{2}-1}}\left[C_{1}-C_{2}\right] & \text { for qdS, }\end{cases} \\
& \frac{i}{\pi} \sqrt{-\eta}\left[\frac{2}{\sqrt{5}}-\gamma+\frac{\sqrt{5}}{4}\left(\gamma^{2}+\frac{\pi^{2}}{6}\right)\right. \\
& \left.-\frac{5}{24}\left(\gamma^{3}+\frac{\gamma \pi^{2}}{2}+2 \zeta(3)\right)+\cdots\right]\left(\frac{1+\Delta}{2}\right)^{-\frac{\sqrt{5}}{2}}\left[C_{1}-C_{2}\right] \\
& h_{k}(\eta) \stackrel{\left|k c_{S} \eta\right| \approx 1-\Delta(\rightarrow 0)}{=} \\
& \frac{i}{\pi} \sqrt{-\eta}\left[\frac{1}{\left\{\sqrt{v^{2}-1}\right\}}-\gamma+\frac{1}{2}\left(\gamma^{2}+\frac{\pi^{2}}{6}\right)\left\{\sqrt{v^{2}-1}\right\}\right. \\
& \left.-\frac{1}{6}\left(\gamma^{3}+\frac{\gamma \pi^{2}}{2}+2 \zeta(3)\right)\left\{\sqrt{v^{2}-1}\right\}^{2}+\cdots\right]\left(\frac{1+\Delta}{2}\right)^{-\sqrt{\nu^{2}-1}}\left[C_{1}-C_{2}\right] \text { for qdS. }
\end{aligned}
$$

Next we assume that the WKB approximation is approximately valid for all times for the solution for the mode function $h_{k}$. In the standard WKB approximation the total solution can be recast in the following form:

$h_{k}(\eta)=\left[D_{1} u_{k}(\eta)+D_{2} \bar{u}_{k}(\eta)\right]$,

where $D_{1}$ and $D_{2}$ are two arbitrary integration constants, which depend on the choice of the initial condition during WKB approximation at early and late time scale. In the present context $u_{k}(\eta)$ and $\bar{u}_{k}(\eta)$ are defined as

$$
\begin{aligned}
& \int \frac{1}{\sqrt{2 \sqrt{c_{S}^{2} k^{2}-\frac{1}{\eta^{2}}}}} \exp \left[i \int^{\eta} \mathrm{d} \eta^{\prime} \sqrt{c_{S}^{2} k^{2}-\frac{1}{\eta^{\prime 2}}}\right] \\
& =\frac{1}{\sqrt{2 \sqrt{c_{S}^{2} k^{2}-\frac{1}{\eta^{2}}}}} \exp \left[i\left(\eta \sqrt{c_{S}^{2} k^{2}-\frac{1}{\eta^{2}}}+\tan ^{-1}\left[\frac{1}{\eta \sqrt{c_{S}^{2} k^{2}-\frac{1}{\eta^{2}}}}\right]\right)\right] \\
& u_{k}(\eta)=\left\{\frac{1}{\sqrt{2 \sqrt{c_{S}^{2} k^{2}-\frac{\left[v^{2}-\frac{5}{4}\right]}{\eta^{2}}}}} \exp \left[i \int^{\eta} \mathrm{d} \eta^{\prime} \sqrt{c_{S}^{2} k^{2}-\frac{\left[v^{2}-\frac{5}{4}\right]}{\eta^{\prime 2}}}\right]\right. \\
& =\frac{1}{\sqrt{2 \sqrt{c_{S}^{2} k^{2}-\frac{\left[v^{2}-\frac{5}{4}\right]}{\eta^{2}}}}} \exp \left[i\left(\eta \sqrt{c_{S}^{2} k^{2}-\frac{\left[v^{2}-\frac{5}{4}\right]}{\eta^{2}}}+\sqrt{v^{2}-\frac{5}{4}} \tan ^{-1}\left[\frac{\sqrt{v^{2}-\frac{5}{4}}}{\eta \sqrt{c_{S}^{2} k^{2}-\frac{\left[v^{2}-\frac{5}{4}\right]}{\eta^{2}}}}\right]\right)\right] \text { for qdS. }
\end{aligned}
$$

$$
\bar{u}_{k}(\eta)= \begin{cases}\frac{1}{\sqrt{2 \sqrt{c_{S}^{2} k^{2}-\frac{1}{\eta^{2}}}}} \exp \left[-i \int^{\eta} \mathrm{d} \eta^{\prime} \sqrt{c_{S}^{2} k^{2}-\frac{1}{\eta^{\prime 2}}}\right] & \text { for dS, } \\ =\frac{1}{\sqrt{2 \sqrt{c_{S}^{2} k^{2}-\frac{1}{\eta^{2}}}}} \exp \left[-i\left(\eta \sqrt{c_{S}^{2} k^{2}-\frac{1}{\eta^{2}}}+\tan ^{-1}\left[\frac{1}{\eta \sqrt{c_{S}^{2} k^{2}-\frac{1}{\eta^{2}}}}\right]\right)\right] & \\ \frac{1}{\sqrt{2 \sqrt{c_{S}^{2} k^{2}-\frac{\left[v^{2}-\frac{5}{4}\right]}{\eta^{2}}}}} \exp \left[-i \int^{\eta} \mathrm{d} \eta^{\prime} \sqrt{c_{S}^{2} k^{2}-\frac{\left[v^{2}-\frac{5}{4}\right]}{\eta^{\prime 2}}}\right] \\ =\frac{1}{\sqrt{2 \sqrt{c_{S}^{2} k^{2}-\frac{\left[v^{2}-\frac{5}{4}\right]}{\eta^{2}}}}} \exp \left[-i\left(\eta \sqrt{c_{S}^{2} k^{2}-\frac{\left[v^{2}-\frac{5}{4}\right]}{\eta^{2}}}+\sqrt{v^{2}-\frac{5}{4}} \tan ^{-1}\left[\frac{\sqrt{v^{2}-\frac{5}{4}}}{\eta \sqrt{c_{S}^{2} k^{2}-\frac{\left[v^{2}-\frac{5}{4}\right]}{\eta^{2}}}}\right]\right)\right] & \text { for qdS. }\end{cases}
$$


where we have written the total solution for the mode $h_{k}$ in terms of two linearly independent solutions. Here it is important to note that both of the solutions are hermitian conjugate of each other. If in the present context the exact solution of the mode $h_{k}$ is expanded with respect to these two linearly independent solutions then particle creation is absent in our EFT setup. In the present context correctness of WKB approximation is guaranteed at very early and very late time scales. In this discussion $u_{k}(\eta)$ is valid at very early time scale and $\bar{u}_{k}(\eta)$ perfectly works in the late time scale.

Now we will explicitly check that the exactness of the above mentioned WKB result derived in Eq. (3.75) with the actual solution of the mode function as presented in Eq. (3.71). As mentioned earlier in FLRW space-time in Fourier space Bogoliubov coefficient $\beta(k)$ measures this exactness for a given setup. The particle creation mechanism and its exact amount is described by finding the Bogoliubov coefficient $\beta(k)$ in Fourier space which in principle measures the exact amount of late times solution $u_{k}(\eta)$, if in the present context we exactly start with the early time scale solution $u_{k}(\eta)$. In our present computation we consider a physical situation where the WKB approximation is correct up to the leading order throughout the cosmological evolution in time scale. In the present context the Bogoliubov coefficient $\beta(k)$ in Fourier space can be computed approximately using the following regularized integral:

$$
\beta\left(k, \tau, \tau^{\prime}, \eta^{\prime}\right)= \begin{cases}\int_{\tau^{\prime}}^{\tau} \mathrm{d} \eta \frac{1}{4 \eta^{6}\left(c_{S}^{2} k^{2}-\frac{1}{\eta^{2}}\right)^{\frac{5}{2}}} \exp \left[2 i \int_{\eta^{\prime}}^{\eta} \mathrm{d} \eta^{\prime \prime} \sqrt{\left.c_{S}^{2} k^{2}-\frac{1}{\eta^{\prime \prime 2}}\right]}\right. & \text { for dS } \\ \int_{\tau^{\prime}}^{\tau} \mathrm{d} \eta \frac{\left[v^{2}-\frac{5}{4}\right]^{2}}{4 \eta^{6}\left(c_{S}^{2} k^{2}-\frac{\left[v^{2}-\frac{5}{4}\right]}{\eta^{2}}\right)^{\frac{5}{2}}} \exp \left[2 i \int_{\eta^{\prime}}^{\eta} \mathrm{d} \eta^{\prime \prime} \sqrt{\left.c_{S}^{2} k^{2}-\frac{\left[v^{2}-\frac{5}{4}\right]}{\eta^{\prime \prime 2}}\right]}\right. \text { for qdS. }\end{cases}
$$

which is not exactly analytically computable. To study the behaviour of this integral we consider here three consecutive physical situations: $\left|k c_{S} \eta\right|<<1,\left|k c_{S} \eta\right| \approx 1-\Delta(\rightarrow 0)$ and $\left|k c_{S} \eta\right|>>1$ for the de Sitter case and the quasi de Sitter case. In the three cases we have

$$
\begin{aligned}
& \text { For dS: } \quad \sqrt{\left\{c_{S}^{2} k^{2}-\frac{1}{\eta^{2}}\right\}} \approx \begin{cases}\frac{i}{\eta} & \text { for }\left|k c_{S} \eta\right|<<1, \\
\frac{i \sqrt{2 \Delta}}{\eta} & \text { for }\left|k c_{S} \eta\right| \approx 1-\Delta(\rightarrow 0), \\
k c_{S} & \text { for }\left|k c_{S} \eta\right|>>1 .\end{cases} \\
& \text { For qdS: } \quad \sqrt{\left\{c_{S}^{2} k^{2}-\left[v^{2}-\frac{5}{4}\right] \frac{1}{\eta^{2}}\right\}} \approx \begin{cases}\frac{i \sqrt{\left[v^{2}-\frac{5}{4}\right]}}{\eta} & \text { for }\left|k c_{S} \eta\right|<<1, \\
\frac{i \sqrt{2 \Delta+\left[v^{2}-\frac{9}{4}\right]}}{\eta} & \text { for }\left|k c_{S} \eta\right| \approx 1-\Delta(\rightarrow 0), \\
k c_{S} & \text { for }\left|k c_{S} \eta\right|>>1 .\end{cases}
\end{aligned}
$$

and further using this result the Bogoliubov coefficient $\beta(k)$ in Fourier space can be expressed as

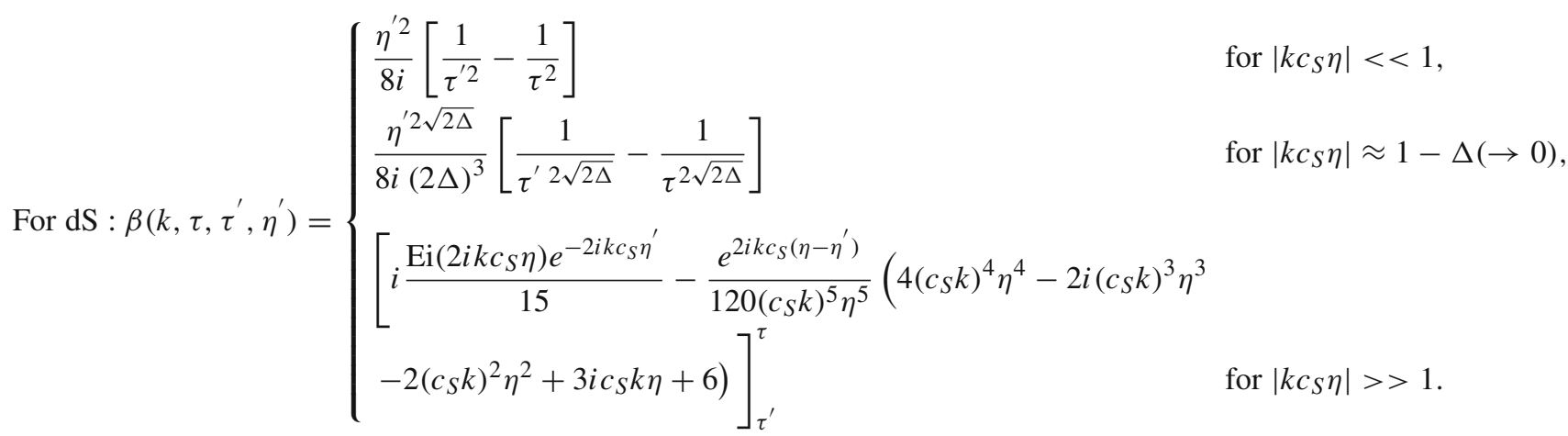


For qdS:

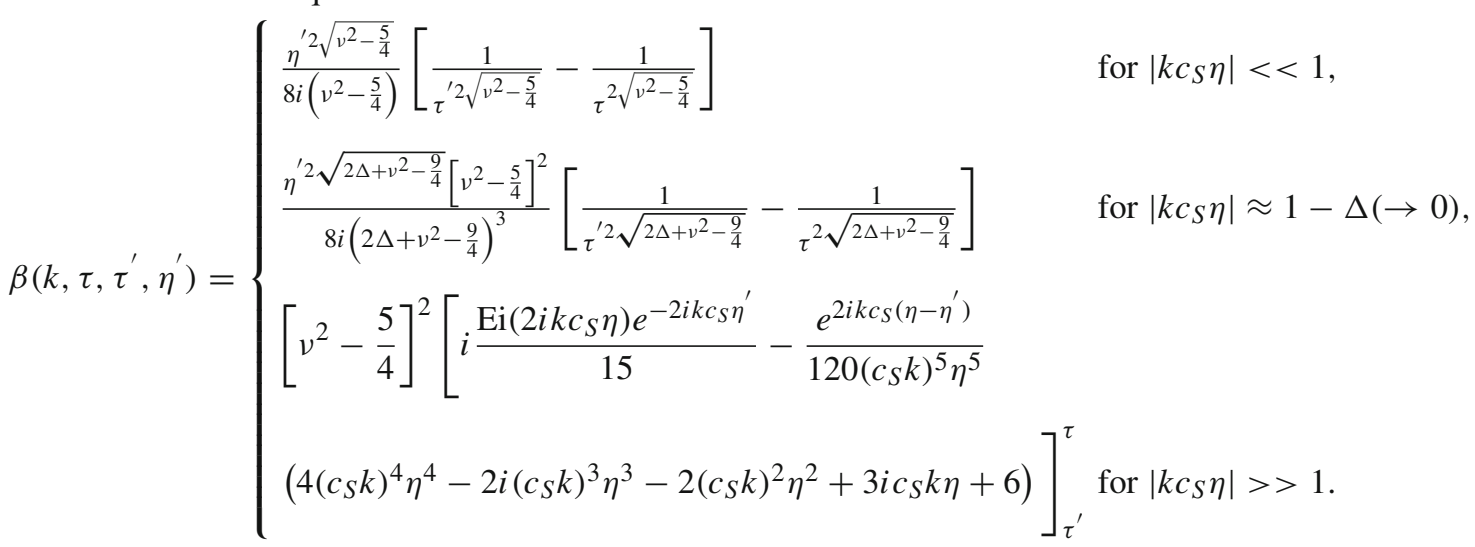

In all the situations described for the de Sitter case and the quasi de Sitter case here the magnitude of the Bogoliubov coefficient $|\beta(k)|$ in Fourier space is considerably small. Specifically it is important to point out here that for the case when $\left|k c_{S} \eta\right|>>1$ the value of the Bogoliubov coefficient $\beta(k)$ in Fourier space is even smaller as the WKB approximated solution is strongly consistent for all time scales. On the other hand in the vicinity of the conformal time scale $\eta \sim \eta_{\text {pair }}$ for $\left|k c_{S} \eta_{\text {pair }}\right|<<1$ the WKB approximated solution is less strongly valid and to validate the solution at this time scale one can neglect the momentum $k$ dependence in the Bogoliubov coefficient $\beta(k)$ in Fourier space. Here $\left|\eta_{\text {pair }}\right|$ characterizes the relative separation between the created particles.

As mentioned earlier here one can use another equivalent way to define the Bogoliubov coefficient $\beta$ in Fourier space by implementing instantaneous Hamiltonian diagonalization method to interpret the results. Using this diagonalized representation the regularized Bogoliubov coefficient $\beta$ in Fourier space can be written as

$\beta_{\text {diag }}\left(k ; \tau, \tau^{\prime}\right)= \begin{cases}\int_{\tau^{\prime}}^{\tau} \mathrm{d} \eta \frac{\exp \left[-2 i \int^{\eta} \mathrm{d} \eta^{\prime} \sqrt{c_{S}^{2} k^{2}-\frac{1}{\eta^{\prime 2}}}\right]}{2 \eta^{3}\left(c_{S}^{2} k^{2}-\frac{1}{\eta^{2}}\right)} & \text { for } \mathrm{d} S, \\ \int_{\tau^{\prime}}^{\tau} \mathrm{d} \eta \frac{\left[v^{2}-\frac{5}{4}\right] \exp \left[-2 i \int^{\eta} \mathrm{d} \eta^{\prime} \sqrt{\left.c_{S}^{2} k^{2}-\frac{\left[v^{2}-\frac{5}{4}\right]}{\eta^{\prime 2}}\right]}\right.}{2 \eta^{3}\left(c_{S}^{2} k^{2}-\frac{\left[v^{2}-\frac{5}{4}\right]}{\eta^{2}}\right)} & \text { for qdS, }\end{cases}$

where $\tau$ and $\tau^{\prime}$ introduced as the conformal time regulator in the present context. In this case as well the Bogoliubov coefficient is not exactly analytically computable. To study the behaviour of this integral we consider here three similar consecutive physical situations for the de Sitter and the quasi de Sitter case as discussed earlier.

For dS:

$$
\beta_{\text {diag }}\left(k ; \tau, \tau^{\prime}\right)= \begin{cases}\frac{1}{4}\left[\tau^{\prime 2}-\tau^{2}\right] & \text { for }\left|k c_{S} \eta\right|<<1, \\ \frac{1}{4(2 \Delta)^{3 / 2}\left[\tau^{\prime 2 \sqrt{2 \Delta}}-\tau^{2 \sqrt{2 \Delta}}\right]} & \text { for }\left|k c_{S} \eta\right| \approx 1-\Delta(\rightarrow 0), \\ {\left[\frac{e^{-2 i k c_{S} \eta}\left(2 i k c_{S} \eta-1\right)}{4\left(c_{S} k\right)^{2} \eta^{2}}-\operatorname{Ei}\left(-2 i k c_{S} \eta\right)\right.} & \text { for }\left|k c_{S} \eta\right|>>1 .\end{cases}
$$


For qdS:

$$
\beta_{\text {diag }}\left(k ; \tau, \tau^{\prime}\right)= \begin{cases}\frac{1}{4\left(v^{2}-\frac{5}{4}\right)}\left[\tau^{\prime 2 \sqrt{v^{2}-\frac{5}{4}}}-\tau^{2 \sqrt{v^{2}-\frac{5}{4}}}\right] & \text { for }\left|k c_{S} \eta\right|<<1, \\ \frac{\left[v^{2}-\frac{5}{4}\right]}{4\left(2 \Delta+v^{2}-\frac{9}{4}\right)^{3 / 2}}\left[\tau^{2 \sqrt{2 \Delta+v^{2}-\frac{9}{4}}}-\tau^{2 \sqrt{2 \Delta+v^{2}-\frac{9}{4}}}\right] & \text { for }\left|k c_{S} \eta\right| \approx 1-\Delta(\rightarrow 0), \\ {\left[v^{2}-\frac{5}{4}\right]^{2}\left[\frac{e^{-2 i k c_{S} \eta}\left(2 i k c_{S} \eta-1\right)}{4\left(c_{S} k\right)^{2} \eta^{2}}-\operatorname{Ei}\left(-2 i k c_{S} \eta\right)\right]_{\tau^{\prime}}^{\tau}} & \text { for }\left|k c_{S} \eta\right|>>1 .\end{cases}
$$

Further using the regularized expressions for the Bogoliubov coefficient $\beta$ in two different representations as mentioned in Eqs. (3.78) and (3.82), and substituting them in Eq. (3.57) we get the following regularized expressions for the Bogoliubov coefficient $\alpha$ in two different representations as given by

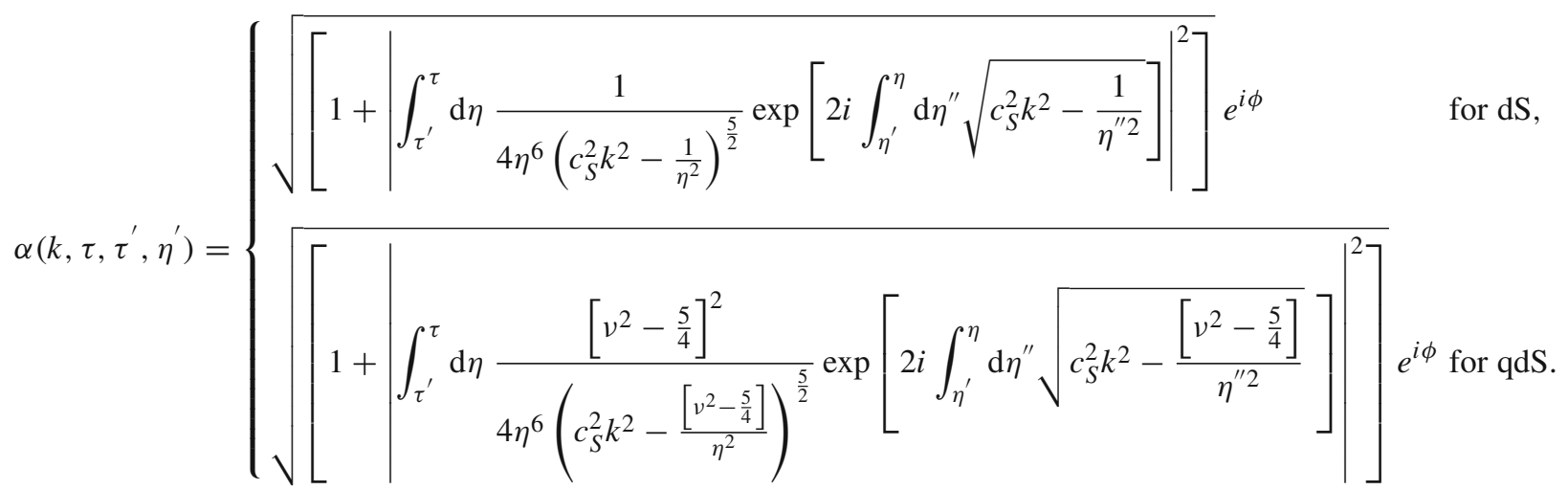

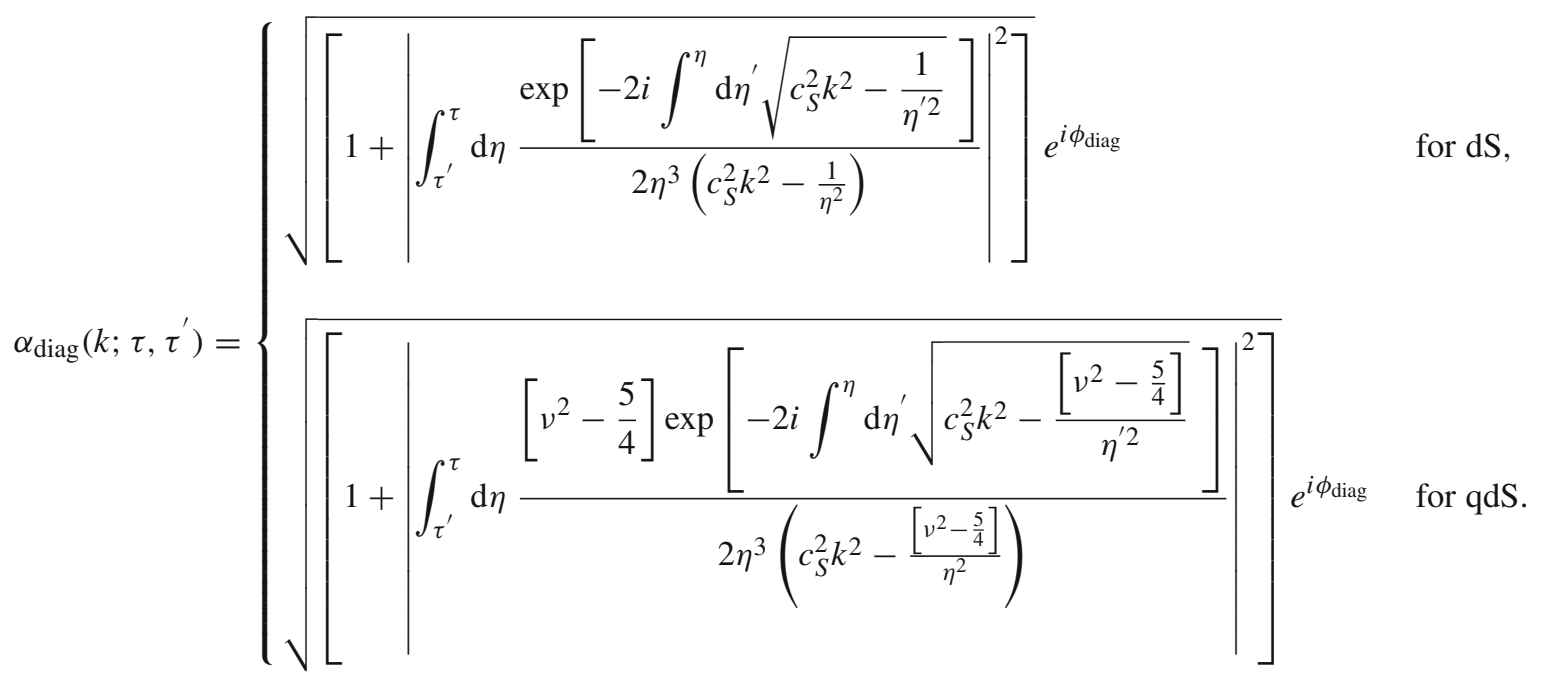

where $\phi$ and $\phi_{\text {diag }}$ are the associated phase factors in two different representations. Here the results are not exactly analytically computable. To study the behaviour of this integral we consider here three consecutive physical situations: $\left|k c_{S} \eta\right|<<1$, $\left|k c_{S} \eta\right| \approx 1-\Delta(\rightarrow 0)$ and $\left|k c_{S} \eta\right|>>1$ for the de Sitter case and the quasi de Sitter case. 
For dS:

$$
\alpha\left(k, \tau, \tau^{\prime}, \eta^{\prime}\right)= \begin{cases}\sqrt{\left[1+\left|\frac{\eta^{\prime 2}}{8 i}\left[\frac{1}{\tau^{\prime 2}}-\frac{1}{\tau^{2}}\right]\right|^{2}\right]} e^{i \phi} & \text { for }\left|k c_{S} \eta\right|<<1, \\ \sqrt{\left[1+\mid \frac{\eta^{\prime 2} \sqrt{2 \Delta}}{8 i(2 \Delta)^{3}}\left[\frac{1}{\tau^{\prime} 2 \sqrt{2 \Delta}}-\frac{1}{\left.\left.\tau^{2 \sqrt{2 \Delta}}\right]\left.\right|^{2}\right]} e^{i \phi}\right.\right.} & \text { for }\left|k c_{S} \eta\right| \approx 1-\Delta(\rightarrow 0), \\ {\left[1+\mid\left[i \frac{\operatorname{Ei}\left(2 i k c_{S} \eta\right) e^{-2 i k c_{S} \eta^{\prime}}}{15}-\frac{e^{2 i k c_{S}\left(\eta-\eta^{\prime}\right)}}{120\left(c_{S} k\right)^{5} \eta^{5}}\left(4\left(c_{S} k\right)^{4} \eta^{4}-2 i\left(c_{S} k\right)^{3} \eta^{3}\right.\right.\right.} & \\ \left.\left.\left.-2\left(c_{S} k\right)^{2} \eta^{2}+3 i c_{S} k \eta+6\right)\right]\left._{\tau^{\prime}}^{\tau}\right|^{2}\right]^{1 / 2} e^{i \phi} & \text { for }\left|k c_{S} \eta\right|>>1 .\end{cases}
$$

For qdS:

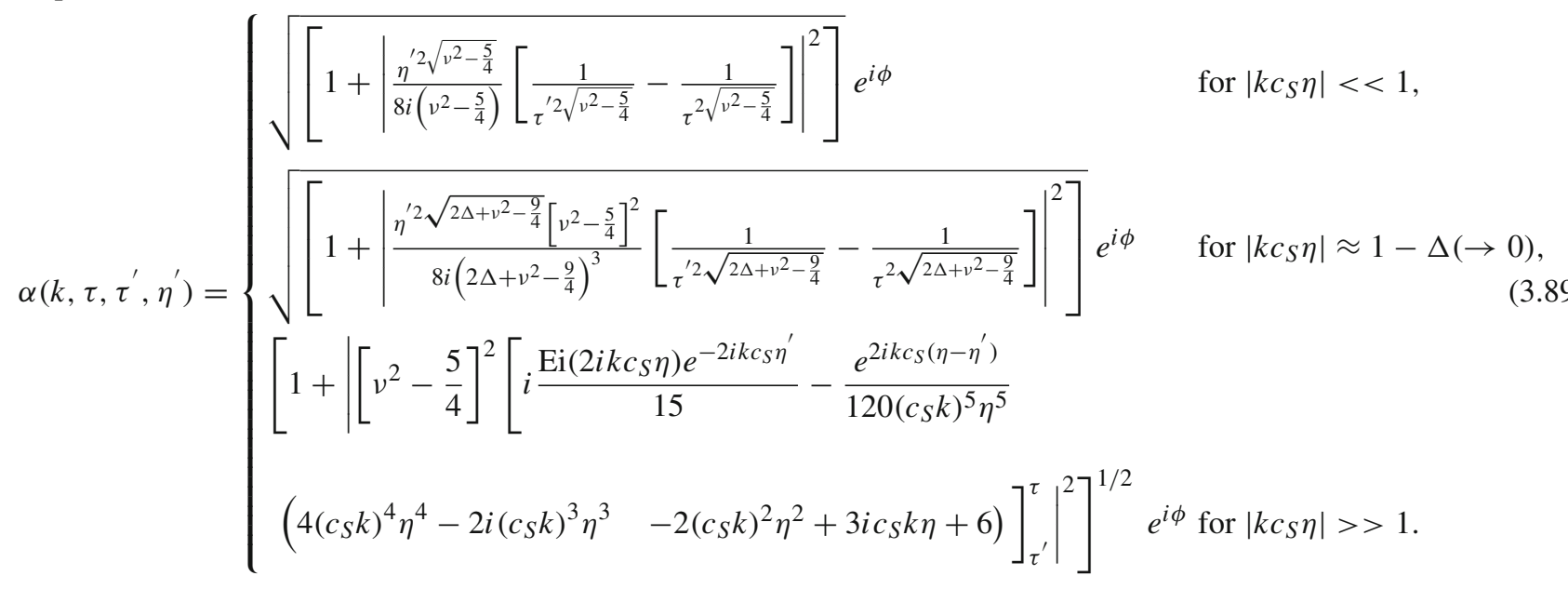

For dS:

$$
\alpha_{\text {diag }}\left(k ; \tau, \tau^{\prime}\right)= \begin{cases}\sqrt{\left[1+\left|\frac{1}{4}\left[\tau^{\prime 2}-\tau^{2}\right]\right|^{2}\right]} e^{i \phi} & \text { for }\left|k c_{S} \eta\right|<<1, \\ \sqrt{\left[1+\left|\frac{1}{4(2 \Delta)^{3 / 2}}\left[\tau^{\prime} 2 \sqrt{2 \Delta}-\tau^{2 \sqrt{2 \Delta}}\right]\right|^{2}\right]} e^{i \phi} & \text { for }\left|k c_{S} \eta\right| \approx 1-\Delta(\rightarrow 0), \\ \sqrt{\left[1+\left|\left[\frac{e^{-2 i k c s^{\eta}\left(2 i k c_{S} \eta-1\right)}}{4\left(c_{S} k\right)^{2} \eta^{2}}-\operatorname{Ei}\left(-2 i k c_{S} \eta\right)\right]_{\tau^{\prime}}\right|^{\tau}\right]} e^{i \phi} & \text { for }\left|k c_{S} \eta\right|>>1 .\end{cases}
$$

For qdS:

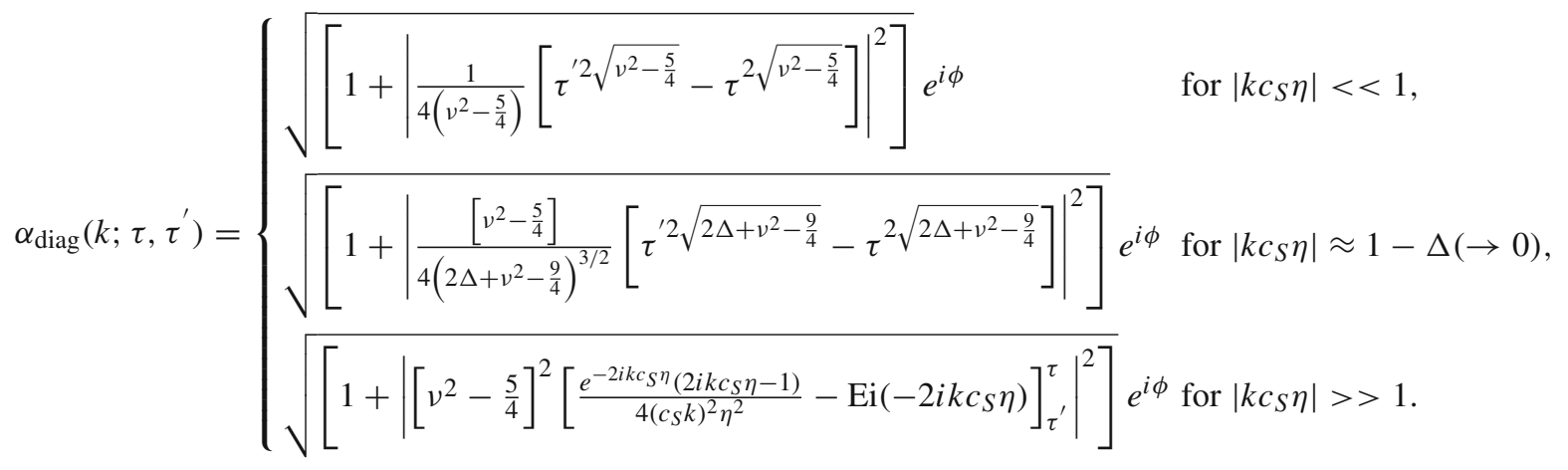


Further using the expressions for the Bogoliubov coefficient $\alpha$ in two different representations and substituting them in Eq. (3.58) we get the following expressions for the reflection and transmission coefficient in two different representations for three consecutive physical situations: $\left|k c_{S} \eta\right|<<1,\left|k c_{S} \eta\right| \approx 1-\Delta(\rightarrow 0)$ and $\left|k c_{S} \eta\right|>>1$ for the de Sitter case and the quasi de Sitter case as given by

For dS:

$$
\begin{aligned}
& \int \frac{\frac{\eta^{\prime 2}}{8 i}\left[\frac{1}{\tau^{\prime 2}}-\frac{1}{\tau^{2}}\right]}{\sqrt{\left[1+\left[\eta^{2}-\frac{1}{\tau^{2}}-\frac{1}{\left.\tau^{2}\right]}\right]^{2}\right.}} e^{-i \phi} \quad \text { for }\left|k c_{S} \eta\right|<<1, \\
& \frac{\frac{\eta^{\prime 2 \sqrt{2 \Delta}}}{8 i(2 \Delta)^{3}}\left[\frac{1}{\tau^{\prime 2 \sqrt{2 \Delta}}}-\frac{1}{\tau^{2 \sqrt{2 \Delta}}}\right]}{\sqrt{\left[1+\mid \frac{\eta^{\prime 2 \sqrt{2 \Delta}}}{8 i(2 \Delta)^{3}}\left[\frac{1}{\tau^{2} 2 \sqrt{2 \Delta}}-\frac{1}{\tau^{2} \sqrt{2 \Delta}}\right]^{2}\right]}} e^{-i \phi} \quad \text { for }\left|k c_{S} \eta\right| \approx 1-\Delta(\rightarrow 0), \\
& \mathcal{R}=\left\{\begin{array}{l}
\sqrt{\left[1+\left|\frac{\eta^{\prime 2} \sqrt{2 \Delta}}{8 i(2 \Delta)^{3}}\left[\frac{1}{\tau^{\prime} 2 \sqrt{2 \Delta}}-\frac{1}{\tau^{2 \sqrt{2 \Delta}}}\right]\right|^{2}\right]} \\
{\left[i \frac{\operatorname{Ei}\left(2 i k c_{S} \eta\right) e^{-2 i k c_{S} \eta^{\prime}}}{15}-\frac{e^{2 i k c_{S}\left(\eta-\eta^{\prime}\right)}}{120\left(c_{S} k\right)^{5} \eta^{5}}\left(4\left(c_{S} k\right)^{4} \eta^{4}-2 i\left(c_{S} k\right)^{3} \eta^{3}\right.\right.}
\end{array}\right. \\
& \left.\left.-2\left(c_{S} k\right)^{2} \eta^{2}+3 i c_{S} k \eta+6\right)\right]_{\tau^{\prime}}^{\tau} /\left[1+\mid\left[i \frac{\mathrm{Ei}\left(2 i k c_{S} \eta\right) e^{-2 i k c_{S} \eta^{\prime}}}{15}-\frac{e^{2 i k c_{S}\left(\eta-\eta^{\prime}\right)}}{120\left(c_{S} k\right)^{5} \eta^{5}}\right.\right. \\
& \left.\left.\times\left(4\left(c_{S} k\right)^{4} \eta^{4}-2 i\left(c_{S} k\right)^{3} \eta^{3}-2\left(c_{S} k\right)^{2} \eta^{2}+3 i c_{S} k \eta+6\right)\right]\left._{\tau^{\prime}}^{\tau}\right|^{2}\right]^{1 / 2} e^{-i \phi} \text { for }\left|k c_{S} \eta\right|>>1 .
\end{aligned}
$$

For dS:

$$
\mathcal{T}= \begin{cases}\frac{1}{\sqrt{\left[1+\left|\frac{\eta^{\prime 2}}{8 i}\left[\frac{1}{\tau^{\prime 2}}-\frac{1}{\tau^{2}}\right]\right|^{2}\right]} e^{-i \phi}} & \text { for }\left|k c_{S} \eta\right|<<1, \\ \sqrt{\left[1+\left|\frac{\eta^{\prime 2} \sqrt{2 \Delta}}{8 i(2 \Delta)^{3}}\left[\frac{1}{\tau^{\prime 2 \sqrt{2 \Delta}}}-\frac{1}{\tau^{2 \sqrt{2 \Delta}}}\right]\right|^{2}\right]} e^{-i \phi} & \text { for }\left|k c_{S} \eta\right| \approx 1-\Delta(\rightarrow 0), \\ 1 /\left[1+\mid\left[i \frac{\operatorname{Ei}\left(2 i k c_{S} \eta\right) e^{-2 i k c_{s} \eta^{\prime}}}{15}-\frac{e^{2 i k c_{S}\left(\eta-\eta^{\prime}\right)}}{120\left(c_{S} k\right)^{5} \eta^{5}}\left(4\left(c_{S} k\right)^{4} \eta^{4}-2 i\left(c_{S} k\right)^{3} \eta^{3}\right.\right.\right. & \\ \left.\left.\left.\left.-2\left(c_{S} k\right)^{2} \eta^{2}+3 i c_{S} k \eta+6\right)\right]_{\tau^{\prime}} \mid\right]^{\tau}\right]^{2 / 2} e^{-i \phi} & \text { for }\left|k c_{S} \eta\right|>>1 .\end{cases}
$$

\section{For qdS:}

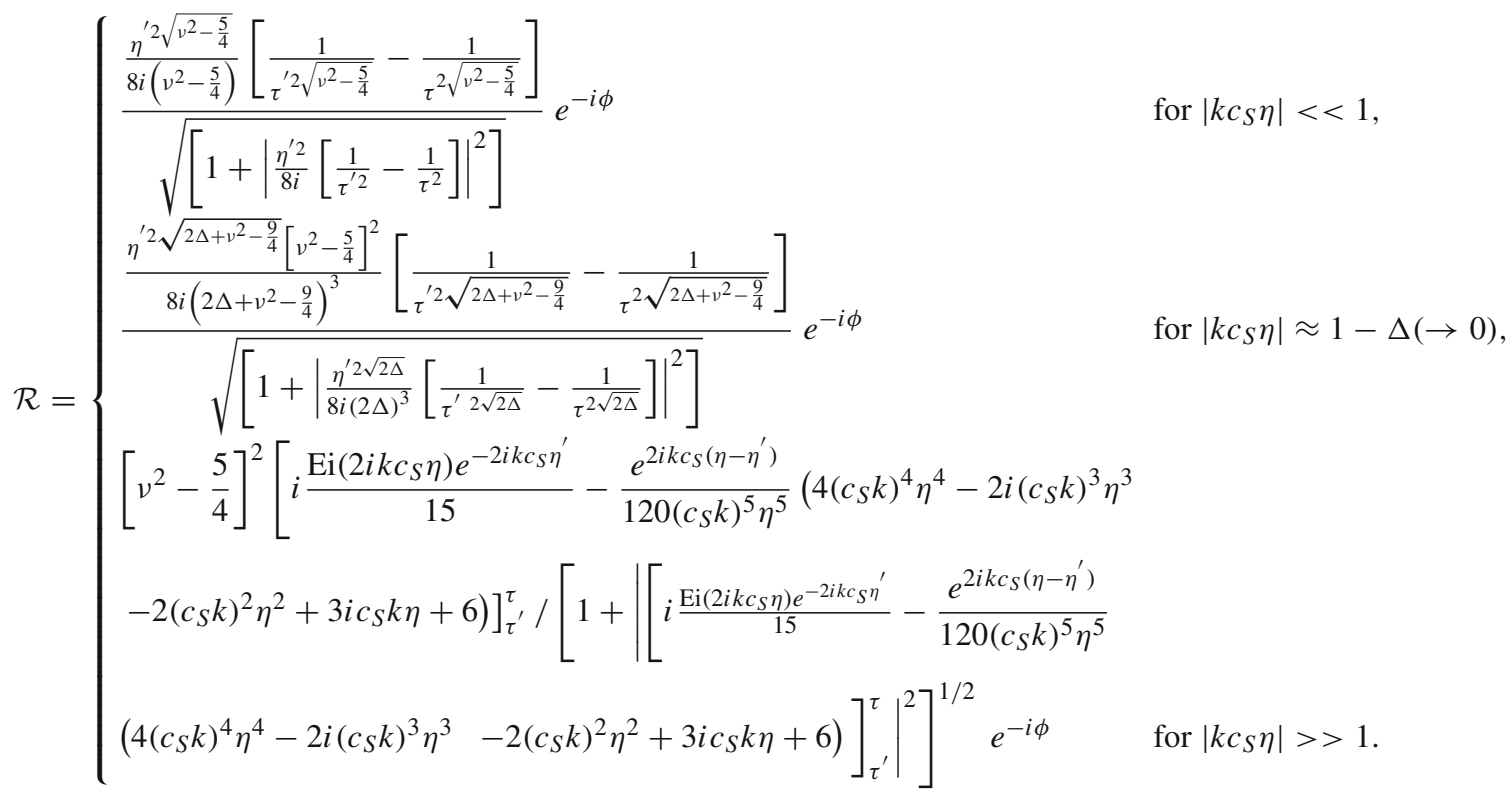


For qdS:

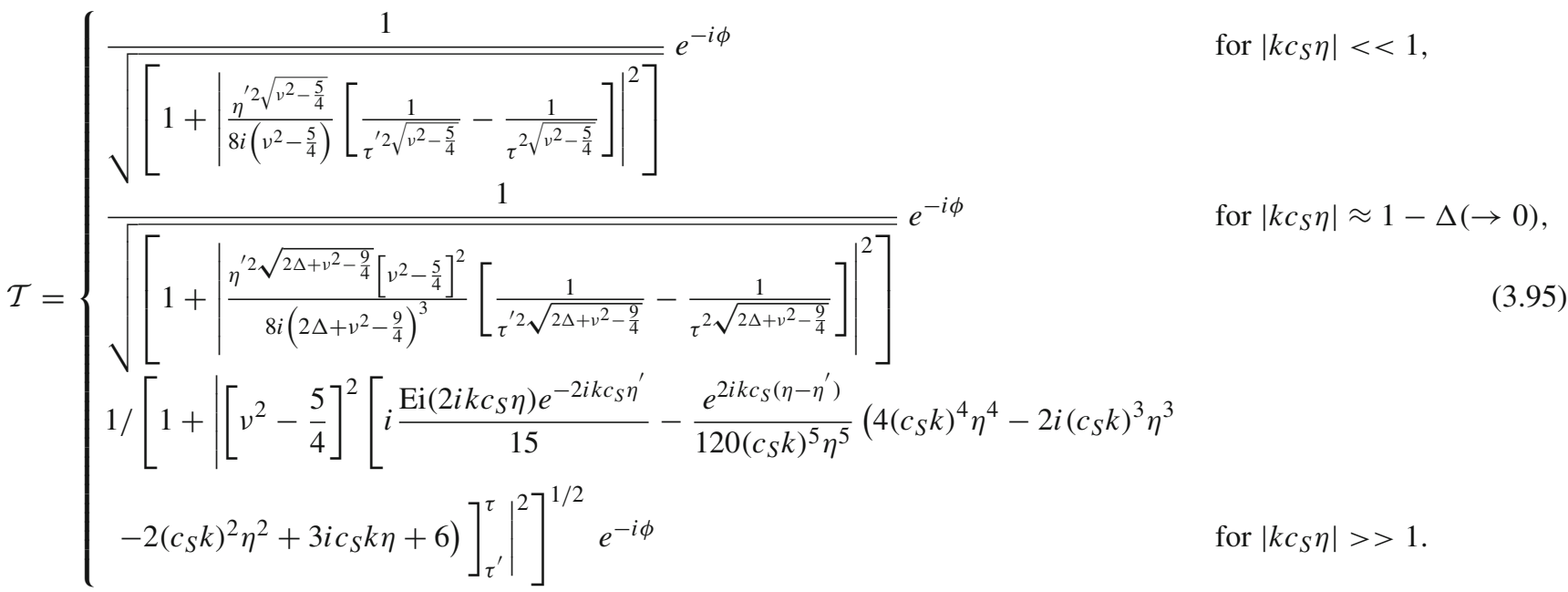

and

\section{For dS:}

$\mathcal{R}_{\text {diag }}\left(k ; \tau, \tau^{\prime}\right)= \begin{cases}\frac{\frac{1}{4}\left[\tau^{\prime 2}-\tau^{2}\right]}{\sqrt{\left[1+\left|\frac{\eta^{\prime 2}}{8 i}\left[\frac{1}{\tau^{\prime 2}}-\frac{1}{\tau^{2}}\right]\right|^{2}\right]} e^{-i \phi}} & \text { for }\left|k c_{S} \eta\right|<<1, \\ \frac{\frac{1}{4(2 \Delta)^{3 / 2}}\left[\tau^{\prime} 2 \sqrt{2 \Delta}-\tau^{2 \sqrt{2 \Delta}]}\right.}{\sqrt{\left[1+\left|\frac{1}{4(2 \Delta)^{3 / 2}}\left[\tau^{\prime} 2 \sqrt{2 \Delta}-\tau^{2 \sqrt{2 \Delta}}\right]\right|^{2}\right]} e^{-i \phi}} & \text { for }\left|k c_{S} \eta\right| \approx 1-\Delta(\rightarrow 0), \\ \frac{\left.4 \frac{e^{-2 i k c s^{\eta}}\left(2 i k c_{S} \eta-1\right)}{4\left(c_{S} k\right)^{2} \eta^{2}}-\operatorname{Ei}\left(-2 i k c_{S} \eta\right)\right]_{\tau^{\prime}}^{\tau}}{\sqrt{\left[1+\left|\left[\frac{e^{-2 i k c_{S} \eta}\left(2 i k c_{S} \eta-1\right)}{4\left(c_{S} k\right)^{2} \eta^{2}}-\operatorname{Ei}\left(-2 i k c_{S} \eta\right)\right]_{\tau^{\prime}}^{\tau}\right|^{2}\right]}} e^{-i \phi} & \text { for }\left|k c_{S} \eta\right|>>1 .\end{cases}$

\section{For dS:}

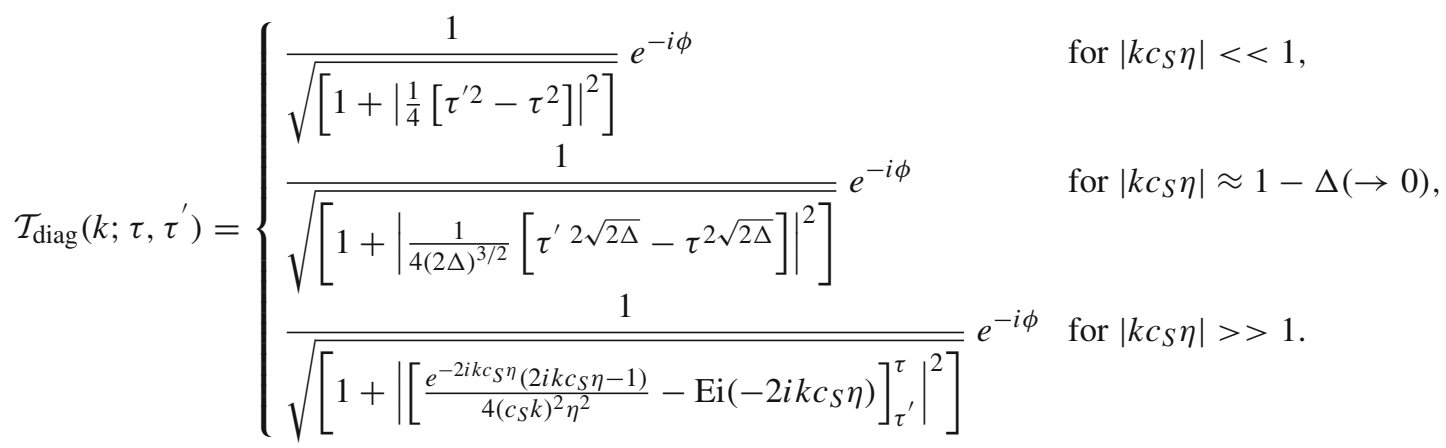


For qdS:

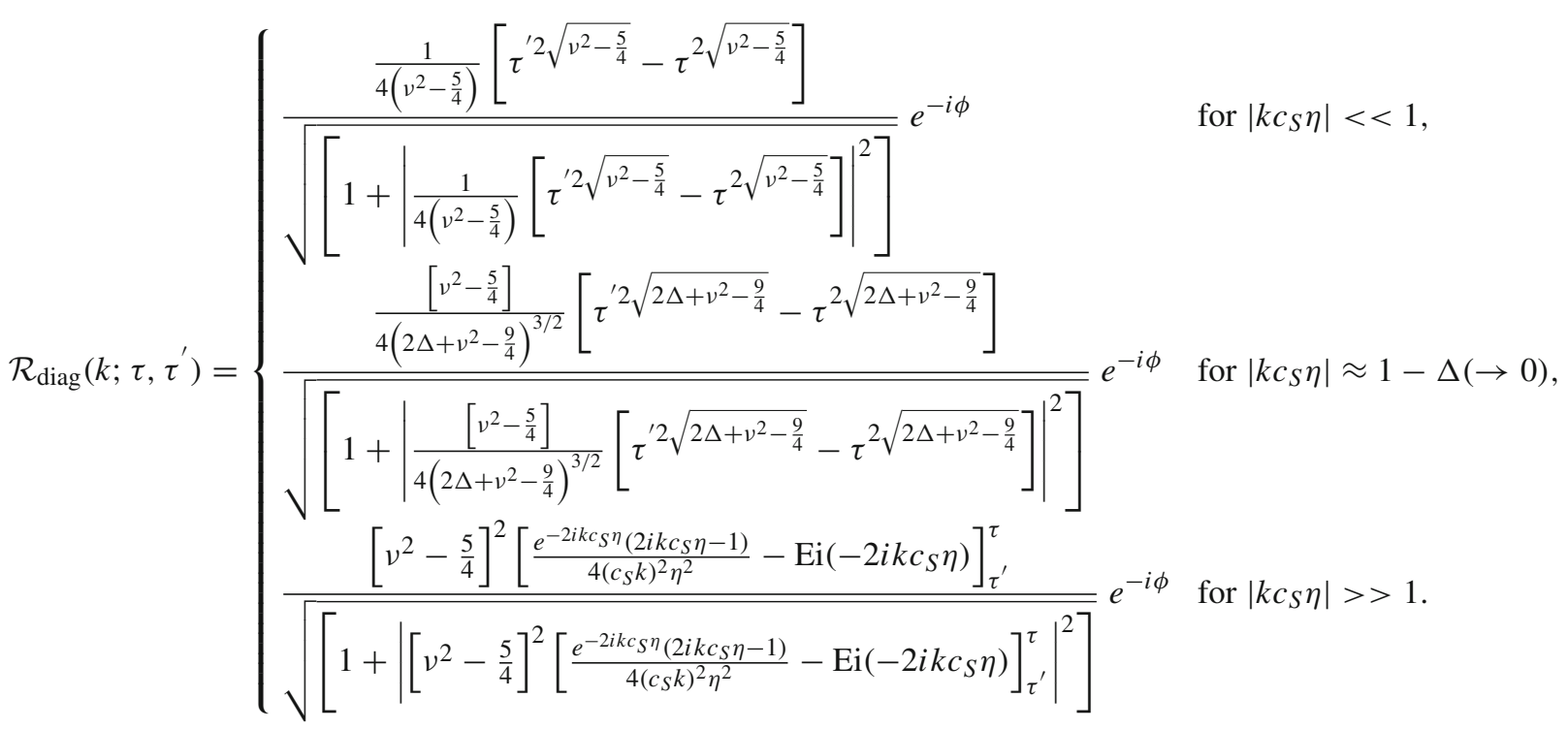

For qdS:

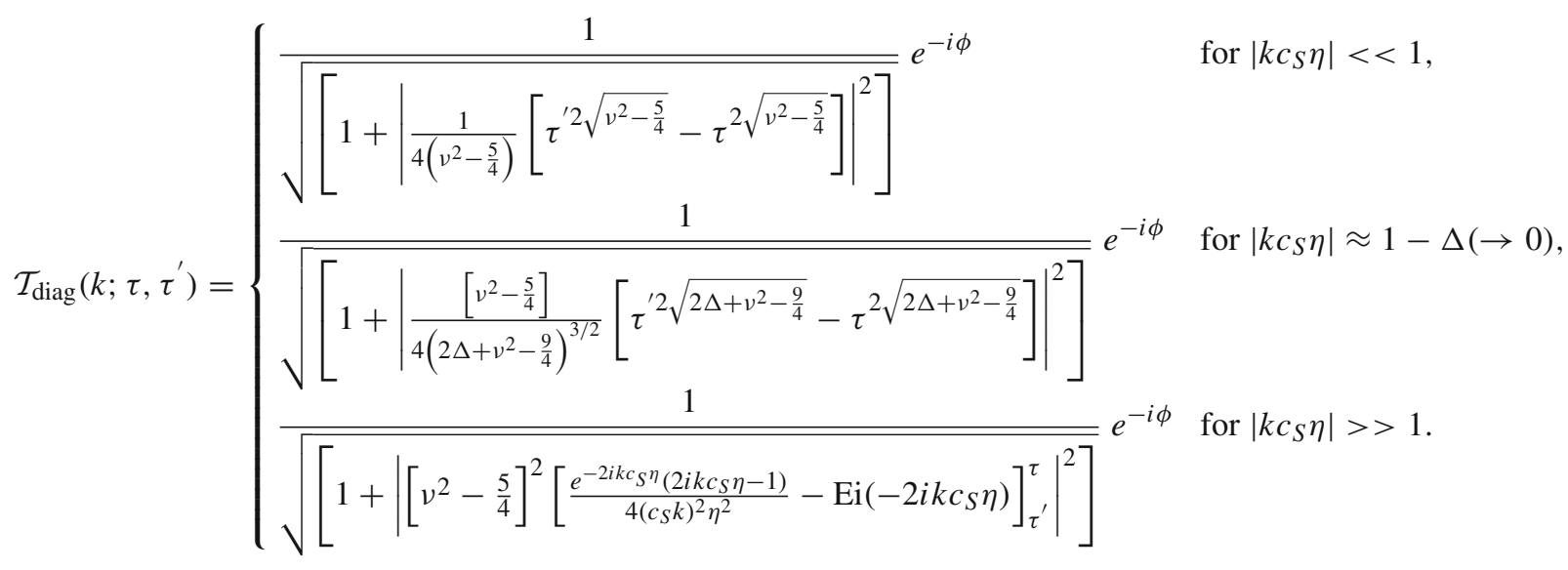

Next the expression for the number of produced particles at time $\tau$ in the two representations can be calculated for de Sitter and quasi de sitter as

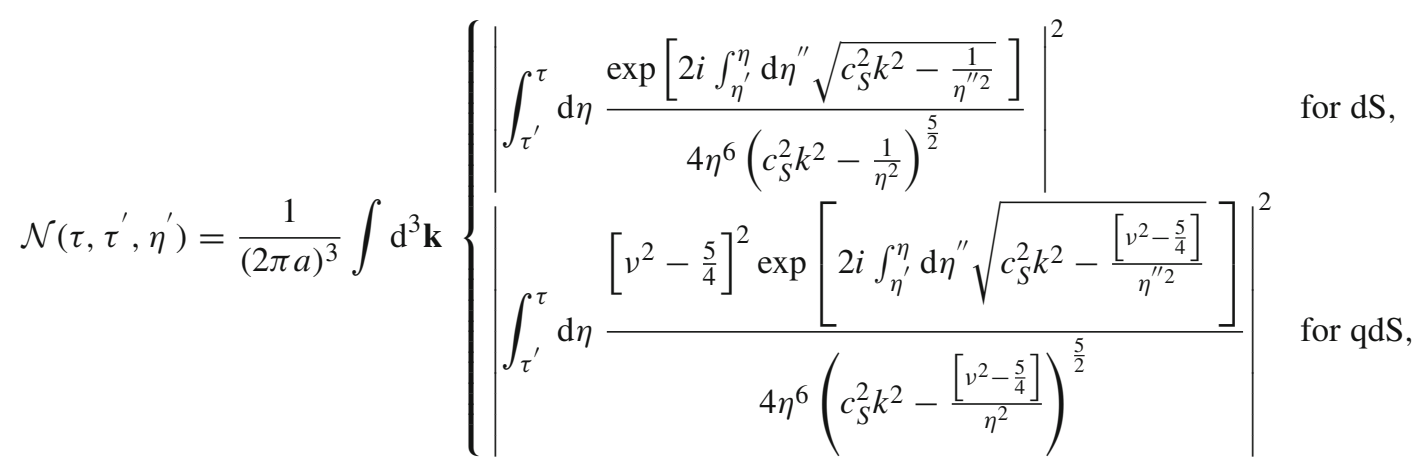




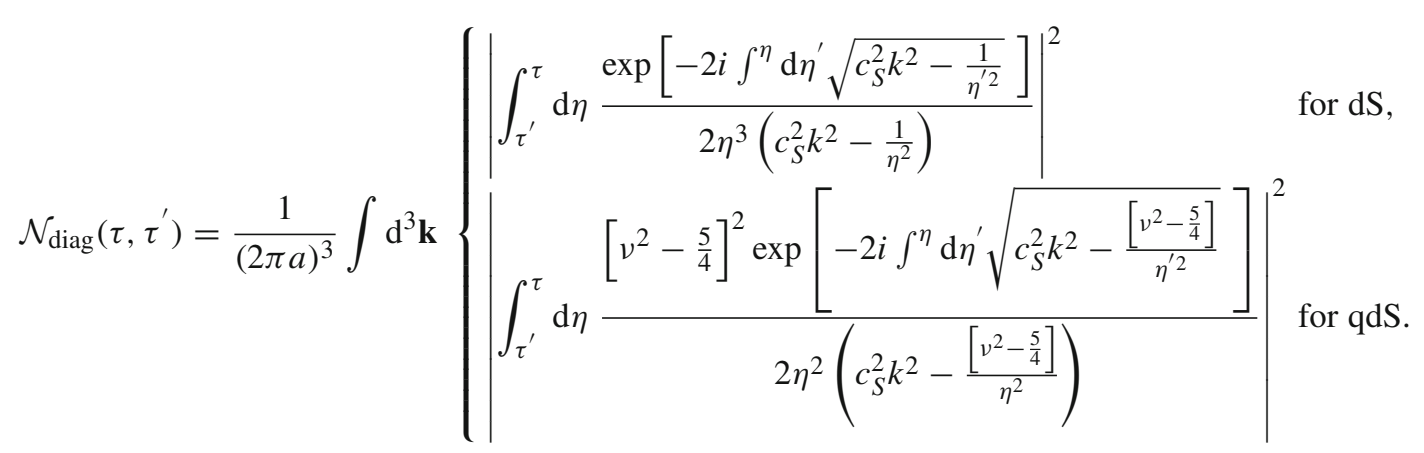

which is not exactly analytically computable. To study the behaviour of this integral we consider here three consecutive physical situations: $\left|k c_{S} \eta\right|<<1,\left|k c_{S} \eta\right| \approx 1-\Delta(\rightarrow 0)$ and $\left|k c_{S} \eta\right|>>1$ for the de Sitter case and the quasi de Sitter case. In the three cases we have

For dS:

$$
\mathcal{N}\left(\tau, \tau^{\prime}, \eta^{\prime}\right)= \begin{cases}\frac{\eta^{\prime 4} V}{64(2 \pi a)^{3}}\left[\frac{1}{\tau^{\prime 2}}-\frac{1}{\tau^{2}}\right]^{2} & \text { for }\left|k c_{S} \eta\right|<<1, \\ \frac{\eta^{\prime 4 \sqrt{2 \Delta}} V}{64(2 \pi a)^{3}(2 \Delta)^{6}}\left[\frac{1}{\tau^{\prime} 2 \sqrt{2 \Delta}}-\frac{1}{\tau^{2 \sqrt{2 \Delta}}}\right]^{2} & \text { for }\left|k c_{S} \eta\right| \approx 1-\Delta(\rightarrow 0), \\ \frac{4 \pi}{(2 \pi a)^{3}} \int_{0}^{\infty} \mathrm{d} k k^{2} \mid\left\{\frac{i e^{-2 i k c_{S} \eta^{\prime}}}{15}\left\{\operatorname{Ei}\left(2 i k c_{S} \tau\right)-\operatorname{Ei}\left(2 i k c_{S} \tau^{\prime}\right)\right\}\right. & \\ -\frac{e^{-2 i k c_{S} \eta^{\prime}}}{120 k^{5}}\left[\frac{e^{2 i k c_{S} \tau}\left(4\left(c_{S} k\right)^{4} \tau^{4}-2 i\left(c_{S} k\right)^{3} \tau^{3}-2\left(c_{S} k\right)^{2} \tau^{2}+3 i k c_{S} \tau+6\right)}{\tau^{5}}\right. & \\ \left.\left.-\frac{e^{2 k c_{S} \tau}\left(4\left(c_{S} k\right)^{4} \tau^{\prime}-2 i\left(c_{S} k\right)^{3} \tau^{\prime 3}-2\left(c_{S} k\right)^{2} \tau^{\prime 2}+3 i k c_{S} \tau^{\prime}+6\right)}{\tau^{\prime 5}}\right]\right\}\left.\right|^{2} & \text { for }\left|k c_{S} \eta\right|>>1 .\end{cases}
$$

For qdS:

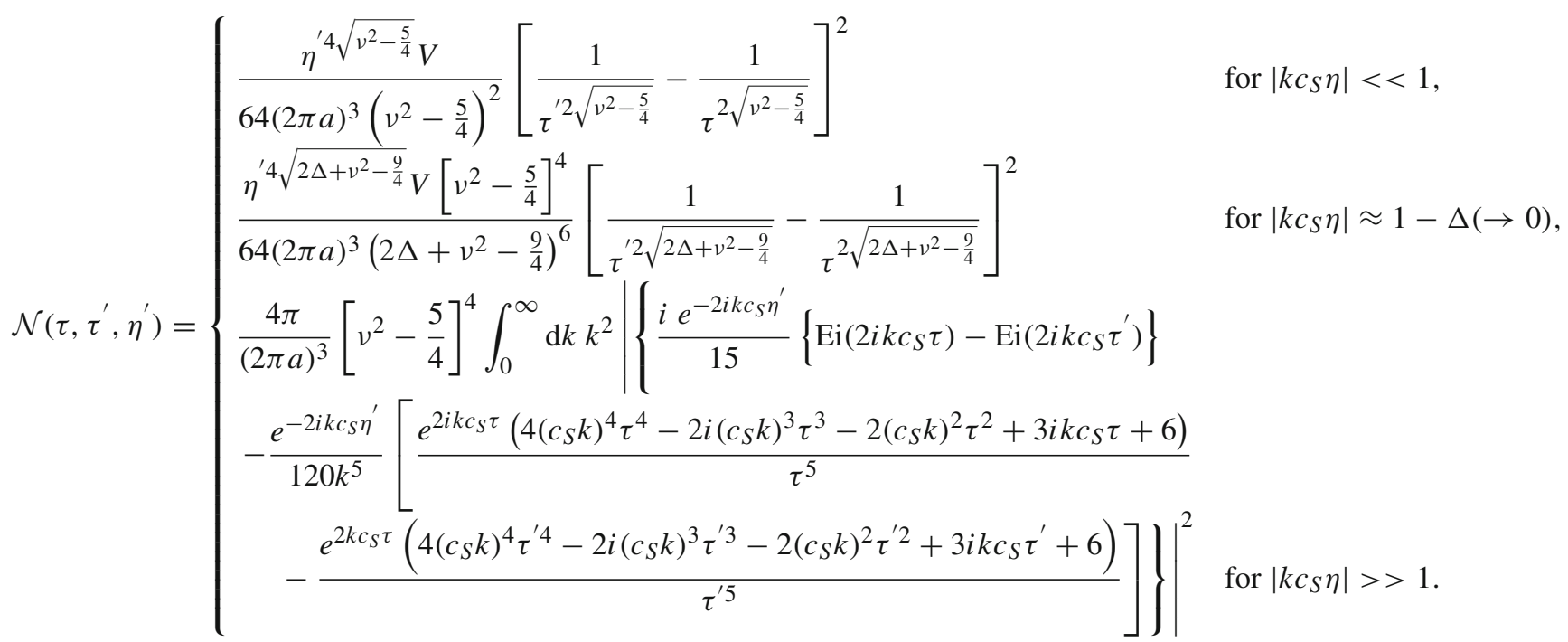


For dS:

$\mathcal{N}_{\text {diag }}\left(\tau, \tau^{\prime}\right)= \begin{cases}\frac{V}{16(2 \pi a)^{3}}\left[\tau^{\prime 2}-\tau^{2}\right]^{2} & \text { for }\left|k c_{S} \eta\right|<<1, \\ \frac{V}{16(2 \pi a)^{3}(2 \Delta)^{3}}\left[\tau^{\prime 2 \sqrt{2 \Delta}}-\tau^{2 \sqrt{2 \Delta}}\right]^{2} & \text { for }\left|k c_{S} \eta\right| \approx 1-\Delta(\rightarrow 0), \\ \frac{4 \pi}{(2 \pi a)^{3}} \int_{0}^{\infty} \mathrm{d} k k^{2} \mid\left[\left\{\frac{e^{-2 i k c_{S} \tau}\left(2 i k c_{S} \tau-1\right)}{4\left(c_{S} k\right)^{2} \tau^{2}}-\frac{e^{-2 i k c_{S} \tau^{\prime}}\left(2 i k c_{S} \tau^{\prime}-1\right)}{4\left(c_{S} k\right)^{2} \tau^{\prime 2}}\right\}\right. & \\ \left.-\left\{\operatorname{Ei}\left(-2 i k c_{S} \tau\right)-\operatorname{Ei}\left(-2 i k c_{S} \tau^{\prime}\right)\right\}\right]\left.\right|^{2} & \text { for }\left|k c_{S} \eta\right|>>1 .\end{cases}$

For qdS:

$\mathcal{N}_{\text {diag }}\left(\tau, \tau^{\prime}\right)= \begin{cases}\frac{V}{16(2 \pi a)^{3}\left(v^{2}-\frac{5}{4}\right)^{2}}\left[\tau^{\prime 2 \sqrt{v^{2}-\frac{5}{4}}}-\tau^{2 \sqrt{v^{2}-\frac{5}{4}}}\right]^{2} & \text { for }\left|k c_{S} \eta\right|<<1, \\ \frac{V\left[v^{2}-\frac{5}{4}\right]^{2}}{16(2 \pi a)^{3}\left(2 \Delta+v^{2}-\frac{9}{4}\right)^{3}}\left[\tau^{\prime 2 \sqrt{2 \Delta+v^{2}-\frac{9}{4}}}-\tau^{2 \sqrt{2 \Delta+v^{2}-\frac{9}{4}}}\right]^{2} & \text { for }\left|k c_{S} \eta\right| \approx 1-\Delta(\rightarrow 0), \\ \frac{4 \pi}{(2 \pi a)^{3}}\left[v^{2}-\frac{5}{4}\right]^{4} \int_{0}^{\infty} \mathrm{d} k k^{2} \mid\left[\left\{\frac{e^{-2 i k c_{S} \tau}\left(2 i k c_{S} \tau-1\right)}{4\left(c_{S} k\right)^{2} \tau^{2}}\right.\right. & \\ \left.-\frac{e^{-2 i k c_{S} \tau^{\prime}}\left(2 i k c_{S} \tau^{\prime}-1\right)}{4\left(c_{S} k\right)^{2} \tau^{\prime 2}}\right\} & \text { for }\left|k c_{S} \eta\right|>>1 .\end{cases}$

Throughout the discussion of total number of particle production we have introduced a symbol $V$ defined as

$V=\int \mathrm{d}^{3} \mathbf{k}$

which physically signifies the total finite volume in momentum space within which the produced particles are occupied.

Finally one can define the total energy density of the produced particles using the following expression:

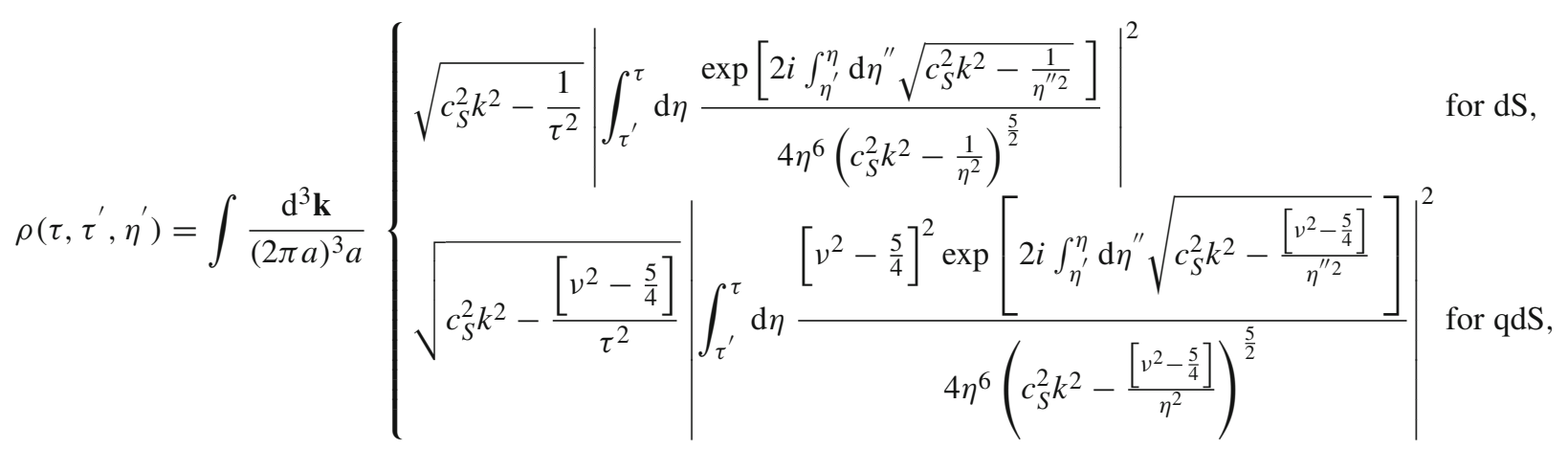




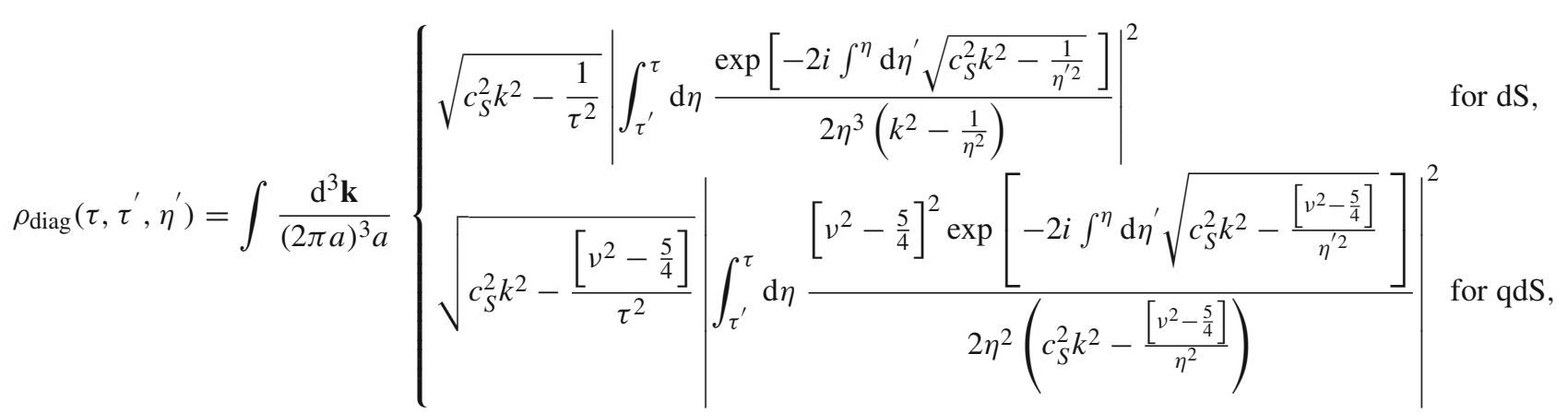

which is not exactly analytically computable. To study the behaviour of this integral we consider here three consecutive physical situations: $\left|k c_{S} \eta\right|<<1,\left|k c_{S} \eta\right| \approx 1-\Delta(\rightarrow 0)$ and $\left|k c_{S} \eta\right|>>1$ for the de Sitter case and the quasi de Sitter case. In the three cases we have

For dS:

$$
\rho\left(\tau, \tau^{\prime}, \eta^{\prime}\right)= \begin{cases}\frac{\eta^{\prime 4} J}{64(2 \pi a)^{3} a}\left[\frac{1}{\tau^{\prime 2}}-\frac{1}{\tau^{2}}\right]^{2} & \text { for }\left|k c_{S} \eta\right|<<1, \\ \frac{\eta^{\prime 4 \sqrt{2 \Delta}} J}{64(2 \pi a)^{3} a(2 \Delta)^{6}}\left[\frac{1}{\tau^{\prime} 2 \sqrt{2 \Delta}}-\frac{1}{\tau^{2 \sqrt{2 \Delta}}}\right]^{2} & \text { for }\left|k c_{S} \eta\right| \approx 1-\Delta(\rightarrow 0), \\ \frac{4 \pi c_{S}}{(2 \pi a)^{3} a} \int_{0}^{\infty} \mathrm{d} k k^{3} \mid\left\{\frac{i e^{-2 i k c_{S} \eta^{\prime}}}{15}\left\{\operatorname{Ei}\left(2 i k c_{S} \tau\right)-\operatorname{Ei}\left(2 i k c_{S} \tau^{\prime}\right)\right\}\right. & \\ -\frac{e^{-2 i k c_{S} \eta^{\prime}}}{120 k^{5}}\left[\frac{e^{2 i k c_{S} \tau}\left(4\left(c_{S} k\right)^{4} \tau^{4}-2 i\left(c_{S} k\right)^{3} \tau^{3}-2\left(c_{S} k\right)^{2} \tau^{2}+3 i\left(c_{S} k\right) \tau+6\right)}{\tau^{5}}\right. & \\ \left.\left.-\frac{e^{2 k c_{S} \tau^{\prime}}\left(4\left(c_{S} k\right)^{4} \tau^{\prime 4}-2 i\left(c_{S} k\right)^{3} \tau^{\prime 3}-2 k^{2} \tau^{\prime 2}+3 i k \tau^{\prime}+6\right)}{\tau^{\prime 5}}\right]\right\}\left.\right|^{2} & \text { for }\left|k c_{S} \eta\right|>>1 .\end{cases}
$$

For qdS:

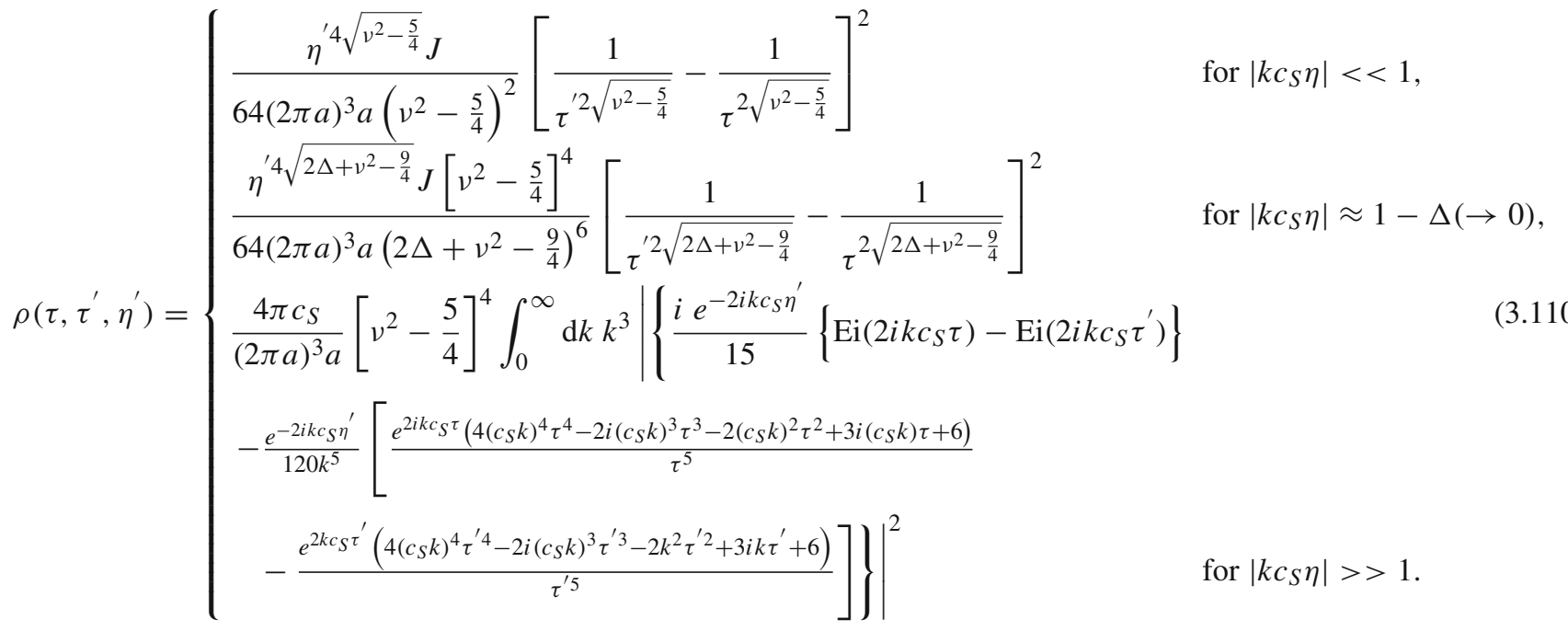


For dS:

$\rho_{\operatorname{diag}}\left(\tau, \tau^{\prime}\right)= \begin{cases}\frac{J}{16(2 \pi a)^{3} a}\left[\tau^{\prime 2}-\tau^{2}\right]^{2} & \text { for }\left|k c_{S} \eta\right|<<1, \\ \frac{J}{16(2 \pi a)^{3} a(2 \Delta)^{3}}\left[\tau^{\prime} 2 \sqrt{2 \Delta}-\tau^{2 \sqrt{2 \Delta}}\right]^{2} & \text { for }\left|k c_{S} \eta\right| \approx 1-\Delta(\rightarrow 0), \\ \frac{4 \pi c_{S}}{(2 \pi a)^{3} a} \int_{0}^{\infty} \mathrm{d} k k^{3} \mid \frac{e^{-2 i k c_{S} \tau}\left(2 i k c_{S} \tau-1\right)}{4\left(c_{S} k \tau\right)^{2}} & \\ -\frac{e^{-2 i k c_{S} \tau^{\prime}}\left(2 i k c_{S} \tau^{\prime}-1\right)}{4\left(c_{S} k \tau^{\prime}\right)^{2}}+\left\{\operatorname{Ei}\left(-2 i k c_{S} \tau\right)-\operatorname{Ei}\left(-2 i k c_{S} \tau^{\prime}\right)\right\} & \text { for }\left|k c_{S} \eta\right|>>1,\end{cases}$

For qdS:

$\rho_{\text {diag }}\left(\tau, \tau^{\prime}, \eta^{\prime}\right)= \begin{cases}\frac{J}{16(2 \pi a)^{3} a}\left[\tau^{\prime 2}-\tau^{2}\right]^{2} & \text { for }\left|k c_{S} \eta\right|<<1, \\ \frac{J}{16(2 \pi a)^{3} a(2 \Delta)^{3}}\left[\tau^{\prime 2 \sqrt{2 \Delta}}-\tau^{2 \sqrt{2 \Delta}}\right]^{2} & \text { for }\left|k c_{S} \eta\right| \approx 1-\Delta(\rightarrow 0), \\ \frac{4 \pi c_{S}}{(2 \pi a)^{3} a}\left[v^{2}-\frac{5}{4}\right]^{4} \int_{0}^{\infty} \mathrm{d} k k^{3} \mid \frac{e^{-2 i k c_{S} \tau}\left(2 i k c_{S} \tau-1\right)}{4\left(c_{S} k \tau\right)^{2}} & \\ -\frac{e^{-2 i k c_{S} \tau^{\prime}}\left(2 i k c_{S} \tau^{\prime}-1\right)}{4\left(c s k \tau^{\prime}\right)^{2}}+\left.\left\{\operatorname{Ei}\left(-2 i k c_{S} \tau\right)-\operatorname{Ei}\left(-2 i k c_{S} \tau^{\prime}\right)\right\}\right|^{2} & \text { for }\left|k c_{S} \eta\right|>>1 .\end{cases}$

Throughout the discussion of the total energy density of the produced particles we have introduced a symbol $J$ defined as

For dS:

$$
J= \begin{cases}\int \mathrm{d}^{3} \mathbf{k} \frac{1}{\tau}=\frac{V}{\tau} & \text { for }\left|k c_{S} \eta\right|<<1, \\ \int \mathrm{d}^{3} \mathbf{k} \frac{\sqrt{2 \Delta}}{\tau}=\frac{\sqrt{2 \Delta} V}{\tau} & \text { for }\left|k c_{S} \eta\right| \approx 1-\Delta(\rightarrow 0), \\ \int \mathrm{d}^{3} \mathbf{k} k c_{S} & \text { for }\left|k c_{S} \eta\right|>>1 .\end{cases}
$$

To study the behaviour of this integral we consider here three consecutive physical situations: $\left|k c_{S} \eta\right|<<1,\left|k c_{S} \eta\right| \approx$ $1-\Delta(\rightarrow 0)$ and $\left|k c_{S} \eta\right|>>1$ for the de Sitter case and the quasi de Sitter case. In the three cases we have

For qdS:

$$
J= \begin{cases}\int \mathrm{d}^{3} \mathbf{k} \frac{\sqrt{\left[v^{2}-\frac{5}{4}\right]}}{\tau}=\frac{V \sqrt{\left[v^{2}-\frac{5}{4}\right]}}{\tau} & \text { for }\left|k c_{S} \eta\right|<<1, \\ \int \mathrm{d}^{3} \mathbf{k} \frac{\sqrt{2 \Delta+\left[v^{2}-\frac{9}{4}\right]}}{\tau}=\frac{\sqrt{2 \Delta+\left[v^{2}-\frac{9}{4}\right]}}{\tau} & \text { for }\left|k c_{S} \eta\right| \approx 1-\Delta(\rightarrow 0), \\ \int \mathrm{d}^{3} \mathbf{k} k c_{S} & \text { for }\left|k c_{S} \eta\right|>>1 .\end{cases}
$$

$J=\int \mathrm{d}^{3} \mathbf{k} p(\tau)= \begin{cases}\int \mathrm{d}^{3} \mathbf{k} \sqrt{c_{S}^{2} k^{2}-\frac{1}{\tau^{2}}} & \text { for } \mathrm{d} S, \\ \int \mathrm{d}^{3} \mathbf{k} \sqrt{c_{S}^{2} k^{2}-\frac{\left[v^{2}-\frac{5}{4}\right]}{\tau^{2}}} & \text { for qdS, }\end{cases}$

which physically signifies the total finite volume weighted by $p(\eta)$ in momentum space within which the produced particles are occupied.
In Figs. 7 and 8, we have explicitly shown the particle creation profile for Case I for two representations.

\subsubsection{Case II: $m>>H$}

Here we set $m=\Upsilon H$, where the parameter $\Upsilon>>1$ in this case. Here the equation of motion for the massive field is

$h_{k}^{\prime \prime}+\left\{c_{S}^{2} k^{2}+\frac{\Upsilon^{2}-2}{\eta^{2}}\right\} h_{k}=0 \quad$ for $\mathrm{d} S$ 


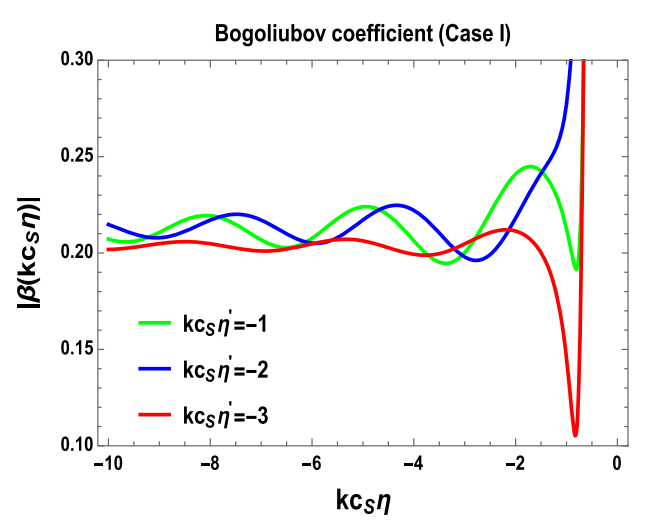

(a)

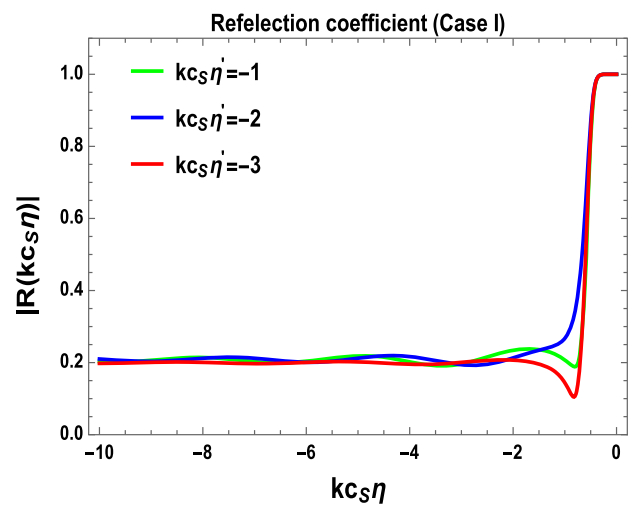

(c)

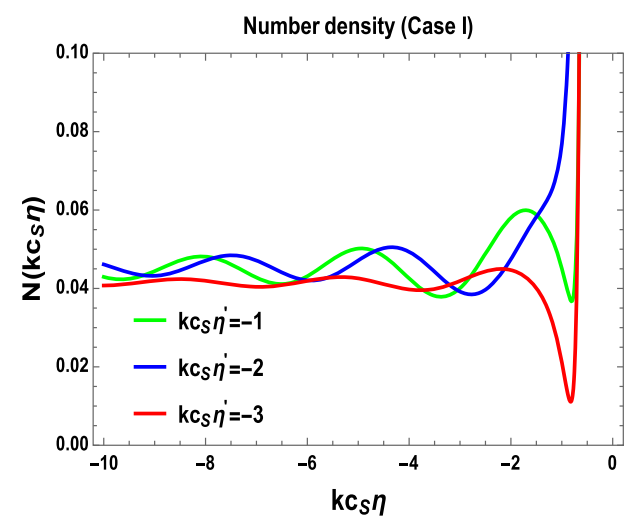

(e)

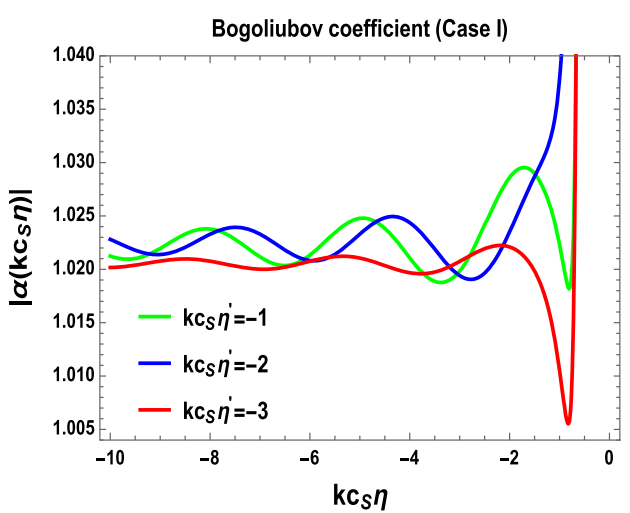

(b)

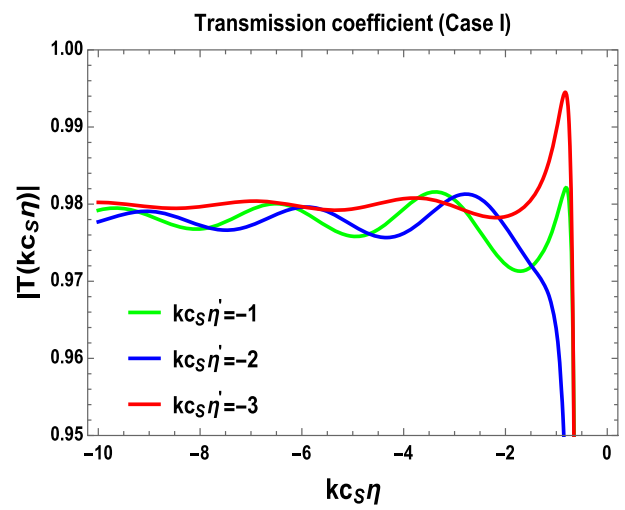

(d)

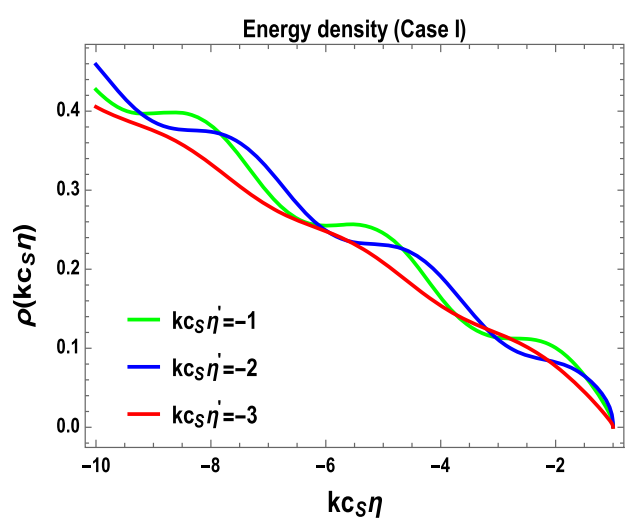

(f)

Fig. 7 Particle creation profile for Case I

$h_{k}^{\prime \prime}+\left\{c_{S}^{2} k^{2}+\frac{\left[\Upsilon^{2}-\left(v^{2}-\frac{1}{4}\right)\right]}{\eta^{2}}\right\} h_{k}=0$ for qdS.

The solution for the mode function for the de Sitter and the quasi de Sitter space can be expressed as

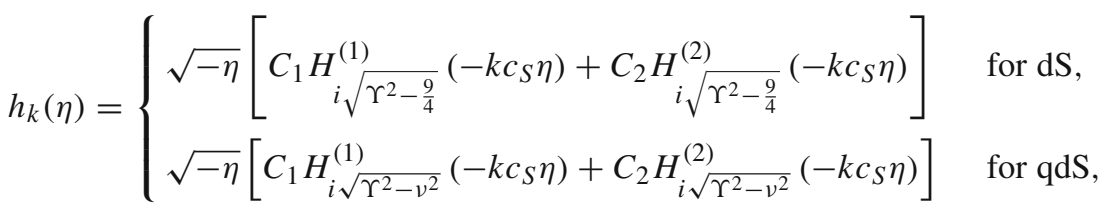




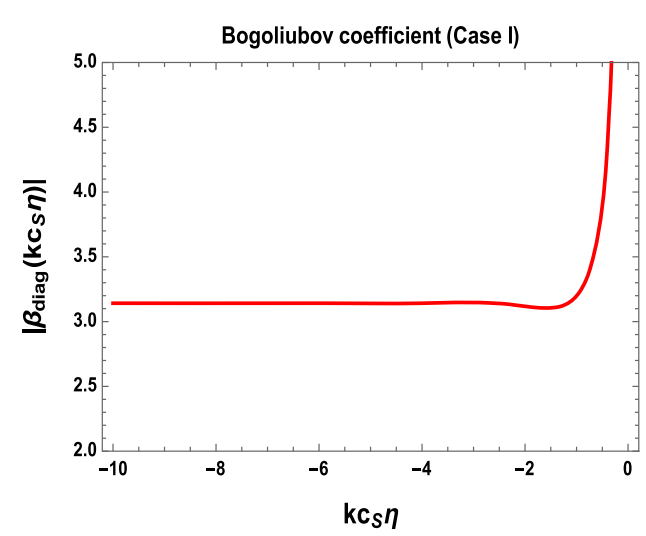

(a)

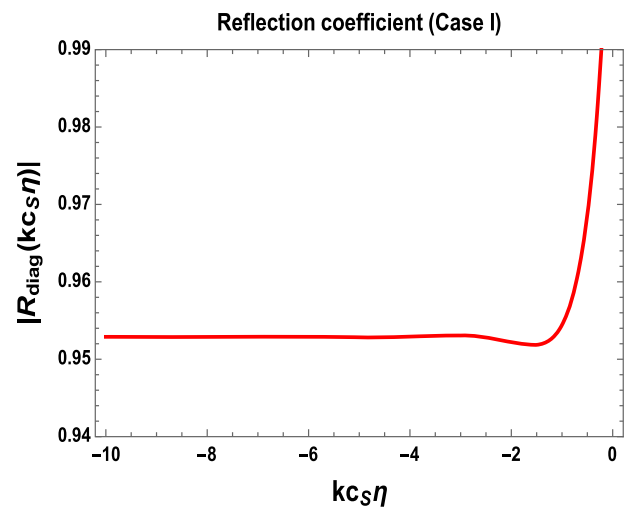

(c)

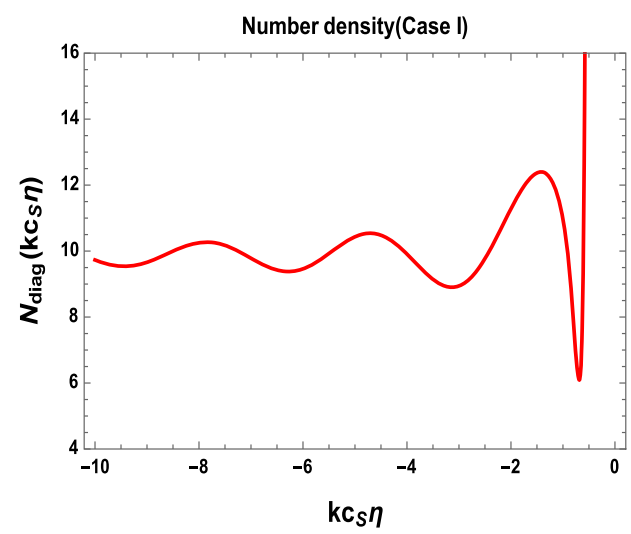

(e)

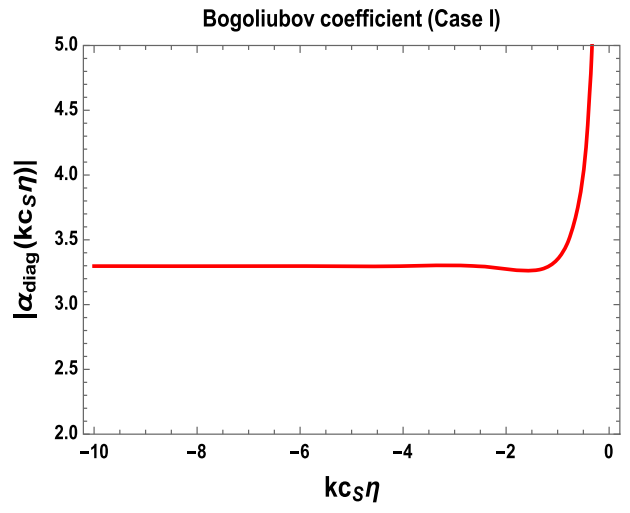

(b)

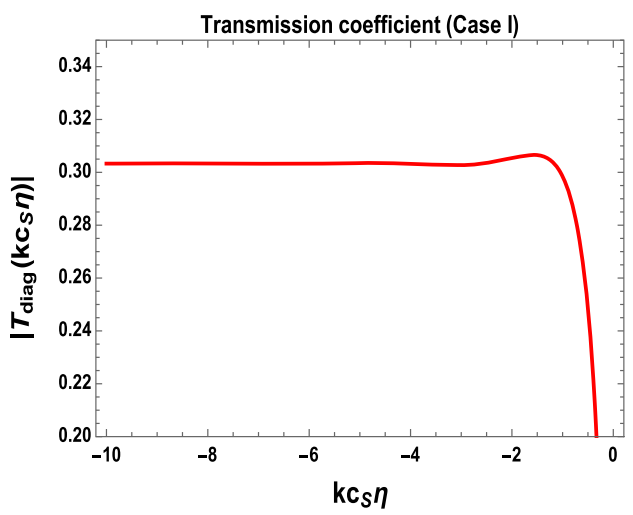

(d)

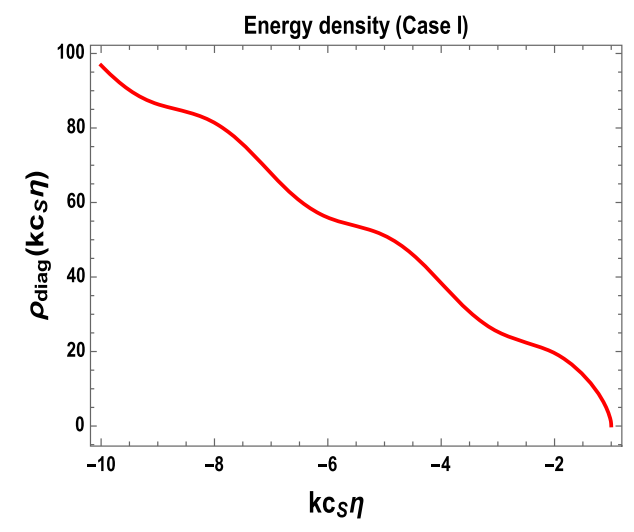

(f)

Fig. 8 Particle creation profile for Case I in diagonalized representation where $C_{1}$ and $C_{2}$ are two arbitrary integration constants, which depend on the choice of the initial condition.

After taking the $k c_{S} \eta \rightarrow-\infty, k c_{S} \eta \rightarrow 0$ and $\left|k c_{S} \eta\right| \approx 1$ limits the most general solution as stated in Eq. (3.118) can be recast as

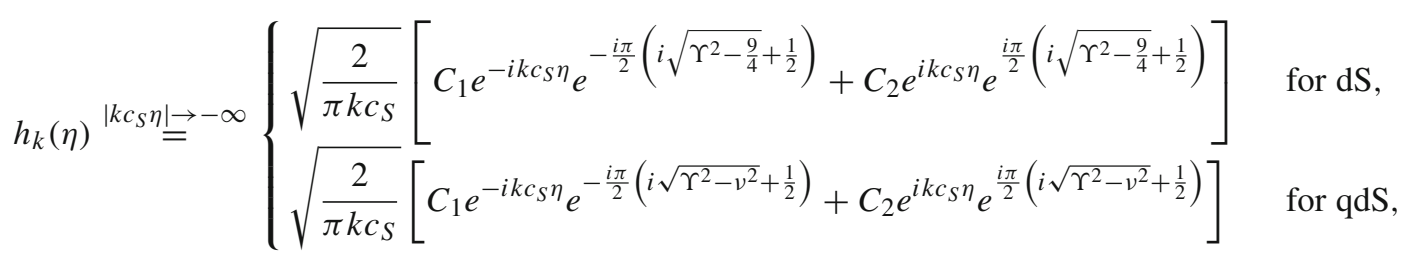


$h_{k}(\eta) \stackrel{\mid k c S \underline{\eta}=\rightarrow 0}{=}\left\{\begin{array}{l}\frac{i \sqrt{-\eta}}{\pi} \Gamma\left(i \sqrt{\Upsilon^{2}-\frac{9}{4}}\right)\left(-\frac{k c_{S} \eta}{2}\right)^{-i \sqrt{\Upsilon^{2}-\frac{9}{4}}}\left[C_{1}-C_{2}\right] \text { for } \mathrm{dS}, \\ \frac{i \sqrt{-\eta}}{\pi} \Gamma\left(i \sqrt{\Upsilon^{2}-v^{2}}\right)\left(-\frac{k c_{S} \eta}{2}\right)^{-i \sqrt{\Upsilon^{2}-v^{2}}}\left[C_{1}-C_{2}\right] \text { for qdS, }\end{array}\right.$

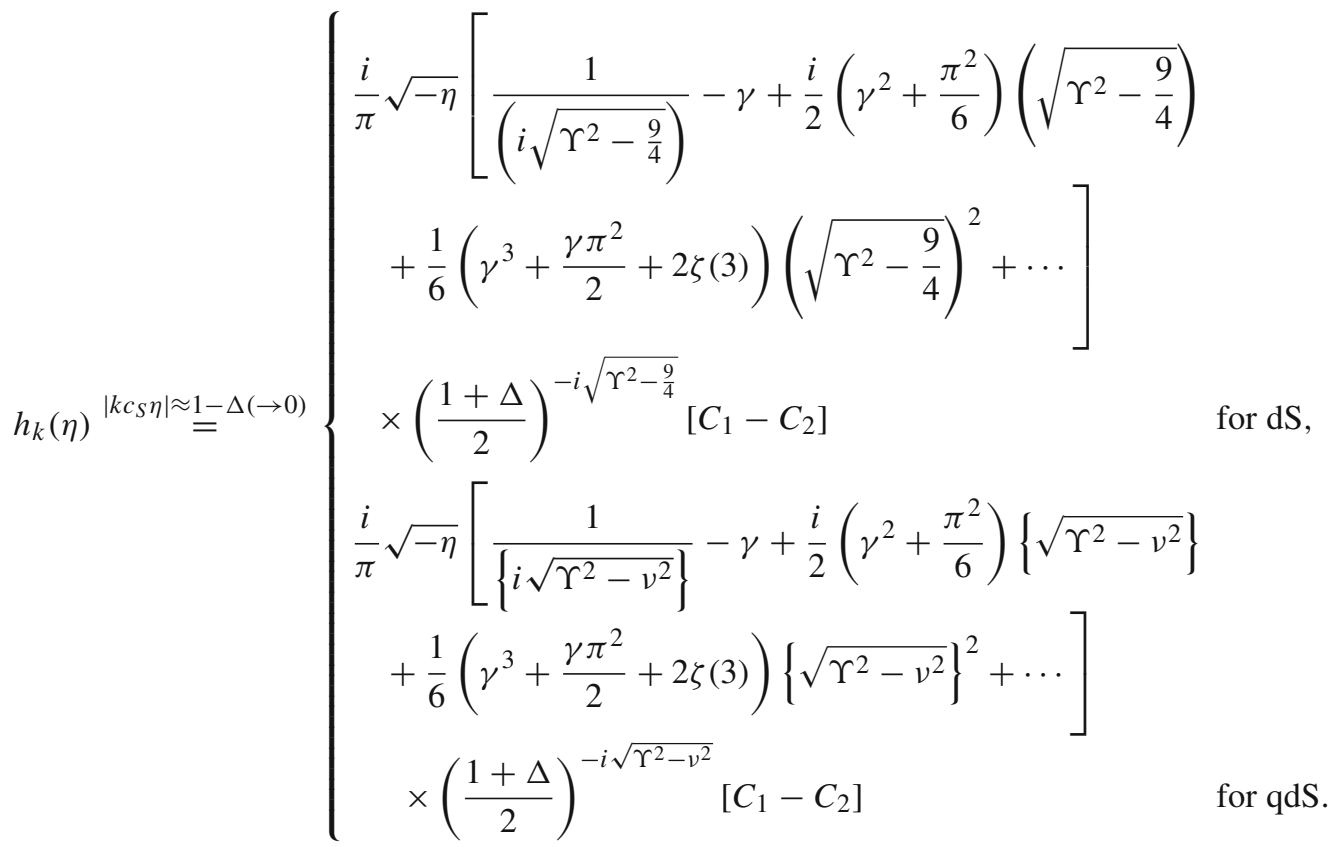

In the standard WKB approximation the total solution can be recast in the following form:

$h_{k}(\eta)=\left[D_{1} u_{k}(\eta)+D_{2} \bar{u}_{k}(\eta)\right]$,

where $D_{1}$ and $D_{2}$ are two arbitrary integration constants, which depend on the choice of the initial condition during WKB approximation at early and late time scale. In the present context $u_{k}(\eta)$ and $\bar{u}_{k}(\eta)$ are defined as

$$
\begin{aligned}
& \int \frac{1}{\sqrt{2 \sqrt{c_{S}^{2} k^{2}+\frac{\Upsilon^{2}-2}{\eta^{2}}}}} \exp \left[i \int^{\eta} \mathrm{d} \eta^{\prime} \sqrt{c_{S}^{2} k^{2}+\frac{\Upsilon^{2}-2}{\eta^{\prime 2}}}\right] \\
& =\frac{1}{\sqrt{2 \sqrt{c_{S}^{2} k^{2}+\frac{\Upsilon^{2}-2}{\eta^{2}}}}} \exp \left[i\left(\eta \sqrt{c_{S}^{2} k^{2}+\frac{\Upsilon^{2}-2}{\eta^{2}}}+\sqrt{\Upsilon^{2}-2} \ln \left[\frac{2}{\eta \sqrt{\Upsilon^{2}-2}}+\frac{2 \sqrt{c_{S}^{2} k^{2}+\frac{\Upsilon^{2}-2}{\eta^{2}}}}{\left(\Upsilon^{2}-2\right)}\right]\right)\right] \\
& u_{k}(\eta)=\left\{\frac{1}{\sqrt{2 \sqrt{c_{S}^{2} k^{2}+\frac{\left[\Upsilon^{2}-\left(v^{2}-\frac{1}{4}\right)\right]}{\eta^{2}}}}} \exp \left[i \int^{\eta} \mathrm{d} \eta^{\prime} \sqrt{c_{S}^{2} k^{2}+\frac{\left[\Upsilon^{2}-\left(v^{2}-\frac{1}{4}\right)\right]}{\eta^{\prime 2}}}\right]\right. \\
& =\frac{1}{\sqrt{2 \sqrt{c_{S}^{2} k^{2}+\frac{\left[\Upsilon^{2}-\left(v^{2}-\frac{1}{4}\right)\right]}{\eta^{2}}}}} \exp \left[i \left[\eta \sqrt{c_{S}^{2} k^{2}+\frac{\left[\Upsilon^{2}-\left(v^{2}-\frac{1}{4}\right)\right.}{\eta^{2}}}\right.\right. \\
& \left.\left.+\sqrt{\Upsilon^{2}-\left(v^{2}-\frac{1}{4}\right)} \ln \left[\frac{2}{\eta \sqrt{\Upsilon^{2}-\left(v^{2}-\frac{1}{4}\right)}}+\frac{2 \sqrt{c_{S}^{2} k^{2}+\frac{\Upsilon^{2}-\left(v^{2}-\frac{1}{4}\right)}{\eta^{2}}}}{\left(\Upsilon^{2}-\left(v^{2}-\frac{1}{4}\right)\right)}\right]\right)\right]
\end{aligned}
$$
for dS, 


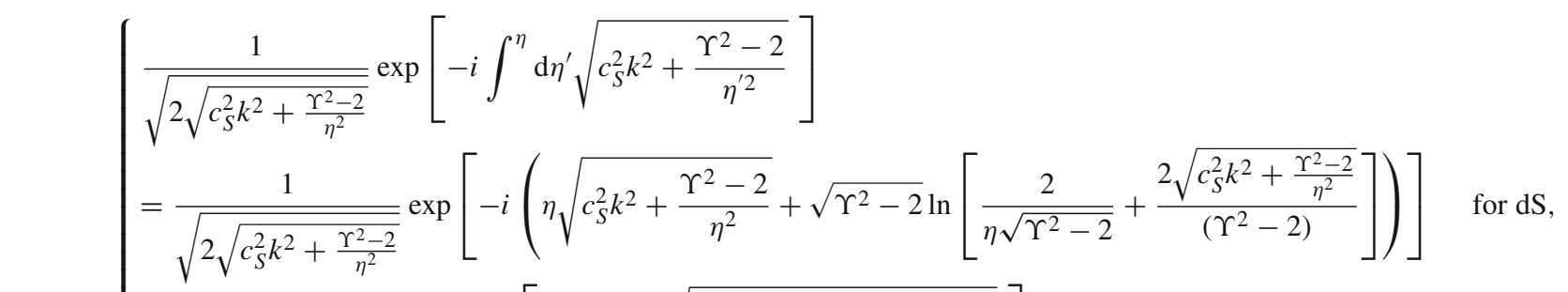

$$
\begin{aligned}
& \bar{u}_{k}(\eta)=\left\{\frac{1}{\sqrt{2 \sqrt{c_{S}^{2} k^{2}+\frac{\left[\Upsilon^{2}-\left(v^{2}-\frac{1}{4}\right)\right]}{\eta^{2}}}}} \exp \left[-i \int^{\eta} \mathrm{d} \eta^{\prime} \sqrt{c_{S}^{2} k^{2}+\frac{\left[\Upsilon^{2}-\left(v^{2}-\frac{1}{4}\right)\right]}{\eta^{\prime 2}}}\right]\right. \\
& =\frac{1}{\sqrt{2 \sqrt{c_{S}^{2} k^{2}+\frac{\left[\Upsilon^{2}-\left(v^{2}-\frac{1}{4}\right)\right]}{\eta^{2}}}}} \exp \left[-i\left(\eta \sqrt{c_{S}^{2} k^{2}+\frac{\left[\Upsilon^{2}-\left(v^{2}-\frac{1}{4}\right)\right]}{\eta^{2}}}\right.\right. \\
& \left.\left.+\sqrt{\Upsilon^{2}-\left(v^{2}-\frac{1}{4}\right)} \ln \left[\frac{2}{\eta \sqrt{\Upsilon^{2}-\left(v^{2}-\frac{1}{4}\right)}}+\frac{2 \sqrt{c_{S}^{2} k^{2}+\frac{\Upsilon^{2}-\left(v^{2}-\frac{1}{4}\right)}{\eta^{2}}}}{\left(\Upsilon^{2}-\left(v^{2}-\frac{1}{4}\right)\right)}\right]\right)\right]
\end{aligned}
$$

where we have written the total solution for the mode $h_{k}$ in terms of two linearly independent solutions. Here it is important to note that both of the solutions are hermitian conjugate of each other. If in the present context the exact solution of the mode $h_{k}$ is expanded with respect to these two linearly independent solutions then particle creation is absent in our EFT setup. In the present context correctness of WKB approximation is guaranteed at very early and very late time scales. In this discussion $u_{k}(\eta)$ is valid at very early time scale and $\bar{u}_{k}(\eta)$ perfectly works in the late time scale. coefficient $\beta(k)$ in Fourier space which in principle measures the exact amount of late times solution $u_{k}(\eta)$, if in the present context we exactly start with the early time scale solution $u_{k}(\eta)$. In our present computation we consider a physical situation where the WKB approximation is correct up to the leading order throughout the cosmological evolution in time scale. In the present context the Bogoliubov coefficient $\beta(k)$ in Fourier space can be computed approximately using the following regularized integral:

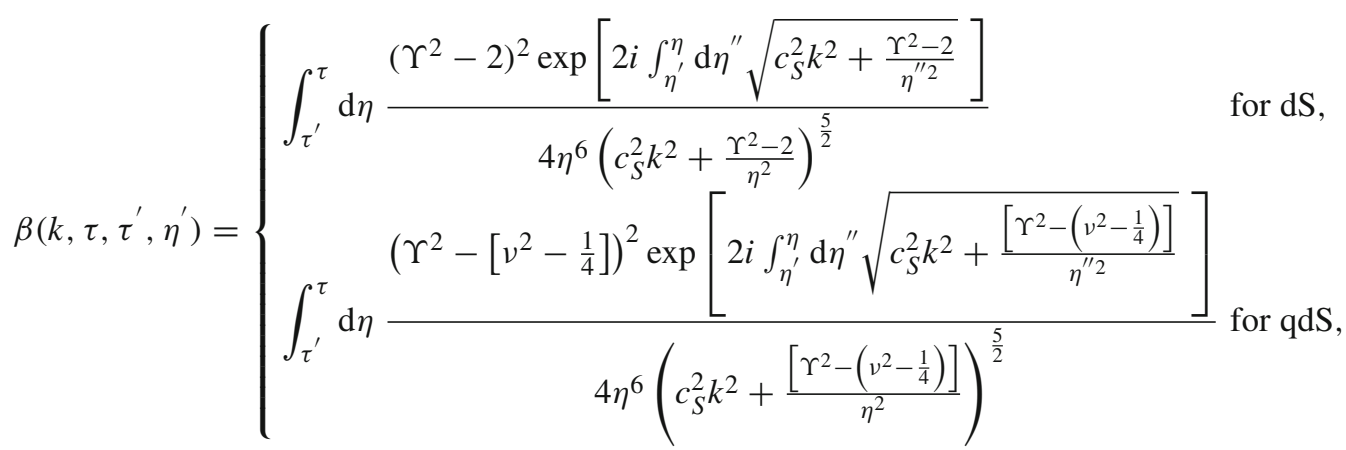

Now we will explicitly check that the exactness of the above mentioned WKB result derived in Eq. (3.122) with the actual solution of the mode function as presented in Eq. (3.118). As mentioned earlier in FLRW space-time in Fourier space Bogoliubov coefficient $\beta(k)$ measures this exactness for a given setup. The particle creation mechanism and its exact amount is described by finding the Bogoliubov which is not exactly analytically computable. To study the behaviour of this integral we consider here three consecutive physical situations: $\left|k c_{S} \eta\right|<<1,\left|k c_{S} \eta\right| \approx 1-\Delta(\rightarrow 0)$ and $\left|k c_{S} \eta\right|>>1$ for the de Sitter case and the quasi de Sitter case. In the three cases we have 
For dS:

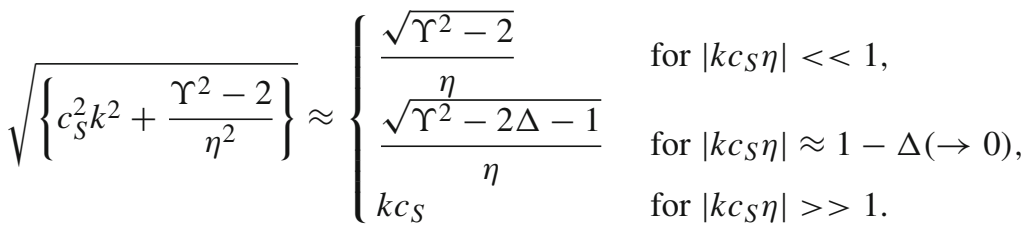

For qdS:

$$
\sqrt{\left\{c_{S}^{2} k^{2}+\frac{\left[\Upsilon^{2}-\left(v^{2}-\frac{1}{4}\right)\right]}{\eta^{2}}\right\}} \approx \begin{cases}\frac{\sqrt{\Upsilon^{2}-\left(v^{2}-\frac{1}{4}\right)}}{\eta} & \text { for }\left|k c_{S} \eta\right|<<1, \\ \frac{\sqrt{\Upsilon^{2}-2 \Delta-\left(v^{2}-\frac{5}{4}\right)}}{\eta} & \text { for }\left|k c_{S} \eta\right| \approx 1-\Delta(\rightarrow 0), \\ k c_{S} & \text { for }\left|k c_{S} \eta\right|>>1,\end{cases}
$$

and further using this result the Bogoliubov coefficient $\beta(k)$ in Fourier space can be expressed as

For dS:

$$
\beta\left(k, \tau, \tau^{\prime}, \eta^{\prime}\right)= \begin{cases}\frac{\left[\tau^{2 i \sqrt{\Upsilon^{2}-2}}-\tau^{\prime 2 i \sqrt{\Upsilon^{2}-2}}\right]}{8 i\left(\Upsilon^{2}-2\right) \eta^{\prime 2 i \sqrt{\Upsilon^{2}-2}}} & \text { for }\left|k c_{S} \eta\right|<<1, \\ \frac{\left(\Upsilon^{2}-2\right)^{2}\left[\tau^{2 i \sqrt{\Upsilon^{2}-2 \Delta-1}}-\tau^{\left.\prime 2 i \sqrt{\Upsilon^{2}-2 \Delta-1}\right]}\right.}{8 i\left(\Upsilon^{2}-2 \Delta-1\right)^{3} \eta^{\prime 2 i \sqrt{\Upsilon^{2}-2 \Delta-1}}} & \text { for }\left|k c_{S} \eta\right| \approx 1-\Delta(\rightarrow 0), \\ \left(\Upsilon^{2}-2\right)^{2}\left[i \frac{\operatorname{Ei}\left(2 i k c_{S} \eta\right) e^{-2 i k c_{S} \eta^{\prime}}}{15}-\frac{e^{2 i k c_{S}\left(\eta-\eta^{\prime}\right)}}{120\left(c_{S} k\right)^{5} \eta^{5}}\right. & \\ \times\left(4\left(c_{S} k\right)^{4} \eta^{4}-2 i\left(c_{S} k\right)^{3} \eta^{3}-2\left(c_{S} k\right)^{2} \eta^{2}+3 i c_{S} k \eta+6\right) & ]_{\tau^{\prime}}^{\tau} \text { for }\left|k c_{S} \eta\right|>>1 .\end{cases}
$$

For qdS:

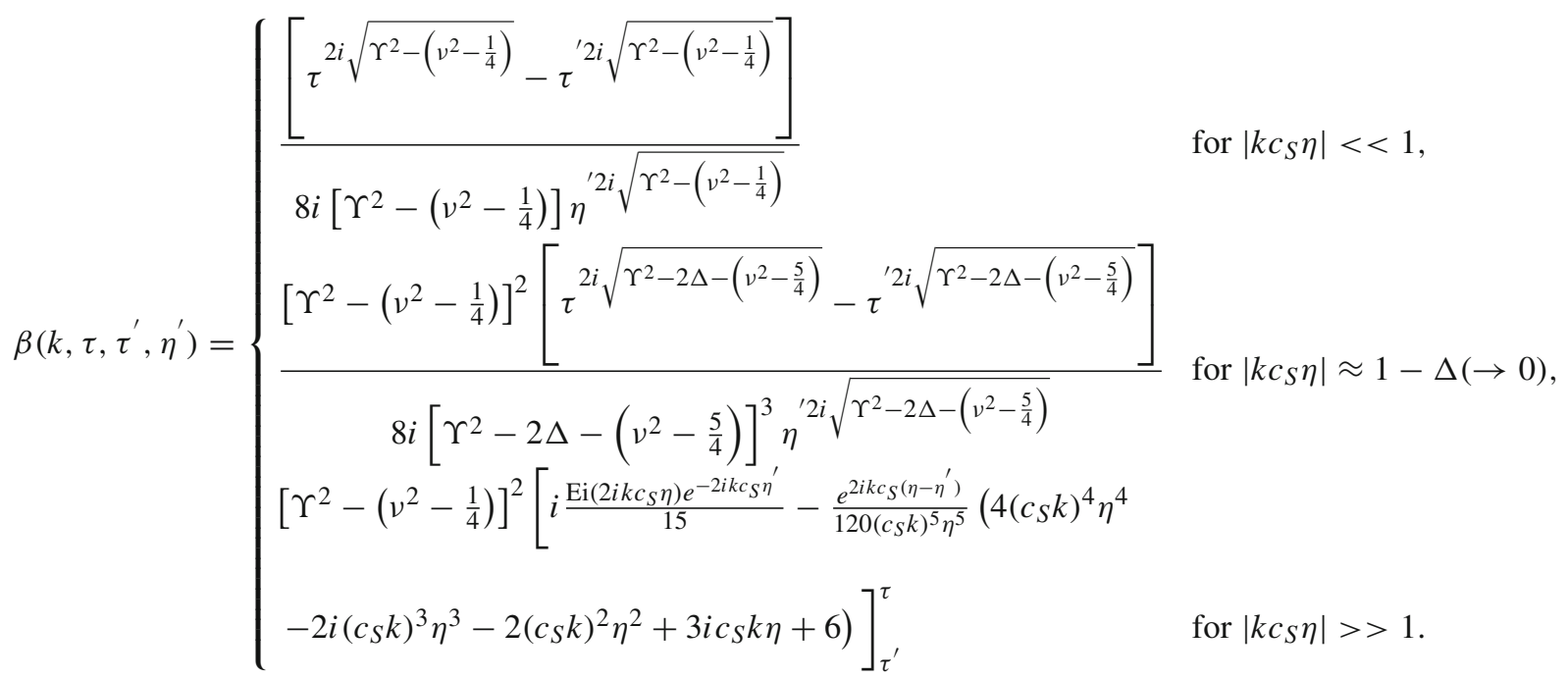


In all the situations described for the de Sitter and the quasi de Sitter case here the magnitude of the Bogoliubov coefficient $|\beta(k)|$ in Fourier space is considerably small. Specifically it is important to point out here that for the case when $\left|k c_{S} \eta\right|>>1$ the value of the Bogoliubov coefficient $\beta(k)$ in Fourier space is even smaller as the WKB approximated solution is strongly consistent for all time scales. On the other hand in the vicinity of the conformal time scale $\eta \sim \eta_{\text {pair }}$ for $\left|k c_{S} \eta_{\text {pair }}\right|<<1$ the WKB approximated solution is less strongly valid and to validate the solution at this time scale one can neglect the momentum $k$ dependence in the Bogoliubov coefficient $\beta(k)$ in Fourier space. Here $\left|\eta_{\text {pair }}\right|$ characterizes the relative separation between the created particles.

As mentioned earlier here one can use another equivalent way to define the Bogoliubov coefficient $\beta$ in Fourier space by implementing an instantaneous Hamiltonian diagonalization method to interpret the results. Using this diagonalized representation the regularized Bogoliubov coefficient $\beta$ in Fourier space can be written as

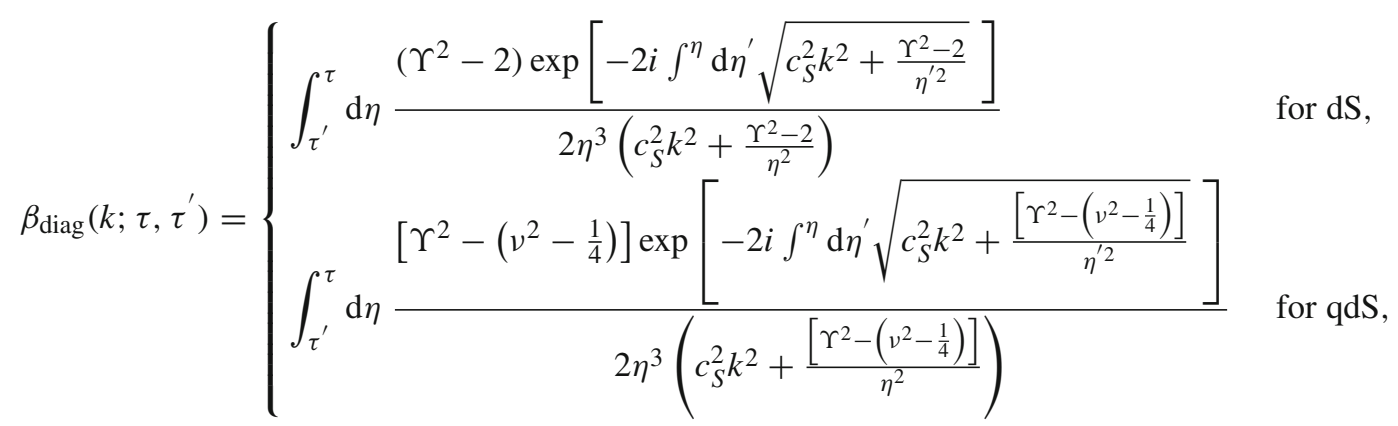

where $\tau$ and $\tau^{\prime}$ are introduced as the conformal time regulator in the present context. In this case as well the Bogoliubov coefficient is not exactly analytically computable. To study the behaviour of this integral we consider here three similar consecutive physical situations for the de Sitter and the quasi de Sitter case as discussed earlier.

For dS:

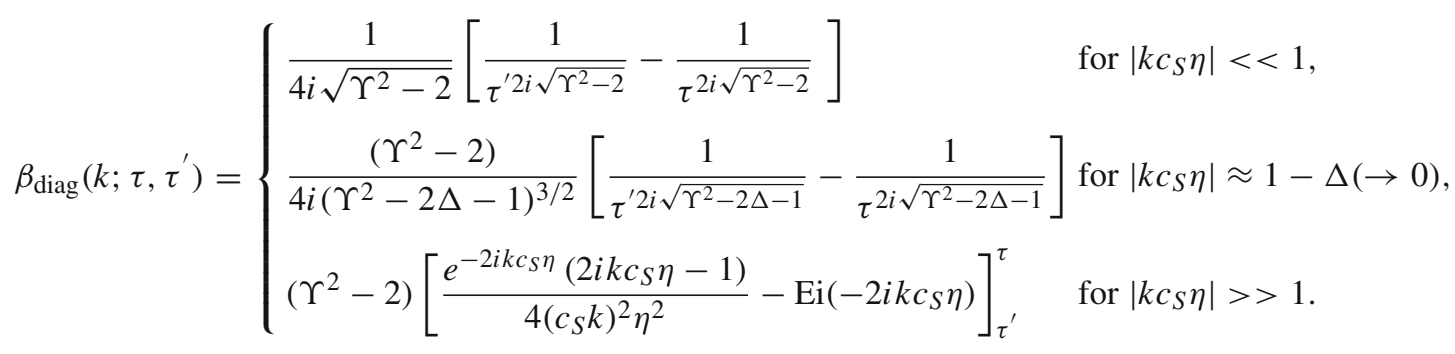

For qdS:

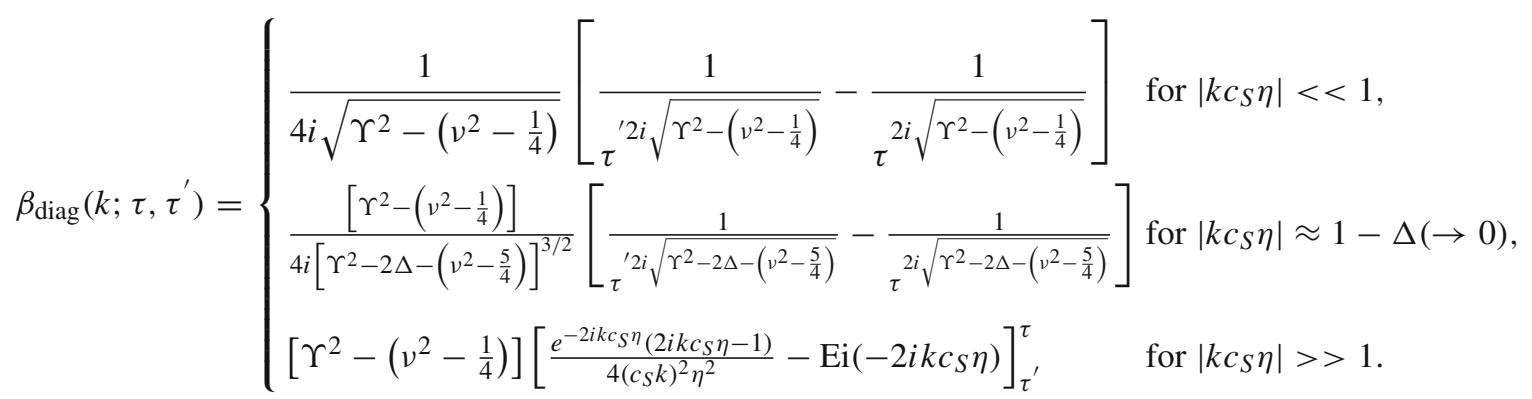

Further using the regularized expressions for the Bogoliubov coefficient $\beta$ in two different representations as mentioned in Eqs. (3.125) and (3.129), and substituting them in Eq. (3.57) we get the following regularized expressions for the Bogoliubov coefficient $\alpha$ in two different representations as given by 


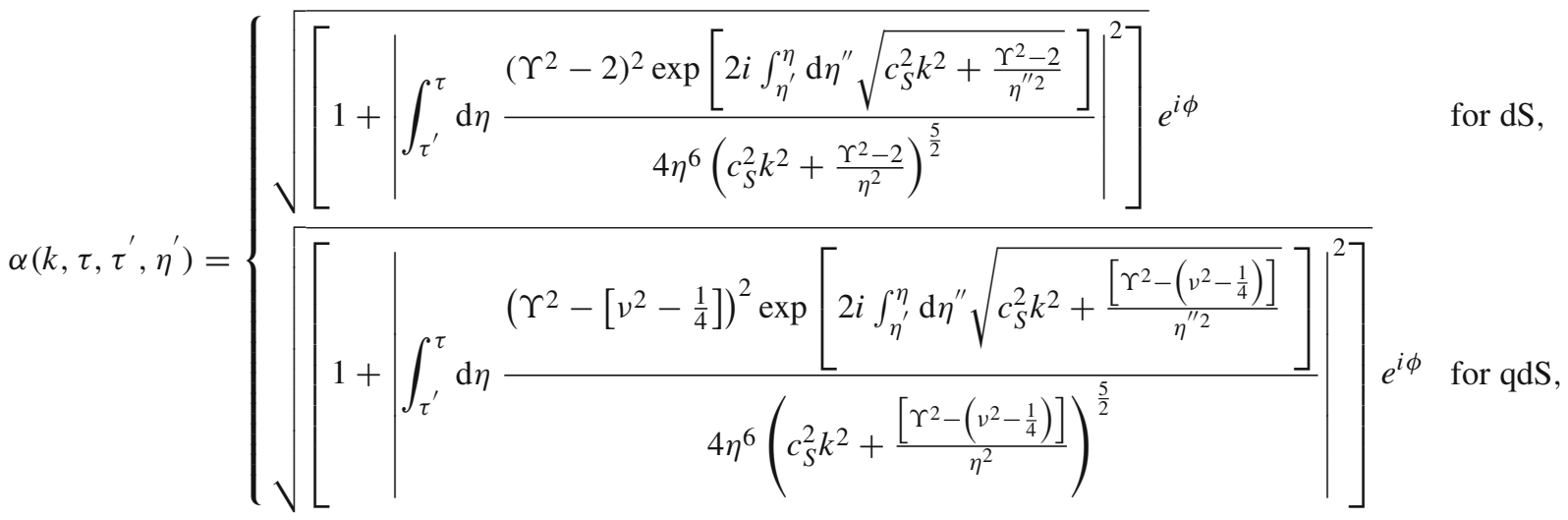

$\alpha_{\text {diag }}\left(k ; \tau, \tau^{\prime}\right)= \begin{cases}\sqrt{\left[1+\left|\int_{\tau^{\prime}}^{\tau} \mathrm{d} \eta \frac{\left(\Upsilon^{2}-2\right) \exp \left[-2 i \int^{\eta} \mathrm{d} \eta^{\prime} \sqrt{c_{S}^{2} k^{2}+\frac{\Upsilon^{2}-2}{\eta^{\prime 2}}}\right]}{2 \eta^{3}\left(c_{S}^{2} k^{2}+\frac{\Upsilon^{2}-2}{\eta^{2}}\right)}\right|^{2}\right]} e^{i \phi_{\text {diag }}} & \text { for dS, } \\ \sqrt{\left[1+\mid \int_{\tau^{\prime}}^{\tau} \mathrm{d} \eta \frac{\left[\Upsilon^{2}-\left(v^{2}-\frac{1}{4}\right)\right] \exp \left[-2 i \int^{\eta} \mathrm{d} \eta^{\prime} \sqrt{\left.c_{S}^{2} k^{2}+\frac{\left[\Upsilon^{2}-\left(v^{2}-\frac{1}{4}\right)\right]}{\eta^{\prime 2}}\right]}\right]}{2 \eta^{3}\left(c_{S}^{2} k^{2}+\frac{\left[\Upsilon^{2}-\left(v^{2}-\frac{1}{4}\right)\right]}{\eta^{2}}\right)}\right]} e^{i \phi_{\text {diag }}} & \text { for qdS, }\end{cases}$

where $\phi$ and $\phi_{\text {diag }}$ are the associated phase factors in two different representations. Here the results are not exactly analytically computable. To study the behaviour of this integral we consider here three consecutive physical situations: $\left|k c_{S} \eta\right|<<1$, $\left|k c_{S} \eta\right| \approx 1-\Delta(\rightarrow 0)$ and $\left|k c_{S} \eta\right|>>1$ for the de Sitter case and the quasi de Sitter case.

For dS:

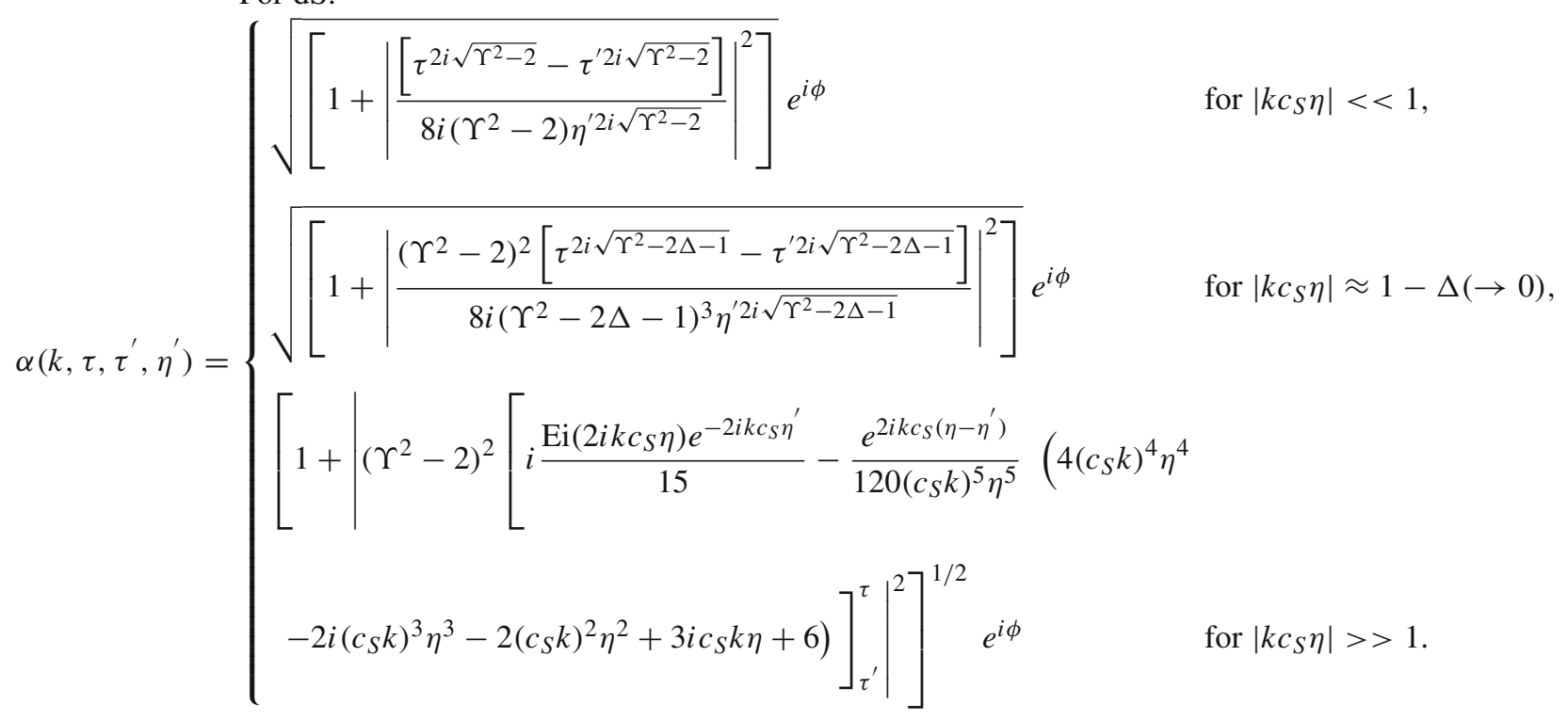


For qdS:

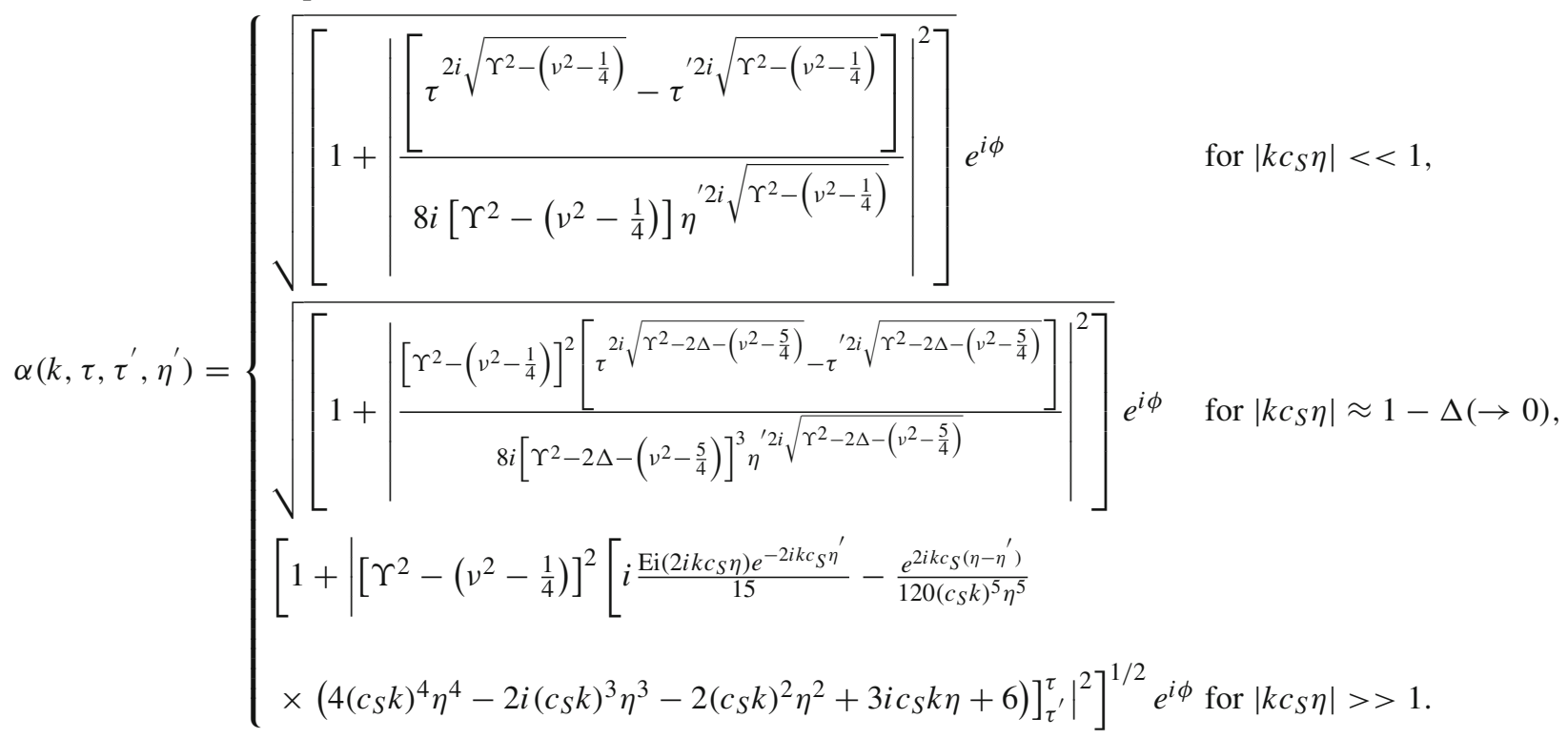

For dS:

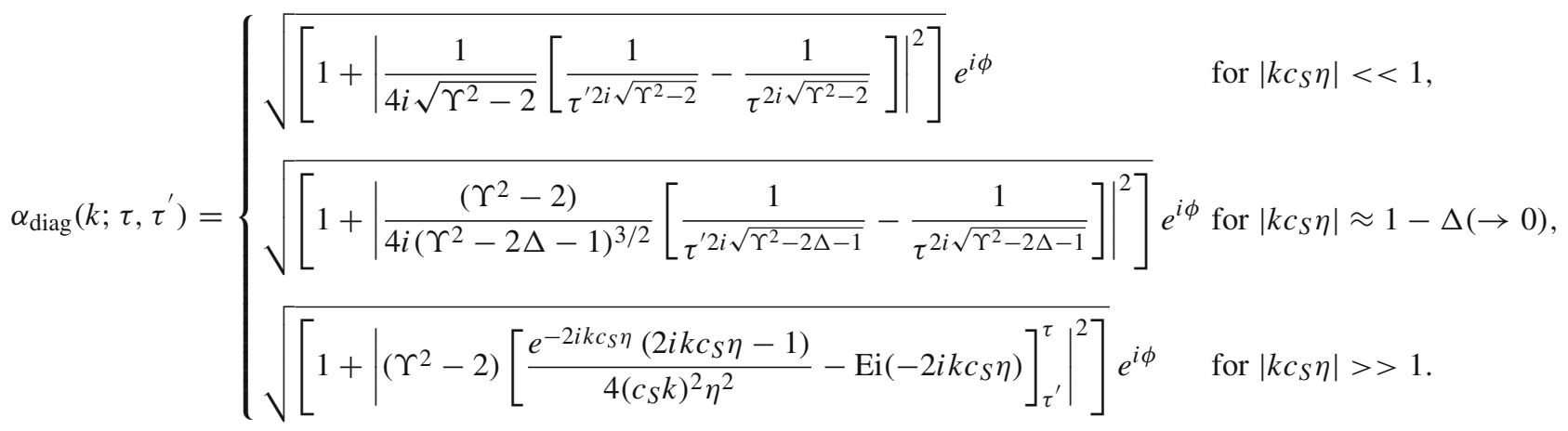

For qdS:

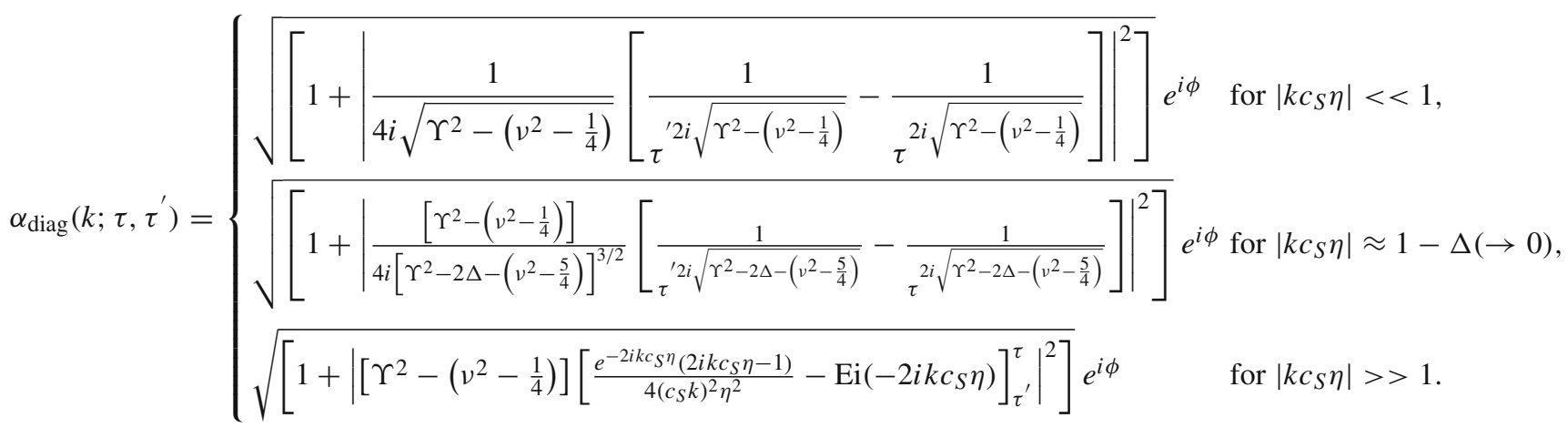

Further using the expressions for the Bogoliubov coefficient $\alpha$ in two different representations and substituting them in Eq. (3.58) we get the following expressions for the reflection and transmission coefficient in two different representations for 
three consecutive physical situations: $\left|k c_{S} \eta\right|<<1,\left|k c_{S} \eta\right| \approx 1-\Delta(\rightarrow 0)$ and $\left|k c_{S} \eta\right|>>1$ for the de Sitter case and the quasi de Sitter case as given by

For dS:

$$
\begin{aligned}
& \int \frac{\frac{\left[\tau^{2 i \sqrt{\Upsilon^{2}-2}}-\tau^{\prime 2 i \sqrt{\Upsilon^{2}-2}}\right]}{8 i\left(\Upsilon^{2}-2\right) \eta^{\prime 2 i \sqrt{\Upsilon^{2}-2}}}}{\left.\left.\sqrt{\left[1\left[\tau^{2 i \sqrt{\Upsilon^{2}-2}}-\tau^{\prime 2} \sqrt{\Upsilon^{2}-2}\right]\right.}\right|^{2}\right]} e^{-i \phi} \quad \text { for }\left|k c_{S} \eta\right|<<1,
\end{aligned}
$$

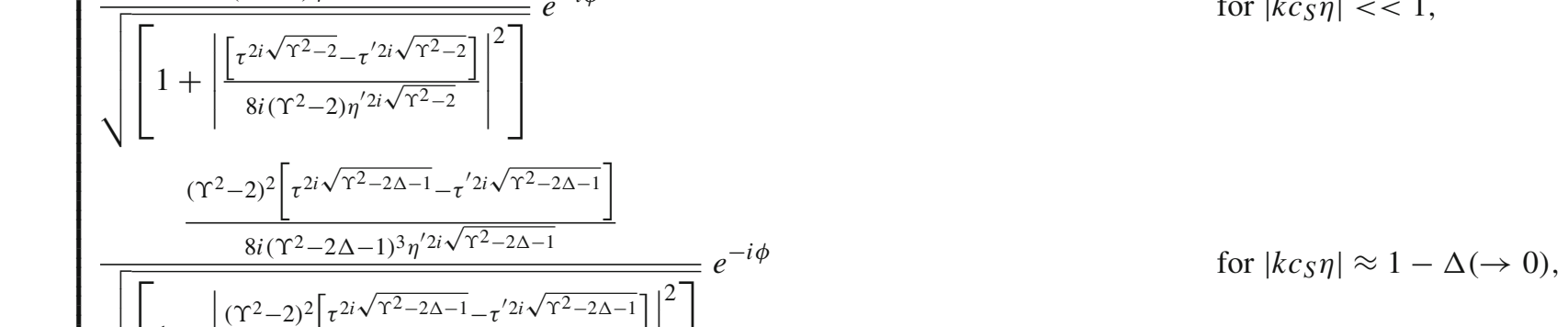

$$
\begin{aligned}
& \mathcal{R}=\left\{\sqrt{\left[1+\left|\frac{\left(\Upsilon^{2}-2\right)^{2}\left[\tau^{2 i} \sqrt{\Upsilon^{2}-2 \Delta-1}-\tau^{2 i} \sqrt{\Upsilon^{2}-2 \Delta-1}\right.}{8 i\left(\Upsilon^{2}-2 \Delta-1\right)^{3} \eta^{\prime 2 i} \sqrt{\Upsilon^{2}-2 \Delta-1}}\right|\right.} \mid\right] \\
& \left(\Upsilon^{2}-2\right)^{2}\left[i \frac{\operatorname{Ei}\left(2 i k c_{S} \eta\right) e^{-2 i k c_{S} \eta^{\prime}}}{15}-\frac{e^{2 i k c_{S}\left(\eta-\eta^{\prime}\right)}}{120\left(c_{S} k\right)^{5} \eta^{5}}\left(4\left(c_{S} k\right)^{4} \eta^{4}-2 i\left(c_{S} k\right)^{3} \eta^{3}\right.\right. \\
& \left.\left.-2\left(c_{S} k\right)^{2} \eta^{2}+3 i c_{S} k \eta+6\right)\right]_{\tau^{\prime}}^{\tau} /\left[1+\mid\left(\Upsilon^{2}-2\right)^{2}\left[i \frac{\operatorname{Ei}\left(2 i k c_{S} \eta\right) e^{-2 i k c} \eta^{\prime} \eta^{\prime}}{15}\right.\right. \\
& \left.\left.-\frac{e^{2 i k c_{S}\left(\eta-\eta^{\prime}\right)}}{120\left(c_{S} k\right)^{5} \eta^{5}}\left(4\left(c_{S} k\right)^{4} \eta^{4}-2 i\left(c_{S} k\right)^{3} \eta^{3}-2\left(c_{S} k\right)^{2} \eta^{2}+3 i c_{S} k \eta+6\right)\right]\left._{\tau^{\prime}}^{\tau}\right|^{2}\right]^{1 / 2} e^{-i \phi} \text { for }\left|k c_{S} \eta\right|>>1
\end{aligned}
$$

For dS:

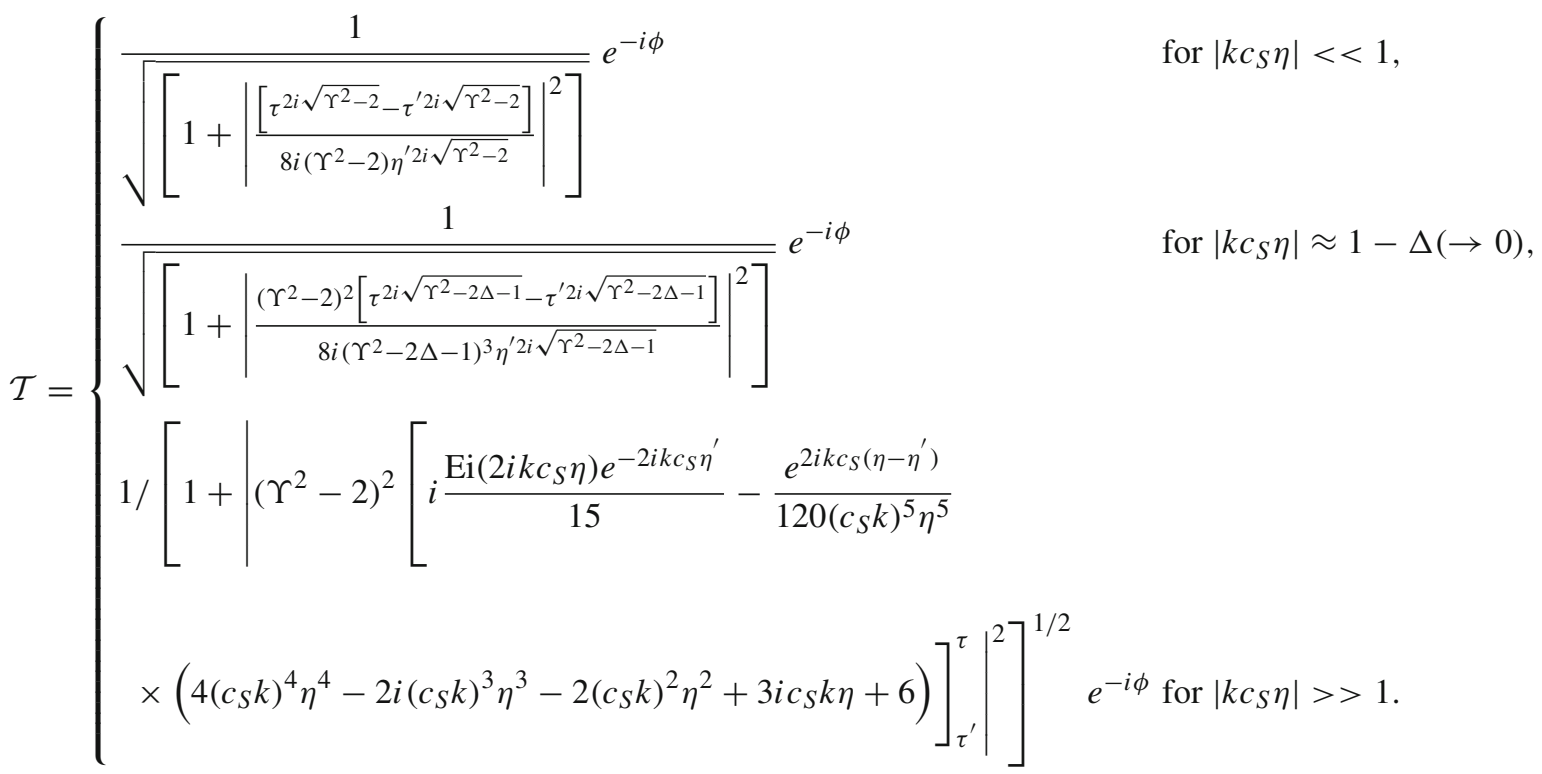


For qdS:

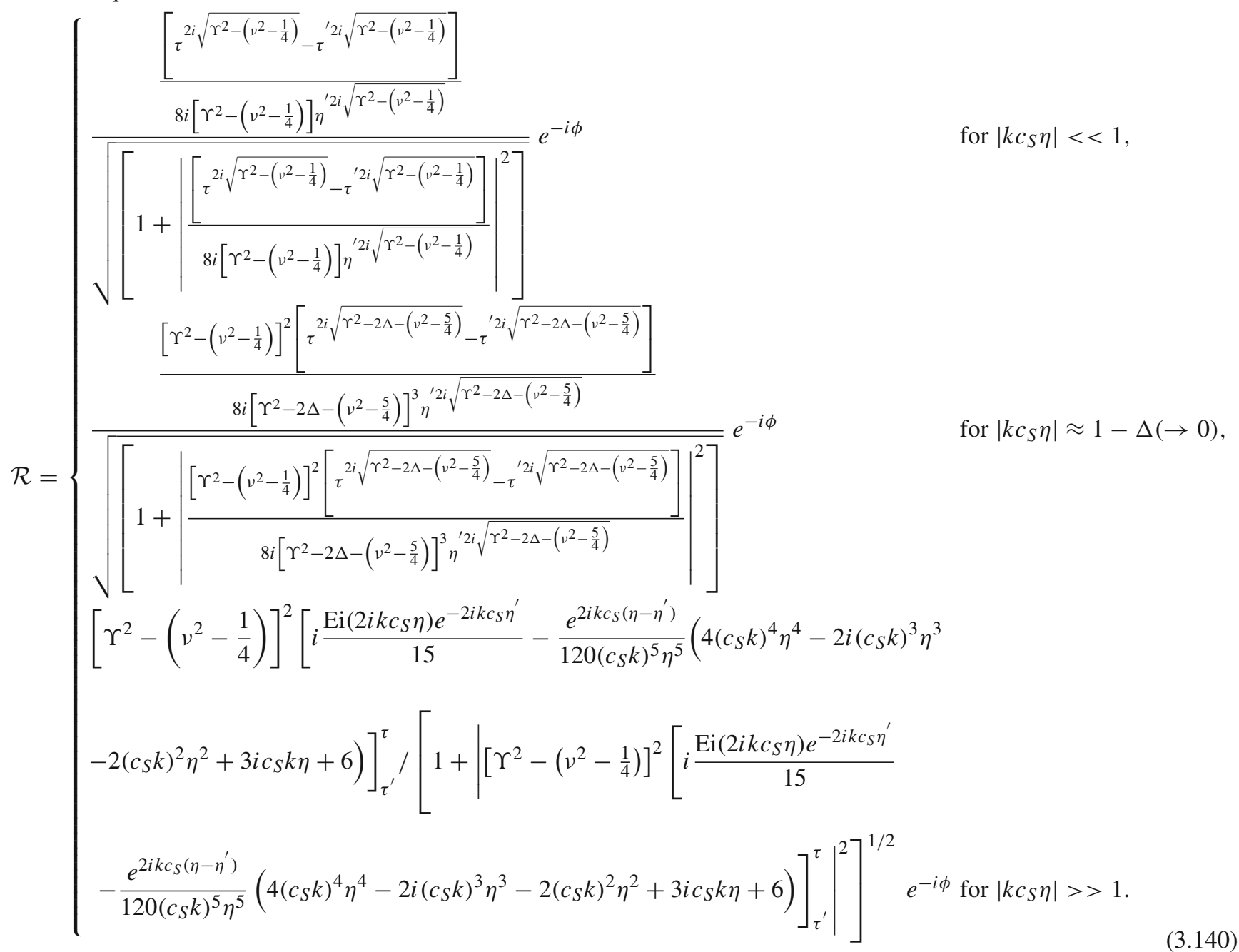

For qdS:

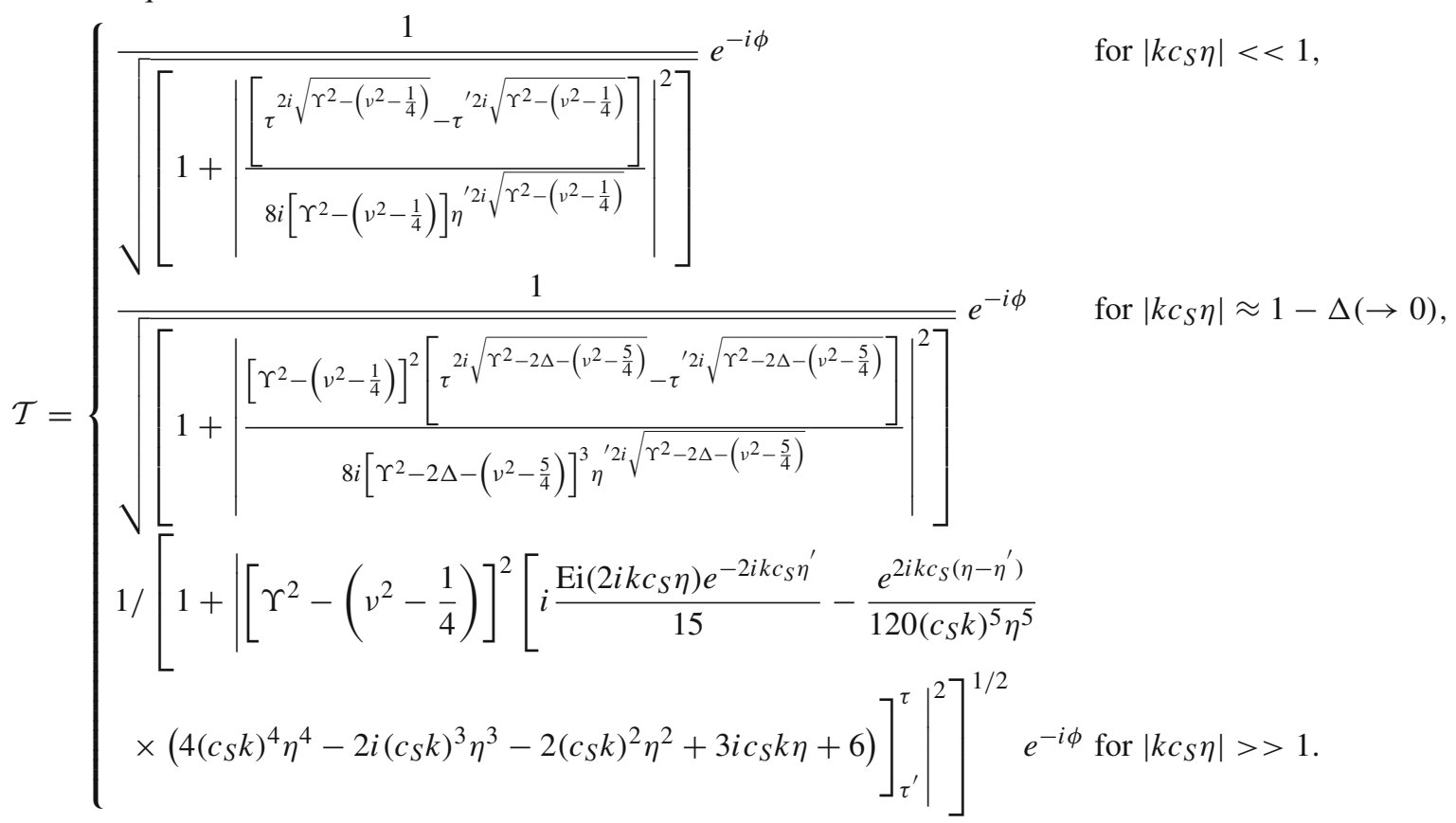


and

For dS:

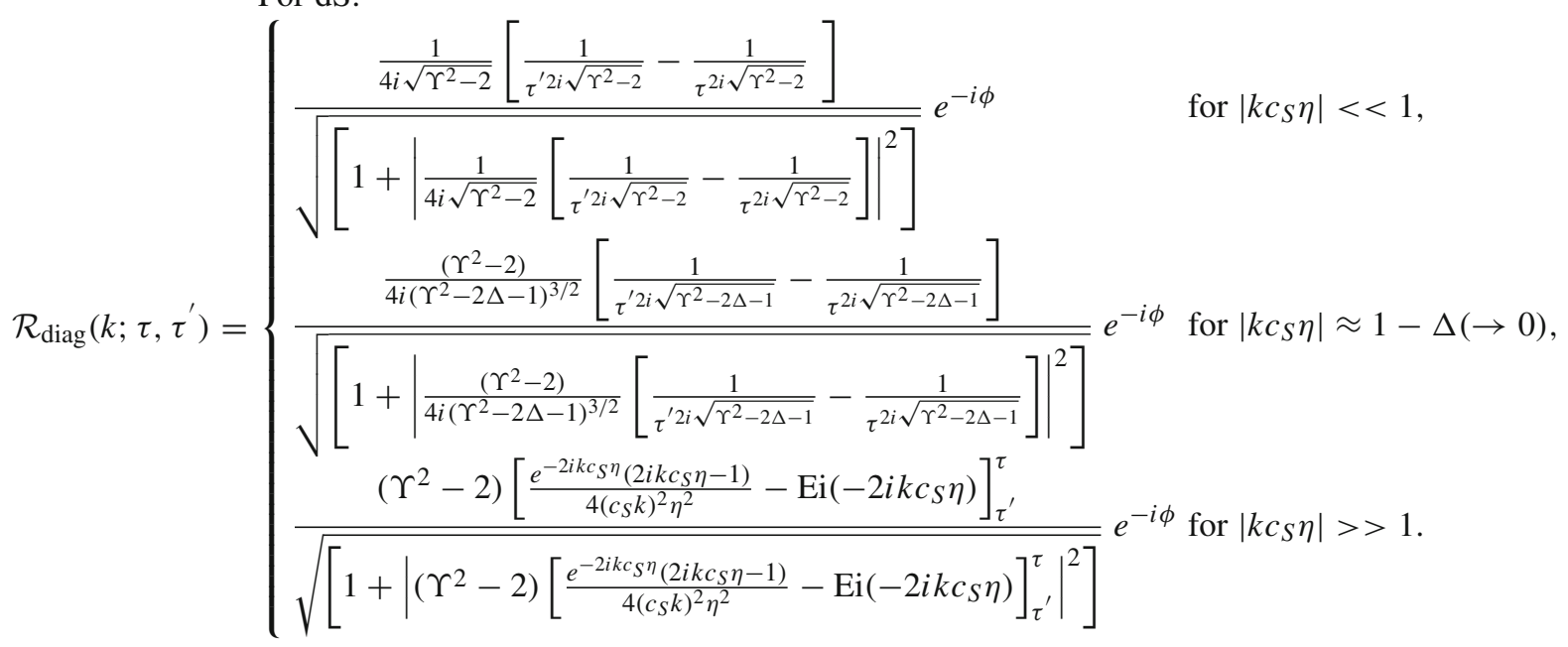

For dS:

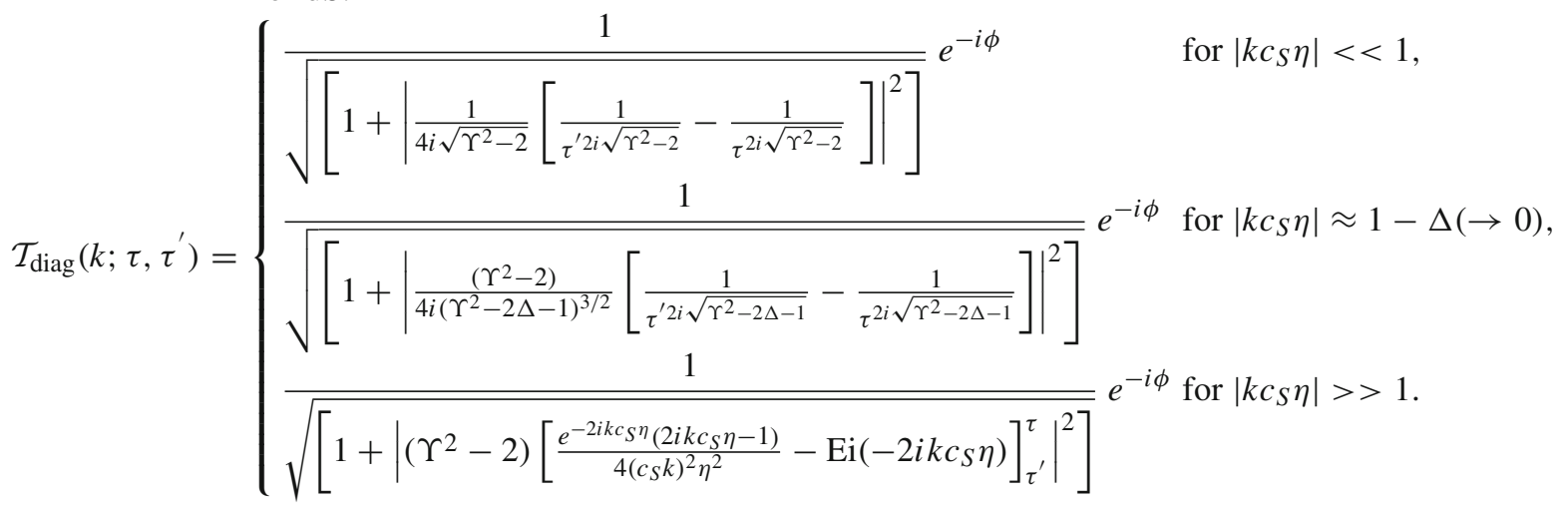

For qdS:

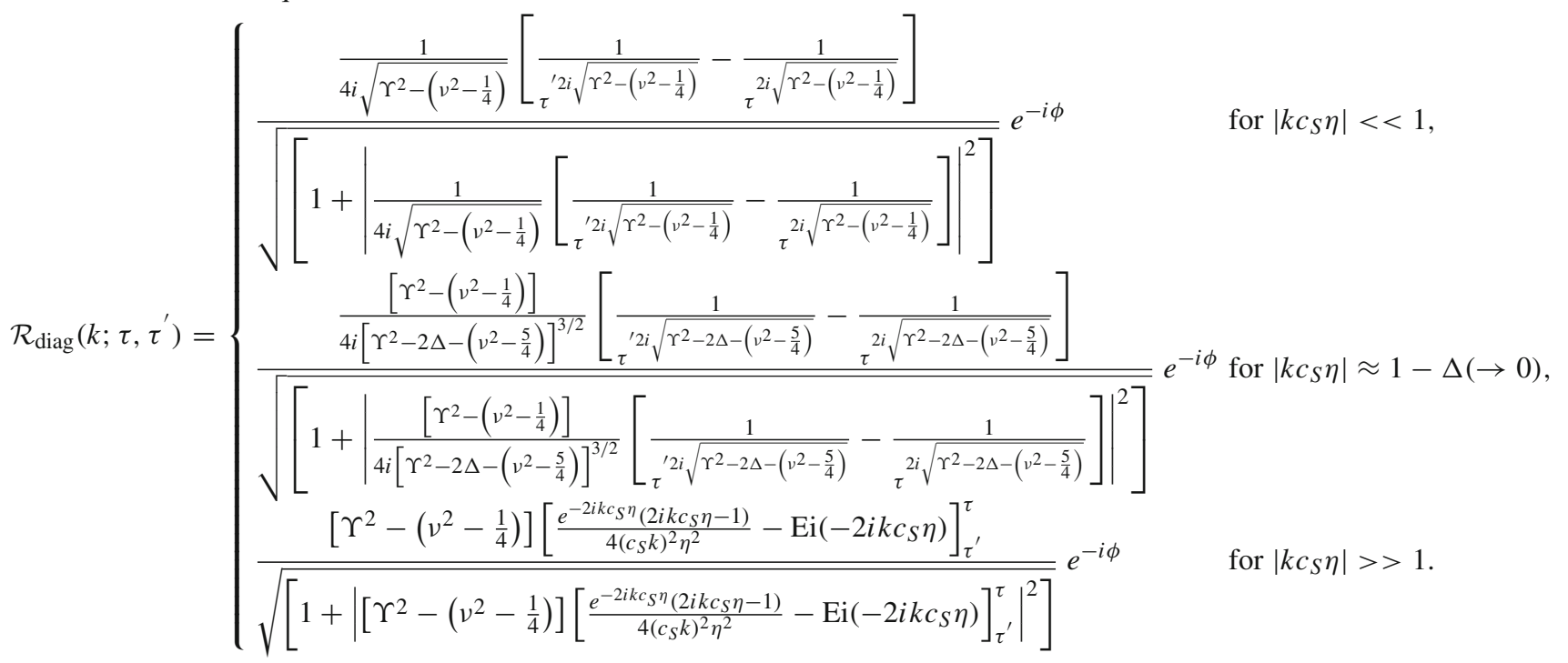


For qdS:

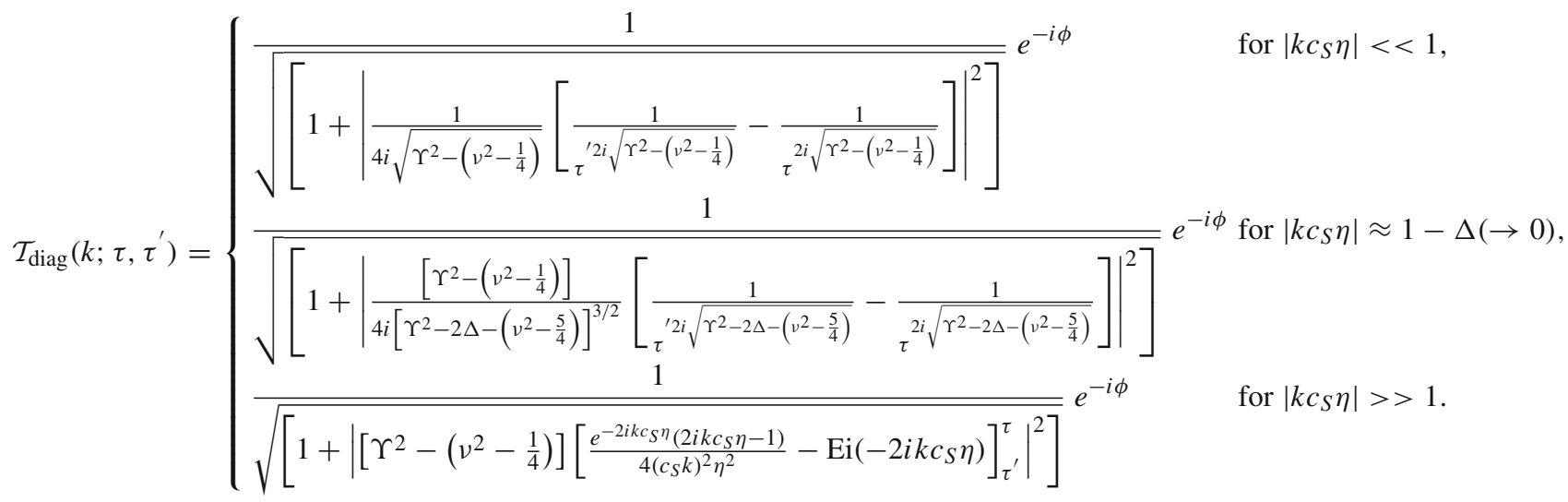

Next the expression for the number of produced particles at time $\tau$ can be calculated from the following expression:

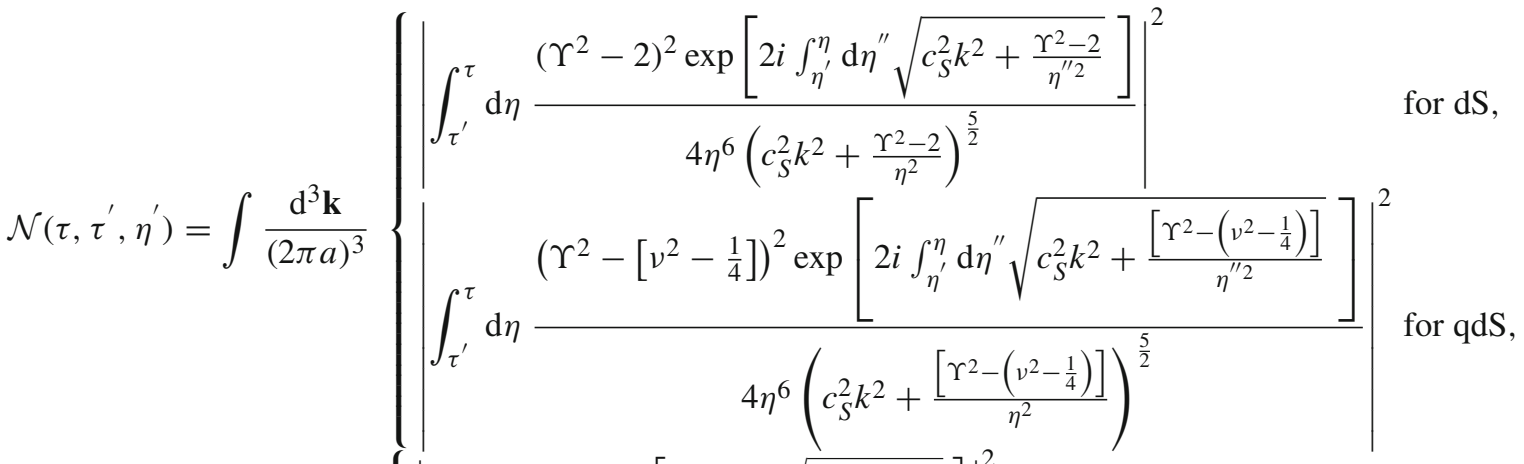

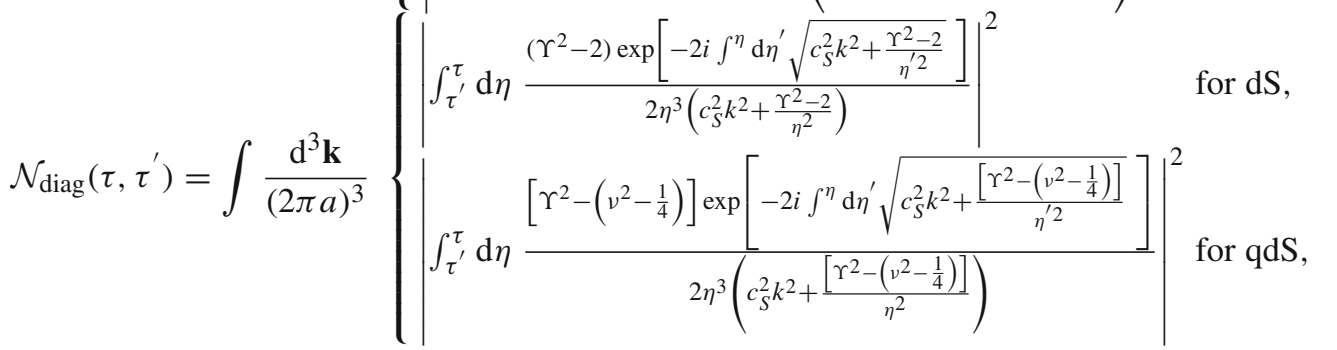

which is not exactly analytically computable. To study the behaviour of this integral we consider here three consecutive physical situations: $\left|k c_{S} \eta\right|<<1,\left|k c_{S} \eta\right| \approx 1-\Delta(\rightarrow 0)$ and $\left|k c_{S} \eta\right|>>1$ for the de Sitter case and the quasi de Sitter case. In the three cases we have

For dS:

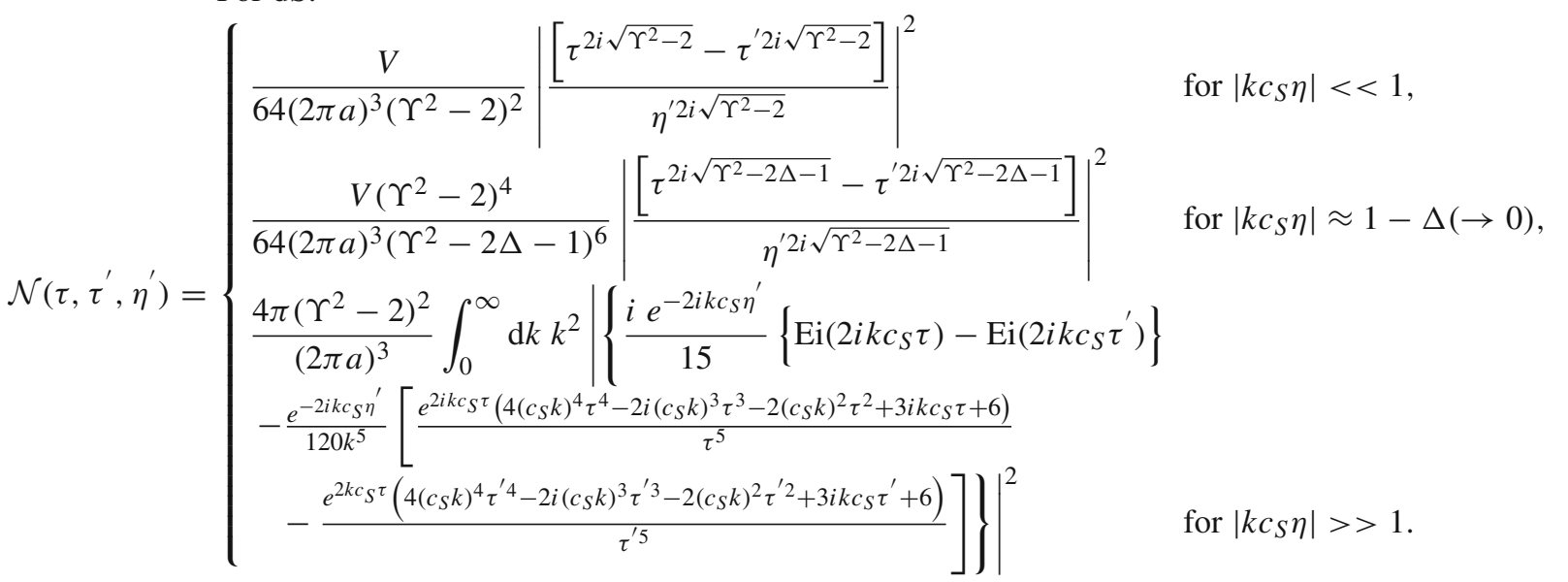


For qdS:

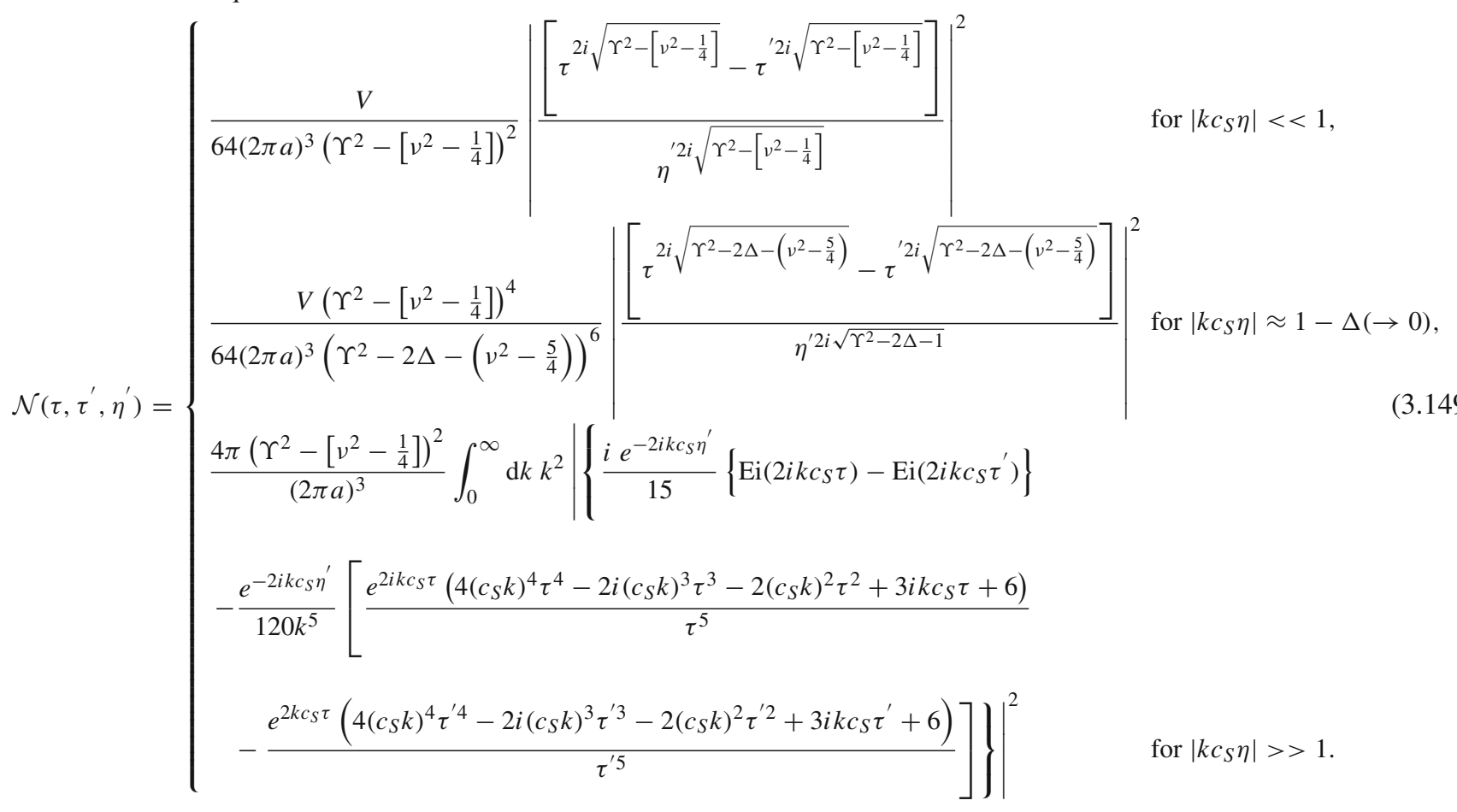

For dS:

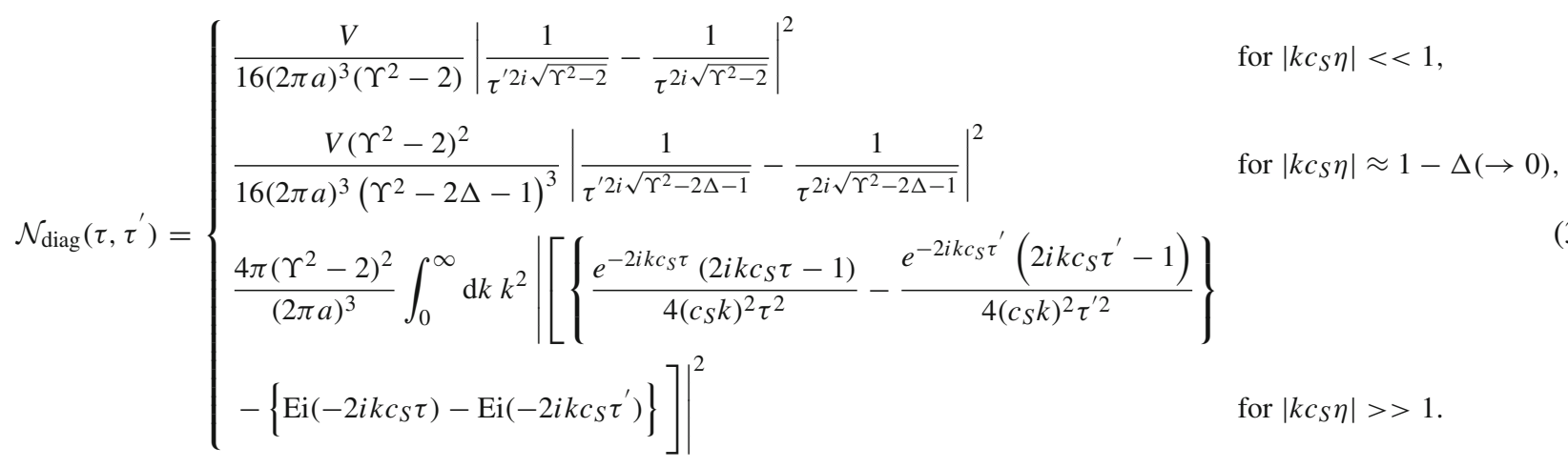

For qdS:

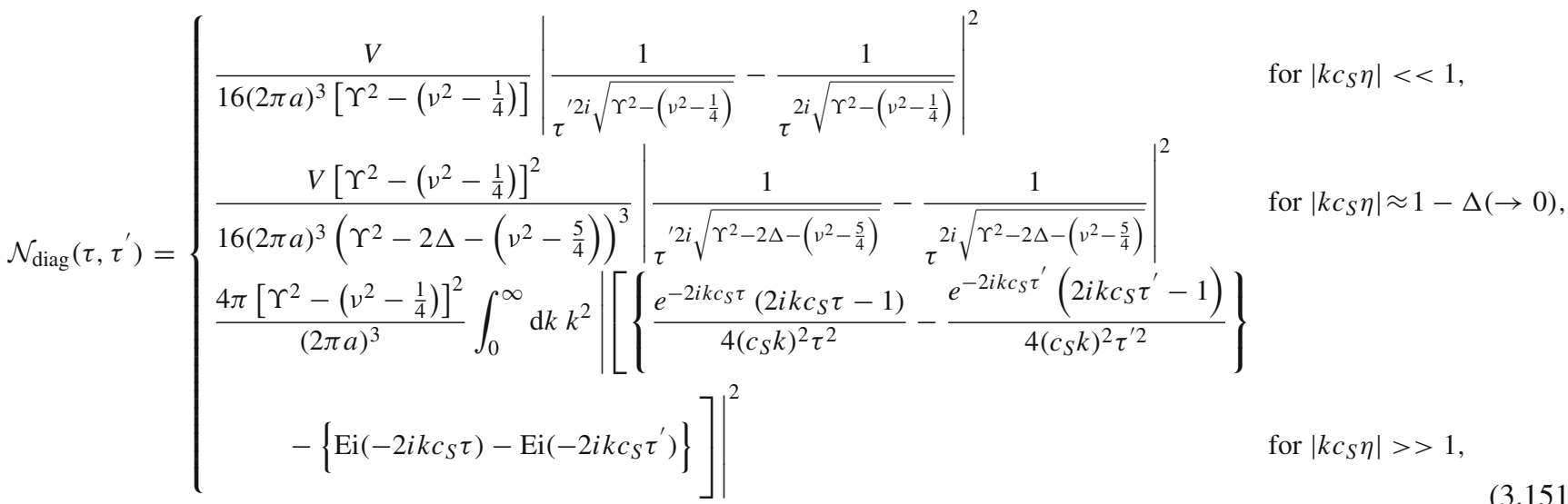

where $V$ is defined in the earlier subsection. 
Finally one can define the total energy density of the produced particles using the following expression: $\rho\left(\tau, \tau^{\prime}, \eta^{\prime}\right)$

$$
=\int \frac{\mathrm{d}^{3} \mathbf{k}}{(2 \pi a)^{3} a}\left\{\begin{array}{l}
\sqrt{c_{S}^{2} k^{2}+\frac{\Upsilon^{2}-2}{\eta^{2}}}\left|\int_{\tau^{\prime}}^{\tau} \mathrm{d} \eta \frac{\left(\Upsilon^{2}-2\right) \exp \left[-2 i \int^{\eta} \mathrm{d} \eta^{\prime} \sqrt{c_{S}^{2} k^{2}+\frac{\Upsilon^{2}-2}{\eta^{\prime 2}}}\right]}{2 \eta^{3}\left(c_{S}^{2} k^{2}+\frac{\Upsilon^{2}-2}{\eta^{2}}\right)}\right|^{2} \\
\sqrt{c_{S}^{2} k^{2}+\frac{\left[\Upsilon^{2}-\left(v^{2}-\frac{1}{4}\right)\right]}{\eta^{2}}} \mid \int_{\tau^{\prime}}^{\tau} \mathrm{d} \eta \frac{\left[\Upsilon^{2}-\left(v^{2}-\frac{1}{4}\right)\right] \exp \left[-\left.2 i \int^{\eta} \mathrm{d} \eta^{\prime} \sqrt{\left.c_{S}^{2} k^{2}+\frac{\left[\Upsilon^{2}-\left(v^{2}-\frac{1}{4}\right)\right]}{\eta^{\prime 2}}\right]}\right|^{2}\right.}{2 \eta^{3}\left(c_{S}^{2} k^{2}+\frac{\left[\Upsilon^{2}-\left(v^{2}-\frac{1}{4}\right)\right]}{\eta^{2}}\right)} \text { for qdS, } \mathrm{dS},
\end{array}\right.
$$

$$
\begin{aligned}
& \rho_{\text {diag }}\left(\tau, \tau^{\prime}, \eta^{\prime}\right)
\end{aligned}
$$

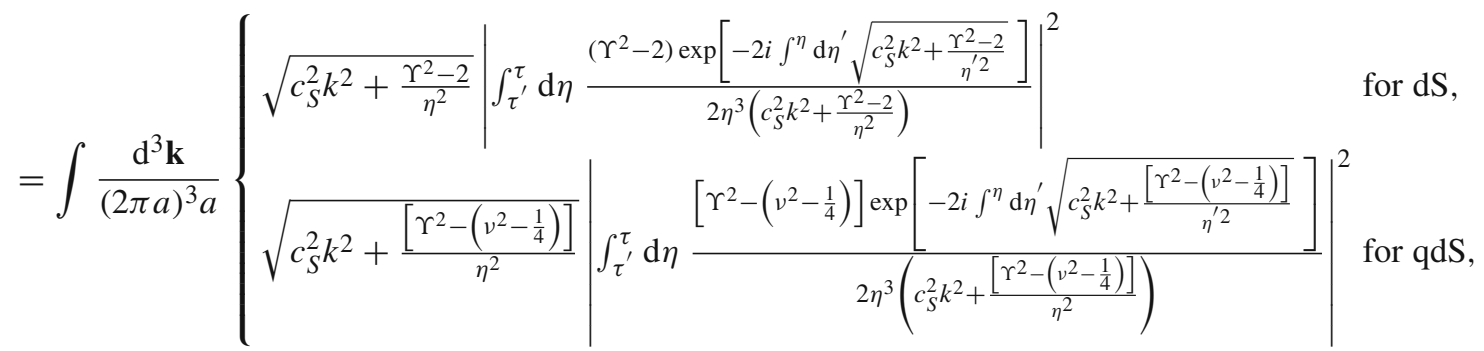

which is not exactly analytically computable. To study the behaviour of this integral we consider here three consecutive physical situations: $\left|k c_{S} \eta\right|<<1,\left|k c_{S} \eta\right| \approx 1-\Delta(\rightarrow 0)$ and $\left|k c_{S} \eta\right|>>1$ for the de Sitter case and the quasi de Sitter case. In the three cases we have

For dS:

$$
\begin{aligned}
& \int \frac{J}{64(2 \pi a)^{3} a\left(\Upsilon^{2}-2\right)^{2}}\left|\frac{\left[\tau^{2 i \sqrt{\Upsilon^{2}-2}}-\tau^{\prime 2 i \sqrt{\Upsilon^{2}-2}}\right]}{\eta^{\prime 2 i \sqrt{\Upsilon^{2}-2}}}\right|^{2} \quad \text { for }\left|k c_{S} \eta\right|<<1, \\
& \frac{J\left(\Upsilon^{2}-2\right)^{4}}{64(2 \pi a)^{3} a\left(\Upsilon^{2}-2 \Delta-1\right)^{6}}\left|\frac{\left[\tau^{2 i \sqrt{\Upsilon^{2}-2 \Delta-1}}-\tau^{\prime 2 i \sqrt{\Upsilon^{2}-2 \Delta-1}}\right]}{\eta^{\prime 2 i \sqrt{\Upsilon^{2}-2 \Delta-1}}}\right|^{2} \text { for }\left|k c_{S} \eta\right| \approx 1-\Delta(\rightarrow 0), \\
& \rho\left(\tau, \tau^{\prime}, \eta^{\prime}\right)=\left\{\frac{4 \pi c_{S}\left(\Upsilon^{2}-2\right)^{2}}{(2 \pi a)^{3} a} \int_{0}^{\infty} \mathrm{d} k k^{3} \mid\left\{\frac{i e^{-2 i k c_{S} \eta^{\prime}}}{15}\left\{\operatorname{Ei}\left(2 i k c_{S} \tau\right)-\operatorname{Ei}\left(2 i k c_{S} \tau^{\prime}\right)\right\}\right.\right. \\
& -\frac{e^{-2 i k c_{S} \eta^{\prime}}}{120 k^{5}}\left[\frac{e^{2 i k c_{S} \tau}\left(4\left(c_{S} k\right)^{4} \tau^{4}-2 i\left(c_{S} k\right)^{3} \tau^{3}-2\left(c_{S} k\right)^{2} \tau^{2}+3 i\left(c_{S} k\right) \tau+6\right)}{\tau^{5}}\right. \\
& \left.-\frac{e^{2 k c_{S} \tau^{\prime}}\left(4\left(c_{S} k\right)^{4} \tau^{\prime 4}-2 i\left(c_{S} k\right)^{3} \tau^{\prime 3}-2 k^{2} \tau^{\prime 2}+3 i k \tau^{\prime}+6\right)}{\tau^{\prime 5}}\right]\left.\right|^{2} \quad \text { for }\left|k c_{S} \eta\right|>>1
\end{aligned}
$$


For qdS:

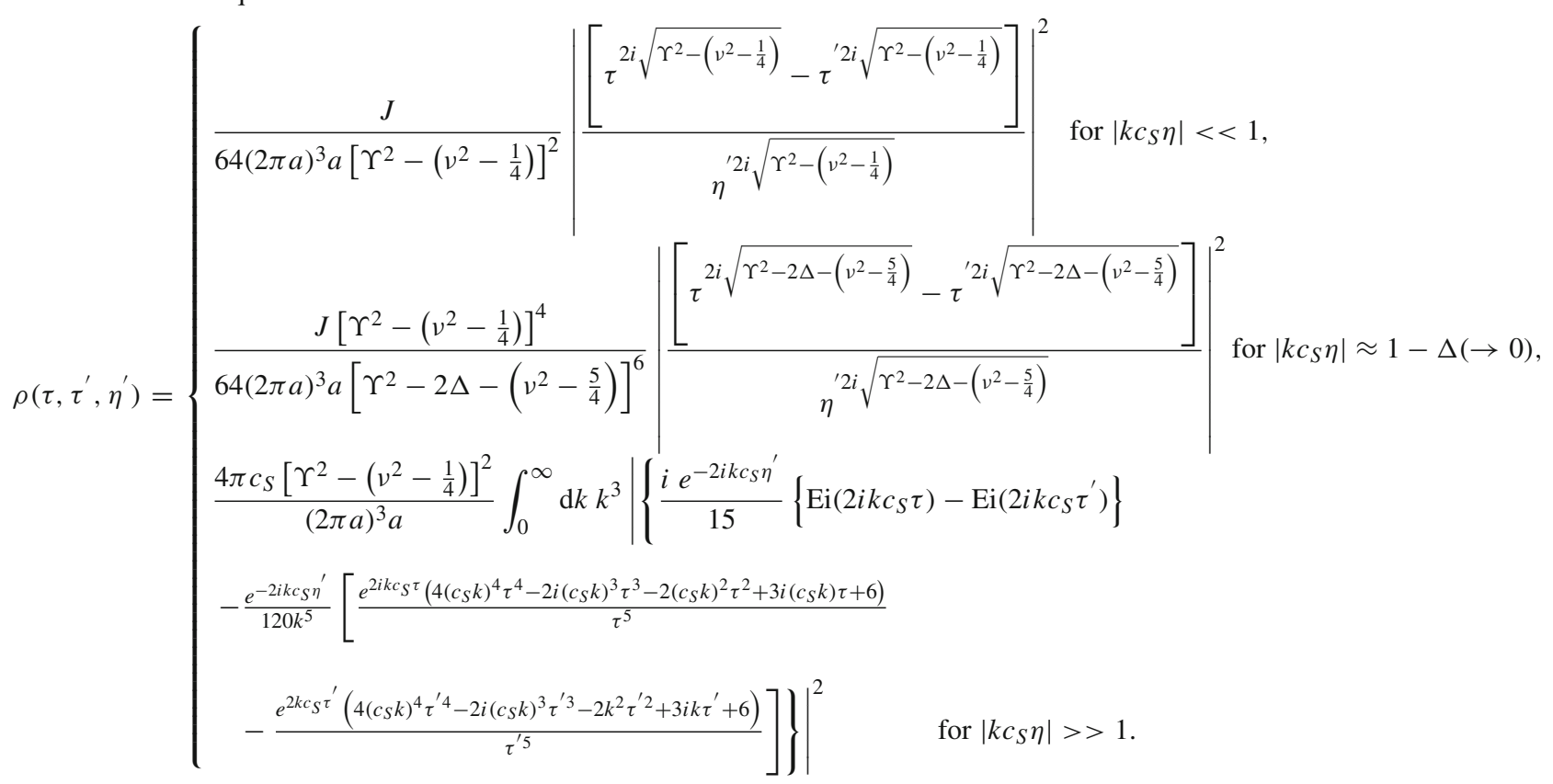

For dS:

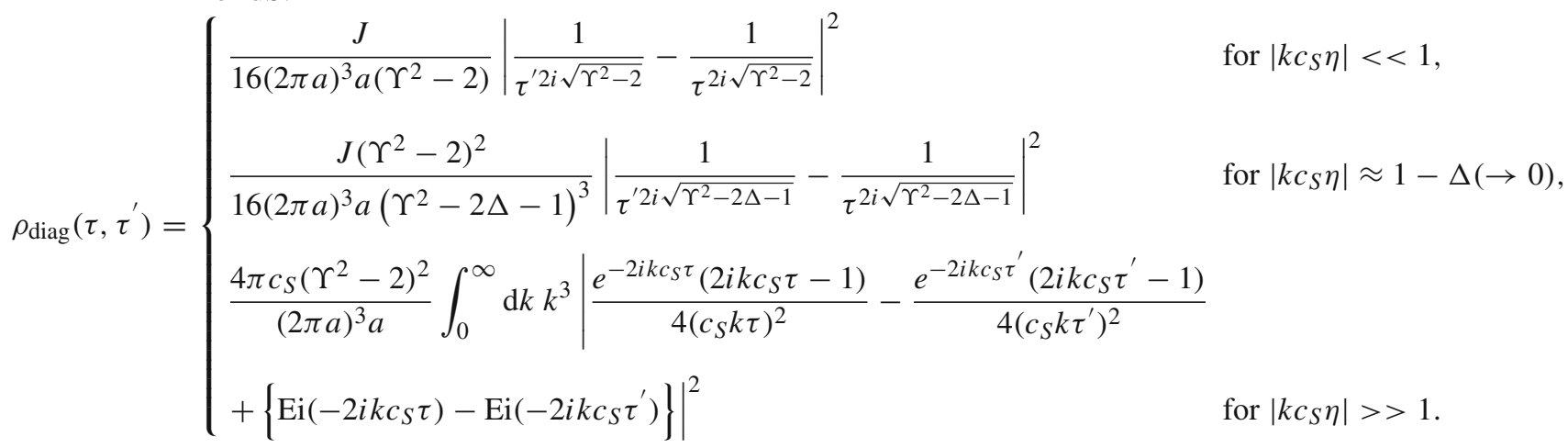

For qdS:

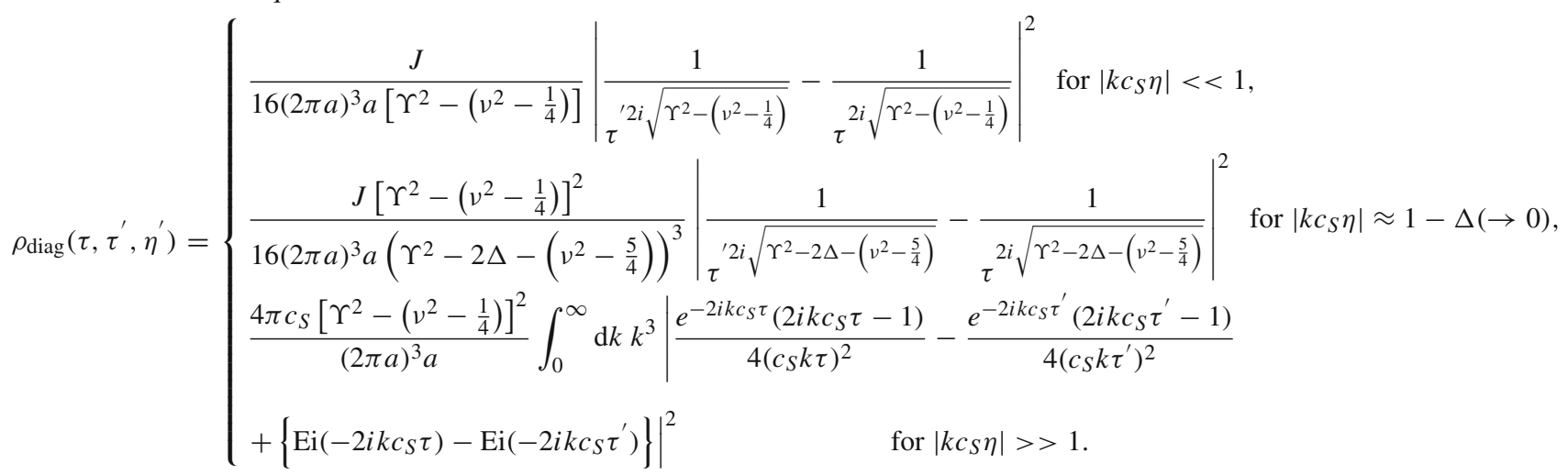


Throughout the discussion of the total energy density of the produced particles we have introduced a symbol $J$ defined as

$J=\int \mathrm{d}^{3} \mathbf{k} p(\tau)= \begin{cases}\int \mathrm{d}^{3} \mathbf{k} \sqrt{c_{S}^{2} k^{2}+\frac{\Upsilon^{2}-2}{\tau^{2}}} & \text { for } \mathrm{d} S, \\ \int \mathrm{d}^{3} \mathbf{k} \sqrt{c_{S}^{2} k^{2}+\frac{\left[\Upsilon^{2}-\left(v^{2}-\frac{1}{4}\right)\right]}{\tau^{2}}} & \text { for qdS. }\end{cases}$

which physically signifies the total finite volume weighted by $p(\eta)$ in momentum space within which the produced particles are occupied.

To study the behaviour of this integral we consider here three consecutive physical situations: $\left|k c_{S} \eta\right|<<1,\left|k c_{S} \eta\right| \approx$ $1-\Delta(\rightarrow 0)$ and $\left|k c_{S} \eta\right|>>1$ for the de Sitter case and the quasi de Sitter case. In the three cases we have

For dS:

$J= \begin{cases}\int \mathrm{d}^{3} \mathbf{k} \frac{\sqrt{\Upsilon^{2}-2}}{\tau}=\frac{V \sqrt{\Upsilon^{2}-2}}{\tau} & \text { for }\left|k c_{S} \eta\right|<<1, \\ \int \mathrm{d}^{3} \mathbf{k} \frac{\sqrt{\Upsilon^{2}-2 \Delta-1}}{\tau}=\frac{\sqrt{\Upsilon^{2}-2 \Delta-1} V}{\tau} & \text { for }\left|k c_{S} \eta\right| \approx 1-\Delta(\rightarrow 0), \\ \int \mathrm{d}^{3} \mathbf{k} k c_{S} & \text { for }\left|k c_{S} \eta\right|>>1 .\end{cases}$

For qdS:

$J= \begin{cases}\int \mathrm{d}^{3} \mathbf{k} \frac{\sqrt{\Upsilon^{2}-\left(v^{2}-\frac{1}{4}\right)}}{\tau}=\frac{V \sqrt{\Upsilon^{2}-\left(v^{2}-\frac{1}{4}\right)}}{\tau} & \text { for }\left|k c_{S} \eta\right|<<1, \\ \int \mathrm{d}^{3} \mathbf{k} \frac{\sqrt{\Upsilon^{2}-2 \Delta-\left(v^{2}-\frac{5}{4}\right)}}{\tau}=\frac{\sqrt{\Upsilon^{2}-2 \Delta-\left(v^{2}-\frac{5}{4}\right)} V}{\tau} & \text { for }\left|k c_{S} \eta\right| \approx 1-\Delta(\rightarrow 0), \\ \int \mathrm{d}^{3} \mathbf{k} k c_{S} & \text { for }\left|k c_{S} \eta\right|>>1 .\end{cases}$

In Figs. 9 and 10, we have explicitly shown the particle creation profile for Case II for two representations.

\subsubsection{Case III: $m<<H$}

Here we set $m<<H$ for the computation. Here the equation of motion for the field with mass $m<<H$ is given by

$$
\begin{array}{cc}
h_{k}^{\prime \prime}+\left\{c_{S}^{2} k^{2}-\frac{2}{\eta^{2}}\right\} h_{k}=0 & \text { for dS } \\
h_{k}^{\prime \prime}+\left\{c_{S}^{2} k^{2}-\frac{\left(v^{2}-\frac{1}{4}\right)}{\eta^{2}}\right\} h_{k}=0 & \text { for qdS }
\end{array}
$$

The solution for the mode function for the de Sitter and the quasi de Sitter space can be expressed as

$h_{k}(\eta)= \begin{cases}\sqrt{-\eta}\left[C_{1} H_{3 / 2}^{(1)}\left(-k c_{S} \eta\right)+C_{2} H_{3 / 2}^{(2)}\left(-k c_{S} \eta\right)\right] & \text { for dS, } \\ \sqrt{-\eta}\left[C_{1} H_{v}^{(1)}\left(-k c_{S} \eta\right)+C_{2} H_{v}^{(2)}\left(-k c_{S} \eta\right)\right] & \text { for qdS }\end{cases}$

where $C_{1}$ and $C_{2}$ are two arbitrary integration constants, which depend on the choice of the initial condition.

After taking the $k c_{S} \eta \rightarrow-\infty, k c_{S} \eta \rightarrow 0$ and $\left|k c_{S} \eta\right| \approx 1$ limits the most general solution as stated in Eq. (3.163) can be recast as 


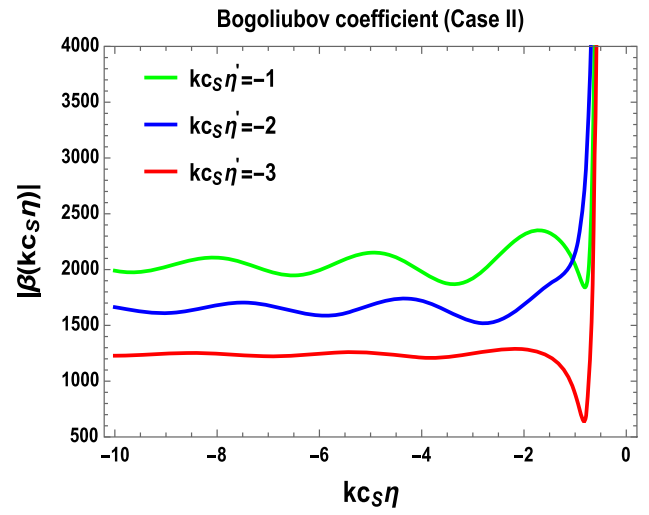

(a)

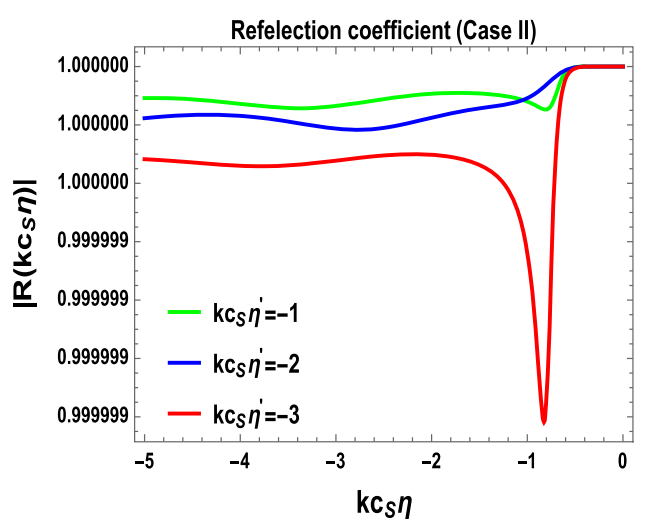

(c)

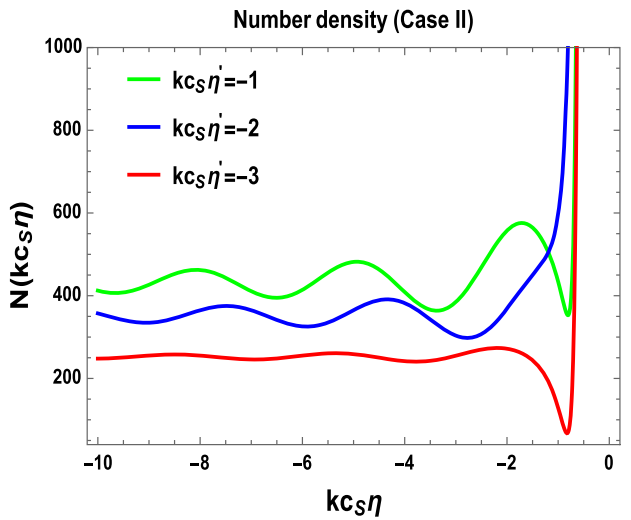

(e)

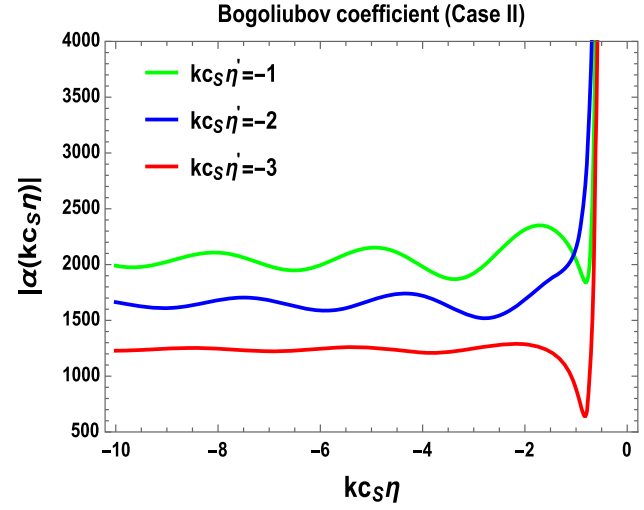

(b)

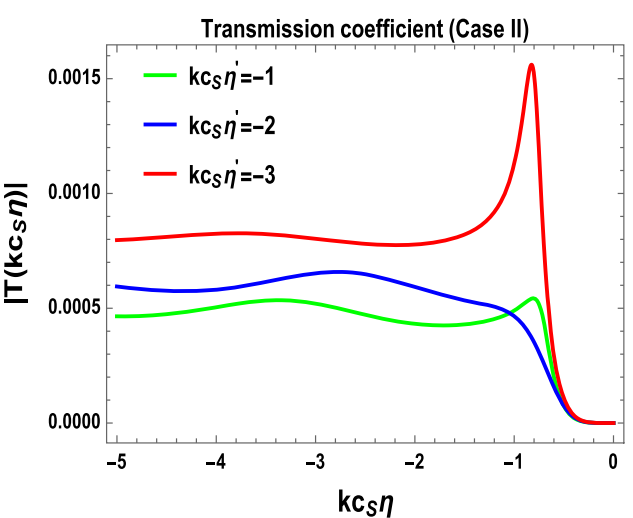

(d)

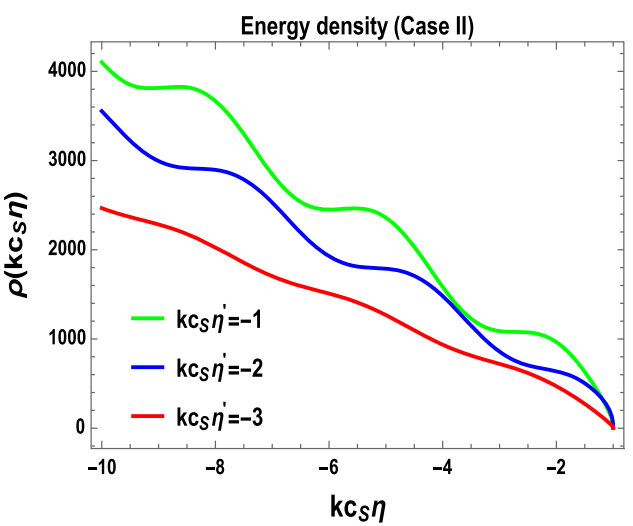

(f)

Fig. 9 Particle creation profile for Case II

$h_{k}(\eta) \stackrel{\left|k c_{S} \eta\right| \rightarrow-\infty}{=} \begin{cases}-\sqrt{\frac{2}{\pi k c_{S}}}\left[C_{1} e^{-i k c_{S} \eta}+C_{2} e^{i k c_{S} \eta}\right] & \text { for } \mathrm{dS}, \\ \sqrt{\frac{2}{\pi k c_{S}}}\left[C_{1} e^{-i k c_{S} \eta} e^{-\frac{i \pi}{2}\left(v+\frac{1}{2}\right)}+C_{2} e^{i k c_{S} \eta} e^{\left.\frac{i \pi}{2}\left(v+\frac{1}{2}\right)\right]}\right] & \text { for } \mathrm{qdS},\end{cases}$ 


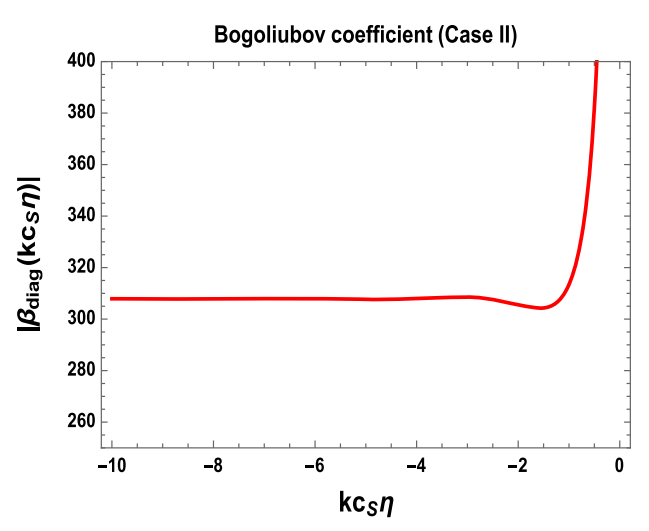

(a)

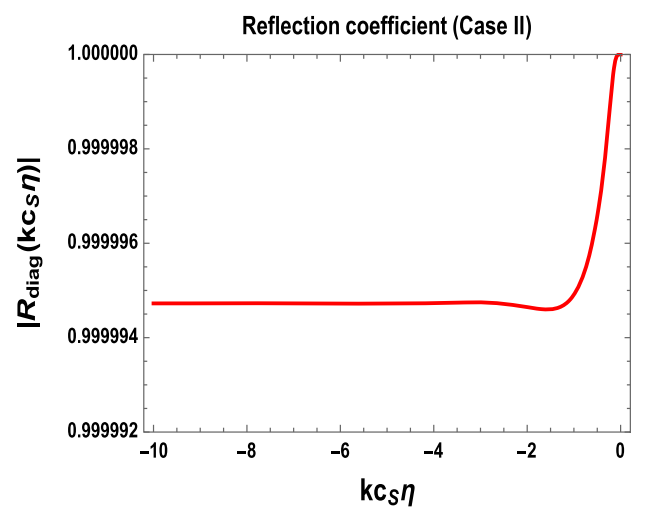

(c)

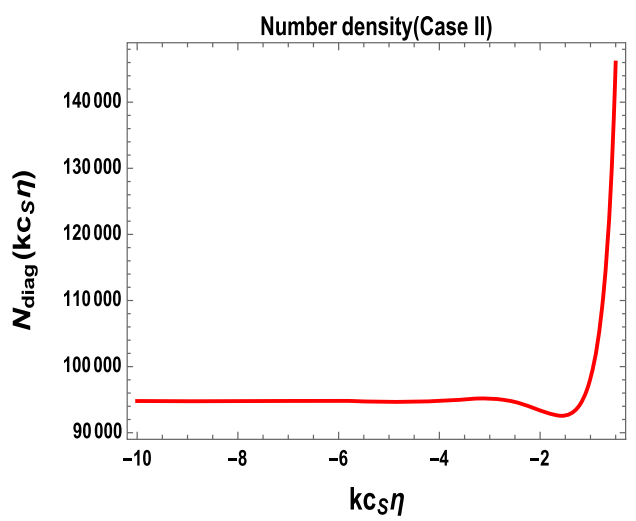

(e)

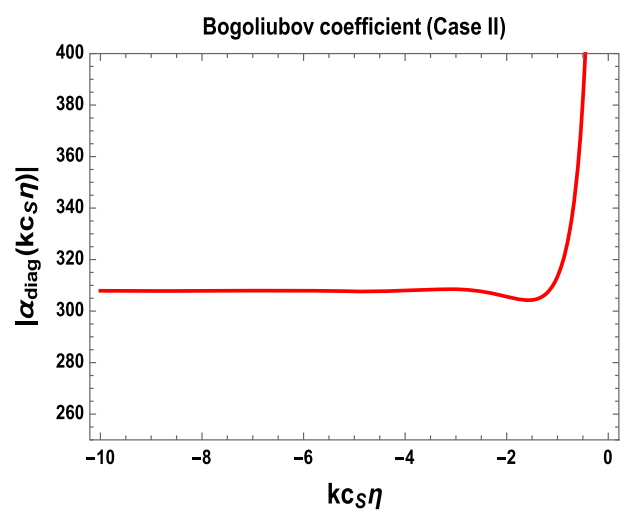

(b)

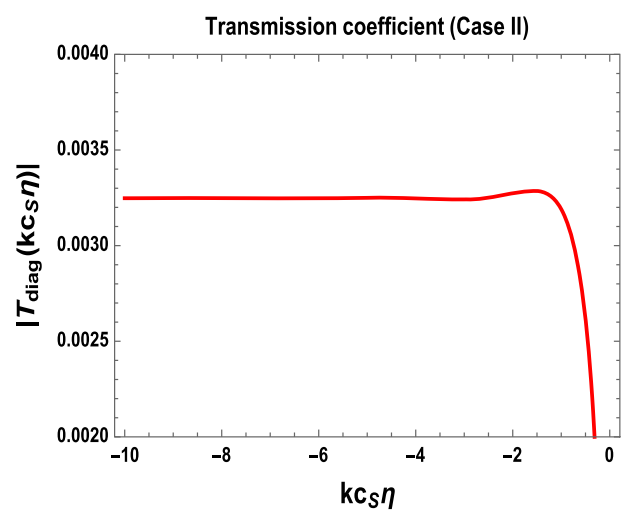

(d)

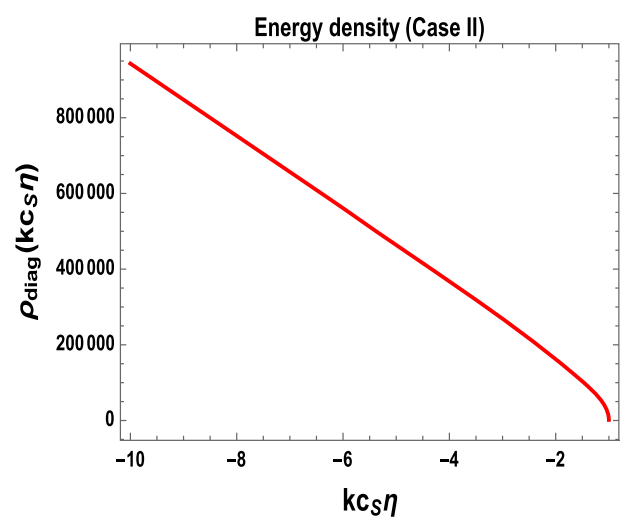

(f)

Fig. 10 Particle creation profile for Case II in diagonalized representation

$$
h_{k}(\eta) \stackrel{\left|k c_{S} \eta\right| \rightarrow 0}{=}\left\{\begin{array}{l}
\frac{i \sqrt{-\eta}}{2 \sqrt{\pi}}\left(-\frac{k c_{S} \eta}{2}\right)^{-\frac{3}{2}}\left[C_{1}-C_{2}\right] \quad \text { for } \mathrm{dS}, \\
\frac{i \sqrt{-\eta}}{\pi} \Gamma(v)\left(-\frac{k c_{S} \eta}{2}\right)^{-v}\left[C_{1}-C_{2}\right] \text { for qdS, }
\end{array}\right.
$$




$$
h_{k}(\eta) \stackrel{|k c s \eta| \approx 1-\Delta \rightarrow 0)}{=} \begin{cases}\frac{i}{\pi} \sqrt{-\eta}\left[\frac{2}{3}-\gamma+\frac{3}{4}\left(\gamma^{2}+\frac{\pi^{2}}{6}\right)-\frac{9}{24}\left(\gamma^{3}+\frac{\gamma \pi^{2}}{2}+2 \zeta(3)\right)+\cdots\right] \\ \times\left(\frac{1+\Delta}{2}\right)^{-\frac{3}{2}}\left[C_{1}-C_{2}\right] & \text { for dS, } \\ \frac{i}{\pi} \sqrt{-\eta}\left[\frac{1}{v}-\gamma+\frac{\nu}{2}\left(\gamma^{2}+\frac{\pi^{2}}{6}\right)-\frac{\nu^{2}}{6}\left(\gamma^{3}+\frac{\gamma \pi^{2}}{2}+2 \zeta(3)\right)+\cdots\right] & \text { for qdS. } \\ \times\left(\frac{1+\Delta}{2}\right)^{-v}\left[C_{1}-C_{2}\right] & \text {. }\end{cases}
$$

In the standard WKB approximation the total solution can be recast in the following form:

$h_{k}(\eta)=\left[D_{1} u_{k}(\eta)+D_{2} \bar{u}_{k}(\eta)\right]$,

where $D_{1}$ and $D_{2}$ are two arbitrary integration constants, which depend on the choice of the initial condition during WKB approximation at early and late time scale. In the present context $u_{k}(\eta)$ and $\bar{u}_{k}(\eta)$ are defined as

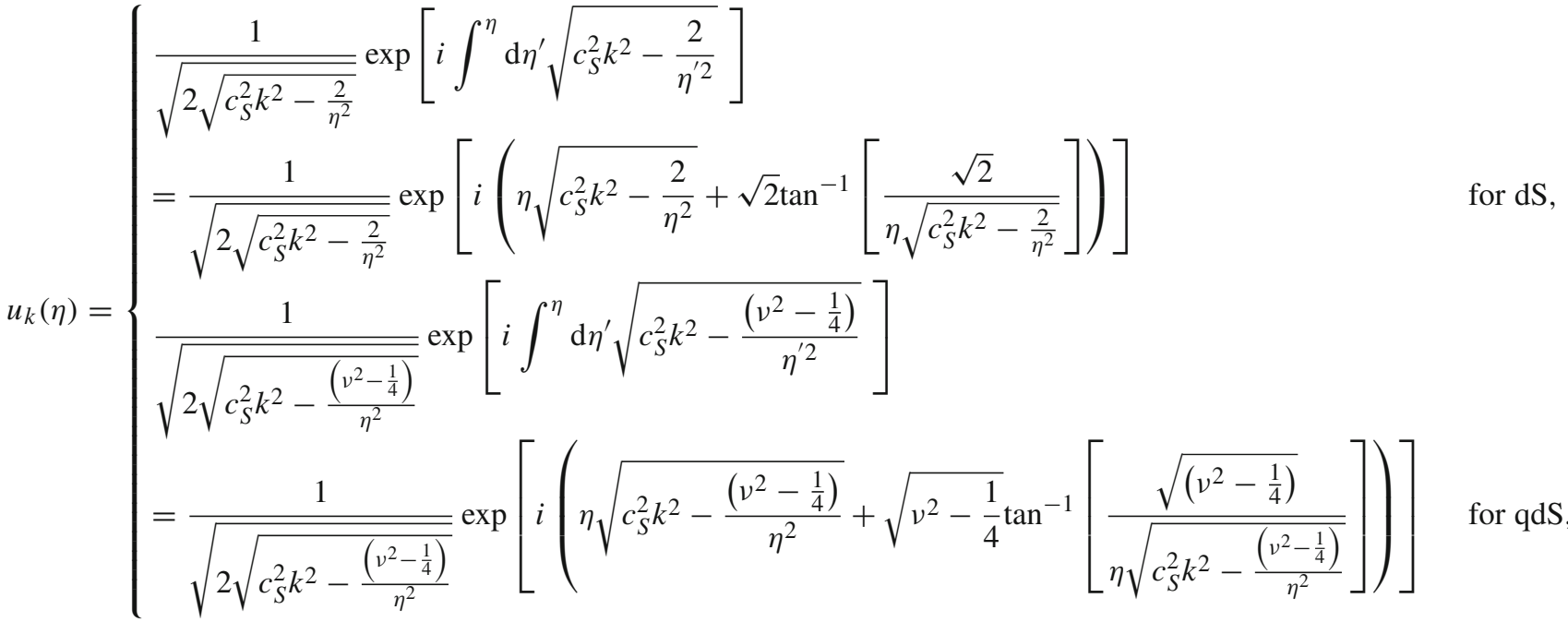

$$
\begin{aligned}
& \bar{u}_{k}(\eta)=\left\{\begin{array}{l}
\frac{1}{\sqrt{2 \sqrt{c_{S}^{2} k^{2}-\frac{2}{\eta^{2}}}}} \exp \left[-i \int^{\eta} \mathrm{d} \eta^{\prime} \sqrt{c_{S}^{2} k^{2}-\frac{2}{\eta^{\prime 2}}}\right] \\
=\frac{1}{\sqrt{2 \sqrt{c_{S}^{2} k^{2}-\frac{2}{\eta^{2}}}}} \exp \left[-i\left(\eta \sqrt{c_{S}^{2} k^{2}-\frac{2}{\eta^{2}}}+\sqrt{2} \tan ^{-1}\left[\frac{\sqrt{2}}{\eta \sqrt{c_{S}^{2} k^{2}-\frac{2}{\eta^{2}}}}\right]\right)\right] \\
\frac{1}{\sqrt{2 \sqrt{c_{S}^{2} k^{2}-\frac{\left(v^{2}-\frac{1}{4}\right)}{\eta^{2}}}}} \exp \left[-i \int^{\eta} \mathrm{d} \eta^{\prime} \sqrt{c_{S}^{2} k^{2}-\frac{\left(v^{2}-\frac{1}{4}\right)}{\eta^{\prime 2}}}\right] \\
=\frac{1}{\sqrt{2 \sqrt{c_{S}^{2} k^{2}-\frac{\left(v^{2}-\frac{1}{4}\right)}{\eta^{2}}}}} \exp \left[-i\left(\eta \sqrt{c_{S}^{2} k^{2}-\frac{\left(v^{2}-\frac{1}{4}\right)}{\eta^{2}}}+\sqrt{v^{2}-\frac{1}{4}} \tan ^{-1}\left[\frac{\sqrt{\left(v^{2}-\frac{1}{4}\right)}}{\eta \sqrt{c_{S}^{2} k^{2}-\frac{\left(v^{2}-\frac{1}{4}\right)}{\eta^{2}}}}\right]\right)\right]
\end{array}\right.
\end{aligned}
$$

where we have written the total solution for the mode $h_{k}$ in terms of two linearly independent solutions. Here it is important to note that both of the solutions are hermitian conjugate of each other. If in the present context the exact solution of the mode $h_{k}$ is expanded with respect to these two linearly independent solutions then particle creation is absent in our EFT setup. In 
the present context correctness of WKB approximation is guaranteed at very early and very late time scales. In this discussion $u_{k}(\eta)$ is valid at very early time scale and $\bar{u}_{k}(\eta)$ perfectly works in the late time scale.

Now we will explicitly check that the exactness of the above mentioned WKB result derived in Eq. (3.167) with the actual solution of the mode function as presented in Eq. (3.163). As mentioned earlier in FLRW space-time in Fourier space Bogoliubov coefficient $\beta(k)$ measures this exactness for a given setup. The particle creation mechanism and its exact amount is described by finding the Bogoliubov coefficient $\beta(k)$ in Fourier space which in principle measures the exact amount of late times solution $u_{k}(\eta)$, if in the present context we exactly start with the early time scale solution $u_{k}(\eta)$. In our present computation we consider a physical situation where the WKB approximation is correct up to the leading order throughout the cosmological evolution in time scale. In the present context the Bogoliubov coefficient $\beta(k)$ in Fourier space can be computed approximately using the following regularized integral:

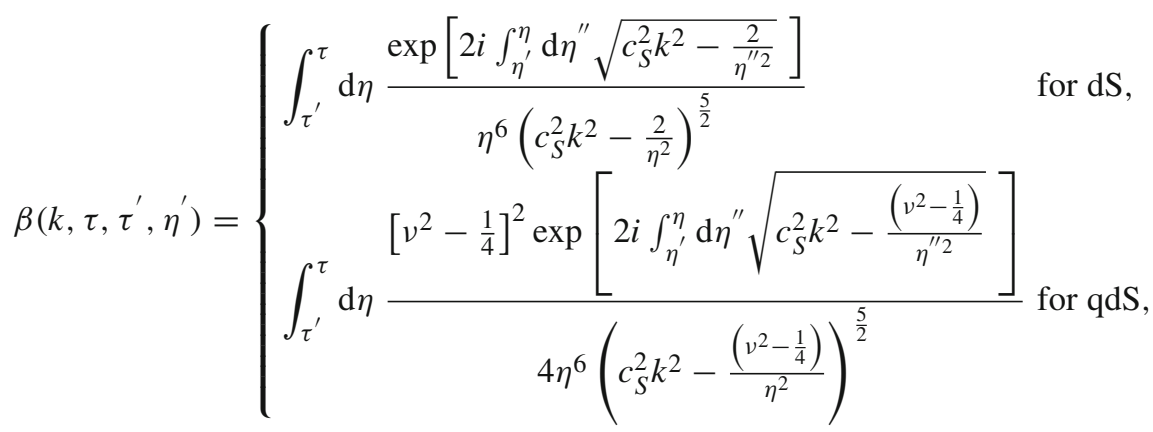

which is not exactly analytically computable. To study the behaviour of this integral we consider here three consecutive physical situations: $\left|k c_{S} \eta\right|<<1,\left|k c_{S} \eta\right| \approx 1-\Delta(\rightarrow 0)$ and $\left|k c_{S} \eta\right|>>1$ for the de Sitter case and the quasi de Sitter case. In the three cases we have

For dS: $\quad \sqrt{\left\{c_{S}^{2} k^{2}-\frac{2}{\eta^{2}}\right\}} \approx \begin{cases}\frac{i \sqrt{2}}{\eta} & \text { for }\left|k c_{S} \eta\right|<<1, \\ \frac{i \sqrt{2 \Delta+1}}{\eta} & \text { for }\left|k c_{S} \eta\right| \approx 1-\Delta(\rightarrow 0), \\ k c_{S} & \text { for }\left|k c_{S} \eta\right|>>1 .\end{cases}$

For qdS: $\quad \sqrt{\left\{c_{S}^{2} k^{2}-\frac{\left(v^{2}-\frac{1}{4}\right)}{\eta^{2}}\right\}} \approx \begin{cases}\frac{i \sqrt{\left(v^{2}-\frac{1}{4}\right)}}{\eta} & \text { for }\left|k c_{S} \eta\right|<<1, \\ \frac{i \sqrt{2 \Delta+\left(v^{2}-\frac{5}{4}\right)}}{\eta} & \text { for }\left|k c_{S} \eta\right| \approx 1-\Delta(\rightarrow 0), \\ k c_{S} & \text { for }\left|k c_{S} \eta\right|>>1,\end{cases}$

and further using this result the Bogoliubov coefficient $\beta(k)$ in Fourier space can be expressed as

For dS:

$$
\beta\left(k, \tau, \tau^{\prime}, \eta^{\prime}\right)= \begin{cases}\frac{\eta^{2 \sqrt{2}}}{16 i}\left[\frac{1}{\tau^{\prime 2 \sqrt{2}}}-\frac{1}{\tau^{2 \sqrt{2}}}\right] & \text { for }\left|k c_{S} \eta\right|<<1, \\ \frac{\eta^{\prime 2 \sqrt{2 \Delta+1}}\left[\frac { 1 } { 2 i ( 2 \Delta + 1 ) ^ { 3 } } \left[\frac{1}{\tau^{\prime 2 \sqrt{2 \Delta+1}}}-\frac{1}{\left.\tau^{2 \sqrt{2 \Delta+1}}\right]}\right.\right.}{\left[\frac{\operatorname{Ei}\left(2 i k c_{S} \eta\right) e^{-2 i k c_{S} \eta^{\prime}}}{15}-\frac{e^{2 i k c_{S}\left(\eta-\eta^{\prime}\right)}}{120\left(c_{S} k\right)^{5} \eta^{5}}\right.} & \text { for }\left|k c_{S} \eta\right| \approx 1-\Delta(\rightarrow 0), \\ \times\left(4\left(c_{S} k\right)^{4} \eta^{4}-2 i\left(c_{S} k\right)^{3} \eta^{3}-2\left(c_{S} k\right)^{2} \eta^{2}+3 i c_{S} k \eta+6\right) & ]_{\tau^{\prime}}^{\tau}\end{cases}
$$


For qdS:

$\beta\left(k, \tau, \tau^{\prime}, \eta^{\prime}\right)= \begin{cases}\frac{\eta^{2 \sqrt{v^{2}-\frac{1}{4}}}}{8 i\left(v^{2}-\frac{1}{4}\right)}\left[\frac{1}{\tau^{\prime 2 \sqrt{v^{2}-\frac{1}{4}}}}-\frac{1}{\left.\tau^{2 \sqrt{v^{2}-\frac{1}{4}}}\right]}\right. & \text { for }\left|k c_{S} \eta\right|<1, \\ \frac{\left(v^{2}-\frac{1}{4}\right)^{2} \eta^{2} \sqrt{2 \Delta+\left(v^{2}-\frac{5}{4}\right)}}{8 i\left[2 \Delta+\left(v^{2}-\frac{5}{4}\right)\right]^{3}}\left[\frac{1}{\left.\tau^{\prime 2 \sqrt{2 \Delta+\left(v^{2}-\frac{5}{4}\right)}}-\frac{1}{\tau^{2} \sqrt{2 \Delta+\left(v^{2}-\frac{5}{4}\right)}}\right]}\right. & \text { for }\left|k c_{S} \eta\right| \approx 1-\Delta(\rightarrow 0), \\ \left(v^{2}-\frac{1}{4}\right)^{2}\left[i \frac{\operatorname{Ei}\left(2 i k c_{S} \eta\right) e^{-2 i k c s \eta^{\prime}}}{15}-\frac{e^{2 i k c_{S}\left(\eta-\eta^{\prime}\right)}}{120\left(c_{S} k\right)^{5} \eta^{5}}\left(4\left(c_{S} k\right)^{4} \eta^{4}-2 i\left(c_{S} k\right)^{3} \eta^{3}\right.\right. & \\ \left.-2\left(c_{S} k\right)^{2} \eta^{2}+3 i c_{S} k \eta+6\right) & \text { for }\left|k c_{S} \eta\right|>>1 .\end{cases}$

In all the situations described for the de Sitter and the quasi de Sitter case here the magnitude of the Bogoliubov coefficient $|\beta(k)|$ in Fourier space is considerably small. Specifically it is important to point out here that for the case when $\left|k c_{S} \eta\right|>>1$ the value of the Bogoliubov coefficient $\beta(k)$ in Fourier space is even smaller as the WKB approximated solution is strongly consistent for all time scales. On the other hand in the vicinity of the conformal time scale $\eta \sim \eta_{\text {pair }}$ for $\left|k c_{S} \eta_{\text {pair }}\right|<<1$ the WKB approximated solution is less strongly valid and to validate the solution at this time scale one can neglect the momentum $k$ dependence in the Bogoliubov coefficient $\beta(k)$ in Fourier space. Here $\left|\eta_{\text {pair }}\right|$ characterizes the relative separation between the created particles.

As mentioned earlier here one can use another equivalent way to define the Bogoliubov coefficient $\beta$ in Fourier space by implementing instantaneous Hamiltonian diagonalization method to interpret the results. Using this diagonalized representation the regularized Bogoliubov coefficient $\beta$ in Fourier space can be written as

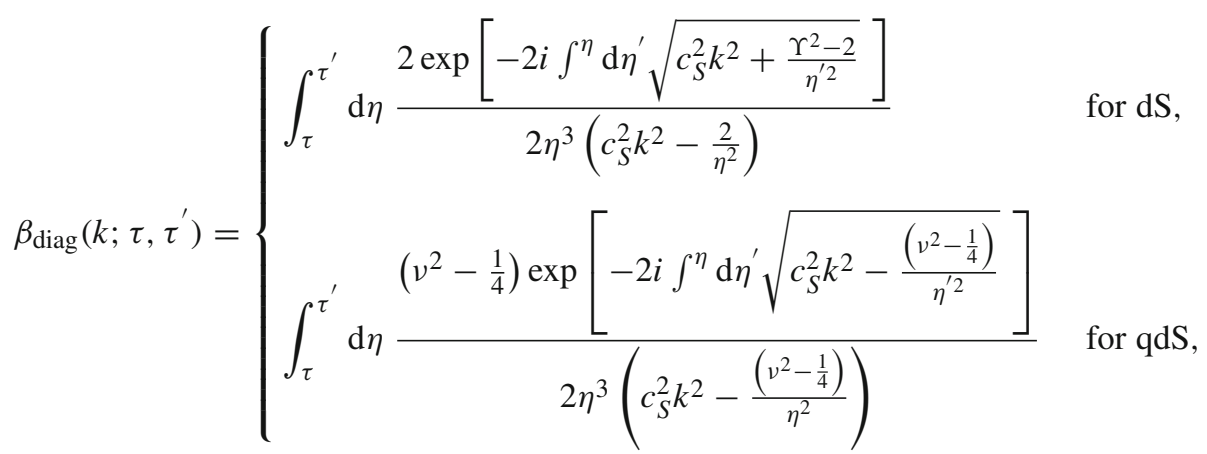

where $\tau$ and $\tau^{\prime}$ are introduced as the conformal time regulator in the present context. In this case as well the Bogoliubov coefficient is not exactly analytically computable. To study the behaviour of this integral we consider here three similar consecutive physical situations for the de Sitter and the quasi de Sitter case as discussed earlier.

For dS:

$\beta_{\text {diag }}\left(k ; \tau, \tau^{\prime}\right)= \begin{cases}\frac{1}{4 \sqrt{2}}\left[\tau^{2 \sqrt{2}}-\tau^{\prime 2 \sqrt{2}}\right] & \text { for }\left|k c_{S} \eta\right|<<1, \\ \frac{1}{2 i(2 \Delta+1)^{3 / 2}\left[\tau^{2 \sqrt{2 \Delta+1}}-\tau^{\prime 2 \sqrt{2 \Delta+1}]}\right.} & \text { for }\left|k c_{S} \eta\right| \approx 1-\Delta(\rightarrow 0), \\ -2\left[\frac{e^{-2 i k c_{S} \eta}\left(2 i k c_{S} \eta-1\right)}{4\left(c_{S} k\right)^{2} \eta^{2}}-\operatorname{Ei}\left(-2 i k c_{S} \eta\right)\right]_{\tau^{\prime}}^{\tau} & \text { for }\left|k c_{S} \eta\right|>>1 .\end{cases}$ 
For qdS:

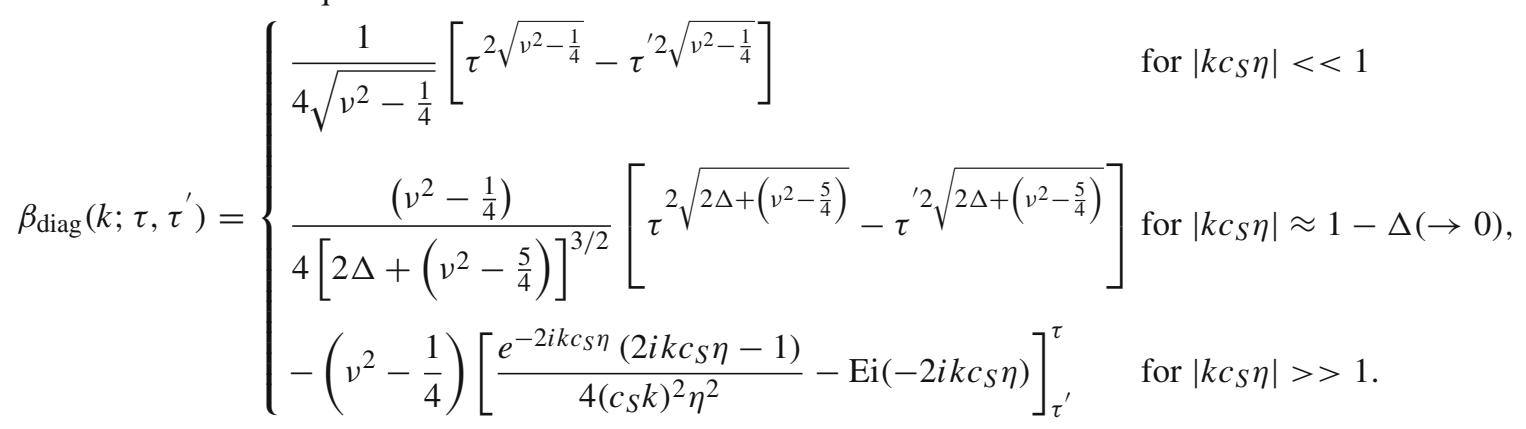

Further using the regularized expressions for the Bogoliubov coefficient $\beta$ in two different representations as mentioned in Eqs. (3.170) and (3.175), and substituting them in Eq. (3.57) we get the following regularized expressions for the Bogoliubov coefficient $\alpha$ in two different representations as given by

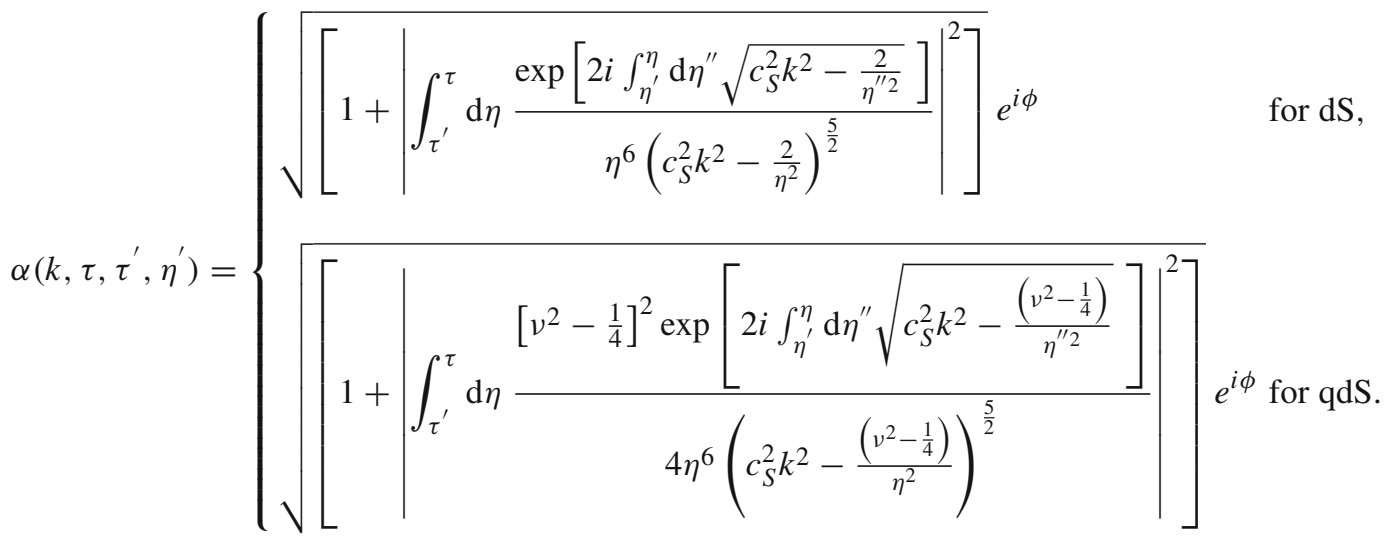

$\alpha_{\text {diag }}\left(k ; \tau, \tau^{\prime}\right)= \begin{cases}\sqrt{\left[\sqrt{\left[\left.\int_{\tau}^{\tau^{\prime}} \mathrm{d} \eta \frac{2 \exp \left[-2 i \int^{\eta} \mathrm{d} \eta^{\prime} \sqrt{c_{S}^{2} k^{2}+\frac{\Upsilon^{2}-2}{\eta^{\prime 2}}}\right]}{2 \eta^{3}\left(c_{S}^{2} k^{2}-\frac{2}{\eta^{2}}\right)}\right|^{2}\right]} e^{i \phi_{\text {diag }}}\right.} & \text { for dS, } \\ \sqrt{\left[1+\mid \int_{\tau}^{\tau^{\prime}} \mathrm{d} \eta \frac{\left(v^{2}-\frac{1}{4}\right) \exp \left[-2 i \int^{\eta} \mathrm{d} \eta^{\prime} \sqrt{c_{S}^{2} k^{2}-\frac{\left(v^{2}-\frac{1}{4}\right)}{\eta^{\prime 2}}}\right]}{2 \eta^{3}\left(c_{S}^{2} k^{2}-\frac{\left(v^{2}-\frac{1}{4}\right)}{\eta^{2}}\right)}\right]} e^{i \phi_{\text {diag }}} & \text { for qdS, }\end{cases}$

where $\phi$ and $\phi_{\text {diag }}$ are the associated phase factors in two different representations. Here the results are not exactly analytically computable. To study the behaviour of this integral we consider here three consecutive physical situations: $\left|k c_{S} \eta\right|<<1$, $\left|k c_{S} \eta\right| \approx 1-\Delta(\rightarrow 0)$ and $\left|k c_{S} \eta\right|>>1$ for the de Sitter case and the quasi de Sitter case. 
For dS:

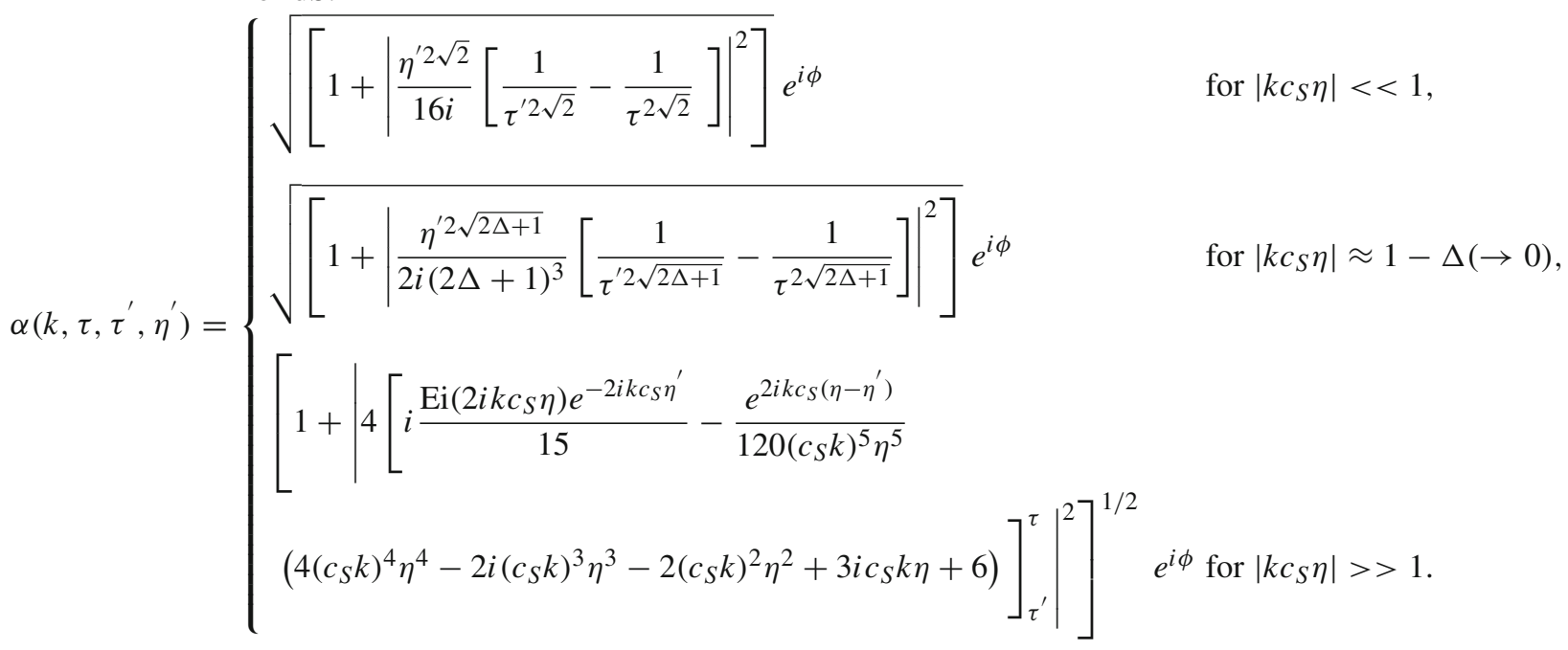

For qdS:

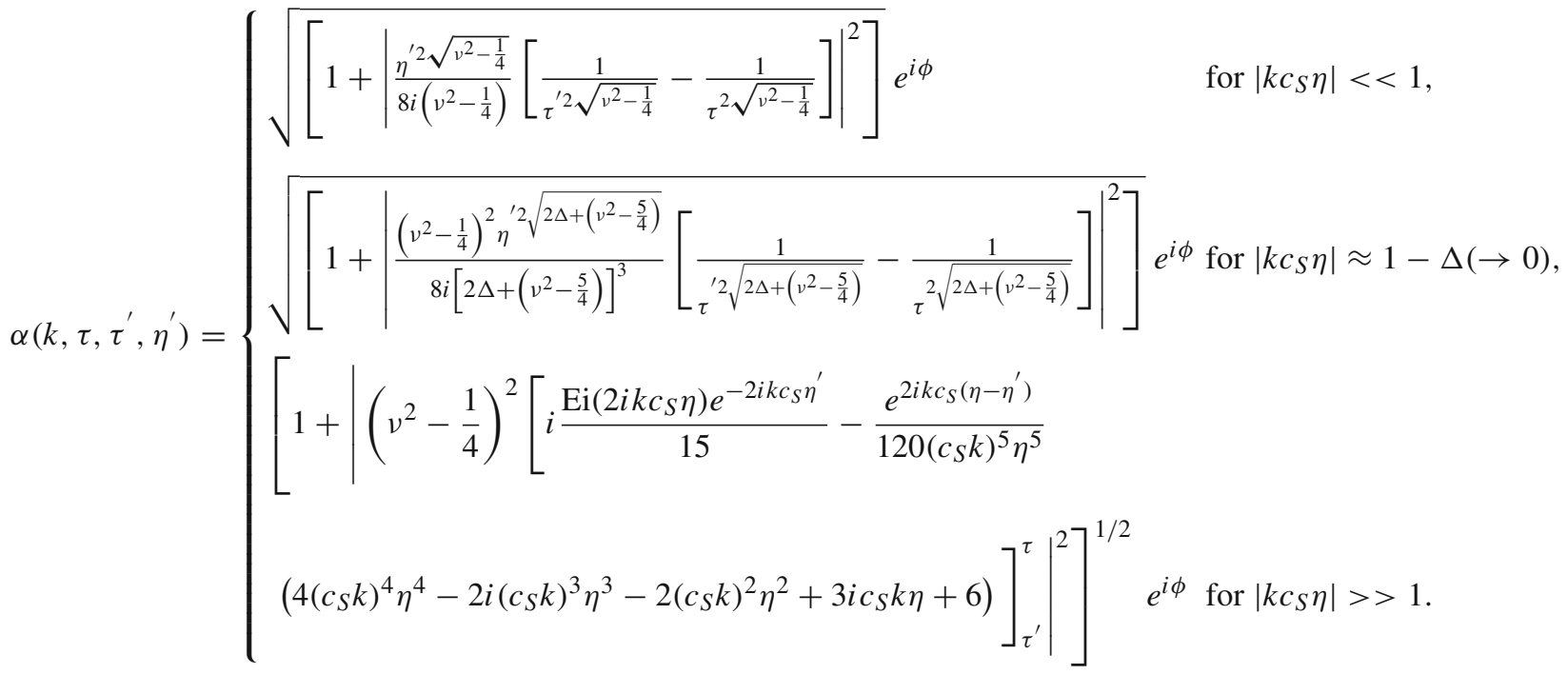

For dS:

$$
\alpha_{\text {diag }}\left(k ; \tau, \tau^{\prime}\right)= \begin{cases}\sqrt{\left[\frac{}{\left[1+\left|\frac{1}{4 \sqrt{2}}\left[\tau^{2 \sqrt{2}}-\tau^{\prime 2 \sqrt{2}}\right]\right|^{2}\right]} e^{i \phi}\right.} & \text { for }\left|k c_{S} \eta\right|<<1, \\ \sqrt{\left[1+\mid \frac{1}{2 i(2 \Delta+1)^{3 / 2}}\left[\tau^{2 \sqrt{2 \Delta+1}}-\tau^{\left.\prime 2 \sqrt{2 \Delta+1}]\left.\right|^{2}\right]} e^{i \phi}\right.\right.} & \text { for }\left|k c_{S} \eta\right| \approx 1-\Delta(\rightarrow 0), \\ \sqrt{\left[1+\left|-2\left[\frac{e^{-2 i k c_{S} \eta}\left(2 i k c_{S} \eta-1\right)}{4\left(c_{S} k\right)^{2} \eta^{2}}-\operatorname{Ei}\left(-2 i k c_{S} \eta\right)\right]_{\tau^{\prime}}\right|^{2}\right]} e^{i \phi} \text { for }\left|k c_{S} \eta\right|>>1 .\end{cases}
$$


For qdS:

$$
\alpha_{\text {diag }}\left(k ; \tau, \tau^{\prime}\right)= \begin{cases}\sqrt{\left[1+\mid \frac{1}{4 \sqrt{v^{2}-\frac{1}{4}}}\left[\tau^{2 \sqrt{v^{2}-\frac{1}{4}}}-\tau^{\left.\left.\prime 2 \sqrt{v^{2}-\frac{1}{4}}\right]\left.\right|^{2}\right]} e^{i \phi}\right.\right.} & \text { for }\left|k c_{S} \eta\right|<<1, \\ \sqrt{\left[1+\mid \frac{\left(v^{2}-\frac{1}{4}\right)}{4\left[2 \Delta+\left(v^{2}-\frac{5}{4}\right)\right]^{3 / 2}}\left[\tau^{2 \sqrt{2 \Delta+\left(v^{2}-\frac{5}{4}\right)}}-\tau^{\left.2 \sqrt{2 \Delta+\left(v^{2}-\frac{5}{4}\right)}\right]}\right]\right.} & e^{i \phi} \text { for }\left|k c_{S} \eta\right| \approx 1-\Delta(\rightarrow 0), \\ \sqrt{\left[1+\left|-\left(v^{2}-\frac{1}{4}\right)\left[\frac{e^{-2 i k c_{S} \eta}\left(2 i k c_{S} \eta-1\right)}{4\left(c_{S} k\right)^{2} \eta^{2}}-\operatorname{Ei}\left(-2 i k c_{S} \eta\right)\right]_{\tau^{\prime}}\right|^{2}\right]} e^{i \phi} & \text { for }\left|k c_{S} \eta\right|>>1 .\end{cases}
$$

Further using the expressions for the Bogoliubov coefficient $\alpha$ in two different representations and substituting them in Eq. (3.58) we get the following expressions for the reflection and transmission coefficient in two different representations for three consecutive physical situations: $\left|k c_{S} \eta\right|<<1,\left|k c_{S} \eta\right| \approx 1-\Delta(\rightarrow 0)$ and $\left|k c_{S} \eta\right|>>1$ for the de Sitter case and the quasi de Sitter case as given by

For dS:

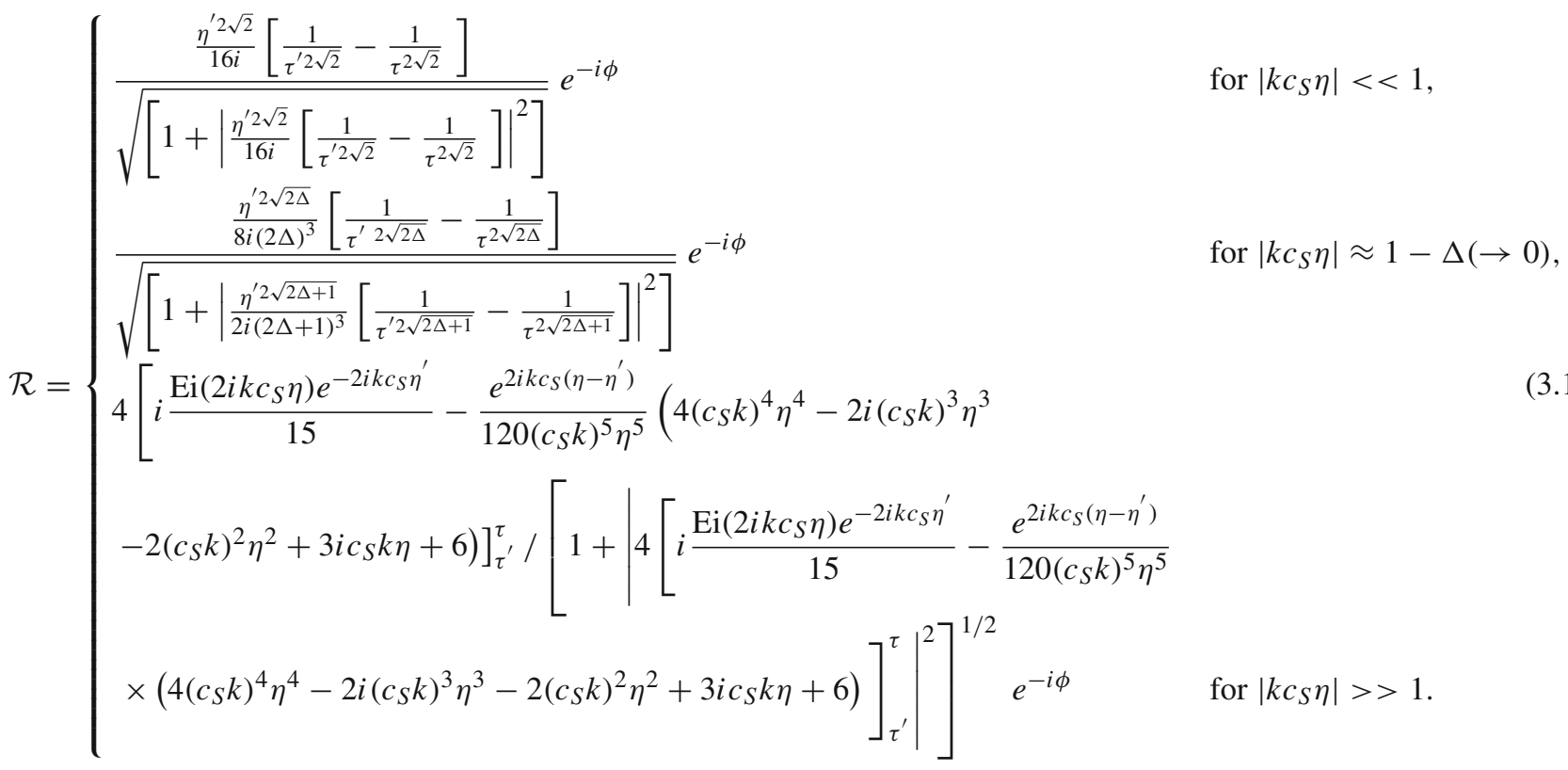

For dS:

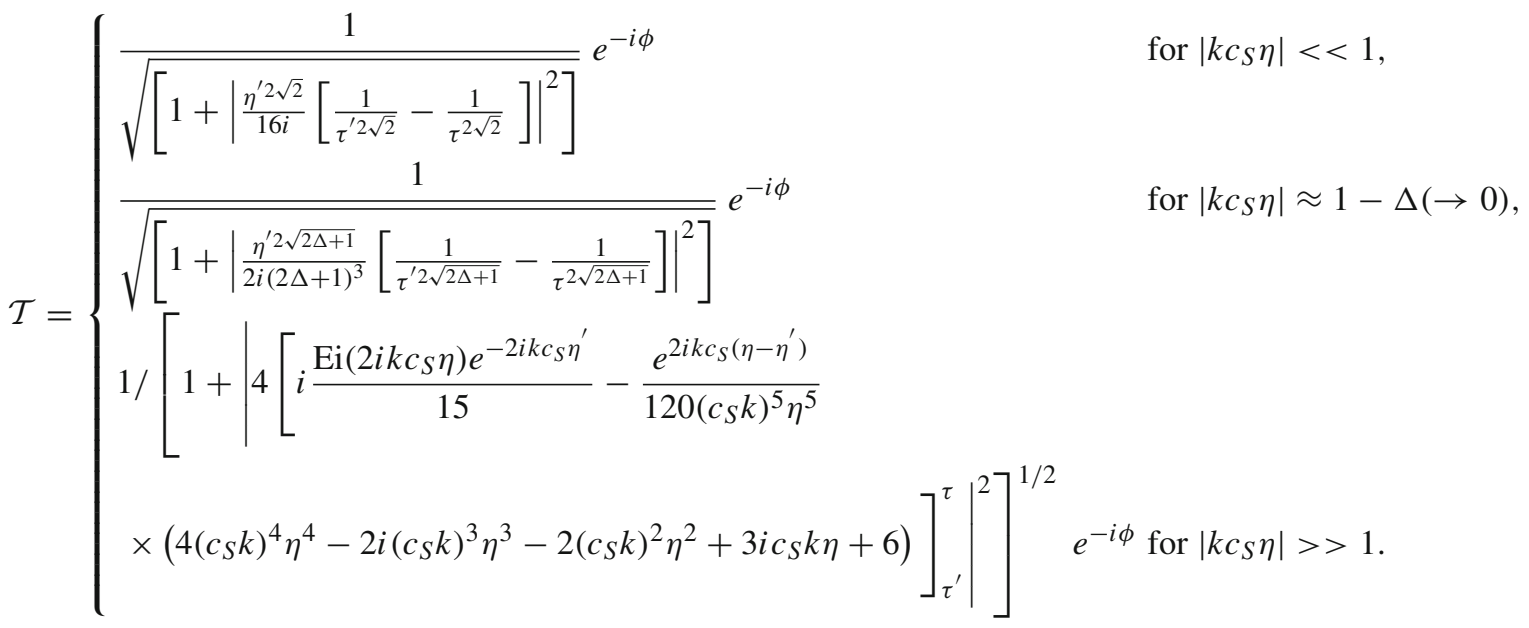


For qdS:

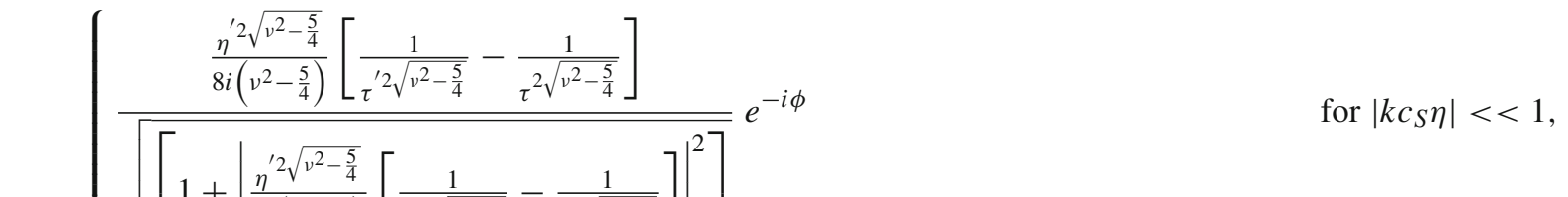

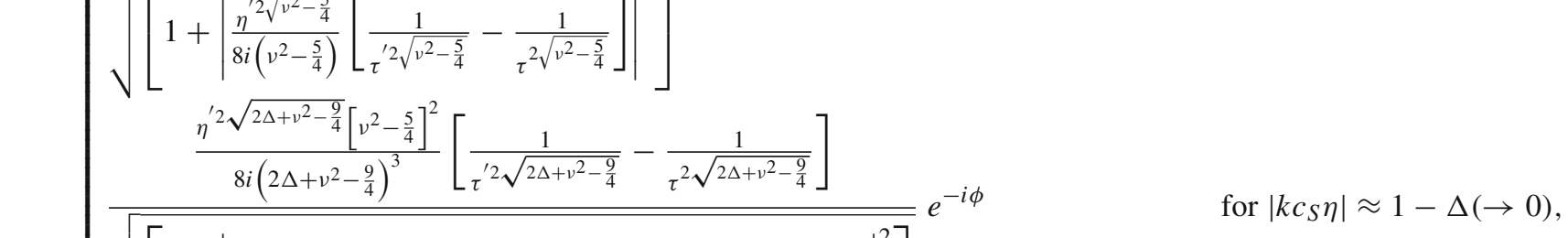

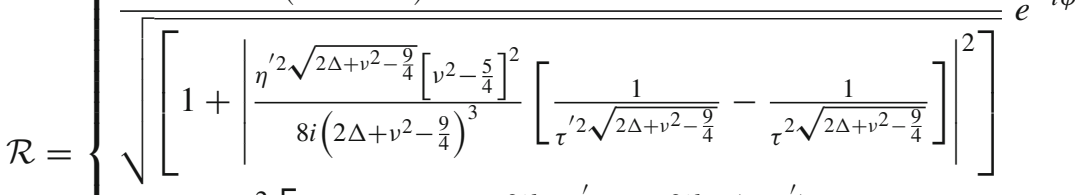

$$
\begin{aligned}
& {\left[v^{2}-\frac{5}{4}\right]^{2}\left[i \frac{\operatorname{Ei}\left(2 i k c_{S} \eta\right) e^{-2 i k c_{S} \eta^{\prime}}}{15}-\frac{e^{2 i k c_{S}\left(\eta-\eta^{\prime}\right)}}{120\left(c_{S} k\right)^{5} \eta^{5}}\left(4\left(c_{S} k\right)^{4} \eta^{4}-2 i\left(c_{S} k\right)^{3} \eta^{3}\right.\right.} \\
& \left.\left.-2\left(c_{S} k\right)^{2} \eta^{2}+3 i c_{S} k \eta+6\right)\right]_{\tau^{\prime}}^{\tau} /\left[1+\mid\left[v^{2}-\frac{5}{4}\right]^{2}\left[i \frac{E \mathrm{E}\left(2 i k c_{S} \eta\right) e^{-2 i k c_{S} \eta^{\prime}}}{15}\right.\right. \\
& \left.\left.-\frac{e^{2 i k c_{S}\left(\eta-\eta^{\prime}\right)}}{120\left(c_{S} k\right)^{5} \eta^{5}}\left(4\left(c_{S} k\right)^{4} \eta^{4}-2 i\left(c_{S} k\right)^{3} \eta^{3}-2\left(c_{S} k\right)^{2} \eta^{2}+3 i c_{S} k \eta+6\right)\right]\left._{\tau^{\prime}}^{\tau}\right|^{2}\right]^{1 / 2} e^{-i \phi} \text { for }\left|k c_{S} \eta\right|>>1
\end{aligned}
$$

For qdS:

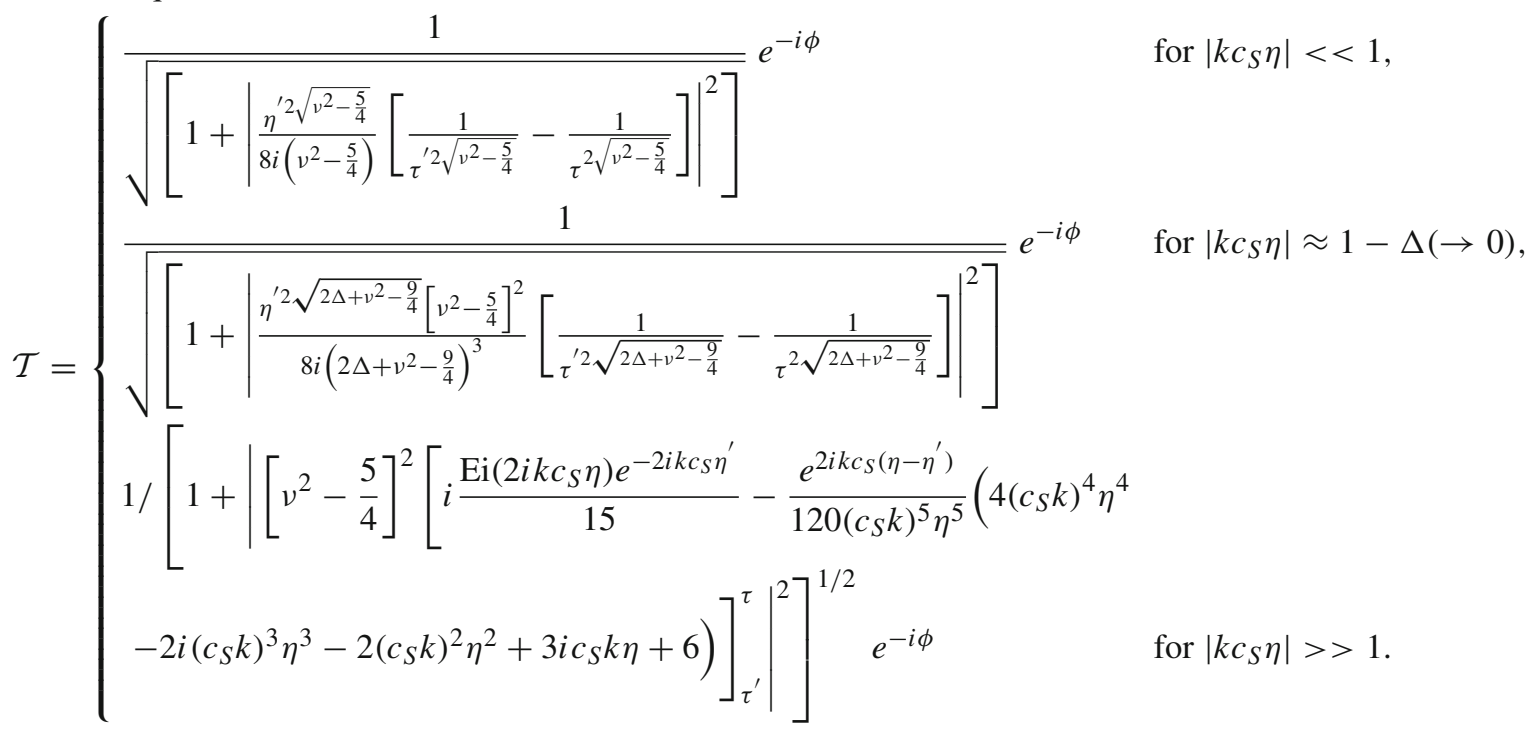


For dS:

$\mathcal{R}_{\operatorname{diag}}\left(k ; \tau, \tau^{\prime}\right)= \begin{cases}\frac{\frac{1}{4 \sqrt{2}}\left[\tau^{2 \sqrt{2}}-\tau^{\prime 2 \sqrt{2}}\right]}{\sqrt{\left[1+\left|\frac{1}{4 \sqrt{2}}\left[\tau^{2 \sqrt{2}}-\tau^{\prime 2 \sqrt{2}}\right]\right|^{2}\right]} e^{-i \phi}} & \text { for }\left|k c_{S} \eta\right|<<1, \\ \frac{\frac{1}{2 i(2 \Delta+1)^{3 / 2}}\left[\tau^{2 \sqrt{2 \Delta+1}}-\tau^{\prime 2 \sqrt{2 \Delta+1}]}\right.}{\sqrt{\left[1+\left|\frac{1}{2 i(2 \Delta+1)^{3 / 2}}\left[\tau^{2 \sqrt{2 \Delta+1}}-\tau^{\prime 2 \sqrt{2 \Delta+1}}\right]\right|^{2}\right]}} e^{-i \phi} & \text { for }\left|k c_{S} \eta\right| \approx 1-\Delta(\rightarrow 0), \\ \frac{-2\left[\frac{e^{-2 i k c c^{\eta}}\left(2 i k c_{S} \eta-1\right)}{4\left(c_{S} k\right)^{2} \eta^{2}}-\operatorname{Ei}\left(-2 i k c_{S} \eta\right)\right]_{\tau^{\prime}}^{\tau}}{\left.\left.\sqrt{\left[1+\left|-2\left[\frac{e^{-2 i k c_{S} \eta}\left(2 i k c_{S} \eta-1\right)}{4\left(c_{S} k\right)^{2} \eta^{2}}-\operatorname{Ei}\left(-2 i k c_{S} \eta\right)\right]_{\tau^{\prime}}^{\tau}\right|\right.}\right|^{2}\right]} & \text { for }\left|k c_{S} \eta\right|>>1 .\end{cases}$

For dS:

$\mathcal{T}_{\text {diag }}\left(k ; \tau, \tau^{\prime}\right)= \begin{cases}\frac{1}{\sqrt{\left[1+\left|\frac{1}{4 \sqrt{2}}\left[\tau^{2 \sqrt{2}}-\tau^{\prime 2 \sqrt{2}}\right]\right|^{2}\right]} e^{-i \phi}} & \text { for }\left|k c_{S} \eta\right|<<1, \\ \frac{1}{\sqrt{\left[1+\mid \frac{1}{2 i(2 \Delta+1)^{3 / 2}}\left[\tau^{2 \sqrt{2 \Delta+1}}-\tau^{\left.\prime 2 \sqrt{2 \Delta+1}]\left.\right|^{2}\right]}\right.\right.} e^{-i \phi}} & \text { for }\left|k c_{S} \eta\right| \approx 1-\Delta(\rightarrow 0), \\ \frac{1}{\left.\left.\sqrt{\left[1+\left|-2\left[\frac{e^{-2 i k c S^{\eta}\left(2 i k c_{S} \eta-1\right)}}{4\left(c_{S} k\right)^{2} \eta^{2}}-\operatorname{Ei}\left(-2 i k c_{S} \eta\right)\right]^{\tau}\right|^{\prime}\right.}\right|^{2}\right]} e^{-i \phi} & \text { for }\left|k c_{S} \eta\right|>>1 .\end{cases}$

For qdS:

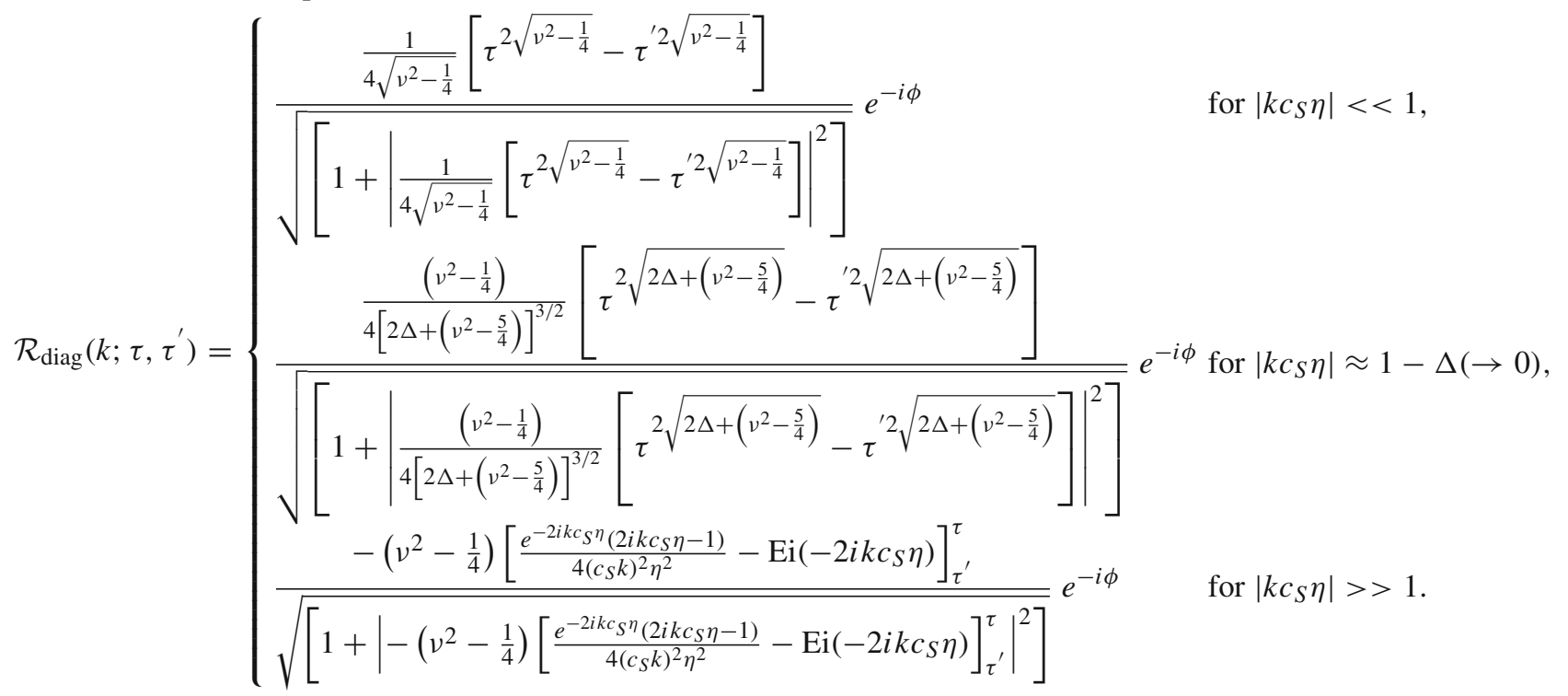


For qdS:

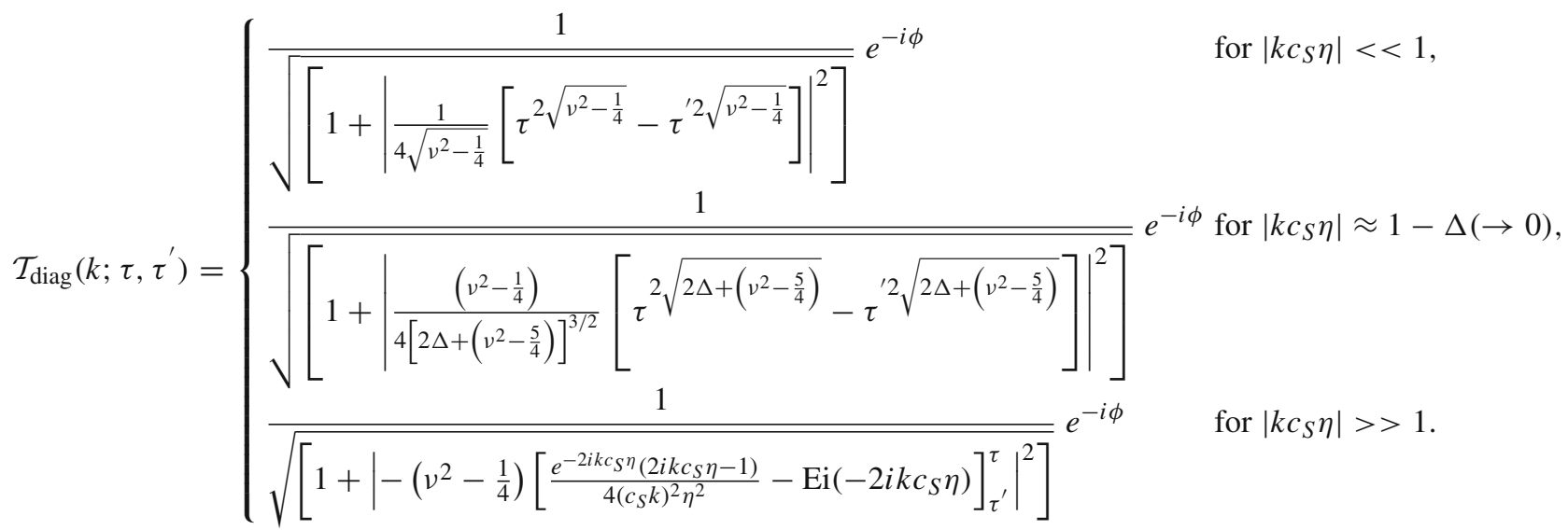

Next the expression for the number of produced particles at time $\tau$ can be calculated from the following formula:

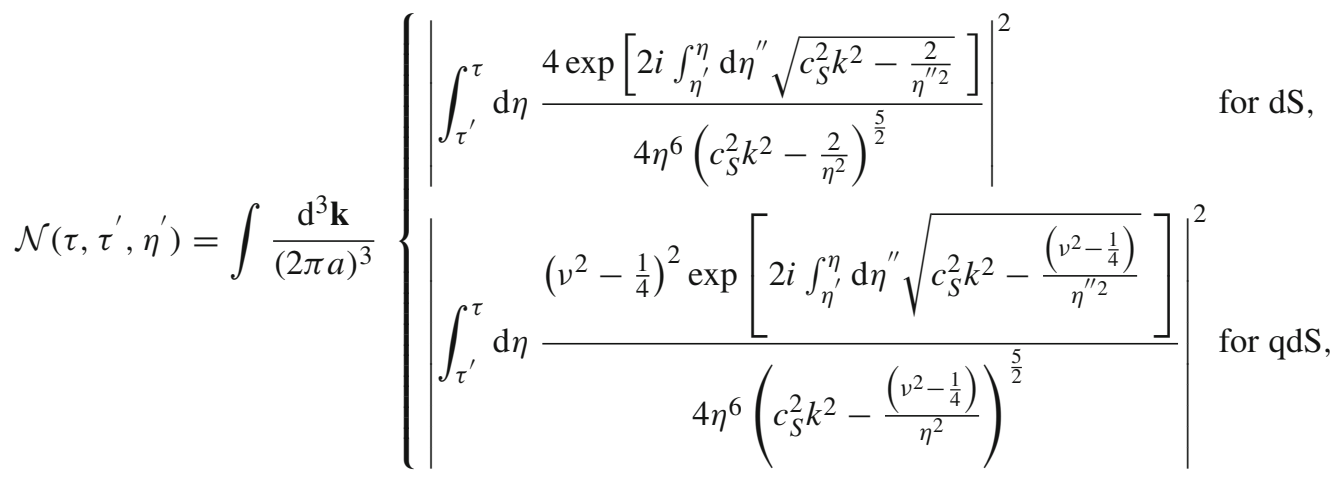

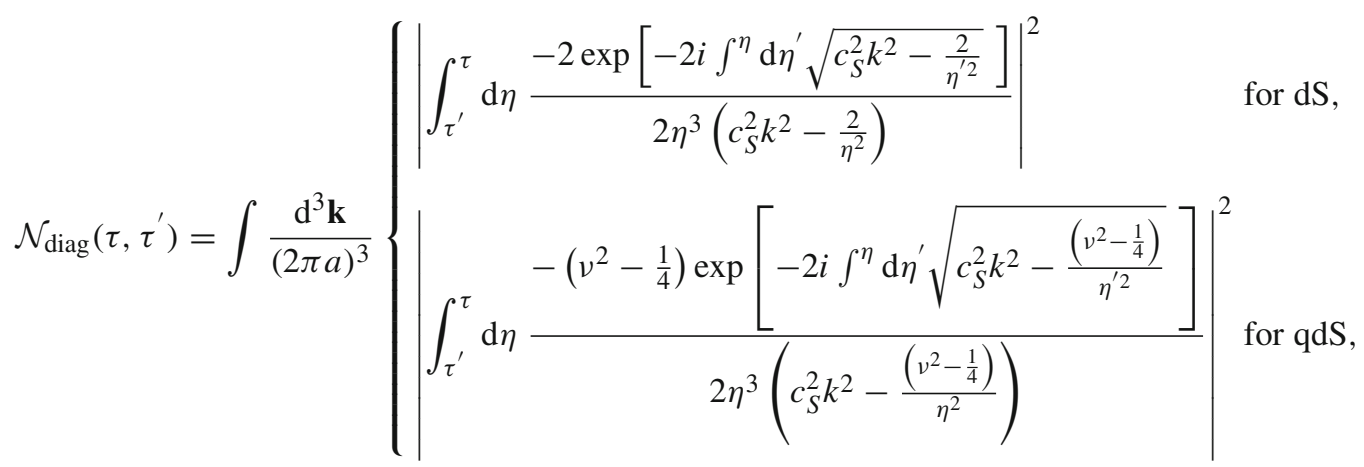

which is not exactly analytically computable. To study the behaviour of this integral we consider here three consecutive physical situations: $\left|k c_{S} \eta\right|<<1,\left|k c_{S} \eta\right| \approx 1-\Delta(\rightarrow 0)$ and $\left|k c_{S} \eta\right|>>1$ for the de Sitter case and the quasi de Sitter case. In the three cases we have the following. 
For dS:

$\mathcal{N}\left(\tau, \tau^{\prime}, \eta^{\prime}\right)$

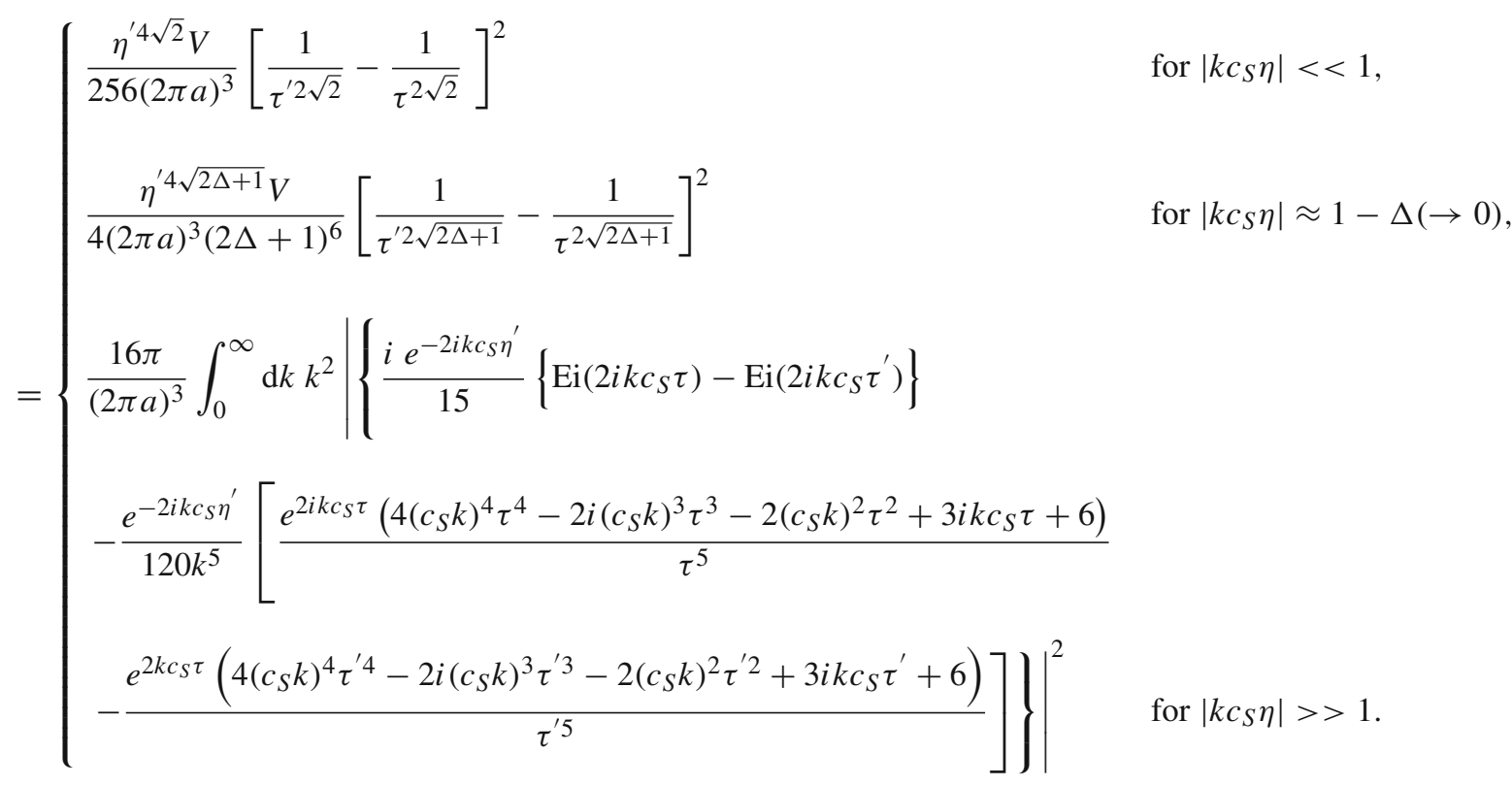

For qdS:

$$
\mathcal{N}\left(\tau, \tau^{\prime}, \eta^{\prime}\right)= \begin{cases}\frac{\eta^{\prime 4 \sqrt{v^{2}-\frac{1}{4}} V}}{64(2 \pi a)^{3}\left(v^{2}-\frac{1}{4}\right)^{2}}\left[\frac{1}{\tau^{\prime 2 \sqrt{v^{2}-\frac{1}{4}}}}-\frac{1}{\tau^{2} \sqrt{v^{2}-\frac{1}{4}}}\right]^{2} & \text { for }\left|k c_{S} \eta\right|<<1, \\ \frac{\eta^{4} \sqrt{2 \Delta+\left(v^{2}-\frac{5}{4}\right)} V\left(v^{2}-\frac{1}{4}\right)^{4}}{64(2 \pi a)^{3}\left[2 \Delta+\left(v^{2}-\frac{5}{4}\right)\right]^{6}}\left[\frac{1}{\tau^{\prime 2} \sqrt{2 \Delta+\left(v^{2}-\frac{5}{4}\right)}}-\frac{1}{\tau^{2} \sqrt{2 \Delta+\left(v^{2}-\frac{5}{4}\right)}}\right]^{2} & \text { for }\left|k c_{S} \eta\right| \approx 1-\Delta(\rightarrow 0), \\ -\frac{e^{-2 i k c_{S} \eta^{\prime}}}{120 k^{5}}\left[\frac{e^{2 i k c_{S} \tau}\left(4\left(c_{S} k\right)^{4} \tau^{4}-2 i\left(c_{S} k\right)^{3} \tau^{3}-2\left(c_{S} k\right)^{2} \tau^{2}+3 i k c_{S} \tau+6\right)}{\tau^{5}} \int_{0}^{\infty} \mathrm{d} k k^{2} \mid\left\{\frac{i e^{-2 i k s_{S} \eta^{\prime}}}{15}\left\{\operatorname{Ei}\left(2 i k c_{S} \tau\right)-\operatorname{Ei}\left(2 i k c_{S} \tau^{\prime}\right)\right\}\right.\right. & \\ \left.\left.-\frac{e^{2 k c_{S} \tau}\left(4\left(c_{S} k\right)^{4} \tau^{\prime 4}-2 i\left(c_{S} k\right)^{3} \tau^{\prime 3}-2\left(c_{S} k\right)^{2} \tau^{\prime 2}+3 i k c_{S} \tau^{\prime}+6\right)}{\tau^{\prime 5}}\right]\right\}\left.\right|^{2} & \text { for }\left|k c_{S} \eta\right|>>1 .\end{cases}
$$


For dS:

$\mathcal{N}_{\text {diag }}\left(\tau, \tau^{\prime}\right)= \begin{cases}\frac{V}{32(2 \pi a)^{3}}\left[\tau^{2 \sqrt{2}}-\tau^{\prime 2 \sqrt{2}}\right]^{2} & \text { for }\left|k c_{S} \eta\right|<<1, \\ \frac{V}{4(2 \pi a)^{3}(2 \Delta+1)^{3}}\left[\tau^{2 \sqrt{2 \Delta+1}}-\tau^{\prime 2 \sqrt{2 \Delta+1}}\right]^{2} & \text { for }\left|k c_{S} \eta\right| \approx 1-\Delta(\rightarrow 0), \\ \frac{16 \pi}{(2 \pi a)^{3}} \int_{0}^{\infty} \mathrm{d} k k^{2} \mid\left[\left\{\frac{e^{-2 i k c_{S} \tau}\left(2 i k c_{S} \tau-1\right)}{4\left(c_{S} k\right)^{2} \tau^{2}}-\frac{e^{-2 i k c_{S} \tau^{\prime}}\left(2 i k c_{S} \tau^{\prime}-1\right)}{4\left(c_{S} k\right)^{2} \tau^{\prime 2}}\right\}\right. & \\ \left.-\left\{\operatorname{Ei}\left(-2 i k c_{S} \tau\right)-\operatorname{Ei}\left(-2 i k c_{S} \tau^{\prime}\right)\right\}\right]\left.\right|^{2} & \left.\text { for }\left|k c_{S} \eta\right|>>1.197\right)\end{cases}$

For qdS:

$\mathcal{N}_{\text {diag }}\left(\tau, \tau^{\prime}\right)= \begin{cases}\frac{V}{16(2 \pi a)^{3}\left(v^{2}-\frac{1}{4}\right)}\left[\tau^{2 \sqrt{v^{2}-\frac{1}{4}}}-\tau^{\prime 2 \sqrt{v^{2}-\frac{1}{4}}}\right]^{2} & \text { for }\left|k c_{S} \eta\right|<<1, \\ \frac{V\left(v^{2}-\frac{1}{4}\right)^{2}}{16(2 \pi a)^{3}\left[2 \Delta+\left(v^{2}-\frac{5}{4}\right)\right]^{3}}\left[\tau^{\left.2 \sqrt{2 \Delta+\left(v^{2}-\frac{5}{4}\right)}-\tau^{\prime 2 \sqrt{2 \Delta+\left(v^{2}-\frac{5}{4}\right)}}\right]^{2}}\right. & \text { for }\left|k c_{S} \eta\right| \approx 1-\Delta(\rightarrow 0), \\ \frac{4 \pi\left(v^{2}-\frac{1}{4}\right)^{2}}{(2 \pi a)^{3}} \int_{0}^{\infty} \mathrm{d} k k^{2} \mid\left[\left\{\frac{e^{-2 i k c_{S} \tau}\left(2 i k c_{S} \tau-1\right)}{4\left(c_{S} k\right)^{2} \tau^{2}}-\frac{e^{-2 i k c_{S} \tau^{\prime}}\left(2 i k c_{S} \tau^{\prime}-1\right)}{4\left(c_{S} k\right)^{2} \tau^{\prime 2}}\right\}\right. & \\ \left.-\left\{\operatorname{Ei}\left(-2 i k c_{S} \tau\right)-\operatorname{Ei}\left(-2 i k c_{S} \tau^{\prime}\right)\right\}\right]\left.\right|^{2} & \text { for }\left|k c_{S} \eta\right|>>1,\end{cases}$

where $V$ is introduced earlier.

Finally one can define the total energy density of the produced particles using the following expression:

$$
\begin{aligned}
& \rho\left(\tau, \tau^{\prime}, \eta^{\prime}\right) \\
& =\int \frac{d^{3} \mathbf{k}}{(2 \pi a)^{3} a}\left\{\begin{array}{l}
\sqrt{c_{S}^{2} k^{2}-\frac{2}{\eta^{2}}}\left|\int_{\tau^{\prime}}^{\tau} \mathrm{d} \eta \frac{-2 \exp \left[-2 i \int^{\eta} \mathrm{d} \eta^{\prime} \sqrt{c_{S}^{2} k^{2}-\frac{2}{\eta^{\prime 2}}}\right]}{2 \eta^{3}\left(c_{S}^{2} k^{2}-\frac{2}{\eta^{2}}\right)}\right|^{2} \\
\sqrt{c_{S}^{2} k^{2}-\frac{\left(v^{2}-\frac{1}{4}\right)}{\eta^{2}}}\left|\int_{\tau^{\prime}}^{\tau} \mathrm{d} \eta \frac{-\left(v^{2}-\frac{1}{4}\right) \exp \left[-2 i \int^{\eta} \mathrm{d} \eta^{\prime} \sqrt{c_{S}^{2} k^{2}-\frac{\left(v^{2}-\frac{1}{4}\right)}{\eta^{\prime 2}}}\right]}{2 \eta^{3}\left(c_{S}^{2} k^{2}-\frac{\left(v^{2}-\frac{1}{4}\right)}{\eta^{2}}\right)}\right|^{2} \text { for dS, }
\end{array}\right.
\end{aligned}
$$




$$
\begin{aligned}
& \rho_{\text {diag }}\left(\tau, \tau^{\prime}, \eta^{\prime}\right)
\end{aligned}
$$

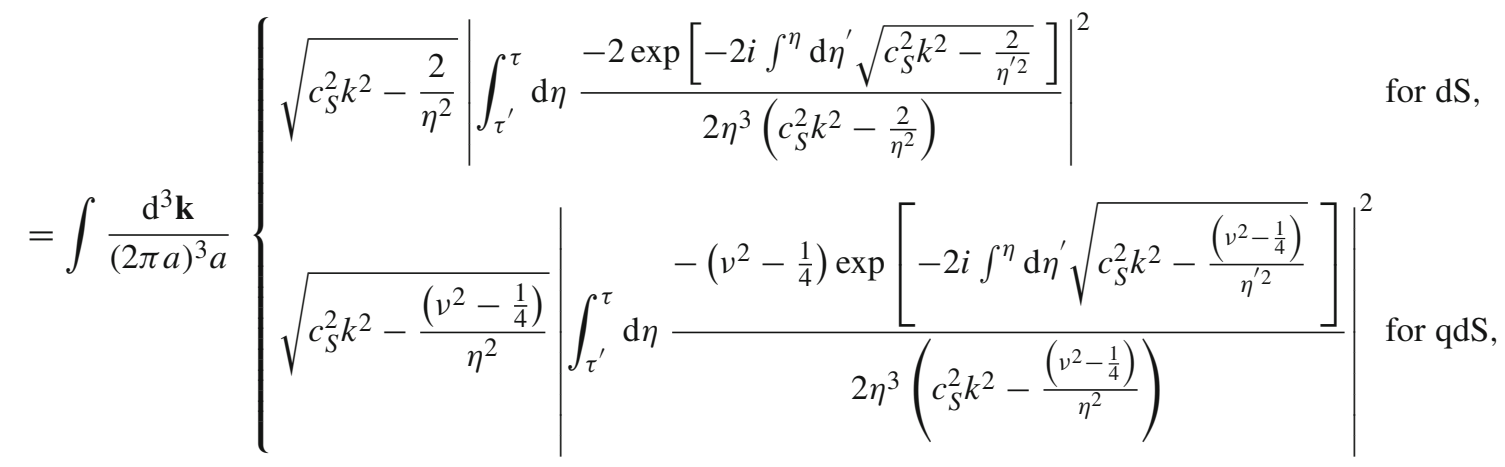

which is not exactly analytically computable. To study the behaviour of this integral we consider here three consecutive physical situations: $\left|k c_{S} \eta\right|<<1,\left|k c_{S} \eta\right| \approx 1-\Delta(\rightarrow 0)$ and $\left|k c_{S} \eta\right|>>1$ for the de Sitter case and the quasi de Sitter case. In the three cases we have

For dS:

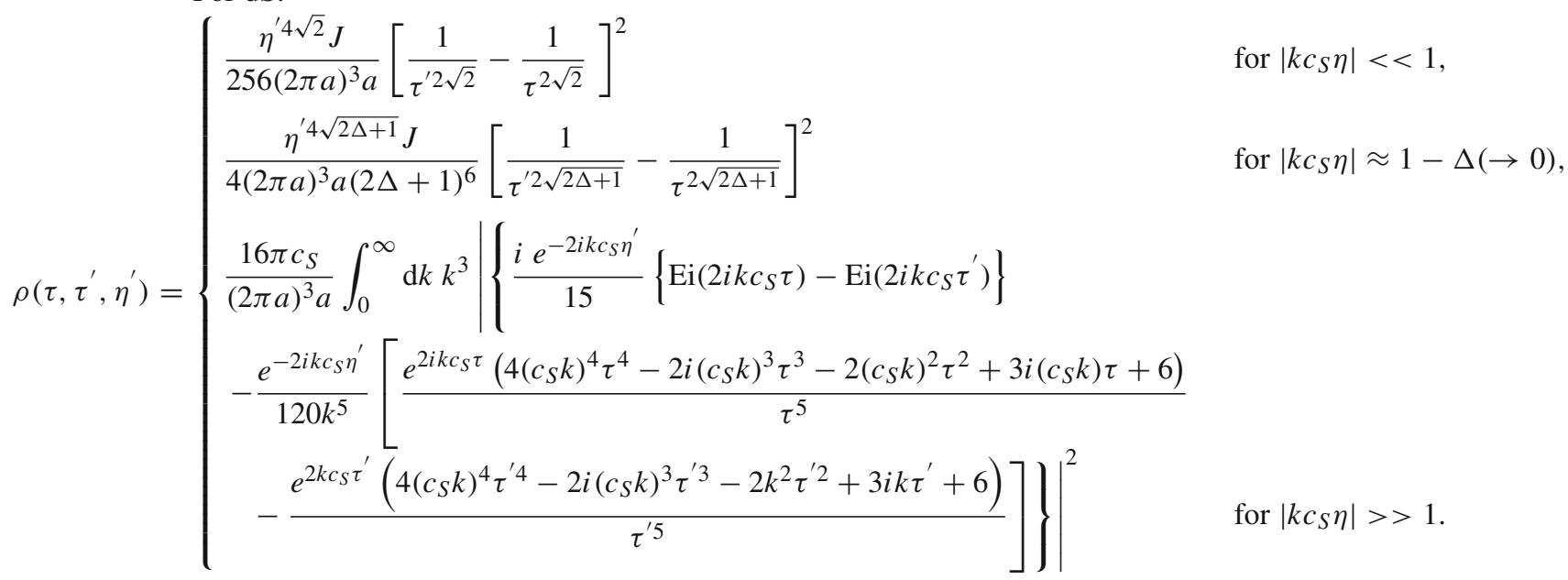

For qdS:

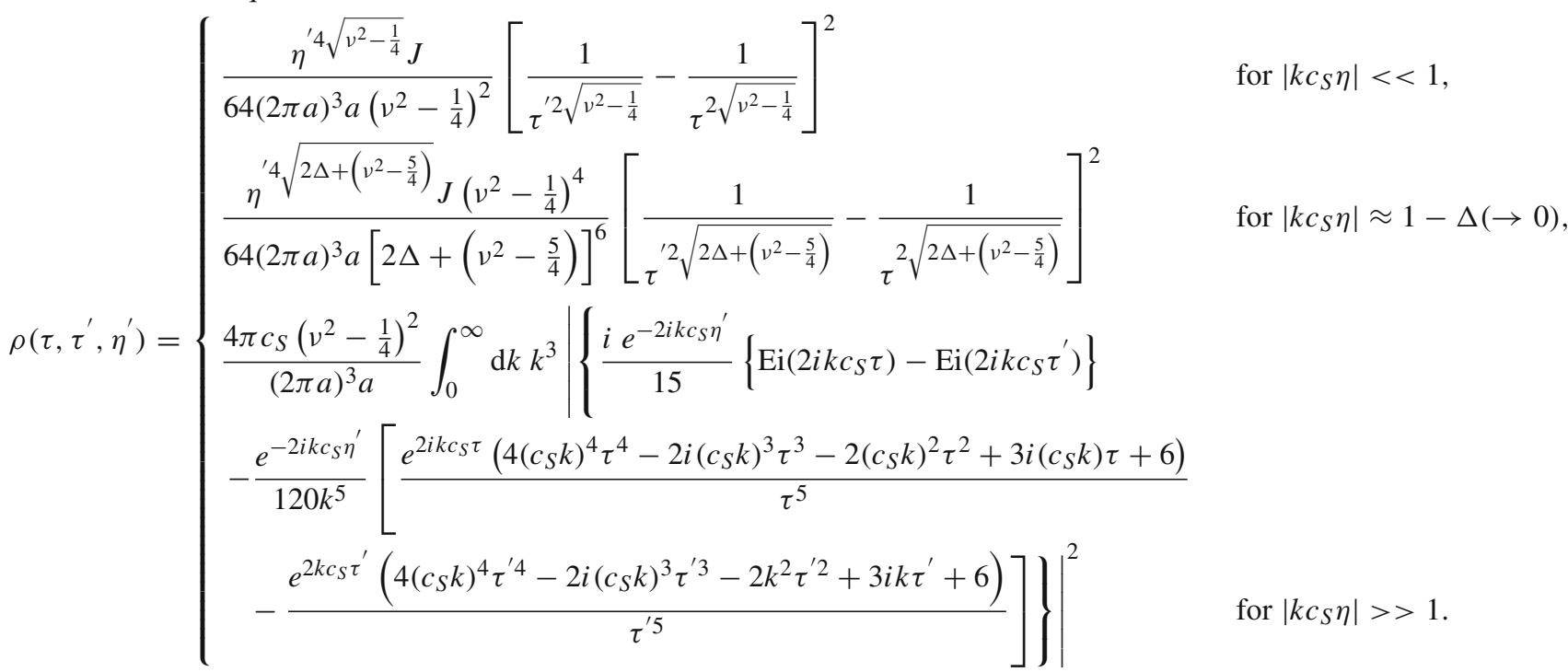


For dS:

$$
\rho_{\text {diag }}\left(\tau, \tau^{\prime}\right)= \begin{cases}\frac{J}{32(2 \pi a)^{3} a}\left[\tau^{2 \sqrt{2}}-\tau^{\prime 2 \sqrt{2}}\right]^{2} & \text { for }\left|k c_{S} \eta\right|<<1, \\ \frac{J}{4(2 \pi a)^{3} a(2 \Delta+1)^{3}}\left[\tau^{2 \sqrt{2 \Delta+1}}-\tau^{\prime 2 \sqrt{2 \Delta+1}}\right]^{2} & \text { for }\left|k c_{S} \eta\right| \approx 1-\Delta(\rightarrow 0), \\ \frac{16 \pi c_{S}}{(2 \pi a)^{3} a} \int_{0}^{\infty} \mathrm{d} k k^{3} \mid \frac{e^{-2 i k c_{S} \tau}\left(2 i k c_{S} \tau-1\right)}{4\left(c_{S} k \tau\right)^{2}}-\frac{e^{-2 i k c_{S} \tau^{\prime}}\left(2 i k c_{S} \tau^{\prime}-1\right)}{4\left(c_{S} k \tau^{\prime}\right)^{2}} & \text { for }\left|k c_{S} \eta\right|>>1 . \\ +\left.\left\{\operatorname{Ei}\left(-2 i k c_{S} \tau\right)-\operatorname{Ei}\left(-2 i k c_{S} \tau^{\prime}\right)\right\}\right|^{2} & \end{cases}
$$

For qdS:

$$
\rho_{\text {diag }}\left(\tau, \tau^{\prime}, \eta^{\prime}\right)= \begin{cases}\frac{J}{16(2 \pi a)^{3} a\left(v^{2}-\frac{1}{4}\right)}\left[\tau^{2 \sqrt{v^{2}-\frac{1}{4}}}-\tau^{\prime 2 \sqrt{v^{2}-\frac{1}{4}}}\right]^{2} & \text { for }\left|k c_{S} \eta\right|<<1, \\ \frac{J\left(v^{2}-\frac{1}{4}\right)^{2}}{16(2 \pi a)^{3} a\left[2 \Delta+\left(v^{2}-\frac{5}{4}\right)\right]^{3}}\left[\tau^{2 \sqrt{2 \Delta+\left(v^{2}-\frac{5}{4}\right)}}-\tau^{\prime 2 \sqrt{2 \Delta+\left(v^{2}-\frac{5}{4}\right)}}\right]^{2} & \text { for }\left|k c_{S} \eta\right| \approx 1-\Delta(\rightarrow 0), \\ \frac{4 \pi c_{S}\left(v^{2}-\frac{1}{4}\right)^{2}}{(2 \pi a)^{3} a} \int_{0}^{\infty} \mathrm{d} k k^{3} \mid \frac{e^{-2 i k c_{S} \tau}\left(2 i k c_{S} \tau-1\right)}{4\left(c_{S} k \tau\right)^{2}}-\frac{e^{-2 i k c_{S} \tau^{\prime}}\left(2 i k c_{S} \tau^{\prime}-1\right)}{4\left(c_{S} k \tau^{\prime}\right)^{2}} & \\ +\left.\left\{\operatorname{Ei}\left(-2 i k c_{S} \tau\right)-\operatorname{Ei}\left(-2 i k c_{S} \tau^{\prime}\right)\right\}\right|^{2} & \text { for }\left|k c_{S} \eta\right|>>1 .\end{cases}
$$

Throughout the discussion of the total energy density of the produced particles we have introduced a symbol $J$ defined as

$J=\int \mathrm{d}^{3} \mathbf{k} p(\tau)= \begin{cases}\int \mathrm{d}^{3} \mathbf{k} \sqrt{c_{S}^{2} k^{2}-\frac{2}{\tau^{2}}} & \text { for } \mathrm{dS}, \\ \int \mathrm{d}^{3} \mathbf{k} \sqrt{c_{S}^{2} k^{2}-\frac{\left(v^{2}-\frac{1}{4}\right)}{\tau^{2}}} & \text { for qdS, }\end{cases}$

which physically signifies the total finite volume weighted by $p(\eta)$ in momentum space within which the produced particles are occupied.

To study the behaviour of this integral we consider here three consecutive physical situations: $\left|k c_{S} \eta\right|<<1,\left|k c_{S} \eta\right| \approx$ $1-\Delta(\rightarrow 0)$ and $\left|k c_{S} \eta\right|>>1$ for the de Sitter case and the quasi de Sitter case. In the three cases we have

For dS:

$J= \begin{cases}\int \mathrm{d}^{3} \mathbf{k} \frac{\sqrt{2}}{\tau}=\frac{V \sqrt{2}}{\tau} & \text { for }\left|k c_{S} \eta\right|<<1, \\ \int \mathrm{d}^{3} \mathbf{k} \frac{\sqrt{2 \Delta+1}}{\tau}=\frac{\sqrt{2 \Delta+1} V}{\tau} & \text { for }\left|k c_{S} \eta\right| \approx 1-\Delta(\rightarrow 0), \\ \int \mathrm{d}^{3} \mathbf{k} k c_{S} & \text { for }\left|k c_{S} \eta\right|>>1 .\end{cases}$

For qdS:

$J= \begin{cases}\int \mathrm{d}^{3} \mathbf{k} \frac{\sqrt{\left(v^{2}-\frac{1}{4}\right)}}{\tau}=\frac{V \sqrt{\left(v^{2}-\frac{1}{4}\right)}}{\tau} & \text { for }\left|k c_{S} \eta\right|<<1, \\ \int \mathrm{d}^{3} \mathbf{k} \frac{\sqrt{2 \Delta+\left(v^{2}-\frac{5}{4}\right)}}{\tau}=\frac{\sqrt{2 \Delta+\left(v^{2}-\frac{5}{4}\right)} V}{\tau} & \text { for }\left|k c_{S} \eta\right| \approx 1-\Delta(\rightarrow 0), \\ \int \mathrm{d}^{3} \mathbf{k} k c_{S} & \text { for }\left|k c_{S} \eta\right|>>1 .\end{cases}$ 


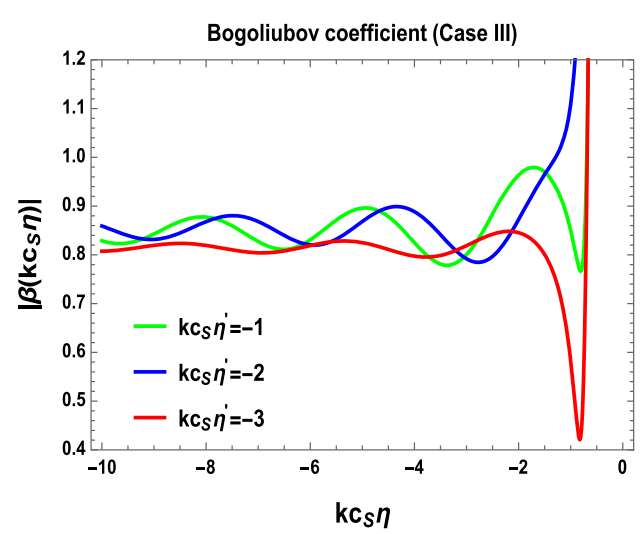

(a)

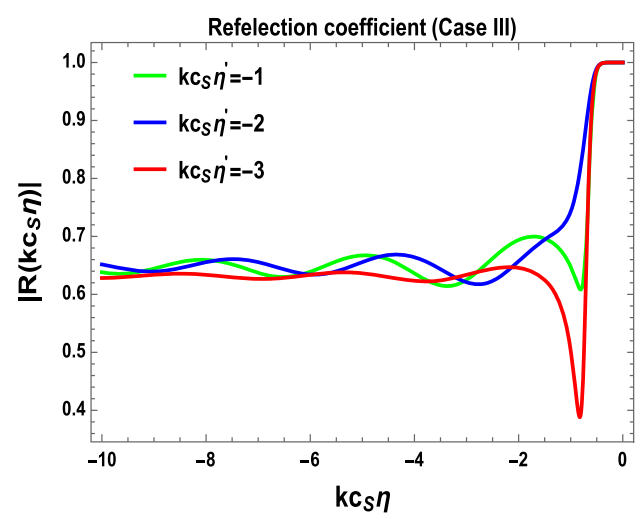

(c)

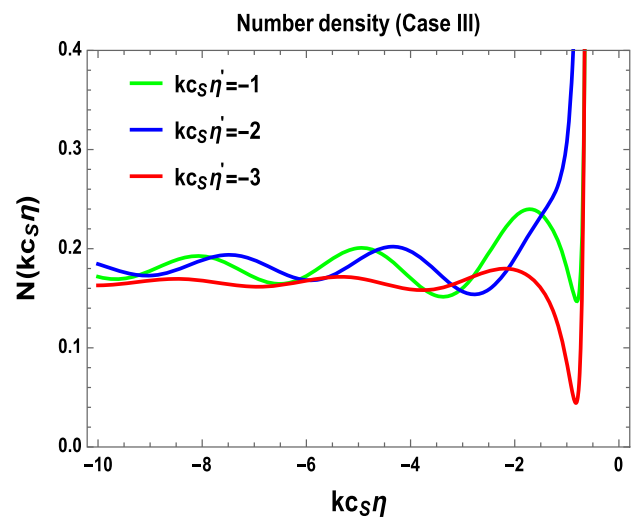

(e)

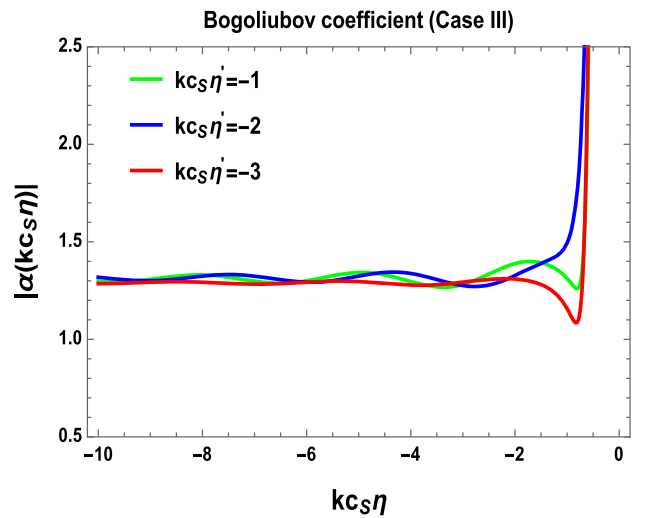

(b)

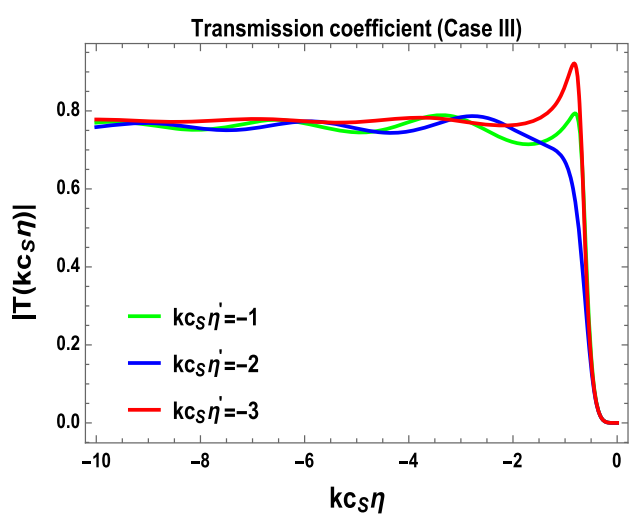

(d)

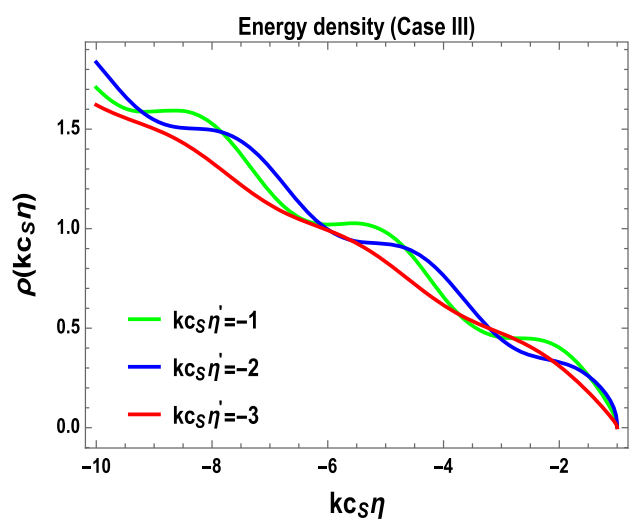

(f)

Fig. 11 Particle creation profile for Case III

$$
S=S_{1}+S_{2}
$$

In Figs. 11 and 12, we have explicitly shown the particle creation profile for Case III for two representations.

\subsection{Cosmological scalar curvature fluctuations from new massive particles}

To describe the effect of the massive particles on the scalar curvature fluctuations here we start with the second order action derived in the framework of effective field theory as where $S_{1}$ and $S_{2}$ is given by

$$
\begin{aligned}
S_{1} & =\frac{1}{2} \int \mathrm{d} \eta \mathrm{d}^{3} x \frac{2 \epsilon M_{p}^{2}}{\tilde{c}_{S}^{2} H^{2}}\left[\frac{\left(\partial_{\eta} \zeta\right)^{2}-c_{S}^{2}\left(\partial_{i} \zeta\right)^{2}}{\eta^{2}}-\frac{m_{\mathrm{inf}}^{2}}{H^{2} \eta^{2}}\right] \\
S_{2} & =-\int \frac{\mathrm{d} \eta}{\tilde{c}_{S} H} m(\eta) \partial_{\eta} \zeta(\eta, \mathbf{x}=0)
\end{aligned}
$$

Here the action contains the following crucial information: 


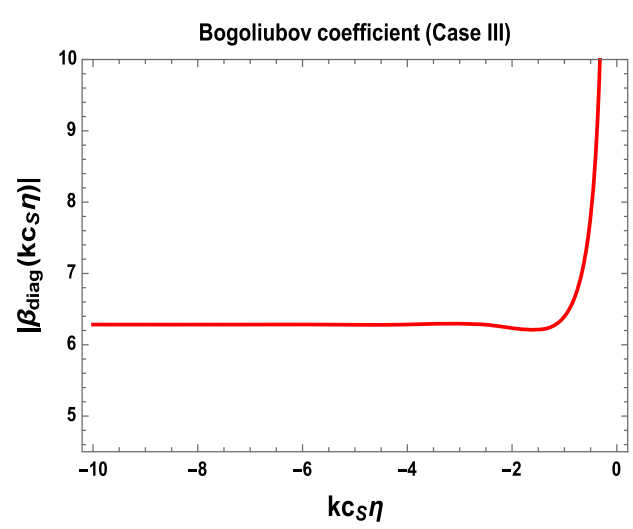

(a)

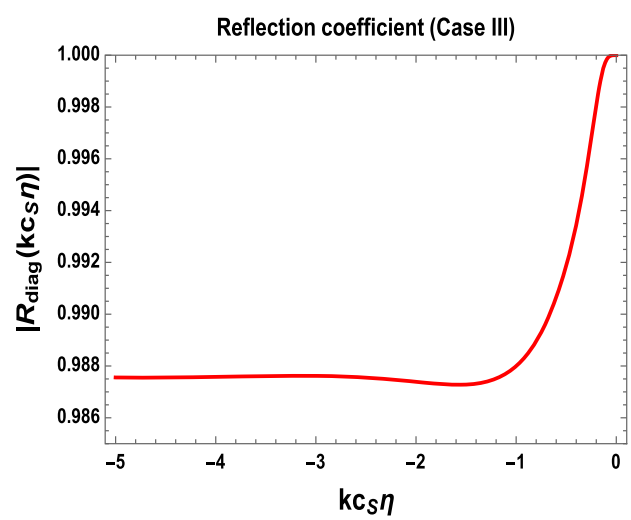

(c)

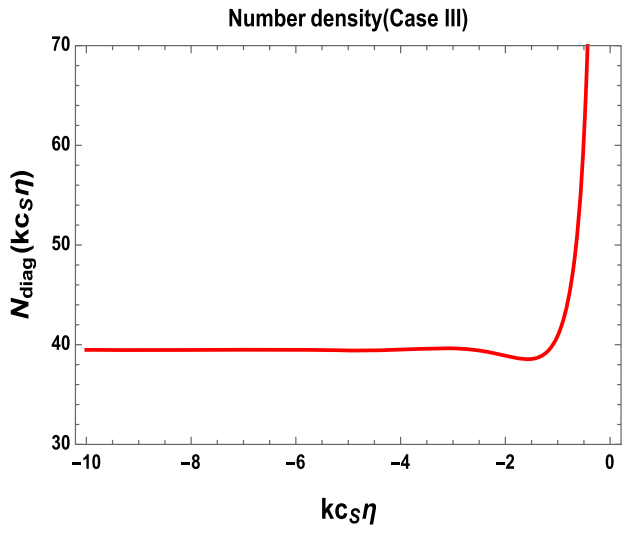

(e)

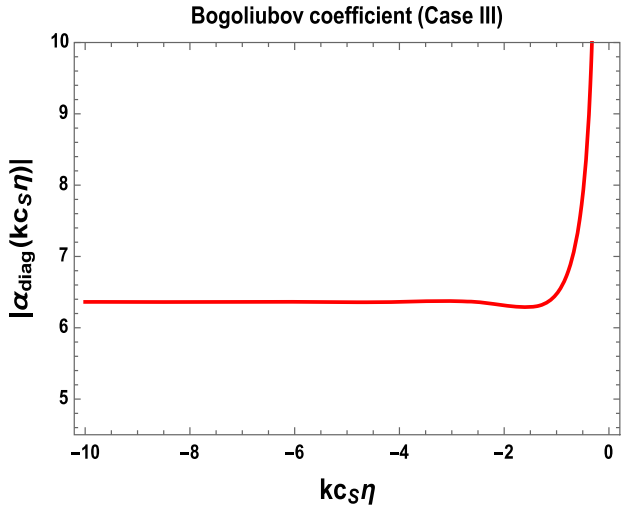

(b)

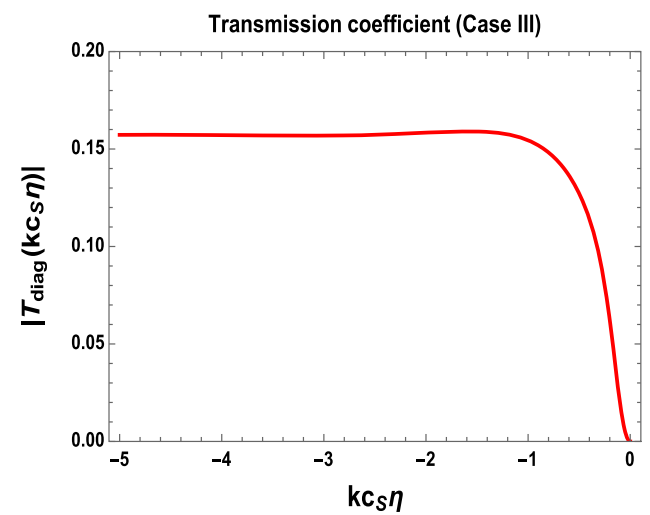

(d)

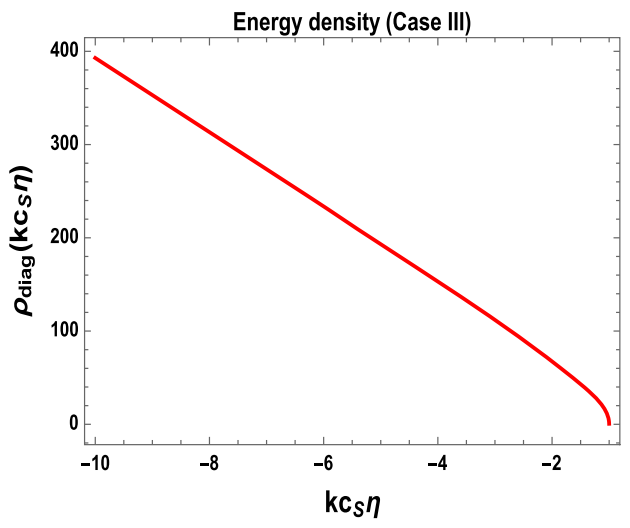

(f)

Fig. 12 Particle creation profile for Case III in diagonalized representation

- The term $S_{2}$ contains the effect of massive particle as explicitly the mass factor appears here. In the most general picture the mass parameter $m(\eta)$ is a function of conformal time $\eta$. Additionally it is important to note that the term $S_{2}$ in the effective action for curvature fluctuation represents specific interaction term in which inflaton field is interacting with the heavy fields. This implies that the time-dependent coupling $m(\eta)$ mimics the role of coupling constant in the present context.
- In the inflationary mass term we have neglected the other contributions from the effective potential. This is a complicated inflationary model as it contains both an inflaton and a heavy field with a time-dependent coupling $m(\eta)$. For this reason initially we keep the mass contribution from the inflationary sector. But it is important to note that, in the most simple inflationary models, one can neglect the mass contribution as well, because in all those cases $m_{\text {inf }}<<H$ approximation is valid. But due 
to the presence of the mass term of the inflaton equation of motion also modified and this will further appear in the solutions as well.

- Here $c_{S}$ is the effective sound speed parameter and $\tilde{c}_{S}$ is the actual sound speed as introduced in the previous section of this paper.

- When all the effective field theoretic interactions are absent in that case both $c_{S} \sim \tilde{c}_{S}=1$ and one can get back the results for canonical slow-roll models.

- On the other hand when the previously mentioned effective field theoretic interactions are switched on within the present description, one can accommodate the noncanonical as well as non-minimal interactions. In that case both $c_{S}$ and $\tilde{c}_{S}$ are less than unity and in such a situation one can always constrain the sound speed parameter as well the strength of the effective field theoretic interactions using observational probes (Planck 2015 data).

- In the case of canonical interactions one can easily compare the present setup with effective time varying mass parameter with the axions with time varying decay constant.

- For $m<<H$, the last term in the above mentioned effective action is absent and in that case the reduced form of the action will enable one to explain the effective field of inflation in the presence of previously mentioned nontrivial effective interactions. Once we switch off all such interactions the above action mimics the case for single field slow-roll inflation.

- Here first we derive the results for arbitrary parametrization of $m / H$ and then discuss the results for $m \approx H$, $m<<H, m>>H$. Also we derive the results for arbitrary choice of initial conditions. Then we discuss the results for Buch Davies vacuum, $\alpha$ vacuum and another specific choice of vacuum which we explicitly discussed in this section.

To extract further informations from Eq. (3.208), first of all one needs to write down the second order action by applying Fourier transform. For this the Fourier transform of the curvature perturbation $\zeta(\eta, \mathbf{x})$ is defined as

$\zeta(\eta, \mathbf{x})=\int \frac{\mathrm{d}^{3} k}{(2 \pi)^{3}} \zeta_{\mathbf{k}}(\eta) \exp (i \mathbf{k} \cdot \mathbf{x})$,

where $\zeta_{\mathbf{k}}(\eta)$ is the time-dependent part of the curvature fluctuation after Fourier transform and can be expressed in terms of the normalized time-dependent scalar mode function $h_{\mathbf{k}}(\eta)$ as

$\zeta_{\mathbf{k}}(\eta)=\frac{h_{\mathbf{k}}(\eta)}{z M_{p}}=\frac{h(\eta, \mathbf{k}) a(\mathbf{k})+h^{*}(\eta,-\mathbf{k}) a^{\dagger}(-\mathbf{k})}{z M_{p}}$ where $z$ is the Mukhanov-Sasaki variable defined as

$z=\frac{a \sqrt{2 \epsilon}}{\tilde{c}_{S}}$

and $a(\mathbf{k})$ and $a^{\dagger}(\mathbf{k})$ are the creation and annihilation operator satisfying the following commutation relations:

$$
\begin{aligned}
{\left[a(\mathbf{k}), a^{\dagger}\left(-\mathbf{k}^{\prime}\right)\right] } & =(2 \pi)^{3} \delta^{3}\left(\mathbf{k}+\mathbf{k}^{\prime}\right), \\
{\left[a(\mathbf{k}), a\left(\mathbf{k}^{\prime}\right)\right] } & =0, \\
{\left[a^{\dagger}(\mathbf{k}), a^{\dagger}\left(\mathbf{k}^{\prime}\right)\right] } & =0 .
\end{aligned}
$$

Additionally it is important to mention here that the exact solution and its WKB approximated results for the timedependent scalar mode function $h_{\mathbf{k}}(\eta)$ are explicitly derived in the previous section.

Presently our prime objective is to compute the VEV of the curvature fluctuation in momentum space in the presence of the mass contribution $S_{2}$ with respect to the arbitrary choice of vacuum, which leads to an important contribution to the Bell inequalities or violation in the context of primordial cosmology. Using the interaction picture the one point function of the curvature fluctuation in momentum space can be expressed as

$\left\langle\zeta_{k}(\eta=0)\right\rangle=-i \int_{-\infty}^{0} \mathrm{~d} \eta a(\eta)\left\langle 0\left|\left[\zeta_{\mathbf{k}}(0), H_{i n t}(\eta)\right]\right| 0\right\rangle$

where $a(\eta)$ is the scale factor defined in the earlier section in terms of the Hubble parameter $H$ and the conformal time scale $\eta$. In the interaction picture the Hamiltonian can written as

$H_{i n t}(\eta)=-\frac{m}{\tilde{c}_{S} H} \partial_{\eta} \zeta(\eta, \mathbf{x}=0)$,

which gives the primary information to compute the explicit expression for the one point function or more precisely the Bell inequality violation in the present context. After applying a Fourier transform in Eq. (3.218) we get the following expression:

$$
\begin{aligned}
H_{i n t}= & -\int \frac{\mathrm{d}^{3} k}{(2 \pi)^{3} \tilde{c}_{S} z M_{p}} \frac{m}{H} \\
& \times\left[h^{\prime}(\eta, \mathbf{k}) a(\mathbf{k})+h^{\dagger^{\prime}}(\eta,-\mathbf{k}) a^{\dagger}(-\mathbf{k})\right]
\end{aligned}
$$

and further substituting Eq. (3.219) in Eq. (3.217) finally we get

$$
\begin{aligned}
\left\langle\zeta_{\mathbf{k}}(\eta=0)\right\rangle= & -i \int_{-\infty}^{0} \mathrm{~d} \eta \frac{a(\eta)}{z^{2} M_{p}^{2}} \frac{m}{\tilde{c}_{S}}\left(h_{\mathbf{k}}(0) h_{\mathbf{k}}^{\dagger^{\prime}}(\eta)\right. \\
& \left.-h_{-\mathbf{k}}^{\dagger}(0) h_{-\mathbf{k}}^{\prime}(\eta)\right)
\end{aligned}
$$


where $h_{\mathbf{k}}(\eta)$ is the exact solution of the mode function as explicitly computed in the earlier section of this paper. Now sometimes it happens that the exact solution of mode function is not exactly defined at $\eta=0$ point. To avoid such a complexity in the present computation for the sake of clarity here we introduce an Infra-Red (IR) cut-off regulator $\xi$ in the conformal time integral and consequently Eq. (3.220) can be recast as

$\left\langle\zeta_{\mathbf{k}}(\eta=\xi \rightarrow 0)\right\rangle=-i \lim _{k c_{S} \xi \rightarrow 0} \int_{-\infty}^{\xi} \mathrm{d} \eta \frac{a(\eta)}{z^{2} M_{p}^{2}} \frac{m}{\tilde{c}_{S}} \times\left(h_{\mathbf{k}}(\xi) h_{\mathbf{k}}^{\dagger^{\prime}}(\eta)-h_{-\mathbf{k}}^{\dagger}(\xi) h_{-\mathbf{k}}^{\prime}(\eta)\right)$.

Further substituting the explicit form of the scalar mode functions computed from the exact solution we get the following generalized expression for the one point function of the curvature fluctuation in momentum space:

$$
\left\langle\zeta_{\mathbf{k}}(\eta=\xi \rightarrow 0)\right\rangle=-i \lim _{k c_{S} \xi \rightarrow 0} \int_{-\infty}^{\xi} \mathrm{d} \eta \frac{a(\eta)}{z^{2} M_{p}^{2}} \frac{m}{\tilde{c}_{S}} \sum_{i=1}^{2} \sum_{j=1}^{2} C_{i}^{*} C_{j} \mathcal{A}_{i j}(\eta, k),
$$

where the conformal time-dependent functions $\mathcal{A}_{i j} \forall i, j=1,2$ in momentum space are defined as

$$
\begin{aligned}
\mathcal{A}_{11}(\eta, k)= & \sqrt{\xi \eta}\left[H_{\Lambda}^{(1)}\left(-k c_{S} \xi\right) H_{\Lambda}^{(1) *^{\prime}}\left(-k c_{S} \eta\right)-H_{\Lambda}^{(1) *}\left(k c_{S} \xi\right) H_{\Lambda}^{(1)^{\prime}}\left(k c_{S} \eta\right)\right] \\
& -\frac{1}{2} \sqrt{\frac{\xi}{\eta}}\left[H_{\Lambda}^{(1)}\left(-k c_{S} \xi\right) H_{\Lambda}^{(1) *}\left(-k c_{S} \eta\right)-H_{\Lambda}^{(1) *}\left(k c_{S} \xi\right) H_{\Lambda}^{(1)}\left(k c_{S} \eta\right)\right], \\
\mathcal{A}_{22}(\eta, k)= & \sqrt{\xi \eta}\left[H_{\Lambda}^{(2)}\left(-k c_{S} \xi\right) H_{\Lambda}^{(2) *^{\prime}}\left(-k c_{S} \eta\right)-H_{\Lambda}^{(2) *}\left(k c_{S} \xi\right) H_{\Lambda}^{(2)^{\prime}}\left(k c_{S} \eta\right)\right] \\
& -\frac{1}{2} \sqrt{\frac{\xi}{\eta}}\left[H_{\Lambda}^{(2)}\left(-k c_{S} \xi\right) H_{\Lambda}^{(2) *}\left(-k c_{S} \eta\right)-H_{\Lambda}^{(2) *}\left(k c_{S} \xi\right) H_{\Lambda}^{(2)}\left(k c_{S} \eta\right)\right], \\
\mathcal{A}_{12}(\eta, k)= & \sqrt{\xi \eta}\left[H_{\Lambda}^{(1)}\left(-k c_{S} \xi\right) H_{\Lambda}^{(2) *^{\prime}}\left(-k c_{S} \eta\right)-H_{\Lambda}^{(1) *}\left(k c_{S} \xi\right) H_{\Lambda}^{(2)^{\prime}}\left(k c_{S} \eta\right)\right] \\
& -\frac{1}{2} \sqrt{\frac{\xi}{\eta}}\left[H_{\Lambda}^{(1)}\left(-k c_{S} \xi\right) H_{\Lambda}^{(2) *}\left(-k c_{S} \eta\right)-H_{\Lambda}^{(1) *}\left(k c_{S} \xi\right) H_{\Lambda}^{(2)}\left(k c_{S} \eta\right)\right], \\
\mathcal{A}_{21}(\eta, k)= & \sqrt{\xi \eta}\left[H_{\Lambda}^{(2)}\left(-k c_{S} \xi\right) H_{\Lambda}^{(1) *^{\prime}}\left(-k c_{S} \eta\right)-H_{\Lambda}^{(2) *}\left(k c_{S} \xi\right) H_{\Lambda}^{(1)^{\prime}}\left(k c_{S} \eta\right)\right] \\
& -\frac{1}{2} \sqrt{\frac{\xi}{\eta}}\left[H_{\Lambda}^{(2)}\left(-k c_{S} \xi\right) H_{\Lambda}^{(1) *}\left(-k c_{S} \eta\right)-H_{\Lambda}^{(2) *}\left(k c_{S} \xi\right) H_{\Lambda}^{(1)}\left(k c_{S} \eta\right)\right],
\end{aligned}
$$

the parameter $\Lambda$ is defined in Eq. (3.31) for dS and quasi dS case. Here after taking the limit $k c_{S} \xi \rightarrow 0$ the conformal time-dependent functions $\mathcal{A}_{i j} \forall i, j=1,2$ in momentum space can be recast as

$$
\begin{aligned}
\lim _{k c_{S} \xi \rightarrow 0} \mathcal{A}_{11}(\eta, k)=\frac{i}{\pi} \Gamma(\Lambda) \sqrt{\eta \xi}\left[\left(-\frac{k c_{S} \xi}{2}\right)^{-\Lambda} H_{\Lambda}^{(1) *^{\prime}}\left(-k c_{S} \eta\right)+\left(\frac{k c_{S} \xi}{2}\right)^{-\Lambda} H_{\Lambda}^{(1)^{\prime}}\left(k c_{S} \eta\right)\right] & -i \sqrt{\xi} \\
& -\frac{k \sqrt{\eta}}{2 \pi}(\Lambda)\left[\left(-\frac{k c_{S} \xi}{2}\right)^{-\Lambda} H_{\Lambda}^{(1) *}\left(-k c_{S} \eta\right)+\left(\frac{k c_{S} \xi}{2}\right)^{-\Lambda} H_{\Lambda}^{(1)}\left(k c_{S} \eta\right)\right], \\
\lim _{k c_{S} \xi \rightarrow 0} \mathcal{A}_{22}(\eta, k)=- & \frac{i}{\pi} \Gamma(\Lambda) \sqrt{\eta \xi}\left[\left(-\frac{k c_{S} \xi}{2}\right)^{-\Lambda} H_{\Lambda}^{(2) *^{\prime}}\left(-k c_{S} \eta\right)+\left(\frac{k c_{S} \xi}{2}\right)^{-\Lambda} H_{\Lambda}^{(2)^{\prime}}\left(k c_{S} \eta\right)\right] \\
& +\frac{i \sqrt{\xi}}{2 \pi \sqrt{\eta}} \Gamma(\Lambda)\left[\left(-\frac{k c_{S} \xi}{2}\right)^{-\Lambda} H_{\Lambda}^{(2) *}\left(-k c_{S} \eta\right)+\left(\frac{k c_{S} \xi}{2}\right)^{-\Lambda} H_{\Lambda}^{(2)}\left(k c_{S} \eta\right)\right], \\
\lim _{k c_{S} \xi \rightarrow 0} \mathcal{A}_{12}(\eta, k)= & \frac{i}{\pi} \Gamma(\Lambda) \sqrt{\eta \xi}\left[\left(-\frac{k c_{S} \xi}{2}\right)^{-\Lambda} H_{\Lambda}^{(2) *^{\prime}}\left(-k c_{S} \eta\right)+\left(\frac{k c_{S} \xi}{2}\right)^{-\Lambda} H_{\Lambda}^{(2)^{\prime}}\left(k c_{S} \eta\right)\right] \\
& -\frac{i \sqrt{\xi}}{2 \pi \sqrt{\eta}} \Gamma(\Lambda)\left[\left(-\frac{k c_{S} \xi}{2}\right)^{-\Lambda} H_{\Lambda}^{(2) *}\left(-k c_{S} \eta\right)+\left(\frac{k c_{S} \xi}{2}\right)^{-\Lambda} H_{\Lambda}^{(2)}\left(k c_{S} \eta\right)\right],
\end{aligned}
$$




$$
\begin{aligned}
\lim _{k c_{S} \xi \rightarrow 0} \mathcal{A}_{21}(\eta, k)=- & \frac{i}{\pi} \Gamma(\Lambda) \sqrt{\eta \xi}\left[\left(-\frac{k c_{S} \xi}{2}\right)^{-\Lambda} H_{\Lambda}^{(1) *^{\prime}}\left(-k c_{S} \eta\right)+\left(\frac{k c_{S} \xi}{2}\right)^{-\Lambda} H_{\Lambda}^{(1)^{\prime}}\left(k c_{S} \eta\right)\right] \\
& +\frac{i \sqrt{\xi}}{2 \pi \sqrt{\eta}} \Gamma(\Lambda)\left[\left(-\frac{k c_{S} \xi}{2}\right)^{-\Lambda} H_{\Lambda}^{(1) *}\left(-k c_{S} \eta\right)+\left(\frac{k c_{S} \xi}{2}\right)^{-\Lambda} H_{\Lambda}^{(1)}\left(k c_{S} \eta\right)\right] .
\end{aligned}
$$

For further simplification we consider here the two limiting cases $\left|k c_{S} \eta\right| \rightarrow-\infty,\left|k c_{S} \eta\right| \rightarrow 0$ and $\left|k c_{S} \eta\right| \approx 1$, which are physically acceptable in the present context. First of we consider the results for $\left|k c_{S} \eta\right| \rightarrow-\infty$. In this case we get

$$
\begin{aligned}
& \left\langle\zeta_{\mathbf{k}}(\eta=0)\right\rangle_{\left|k c_{S} \eta\right| \rightarrow-\infty}=\frac{2}{M_{p}^{2} \pi} \int_{-\infty}^{0} \mathrm{~d} \eta \frac{\tilde{c}_{S} H}{a(\eta) \epsilon k c_{S}} \frac{m}{H}\left[\left|C_{2}\right|^{2} e^{-i k c_{S} \eta}-\left|C_{1}\right|^{2} e^{i k c_{S} \eta}\right. \\
& \left.-i\left(C_{1}^{*} C_{2} e^{i \pi\left(\Lambda+\frac{1}{2}\right)}+C_{1} C_{2}^{*} e^{-i \pi\left(\Lambda+\frac{1}{2}\right)}\right) \sin k c_{S} \eta\right] \\
& \begin{cases}-\frac{1}{M_{p}^{2}} \int_{-\infty}^{0} \mathrm{~d} \eta \frac{\tilde{c}_{S} H}{a(\eta) \epsilon k c_{S}} \frac{m}{H} e^{i k c_{S} \eta} & \text { for Bunch Davies vacu } \\
\frac{2}{M_{p}^{2} \pi} \int_{-\infty}^{0} \mathrm{~d} \eta \frac{\tilde{c}_{S} H}{a(\eta) \epsilon k c_{S}} \frac{m}{H}\left[\sinh ^{2} \alpha e^{i k c_{S} \eta}-\cosh ^{2} \alpha e^{-i k c_{S} \eta}\right. & \\
\left.+i \sinh 2 \alpha \cos \left(\pi\left(\Lambda+\frac{1}{2}\right)+\delta\right) \sin k c_{S} \eta\right] & \text { for } \alpha \text { vacua Type-I } \\
\frac{2\left|N_{\alpha}\right|^{2}}{M_{p}^{2} \pi} \int_{-\infty}^{0} \mathrm{~d} \eta \frac{\tilde{c}_{S} H}{a(\eta) \epsilon k c_{S}} \frac{m}{H}\left[e^{\alpha+\alpha^{*}} e^{i k c_{S} \eta}-e^{-i k c_{S} \eta}\right. & \\
\left.+i\left(e^{\alpha} e^{i \pi\left(\Lambda+\frac{1}{2}\right)}+e^{\alpha^{*}} e^{-i \pi\left(\Lambda+\frac{1}{2}\right)}\right) \sin k c_{S} \eta\right] & \text { for } \alpha \text { vacua Type-II } \\
\frac{8 i|C|^{2}}{M_{p}^{2} \pi} \int_{-\infty}^{0} \mathrm{~d} \eta \frac{\tilde{c}_{S} H}{a(\eta) \epsilon k c_{S}} \frac{m}{H} \sin k c_{S} \eta \cos ^{2} \frac{\pi}{2}\left(\Lambda+\frac{l}{2}\right) & \text { for special vacua. }\end{cases}
\end{aligned}
$$

On the other hand for $\left|k c_{S} \eta\right| \rightarrow 0$ we get the following simplified expression:

$$
\begin{aligned}
\left\langle\zeta_{\mathbf{k}}(\eta=\xi \rightarrow 0)\right\rangle_{\left|k c_{S} \eta\right| \rightarrow 0}= & \frac{4 \pi \sqrt{\xi}}{M_{p}^{2}}\left(C_{1}^{*} C_{2}+C_{1} C_{2}^{*}-\left|C_{1}\right|^{2}-\left|C_{2}\right|^{2}\right) \int_{-\infty}^{\xi} \mathrm{d} \eta \frac{\tilde{c}_{S}^{2} H}{a(\eta)(2 \pi)^{3} \epsilon \sqrt{-\eta}} \frac{m}{H \tilde{c}_{S}} \\
& \times\left(\Lambda-\frac{1}{2}\right)\left[\left(-\frac{k c_{S} \eta}{2}\right)^{-\Lambda}\left(-\frac{k c_{S} \xi}{2}\right)^{-\Lambda}+\left(\frac{k c_{S} \eta}{2}\right)^{-\Lambda}\left(\frac{k c_{S} \xi}{2}\right)^{-\Lambda}\right] \\
= & \frac{I_{\xi}}{(2 \pi)^{3}} \times\left\{\begin{array}{lr}
-\frac{2 \pi^{2} \sqrt{\xi}}{M_{p}^{2}} & \text { for Bunch Davies vacua, } \\
\frac{4 \pi \sqrt{\xi}}{M_{p}^{2}}\left(\cos \delta \sinh 2 \alpha-\cosh ^{2} \alpha-\sinh ^{2} \alpha\right) & \text { for } \alpha \text { vacua Type-I, } \\
\frac{4 \pi \sqrt{\xi}\left|N_{\alpha}\right|^{2}}{M_{p}^{2}}\left(e^{\alpha}+e^{\alpha^{*}}-e^{\alpha+\alpha^{*}}-1\right) & \text { for } \alpha \text { vacua Type-II, } \\
0 & \text { for special vacua, }
\end{array}\right.
\end{aligned}
$$

where the integral $I_{\xi}$ is defined as

$$
I_{\xi}=\int_{-\infty}^{\xi} \mathrm{d} \eta \frac{\tilde{c}_{S}^{2} H}{a(\eta)(2 \pi)^{3} \epsilon \sqrt{-\eta}} \frac{m}{H \tilde{c}_{S}}\left(\Lambda-\frac{1}{2}\right)\left[\left(\frac{k c_{S} \eta}{2}\right)^{-\Lambda}\left(-\frac{k c_{S} \xi}{2}\right)^{-\Lambda}+\left(\frac{k c_{S} \eta}{2}\right)^{-\Lambda}\left(\frac{k c_{S} \xi}{2}\right)^{-\Lambda}\right] .
$$


Finally on the other hand for $\left|k c_{S} \eta\right| \approx 1$ we get the following simplified expression:

$$
\begin{aligned}
\left\langle\zeta_{\mathbf{k}}(\eta=\xi \rightarrow 0)\right\rangle_{\left|k c_{S} \eta\right| \approx 1}=\frac{4 \pi \sqrt{\xi}}{M_{p}^{2}}\left(C_{1}^{*} C_{2}+C_{1} C_{2}^{*}-\left|C_{1}\right|^{2}-\left|C_{2}\right|^{2}\right) \int_{-\infty}^{\xi} \mathrm{d} \eta \frac{\tilde{c}_{S}^{2} H}{a(\eta)(2 \pi)^{3} \epsilon \sqrt{-\eta}} \frac{m}{H \tilde{c}_{S}} \\
\times\left(\Lambda-\frac{1}{2}\right)\left(\frac{1}{2}\right)^{-\Lambda}\left[\left(-\frac{k c_{S} \xi}{2}\right)^{-\Lambda}+(-1)^{-\Lambda}\left(\frac{k c_{S} \xi}{2}\right)^{-\Lambda}\right] \\
=J_{\xi} \times \begin{cases}\frac{4 \pi \sqrt{\xi}}{M_{p}^{2}}\left(\cos \delta \sinh 2 \alpha-\cosh ^{2} \alpha-\sinh ^{2} \alpha\right) & \text { for } \alpha \text { vacua Type-I, Davies vacua }, \\
\frac{4 \pi \sqrt{\xi}\left|N_{\alpha}\right|^{2}}{M_{p}^{2}}\left(e^{\alpha}+e^{\alpha^{*}}-e^{\alpha+\alpha^{*}}-1\right) & \text { for } \alpha \text { vacua Type-III, } \\
0 \quad \text { for special vacua, }\end{cases}
\end{aligned}
$$

where the integral $J_{\xi}$ is defined as

$J_{\xi}=\int_{-\infty}^{\xi} \mathrm{d} \eta \frac{\tilde{c}_{S}^{2} H}{a(\eta)(2 \pi)^{3} \epsilon \sqrt{-\eta}} \frac{m}{H \tilde{c}_{S}}\left(\Lambda-\frac{1}{2}\right)\left(\frac{1}{2}\right)^{-\Lambda}\left[\left(-\frac{k c_{S} \xi}{2}\right)^{-\Lambda}+(-1)^{-\Lambda}\left(\frac{k c_{S} \xi}{2}\right)^{-\Lambda}\right]$

Now to analyze the behaviour of the expectation value of scalar curvature perturbation in position space we need to take the Fourier transform of the expectation value of the scalar curvature perturbation already computed in momentum space. For the most general solution we get the following result:

$\langle\zeta(\mathbf{x}, \eta=0)\rangle=-i \int \frac{\mathrm{d}^{3} k}{(2 \pi)^{3}} \int_{-\infty}^{0} \mathrm{~d} \eta \frac{a(\eta)}{z^{2} M_{p}^{2}} \frac{m}{\tilde{c}_{S}} e^{i \mathbf{k} \cdot \mathbf{x}}\left(h_{\mathbf{k}}(0) h_{\mathbf{k}}^{\dagger^{\prime}}(\eta)-h_{-\mathbf{k}}^{\dagger}(0) h_{-\mathbf{k}}^{\prime}(\eta)\right)$

where $h_{\mathbf{k}}(\eta)$ is the exact solution of the mode function as explicitly computed in the earlier section of this paper. Following the previous methodology here we also introduce a Infra-Red (IR) cut-off regulator $\xi$ in the conformal time integral and consequently Eq. (3.236) can be recast in the following form:

$\langle\zeta(\mathbf{x}, \eta=0)\rangle=-i \lim _{k c_{S} \xi \rightarrow 0} \int \frac{d^{3} k}{(2 \pi)^{3}} \int_{-\infty}^{\xi} \mathrm{d} \eta \frac{a(\eta)}{z^{2} M_{p}^{2}} \frac{m}{H \tilde{c}_{S}} e^{i \mathbf{k} \cdot \mathbf{x}}\left(h_{\mathbf{k}}(\xi) h_{\mathbf{k}}^{\dagger^{\prime}}(\eta)-h_{-\mathbf{k}}^{\dagger}(\xi) h_{-\mathbf{k}}^{\prime}(\eta)\right)$.

Further substituting the explicit form of the scalar mode functions computed from the exact solution we get the following simplified expression for the one point function of the curvature fluctuation in position space:

$$
\langle\zeta(\mathbf{x}, \eta=0)\rangle=-i \lim _{k c_{S} \xi \rightarrow 0} \int \frac{\mathrm{d}^{3} k}{(2 \pi)^{3}} \int_{-\infty}^{\xi} \mathrm{d} \eta \frac{a(\eta)}{z^{2} M_{p}^{2}} \frac{m}{\tilde{c}_{S}} \sum_{i=1}^{2} \sum_{j=1}^{2} C_{i}^{*} C_{j} \mathcal{A}_{i j} e^{i \mathbf{k} . \mathbf{x}}
$$

where the conformal time-dependent functions $\mathcal{A}_{i j} \forall i, j=1,2$ in momentum space have already been defined earlier.

Similarly in position space the representative expressions for the expectation value of the scalar curvature perturbation along with the three limiting cases $\left|k c_{S} \eta\right| \rightarrow-\infty,\left|k c_{S} \eta\right| \rightarrow 0$ and $\left|k c_{S} \eta\right| \approx 1$ are given by 


$$
\begin{aligned}
& \langle\zeta(\mathbf{x}, \eta=0)\rangle_{\left|k c_{S} \eta\right| \rightarrow-\infty} \approx-\frac{2 H \tilde{c}_{S}}{M_{p}^{2} \epsilon \pi c_{S}}\left[\left|C_{2}\right|^{2} O_{1}-\left|C_{1}\right|^{2} O_{2}-i\left(C_{1}^{*} C_{2} e^{i \pi\left(\Lambda+\frac{1}{2}\right)}+C_{1} C_{2}^{*} e^{-i \pi\left(\Lambda+\frac{1}{2}\right)}\right) O_{3}\right] \\
& \begin{cases}\frac{H \tilde{c}_{S}}{M_{p}^{2} \epsilon c_{S}} O_{2} & \text { for Bunch Davies, } \\
-\frac{2 H \tilde{c}_{S}}{M_{p}^{2} \epsilon \pi c_{S}}\left[\sinh ^{2} \alpha O_{1}-\cosh ^{2} \alpha O_{2}\right. & \\
\left.-i \sinh 2 \alpha \cos \left(\pi\left(\Lambda+\frac{1}{2}\right)+\delta\right) O_{3}\right] & \text { for } \alpha \text { vacua Type-I, } \\
-\frac{2\left|N_{\alpha}\right|^{2} \tilde{c}_{S} H}{M_{p}^{2} \epsilon \pi c_{S}}\left[e^{\alpha+\alpha^{*}} O_{1}-O_{2}\right. & \\
\left.-i\left(e^{\alpha} e^{i \pi\left(\Lambda+\frac{1}{2}\right)}+e^{\alpha^{*}} e^{-i \pi\left(\Lambda+\frac{1}{2}\right)}\right) O_{3}\right] & \text { for } \alpha \text { vacua Type-II, } \\
-\frac{8 i H|C|^{2} \tilde{c}_{S}}{M_{p}^{2} \epsilon \pi c_{S}} \cos ^{2} \frac{\pi}{2}\left(\Lambda+\frac{1}{2}\right) O_{3} & \text { for special vacua, }\end{cases}
\end{aligned}
$$

$\langle\zeta(\mathbf{x}, \eta=\xi \rightarrow 0)\rangle_{\left|k c_{S} \eta\right| \rightarrow 0}=\frac{H \sqrt{\xi} \tilde{c}_{S}}{2 M_{p}^{2} \epsilon \pi^{2}}\left(C_{1}^{*} C_{2}+C_{1} C_{2}^{*}-\left|C_{1}\right|^{2}-\left|C_{2}\right|^{2}\right) O_{4}^{\xi \theta}$

$$
=O_{4}^{\xi \theta} \times \begin{cases}-\frac{2 H \pi^{2} \sqrt{\xi} \tilde{c}_{S}}{M_{p}^{2} \epsilon} & \text { for Bunch Davies vacua } \\ \frac{H \sqrt{\xi} \tilde{c}_{S}}{2 M_{p}^{2} \pi^{2} \epsilon}\left(\cos \delta \sinh 2 \alpha-\cosh ^{2} \alpha-\sinh ^{2} \alpha\right) & \text { for } \alpha \text { vacua Type-I } \\ \frac{H \sqrt{\xi}\left|N_{\alpha}\right|^{2} \tilde{c}_{S}}{2 M_{p}^{2} \pi^{2} \epsilon}\left(e^{\alpha}+e^{\alpha^{*}}-e^{\alpha+\alpha^{*}}-1\right) & \text { for } \alpha \text { vacua Type-III } \\ 0 & \text { for special vacua }\end{cases}
$$

$\langle\zeta(\mathbf{x}, \eta=\xi \rightarrow 0)\rangle_{\left|k c_{S} \eta\right| \approx 1}=\frac{H \sqrt{\xi} \tilde{c}_{S}}{2 M_{p}^{2} \epsilon \pi^{2}}\left(C_{1}^{*} C_{2}+C_{1} C_{2}^{*}-\left|C_{1}\right|^{2}-\left|C_{2}\right|^{2}\right) O_{5}^{\xi \theta}$

$$
=O_{5}^{\xi \theta} \times \begin{cases}-\frac{2 H \pi^{2} \sqrt{\xi} \tilde{c}_{S}}{M_{p}^{2} \epsilon} & \text { for Bunch Davies vacua, } \\ \frac{H \sqrt{\xi} \tilde{c}_{S}}{2 M_{p}^{2} \pi^{2} \epsilon}\left(\cos \delta \sinh 2 \alpha-\cosh ^{2} \alpha-\sinh ^{2} \alpha\right) & \text { for } \alpha \text { vacua Type-I, } \\ \frac{H \sqrt{\xi}\left|N_{\alpha}\right|^{2} \tilde{c}_{S}}{2 M_{p}^{2} \pi^{2} \epsilon}\left(e^{\alpha}+e^{\alpha^{*}}-e^{\alpha+\alpha^{*}}-1\right) & \text { for } \alpha \text { vacua Type-II }, \\ 0 & \text { for special vacua, }\end{cases}
$$

where we introduce the following integrals $O_{1}, O_{2}, O_{3}, O_{4}^{\xi \theta}, O_{5}^{\xi \theta}$ are given by

$O_{1}=\int \frac{d^{3} k}{(2 \pi)^{3}} e^{i \mathbf{k} \cdot \mathbf{x}} \int_{-\infty}^{0} \mathrm{~d} \eta \frac{\eta}{k} m(\eta) e^{-i k c_{S} \eta}$ 


$$
\begin{aligned}
O_{2}= & \int \frac{\mathrm{d}^{3} k}{(2 \pi)^{3}} e^{i \mathbf{k} \cdot \mathbf{x}} \int_{-\infty}^{0} \mathrm{~d} \eta \frac{\eta}{k} m(\eta) e^{i k c_{S} \eta} \\
O_{3}= & \int \frac{\mathrm{d}^{3} k}{(2 \pi)^{3}} e^{i \mathbf{k} \cdot \mathbf{x}} \int_{-\infty}^{0} \mathrm{~d} \eta \frac{\eta}{k} m(\eta) \sin k c_{S} \eta \\
O_{4}^{\xi \theta}= & \int \frac{\mathrm{d}^{3} k}{(2 \pi)^{3}} e^{i \mathbf{k} \cdot \mathbf{x}} \int_{-\infty}^{\xi} \mathrm{d} \eta \frac{1}{a(\eta) \sqrt{-\eta}} \frac{m}{H}\left(\Lambda-\frac{1}{2}\right) \\
& \times\left[\left(-\frac{k c_{S} \eta}{2}\right)^{-\Lambda}\left(-\frac{k c_{S} \xi}{2}\right)^{-\Lambda}\right. \\
& \left.+\left(\frac{k c_{S} \eta}{2}\right)^{-\Lambda}\left(\frac{k c_{S} \xi}{2}\right)^{-\Lambda}\right], \\
O_{5}^{\xi \theta}= & \int \frac{\mathrm{d}^{3} k}{(2 \pi)^{3}} e^{i \mathbf{k} \cdot \mathbf{x}} \int_{-\infty}^{\xi} \mathrm{d} \eta \frac{1}{a(\eta) \sqrt{-\eta}} \frac{m}{H}\left(\Lambda-\frac{1}{2}\right)\left(\frac{1}{2}\right)^{-\Lambda} \\
& \times\left[\left(-\frac{k c_{S} \xi}{2}\right)^{-\Lambda}+(-1)^{-\Lambda}\left(\frac{k c_{S} \xi}{2}\right)^{-\Lambda}\right]
\end{aligned}
$$

Now to compute these momentum integrals we have the following sets of assumptions:

1. We choose spherical polar coordinate for the computation of momentum volume integral.

2. We take two situations where $\mathbf{k}$ and $\mathbf{x}$ are parallel and having an angle $\Theta$ in between them. For the first case

$\mathbf{k} \cdot \mathbf{x}=k x$

and for the second case we have

$\mathbf{k} \cdot \mathbf{x}=k x \cos \Theta$,

where the range of the angular parameter is lying within the window $\Theta_{1} \leq \Theta \leq \Theta_{2}$, where $\Theta_{1}$ and $\Theta_{2}$ are two cut-offs in the angular coordinate which are introduced to regularize the momentum integrals in the present context. Additionally it is also important to note that for the first case the volume element for the momentum integration is given by $\mathrm{d}^{3} k=4 \pi k^{2} d k$ and for the second case the volume element for the momentum integration is considered as $\mathrm{d}^{3} k=k^{2} \sin \Theta \mathrm{d} k \mathrm{~d} \Theta \mathrm{d} \phi$, where $\phi$ is called the azimuthal coordinate; it is lying within the window $0<\phi<2 \pi$.

3. Last but not the least, to perform volume integration in momentum space for the two point function we need to introduce a momentum IR cut-off at

$$
k_{I R}=\frac{1}{L_{I R}} .
$$

For Case I and Case II we get the following results:

\section{For Case I}

$$
\begin{aligned}
O_{1} & =\frac{1}{2 \pi^{2}} \int_{0}^{\infty} \mathrm{d} k \int_{-\infty}^{0} \mathrm{~d} \eta \eta k m(\eta) e^{i k\left(x-c_{S} \eta\right)} \\
& =-\frac{i}{2 \pi^{2} c_{S}^{2}} m\left(\eta=-|\mathbf{x}| / c_{S}\right), \\
O_{2} & =\frac{1}{2 \pi^{2}} \int_{0}^{\infty} \mathrm{d} k \int_{-\infty}^{0} \mathrm{~d} \eta \eta k m(\eta) e^{i k\left(x+c_{S} \eta\right)} \\
& =\frac{i}{2 \pi^{2} c_{S}^{2}} m\left(\eta=-|\mathbf{x}| / c_{S}\right), \\
O_{3} & =\frac{1}{2 \pi^{2}} \int_{0}^{\infty} \mathrm{d} k \int_{-\infty}^{0} \mathrm{~d} \eta \eta k m(\eta) e^{i k x} \sin k c_{S} \eta \\
& =\frac{1}{2 \pi^{2} c_{S}^{2}} m\left(\eta=-|\mathbf{x}| / c_{S}\right),
\end{aligned}
$$

$$
\begin{aligned}
O_{4}^{\xi \theta}= & \frac{1}{2 \pi^{2}} \int_{0}^{\infty} \mathrm{d} k k^{2} e^{i k x} \int_{-\infty}^{\xi} \mathrm{d} \eta \frac{\tilde{c}_{S}^{2} H}{a(\eta) \epsilon \sqrt{-\eta}} \frac{m}{H \tilde{c}_{S}} \\
& \times\left(\Lambda-\frac{1}{2}\right)\left[\left(-\frac{k c_{S} \eta}{2}\right)^{-\Lambda}\left(-\frac{k c_{S} \xi}{2}\right)^{-\Lambda}\right. \\
& \left.+\left(\frac{k c_{S} \eta}{2}\right)^{-\Lambda}\left(\frac{k c_{S} \xi}{2}\right)^{-\Lambda}\right]
\end{aligned}
$$

$$
O_{5}^{\xi \theta}=\frac{1}{2 \pi^{2}} \int_{0}^{\infty} \mathrm{d} k e^{i k x} k^{2-\Lambda}
$$

$$
\begin{aligned}
& \times \int_{-\infty}^{\xi} \mathrm{d} \eta \frac{\tilde{c}_{S}^{2} H}{a(\eta) \epsilon \sqrt{-\eta}} \frac{m}{H \tilde{c}_{S}}\left(\Lambda-\frac{1}{2}\right)\left(\frac{1}{2}\right)^{-\Lambda} \\
& \times\left[\left(-\frac{c_{S} \xi}{2}\right)^{-\Lambda}+(-1)^{-\Lambda}\left(\frac{c_{S} \xi}{2}\right)^{-\Lambda}\right] .
\end{aligned}
$$

For Case II

$$
\begin{aligned}
O_{1} & =\frac{1}{4 \pi^{2}} \int_{0}^{\infty} \mathrm{d} k \int_{-\infty}^{0} \mathrm{~d} \eta \int_{\Theta_{1}}^{\Theta_{2}} \mathrm{~d} \Theta \eta k m(\eta) e^{i k(x \cos \Theta-c s \eta)} \\
& =-\int_{\Theta_{1}}^{\Theta_{2}} \mathrm{~d} \Theta \frac{i m\left(\eta=-\frac{|\mathbf{x}| \cos \Theta}{c_{S}}\right)}{4 \pi^{2} c_{S}^{2}} \cos \Theta \\
O_{2} & =\frac{1}{4 \pi^{2}} \int_{0}^{\infty} \mathrm{d} k \int_{-\infty}^{0} \mathrm{~d} \eta \int_{\Theta_{1}}^{\Theta_{2}} \mathrm{~d} \Theta \eta k m(\eta) e^{i k(x \cos \Theta+c s \eta)} \\
& =\int_{\Theta_{1}}^{\Theta_{2}} \mathrm{~d} \Theta \frac{i m\left(\eta=-\frac{|\mathbf{x}| \cos \Theta}{c_{S}}\right)}{4 \pi^{2} c_{S}^{2}} \cos \Theta
\end{aligned}
$$




$$
\begin{aligned}
O_{3}= & \frac{1}{4 \pi^{2}} \int_{0}^{\infty} \mathrm{d} k \int_{-\infty}^{0} \mathrm{~d} \eta \int_{\Theta_{1}}^{\Theta_{2}} \mathrm{~d} \Theta \eta k m(\eta) e^{i k x \cos \Theta} \sin k c_{S} \eta \\
= & \int_{\Theta_{1}}^{\Theta_{2}} \mathrm{~d} \Theta \frac{m\left(\eta=-\frac{|\mathbf{x}| \cos \Theta}{c_{S}}\right)}{4 \pi^{2} c_{S}^{2}} \cos \Theta, \\
O_{4}^{\xi \theta}= & \frac{1}{4 \pi^{2}} \int_{0}^{\infty} \mathrm{d} k \int_{-\infty}^{\xi} \mathrm{d} \eta \int_{\Theta_{1}}^{\Theta_{2}} \mathrm{~d} \Theta k^{2} e^{i k x \cos \Theta} \\
& \times \frac{\tilde{c}_{S}^{2} H}{a(\eta) \epsilon \sqrt{-\eta}} \frac{m}{H \tilde{c}_{S}}\left(\Lambda-\frac{1}{2}\right) \\
& \times\left[\left(-\frac{k c_{S} \eta}{2}\right)^{-\Lambda}\left(-\frac{k c_{S} \xi}{2}\right)\right. \\
& \left.+\left(\frac{k c_{S} \eta}{2}\right)^{-\Lambda}\left(\frac{k c_{S} \xi}{2}\right)^{-\Lambda}\right], \\
O_{5}^{\xi \theta}= & \frac{1}{4 \pi^{2}} \int_{0}^{\infty} \mathrm{d} k \int_{\Theta_{1}}^{\Theta_{2}} \mathrm{~d} \Theta e^{i k x} \cos \Theta k^{2-\Lambda} \\
& \times \int_{-\infty}^{\xi} \mathrm{d} \eta \frac{\tilde{c}_{S}^{2} H}{a(\eta) \epsilon \sqrt{-\eta}} \frac{m}{H \tilde{c}_{S}}\left(\Lambda-\frac{1}{2}\right)\left(\frac{1}{2}\right)^{-\Lambda} \\
& \times\left[\left(-\frac{c_{S} \xi}{2}\right)^{-\Lambda}+(-1)^{-\Lambda}\left(\frac{c_{S} \xi}{2}\right)^{-\Lambda}\right]
\end{aligned}
$$

where $\Theta_{1}$ and $\Theta_{2}$ play the role of angular regulator in the present context.

Now our objective is to compute the expression for the two point correlation function from scalar curvature perturbation. Following the previously mentioned computational technique of the in-in formalism, which is commonly known as the Swinger-Keyldish formalism, here we compute the expression for the two point correlation function from scalar curvature perturbation. Using the interaction picture the two point correlation function of the curvature fluctuation in momentum space can be expressed as

$$
\left\langle\zeta_{\mathbf{k}}(\eta) \zeta_{\mathbf{q}}(\eta)\right\rangle=(2 \pi)^{3} \delta^{3}(\mathbf{k}+\mathbf{q}) \frac{2 \pi^{2}}{k^{3}} \Delta_{\zeta}(k),
$$

where the primordial power spectrum for the scalar mode at any arbitrary momentum scale can be written as

$$
\begin{aligned}
\Delta_{\zeta}(k) & =\frac{k^{3}\left|h_{\mathbf{k}}\right|^{2}}{2 \pi^{2} z^{2} M_{p}^{2}} \\
& =\frac{\left(-k \eta \tilde{c}_{S}\right)^{3} H^{2}}{4 \tilde{c}_{S} \in \pi^{2} M_{p}^{2}} \sum_{i=1}^{2} \sum_{j=1}^{2} C_{i}^{*} C_{j} U_{i j}(\eta, k),
\end{aligned}
$$

where $U_{i j} \forall i, j=1,2$ are defined as

$$
\begin{aligned}
& U_{11}(\eta, k)=H_{\Lambda}^{(1) *}\left(-k c_{S} \eta\right) H_{\Lambda}^{(1)}\left(-k c_{S} \eta\right), \\
& U_{12}(\eta, k)=H_{\Lambda}^{(1) *}\left(-k c_{S} \eta\right) H_{\Lambda}^{(2)}\left(-k c_{S} \eta\right), \\
& U_{21}(\eta, k)=H_{\Lambda}^{(2) *}\left(-k c_{S} \eta\right) H_{\Lambda}^{(1)}\left(-k c_{S} \eta\right), \\
& U_{22}(\eta, k)=H_{\Lambda}^{(2) *}\left(-k c_{S} \eta\right) H_{\Lambda}^{(2)}\left(-k c_{S} \eta\right) .
\end{aligned}
$$

For further simplification we consider here three limiting cases $\left|k c_{S} \eta\right| \rightarrow-\infty,\left|k c_{S} \eta\right| \rightarrow 0$ and $\left|k c_{S} \eta\right| \approx 1$, which are physically acceptable in the present context. First of we consider the results for $\left|k c_{S} \eta\right| \rightarrow-\infty$. In this case we get

$$
\begin{aligned}
& \left\langle\zeta_{\mathbf{k}}(\eta) \zeta_{\mathbf{q}}(\eta)\right\rangle_{\left|k c_{S} \eta\right| \rightarrow-\infty} \\
& =(2 \pi)^{3} \delta^{3}(\mathbf{k}+\mathbf{q}) \frac{2 \pi^{2}}{k^{3}}\left[\Delta_{\zeta}(k)\right]_{\left|k c_{S} \eta\right| \rightarrow-\infty},
\end{aligned}
$$

where the primordial power spectrum for the scalar mode at $\left|k c_{S} \eta\right| \rightarrow-\infty$ can be written as

$$
\begin{aligned}
& {\left[\Delta_{\zeta}(k)\right]_{\left|k c_{S} \eta\right| \rightarrow-\infty} \approx \frac{H^{2}}{2 \epsilon M_{p}^{2}} \frac{k^{2} \eta^{2} \tilde{c}_{S}^{2}}{c_{S} \pi^{3}}\left[\left|C_{2}\right|^{2}+\left|C_{1}\right|^{2}+\left(C_{1}^{*} C_{2} e^{2 i k c_{S} \eta} e^{i \pi\left(\Lambda+\frac{1}{2}\right)}+C_{1} C_{2}^{*} e^{-2 i k c_{S} \eta} e^{-i \pi\left(\Lambda+\frac{1}{2}\right)}\right)\right]} \\
& = \begin{cases}\frac{H^{2}}{2 \epsilon M_{p}^{2} c_{S}} \frac{\left(-k \eta \tilde{c}_{S}\right)^{2}}{2 \pi^{2}} & \text { for Bunch Davies, } \\
\frac{H^{2}}{2 \epsilon M_{p}^{2} c_{S}} \frac{\left(-k \tilde{c}_{S} \eta\right)^{2}}{\pi^{3}}\left[\sinh ^{2} \alpha+\cosh ^{2} \alpha\right. & \\
\left.+\sinh 2 \alpha \cos \left(2 k c_{S} \eta+\pi\left(\Lambda+\frac{l}{2}\right)+\delta\right)\right] & \text { for } \alpha \text { vacua Type-I, } \\
\frac{H^{2}}{2 \epsilon M_{p}^{2} c_{S}} \frac{4\left(-k \tilde{c}_{S} \eta\right)^{2}\left|N_{\alpha}\right|^{2}}{\pi^{3}} \cos ^{2}\left(k c_{S} \eta+\frac{\pi}{2}\left(\Lambda+\frac{l}{2}\right)-i \frac{\alpha}{2}\right) & \text { for } \alpha \text { vacua Type-II, } \\
\frac{H^{2}}{2 \epsilon M_{p}^{2} c_{S}} \frac{4\left(-k \tilde{c}_{S} \eta\right)^{2}}{\pi^{3}} \cos ^{2}\left(k c_{S} \eta+\frac{\pi}{2}\left(\Lambda+\frac{l}{2}\right)\right) & \text { for special vacua. }\end{cases}
\end{aligned}
$$


Next we consider the results for $\left|k c_{S} \eta\right| \rightarrow 0$. In this case we get

$\left\langle\zeta_{\mathbf{k}}(\eta) \zeta_{\mathbf{q}}(\eta)\right\rangle_{\left|k c_{S} \eta\right| \rightarrow 0}=(2 \pi)^{3} \delta^{3}(\mathbf{k}+\mathbf{q}) \frac{2 \pi^{2}}{k^{3}}\left[\Delta_{\zeta}(k)\right]_{\left|k c_{S} \eta\right| \rightarrow 0}$,

where the primordial power spectrum for the scalar mode at $\left|k c_{S} \eta\right| \rightarrow 0$ can be written as

$$
\begin{aligned}
{\left[\Delta_{\zeta}(k)\right]_{\left|k c_{S} \eta\right| \rightarrow 0} \approx } & \frac{H^{2}}{2 \epsilon M_{p}^{2}} \frac{\left(-k \eta \tilde{c}_{S}\right)^{3}}{2 \tilde{c}_{S} \pi^{4}} \Gamma^{2}(\Lambda)\left(-\frac{k c_{S} \eta}{2}\right)^{-2 \Lambda}\left[\left|C_{2}\right|^{2}+\left|C_{1}\right|^{2}-\left(C_{1}^{*} C_{2}+C_{1} C_{2}^{*}\right)\right] \\
& = \begin{cases}\frac{H^{2}}{2 \epsilon M_{p}^{2}} \frac{\left(-k \eta c_{S}\right)^{3-2 \Lambda}}{2^{2(2-\Lambda)} \pi^{2}} \frac{\tilde{c}_{S}^{2}}{c_{S}^{3}}\left|\frac{\Gamma(\Lambda)}{\Gamma\left(\frac{3}{2}\right)}\right|^{2} & \text { for Bunch Davies, } \\
\frac{H^{2}}{2 \epsilon M_{p}^{2}} \frac{\left(-k \eta c_{S}\right)^{3-2 \Lambda}}{2^{(3-2 \Lambda)} \pi^{3}} \frac{\tilde{c}_{S}^{2}}{c_{S}^{3}}\left|\frac{\Gamma(\Lambda)}{\Gamma\left(\frac{3}{2}\right)}\right|^{2}\left[\sinh ^{2} \alpha+\cosh ^{2} \alpha-\sinh 2 \alpha \cos \delta\right] & \text { for } \alpha \text { vacua Type-I, } \\
\frac{H^{2}}{2 \epsilon M_{p}^{2}} \frac{\left(-k \eta c_{S}\right)^{3-2 \Lambda}\left|N_{\alpha}\right|^{2}}{2^{(1-2 \Lambda)} \pi^{3}} \frac{\tilde{c}_{S}^{2}}{c_{S}^{3}}\left|\frac{\Gamma(\Lambda)}{\Gamma\left(\frac{3}{2}\right)}\right|^{2} \sin ^{2} \frac{\alpha}{2} & \text { for } \alpha \text { vacua Type-II, } \\
0 & \text { for special vacua. }\end{cases}
\end{aligned}
$$

Finally we consider the results for $\left|k c_{S} \eta\right| \approx 1$. In this case we get

$$
\left\langle\zeta_{\mathbf{k}}(\eta=0) \zeta_{\mathbf{q}}(\eta=0)\right\rangle_{\left|k c_{S} \eta\right| \approx 1}=(2 \pi)^{3} \delta^{3}(\mathbf{k}+\mathbf{q}) \frac{2 \pi^{2}}{k^{3}}\left[\Delta_{\zeta}(k)\right]_{\left|k c_{S} \eta\right| \approx 1},
$$

where the primordial power spectrum for the scalar mode at $\left|k c_{S} \eta\right| \approx 1$ can be written as

$$
\begin{aligned}
{\left[\Delta_{\zeta}(k)\right]_{\left|k c_{S} \eta\right| \approx 1} \approx } & \frac{H^{2}}{2 \epsilon M_{p}^{2}} \frac{1}{2^{2(1-\Lambda)} \pi^{3}} \frac{\tilde{c}_{S}^{2}}{c_{S}^{3}}\left|\frac{\Gamma(\Lambda)}{\Gamma\left(\frac{3}{2}\right)}\right|^{2}\left[\left|C_{2}\right|^{2}+\left|C_{1}\right|^{2}-\left(C_{1}^{*} C_{2}+C_{1} C_{2}^{*}\right)\right] \\
& = \begin{cases}\frac{H^{2}}{2 \epsilon M_{p}^{2}} \frac{1}{2^{2(2-\Lambda)} \pi^{2}} \frac{\tilde{c}_{S}^{2}}{c_{S}^{3}}\left|\frac{\Gamma(\Lambda)}{\Gamma\left(\frac{3}{2}\right)}\right|^{2} & \text { for Bunch Davies, } \\
\frac{H^{2}}{2 \epsilon M_{p}^{2}} \frac{1}{2^{(3-2 \Lambda)} \pi^{3}} \frac{\tilde{c}_{S}^{2}}{c_{S}^{3}}\left|\frac{\Gamma(\Lambda)}{\Gamma\left(\frac{3}{2}\right)}\right|^{2}\left[\sinh ^{2} \alpha+\cosh ^{2} \alpha-\sinh 2 \alpha \cos \delta\right] & \text { for } \alpha \text { vacua Type-I, } \\
\frac{H^{2}}{2 \epsilon M_{p}^{2}} \frac{\left|N_{\alpha}\right|^{2}}{2^{(1-2 \Lambda)} \pi^{3}} \frac{\tilde{c}_{S}^{2}}{c_{S}^{3}}\left|\frac{\Gamma(\Lambda)}{\Gamma\left(\frac{3}{2}\right)}\right|^{2} \sin ^{2} \frac{\alpha}{2} & \text { for } \alpha \text { vacua Type-II, } \\
0 & \text { for special vacua. }\end{cases}
\end{aligned}
$$

Now to analyze the behaviour of the two point correlation function of the scalar curvature perturbation in position space we need to take the Fourier transform of the two point correlation function of the scalar curvature perturbation already computed in momentum space. For the most general solution we get the following result:

$\langle\zeta(\mathbf{x}, \eta) \zeta(\mathbf{y}, \eta)\rangle=\int \frac{\mathrm{d}^{3} k}{(2 \pi)^{3}} e^{i \mathbf{k} .(\mathbf{x}-\mathbf{y})} \frac{\left(-\eta \tilde{c}_{S}\right)^{3}}{2 \tilde{c}_{S} \in M_{p}^{2}} \sum_{i=1}^{2} \sum_{j=1}^{2} C_{i}^{*} C_{j} U_{i j}$,

where the conformal time-dependent functions $U_{i j} \forall i, j=1,2$ in momentum space have already been defined earlier.

Similarly in position space the representative expressions for the expectation value of the scalar curvature perturbation along with limiting case $\left|k c_{S} \eta\right| \rightarrow-\infty$ is given by 
$\langle\zeta(\mathbf{x}, \eta) \zeta(\mathbf{y}, \eta)\rangle_{\left|k c_{S} \eta\right| \rightarrow-\infty} \approx \frac{1}{4 \pi^{4}} \frac{H^{2}}{2 \epsilon M_{p}^{2}} \frac{\eta^{2} \tilde{c}_{S}^{2}}{c_{S}}\left[\left(\left|C_{2}\right|^{2}+\left|C_{1}\right|^{2}\right) J_{1}+\left(C_{1}^{*} C_{2} e^{i \pi\left(\Lambda+\frac{1}{2}\right)} J_{2}+C_{1} C_{2}^{*} e^{-i \pi\left(\Lambda+\frac{1}{2}\right)} J_{3}\right)\right]$

$$
= \begin{cases}\frac{H^{2}}{2 \epsilon M_{p}^{2} c_{S}} \frac{\left(\eta \tilde{c}_{S}\right)^{2}}{(2 \pi)^{3}} J_{1} & \text { for Bunch Davies, } \\ \frac{H^{2}}{2 \epsilon M_{p}^{2} c_{S}} \frac{\left(\tilde{c}_{S} \eta\right)^{2}}{4 \pi^{4}}\left[\left(\sinh ^{2} \alpha+\cosh ^{2} \alpha\right) J_{1}\right. & \\ \left.\quad+\frac{1}{2} \sinh 2 \alpha\left(e^{i\left(\pi\left(\Lambda+\frac{1}{2}\right)+\delta\right)} J_{2}+e^{-i\left(\pi\left(\Lambda+\frac{1}{2}\right)+\delta\right)}\right) J_{3}\right] & \text { for } \alpha \text { vacua Type-I, } \\ \frac{H^{2}}{2 \epsilon M_{p}^{2} c_{S}} \frac{\left(\tilde{c}_{S} \eta\right)^{2}\left|N_{\alpha}\right|^{2}}{2 \pi^{4}}\left[J_{1}+\left(e^{i \pi\left(\Lambda+\frac{1}{2}\right)} e^{\alpha} J_{2}+e^{-i \pi\left(\Lambda+\frac{1}{2}\right)} e^{-\alpha} J_{3}\right)\right] & \text { for } \alpha \text { vacua Type-II }, \\ \frac{H^{2}}{2 \epsilon M_{p}^{2} c_{S}} \frac{\left(\tilde{c}_{S} \eta\right)^{2}}{2 \pi^{4}}\left[J_{1}+\left(e^{i \pi\left(\Lambda+\frac{1}{2}\right)} e^{\alpha} J_{2}+e^{-i \pi\left(\Lambda+\frac{1}{2}\right)} e^{-\alpha} J_{3}\right)\right] & \text { for special vacua, }\end{cases}
$$

where the momentum integrals $J_{1}, J_{2}$ and $J_{3}$ are defined in the following:

$$
\begin{aligned}
& J_{1}=\int \mathrm{d}^{3} k \frac{e^{i \mathbf{k} \cdot(\mathbf{x}-\mathbf{y})}}{k}, \\
& J_{2}=\int \mathrm{d}^{3} k \frac{e^{i \mathbf{k} .(\mathbf{x}-\mathbf{y})}}{k} e^{2 i k c_{S} \eta}, \\
& J_{3}=\int \mathrm{d}^{3} k \frac{e^{i \mathbf{k} \cdot(\mathbf{x}-\mathbf{y})}}{k} e^{-2 i k c_{S} \eta} .
\end{aligned}
$$

To compute this integrals we follow the same assumptions as mentioned for the computation of VEV of curvature perturbation in position space. Here we have the following results:

For Case I

$$
\begin{aligned}
& J_{1}=\int_{0}^{\infty} \mathrm{d} k k e^{i k(|\mathbf{x}-\mathbf{y}|)}=-\frac{4 \pi}{|\mathbf{x}-\mathbf{y}|^{2}}, \\
& J_{2}=\int_{0}^{\infty} \mathrm{d} k k e^{i k\left(|\mathbf{x}-\mathbf{y}|+2 c_{S} \eta\right)}=-\frac{4 \pi}{\left(|\mathbf{x}-\mathbf{y}|+2 c_{S} \eta\right)^{2}}, \\
& J_{3}=\int_{0}^{\infty} \mathrm{d} k k e^{i k\left(|\mathbf{x}-\mathbf{y}|-2 c_{S} \eta\right)}=-\frac{4 \pi}{\left(|\mathbf{x}-\mathbf{y}|-2 c_{S} \eta\right)^{2}} .
\end{aligned}
$$

For Case II

$$
\begin{aligned}
J_{1}= & \int_{0}^{\infty} \mathrm{d} k \int_{\Theta_{1}}^{\Theta_{2}} \mathrm{~d} \Theta k e^{i k x \cos \Theta}=\frac{2 \pi\left(\tan \Theta_{1}-\tan \Theta_{2}\right)}{|\mathbf{x}-\mathbf{y}|^{2}}, \\
J_{2}= & \int_{0}^{\infty} \mathrm{d} k \int_{\Theta_{1}}^{\Theta_{2}} \mathrm{~d} \Theta k e^{i k(x \cos \Theta+2 c s \eta)} \\
= & \frac{2 \pi|\mathbf{x}-\mathbf{y}|}{\left(|\mathbf{x}-\mathbf{y}|^{2}-4 c_{S}^{2} \eta^{2}\right)}\left\{\left[\frac{\sin \Theta_{1}}{\left(|\mathbf{x}-\mathbf{y}| \cos \Theta_{1}+2 c_{S} \eta\right)}-\frac{\sin \Theta_{2}}{\left(|\mathbf{x}-\mathbf{y}| \cos \Theta_{2}+2 c_{S} \eta\right)}\right]\right. \\
& \left.+\frac{4 c_{S} \eta}{\sqrt{|\mathbf{x}-\mathbf{y}|^{2}-4 c_{S}^{2} \eta^{2}}}\left[\tanh ^{-1}\left(\frac{\left(2 c_{S} \eta-|\mathbf{x}-\mathbf{y}|\right) \tan \frac{\Theta_{1}}{2}}{\sqrt{|\mathbf{x}-\mathbf{y}|^{2}-4 c_{S}^{2} \eta^{2}}}\right)-\tanh ^{-1}\left(\frac{\left(2 c_{S} \eta-|\mathbf{x}-\mathbf{y}|\right) \tan \frac{\Theta_{2}}{2}}{\sqrt{|\mathbf{x}-\mathbf{y}|^{2}-4 c_{S}^{2} \eta^{2}}}\right)\right]\right\},
\end{aligned}
$$




$$
\begin{aligned}
J_{3}= & \int_{0}^{\infty} \mathrm{d} k \int_{\Theta_{1}}^{\Theta_{2}} \mathrm{~d} \Theta k e^{i k\left(x \cos \Theta-2 c_{S} \eta\right)} \\
= & \frac{2 \pi|\mathbf{x}-\mathbf{y}|}{\left(|\mathbf{x}-\mathbf{y}|^{2}-4 c_{S}^{2} \eta^{2}\right)}\left\{\left[\frac{\sin \Theta_{1}}{\left(|\mathbf{x}-\mathbf{y}| \cos \Theta_{1}-2 c_{S} \eta\right)}-\frac{\sin \Theta_{2}}{\left(|\mathbf{x}-\mathbf{y}| \cos \Theta_{2}-2 c_{S} \eta\right)}\right]\right. \\
& \left.+\frac{4 c_{S} \eta}{\sqrt{|\mathbf{x}-\mathbf{y}|^{2}-4 c_{S}^{2} \eta^{2}}}\left[\tanh ^{-1}\left(\frac{\left(2 c_{S} \eta+|\mathbf{x}-\mathbf{y}|\right) \tan \frac{\Theta_{1}}{2}}{\sqrt{|\mathbf{x}-\mathbf{y}|^{2}-4 c_{S}^{2} \eta^{2}}}\right)-\tanh ^{-1}\left(\frac{\left(2 c_{S} \eta+|\mathbf{x}-\mathbf{y}|\right) \tan \frac{\Theta_{2}}{2}}{\sqrt{|\mathbf{x}-\mathbf{y}|^{2}-4 c_{S}^{2} \eta^{2}}}\right)\right]\right\} .
\end{aligned}
$$

Here by setting $\mathbf{y}=0$ one can derive the results for $\langle\zeta(\mathbf{x}, \eta) \zeta(0, \eta)\rangle$ at conformal time scale $\eta$ with $\left|k c_{S} \eta\right| \rightarrow-\infty$. Next we consider the results for $\left|k c_{S} \eta\right| \rightarrow 0$. In this case we get

$$
\begin{aligned}
&\langle\zeta(\mathbf{x}, \eta) \zeta(\mathbf{y}, \eta)\rangle_{|k c s \eta| \rightarrow 0} \approx \frac{H^{2}}{2 \epsilon M_{p}^{2}} \frac{\left(-\eta c_{S}\right)^{3-2 \Lambda}}{2^{2(2-\Lambda)} \pi^{4}} \frac{\tilde{c}_{S}^{2}}{c_{S}^{3}}\left|\frac{\Gamma(\Lambda)}{\Gamma\left(\frac{3}{2}\right)}\right|^{2}\left[\left(\left|C_{2}\right|^{2}+\left|C_{1}\right|^{2}\right)-\left(C_{1}^{*} C_{2}+C_{1} C_{2}^{*}\right)\right] K_{I} \\
&=K_{I} \times \begin{cases}\frac{H^{2}}{2 \epsilon M_{p}^{2}} \frac{\left(-\eta c_{S}\right)^{3-2 \Lambda}}{2^{2(3-\Lambda)} \pi^{3}} \frac{\tilde{c}_{S}^{2}}{c_{S}^{3}}\left|\frac{\Gamma(\Lambda)}{\Gamma\left(\frac{3}{2}\right)}\right|^{2} & \text { for Bunch Davies, } \\
\frac{H^{2}}{2 \epsilon M_{p}^{2}} \frac{\left(-\eta c_{S}\right)^{3-2 \Lambda}}{2^{(5-2 \Lambda)} \pi^{4}} \frac{\tilde{c}_{S}^{2}}{c_{S}^{3}}\left|\frac{\Gamma(\Lambda)}{\Gamma\left(\frac{3}{2}\right)}\right|^{2}\left[\sinh ^{2} \alpha+\cosh ^{2} \alpha\right. & \text { for } \alpha \text { vacua Type-I, } \\
-\sinh \frac{2 \alpha \cos \delta]}{2 \epsilon M_{p}^{2}} \frac{\left(-\eta c_{S}\right)^{3-2 \Lambda}\left|N_{\alpha}\right|^{2}}{2^{(3-2 \Lambda)} \pi^{4}} \frac{\tilde{c}_{S}^{2}}{c_{S}^{3}}\left|\frac{\Gamma(\Lambda)}{\Gamma\left(\frac{3}{2}\right)}\right|^{2} \sin ^{2} \frac{\alpha}{2} & \text { for } \alpha \text { vacua Type-II, } \\
0 & \text { for special vacua, }\end{cases}
\end{aligned}
$$

where the momentum integrals $K_{I}$ are defined in the following:

$K_{I}=\int \mathrm{d}^{3} k k^{1-2 \Lambda} e^{i \mathbf{k} \cdot(\mathbf{x}-\mathbf{y})}$.

To compute this integral we follow the same assumptions as mentioned for the computation of VEV of curvature perturbation in position space. Here we have the following results:

For Case I: $\quad K_{I}=4 \pi \int_{0}^{\infty} \mathrm{d} k k^{3-2 \Lambda} e^{i k|\mathbf{x}-\mathbf{y}|}=4 \pi\left(\frac{i}{|\mathbf{x}-\mathbf{y}|}\right)^{3-2 \Lambda} \Gamma(3-2 \Lambda)$.

For Case II: $\quad K_{I}=2 \pi \int_{0}^{\infty} \mathrm{d} k \int_{\Theta_{2}}^{\Theta_{2}} \mathrm{~d} \Theta k^{3-2 \Lambda} e^{i k|\mathbf{x}-\mathbf{y}| \cos \Theta}$

$$
\begin{aligned}
=2 \pi & \frac{\Gamma(4-2 \Lambda)(-i)^{2 \Lambda}}{(3-2 \Lambda)|\mathbf{x}-\mathbf{y}|^{4-2 \Lambda}}\left[\left(\sec \Theta_{1}\right)^{3-2 \Lambda}{ }_{2} F_{1}\left(\frac{1}{2}, \Lambda-\frac{3}{2} ; \Lambda-\frac{1}{2} ; \cos ^{2} \Theta_{1}\right)\right. \\
& \left.-\left(\sec \Theta_{2}\right)^{3-2 \Lambda}{ }_{2} F_{1}\left(\frac{1}{2}, \Lambda-\frac{3}{2} ; \Lambda-\frac{1}{2} ; \cos ^{2} \Theta_{2}\right)\right] .
\end{aligned}
$$

Here by setting $\mathbf{y}=0$ one can derive the results for $\langle\zeta(\mathbf{x}, \eta) \zeta(0, \eta)\rangle$ at conformal time scale $\eta$ with $\left|k c_{S} \eta\right| \rightarrow 0$. 
Finally we consider the results for $\left|k c_{S} \eta\right| \approx 1$. In this case we get

$$
\begin{aligned}
\langle\zeta(\mathbf{x}, \eta=0) \zeta(\mathbf{y}, \eta=0)\rangle_{\left|k c_{S} \eta\right| \approx 1} \approx \frac{H^{2}}{2 \epsilon M_{p}^{2}} \frac{1}{2^{2(2-\Lambda)} \pi^{4}} \frac{\tilde{c}_{S}^{2}}{c_{S}^{3}}\left|\frac{\Gamma(\Lambda)}{\Gamma\left(\frac{3}{2}\right)}\right|^{2}\left[\left(\left|C_{2}\right|^{2}+\left|C_{1}\right|^{2}\right)-\left(C_{1}^{*} C_{2}+C_{1} C_{2}^{*}\right)\right] Z_{I} & \text { for Bunch Davies, } \\
& =Z_{I} \times \begin{cases}\frac{H^{2}}{2 \epsilon M_{p}^{2}} \frac{1}{2^{2(3-\Lambda)} \pi^{3}} \frac{\tilde{c}_{S}^{2}}{c_{S}^{3}}\left|\frac{\Gamma(\Lambda)}{\Gamma\left(\frac{3}{2}\right)}\right|^{2} \\
\frac{H^{2}}{2 \epsilon M_{p}^{2}} \frac{1}{2^{(5-2 \Lambda)} \pi^{4}} \frac{\tilde{c}_{S}^{2}}{c_{S}^{3}}\left|\frac{\Gamma(\Lambda)}{\Gamma\left(\frac{3}{2}\right)}\right|^{2}\left[\sinh ^{2} \alpha+\cosh ^{2} \alpha\right. & \text { for } \alpha \text { vacua Type-I, } \\
\frac{H^{2}}{2 \epsilon M_{p}^{2}} \frac{\left|N_{\alpha}\right|^{2}}{2^{(3-2 \Lambda)} \pi^{4}} \frac{\tilde{c}_{S}^{2}}{c_{S}^{3}}\left|\frac{\Gamma(\Lambda)}{\Gamma\left(\frac{3}{2}\right)}\right|^{2} \sin ^{2} \frac{\alpha}{2} & \text { for } \alpha \text { vacua Type-II, } \\
0 \quad & \text { for special vacua, }\end{cases}
\end{aligned}
$$

where the momentum integrals $Z_{I}$ are defined in the following:

$Z_{I}=\int \mathrm{d}^{3} k \frac{e^{i \mathbf{k} \cdot(\mathbf{x}-\mathbf{y})}}{k^{3}}$.

To compute this integral we follow the same assumptions as mentioned for the computation of VEV of curvature perturbation in position space. Additionally we introduce an infrared cut-off $L_{I R}$ to regularize the integral. Here we have the following results:

For Case I: $\quad Z_{I}=4 \pi \int_{1 / L_{I R}}^{\infty} \mathrm{d} k \frac{e^{i k|\mathbf{x}-\mathbf{y}|}}{k} \approx 4 \pi\left\{\ln \left(\frac{L_{I R}}{|\mathbf{x}-\mathbf{y}|}\right)-\gamma_{E}\right\}$.

For Case II: $\quad Z_{I}=2 \pi \int_{1 / L_{I R}}^{\infty} \mathrm{d} k \int_{\Theta_{1}}^{\Theta_{2}} \mathrm{~d} \Theta \frac{e^{i k|\mathbf{x}-\mathbf{y}| \cos \Theta}}{k}$

$$
\begin{aligned}
= & 4 \pi\left[( \Theta _ { 2 } - \Theta _ { 1 } ) \left\{\ln \left(\frac{L_{I R}}{|\mathbf{x}-\mathbf{y}|}\right)+\ln \left(\frac{\cos \Theta_{2}}{\cos \Theta_{1}}\right)-\gamma_{E}\right.\right. \\
& \left.\left.+\operatorname{Ei}\left(\frac{i|\mathbf{x}-\mathbf{y}| \cos \Theta_{2}}{L_{I R}}\right)-\operatorname{Ei}\left(\frac{i|\mathbf{x}-\mathbf{y}| \cos \Theta_{2}}{L_{I R}}\right)\right\}-\int_{\Theta_{1}}^{\Theta_{2}} \mathrm{~d} \Theta \operatorname{Ei}\left(\frac{i|\mathbf{x}-\mathbf{y}| \cos \Theta}{L_{I R}}\right)\right] .
\end{aligned}
$$

Here by setting $\mathbf{y}=0$ one can derive the results for $\langle\zeta(\mathbf{x}, \eta=0) \zeta(0, \eta=0)\rangle$ at conformal time scale $\eta$ with $\left|k c_{S} \eta\right| \rightarrow-\infty$.

Now our prime objective is the following:

- to derive an exact connection between the computed VEV and two point function of the scalar curvature perturbation in the presence of mass parameter $m(\eta)$,

- to derive the exact connection between the VEV of the curvature perturbation with the real cosmological observables,

- to put an additional constraint on the primordial cosmological setup in the presence of new mass parameter $m(\eta)$ within the prescription of EFT,

- to check the future possibility of detecting VEV of the curvature perturbation in the cosmological experiments and to put stringent bound on the Wilsonian operators of the background EFT framework,

- to give a theoretical understanding of the new cosmological quantity i.e. VEV of the curvature perturbation in the presence of mass parameter $m(\eta)$, using which it may be possible to break the degeneracy between various cosmological parameters and once can able to discriminate between various models in primordial cosmology. ${ }^{12}$

\footnotetext{
$\overline{12}$ Implementing the present techniques it is also possible to derive a direct connection between the primordial non-Gaussianity computed from the three point and one point scalar fluctuations for the newly introduced mass parameter $m(\eta)$. We will explore this possibility in near future in great detail within the context of EFT.
} 
To establish such a clear connection in momentum space we write down the following sets of new consistency relations in primordial cosmology for the three limiting cases $\left|k c_{S} \eta\right| \rightarrow-\infty,\left|k c_{S} \eta\right| \rightarrow 0$ and $\left|k c_{S} \eta\right| \approx 1$ :

$$
\begin{gathered}
\left\langle\zeta_{\mathbf{k}}(\eta=0)\right\rangle_{\left|k c_{S} \eta\right| \rightarrow-\infty}=\left\langle\zeta_{\mathbf{k}}(\eta=0) \zeta_{\mathbf{q}}(\eta=0)\right\rangle_{\left|k c_{S} \eta\right| \rightarrow-\infty}^{\prime} \times \frac{2 k}{\eta^{2} \tilde{c}_{S} H} \mathcal{I}_{1}, \\
\left\langle\zeta_{\mathbf{k}}(\eta=\xi \rightarrow 0)\right\rangle_{\left|k c_{S} \eta\right| \rightarrow 0}=\left\langle\zeta_{\mathbf{k}}(\eta=\xi \rightarrow 0) \zeta_{\mathbf{q}}(\eta=\xi \rightarrow 0)\right\rangle_{\left|k c_{S} \eta\right| \rightarrow 0}^{\prime} \times \frac{2^{2-2 \Lambda} k^{2 \Lambda} c_{S}^{2 \Lambda}}{(-\eta)^{3-2 \Lambda} H \pi}\left|\frac{\Gamma\left(\frac{3}{2}\right)}{\Gamma(\Lambda)}\right|^{2} \mathcal{I}_{2}, \\
\left\langle\zeta_{\mathbf{k}}(\eta=0)\right\rangle_{|k c s \eta| \approx 1}=\left\langle\zeta_{\mathbf{k}}(\eta=0) \zeta_{\mathbf{q}}(\eta=0)\right\rangle_{\left|k c_{S} \eta\right| \approx 1}^{\prime} \times \frac{2^{2-2 \Lambda} k^{3} c_{S}^{3}}{H \pi}\left|\frac{\Gamma\left(\frac{3}{2}\right)}{\Gamma(\Lambda)}\right|^{2} \mathcal{I}_{3},
\end{gathered}
$$

where $\left\langle\zeta_{\mathbf{k}}(\eta=0) \zeta_{\mathbf{q}}(\eta=0)\right\rangle_{\left|k c_{S} \eta\right| \rightarrow-\infty}^{\prime},\left\langle\zeta_{\mathbf{k}}(\eta=0) \zeta_{\mathbf{q}}(\eta=0)\right\rangle_{\left|k c_{S} \eta\right| \rightarrow 0}^{\prime}$ and $\left\langle\zeta_{\mathbf{k}}(\eta=0) \zeta_{\mathbf{q}}(\eta=0)\right\rangle_{\left|k c_{S} \eta\right| \approx 1}^{\prime}$ are defined as

$$
\begin{gathered}
\left\langle\zeta_{\mathbf{k}}(\eta=0) \zeta_{\mathbf{q}}(\eta=0)\right\rangle_{\left|k c_{S} \eta\right| \rightarrow-\infty}^{\prime}=\frac{\left\langle\zeta_{\mathbf{k}}(\eta=0) \zeta_{\mathbf{q}}(\eta=0)\right\rangle_{\left|k c_{s} \eta\right| \rightarrow-\infty}}{(2 \pi)^{3} \delta^{3}(\mathbf{k}+\mathbf{q})}, \\
\left\langle\zeta_{\mathbf{k}}(\eta=0) \zeta_{\mathbf{q}}(\eta=0)\right\rangle_{\left|k c_{S} \eta\right| \rightarrow 0}^{\prime}=\frac{\left\langle\zeta_{\mathbf{k}}(\eta=0) \zeta_{\mathbf{q}}(\eta=0)\right\rangle_{|k c s \eta| \rightarrow 0}}{(2 \pi)^{3} \delta^{3}(\mathbf{k}+\mathbf{q})} \\
\left\langle\zeta_{\mathbf{k}}(\eta=0) \zeta_{\mathbf{q}}(\eta=0)\right\rangle_{\left|k c_{S} \eta\right| \approx 1}^{\prime}=\frac{\left\langle\zeta_{\mathbf{k}}(\eta=0) \zeta_{\mathbf{q}}(\eta=0)\right\rangle_{\left|k c_{S} \eta\right| \approx 1}}{(2 \pi)^{3} \delta^{3}(\mathbf{k}+\mathbf{q})}
\end{gathered}
$$

Let us now define a new cosmological observable $\hat{O}_{\text {obs }}$ in the three limiting cases $\left|k c_{S} \eta\right| \rightarrow-\infty,\left|k c_{S} \eta\right| \rightarrow 0$ and $\left|k c_{S} \eta\right| \approx 1$ as

$$
\begin{aligned}
& \hat{O}_{\text {obs }} \stackrel{\left|k c_{S} \eta\right| \rightarrow-\infty}{=} \frac{2 \tilde{c}_{S}}{\left(-k \eta \tilde{c}_{S}\right)^{2} H} \mathcal{I}_{1}, \\
& \hat{O}_{\text {obs }} \stackrel{\left|k c_{S} \eta\right| \rightarrow 0}{=} \frac{2^{3-2 \Lambda} c_{S}^{3} \pi}{\left(-k \eta c_{S}\right)^{3-2 \Lambda} H}\left|\frac{\Gamma\left(\frac{3}{2}\right)}{\Gamma(\Lambda)}\right|^{2} \mathcal{I}_{2}, \\
& \hat{O}_{\text {obs }} \stackrel{\left|k c_{S} \eta\right| \approx 1}{=} \frac{2^{3-2 \Lambda} c_{S}^{3} \pi}{H}\left|\frac{\Gamma\left(\frac{3}{2}\right)}{\Gamma(\Lambda)}\right|^{2} \mathcal{I}_{3},
\end{aligned}
$$

where $\mathcal{I}_{1}, \mathcal{I}_{2}$ and $\mathcal{I}_{3}$ are defined as

$$
\begin{aligned}
\mathcal{I}_{1} & =-\frac{\int_{-\infty}^{0} \mathrm{~d} \eta \frac{\eta}{k} m\left[\left|C_{2}\right|^{2} e^{-i k c_{S} \eta}-\left|C_{1}\right|^{2} e^{i k c_{S} \eta}-i\left(C_{1}^{*} C_{2} e^{i \pi\left(\Lambda+\frac{1}{2}\right)}+C_{1} C_{2}^{*} e^{-i \pi\left(\Lambda+\frac{1}{2}\right)}\right) \sin k c_{S} \eta\right]}{\left[\left|C_{2}\right|^{2}+\left|C_{1}\right|^{2}+\left(C_{1}^{*} C_{2} e^{2 i k c_{S} \eta} e^{i \pi\left(\Lambda+\frac{1}{2}\right)}+C_{1} C_{2}^{*} e^{-2 i k c_{S} \eta} e^{-i \pi\left(\Lambda+\frac{1}{2}\right)}\right)\right]} \\
& = \begin{cases}\int_{-\infty}^{0} \mathrm{~d} \eta \frac{\eta}{k} m e^{i k c_{S} \eta} & \text { for Bunch Davies, } \\
-\frac{\int_{-\infty}^{0} \mathrm{~d} \eta \frac{\eta}{k} m\left[\sinh ^{2} \alpha e^{i k c_{S} \eta}-\cosh ^{2} \alpha e^{-i k c_{S} \eta}+i \sinh 2 \alpha \cos \left(\pi\left(\Lambda+\frac{1}{2}\right)+\delta\right) \sin k c_{S} \eta\right]}{\left[\sinh ^{2} \alpha+\cosh ^{2} \alpha+\sinh 2 \alpha \cos \left(2 k c_{S} \eta+\pi\left(\Lambda+\frac{1}{2}\right)+\delta\right)\right]} & \text { for } \alpha \text { vacua Type-I, } \\
-\frac{\int_{-\infty}^{0} \mathrm{~d} \eta \frac{\eta}{k} m\left[e^{\alpha+\alpha^{*}} e^{i k c_{S} \eta}-e^{-i k c_{S} \eta}+i\left(e^{\alpha} e^{i \pi\left(\Lambda+\frac{1}{2}\right)}+e^{\alpha^{*}} e^{-i \pi\left(\Lambda+\frac{1}{2}\right)}\right) \sin k c_{S} \eta\right]}{4 \cos ^{2}\left(k c_{S} \eta+\frac{\pi}{2}\left(\Lambda+\frac{1}{2}\right)-i \frac{\alpha}{2}\right)} & \text { for } \alpha \text { vacua Type-II } \\
-\frac{i \pi^{2} \int_{-\infty}^{0} \mathrm{~d} \eta \frac{\eta}{k} m \sin k c_{S} \eta \cos ^{2} \frac{\pi}{2}\left(\Lambda+\frac{1}{2}\right)}{\cos ^{2}\left(k c_{S} \eta+\frac{\pi}{2}\left(\Lambda+\frac{1}{2}\right)\right)} & \text { for special vacua, }\end{cases}
\end{aligned}
$$




$$
\begin{aligned}
& \mathcal{I}_{2}=\sqrt{\xi} \int_{-\infty}^{\xi} \mathrm{d} \eta \sqrt{-\eta} m\left(\Lambda-\frac{1}{2}\right) \\
& \times\left[\left(-\frac{k c_{S} \eta}{2}\right)^{-\Lambda}\left(-\frac{k c_{S} \xi}{2}\right)^{-\Lambda}+\left(\frac{k c_{S} \eta}{2}\right)^{-\Lambda}\left(\frac{k c_{S} \xi}{2}\right)^{-\Lambda}\right] \\
& \mathcal{I}_{3}=\sqrt{\xi} \int_{-\infty}^{\xi} \mathrm{d} \eta \sqrt{-\eta} m\left(\Lambda-\frac{1}{2}\right) 2^{1+\Lambda}\left(\frac{k c_{S} \xi}{2}\right)^{-\Lambda}(-1)^{-\Lambda} .
\end{aligned}
$$

Next we write down the expression for new cosmological observable $\hat{O}_{\text {obs }}$ in terms of all other known cosmological quantities

$\hat{O}_{\text {obs }} \stackrel{\left|k c_{S \eta}\right| \approx 1}{=} \frac{2^{n_{\zeta}-1} c_{S}^{3} \pi}{H}\left|\frac{\Gamma\left(\frac{3}{2}\right)}{\Gamma\left(\frac{4-n_{\zeta}}{2}\right)}\right|^{2} \mathcal{I}_{3}\left(\Lambda=\frac{4-n_{\zeta}}{2}\right)$,

where the spectral tilt for scalar fluctuations is given by

$$
\begin{gathered}
n_{\zeta}-1 \equiv\left(\frac{\mathrm{d} \ln \Delta_{\zeta}(k)}{\mathrm{d} \ln k}\right)_{\left|k c_{S} \eta\right| \approx 1} \approx 3-2 \Lambda \\
=\left\{\begin{array}{l}
3-2 \sqrt{\frac{9}{4}-\frac{m_{\text {inf }}^{2}}{H^{2}}} \text { for de Sitter, } \\
3-2 \sqrt{v^{2}-\frac{m_{\text {inf }}^{2}}{H^{2}}} \text { for quasi de Sitter. }
\end{array}\right.
\end{gathered}
$$

One can approximately consider a phenomenological situation where the inflaton and the new particles are exactly identical. Technically this means they have comparable masses, i.e. $m_{\text {inf }} \approx m$. In that situation the spectral tilt for the scalar fluctuations is given by

$$
\begin{aligned}
& n_{\zeta}-1 \equiv\left(\frac{\mathrm{d} \ln \Delta_{\zeta}(k)}{\mathrm{d} \ln k}\right)_{\left|k c_{S} \eta\right| \approx 1} \\
& \approx\left\{\begin{array}{l}
3-2 \sqrt{\frac{9}{4}-\frac{m^{2}}{H^{2}}} \text { for de Sitter, } \\
3-2 \sqrt{v^{2}-\frac{m^{2}}{H^{2}}} \text { for quasi de Sitter. }
\end{array}\right.
\end{aligned}
$$

As the value of the scalar spectral tilt $n_{\zeta}$ is known from observation, one can easily give the estimate of the value of the ratio of the mass parameter $m$ with Hubble scale during inflation $H$ as

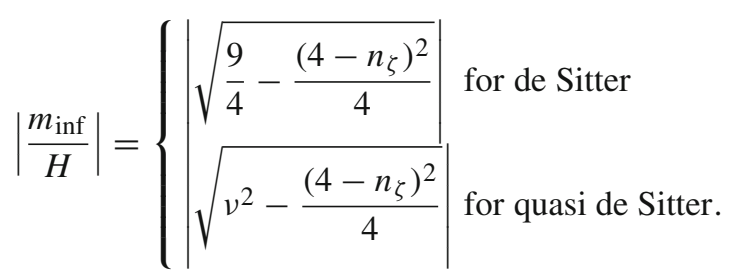

In the approximated situation as mentioned earlier one can similarly give an estimate of the value of the ratio of the mass parameter $m$ with Hubble scale during inflation $H$ as

$$
\left|\frac{m}{H}\right|= \begin{cases}\left|\sqrt{\frac{9}{4}-\frac{\left(4-n_{\zeta}\right)^{2}}{4}}\right| & \text { for de Sitter } \\ \sqrt{\sqrt{v^{2}-\frac{\left(4-n_{\zeta}\right)^{2}}{4}}} \mid & \text { for quasi de Sitter. }\end{cases}
$$

Additionally, in this context the integral $\mathcal{I}_{3}\left(\Lambda=\frac{4-n_{\zeta}}{2}\right)$ is defined in terms of the scalar spectral tilt as

$$
\begin{aligned}
\mathcal{I}_{3}= & \sqrt{\xi} \int_{-\infty}^{\xi} \mathrm{d} \eta \sqrt{-\eta} m\left(\frac{3-n_{\zeta}}{2}\right) 2^{3-\frac{n_{\zeta}}{2}} \\
& \times\left(\frac{k c_{S} \xi}{2}\right)^{\frac{n_{\zeta}-1}{2}}(-1)^{\frac{n_{\zeta}-1}{2}} .
\end{aligned}
$$

Now our main claim is that, if in the near future with sufficient statistical accuracy one can measure the new cosmological parameter $\hat{O}_{\text {obs }}$, then using Eq. (3.303) one can further write the expression for the scale of inflation in the presence of new particle with mass $m$ as

$H=\frac{2^{n_{\zeta}-1} c_{S}^{3} \pi}{\hat{O}_{\mathrm{obs}}}\left|\frac{\Gamma\left(\frac{3}{2}\right)}{\Gamma\left(\frac{4-n_{\zeta}}{2}\right)}\right|^{2} \mathcal{I}_{3}\left(\Lambda=\frac{4-n_{\zeta}}{2}\right)$,

where all the observables are computed at the horizon crossing $\left|k c_{S} \eta\right| \approx 1$ and the temporal IR cut-off $\xi$ should be fixed at the pivot scale $k_{*}$ where $\left|k c_{S} \eta\right| \approx 1$ condition is additionally satisfied. Here the main advantage of this expression is that we do not need any prior knowledge or any equivalent information from the tensor-to-scalar ratio $r$. This implies that even though we are not able to detect the signatures of primordial gravitational waves through tensor-to-scalar ratio $r$ it is possible to quantify exactly the scale of inflationary paradigm and comment on the new physics associated with this scenario. An additional advantage of such relation is that the computation is applicable for any models of inflation as it is based on the background EFT framework.

Next we express the scale of inflation in terms of the amplitude of scalar power spectrum and the scalar spectral tilt as 


$$
\begin{aligned}
& H \approx \sqrt{\epsilon} \frac{2^{\frac{n_{\zeta}-1}{2}} \pi^{3 / 2} \sqrt{\left[\Delta_{\zeta}(k)\right]_{\left|k c_{S} \eta\right| \approx 1}}}{\sqrt{\left[\left|C_{2}\right|^{2}+\left|C_{1}\right|^{2}-\left(C_{1}^{*} C_{2}+C_{1} C_{2}^{*}\right)\right]}} \frac{c_{S}^{3 / 2}}{\tilde{c}_{S}}\left|\frac{\Gamma\left(\frac{3}{2}\right)}{\Gamma\left(\frac{4-n_{\zeta}}{2}\right)}\right| M_{p}
\end{aligned}
$$

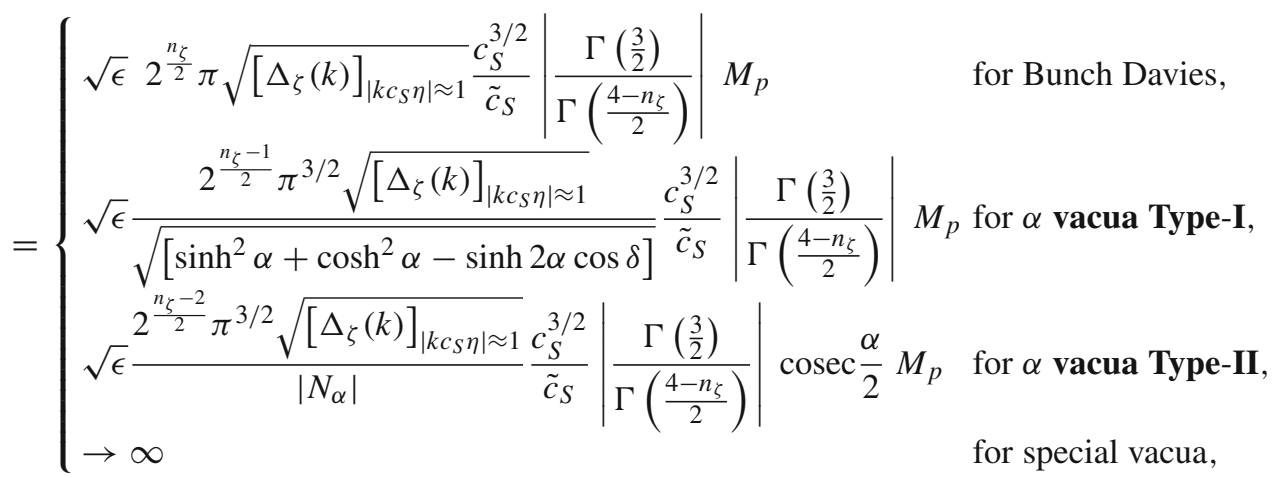

where we do not need any information from the VEV of the scalar fluctuation. Before going to the further details also here it is important to mention that in Eqs. (3.309) and (3.310) and the following parameters are known from the Planck 2015 data set within $2 \sigma$ C.L.:

1. Amplitude of the scalar power spectrum:

$$
\ln \left(10^{10}\left[\Delta_{\zeta}(k)\right]_{\left|k c_{S} \eta\right| \approx 1}\right)=3.089 \pm 0.036
$$

2. Scalar spectral tilt:

$$
n_{\zeta}=0.9569 \pm 0.0077
$$

3. Sound speed:

$$
0.02<\tilde{c}_{S}<1
$$

As using both Eqs. (3.309) and (3.310) one can predict the scale of inflation, here one can compare both of them to compute the value of the first slow-roll parameter $\epsilon$ in a model independent way in the background of EFT framework. After doing this here we get the following expression for the slow-roll parameter $\epsilon$ as given by

$$
\begin{aligned}
\epsilon & \approx \frac{2^{n_{\zeta}-1} c_{S}^{3} \tilde{c}_{S}^{2}\left[\left|C_{2}\right|^{2}+\left|C_{1}\right|^{2}-\left(C_{1}^{*} C_{2}+C_{1} C_{2}^{*}\right)\right]}{\left[\Delta_{\zeta}(k)\right]_{\left|k c_{S} \eta\right| \approx 1} \hat{O}_{\mathrm{obs}}^{2} M_{p}^{2} \pi}\left|\frac{\Gamma\left(\frac{3}{2}\right)}{\Gamma\left(\frac{4-n_{\zeta}}{2}\right)}\right|^{2} \mathcal{I}_{3}^{2}\left(\Lambda=\frac{4-n_{\zeta}}{2}\right) \\
& = \begin{cases}\frac{2^{n_{\zeta}-2} c_{S}^{3} \tilde{c}_{S}^{2}}{\left[\Delta_{\zeta}(k)\right]_{\left|k c_{S} \eta\right| \approx 1} \hat{O}_{\mathrm{obs}}^{2} M_{p}^{2}}\left|\frac{\Gamma\left(\frac{3}{2}\right)}{\Gamma\left(\frac{4-n_{\zeta}}{2}\right)}\right|^{2} \mathcal{I}_{3}^{2}\left(\Lambda=\frac{4-n_{\zeta}}{2}\right) & \text { for Bunch Davies, } \\
\frac{2^{n_{\zeta}-1} c_{S}^{3} \tilde{c}_{S}^{2}\left[\sinh ^{2} \alpha+\cosh ^{2} \alpha-\sinh 2 \alpha \cos \delta\right]}{\left[\Delta_{\zeta}(k)\right]_{\left|k c_{S} \eta\right| \approx 1} \hat{O}_{\mathrm{obs}}^{2} M_{p}^{2} \pi}\left|\frac{\Gamma\left(\frac{3}{2}\right)}{\Gamma\left(\frac{4-n_{\zeta}}{2}\right)}\right|^{2} \mathcal{I}_{3}^{2}\left(\Lambda=\frac{4-n_{\zeta}}{2}\right) & \text { for } \alpha \text { vacua Type-I, } \\
\frac{2^{n_{\zeta}+1} c_{S}^{3} \tilde{c}_{S}^{2}\left|N_{\alpha}\right|^{2}}{\left[\Delta_{\zeta}(k)\right]_{\left|k c_{S} \eta\right| \approx 1} \hat{O}_{\mathrm{obs}}^{2} M_{p}^{2} \pi}\left|\frac{\Gamma\left(\frac{3}{2}\right)}{\Gamma\left(\frac{4-n_{\zeta}}{2}\right)}\right|^{2} \mathcal{I}_{3}^{2}\left(\Lambda=\frac{4-n_{\zeta}}{2}\right) \sin ^{2} \frac{\alpha}{2} & \text { for } \alpha \text { vacua Type-II, } \\
\rightarrow 0 & \text { for special vacua. }\end{cases}
\end{aligned}
$$

On the other hand within the framework of EFT using the slow-roll parameter $\epsilon$ one can write down a consistency relation for the tensor-to-scalar ratio $r$ in the presence of sound speed $\tilde{c}_{S}$ as 
$r=\frac{\left[\Delta_{h}(k)\right]_{\left|k c_{S} \eta\right| \approx 1}}{\left[\Delta_{\zeta}(k)\right]_{\left|k c_{S} \eta\right| \approx 1}}=16 \epsilon \tilde{c}_{S}$,

where $\left[\Delta_{h}(k)\right]_{\left|k c_{S} \eta\right| \approx 1}$ and $\left[\Delta_{\zeta}(k)\right]_{\left|k c_{S} \eta\right| \approx 1}$ signify the amplitude of tensor and scalar fluctuations. Further using Eq. (3.315) in Eq. (3.317) we get the following expression for the tensorto-scalar ratio and the amplitude of tensor fluctuation in a model-independent fashion as gravity waves in a model-independent way. Here we only need to measure the mass of the particles participating in evolution of universe and for serve this purpose we need to measure the value of the VEV of the scalar fluctuations or the one point function or more precisely a new cosmological observable depicted by $\hat{O}_{\text {obs }}$ as introduced in the context of EFT. Additionally, it is important to mention here that the final analytical expression for the scale

$$
\begin{aligned}
r \approx \frac{2^{n_{\zeta}+3} c_{S}^{3} \tilde{c}_{S}^{3}\left[\left|C_{2}\right|^{2}+\left|C_{1}\right|^{2}-\left(C_{1}^{*} C_{2}+C_{1} C_{2}^{*}\right)\right]}{\left[\Delta_{\zeta}(k)\right]_{\left|k c_{S} \eta\right| \approx 1} \hat{O}_{\mathrm{obs}}^{2} M_{p}^{2} \pi}\left|\frac{\Gamma\left(\frac{3}{2}\right)}{\Gamma\left(\frac{4-n_{\zeta}}{2}\right)}\right|^{2} \mathcal{I}_{3}^{2}\left(\Lambda=\frac{4-n_{\zeta}}{2}\right) & \text { for Bunch Davies, } \\
& = \begin{cases}\frac{2^{n_{\zeta}+2} c_{S}^{3} \tilde{c}_{S}^{3}}{\left[\Delta_{\zeta}(k)\right]_{\left|k c_{S} \eta\right| \approx 1} \hat{O}_{\mathrm{obs}}^{2} M_{p}^{2}}\left|\frac{\Gamma\left(\frac{3}{2}\right)}{\Gamma\left(\frac{4-n_{\zeta}}{2}\right)}\right|^{2} \mathcal{I}_{3}^{2}\left(\Lambda=\frac{4-n_{\zeta}}{2}\right) & \text { for } \alpha \text { vacua Type-II, } \\
\frac{2^{n_{\zeta}+3} c_{S}^{3} \tilde{c}_{S}^{3}\left[\sinh ^{2} \alpha+\cosh ^{2} \alpha-\sinh 2 \alpha \cos \delta\right]}{\left[\Delta_{\zeta}(k)\right]_{\left|k c_{S} \eta\right| \approx 1} \hat{O}_{\mathrm{obs}}^{2} M_{p}^{2} \pi}\left|\frac{\Gamma\left(\frac{3}{2}\right)}{\Gamma\left(\frac{4-n_{\zeta}}{2}\right)}\right|^{2} \mathcal{I}_{3}^{2}\left(\Lambda=\frac{4-n_{\zeta}}{2}\right) & \text { for } \alpha \text { vacua Type-I, } \\
\frac{2^{n_{\zeta}+5} c_{S}^{3} \tilde{c}_{S}^{3}\left|N_{\alpha}\right|^{2}}{\left[\Delta_{\zeta}(k)\right]_{\left|k c_{S} \eta\right| \approx 1} \hat{O}_{\mathrm{obs}}^{2} M_{p}^{2} \pi}\left|\frac{\Gamma\left(\frac{3}{2}\right)}{\Gamma\left(\frac{4-n_{\zeta}}{2}\right)}\right|^{2} \mathcal{I}_{3}^{2}\left(\Lambda=\frac{4-n_{\zeta}}{2}\right) \sin ^{2} \frac{\alpha}{2} & \text { for special vacua, } \\
\rightarrow 0 & \end{cases}
\end{aligned}
$$

and

$$
\begin{aligned}
{\left[\Delta_{h}(k)\right]_{\left|k c_{S} \eta\right| \approx 1} \approx \frac{2^{n_{\zeta}+3} c_{S}^{3} \tilde{c}_{S}^{3}\left[\left|C_{2}\right|^{2}+\left|C_{1}\right|^{2}-\left(C_{1}^{*} C_{2}+C_{1} C_{2}^{*}\right)\right]}{\hat{O}_{\mathrm{obs}}^{2} M_{p}^{2} \pi}\left|\frac{\Gamma\left(\frac{3}{2}\right)}{\Gamma\left(\frac{4-n_{\zeta}}{2}\right)}\right|^{2} \mathcal{I}_{3}^{2}\left(\Lambda=\frac{4-n_{\zeta}}{2}\right) } & \text { for Bunch Davies, } \\
& = \begin{cases}\frac{2^{n_{\zeta}+2} c_{S}^{3} \tilde{c}_{S}^{3}}{\hat{O}_{\mathrm{obs}}^{2} M_{p}^{2}}\left|\frac{\Gamma\left(\frac{3}{2}\right)}{\Gamma\left(\frac{4-n_{\zeta}}{2}\right)}\right|^{2} \mathcal{I}_{3}^{2}\left(\Lambda=\frac{4-n_{\zeta}}{2}\right) & \text { for } \alpha \text { vacua Type-I, } \\
\times\left|\frac{\Gamma\left(\frac{3}{2}\right)}{\Gamma\left(\frac{4-n_{\zeta}}{2}\right)}\right|^{2} \mathcal{I}_{3}^{2}\left(\Lambda=\frac{4-n_{\zeta}}{2}\right) & \hat{O}_{\mathrm{obs}}^{2} M_{p}^{2} \pi \\
\frac{2^{n_{\zeta}+3} c_{S}^{3} \tilde{c}_{S}^{3}\left[\sinh ^{2} \alpha+\cosh ^{2} \alpha-\sinh 2 \alpha \cos \delta\right]}{\hat{O}_{\mathrm{obs}}^{2} M_{p}^{2} \pi} \pi^{5}\left|N_{\alpha}\right|^{2}\left|\frac{\Gamma\left(\frac{3}{2}\right)}{\Gamma\left(\frac{4-n_{\zeta}}{2}\right)}\right|^{2} \mathcal{I}_{3}^{2}\left(\Lambda=\frac{4-n_{\zeta}}{2}\right) \sin ^{2} \frac{\alpha}{2} \text { for } \alpha \text { vacua Type-II, } \\
\rightarrow 0 & \text { for special vacua. }\end{cases}
\end{aligned}
$$

Here in summary from the derived results we get the following information:

- Scale of inflation and the associated new physics can be predicted without the prior knowledge of primordial of inflation is independent of the choice of any initial condition during inflation.

- Here one can give an estimate of scaled mass parameter $m_{\text {inf }} / H$ and $m / H$ in terms of the scalar spectral tilt $n_{\zeta}$ for the de Sitter case and the quasi de Sitter case. 
- One can also compute the expression for the scale of inflation in therms of known inflationary observables for a proper choice of initial condition.

- Further if we demand that the scale of inflation computed from both techniques should give unique result then by comparing both of the expressions we derive the analytical model-independent expression for the first slow-roll parameter $\epsilon$ within the framework of EFT.

- Further using the consistency relations valid in the context of inflation one can further derive the expression for both tensor-to-scalar ratio $r$ and the amplitude of the tensor fluctuations in a model-independent way.

- In the case of single field slow-roll models of inflation the amount of non-Gaussianity is proportional to the first slow-roll parameter $\epsilon$ or more precisely with the primordial gravity waves through tensor-to-scalar ratio $r$. So using the prescribed methodology mentioned in this paper one can easily derive the model independent expression for the non-Gaussian amplitude in terms of the time-dependent mass parameter within the framework of EFT. We will report soon on these aspects.

- It is important to mention here that if we use the constraint on the scalar spectral tilt as obtained from the Planck 2015 data we get the following $2 \sigma$ bound on the magnitude of the mass parameter of the inflaton field:

For de Sitter:

$0.23<\left|\frac{m_{\mathrm{inf}}}{H}\right|_{k c_{S} \eta \approx 1}<0.28$,

For quasi de Sitter:

$$
\begin{aligned}
& 0.23 \times\left|\sqrt{1-56.18\left(\epsilon+\frac{\eta}{2}+\frac{s}{2}\right)}\right|<\left|\frac{m_{\text {inf }}}{H}\right|_{k c_{S} \eta \approx 1} \\
& <0.28 \times\left|\sqrt{1-39.06\left(\epsilon+\frac{\eta}{2}+\frac{s}{2}\right)}\right| .
\end{aligned}
$$

and for the approximated situation where $m_{\mathrm{inf}} \approx m$ we get the following $2 \sigma$ bound on the magnitude of the mass parameter of the new heavy particles:

For de Sitter:

$0.23<\left|\frac{m}{H}\right|_{k c_{S} \eta \approx 1}<0.28$,
For quasi de Sitter:

$$
\begin{aligned}
& 0.23 \times\left|\sqrt{1-56.18\left(\epsilon+\frac{\eta}{2}+\frac{s}{2}\right)}\right|<\left|\frac{m}{H}\right|_{k c_{S} \eta \approx 1} \\
& <0.28 \times\left|\sqrt{1-39.06\left(\epsilon+\frac{\eta}{2}+\frac{s}{2}\right)}\right| .
\end{aligned}
$$

The importance of the obtained bound on the mass parameter of the new heavy particles can be justified in a following manner:

1. If the contribution from the inflaton field mass term or in the special case if the heavy massive field is very negligible then in that situation for de Sitter case we get the feature of exact scale invariance of the primordial power spectrum. But as the various observational probes confirms the fact that primordial power spectrum for scalar fluctuations are not exactly scale invariant, it is required to use quasi de Sitter approximation in the present context. As in the special case mass of the heavy field is negligibly small, this implies that the amount of Bell violation is also negligibly small.

2. From the above mentioned bound it is clear that to get nearly scale invariant primordial power spectrum non-negligible contribution in the inflaton field mass term and for the special case heavy field mass is necessarily required in the de Sitter case and the quasi de Sitter case. Most importantly in the de Sitter case also we get the nearly scale invariant feature in the primordial power spectrum in this case. The nonnegligible mass contribution from the obtained bound also implies that the amount of Bell violation in the primordial universe is not negligibly small.

- On the other hand if in the near future any observational probe can measure the value of the tensor-to-scalar ratio and primordial non-Gaussianity then also there is a possibility to give an estimate of the newly introduced massive particle and the new cosmological observable depicted by $\hat{O}_{\text {obs }}$. This will surely quantify the amount of Bell violation in early universe cosmology.

\subsubsection{Case I: $m \approx H$}

For further simplification we consider here $m \approx H$ along with the two limiting cases $\left|k c_{S} \eta\right| \rightarrow-\infty,\left|k c_{S} \eta\right| \rightarrow 0$ and $\left|k c_{S} \eta\right| \approx 1$, which are physically acceptable in the present context: 


$$
\begin{aligned}
& \left\langle\zeta_{\mathbf{k}}(\eta=0)\right\rangle_{\left|k c_{S} \eta\right| \rightarrow-\infty} \approx-\frac{16 \pi^{2} H \tilde{c}_{S}}{M_{p}^{2} \epsilon c_{S}}\left[\left|C_{2}\right|^{2} I_{1}-\left|C_{1}\right|^{2} I_{2}-i\left(C_{1}^{*} C_{2} e^{i \pi\left(\Lambda+\frac{1}{2}\right)}+C_{1} C_{2}^{*} e^{-i \pi\left(\Lambda+\frac{1}{2}\right)}\right) I_{3}\right] \\
& \left\{\begin{array}{cc}
\frac{8 \pi^{3} H \tilde{c}_{S}}{M_{p}^{2} \epsilon c_{S}} I_{2} & \text { for Bunch Davies vacua } \\
-\frac{16 \pi^{2} H \tilde{c}_{S}\left[\sinh ^{2} \alpha I_{1}-\cosh ^{2} \alpha I_{2}\right.}{M_{p}^{2} \epsilon c_{S}} & \\
\left.-i \sinh 2 \alpha \cos \left(\pi\left(\Lambda+\frac{1}{2}\right)+\delta\right) I_{3}\right] & \text { for } \alpha \text { vacua Type-I } \\
-\frac{16 \pi^{2}\left|N_{\alpha}\right|^{2} H \tilde{c}_{S}}{M_{p}^{2} \epsilon c_{S}}\left[e^{\alpha+\alpha^{*}} I_{1}-I_{2}\right. & \\
\left.-i\left(e^{\alpha} e^{i \pi\left(\Lambda+\frac{1}{2}\right)}+e^{\alpha^{*}} e^{-i \pi\left(\Lambda+\frac{1}{2}\right)}\right) I_{3}\right] & \text { for } \alpha \text { vacua Type-II } \\
-\frac{64 i \pi^{2}|C|^{2} H \tilde{c}_{S}}{M_{p}^{2} \epsilon c_{S}} \cos ^{2} \frac{\pi}{2}\left(\Lambda+\frac{1}{2}\right) I_{3} & \text { for special vacua, }
\end{array}\right. \\
& \left\langle\zeta_{\mathbf{k}}(\eta=\xi \rightarrow 0)\right\rangle_{\left|k c_{S} \eta\right| \rightarrow 0}=\frac{4 \pi \sqrt{\xi} H \tilde{c}_{S}}{M_{p}^{2} \epsilon}\left(C_{1}^{*} C_{2}+C_{1} C_{2}^{*}-\left|C_{1}\right|^{2}-\left|C_{2}\right|^{2}\right) I_{4}^{\xi \theta_{1}} \\
& \left(-\frac{2 \pi^{2} \sqrt{\xi} H \tilde{c}_{S}}{M_{p}^{2} \epsilon} \quad\right. \text { for Bunch Davies vacua } \\
& =I_{4}^{\xi \theta_{1}} \times \begin{cases}\frac{4 \pi \sqrt{\xi} H \tilde{c}_{S}}{M_{p}^{2} \epsilon}\left(\cos \delta \sinh 2 \alpha-\cosh ^{2} \alpha-\sinh ^{2} \alpha\right) & \text { for } \alpha \text { vacua Type-I, } \\
\frac{4 \pi \sqrt{\xi}\left|N_{\alpha}\right|^{2} H \tilde{c}_{S}}{M_{p}^{2} \epsilon}\left(e^{\alpha}+e^{\alpha^{*}}-e^{\alpha+\alpha^{*}}-1\right) & \text { for } \alpha \text { vacua Type-II } \\
0 & \text { for special vacua }\end{cases} \\
& \left\langle\zeta_{\mathbf{k}}(\eta=\xi \rightarrow 0)\right\rangle_{\left|k c_{S} \eta\right| \approx 1}=\frac{4 \pi \sqrt{\xi} H \tilde{c}_{S}}{M_{p}^{2} \epsilon}\left(C_{1}^{*} C_{2}+C_{1} C_{2}^{*}-\left|C_{1}\right|^{2}-\left|C_{2}\right|^{2}\right) I_{5}^{\xi \theta_{2}} \\
& =I_{5}^{\xi \theta_{2}} \times \begin{cases}-\frac{2 \pi^{2} \sqrt{\xi} H \tilde{c}_{S}}{M_{p}^{2} \epsilon} & \text { for Bunch Davies vacua } \\
\frac{4 \pi \sqrt{\xi} H \tilde{c}_{S}}{M_{p}^{2} \epsilon}\left(\cos \delta \sinh 2 \alpha-\cosh ^{2} \alpha-\sinh ^{2} \alpha\right) & \text { for } \alpha \text { vacua Type-I, } \\
\frac{4 \pi \sqrt{\xi}\left|N_{\alpha}\right|^{2} H \tilde{c}_{S}}{M_{p}^{2} \epsilon}\left(e^{\alpha}+e^{\alpha^{*}}-e^{\alpha+\alpha^{*}}-1\right) & \text { for } \alpha \text { vacua Type-II } \\
0 & \text { for special vacua }\end{cases}
\end{aligned}
$$

where we introduce the following integrals $I_{1}, I_{2}, I_{3}, I_{4}^{\xi \theta_{1}}, I_{4}^{\xi \theta_{2}}$ as given by

$$
\begin{aligned}
& I_{1}=\frac{1}{(2 \pi)^{3}} \int_{-\infty}^{0} \mathrm{~d} \eta \frac{\eta}{k} H e^{-i k c_{S} \eta} \\
& I_{2}=\frac{1}{(2 \pi)^{3}} \int_{-\infty}^{0} \mathrm{~d} \eta \frac{\eta}{k} H e^{i k c_{S} \eta}
\end{aligned}
$$




$$
\begin{aligned}
I_{3} & =\frac{1}{(2 \pi)^{3}} \int_{-\infty}^{0} \mathrm{~d} \eta \frac{\eta}{k} H \sin k c_{S} \eta, \\
I_{4}^{\xi \theta_{1}} & =-i \frac{H}{(2 \pi)^{3}} \frac{\left(\Lambda-\frac{1}{2}\right)}{\left(\Lambda-\frac{3}{2}\right)}\left[1+(-1)^{-2 \Lambda}\right]\left(\frac{k c_{S}}{2}\right)^{-2 \Lambda} \xi^{-\Lambda}\left[\xi^{\frac{3}{2}-\Lambda}+(-1)^{\frac{1}{2}-\Lambda} \theta_{1}^{\frac{3}{2}-\Lambda}\right], \\
I_{5}^{\xi \theta_{2}} & =-i \frac{H}{(2 \pi)^{3}} \frac{\left(\Lambda-\frac{1}{2}\right)}{\left(\Lambda-\frac{3}{2}\right)}\left[1+(-1)^{-2 \Lambda}\right]\left(-\frac{1}{2 \theta_{2}}\right)^{-2 \Lambda} \xi^{-\Lambda}\left[\xi^{\frac{3}{2}-\Lambda}+(-1)^{\frac{1}{2}-\Lambda} \theta_{2}^{\frac{3}{2}-\Lambda}\right]
\end{aligned}
$$

and here we introduce a UV cut-off regulator $\theta_{1}$ and $\theta_{2}$ in $I_{4}^{\xi \theta_{1}}$ and $I_{4}^{\xi \theta_{2}}$ respectively. Additionally, it is important to note that here the parameter $\Lambda$ is given by ${ }^{13}$ :

$$
\begin{aligned}
& \text { For } m_{\mathrm{inf}}<+H: \quad \Lambda \approx \begin{cases}\frac{3}{2} & \text { for dS, } \\
v & \text { for qdS. }\end{cases} \\
& \text { For } m_{\mathrm{inf}} \approx H: \quad \Lambda \approx \begin{cases}\frac{\sqrt{5}}{2} & \text { for dS }, \\
\sqrt{v^{2}-1} & \text { for qdS. }\end{cases}
\end{aligned}
$$

Similarly in position space the representative expressions for the expectation value of the scalar curvature perturbation for $m \approx H$ along with the three limiting cases $\left|k c_{S} \eta\right| \rightarrow-\infty,\left|k c_{S} \eta\right| \rightarrow 0$ and $\left|k c_{S} \eta\right| \approx 1$ are given by

$$
\begin{aligned}
& \langle\zeta(\mathbf{x}, \eta=0)\rangle_{\left|k c_{S} \eta\right| \rightarrow-\infty} \approx-\frac{2 H \tilde{c}_{S}}{M_{p}^{2} \epsilon \pi c_{S}}\left[\left|C_{2}\right|^{2} B_{1}-\left|C_{1}\right|^{2} B_{2}-i\left(C_{1}^{*} C_{2} e^{i \pi\left(\Lambda+\frac{1}{2}\right)}+C_{1} C_{2}^{*} e^{-i \pi\left(\Lambda+\frac{1}{2}\right)}\right) B_{3}\right] \\
& = \begin{cases}\frac{H \tilde{c}_{S}}{M_{p}^{2} \epsilon c_{S}} B_{2} & \text { for Bunch Davies, } \\
-\frac{2 H \tilde{c}_{S}}{M_{p}^{2} \epsilon \pi c_{S}}\left[\sinh ^{2} \alpha B_{1}-\cosh ^{2} \alpha B_{2}-i \sinh 2 \alpha \cos \left(\pi\left(\Lambda+\frac{1}{2}\right)+\delta\right) B_{3}\right] & \text { for } \alpha \text { vacua Type-I, } \\
-\frac{2\left|N_{\alpha}\right|^{2} H \tilde{c}_{S}}{M_{p}^{2} \epsilon \pi c_{S}}\left[e^{\alpha+\alpha^{*}} B_{1}-B_{2}-i\left(e^{\alpha} e^{i \pi\left(\Lambda+\frac{1}{2}\right)}+e^{\alpha^{*}} e^{-i \pi\left(\Lambda+\frac{1}{2}\right)}\right) B_{3}\right] & \text { for } \alpha \text { vacua Type-II, } \\
-\frac{8 i|C|^{2} H \tilde{c}_{S}}{M_{p}^{2} \epsilon \pi c_{S}} \cos ^{2} \frac{\pi}{2}\left(\Lambda+\frac{1}{2}\right) B_{3} & \text { for special vacua, }\end{cases} \\
& \langle\zeta(\mathbf{x}, \eta=\xi \rightarrow 0)\rangle_{|k c s \eta| \rightarrow 0}=\frac{\sqrt{\xi} H \tilde{c}_{S}}{2 M_{p}^{2} \epsilon \pi^{2}}\left(C_{1}^{*} C_{2}+C_{1} C_{2}^{*}-\left|C_{1}\right|^{2}-\left|C_{2}\right|^{2}\right) B_{4}^{\xi \theta_{1}} \\
& =B_{4}^{\xi \theta_{1}} \times \begin{cases}-\frac{2 \pi^{2} \sqrt{\xi} H \tilde{c}_{S}}{M_{p}^{2} \epsilon} & \text { for Bunch Davies vacua } \\
\frac{\sqrt{\xi} H \tilde{c}_{S}}{2 M_{p}^{2} \pi^{2} \epsilon}\left(\cos \delta \sinh 2 \alpha-\cosh ^{2} \alpha-\sinh ^{2} \alpha\right) & \text { for } \alpha \text { vacua Type-I, } \\
\frac{\sqrt{\xi}\left|N_{\alpha}\right|^{2} H \tilde{c}_{S}}{2 M_{p}^{2} \pi^{2} \epsilon}\left(e^{\alpha}+e^{\alpha^{*}}-e^{\alpha+\alpha^{*}}-1\right) & \text { for } \alpha \text { vacua Type-II }, \\
0 & \text { for special vacua },\end{cases}
\end{aligned}
$$

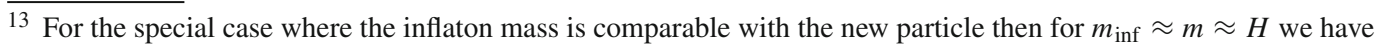

$\Lambda \approx \begin{cases}\frac{\sqrt{5}}{2} & \text { for dS }, \\ \sqrt{v^{2}-1} & \text { for qdS. }\end{cases}$ 


$$
\begin{aligned}
\langle\zeta(\mathbf{x}, \eta=\xi \rightarrow 0)\rangle_{\left|k c_{S} \eta\right| \approx 1} & =\frac{\sqrt{\xi} H \tilde{c}_{S}}{2 M_{p}^{2} \epsilon \pi^{2}}\left(C_{1}^{*} C_{2}+C_{1} C_{2}^{*}-\left|C_{1}\right|^{2}-\left|C_{2}\right|^{2}\right) B_{5}^{\xi \theta_{2}} \\
& =B_{5}^{\xi \theta_{2}} \times \begin{cases}-\frac{2 \pi^{2} \sqrt{\xi} H \tilde{c}_{S}}{M_{p}^{2} \epsilon} & \text { for Bunch Davies vacua, } \\
\frac{\sqrt{\xi} H \tilde{c}_{S}}{2 M_{p}^{2} \pi^{2} \epsilon}\left(\cos \delta \sinh 2 \alpha-\cosh ^{2} \alpha-\sinh ^{2} \alpha\right) & \text { for } \alpha \text { vacua Type-I, } \\
\frac{\sqrt{\xi}\left|N_{\alpha}\right|^{2} H \tilde{c}_{S}}{2 M_{p}^{2} \pi^{2} \epsilon}\left(e^{\alpha}+e^{\alpha^{*}}-e^{\alpha+\alpha^{*}}-1\right) & \text { for } \alpha \text { vacua Type-II, } \\
0 & \text { for special vacua, }\end{cases}
\end{aligned}
$$

where we introduce the integrals $B_{1}, B_{2}, B_{3}, B_{4}^{\xi \theta}, B_{5}^{\xi \theta}$ as given by

$$
\begin{aligned}
B_{1}= & \frac{1}{(2 \pi)^{3}} \int \mathrm{d}^{3} k e^{i \mathbf{k} \cdot \mathbf{x}} \int_{-\infty}^{0} \mathrm{~d} \eta \frac{\eta}{k} H e^{-i k c_{S} \eta}, \\
B_{2}= & \frac{1}{(2 \pi)^{3}} \int \mathrm{d}^{3} k e^{i \mathbf{k} \cdot \mathbf{x}} \int_{-\infty}^{0} \mathrm{~d} \eta \frac{\eta}{k} H e^{i k c_{S} \eta}, \\
B_{3}= & \frac{1}{(2 \pi)^{3}} \int \mathrm{d}^{3} k e^{i \mathbf{k} \cdot \mathbf{x}} \int_{-\infty}^{0} \mathrm{~d} \eta \frac{\eta}{k} H \sin k c_{S} \eta, \\
B_{4}^{\xi \theta_{1}=} & -i \frac{H}{(2 \pi)^{3}} \frac{\left(\Lambda-\frac{1}{2}\right)}{\left(\Lambda-\frac{3}{2}\right)}\left[1+(-1)^{-2 \Lambda}\right]\left(\frac{c_{S}}{2}\right)^{-2 \Lambda} \\
& \times \xi^{-\Lambda}\left[\xi^{\frac{3}{2}-\Lambda}+(-1)^{\frac{1}{2}-\Lambda} \theta_{1}^{\frac{3}{2}-\Lambda}\right] \int \mathrm{d}^{3} k e^{i \mathbf{k} \cdot \mathbf{x} k^{-2 \Lambda}}, \\
B_{5}^{\xi \theta_{2}=} & -i \frac{H}{(2 \pi)^{3}} \frac{\left(\Lambda-\frac{1}{2}\right)}{\left(\Lambda-\frac{3}{2}\right)}\left[1+(-1)^{-2 \Lambda}\right]\left(-\frac{1}{2 \theta_{2}}\right)^{-2 \Lambda} \\
& \times \xi^{-\Lambda}\left[\xi^{\frac{3}{2}-\Lambda}+(-1)^{\frac{1}{2}-\Lambda} \theta_{2}^{\frac{3}{2}-\Lambda}\right] \int \mathrm{d}^{3} k e^{i \mathbf{k} \cdot \mathbf{x} .}
\end{aligned}
$$

Now using the previously mentioned assumptions to compute the momentum integral for Case I and Case II we get the following results:

\section{For Case I:}

$$
\begin{aligned}
B_{1} & =\frac{1}{2 \pi^{2}} \int_{0}^{\infty} \mathrm{d} k \int_{-\infty}^{0} \mathrm{~d} \eta \eta k H e^{i k\left(x-c_{S} \eta\right)} \\
& =-\frac{i H\left(\eta=-|\mathbf{x}| / c_{S}\right)}{2 \pi^{2} c_{S}^{2}}, \\
B_{2} & =\frac{1}{2 \pi^{2}} \int_{0}^{\infty} \mathrm{d} k \int_{-\infty}^{0} \mathrm{~d} \eta \eta k H e^{i k\left(x+c_{S} \eta\right)} \\
& =\frac{i H\left(\eta=-|\mathbf{x}| / c_{S}\right)}{2 \pi^{2} c_{S}^{2}},
\end{aligned}
$$

$$
\begin{aligned}
B_{3}= & \frac{1}{2 \pi^{2}} \int_{0}^{\infty} \mathrm{d} k \int_{-\infty}^{0} \mathrm{~d} \eta \eta k H e^{i k x} \sin k c_{S} \eta \\
= & \frac{H\left(\eta=-|\mathbf{x}| / c_{S}\right)}{2 \pi^{2} c_{S}^{2}}, \\
B_{4}^{\xi \theta_{1}}= & -i \frac{H \Gamma(3-2 \Lambda)}{(2 \pi)^{3}} \frac{\left(\Lambda-\frac{1}{2}\right)}{\left(\Lambda-\frac{3}{2}\right)}\left[1+(-1)^{-2 \Lambda}\right]\left(\frac{c_{S}}{2}\right)^{-2 \Lambda} \\
& \times \xi^{-\Lambda}\left[\xi^{\frac{3}{2}-\Lambda}+(-1)^{\frac{1}{2}-\Lambda} \theta^{\frac{3}{2}-\Lambda}\right](-i x)^{2 \Lambda-3} \\
B_{5}^{\xi \theta_{2}}= & -2 \frac{H}{(2 \pi)^{3}} \frac{\left(\Lambda-\frac{1}{2}\right)}{\left(\Lambda-\frac{3}{2}\right)}\left[1+(-1)^{-2 \Lambda}\right]\left(-\frac{1}{2 \theta_{2}}\right)^{-2 \Lambda} \\
& \times \xi^{-\Lambda}\left[\xi^{\frac{3}{2}-\Lambda}+(-1)^{\frac{1}{2}-\Lambda} \theta^{\frac{3}{2}-\Lambda}\right] x^{-3} .
\end{aligned}
$$

\section{For Case II:}

$$
\begin{aligned}
B_{1} & =\frac{1}{4 \pi^{2}} \int_{0}^{\infty} \mathrm{d} k \int_{-\infty}^{0} \mathrm{~d} \eta \int_{\Theta_{1}}^{\Theta_{2}} \mathrm{~d} \Theta \eta k H e^{i k\left(x \cos \Theta+c_{S} \eta\right)} \\
& =-\int_{\Theta_{1}}^{\Theta_{2}} \mathrm{~d} \Theta \frac{i H\left(\eta=-\frac{|\mathbf{x}| \cos \Theta}{c_{S}}\right)}{4 \pi^{2} c_{S}^{2}} \cos \Theta
\end{aligned}
$$

$$
\begin{aligned}
B_{2} & =\frac{1}{4 \pi^{2}} \int_{0}^{\infty} \mathrm{d} k \int_{-\infty}^{0} \mathrm{~d} \eta \int_{\Theta_{1}}^{\Theta_{2}} \mathrm{~d} \Theta \eta k e^{i k\left(x \cos \Theta+c_{S} \eta\right)} \\
& =\int_{\Theta_{1}}^{\Theta_{2}} \mathrm{~d} \Theta \frac{i H\left(\eta=-\frac{|\mathbf{x}| \cos \Theta}{c_{S}}\right)}{4 \pi^{2} c_{S}^{2}} \cos \Theta
\end{aligned}
$$

$$
\begin{aligned}
B_{3}= & \frac{1}{4 \pi^{2}} \int_{0}^{\infty} \mathrm{d} k \int_{-\infty}^{0} \mathrm{~d} \eta \int_{\Theta_{1}}^{\Theta_{2}} \mathrm{~d} \Theta \eta k e^{i k x \cos \Theta} \sin k c_{S} \eta \\
= & \int_{\Theta_{1}}^{\Theta_{2}} \mathrm{~d} \Theta \frac{H\left(\eta=-\frac{|\mathbf{x}| \cos \Theta}{c_{S}}\right)}{4 \pi^{2} c_{S}^{2}} \cos \Theta \\
B_{4}^{\xi \theta_{1}}= & -i \frac{H}{(2 \pi)^{3}} \frac{\left(\Lambda-\frac{1}{2}\right)}{\left(\Lambda-\frac{3}{2}\right)}\left[1+(-1)^{-2 \Lambda}\right]\left(\frac{c_{S}}{2}\right)^{-2 \Lambda} \\
& \times \xi^{-\Lambda}\left[\xi^{\frac{3}{2}-\Lambda}+(-1)^{\frac{1}{2}-\Lambda} \theta_{1}^{\frac{3}{2}-\Lambda}\right] Q_{1}
\end{aligned}
$$




$$
\begin{aligned}
B_{5}^{\xi \theta_{2}}= & -i \frac{H}{(2 \pi)^{3}} \frac{\left(\Lambda-\frac{1}{2}\right)}{\left(\Lambda-\frac{3}{2}\right)}\left[1+(-1)^{-2 \Lambda}\right]\left(-\frac{1}{2 \theta_{2}}\right)^{-2 \Lambda} \\
& \times \xi^{-\Lambda}\left[\xi^{\frac{3}{2}-\Lambda}+(-1)^{\frac{1}{2}-\Lambda} \theta_{2}^{\frac{3}{2}-\Lambda}\right] Q_{2}
\end{aligned}
$$

where $\Theta_{1}$ and $\Theta_{2}$ play the role of angular regulator in the present context and $Q_{1}$ and $Q_{2}$ are defined as

$$
\begin{aligned}
Q_{1}= & \int_{0}^{\pi} \int_{0}^{\infty} \mathrm{d} \Theta \mathrm{d} k e^{i k x \cos \Theta} k^{2-2 \Lambda} \\
= & \frac{i \sqrt{\pi}(-i x)^{2 \Lambda} \sec (2 \pi \Lambda) \Gamma(3-2 \Lambda)}{8 x^{3} \Gamma^{2}(2-\Lambda) \Gamma(2 \Lambda-2) \Gamma\left(2 \Lambda-\frac{3}{2}\right)} \\
& \times\left[\pi^{3 / 2} 4^{\Lambda} \Gamma\left(2 \Lambda-\frac{3}{2}\right)+4\left(e^{4 i \pi \Lambda}-1\right) 2\right. \\
& \times F_{1}\left(\frac{1}{2}, 2 \Lambda-2 ; 2 \Lambda-\frac{3}{2} ;-1\right) \\
& \left.\times \Gamma^{2}(2-\Lambda) \Gamma^{2}(2 \Lambda-2)\right]
\end{aligned}
$$

limiting cases $\left|k c_{S} \eta\right| \rightarrow-\infty,\left|k c_{S} \eta\right| \rightarrow 0$ and $\left|k c_{S} \eta\right| \approx 1$ as given by

$$
\left[\hat{O}_{\mathrm{obs}}\right]_{m \approx H} \stackrel{\left|k c_{S} \eta\right| \rightarrow-\infty}{=} \frac{2 \tilde{c}_{S}}{\left(-k \eta \tilde{c}_{S}\right)^{2} H} \mathcal{I}_{1}^{I},
$$

$$
\left[\hat{O}_{\mathrm{obs}}\right]_{m \approx H} \stackrel{\left|k c_{S} \eta\right| \rightarrow 0}{=} \frac{2^{3-2 \Lambda} c_{S}^{3} \pi}{\left(-k \eta c_{S}\right)^{3-2 \Lambda} H}\left|\frac{\Gamma\left(\frac{3}{2}\right)}{\Gamma(\Lambda)}\right|^{2} \mathcal{I}_{2}^{I} \text {, }
$$$$
\left[\hat{O}_{\mathrm{obs}}\right]_{m \approx H} \stackrel{\left|k c_{S} \eta\right| \approx 1}{=} \frac{2^{3-2 \Lambda} c_{S}^{3} \pi}{H}\left|\frac{\Gamma\left(\frac{3}{2}\right)}{\Gamma(\Lambda)}\right|^{2} \mathcal{I}_{3}^{I},
$$

where for $m \approx H$ the integrals $\mathcal{I}_{1}^{I}, \mathcal{I}_{2}^{I}$ and $\mathcal{I}_{3}^{I}$ are given by the following expressions:

$$
\begin{aligned}
\mathcal{I}_{1}^{I}= & -H \frac{\left[\left|C_{2}\right|^{2} I_{1}-\left|C_{1}\right|^{2} I_{2}-i\left(C_{1}^{*} C_{2} e^{i \pi\left(\Lambda+\frac{1}{2}\right)}+C_{1} C_{2}^{*} e^{-i \pi\left(\Lambda+\frac{1}{2}\right)}\right) I_{3}\right]}{\left[\left|C_{2}\right|^{2}+\left|C_{1}\right|^{2}+\left(C_{1}^{*} C_{2} e^{2 i k c_{S} \eta} e^{i \pi\left(\Lambda+\frac{1}{2}\right)}+C_{1} C_{2}^{*} e^{-2 i k c_{S} \eta} e^{-i \pi\left(\Lambda+\frac{1}{2}\right)}\right)\right]} \\
& = \begin{cases}H I_{2} & \text { for Bunch Davies, } \\
-H \frac{\left[\sinh ^{2} \alpha I_{1}-\cosh ^{2} \alpha I_{2}+i \sinh 2 \alpha \cos \left(\pi\left(\Lambda+\frac{1}{2}\right)+\delta\right) I_{3}\right]}{\left[\sinh ^{2} \alpha+\cosh ^{2} \alpha+\sinh 2 \alpha \cos \left(2 k c_{S} \eta+\pi\left(\Lambda+\frac{1}{2}\right)+\delta\right)\right]} & \text { for } \alpha \text { vacua Type-I, } \\
-H \frac{\left[e^{\alpha+\alpha^{*}} I_{1}-I_{2}+i\left(e^{\alpha} e^{i \pi\left(\Lambda+\frac{1}{2}\right)}+e^{\alpha^{*}} e^{-i \pi\left(\Lambda+\frac{1}{2}\right)}\right) I_{3}\right]}{4 \cos ^{2}\left(k c_{S} \eta+\frac{\pi}{2}\left(\Lambda+\frac{1}{2}\right)-i \frac{\alpha}{2}\right)} & \text { for } \alpha \text { vacua Type-II, } \\
-H \frac{i \pi^{2} I_{3} \cos ^{2} \frac{\pi}{2}\left(\Lambda+\frac{1}{2}\right)}{\cos ^{2}\left(k c_{S} \eta+\frac{\pi}{2}\left(\Lambda+\frac{1}{2}\right)\right)} & \text { for special vacua, }\end{cases}
\end{aligned}
$$

$$
\begin{aligned}
Q_{2}= & \int_{0}^{\pi} \int_{0}^{\infty} d \Theta \mathrm{d} k e^{i k x \cos \Theta} k^{2} \\
= & \frac{i}{x^{3}}\left[\ln \left[\frac{\tan \left(\frac{\pi}{4}+\frac{\Theta_{2}}{2}\right)}{\tan \left(\frac{\pi}{4}+\frac{\Theta_{1}}{2}\right)}\right]\right. \\
& \left.+\sec \Theta_{1} \tan \Theta_{1}-\sec \Theta_{2} \tan \Theta_{2}\right] .
\end{aligned}
$$

Let us now write down the expressions for newly introduced cosmological observable $\hat{O}_{\text {obs }}$ for $m \approx H$ in the three
$\mathcal{I}_{2}=\sqrt{\xi} H I_{4}^{\xi \theta_{1}}$

$\mathcal{I}_{3}=\sqrt{\xi} H I_{5}^{\xi \theta_{2}}$

The result obtained in this section implies that if we take $m \approx H$ then it may be possible to measure the effect of Bell violation in the context of primordial cosmology, specifically for the inflationary paradigm. In this case the scale of inflation is comparable of the order of the mass parameter $m$. In such a case to get unique prediction of the scale of inflation and as well as the nature of the new particle by knowing the impact of one point function or the newly defined observable. 
Additionally it is important to note that, as the result in this case is dependent on the temporal cut-off scale $\xi$, we need to choose this parameter in such a way that the obtained results are consistent with the numerical value of all other inflationary observables as recently observed by Planck.

\subsubsection{Case II: $m>>H$}

For further simplification we consider here $m \approx \Upsilon H$ with $\Upsilon>>1$ and also consider the three limiting cases $\left|k c_{S} \eta\right| \rightarrow$ $-\infty,\left|k c_{S} \eta\right| \rightarrow 0$ and $\left|k c_{S} \eta\right| \approx 1$, which are physically acceptable in the present context. where the integrals $I_{1}, I_{2}, I_{3}, I_{4}^{\xi \theta_{1}}, I_{5}^{\xi \theta_{2}}$ are defined earler. Additionally, it is important to note that here the parameter $\Lambda$ is given by: ${ }^{14}$

For $m_{\mathrm{inf}}<<H: \quad \Lambda \approx \begin{cases}\frac{3}{2} & \text { for } \mathrm{dS}, \\ v & \text { for } \mathrm{qdS} .\end{cases}$

$$
\begin{aligned}
& \left\langle\zeta_{\mathbf{k}}(\eta=0)\right\rangle_{\left|k c_{S} \eta\right| \rightarrow-\infty} \approx-\frac{16 \Upsilon \pi^{2} H \tilde{c}_{S}}{M_{p}^{2} \epsilon}\left[\left|C_{2}\right|^{2} I_{1}-\left|C_{1}\right|^{2} I_{2}-i\left(C_{1}^{*} C_{2} e^{i \pi\left(\Lambda+\frac{1}{2}\right)}+C_{1} C_{2}^{*} e^{-i \pi\left(\Lambda+\frac{1}{2}\right)}\right) I_{3}\right] \\
& = \begin{cases}\frac{8 \Upsilon \pi^{3} H \tilde{c}_{S}}{M_{p}^{2} \epsilon} I_{2} & \text { for Bunch Davies, } \\
-\frac{16 \Upsilon \pi^{2} H \tilde{c_{S}}}{M_{p}^{2} \epsilon}\left[\sinh ^{2} \alpha I_{1}-\cosh ^{2} \alpha I_{2}-i \sinh 2 \alpha \cos \left(\pi\left(\Lambda+\frac{1}{2}\right)+\delta\right) I_{3}\right] & \text { for } \alpha \text { vacua Type-I } \\
-\frac{16 \Upsilon \pi^{2}\left|N_{\alpha}\right|^{2} H \tilde{c}_{S}}{M_{p}^{2} \epsilon}\left[e^{\alpha+\alpha^{*}} I_{1}-I_{2}-i\left(e^{\alpha} e^{i \pi\left(\Lambda+\frac{1}{2}\right)}+e^{\alpha^{*}} e^{-i \pi\left(\Lambda+\frac{1}{2}\right)}\right) I_{3}\right] & \text { for } \alpha \text { vacua Type-II } \\
-\frac{64 i \Upsilon \pi^{2}|C|^{2} H \tilde{c}_{S}}{M_{p}^{2} \epsilon} \cos ^{2} \frac{\pi}{2}\left(\Lambda+\frac{1}{2}\right) I_{3} & \text { for special vacua }\end{cases} \\
& \left\langle\zeta_{\mathbf{k}}(\eta=\xi \rightarrow 0)\right\rangle_{\left|k c_{S} \eta\right| \rightarrow 0}=\frac{4 \Upsilon \pi \sqrt{\xi} H \tilde{c}_{S}}{M_{p}^{2} \epsilon}\left(C_{1}^{*} C_{2}+C_{1} C_{2}^{*}-\left|C_{1}\right|^{2}-\left|C_{2}\right|^{2}\right) I_{4}^{\xi \theta_{1}} \\
& =I_{4}^{\xi \theta_{1}} \times \begin{cases}-\frac{2 \Upsilon \pi^{2} \sqrt{\xi} H \tilde{c}_{S}}{M_{p}^{2} \epsilon} & \text { for Bunch Davies vacua, } \\
\frac{4 \Upsilon \pi \sqrt{\xi} H \tilde{c}_{S}}{M_{p}^{2} \epsilon}\left(\cos \delta \sinh 2 \alpha-\cosh ^{2} \alpha-\sinh ^{2} \alpha\right) & \text { for } \alpha \text { vacua Type-I, } \\
\frac{4 \Upsilon \pi \sqrt{\xi}\left|N_{\alpha}\right|^{2} H \tilde{c}_{S}}{M_{p}^{2} \epsilon}\left(e^{\alpha}+e^{\alpha^{*}}-e^{\alpha+\alpha^{*}}-1\right) & \text { for } \alpha \text { vacua Type-II }, \\
0 & \text { for special vacua, }\end{cases} \\
& \left\langle\zeta_{\mathbf{k}}(\eta=\xi \rightarrow 0)\right\rangle_{\left|k c_{S} \eta\right| \approx 1}=\frac{4 \Upsilon \pi \sqrt{\xi} H \tilde{c}_{S}}{M_{p}^{2} \epsilon}\left(C_{1}^{*} C_{2}+C_{1} C_{2}^{*}-\left|C_{1}\right|^{2}-\left|C_{2}\right|^{2}\right) I_{5}^{\xi \theta_{2}} \\
& =I_{5}^{\xi \theta_{2}} \times \begin{cases}-\frac{2 \Upsilon \pi^{2} \sqrt{\xi} H \tilde{c}_{S}}{M_{p}^{2} \epsilon} & \text { for Bunch Davies vacua, } \\
\frac{4 \Upsilon \pi \sqrt{\xi} H \tilde{c}_{S}}{M_{p}^{2} \epsilon}\left(\cos \delta \sinh 2 \alpha-\cosh ^{2} \alpha-\sinh ^{2} \alpha\right) & \text { for } \alpha \text { vacua Type-I, } \\
\frac{4 \Upsilon \pi \sqrt{\xi}\left|N_{\alpha}\right|^{2} H \tilde{c}_{S}}{M_{p}^{2} \epsilon}\left(e^{\alpha}+e^{\alpha^{*}}-e^{\alpha+\alpha^{*}}-1\right) & \text { for } \alpha \text { vacua Type-II }, \\
0 & \text { for special vacua, }\end{cases}
\end{aligned}
$$

$\overline{{ }^{14} \text { For the case }} m>>H$ the approximation $m_{\mathrm{inf}} \approx m$ is not valid as the mass scale of the inflaton cannot be larger than the scale of inflation. 
Similarly in position space the representative expressions for the expectation value of the scalar curvature perturbation along with two limiting cases $\left|k c_{S} \eta\right| \rightarrow-\infty$ and $\left|k c_{S} \eta\right| \rightarrow 0$ are given by

$$
\begin{aligned}
\langle\zeta(\mathbf{x}, \eta=0)\rangle_{\left|k c_{S} \eta\right| \rightarrow-\infty} \approx & -\frac{2 \Upsilon H \tilde{c}_{S}}{M_{p}^{2} \epsilon \pi}\left[\left|C_{2}\right|^{2} B_{1}-\left|C_{1}\right|^{2} B_{2}-i\left(C_{1}^{*} C_{2} e^{i \pi\left(\Lambda+\frac{1}{2}\right)}+C_{1} C_{2}^{*} e^{-i \pi\left(\Lambda+\frac{1}{2}\right)}\right) B_{3}\right] \\
& =\left\{\begin{array}{cc}
\frac{H \Upsilon \tilde{c}_{S}}{M_{p}^{2} \epsilon} B_{2} & \text { for Bunch Davies, } \\
-\frac{2 H \Upsilon \tilde{c}_{S}}{M_{p}^{2} \epsilon \pi}\left[\sinh ^{2} \alpha B_{1}-\cosh ^{2} \alpha B_{2}\right. & \\
\left.-i \sinh 2 \alpha \cos \left(\pi\left(\Lambda+\frac{1}{2}\right)+\delta\right) B_{3}\right] & \text { for } \alpha \text { vacua Type-I, } \\
-\frac{2\left|N_{\alpha}\right|^{2} H \tilde{c}_{S}}{M_{p}^{2} \epsilon \pi}\left[e^{\alpha+\alpha^{*}} B_{1}-B_{2}\right. & \text { for special vacua, } \\
\left.-i\left(e^{\alpha} e^{i \pi\left(\Lambda+\frac{1}{2}\right)}+e^{\alpha^{*}} e^{-i \pi\left(\Lambda+\frac{1}{2}\right)}\right) B_{3}\right] & \text { for } \alpha \text { vacua Type-II, } \\
-\frac{8 i \Upsilon|C|^{2} H \tilde{c}_{S}}{M_{p}^{2} \epsilon \pi} \cos ^{2} \frac{\pi}{2}\left(\Lambda+\frac{1}{2}\right) B_{3} &
\end{array}\right.
\end{aligned}
$$

$\langle\zeta(\mathbf{x}, \eta=\xi \rightarrow 0)\rangle_{\left|k c_{S} \eta\right| \rightarrow 0}=\frac{\Upsilon \sqrt{\xi} H \tilde{c}_{S}}{2 M_{p}^{2} \epsilon \pi^{2}}\left(C_{1}^{*} C_{2}+C_{1} C_{2}^{*}-\left|C_{1}\right|^{2}-\left|C_{2}\right|^{2}\right) B_{4}^{\xi \theta_{1}}$

$$
=B_{4}^{\xi \theta_{1}} \times \begin{cases}-\frac{2 \Upsilon \pi^{2} \sqrt{\xi} H \tilde{c}_{S}}{M_{p}^{2} \epsilon} & \text { for Bunch Davies vacua } \\ \frac{\Upsilon \sqrt{\xi} H \tilde{c}_{S}}{2 M_{p}^{2} \pi^{2} \epsilon}\left(\cos \delta \sinh 2 \alpha-\cosh ^{2} \alpha-\sinh ^{2} \alpha\right) & \text { for } \alpha \text { vacua Type-I, } \\ \frac{\Upsilon \sqrt{\xi}\left|N_{\alpha}\right|^{2} H \tilde{c}_{S}}{2 M_{p}^{2} \pi^{2} \epsilon}\left(e^{\alpha}+e^{\alpha^{*}}-e^{\alpha+\alpha^{*}}-1\right) & \text { for } \alpha \text { vacua Type-II } \\ 0 & \text { for special vacua }\end{cases}
$$

$\langle\zeta(\mathbf{x}, \eta=\xi \rightarrow 0)\rangle_{\left|k c_{S} \eta\right| \approx 1}=\frac{\Upsilon \sqrt{\xi} H \tilde{c}_{S}}{2 M_{p}^{2} \epsilon \pi^{2}}\left(C_{1}^{*} C_{2}+C_{1} C_{2}^{*}-\left|C_{1}\right|^{2}-\left|C_{2}\right|^{2}\right) B_{5}^{\xi \theta_{2}}$

$$
=B_{5}^{\xi \theta_{2}} \times \begin{cases}-\frac{2 \Upsilon \pi^{2} \sqrt{\xi} H \tilde{c}_{S}}{M_{p}^{2} \epsilon} & \text { for Bunch Davies vacua, } \\ \frac{\Upsilon \sqrt{\xi} H \tilde{c}_{S}}{2 M_{p}^{2} \pi^{2} \epsilon}\left(\cos \delta \sinh 2 \alpha-\cosh ^{2} \alpha-\sinh ^{2} \alpha\right) & \text { for } \alpha \text { vacua Type-I, } \\ \frac{\Upsilon \sqrt{\xi}\left|N_{\alpha}\right|^{2} H \tilde{c}_{S}}{2 M_{p}^{2} \pi^{2} \epsilon}\left(e^{\alpha}+e^{\alpha^{*}}-e^{\alpha+\alpha^{*}}-1\right) & \text { for } \alpha \text { vacua Type-II, } \\ 0 & \text { for special vacua, }\end{cases}
$$

where the integrals $B_{1}, B_{2}, B_{3}, B_{4}^{\xi \theta_{1}}, B_{5}^{\xi \theta_{2}}$ are defined earlier.

Let us now write down the expressions for newly introduced cosmological observable $\hat{O}_{\text {obs }}$ for $m \approx H$ in the three limiting cases $\left|k c_{S} \eta\right| \rightarrow-\infty,\left|k c_{S} \eta\right| \rightarrow 0$ and $\left|k c_{S} \eta\right| \approx 1$ as given by

$\left[\hat{O}_{\mathrm{obs}}\right]_{m>>H} \stackrel{\left|k c_{S} \eta\right| \rightarrow-\infty}{=} \frac{2 \tilde{c}_{S}}{\left(-k \eta \tilde{c}_{S}\right)^{2} H} \mathcal{I}_{1}^{I}$ 
$\left[\hat{O}_{\mathrm{obs}}\right]_{m>>H} \stackrel{\mid k c s \underline{\eta}=\rightarrow 0}{=} \frac{2^{3-2 \Lambda} c_{S}^{3} \pi}{\left(-k \eta c_{S}\right)^{3-2 \Lambda} H}\left|\frac{\Gamma\left(\frac{3}{2}\right)}{\Gamma(\Lambda)}\right|^{2} \mathcal{I}_{2}^{I}$,

$\left[\hat{O}_{\mathrm{obs}}\right]_{m>>H} \stackrel{\left|k c_{S} \eta\right| \approx 1}{=} \frac{2^{3-2 \Lambda} c_{S}^{3} \pi}{H}\left|\frac{\Gamma\left(\frac{3}{2}\right)}{\Gamma(\Lambda)}\right|^{2} \mathcal{I}_{3}^{I}$,

where for $m \approx H$ the integrals $\mathcal{I}_{1}^{I}, \mathcal{I}_{2}^{I}$ and $\mathcal{I}_{3}^{I}$ are given by the following expressions: $\left|k c_{S} \eta\right| \approx 1$, which are physically acceptable in the present context.

$$
\begin{aligned}
\left\langle\zeta_{\mathbf{k}}(\eta=0)\right\rangle_{\left|k c_{S} \eta\right| \rightarrow-\infty} & \approx 0, \\
\left\langle\zeta_{\mathbf{k}}(\eta=\xi \rightarrow 0)\right\rangle_{\left|k c_{S} \eta\right| \rightarrow 0} & \approx 0, \\
\left\langle\zeta_{\mathbf{k}}(\eta=\xi \rightarrow 0)\right\rangle_{\left|k c_{S} \eta\right| \approx 1} & \approx 0 .
\end{aligned}
$$

Additionally, it is important to note that here the parameter $\Lambda$ is given by ${ }^{15}$

$$
\begin{aligned}
& \mathcal{I}_{1}^{I}=-\Upsilon H \frac{\left[\left|C_{2}\right|^{2} I_{1}-\left|C_{1}\right|^{2} I_{2}-i\left(C_{1}^{*} C_{2} e^{i \pi\left(\Lambda+\frac{1}{2}\right)}+C_{1} C_{2}^{*} e^{-i \pi\left(\Lambda+\frac{1}{2}\right)}\right) I_{3}\right]}{\left[\left|C_{2}\right|^{2}+\left|C_{1}\right|^{2}+\left(C_{1}^{*} C_{2} e^{2 i k c_{S} \eta} e^{i \pi\left(\Lambda+\frac{1}{2}\right)}+C_{1} C_{2}^{*} e^{-2 i k c_{S} \eta} e^{-i \pi\left(\Lambda+\frac{1}{2}\right)}\right)\right]} \\
& = \begin{cases}\Upsilon H I_{2} & \text { for Bunch Davies, } \\
-\Upsilon H \frac{\left[\sinh ^{2} \alpha I_{1}-\cosh ^{2} \alpha I_{2}+i \sinh 2 \alpha \cos \left(\pi\left(\Lambda+\frac{1}{2}\right)+\delta\right) I_{3}\right]}{\left[\sinh ^{2} \alpha+\cosh ^{2} \alpha+\sinh 2 \alpha \cos \left(2 k c_{S} \eta+\pi\left(\Lambda+\frac{1}{2}\right)+\delta\right)\right]} & \text { for } \alpha \text { vacua Type-I, } \\
-\Upsilon H \frac{\left[e^{\alpha+\alpha^{*}} I_{1}-I_{2}+i\left(e^{\alpha} e^{i \pi\left(\Lambda+\frac{1}{2}\right)}+e^{\alpha^{*}} e^{-i \pi\left(\Lambda+\frac{1}{2}\right)}\right) I_{3}\right]}{4 \cos ^{2}\left(k c_{S} \eta+\frac{\pi}{2}\left(\Lambda+\frac{l}{2}\right)-i \frac{\alpha}{2}\right)} & \text { for } \alpha \text { vacua Type-II, } \\
-\Upsilon H \frac{i \pi^{2} I_{3} \cos ^{2} \frac{\pi}{2}\left(\Lambda+\frac{1}{2}\right)}{\cos ^{2}\left(k c_{S} \eta+\frac{\pi}{2}\left(\Lambda+\frac{1}{2}\right)\right)} & \text { for special vacua, }\end{cases}
\end{aligned}
$$

$\mathcal{I}_{2}=\sqrt{\xi} \Upsilon H I_{4}^{\xi \theta_{1}}$,

$\mathcal{I}_{3}=\sqrt{\xi} \Upsilon H I_{5}^{\xi \theta_{2}}$.

The results obtained in this section implies that if we take $m>>H$ then it may be possible to measure the effect of Bell violation in the context of primordial cosmology, specifically for the inflationary paradigm. In such a case to get unique prediction of the scale of inflation and associated new physics we need to measure the impact of one point function or the newly defined observable and in such a case we do not need to wait for future observations for primordial gravitational waves and primordial non-Gaussianity to comment on the unique scale of inflation in a model-independent way. But as the result in this case is dependent on the temporal cut-off scale $\xi$, we need to choose this parameter in such a way that the obtained results are consistent with the numerical value of all other inflationary observables as recently observed by Planck.

\subsubsection{Case III: $m<<H$}

For further simplification we consider here $m<<H$ with the three limiting cases $\left|k c_{S} \eta\right| \rightarrow-\infty,\left|k c_{S} \eta\right| \rightarrow 0$ and
For $m_{\mathrm{inf}}<<H: \quad \Lambda \approx \begin{cases}\frac{3}{2} & \text { for dS } \\ v & \text { for qdS }\end{cases}$

For $m_{\mathrm{inf}} \approx H: \quad \Lambda \approx \begin{cases}\frac{\sqrt{5}}{2} & \text { for dS } \\ \sqrt{v^{2}-1} & \text { for qdS. }\end{cases}$

After taking the Fourier transform in position space we get

$$
\begin{aligned}
\langle\zeta(\mathbf{x}, \eta=0)\rangle_{\left|k c_{S} \eta\right| \rightarrow-\infty} & \approx 0, \\
\langle\zeta(\mathbf{x}, \eta=\xi \rightarrow 0)\rangle_{\left|k c_{S} \eta\right| \rightarrow 0} & \approx 0, \\
\langle\zeta(\mathbf{x}, \eta=\xi \rightarrow 0)\rangle_{\left|k c_{S} \eta\right| \approx 1} & \approx 0 .
\end{aligned}
$$

Let us now define a new cosmological observable $\hat{O}_{\text {obs }}$ in the three limiting cases $\left|k c_{S} \eta\right| \rightarrow-\infty,\left|k c_{S} \eta\right| \rightarrow 0$ and $\left|k c_{S} \eta\right| \approx 1$ as

$\hat{O}_{\mathrm{obs}} \stackrel{\left|k c_{S} \eta\right| \rightarrow-\infty}{=} \frac{2 \tilde{c}_{S}}{\left(-k \eta \tilde{c}_{S}\right)^{2} H} \mathcal{I}_{1} \approx 0$,

$\overline{15}$ For the special case where inflaton mass are comparable with the new particle then for $m_{\text {inf }} \approx m<<H$ we have

$\Lambda \approx \begin{cases}\frac{3}{2} & \text { for } \mathrm{dS}, \\ v & \text { for } \mathrm{qdS} .\end{cases}$ 
$\hat{O}_{\mathrm{obs}} \stackrel{\mid k c_{S} \underline{\eta} \rightarrow \rightarrow 0}{=} \frac{2^{3-2 \Lambda} c_{S}^{3} \pi}{\left(-k \eta c_{S}\right)^{3-2 \Lambda} H}\left|\frac{\Gamma\left(\frac{3}{2}\right)}{\Gamma(\Lambda)}\right|^{2} \mathcal{I}_{2} \approx 0$,

$\hat{O}_{\mathrm{obs}} \stackrel{\left|k c_{S} \eta\right| \approx 1}{=} \frac{2^{3-2 \Lambda} c_{S}^{3} \pi}{H}\left|\frac{\Gamma\left(\frac{3}{2}\right)}{\Gamma(\Lambda)}\right|^{2} \mathcal{I}_{3} \approx 0$,

where for $m<<H$ cases $\mathcal{I}_{1}, \mathcal{I}_{2}$ and $\mathcal{I}_{3}$ are defined as

$\mathcal{I}_{1} \approx 0$

$\mathcal{I}_{2} \approx 0$,

$\mathcal{I}_{3} \approx 0$.

The results obtained in this section implies that if we take $m<<H$ then it is not possible to measure the effect of Bell violation in the context of primordial cosmology, specifically for the inflationary paradigm. In such a case to get unique prediction of the scale of inflation and associated new physics we need to wait for future observations for primordial gravitational waves and primordial non-Gaussianity.

\section{Specific example: analogy with axion fluctuations in string theory}

In this section we discuss the axion fluctuations originating from string theory and its exact connection with the present topic of discussion in this paper. Here we discuss the background string theoretic framework and its four dimensional effective field theory version which will participate in the axion fluctuations in primordial cosmology. See Refs. [6780] for further details in this direction.

\subsection{Axion monodromy model}

Let us start our discussion with the canonically normalized string theory originating the axion action:

$S_{\text {axion }}=\int \mathrm{d}^{4} x \sqrt{-g}\left[-\frac{1}{2}(\partial \phi)^{2}-V(\phi)\right]$,

where $\phi$ is the axion field and the corresponding potential from string theory can be expressed as

$$
\begin{aligned}
V(\phi) & =\mu^{3} \phi+\Lambda_{C}^{4} \cos \left(\frac{\phi}{f_{a}}\right) \\
& =\mu^{3}\left[\phi+b f_{a} \cos \left(\frac{\phi}{f_{a}}\right)\right],
\end{aligned}
$$

where we define a new parameter $b$ as

$b=\frac{\Lambda_{C}^{4}}{\mu^{3} f_{a}}$.

Here it is important to mention that the linear part of the axion potential as appearing in Eq. (4.2) has been derived in the context of string theory in Ref. [80], whereas the cosine part of the axion potential has its origin in non-perturbative aspects in string theory [70].

One can also express the axion action by introducing a dimensionless axion field, $\phi=a f_{a}$ as

$S_{\text {axion }}=\int \mathrm{d}^{4} x \sqrt{-g}\left[-\frac{f_{a}^{2}}{2}(\partial a)^{2}-U(a)\right]$,

where the effective axion potential can be recast as

$$
\begin{aligned}
U(a)=V\left(a f_{a}\right) & =\mu^{3} a f_{a}+\Lambda_{C}^{4} \cos a \\
& =\mu^{3} f_{a}[a+b \cos a] .
\end{aligned}
$$

Further introducing conformal time in this computation axion action can be recast as

$S_{\text {axion }}=\int \mathrm{d} \eta \mathrm{d}^{3} x\left[\frac{f_{a}^{2}(\eta)}{2 H^{2}} \frac{\left[\left(\partial_{\eta} a\right)^{2}-\left(\partial_{i} a\right)^{2}\right]}{\eta^{2}}-\frac{U(a)}{H^{4} \eta^{4}}\right]$,

where only mass contribution for the axion field will contribute to the fluctuations and other part can be treated as back-reaction effect which one can neglect due to its smallness.

Additionally, it is important to mention the following information regarding the axion action and the representative theoretical setup:

- In this case the scale $\Lambda_{C}$ is characterized by

$$
\Lambda_{C}=\sqrt{m_{\mathrm{SUSY}} M_{p}} e^{-c S_{\mathrm{inst}}},
$$

where $S_{\text {inst }}$ characterize the action of the instanton which gives rise to the effective potential in the present context, $c$ signifies a constant factor which is of the order of unity, $m_{\text {SUSY }}$ represents the supersymmetry breaking mass scale and $M_{p}$ is the reduced Planck mass, defined as

$$
M_{p}=\frac{L^{3}}{\sqrt{\alpha^{\prime}} g_{s}} \text {. }
$$

Substituting this expression in Eq. (4.7) we finally get

$$
\Lambda_{C}=\sqrt{\frac{m_{\mathrm{SUSY}} L^{3}}{\sqrt{\alpha^{\prime}} g_{s}}} e^{-c S_{\mathrm{inst}}}
$$

- Here the overall scale of the effective potential is given by

$$
V_{0}=\mu^{3} f_{a}=\frac{1}{\alpha^{\prime 2} g_{s}} e^{4 A_{0}}+\frac{R^{2}}{\alpha^{\prime} L^{4}} m_{\mathrm{SUSY}}^{4} e^{2 A_{0}} .
$$

where $e^{A_{0}}$ is the warp factor at the bottom portion of the throat, $\alpha^{\prime}$ is the Regge slope parameter, $g_{s}$ is the string 
coupling constant, $L^{6}$ is the volume factor in string units and $R$ approximately characterizes the radius of the AdS like (Klebanov-Strassler) throat region in which the 5 brane and antibranes are placed.

- Here the second term in the effective potential has a periodicity of $2 \pi f_{a}$ and maintains the shift symmetry $\phi \rightarrow \phi+2 \pi f_{a}$. Here $f_{a}$ characterizes the decay constant. On the other hand, the first term in the effective potential breaks the shift symmetry. This implies that the total effective potential breaks the shift symmetry in the present context.

- Additionally, the total scale of the axion effective potential is determined by the term $\mu^{3}$ after introducing the additional parameter $b$. In the present context in terms of string parameters the factor $b$ can be recast as ${ }^{6}$ :

$$
\begin{aligned}
b & =\frac{\Lambda_{C}^{4}}{\mu^{3} f_{a}}=\left(\frac{m_{\mathrm{SUSY}}^{2} M_{p}^{2} e^{-4 c S_{\mathrm{inst}}}}{\frac{1}{\alpha^{\prime 2} g_{s}} e^{4 A_{0}}+\frac{R^{2}}{\alpha^{\prime} L^{4}} m_{\mathrm{SUSY}}^{4} e^{2 A_{0}}}\right) \\
& =\left(\frac{m_{\mathrm{SUSY}}^{2} \frac{L^{6}}{\alpha^{\prime} g_{s}^{2}} e^{-4 c S_{\mathrm{inst}}}}{\frac{1}{\alpha^{\prime 2} g_{s}} e^{4 A_{0}}+\frac{R^{2}}{\alpha^{\prime} L^{4}} m_{\mathrm{SUSY}}^{4} e^{2 A_{0}}}\right) .
\end{aligned}
$$

Here the warp factor $e^{A_{0}}$ is given by

$$
e^{A_{0}}=\left(\frac{\Lambda_{C}}{m_{\mathrm{SUSY}}}\right)^{2} \frac{L}{R} \sqrt{g_{s} \alpha^{\prime}}=\frac{L^{4}}{m_{\mathrm{SUSY}} R} \sqrt{\frac{\alpha^{\prime}}{g_{s}}} e^{-2 c S_{\mathrm{inst}}}
$$

Substituting the explicit expression for the warp factor in $b$ we finally get

$$
\begin{aligned}
b=\frac{\Lambda_{C}^{4}}{\mu^{3} f_{a}} & =\left(\frac{m_{\mathrm{SUSY}}^{2} \frac{L^{6}}{\alpha^{\prime} g_{s}^{2}} e^{-4 c S_{\text {inst }}}}{\frac{L^{16}}{m_{\mathrm{SUSY}}^{4} g_{s}^{3} R^{4}} e^{-8 c S_{\text {inst }}}+\frac{L^{4}}{g_{s}} m_{\mathrm{SUSY}}^{2} e^{-4 c S_{\text {inst }}}}\right) \\
& =\left(\frac{\frac{L^{2}}{\alpha^{\prime} g_{s}}}{1+\frac{L^{12}}{m_{\mathrm{SUSY}}^{6} g_{s}^{2} R^{4}} e^{-4 c S_{\text {inst }}}}\right)
\end{aligned}
$$

Additionally, the warped down string scale at the bottom the throat is given by

$$
M_{s}=\frac{e^{A_{0}}}{\sqrt{\alpha^{\prime}}}=\left(\frac{\Lambda_{C}}{m_{\mathrm{SUSY}}}\right)^{2} \frac{L}{R} \sqrt{g_{s}}=\frac{L^{4}}{m_{\mathrm{SUSY}} R \sqrt{g_{s}}} e^{-2 c S_{\mathrm{inst}}} .
$$

- Most importantly if one can treat the axion decay constant $f_{a}$ is background conformal time scale dependent or if one interpret this to be parameter of string theory then in our present discussion of the paper it exactly mimics the role of slow-roll parameter $\epsilon$ rescaled with sound speed
$c_{S}$ of the new particle. Most importantly, one can treat such new particles as the axion field originating from string theory.

- In this context we also assume that the axion decay constant $f_{a}$ is inflaton field dependent and this is perfectly consistent with the fact that $f_{a}$ is background conformal time scale dependent.

- In the present context we also assume that the axion decay constant $f_{a}$ initially becomes large compared the Hubble scale during inflation i.e. $f_{a}>>H$ and becomes smaller compared to the Hubble scale i.e. $f_{a}<<H$ during some time interval, a few e-foldings after the massive new particles were created. Then it becomes large again. Here due to the increase in the value of the axion decay constant $f_{a}$ one can suppress the effect of quantum fluctuations at shorter distance scale. As a result in the next setup we get an admissible value for the magnitude of the dimensionless axion field $a=\phi / f_{a}$ at the location of the each created particle from axion. As $f_{a}$ mimics the role of mass parameter $m$ in the present context all the earlier computation for four cases of the choice of mass parameters are valid here.

- In this computation we additionally setup the cosmological detector settings or more precisely the decider variables are adjusted in such a way that it creates an axion field with fluctuations at a characteristic scale controlled by the mutual effects from $\Lambda_{C}^{4}$ and $\mu^{3}$ as introduced earlier. Here the effect from $\Lambda_{C}^{4}$ becomes dominant in the early universe and determines the scale of inflation. On the other hand the effect from the term $\mu^{3}$ becomes larger during late time acceleration of our universe. In the present context, more precisely the energy scales $\Lambda_{C}^{4}$ and $\mu^{3}$ serve the purpose of cosmological constant at early and late times of the evolution of the universe.

- The quantum fluctuations in the dimensionless stringy axionic field $a=\phi / f_{a}$ exactly mimics the role of scalar (curvature) perturbation $\zeta$ as introduced in the earlier section. Additionally it is important to note that the quantum fluctuations in the dimensionless stringy axionic field $a$ are larger at distance scale corresponding to a particular conformal time $x_{\text {dist }} \sim\left|\eta_{c}\right|$, and for distances smaller than $x<x_{\text {dist }} \sim\left|\eta_{c}\right|$ the effects of the quantum fluctuations are also smaller. For this reason, one can interpret such a distance or the corresponding time scale to be the critical one in the present context.

- During the computation detector settings or more precisely the decider variables are chosen in such a way that they will appear locally around each massive particle. Most importantly it is important to mention here that here we also assume that during this process detector settings or more precisely the decider variables are independent from the environment of the other massive particle pair 
in the present context. In our case axion plays the role of such a decider variable.

- In case of axion the cosmological Bell test experimental setup is prepared in such a way that it survives after inflationary epoch up to very late times. After the end of inflation the oscillating and periodic part of the potential $U(a) \sim \Lambda_{C}^{4} \cos a$ gives the mass contribution to the axion field and due to the specific structure of the potential, axion field oscillates at later times after the end of inflation. This additionally implies that in the present context the axion field contributes to explain the dark matter content of the universe. Moreover, apart from explaining fluctuation appearing from curvature perturbation in the context of axion isocurvature fluctuations also contribute to the dark matter. But according to the present day observations, we cannot see such fluctuations at all and here one can interpret that the contribution from the isocurvature fluctuations become highly suppressed in cosmological perturbations to explain the existence of dark matter. But such fluctuations are theoretically useful to determine the initial field value of the dimensionless axion field $a=\phi / f_{a}$.

- The mass contribution to the axion field appearing from the periodic part of the potential $U(a) \sim \Lambda_{C}^{4} \cos a$ is exactly equivalent to the conformal time scale dependent mass parameter $m(\eta)$ in the present context of discussion.

\subsection{Axion effective interaction}

Before going to the further details let us analyze the structure of the effective axionic potential. If we consider the total effective potential then the potential has extrema at $a=a_{0}$ given by the following constraint condition:

$$
\begin{aligned}
U^{\prime}\left(a=a_{0}\right) & =0 \Longrightarrow a_{0}=\frac{\phi_{0}}{f_{a}}=\sin ^{-1}\left(\frac{1}{b}\right) \\
& =\sin ^{-1}\left(\frac{\mu^{3} f_{a}}{\Lambda_{C}^{4}}\right) .
\end{aligned}
$$

Here' signifies derivatives with respect to axion field $a$. In this context the time-dependent effective mass of the dimensionless axion field at extrema $a=a_{0}=\phi_{0} / f_{a}$ is computed from the total axionic effective potential $U(a)$ as

$$
\begin{aligned}
& m_{\text {axion }}^{2}=U^{\prime \prime}\left(a=a_{0}\right)=-\mu^{3} f_{a} b \cos \left(\sin ^{-1}\left(\frac{1}{b}\right)\right) \\
& =-\Lambda_{C}^{4} \cos \left(\sin ^{-1}\left(\frac{\mu^{3} f_{a}}{\Lambda_{C}^{4}}\right)\right) .
\end{aligned}
$$

To get the minima/maxima at the location $a=a_{0}$ we get the following set of possible constraints from the total effective potential:
- For maxima we need:

$$
U^{\prime \prime}\left(a=a_{0}\right)<0
$$

and this implies that in such a situation the axion mass term

$m_{\text {axion }}^{2}<0$

which gives us tachyonic type of instability. Consequently as always $\cos \left(\sin ^{-1}\left(\frac{1}{b}\right)\right)>0$ then here we get the following constraint condition on the axion model parameters:

$\mu^{3} f_{a} b=\Lambda_{C}^{4}>0$

This is possible:

1. If $\mu^{3} f_{a}>0$ and $b>0$ or precisely $\Lambda_{C}^{4}>0$.

2. If $\mu^{3} f_{a}<0$ and $b<0$ or precisely $\Lambda_{C}^{4}>0$.

- For minima we need:

$U^{\prime \prime}\left(a=a_{0}\right)>0$

and this implies that in such a situation the axion mass term

$m_{\text {axion }}^{2}>0$

which avoids tachyonic type of instability and makes the analysis more consistent in the present context. Consequently as always $\cos \left(\sin ^{-1}\left(\frac{1}{b}\right)\right)>0$ then here we get the following constraint condition on the axion model parameters:

$\mu^{3} f_{a} b=\Lambda_{C}^{4}<0$

This is possible:

1. If $\mu^{3} f_{a}>0$ and $b<0$ or precisely $\Lambda_{C}^{4}<0$.

2. If $\mu^{3} f_{a}<0$ and $b>0$ or precisely $\Lambda_{C}^{4}<0$.

On the other hand if we consider the fact that during inflation and in later stages oscillating part of the effective axionic potential contributes larger compared to the linear contribution then for such a periodic structure of the effective potential we get the extrema at $a=a_{0}$ given by the following constraint condition:

$U^{\prime}\left(a=a_{0}\right)=0 \Longrightarrow a_{0}=\frac{\phi_{0}}{f_{a}}=m \pi$,

where $m \subset \mathbf{Z}$. In this context the time-dependent effective mass of the dimensionless axion field at extrema $a=a_{0}=$ 
$\phi_{0} / f_{a}$ is computed from the total axionic effective potential $U(a)$ as

$$
\begin{aligned}
m_{\text {axion }}^{2} & =U^{\prime \prime}\left(a=a_{0}\right)=-\mu^{3} f_{a} b \cos (m \pi) \\
& =-\mu^{3} f_{a} b(-1)^{m} .
\end{aligned}
$$

To get the minima/maxima at the location $a=a_{0}$ we get the following set of possible constraints from the total effective potential:

- For maxima we need:

$U^{\prime \prime}\left(a=a_{0}\right)<0$

and this implies that in such a situation the axion mass term

$m_{\text {axion }}^{2}<0$

which gives us tachyonic type of instability. Consequently we get the following constraint condition on the axion model parameters:

$\mu^{3} f_{a} b(-1)^{m}=\Lambda_{C}^{4}(-1)^{m}>0$

This is possible:

1. If $\mu^{3} f_{a}>0, b>0$ or precisely $\Lambda_{C}^{4}>0$ and $(-1)^{m}>0$ i.e. $m$ is even integer number.

2. If $\mu^{3} f_{a}>0, b<0$ or precisely $\Lambda_{C}^{4}<0$ and $(-1)^{m}<0$ i.e. $m$ is odd integer number.

3. If $\mu^{3} f_{a}<0, b>0$ or precisely $\Lambda_{C}^{4}<0$ and $(-1)^{m}<0$ i.e. $m$ is odd integer number.

4. If $\mu^{3} f_{a}<0, b<0$ or precisely $\Lambda_{C}^{4}>0$ and $(-1)^{m}>0$ i.e. $m$ is even integer number.

- For minima we need:

$U^{\prime \prime}\left(a=a_{0}\right)>0$

and this implies that in such a situation the axion mass term

$m_{\text {axion }}^{2}>0$

which avoids tachyonic type of instability and makes the analysis more consistent in the present context. Consequently we get the following constraint condition on the axion model parameters:

$\mu^{3} f_{a} b(-1)^{m}=\Lambda_{C}^{4}(-1)^{m}<0$

This is possible:
1. If $\mu^{3} f_{a}>0, b>0$ or precisely $\Lambda_{C}^{4}>0$ and $(-1)^{m}<0$ i.e. $m$ is odd integer number.

2. If $\mu^{3} f_{a}>0, b<0$ or precisely $\Lambda_{C}^{4}<0$ and $(-1)^{m}>0$ i.e. $m$ is even integer number.

3. If $\mu^{3} f_{a}<0, b>0$ or precisely $\Lambda_{C}^{4}<0$ and $(-1)^{m}>0$ i.e. $m$ is even integer number.

4. If $\mu^{3} f_{a}<0, b<0$ or precisely $\Lambda_{C}^{4}>0$ and $(-1)^{m}<0$ i.e. $m$ is odd integer number.

Now to comment on the effect of very small back-reaction one can compute the following terms at $a=a_{0}$ :

$$
\begin{aligned}
& U\left(a=a_{0}\right) \\
& \quad= \begin{cases}\mu^{3} f_{a}\left[\sin ^{-1}\left(\frac{1}{b}\right)+b \cos \left(\sin ^{-1}\left(\frac{1}{b}\right)\right)\right] & \text { for total } U(a) \\
\mu^{3} f_{a}\left[m \pi+b(-1)^{m}\right] & \text { for osc. } U(a) .\end{cases}
\end{aligned}
$$

$U^{\prime \prime \prime}\left(a=a_{0}\right)= \begin{cases}\mu^{3} f_{a} & \text { for total } U(a) \\ 0 & \text { for osc. } U(a),\end{cases}$

$$
\begin{aligned}
& U^{\prime \prime \prime \prime}\left(a=a_{0}\right)=\lambda_{\text {self }} \\
& \quad= \begin{cases}\mu^{3} f_{a} b \cos \left(\sin ^{-1}\left(\frac{1}{b}\right)\right) & \text { for total } U(a) \\
\mu^{3} f_{a} b(-1)^{m} & \text { for osc. } U(a) .\end{cases}
\end{aligned}
$$

Here we restrict ourselves to up to fourth derivative terms to make the potential renormalizable. Now if we claim that $\mu^{3} f_{a}$ is small then one can neglect $U\left(a=a_{0}\right), U^{\prime \prime \prime}\left(a=a_{0}\right)$ and self interaction term $U^{\prime \prime \prime \prime}\left(a=a_{0}\right)$ due to small backreaction. Consequently if we take the Taylor expansion of the axion potential around $a=a_{0}$ we get

$U(a) \approx \frac{1}{2} m_{\text {axion }}^{2}\left(a-a_{0}\right)^{2}$

\subsection{Axion creation from quantum fluctuation}

Now to study the effects of fluctuations explicitly let us first write down the equation of motion corresponding to axion field as given by

$\partial_{\eta}\left(\frac{f_{a}^{2}}{H^{2} \eta^{2}}\left(\partial_{\eta} \bar{a}\right)\right)-\frac{f_{a}^{2}}{H^{2} \eta^{2}}\left(\partial_{i}^{2} \bar{a}\right)+\frac{m_{\text {axion }}^{2}}{H^{4} \eta^{4}} \bar{a}=0$,

where we use the fact that $f_{a} / H$ is a conformal timedependent factor and $\bar{a}$ is defined as $\bar{a}=a-a_{0}$. Further taking the following ansatz for the Fourier transformation:

$\bar{a}=\bar{a}(\eta, \mathbf{x})=\int \frac{\mathrm{d}^{3} k}{(2 \pi)^{3}} \bar{a}_{\mathbf{k}}(\eta) \exp (i \mathbf{k} \cdot \mathbf{x})$, 


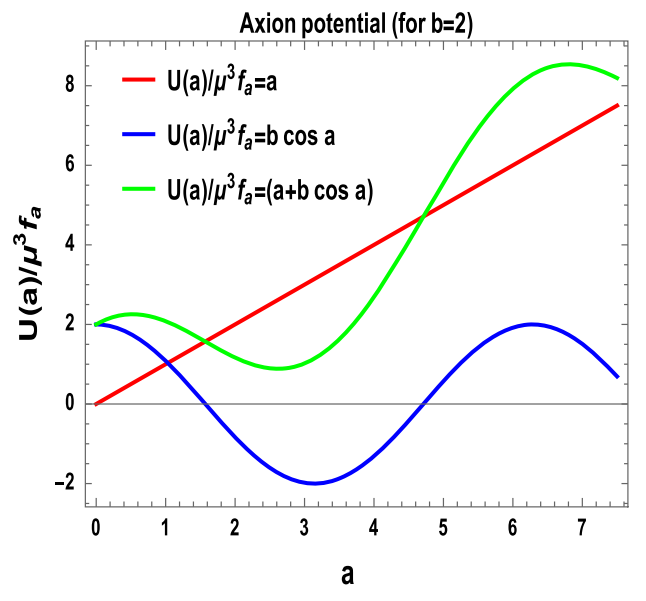

(a) Various parts of potential with $b=2$

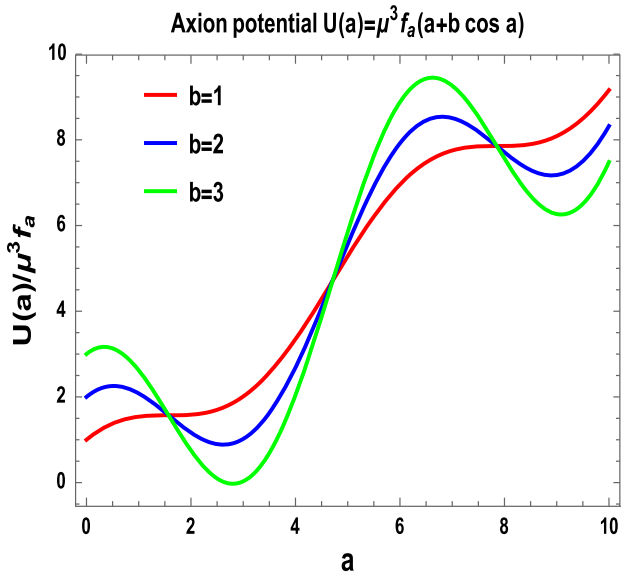

(b) Total potential with $b=1,2,3$

Fig. 13 Behaviour of the axion effective potential

in momentum space the equation of motion can be recast as

$\partial_{\eta}^{2} \vartheta_{\mathbf{k}}+\left(k^{2}-\frac{\partial_{\eta}^{2}\left(\frac{f_{a}^{2}}{H^{2} \eta^{2}}\right)}{\left(\frac{f_{a}^{2}}{H^{2} \eta^{2}}\right)}+\frac{m_{\text {axion }}^{2}}{f_{a}^{2} H^{2} \eta^{2}}\right) \vartheta_{\mathbf{k}}=0$

where introduce a new variable $\vartheta_{\mathbf{k}}$ defined as

$\vartheta_{\mathbf{k}}=\frac{f_{a}^{2}}{H^{2} \eta^{2} M_{p}^{2}} \bar{a}_{\mathbf{k}}$

Here it is important to mention here that to solve the above mentioned mode equation exactly or using the WKB approximation method for axion fluctuation we need to assume some specific structural form of the conformal time scale dependent axion decay constant. Here we take the following parametric profile for the conformal time scale dependent axion decay constant (Figs. 13 and 14):

$$
f_{a}=\sqrt{100-\frac{80}{1+\left(\ln \frac{\eta}{\eta_{c}}\right)^{2}}} H .
$$

For this specific choice of the axion decay constant one can find the following characteristic features:

- For very early times this is constant and we get $f_{a} \approx$ $10 H$.

- For very late times this is constant and we get $f_{a} \approx 10 \mathrm{H}$.

- At conformal time scale $\eta \sim \eta_{c}$, it takes smaller value, $f_{a} \approx 2 \sqrt{5} H$.
- For this specific choice the Mukhanov-Sasaki variable can be computed as

$$
\frac{\partial_{\eta}^{2}\left(\frac{f_{a}^{2}}{H^{2} \eta^{2}}\right)}{\left(\frac{f_{a}^{2}}{H^{2} \eta^{2}}\right)} \approx \begin{cases}\frac{6}{\eta^{2}} & \text { for } \eta \sim \eta_{c}, \text { early \& late } \eta, \\ \frac{6+\Delta_{c}}{\eta^{2}} & \text { for } \eta<\eta_{c} .\end{cases}
$$

- Also for this case the axion mass parameter can be expressed as

$$
\frac{m_{\text {axion }}^{2}}{f_{a}^{2} H^{2}}=-\frac{\Lambda_{C}^{4}}{\left[100-\frac{80}{1+\left(\ln \frac{\eta}{\eta_{c}}\right)^{2}}\right]^{2} H^{4}} \times \Sigma_{C}
$$

where $\Sigma_{C}$ is defined as

$\Sigma_{C}= \begin{cases}\cos \left(\sin ^{-1}\left(\frac{\mu^{3} H}{\Lambda_{C}^{4}} \sqrt{100-\frac{80}{1+\left(\ln \frac{\eta}{\eta_{c}}\right)^{2}}}\right)\right) & \text { for total } U(a) \\ (-1)^{m} & \text { for osc. } U(a) .\end{cases}$

In this context we are interested in the following situations:

1. At very early time scale.

2. At very late time scale.

3. At intermediate scale $\eta<\eta_{c}$.

4. At the characteristic scale $\eta \sim \eta_{c}$.

For all these four physical situations the axion mass parameter can be recast as 


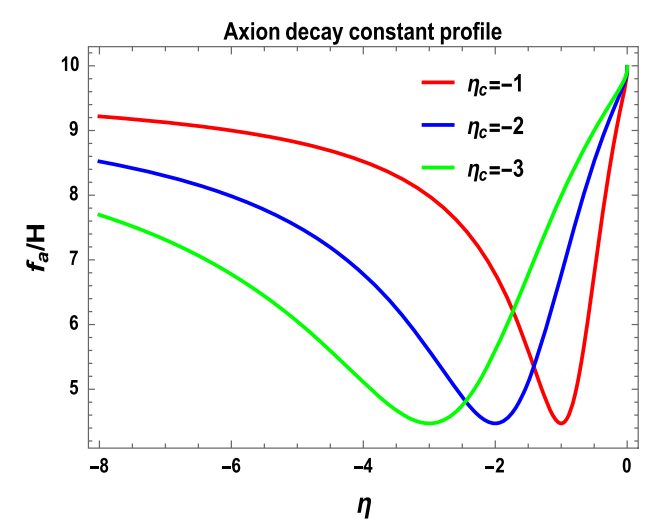

(a) Axion decay constant profile for $\eta_{c}=-1,-2,-3$.

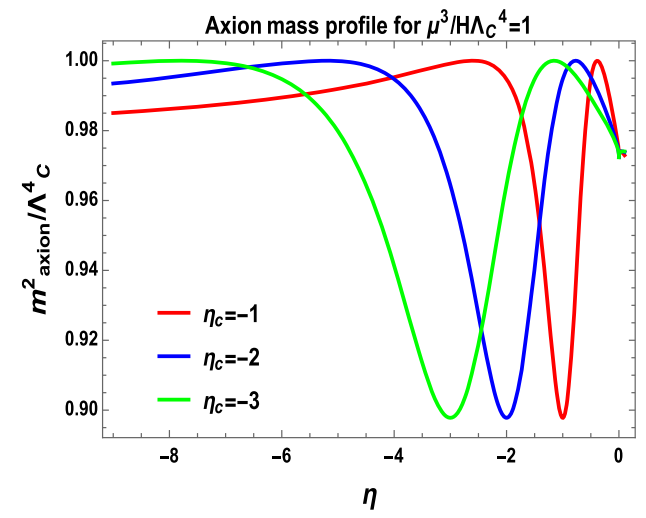

(c) Axion mass profile for $\eta_{c}=-1,-2,-3$.

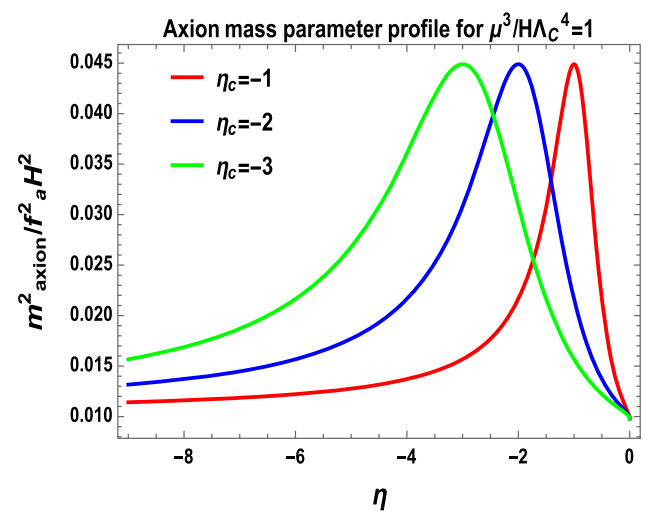

(e) Axion mass parameter profile for $\eta_{c}=-1,-2,-3$.

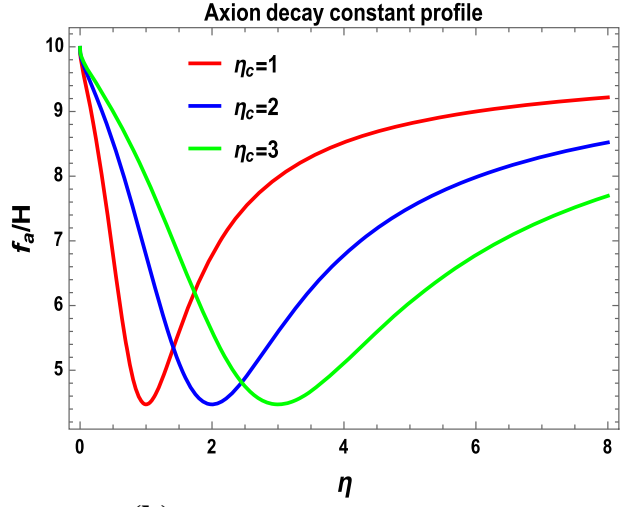

(b) Axion decay constant profile for $\eta_{c}=1,2,3$.

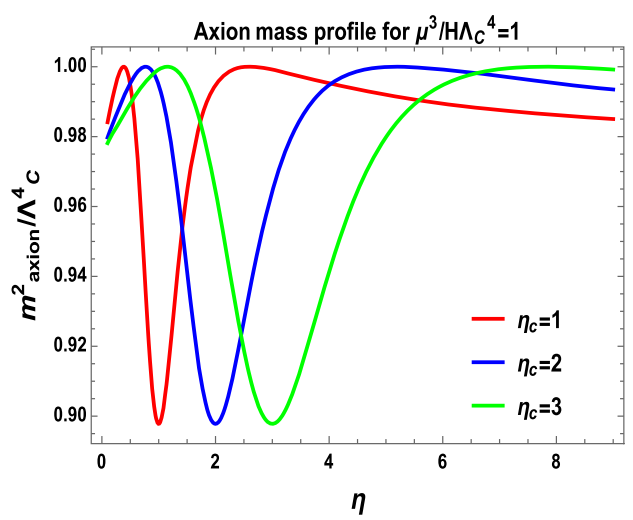

(d) Axion mass profile for $\eta_{c}=1,2,3$.

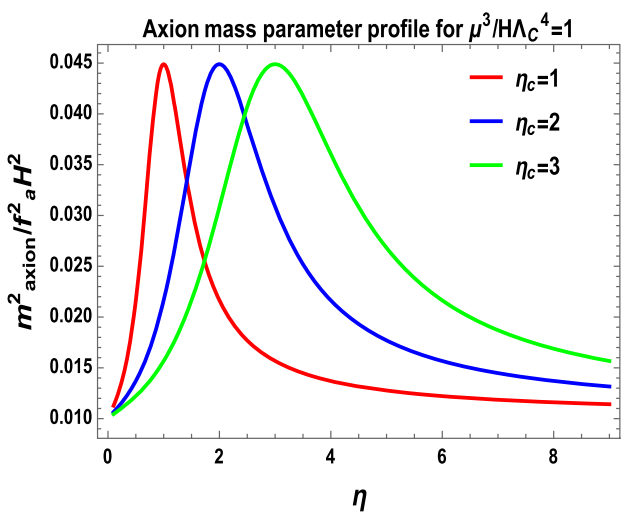

(f) Axion mass parameter profile for $\eta_{c}=1,2,3$.

Fig. 14 Conformal time scale dependent behaviour of axion decay constant $f_{a} / H$, axion mass $m_{\text {axion }}^{2} / \Lambda_{C}^{4}$ and axion mass parameter $m_{\text {axion }}^{2} / f_{a}^{2} H^{2}$ for a given profile 


\section{For total U(a)}

$$
\frac{m_{\text {axion }}^{2}}{f_{a}^{2} H^{2}}: \approx \begin{cases}-\frac{\Lambda_{C}^{4} \times \cos \left(\sin ^{-1}\left(\frac{10 \mu^{3} H}{\Lambda_{C}^{4}}\right)\right)}{10000 H^{4}} & \text { for early \& late } \eta, \\ -\frac{\Lambda_{C}^{4} \times \cos \left(\sin ^{-1}\left(\frac{\mu^{3} H}{\Lambda_{C}^{4}} \sqrt{\left.100-\frac{80}{1+\omega_{C}}\right)}\right)\right.}{\left[100-\frac{80}{1+\omega_{C}}\right]^{2} H^{4}} & \text { for } \eta<\eta_{c} \\ -\frac{\Lambda_{C}^{4} \times \cos \left(\sin ^{-1}\left(\frac{2 \sqrt{5} \mu^{3} H}{\Lambda_{C}^{4}}\right)\right)}{400 H^{4}} & \text { for } \eta \sim \eta_{c} .\end{cases}
$$

For osc. U(a)

$$
\frac{m_{\text {axion }}^{2}}{f_{a}^{2} H^{2}} \approx \begin{cases}-\frac{\Lambda_{C}^{4}}{10000 H^{4}} \times(-1)^{m} & \text { for early \& late } \eta \\ -\frac{\Lambda_{C}^{4}}{\left[100-\frac{80}{1+\omega_{C}}\right]^{2} H^{4}} \times(-1)^{m} & \text { for } \eta<\eta_{c} \\ -\frac{\Lambda_{C}^{4}}{400 H^{4}} \times(-1)^{m} & \text { for } \eta \sim \eta_{c}\end{cases}
$$

where $\omega_{C}$ is a small contribution. As for all the cases the axion mass parameter can be treated as constant factor one can recast the mode equations in the following form:

$$
\begin{gathered}
\vartheta_{\mathbf{k}}^{\prime \prime}+\left(k^{2}+\frac{\left(\frac{m_{\text {axion }}^{2}}{f_{a}^{2} H^{2}}-6\right)}{\eta^{2}}\right) \vartheta_{\mathbf{k}}=0 \quad \text { for } \eta \sim \eta_{\mathbf{c}} \text {, early \& late } \eta, \\
\vartheta_{\mathbf{k}}^{\prime \prime}+\left(k^{2}+\frac{\left(\frac{m_{\text {axion }}^{2}}{f_{a}^{2} H^{2}}-6-\Delta_{c}\right)}{\eta^{2}}\right) \vartheta_{\mathbf{k}}=0 \quad \text { for } \eta<\eta_{\mathbf{c}} .
\end{gathered}
$$

The solutions for the mode function for the above mentioned cases can be expressed as

$$
\vartheta_{\mathbf{k}}(\eta)=\frac{f_{a}^{2}}{H^{2} \eta^{2} M_{p}^{2}} \bar{a}_{\mathbf{k}}=\left\{\begin{array}{l}
\left.\sqrt{-\eta}\left[\begin{array}{l}
C_{1} H^{(1)} \\
\sqrt{\frac{25}{4}-\frac{m_{\text {axion }}^{2}}{f_{a}^{2} H^{2}}}(-k \eta)+C_{2} H^{(2)} \\
\sqrt{\frac{25}{4}-\frac{m_{\text {axion }}^{2}}{f_{a}^{2} H^{2}}}(-k \eta)
\end{array}\right] \begin{array}{r}
\text { for } \eta \sim \eta_{c}, \text { early \& late } \eta, \\
C_{1} H^{(1)} \sqrt{\frac{25}{4}+\Delta_{c}-\frac{m_{\text {axion }}^{2}}{f_{a}^{2} H^{2}}}(-k \eta)+C_{2} H^{(2)} \\
\sqrt{\frac{25}{4}+\Delta_{c}-\frac{m_{\text {axion }}^{2}}{f_{a}^{2} H^{2}}}
\end{array}\right] \text { for } \eta<\eta_{c} .
\end{array}\right.
$$

Here $C_{1}$ and $C_{2}$ are the arbitrary integration constants and the numerical value depend on the choice of the initial condition or more precisely the vacuum.

In the standard WKB approximation the total solution can be recast in the following form:

$\vartheta_{\mathbf{k}}(\eta)=\frac{f_{a}^{2}}{H^{2} \eta^{2} M_{p}^{2}} \bar{a}_{\mathbf{k}}=\left[D_{1} u_{k}(\eta)+D_{2} \bar{u}_{k}(\eta)\right]$, 
where $D_{1}$ and $D_{2}$ are two arbitrary integration constants, which depend on the choice of the initial condition on making the WKB approximation at early and late time scale. In the present context $u_{k}(\eta)$ and $\bar{u}_{k}(\eta)$ are defined as

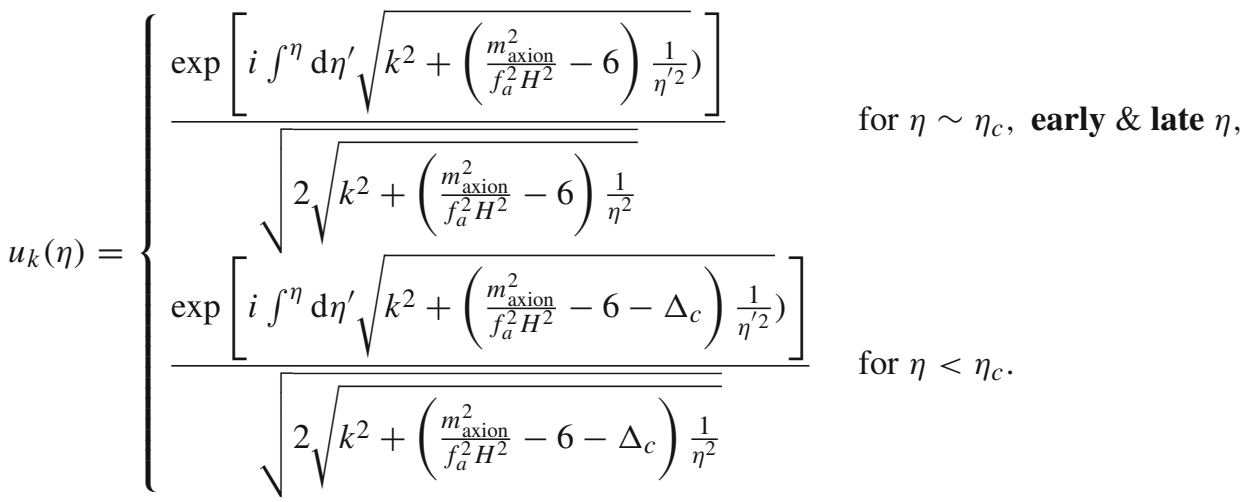

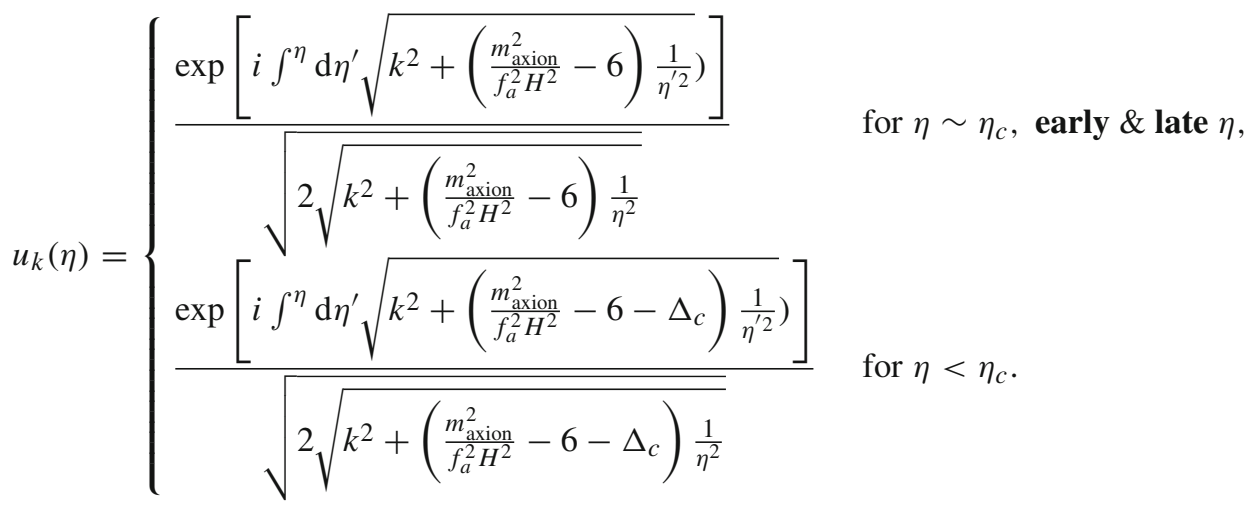

Here in the most general situation the new conformal time-dependent factor $p(\eta)$ is defined as

$$
p(\eta)= \begin{cases}\sqrt{k^{2}+\left(\frac{m_{\text {axion }}^{2}}{f_{a}^{2} H^{2}}-6\right) \frac{1}{\eta^{2}}} & \text { for } \eta \sim \eta_{c}, \text { early \& late } \eta, \\ \sqrt{k^{2}+\left(\frac{m_{\text {axion }}^{2}}{f_{a}^{2} H^{2}}-6-\Delta_{c}\right) \frac{1}{\eta^{2}}} & \text { for } \eta<\eta_{c} .\end{cases}
$$

which we use thoroughly in our computation. Here it is important to mention the expressions for the controlling factor $p(\eta)$ in different regime of solution:

$$
\begin{aligned}
& \text { For } \eta \sim \eta_{\mathbf{c}}, \text { early \& late } \eta: \quad p(\eta) \approx \\
& \begin{cases}\sqrt{k^{2}-\frac{5}{\eta^{2}}} & \text { for } m_{\text {axion }} / f_{a} \approx H \\
\sqrt{k^{2}-\frac{6}{\eta^{2}}} & \text { for } m_{\text {axion }} / f_{a}<<H \\
\sqrt{k^{2}+\left(\lambda^{2}-6\right) \frac{1}{\eta^{2}}} & \text { for } m_{\text {axion }} / f_{a}>>H\end{cases}
\end{aligned}
$$


For $\eta<\eta_{c}: \quad p(\eta) \approx \begin{cases}\sqrt{\sqrt{k^{2}-\left(5+\Delta_{c}\right) \frac{1}{\eta^{2}}}} & \text { for } m_{\mathrm{axion}} / f_{a} \approx H \\ \sqrt{k^{2}-\left(6+\Delta_{c}\right) \frac{1}{\eta^{2}}} & \text { for } m_{\mathrm{axion}} / f_{a}<<H \\ \sqrt{k^{2}+\left(\lambda^{2}-6-\Delta_{c}\right) \frac{1}{\eta^{2}}} & \text { for } m_{\mathrm{axion}} / f_{a}>>H,\end{cases}$

where for $m_{\text {axion }} / f_{a}>>H$ we introduce a new parameter $\lambda$ as, $m_{\text {axion }} / f_{a}=\lambda H$ with $\lambda>>1$. It is important to mention that the general expression for the Bogoliubov coefficient $\beta$ in Fourier space is given by the following approximation:

$$
\beta(k)= \begin{cases}\int_{-\infty}^{0} \mathrm{~d} \eta \frac{\left(\frac{m_{\text {axion }}^{2}}{f_{a}^{2} H^{2}}-6\right)^{2} \exp \left[2 i \int_{-\infty}^{\eta} \mathrm{d} \eta^{\prime} \sqrt{k^{2}+\left(\frac{m_{\text {axion }}^{2}}{f_{a}^{2} H^{2}}-6\right) \frac{1}{\eta^{\prime 2}}}\right]}{4 \eta^{6}\left(k^{2}+\left(\frac{m_{\text {axion }}^{2}}{f_{a}^{2} H^{2}}-6\right) \frac{1}{\eta^{2}}\right)^{5 / 2}} & \text { for } \eta \sim \eta_{c}, \text { early \& late } \eta, \\ \int_{-\infty}^{0} \mathrm{~d} \eta \frac{\left(\frac{m_{\text {axion }}^{2}}{f_{a}^{2} H^{2}}-6-\Delta_{c}\right)^{2} \exp \left[2 i \int_{-\infty}^{\eta} \mathrm{d} \eta^{\prime} \sqrt{k^{2}+\left(\frac{m_{\text {axion }}^{2}}{f_{a}^{2} H^{2}}-6-\Delta_{c}\right) \frac{1}{\eta^{\prime 2}}}\right]}{4 \eta^{6}\left(k^{2}+\left(\frac{m_{\text {axion }}^{2}}{f_{a}^{2} H^{2}}-6-\Delta_{c}\right) \frac{1}{\eta^{2}}\right)^{5 / 2}} & \text { for } \eta<\eta_{c} .\end{cases}
$$

One can use another equivalent way to define the Bogoliubov coefficient $\beta$ in Fourier space by implementing instantaneous Hamiltonian diagonalization method in the present context. Using this diagonalized representation the regularized Bogoliubov coefficient $\beta$ in Fourier space can be written as

$$
\beta_{\text {diag }}\left(k ; \tau, \tau^{\prime}\right)= \begin{cases}\int_{\tau^{\prime}}^{\tau} \mathrm{d} \eta \frac{-\left(\frac{m_{\text {axion }}^{2}}{f_{a}^{2} H^{2}}-6\right) \exp \left[-2 i \int \eta^{\eta} \mathrm{d} \eta^{\prime} \sqrt{k^{2}+\left(\frac{m_{\text {axion }}^{2}}{f_{a}^{2} H^{2}}-6\right) \frac{1}{\eta^{\prime 2}}}\right]}{2 \eta^{3}\left(k^{2}+\left(\frac{m_{\text {axion }}^{2}}{f_{a}^{2} H^{2}}-6\right) \frac{1}{\eta^{2}}\right)} & \text { for } \eta \sim \eta_{c}, \text { early \& late } \eta, \\ \int_{\tau^{\prime}}^{\tau} \mathrm{d} \eta \frac{-\left(\frac{m_{\text {axion }}^{2}}{f_{a}^{2} H^{2}}-6-\Delta_{c}\right) \exp \left[-2 i \int^{\eta} \mathrm{d} \eta^{\prime} \sqrt{k^{2}+\left(\frac{m_{\text {axion }}^{2}}{f_{a}^{2} H^{2}}-6-\Delta_{c}\right) \frac{1}{\eta^{\prime 2}}}\right]}{2 \eta^{3}\left(k^{2}+\left(\frac{m_{\text {axion }}^{2}}{f_{a}^{2} H^{2}}-6-\Delta_{c}\right) \frac{1}{\eta^{2}}\right)} & \text { for } \eta<\eta_{c},\end{cases}
$$

where $\tau$ and $\tau^{\prime}$ introduced as the conformal time regulator in the present context. We will also derive the expressions using Eq. (3.48) in the next two subsections. In the next two subsection we will explicitly discuss two physical possibilities which captures the effect of massive particles in our computation.

Further using the expressions for the Bogoliubov coefficient $\beta$ in two different representations, and substituting them in Eq. (3.57) we get the following expressions for the Bogoliubov coefficient $\alpha$ in two different representations as given by

$$
\alpha(k)=\left\{\begin{array}{l}
\sqrt{\left[1+\mid \int_{-\infty}^{0} \mathrm{~d} \eta \frac{\left(\frac{m_{\text {axion }}^{2}}{f_{a}^{2} H^{2}}-6\right)^{2} \exp \left[2 i \int_{-\infty}^{\eta} \mathrm{d} \eta^{\prime} \sqrt{k^{2}+\left(\frac{m_{\text {axion }}^{2}}{f_{a}^{2} H^{2}}-6\right) \frac{1}{\eta^{\prime 2}}}\right]}{4 \eta^{6}\left(k^{2}+\left(\frac{m_{\text {axion }}^{2}}{f_{a}^{2} H^{2}}-6\right) \frac{1}{\eta^{2}}\right)^{5 / 2}}\right]} e^{i \phi} \quad \text { for } \eta \sim \eta_{c}, \text { early \& late } \eta \\
\sqrt{\left[1+\mid \int_{-\infty}^{0} \mathrm{~d} \eta \frac{\left(\frac{m_{\text {axion }}^{2}}{f_{a}^{2} H^{2}}-6-\Delta_{c}\right)^{2} \exp \left[2 i \int_{-\infty}^{\eta} \mathrm{d} \eta^{\prime} \sqrt{k^{2}+\left(\frac{m_{\text {axion }}^{2}}{f_{a}^{2} H^{2}}-6-\Delta_{c}\right) \frac{1}{\eta^{\prime 2}}}\right]}{4 \eta^{6}\left(k^{2}+\left(\frac{m_{\text {axion }}^{2}}{f_{a}^{2} H^{2}}-6-\Delta_{c}\right) \frac{1}{\eta^{2}}\right)^{5 / 2}}\right]} e^{i \phi \text { for } \eta<\eta_{c} .}
\end{array}\right.
$$




$$
\alpha_{\text {diag }}\left(k ; \tau, \tau^{\prime}\right)= \begin{cases}\sqrt{\left[1+\left|\int_{\tau^{\prime}}^{\tau} \mathrm{d} \eta \frac{-\left(\frac{m_{\text {axion }}^{2}}{f_{a}^{2} H^{2}}-6\right) \exp \left[-2 i \int^{\eta} \mathrm{d} \eta^{\prime} \sqrt{k^{2}+\left(\frac{m_{\text {axion }}^{2}}{f_{a}^{2} H^{2}}-6\right) \frac{1}{\eta^{\prime 2}}}\right]}{2 \eta^{3}\left(k^{2}+\left(\frac{m_{\text {axion }}^{2}}{f_{a}^{2} H^{2}}-6\right) \frac{1}{\eta^{2}}\right)}\right|^{2}\right.} e^{i \phi} & \text { for } \eta \sim \eta_{c}, \text { early \& late } \eta, \\ \sqrt{\left[1+\mid \int_{\tau^{\prime}}^{\tau} \mathrm{d} \eta \frac{-\left(\frac{m_{\text {axion }}^{2}}{f_{a}^{2} H^{2}}-6-\Delta_{c}\right) \exp \left[-2 i \int^{\eta} \mathrm{d} \eta^{\prime} \sqrt{k^{2}+\left(\frac{m_{\text {axion }}^{2}}{f_{a}^{2} H^{2}}-6-\Delta_{c}\right) \frac{1}{\eta^{\prime 2}}}\right]}{2 \eta^{3}\left(k^{2}+\left(\frac{m_{\text {axion }}^{2}}{f_{a}^{2} H^{2}}-6-\Delta_{c}\right) \frac{1}{\eta^{2}}\right)}\right]} e^{i \phi} \text { for } \eta<\eta_{c} .\end{cases}
$$

where $\phi$ and $\phi_{\text {diag }}$ are the associated phase factors in two different representations. Further using the expressions for Bogoliubov coefficient $\alpha$ in two different representations as mentioned in Eqs. (3.59) and (3.60), and substituting them in Eq. (3.58) we get the following expressions for the reflection and transmission coefficient in two different representations as given by

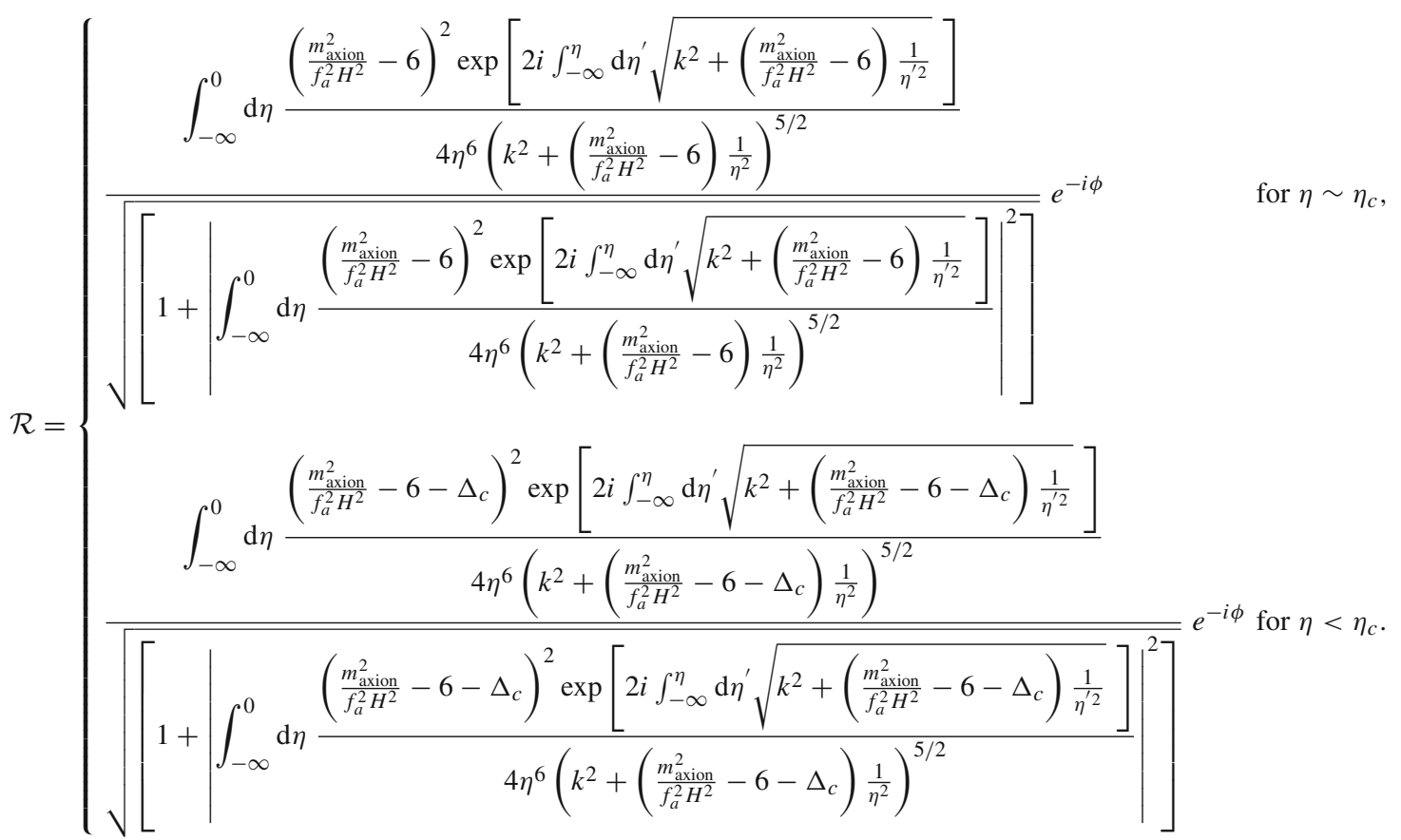

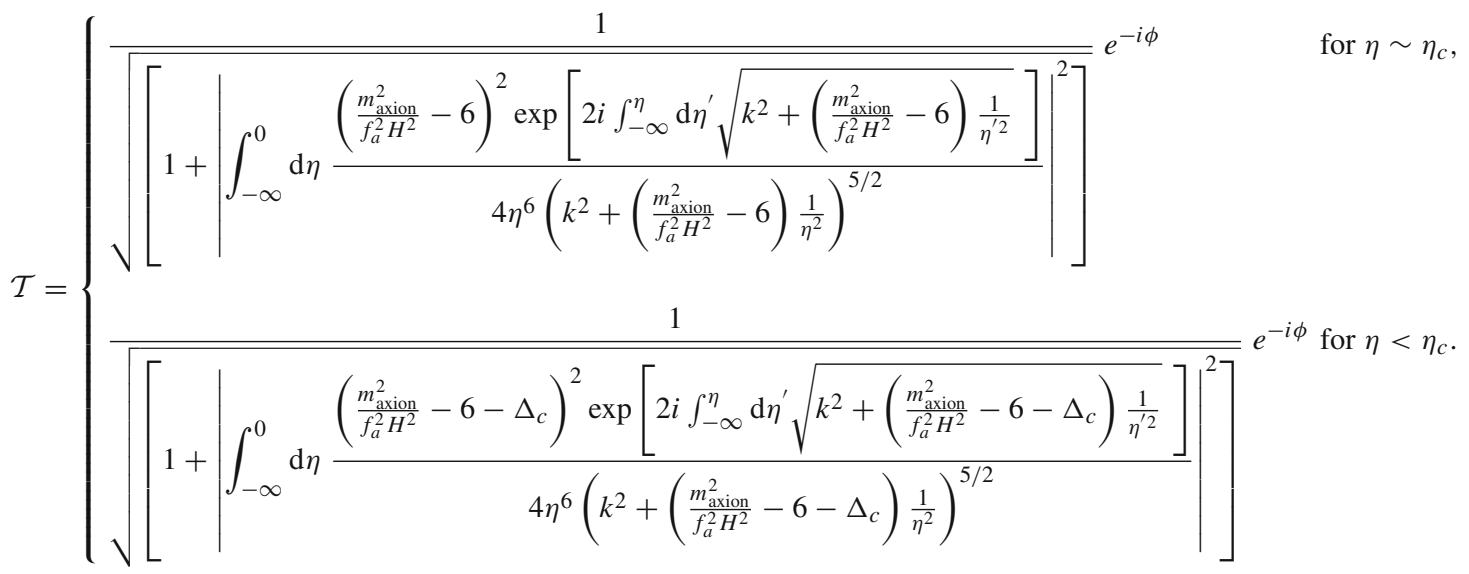




$$
\begin{aligned}
& \iint_{\tau^{\prime}}^{\tau} \mathrm{d} \eta \frac{-\left(\frac{m_{\text {axion }}^{2}}{f_{a}^{2} H^{2}}-6\right) \exp \left[-2 i \int^{\eta} \mathrm{d} \eta^{\prime} \sqrt{k^{2}+\left(\frac{m_{\text {axion }}^{2}}{f_{a}^{2} H^{2}}-6\right) \frac{1}{\eta^{\prime 2}}}\right]}{2 \eta^{3}\left(k^{2}+\left(\frac{m_{\text {axion }}^{2}}{f_{a}^{2} H^{2}}-6\right) \frac{1}{\eta^{2}}\right)} \\
& \mathcal{R}_{\text {digg }}\left(k ; \tau, \tau^{\prime}\right)=\left\{\sqrt{\sqrt{\left[1+\left|\int_{\tau^{\prime}}^{\tau} \mathrm{d} \eta \frac{-\left(\frac{m_{\text {axion }}^{2}}{f_{a}^{2} H^{2}}-6\right) \exp \left[-2 i \int^{\eta} \mathrm{d} \eta^{\prime} \sqrt{\left.k^{2}+\left(\frac{m_{\text {axion }}^{2}}{f_{a}^{2} H^{2}}-6\right) \frac{1}{\eta^{\prime 2}}\right]}\right.}{2 \eta^{3}\left(k^{2}+\left(\frac{m_{\text {axion }}^{2}}{f_{a}^{2} H^{2}}-6\right) \frac{1}{\eta^{2}}\right)}\right|^{2}\right.}} e^{-i \phi}\right. \\
& \int_{\tau^{\prime}}^{\tau} \mathrm{d} \eta \frac{-\left(\frac{m_{\text {axion }}^{2}}{f_{a}^{2} H^{2}}-6-\Delta_{c}\right) \exp \left[-2 i \int^{\eta} \mathrm{d} \eta^{\prime} \sqrt{k^{2}+\left(\frac{m_{\text {axion }}^{2}}{f_{a}^{2} H^{2}}-6-\Delta_{c}\right) \frac{1}{\eta^{\prime 2}}}\right]}{2 \eta^{3}\left(k^{2}+\left(\frac{m_{\text {axion }}^{2}}{f_{a}^{2} H^{2}}-6-\Delta_{c}\right) \frac{1}{\eta^{2}}\right)}
\end{aligned}
$$

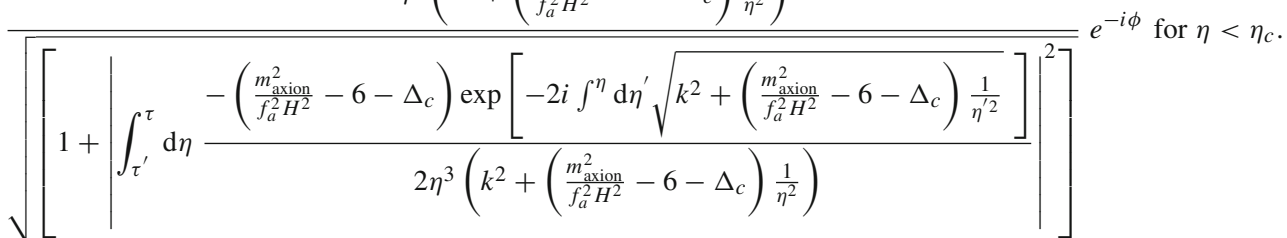

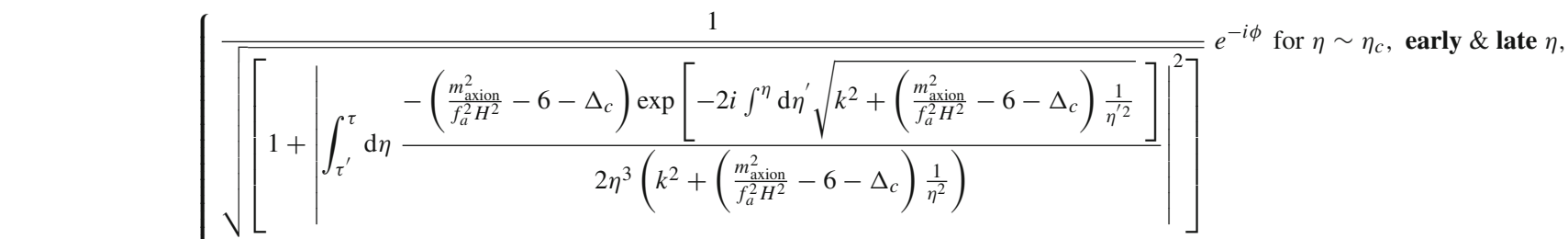

$$
\begin{aligned}
& \mathcal{T}_{\text {diag }}\left(k ; \tau, \tau^{\prime}\right)=\{
\end{aligned}
$$

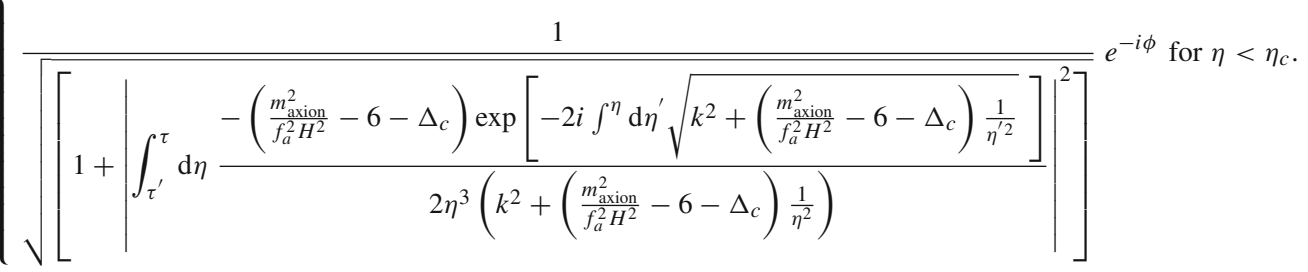

Next the expression for the number of produced particles at time $\tau$ can be calculated in the two representations using from the following formula as

$\mathcal{N}\left(\tau, \tau^{\prime}\right)=\int \frac{\mathrm{d}^{3} \mathbf{k}}{(2 \pi a)^{3}}\left\{\begin{array}{l}\left|\int_{-\infty}^{0} \mathrm{~d} \eta \frac{\left(\frac{m_{\text {axion }}^{2}}{f_{a}^{2} H^{2}}-6\right)^{2} \exp \left[2 i \int_{-\infty}^{\eta} \mathrm{d} \eta^{\prime} \sqrt{k^{2}+\left(\frac{m_{\text {axion }}^{2}}{f_{a}^{2} H^{2}}-6\right) \frac{1}{\eta^{\prime 2}}}\right]}{4 \eta^{6}\left(k^{2}+\left(\frac{m_{\text {axion }}^{2}}{f_{a}^{2} H^{2}}-6\right) \frac{1}{\eta^{2}}\right)^{5 / 2}}\right|^{2} \\ \left|\int_{-\infty}^{0} \mathrm{~d} \eta \frac{\left(\frac{m_{\text {axion }}^{2}}{f_{a}^{2} H^{2}}-6-\Delta_{c}\right)^{2} \exp \left[2 i \int_{-\infty}^{\eta} \mathrm{d} \eta^{\prime} \sqrt{k^{2}+\left(\frac{m_{\text {axion }}^{2}}{f_{a}^{2} H^{2}}-6-\Delta_{c}\right) \frac{1}{\eta^{\prime 2}}}\right]}{4 \eta^{6}\left(k^{2}+\left(\frac{m_{\text {axion }}^{2}}{f_{a}^{2} H^{2}}-6-\Delta_{c}\right) \frac{1}{\eta^{2}}\right)^{5 / 2}}\right|^{2}\end{array}\right.$ for $\eta \sim \eta_{c}$, early \& late $\eta$, 
$\mathcal{N}_{\text {diag }}\left(\tau, \tau^{\prime}\right)=\int \frac{\mathrm{d}^{3} \mathbf{k}}{(2 \pi a)^{3}} \begin{cases}\mid \int_{\tau^{\prime}}^{\tau} \mathrm{d} \eta \frac{-\left(\frac{m_{\text {axion }}^{2}}{f_{a}^{2} H^{2}}-6\right) \exp \left[-2 i \int^{\eta} \mathrm{d} \eta^{\prime} \sqrt{k^{2}+\left(\frac{m_{\text {xion }}^{2}}{f_{a}^{2} H^{2}}-6\right) \frac{1}{\eta^{\prime 2}}}\right]}{2 \eta^{3}\left(k^{2}+\left(\frac{m_{\text {axion }}^{2}}{f_{a}^{2} H^{2}}-6\right) \frac{1}{\eta^{2}}\right)} & \text { for } \eta \sim \eta_{c}, \text { early \& late } \eta, \\ \mid \int_{\tau^{\prime}}^{\tau} \mathrm{d} \eta \frac{-\left(\frac{m_{\text {axion }}^{2}}{f_{a}^{2} H^{2}}-6-\Delta_{c}\right) \exp \left[-2 i \int^{\eta} \mathrm{d} \eta^{\prime} \sqrt{k^{2}+\left(\frac{m_{\text {axion }}^{2}}{f_{a}^{2} H^{2}}-6-\Delta_{c}\right) \frac{1}{\eta^{\prime 2}}}\right.}{2 \eta^{3}\left(k^{2}+\left(\frac{m_{\text {axion }}^{2}}{f_{a}^{2} H^{2}}-6-\Delta_{c}\right) \frac{1}{\eta^{2}}\right)} & \text { for } \eta<\eta_{c} .\end{cases}$

Finally, one can define the total energy density of the produced particles using the following expression:

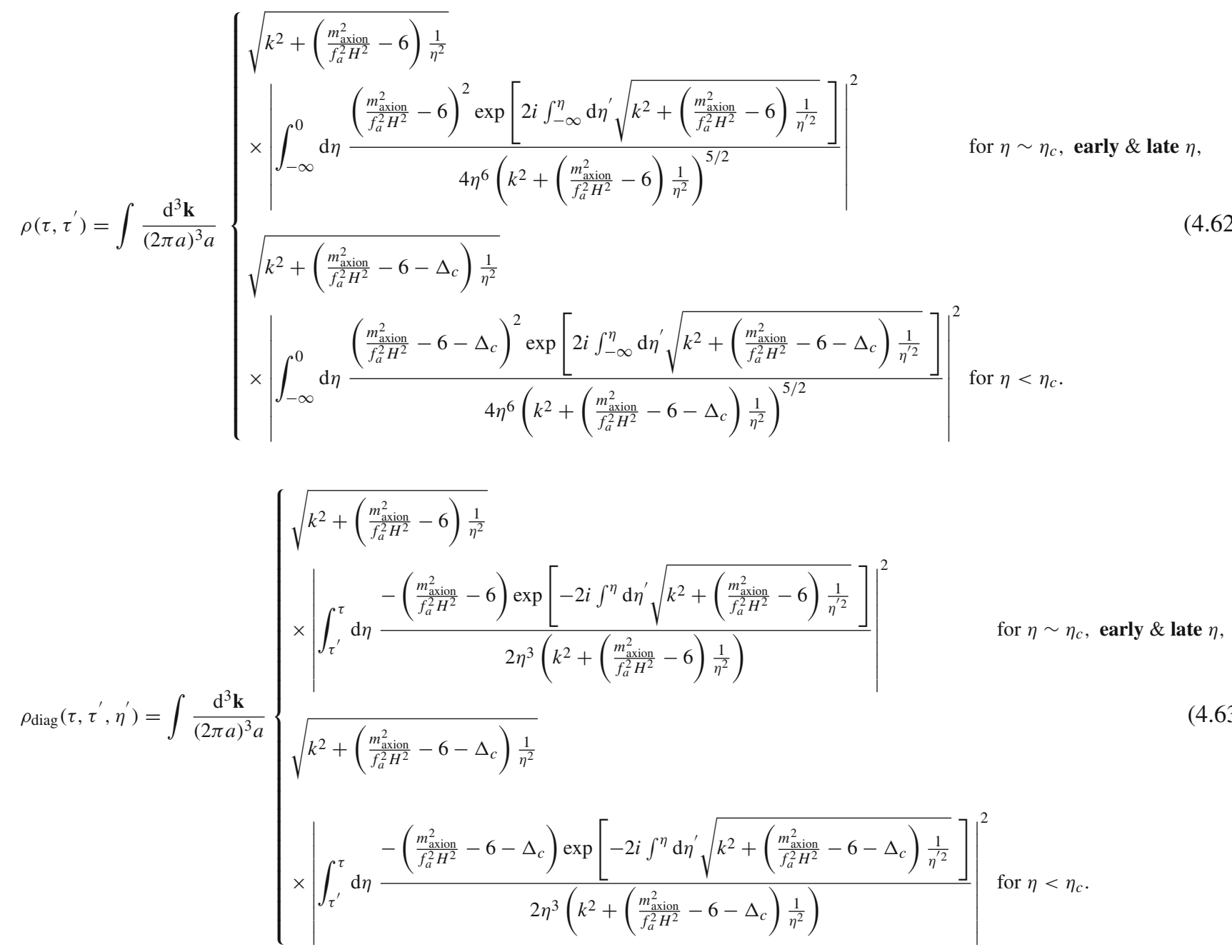

\subsubsection{Case I: $m_{\mathrm{axion}} / f_{a} \approx H$}

The equation of motion for the axion field for $m_{\mathrm{axion}} / f_{a} \approx H$ is given by

$$
\begin{aligned}
\vartheta_{k}^{\prime \prime}+\left\{k^{2}-\frac{5}{\eta^{2}}\right\} \vartheta_{k}=0 & \text { for } \eta \sim \eta_{\mathbf{c}}, \text { early \& late } \eta, \\
\vartheta_{k}^{\prime \prime}+\left\{k^{2}-\left[5+\Delta_{c}\right] \frac{1}{\eta^{2}}\right\} \vartheta_{k}=0 & \text { for } \eta<\eta_{\mathbf{c}} .
\end{aligned}
$$


The solution for the mode function for the de Sitter and the quasi de Sitter space can be expressed as

$\vartheta_{k}(\eta)= \begin{cases}\sqrt{-\eta}\left[C_{1} H_{\sqrt{21 / 2}}^{(1)}(-k \eta)+C_{2} H_{\sqrt{21 / 2}}^{(2)}(-k \eta)\right] & \text { for } \eta \sim \eta_{c}, \text { early \& late } \eta, \\ \sqrt{-\eta}\left[C_{1} H_{\sqrt{\frac{21}{4}+\Delta_{c}}}^{(1)}(-k \eta)+C_{2} H_{\sqrt{\frac{21}{4}+\Delta_{c}}}^{(2)}(-k \eta)\right] & \text { for } \eta<\eta_{c},\end{cases}$

where $C_{1}$ and $C_{2}$ are two arbitrary integration constants, which depend on the choice of the initial condition.

After taking the $k \eta \rightarrow-\infty, k \eta \rightarrow 0$ and $|k \eta| \approx 1-\Delta(\rightarrow 0)$ limits the most general solution as stated in Eq. (4.66) can be recast as

$$
\begin{aligned}
& \vartheta_{k}(\eta) \stackrel{|k \eta| \rightarrow-\infty}{=} \begin{cases}\sqrt{\frac{2}{\pi k}}\left[C_{1} e^{-i k \eta} e^{-\frac{i \pi}{2}\left(\frac{\sqrt{21}+1}{2}\right)}+C_{2} e^{i k \eta} e^{\left.\frac{i \pi}{2}\left(\frac{\sqrt{21}+1}{2}\right)\right]}\right. & \text { for } \eta \sim \eta_{c}, \text { early \& late } \eta, \\
\sqrt{\frac{2}{\pi k}}\left[C_{1} e^{-i k \eta} e^{-\frac{i \pi}{2}\left(\sqrt{\frac{21}{4}+\Delta_{c}}+\frac{1}{2}\right)}+C_{2} e^{i k \eta} e^{\frac{i \pi}{2}\left(\sqrt{\frac{21}{4}+\Delta_{c}}+\frac{1}{2}\right)}\right] & \text { for } \eta<\eta_{c},\end{cases} \\
& \vartheta_{k}(\eta) \stackrel{|k \eta| \rightarrow 0}{=} \begin{cases}\frac{i \sqrt{-\eta}}{\pi} \Gamma\left(\frac{\sqrt{21}}{2}\right)\left(-\frac{k \eta}{2}\right)^{-\frac{\sqrt{21}}{2}}\left[C_{1}-C_{2}\right] & \text { for } \eta \sim \eta_{c}, \text { early \& late } \eta, \\
\frac{i \sqrt{-\eta}}{\pi} \Gamma\left(\sqrt{\frac{21}{4}+\Delta_{c}}\right)\left(-\frac{k \eta}{2}\right)^{-\sqrt{\frac{21}{4}+\Delta_{c}}}\left[C_{1}-C_{2}\right] & \text { for } \eta<\eta_{c} .\end{cases} \\
& \vartheta_{k}(\eta) \stackrel{|k \eta| \approx 1-\Delta(\rightarrow 0)}{=}\left\{\begin{aligned}
\frac{i}{\pi} \sqrt{-\eta} & {\left[\frac{2}{\sqrt{21}}-\gamma+\frac{\sqrt{21}}{4}\left(\gamma^{2}+\frac{\pi^{2}}{6}\right)\right.} \\
& \left.-\frac{7}{8}\left(\gamma^{3}+\frac{\gamma \pi^{2}}{2}+2 \zeta(3)\right)+\cdots\right] \\
& \times\left(\frac{1+\Delta}{2}\right)^{-\frac{\sqrt{21}}{2}}\left[C_{1}-C_{2}\right] \\
\frac{i}{\pi} \sqrt{-\eta} & {\left[\frac{1}{\left\{\sqrt{\frac{21}{4}+\Delta_{c}}\right\}}-\gamma+\frac{1}{2}\left(\gamma^{2}+\frac{\pi^{2}}{6}\right)\left\{\sqrt{\left.\frac{21}{4}+\Delta_{c}\right\}}\right.\right.} \\
& \left.-\frac{1}{6}\left(\gamma^{3}+\frac{\gamma \pi^{2}}{2}+2 \zeta(3)\right)\left\{\sqrt{\frac{21}{4}+\Delta_{c}}\right\}^{2}+\cdots\right] \\
& \times\left(\frac{1+\Delta}{2}\right)^{-\sqrt{\frac{21}{4}+\Delta_{c}}}\left[C_{1}-C_{2}\right]
\end{aligned}\right.
\end{aligned}
$$

Next we assume that the WKB approximation is approximately valid for all times for the solution for the mode function $\vartheta_{k}$. In the standard WKB approximation the total solution can be recast in the following form:

$\vartheta_{k}(\eta)=\left[D_{1} u_{k}(\eta)+D_{2} \bar{u}_{k}(\eta)\right]$,

where $D_{1}$ and $D_{2}$ are two arbitrary integration constants, which depend on the choice of the initial condition during WKB approximation at early and late time scale. In the present context $u_{k}(\eta)$ and $\bar{u}_{k}(\eta)$ are defined as 


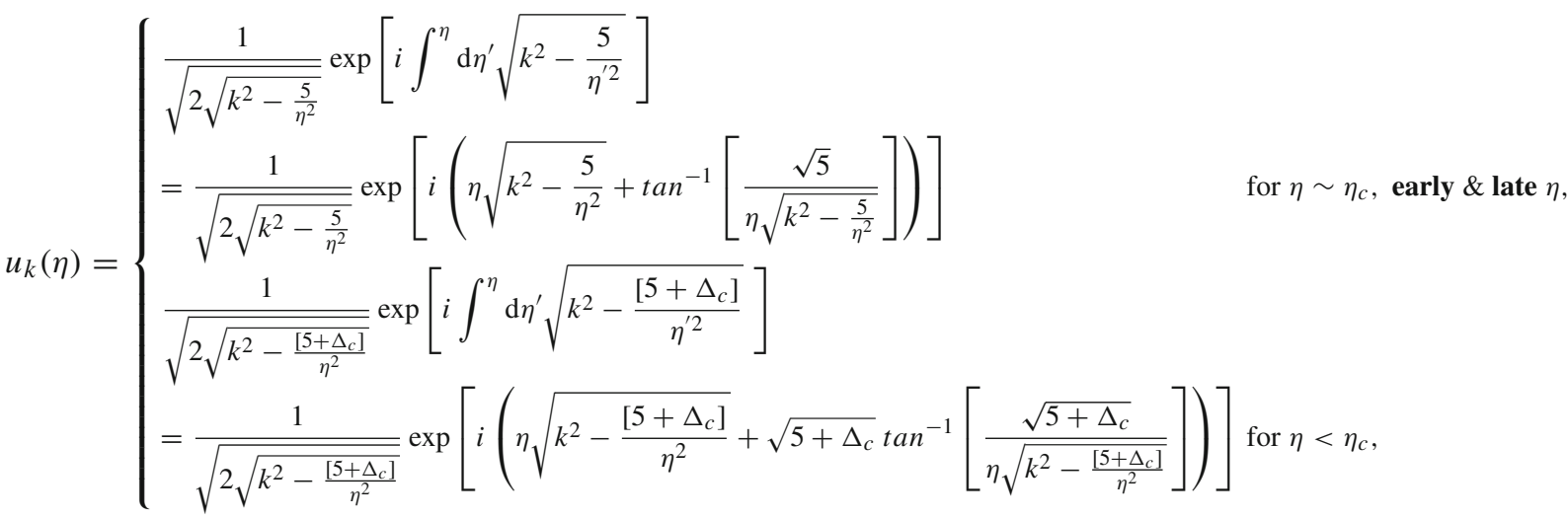

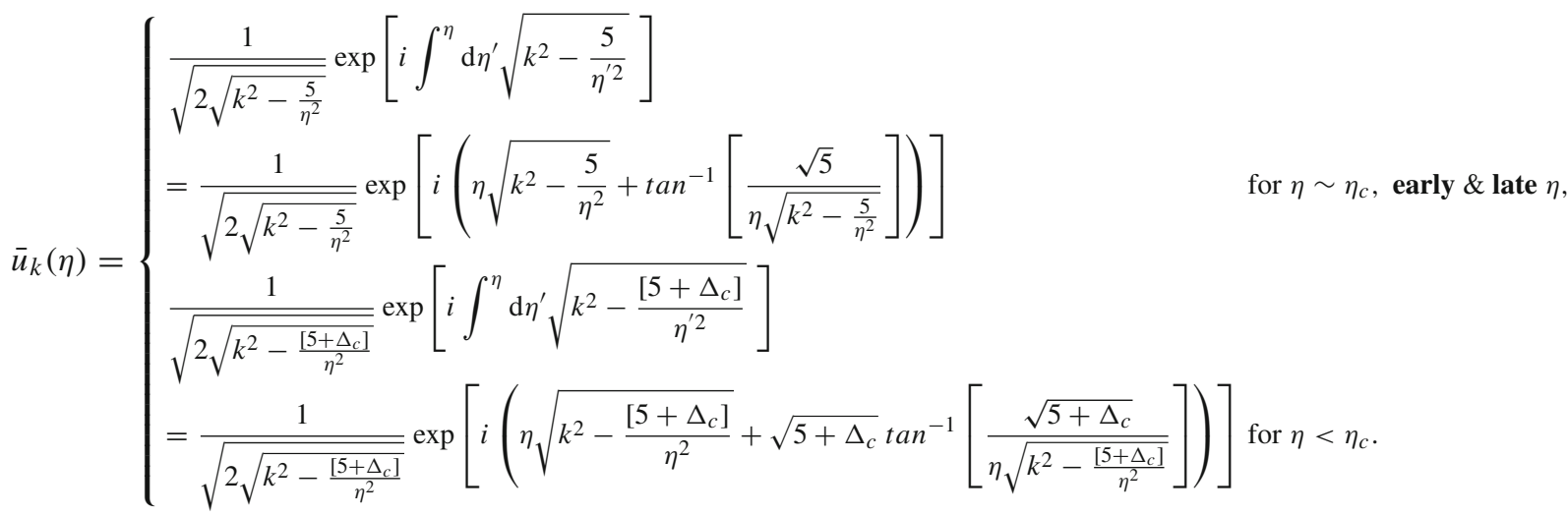

In the present context the Bogoliubov coefficient $\beta(k)$ in Fourier space can be computed approximately using the following expression:

$$
\beta\left(k, \tau, \tau^{\prime}, \eta^{\prime}\right)= \begin{cases}\int_{\tau^{\prime}}^{\tau} \mathrm{d} \eta \frac{25}{4 \eta^{6}\left(k^{2}-\frac{5}{\eta^{2}}\right)^{\frac{5}{2}}} \exp \left[2 i \int_{\eta^{\prime}}^{\eta} \mathrm{d} \eta^{\prime \prime} \sqrt{\left.k^{2}-\frac{5}{\eta^{\prime \prime 2}}\right]}\right. & \text { for } \eta \sim \eta_{c}, \text { early \& late } \eta, \\ \int_{\tau^{\prime}}^{\tau} \mathrm{d} \eta \frac{\left[5+\Delta_{c}\right]^{2}}{4 \eta^{6}\left(k^{2}-\frac{\left[5+\Delta_{c}\right]}{\eta^{2}}\right)^{\frac{5}{2}}} \exp \left[2 i \int_{\eta^{\prime}}^{\eta} \mathrm{d} \eta^{\prime \prime} \sqrt{\left.k^{2}-\frac{\left[5+\Delta_{c}\right]}{\eta^{\prime \prime 2}}\right]}\right. & \text { for } \eta<\eta_{c},\end{cases}
$$

which is not exactly analytically computable. To study the behaviour of this integral we consider here three consecutive physical situations: $|k \eta|<<1,|k \eta| \approx 1-\Delta(\rightarrow 0)$ and $|k \eta|>>1$ for the de Sitter case and the quasi de Sitter case. In the three cases we have

For $\eta \sim \eta_{\mathbf{c}}$, early \& late $\eta: \quad \sqrt{\left\{k^{2}-\frac{5}{\left.\eta^{2}\right\}}\right.} \approx \begin{cases}\frac{i \sqrt{5}}{\eta} & \text { for }|k \eta|<<1, \\ \frac{i \sqrt{2 \Delta+4}}{\eta} & \text { for }|k \eta| \approx 1-\Delta(\rightarrow 0), \\ k & \text { for }|k \eta|>>1 .\end{cases}$ 
For $\eta<\eta_{\mathbf{c}}: \quad \sqrt{\left\{k^{2}-\left[5+\Delta_{c}\right] \frac{1}{\left.\eta^{2}\right\}}\right.} \approx \begin{cases}\frac{i \sqrt{\left[5+\Delta_{c}\right]}}{\eta} & \text { for }|k \eta|<<1, \\ \frac{i \sqrt{2 \Delta+4+\Delta_{c}}}{\eta} & \text { for }|k \eta| \approx 1-\Delta(\rightarrow 0), \\ k & \text { for }|k \eta|>>1,\end{cases}$

and further using this result the Bogoliubov coefficient $\beta(k)$ in Fourier space can be expressed as

$$
\beta\left(k, \tau, \tau^{\prime}, \eta^{\prime}\right)= \begin{cases}\text { For } \eta \sim \eta_{\mathbf{c}}, \text { early \& late } \eta: & \text { for }|k \eta|<<1, \\ \frac{5^{3 / 4} \eta^{\prime 2 \sqrt{5}}}{4\left(\frac{5}{2}+2 \sqrt{5}\right) i}\left[\frac{1}{\tau^{\prime \frac{5}{2}+2 \sqrt{5}}}-\frac{1}{\tau^{\frac{5}{2}+2 \sqrt{5}}}\right] & \text { for }|k \eta| \approx 1-\Delta(\rightarrow 0), \\ 4\left(2 \Delta+4 \eta^{5 / 4}\left(\frac{5}{2}+2 \sqrt{2 \Delta+4}\right.\right. & \frac{1}{25\left[i \frac{\operatorname{Ei}(2 i k \eta) e^{-2 i k \eta^{\prime}}}{15}-\frac{e^{2 i k\left(\eta-\eta^{\prime}\right)}}{120 k^{5} \eta^{5}}\left(4 k^{4} \eta^{4}-2 i k^{3} \eta^{3}-2 k^{2} \eta^{2}+3 i k \eta+6\right)\right]_{\tau^{\prime}}^{\tau}} \text { for }|k \eta|>>1 .\end{cases}
$$

$$
\begin{aligned}
& \text { For } \eta<\eta_{\mathbf{c}} \text { : }
\end{aligned}
$$

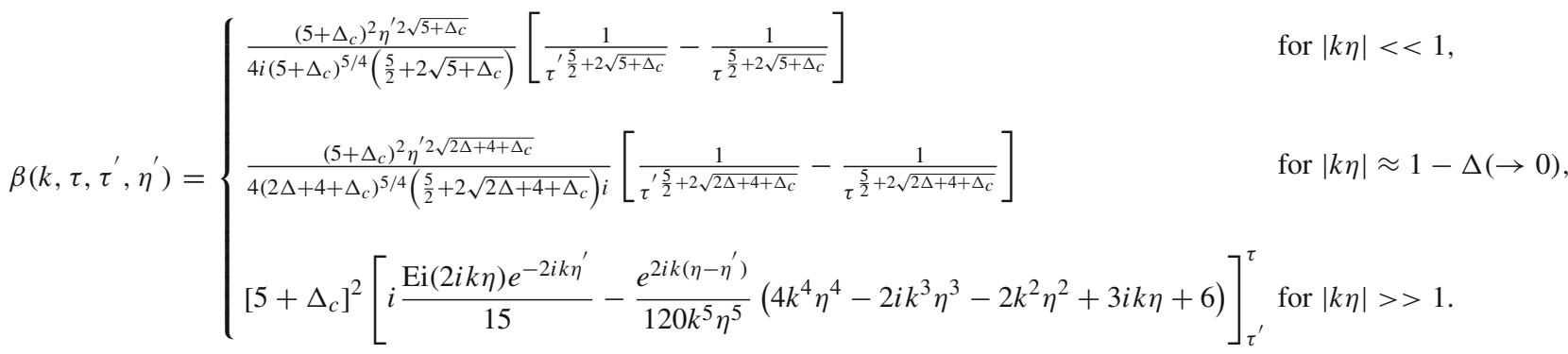

As mentioned earlier here one can use another equivalent way to define the Bogoliubov coefficient $\beta$ in Fourier space by implementing the instantaneous Hamiltonian diagonalization method to interpret the results. Using this diagonalized representation the regularized Bogoliubov coefficient $\beta$ in Fourier space can be written as

$$
\beta_{\text {diag }}\left(k ; \tau, \tau^{\prime}\right)= \begin{cases}\int_{\tau^{\prime}}^{\tau} \mathrm{d} \eta \frac{5 \exp \left[-2 i \int^{\eta} \mathrm{d} \eta^{\prime} \sqrt{k^{2}-\frac{5}{\eta^{\prime 2}}}\right]}{2 \eta^{3}\left(k^{2}-\frac{5}{\eta^{2}}\right)} & \text { for } \eta \sim \eta_{c}, \text { early \& late } \eta, \\ \int_{\tau^{\prime}}^{\tau} \mathrm{d} \eta \frac{\left[5+\Delta_{c}\right] \exp \left[-2 i \int^{\eta} \mathrm{d} \eta^{\prime} \sqrt{k^{2}-\frac{\left[5+\Delta_{c}\right]}{\eta^{\prime 2}}}\right]}{2 \eta^{3}\left(k^{2}-\frac{\left[5+\Delta_{c}\right]}{\eta^{2}}\right)} & \text { for } \eta<\eta_{c},\end{cases}
$$

where $\tau$ and $\tau^{\prime}$ are introduced as the conformal time regulator in the present context. In this case as well the Bogoliubov coefficient is not exactly analytically computable. To study the behaviour of this integral we consider here three similar consecutive physical situations for the de Sitter case and the quasi de Sitter case as discussed earlier. 


\section{For $\eta \sim \eta_{\mathbf{c}}$, early \& late $\eta$ :}

$$
\beta_{\text {diag }}\left(k ; \tau, \tau^{\prime}\right)= \begin{cases}\frac{\sqrt{5}}{2(2 \sqrt{5}-1)}\left[\tau^{\prime 2 \sqrt{5}-1}-\tau^{2 \sqrt{5}-1}\right] & \text { for }|k \eta|<<1, \\ \frac{5}{2 \sqrt{2 \Delta+4}(2 \sqrt{2 \Delta+4}-1)}\left[\tau^{\prime 2 \sqrt{2 \Delta+4}-1}-\tau^{2 \sqrt{2 \Delta+4}-1}\right] & \text { for }|k \eta| \approx 1-\Delta(\rightarrow 0), \\ \frac{5}{2}\left[\frac{e^{-2 i k \eta}(2 i k \eta-1)}{4 k^{2} \eta^{2}}-\operatorname{Ei}(-2 i k \eta)\right]_{\tau^{\prime}}^{\tau} & \text { for }|k \eta|>>1 .\end{cases}
$$

For $\eta<\eta_{\mathbf{c}}$ :

$$
\beta_{\text {diag }}\left(k ; \tau, \tau^{\prime}\right)=\left\{\begin{array}{l}
\frac{\sqrt{5+\Delta_{c}}}{2\left(2 \sqrt{5+\Delta_{c}}-1\right)}\left[\tau^{\prime 2 \sqrt{5+\Delta_{c}}-1}-\tau^{2 \sqrt{5+\Delta_{c}}-1}\right] \text { for }|k \eta|<<1, \\
\frac{\left(5+\Delta_{c}\right)\left[\tau^{\prime 2 \sqrt{2 \Delta+4+\Delta_{c}}-1}-\tau^{2 \sqrt{2 \Delta+4+\Delta_{c}}-1}\right]}{2 \sqrt{2 \Delta+4+\Delta_{c}}\left(2 \sqrt{2 \Delta+4+\Delta_{c}}-1\right)} \text { for }|k \eta| \approx 1-\Delta(\rightarrow 0), \\
\frac{\left(5+\Delta_{c}\right)}{2}\left[\frac{e^{-2 i k \eta}(2 i k \eta-1)}{4 k^{2} \eta^{2}}-\operatorname{Ei}(-2 i k \eta)\right]_{\tau^{\prime}}^{\tau} \text { for }|k \eta|>>1 .
\end{array}\right.
$$

Further using the regularized expressions for the Bogoliubov coefficient $\beta$ in two different representations as mentioned in Eqs. (4.73) and (4.78), and substituting them in Eq. (3.57) we get the following regularized expressions for the Bogoliubov coefficient $\alpha$ in two different representations as given by

$$
\begin{aligned}
& \text { For } \eta \sim \eta_{\mathfrak{c}} \text {, early \& late } \eta \text { : }
\end{aligned}
$$

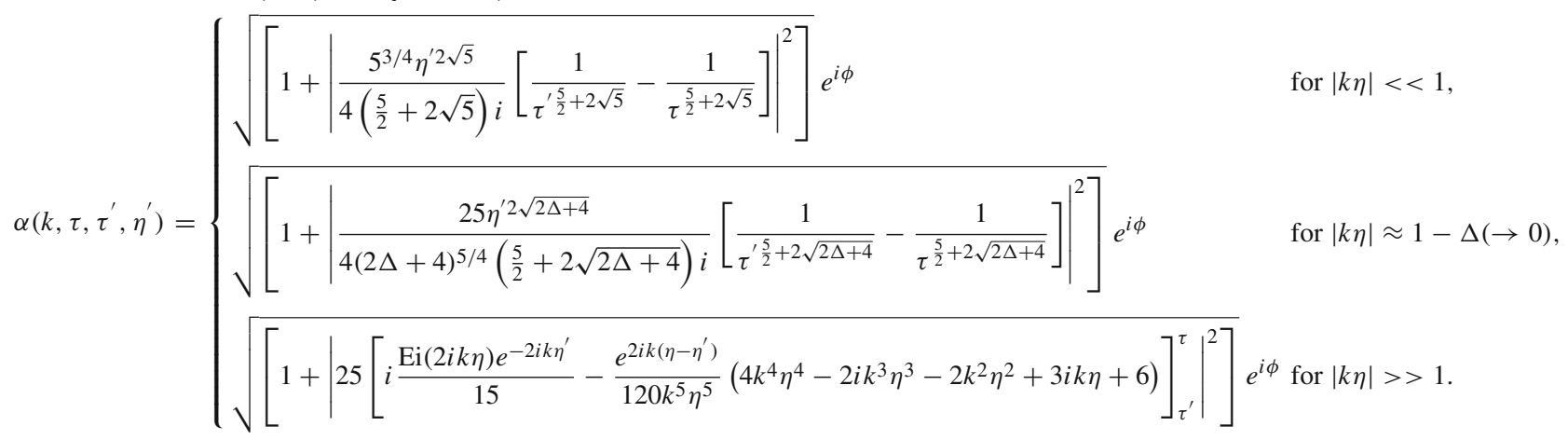

$$
\begin{aligned}
& \text { For } \eta<\eta_{\mathbf{c}} \text { : }
\end{aligned}
$$

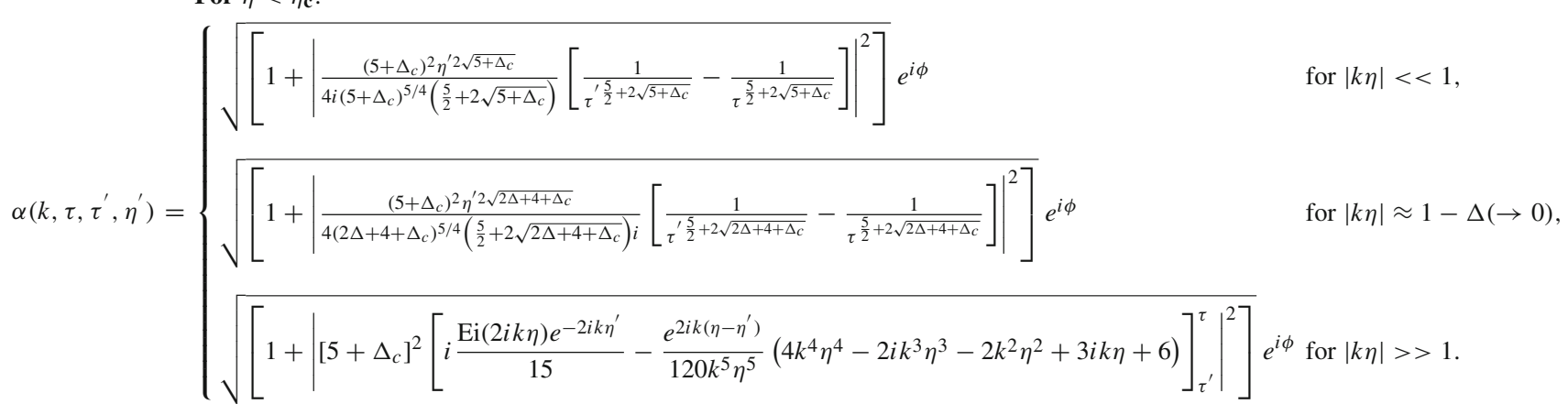


For $\eta \sim \eta_{\mathbf{c}}$, early \& late $\eta$ :

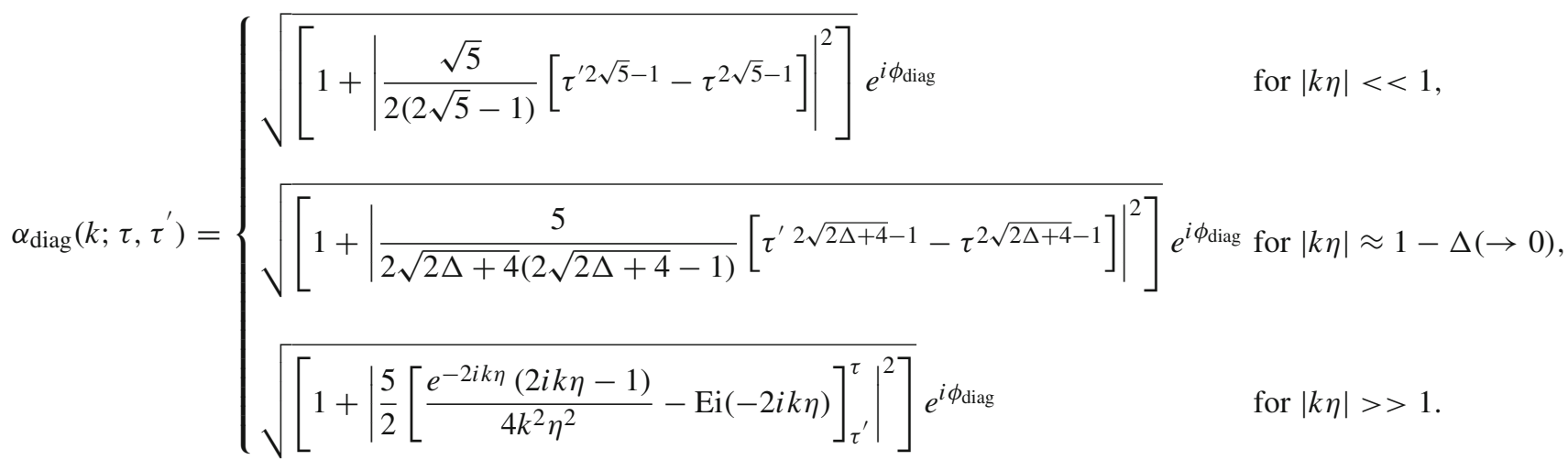

For $\eta<\eta_{\mathbf{c}}$ :

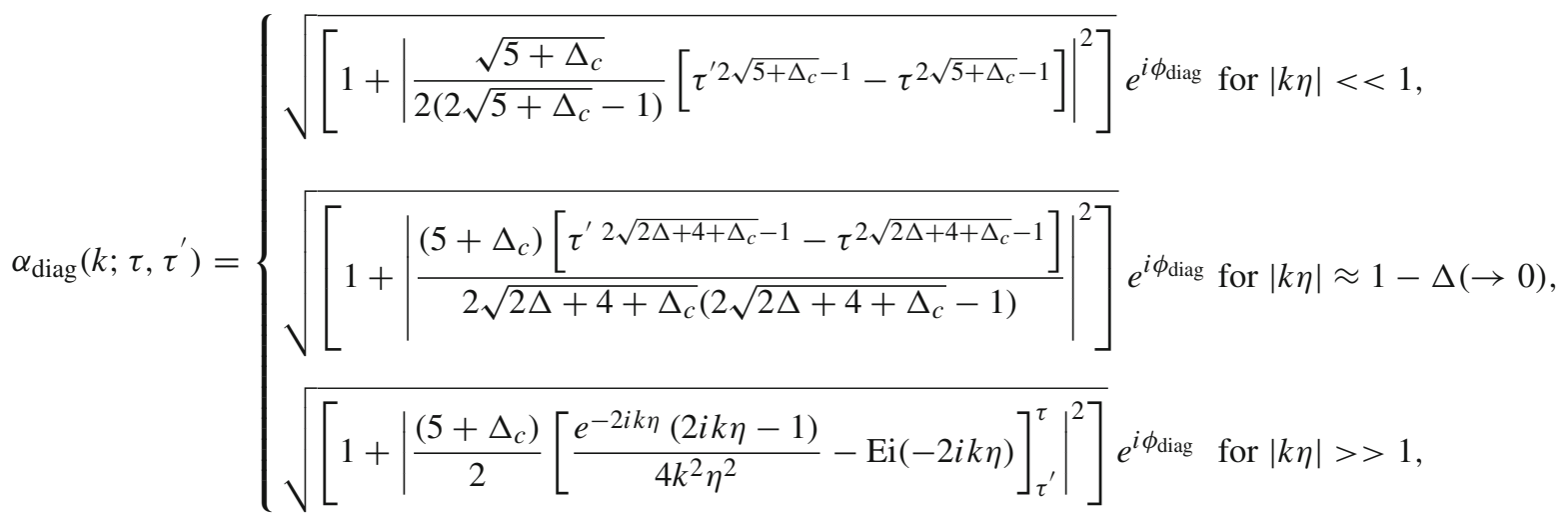

where $\phi$ and $\phi_{\text {diag }}$ are the associated phase factors in two different representations.

Further using the expressions for the Bogoliubov coefficient $\alpha$ in two different representations we get the following expressions for the reflection and transmission coefficient in two different representations for three consecutive physical situations: $|k \eta|<<1,|k \eta| \approx 1-\Delta(\rightarrow 0)$ and $|k \eta|>>1$ for the de Sitter case and the quasi de Sitter case as given by

For $\eta \sim \eta_{\mathbf{c}}$, early \& late $\eta$ :

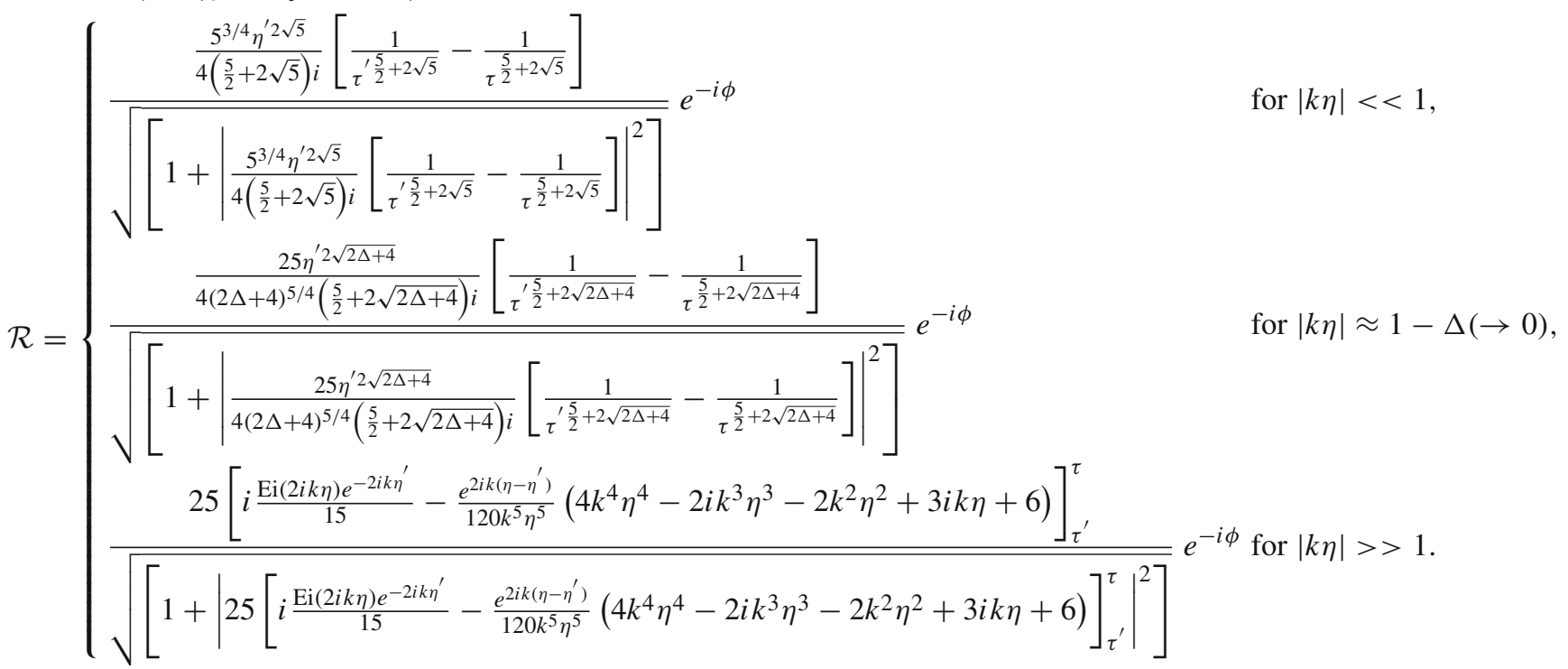


For $\eta \sim \eta_{\mathbf{c}}$, early \& late $\eta$ :

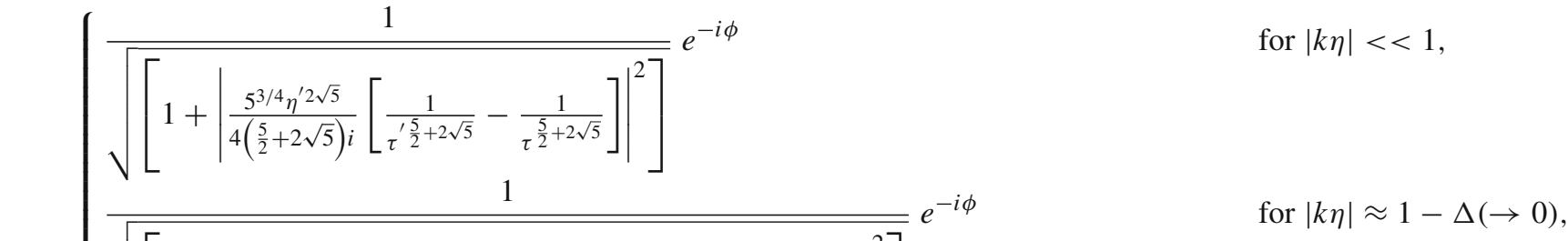

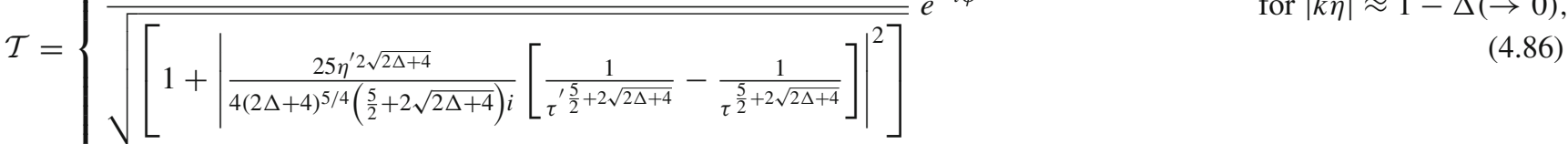

$$
\begin{aligned}
& \frac{1}{\sqrt{\left[1+\left|25\left[i \frac{\mathrm{Ei}(2 i k \eta) e^{-2 i k \eta^{\prime}}}{15}-\frac{e^{2 i k\left(\eta-\eta^{\prime}\right)}}{120 k^{5} \eta^{5}}\left(4 k^{4} \eta^{4}-2 i k^{3} \eta^{3}-2 k^{2} \eta^{2}+3 i k \eta+6\right)\right]_{\tau^{\prime}}^{\tau}\right|^{2}\right]}} e^{-i \phi} \text { for }|k \eta|>>1 \text {. }
\end{aligned}
$$

\section{For $\eta<\eta_{\mathbf{c}}$ :}

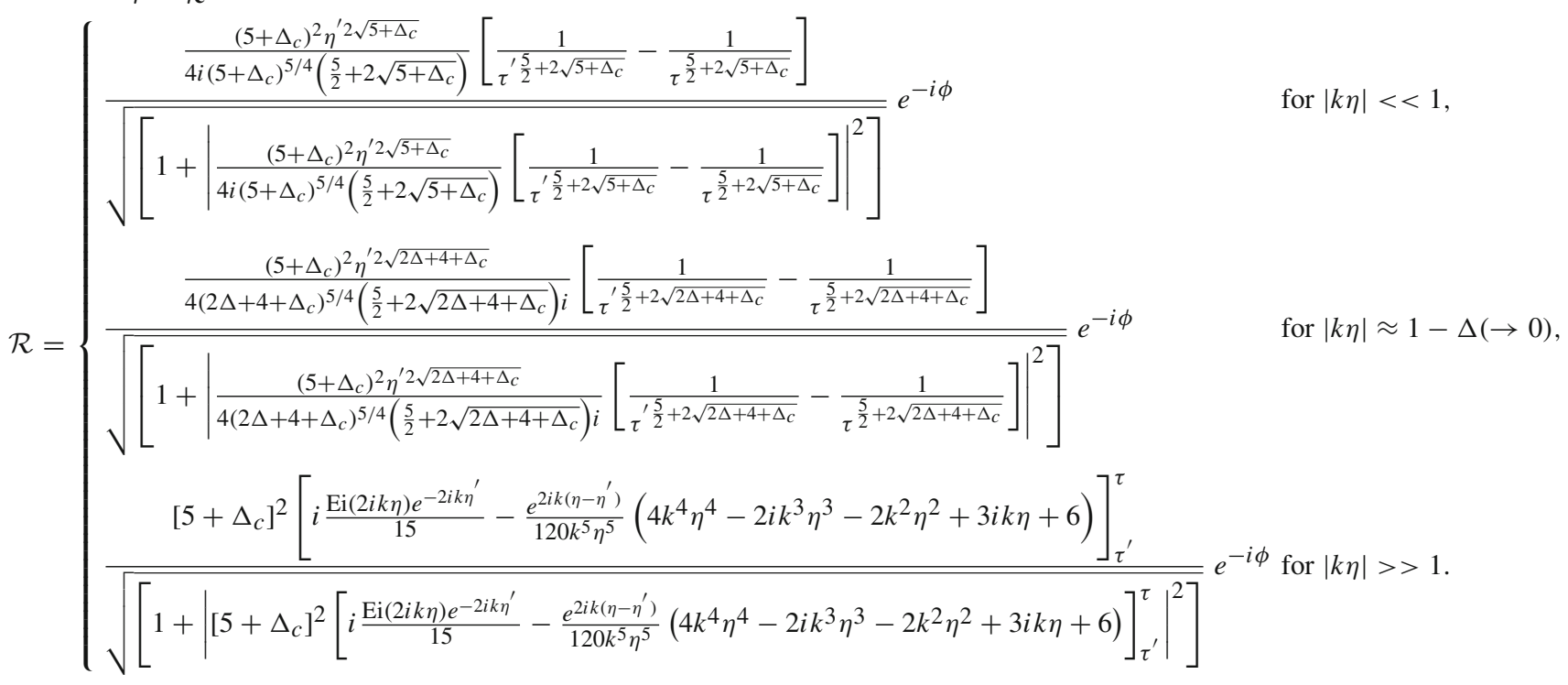

For $\eta<\eta_{\mathbf{c}}$ :

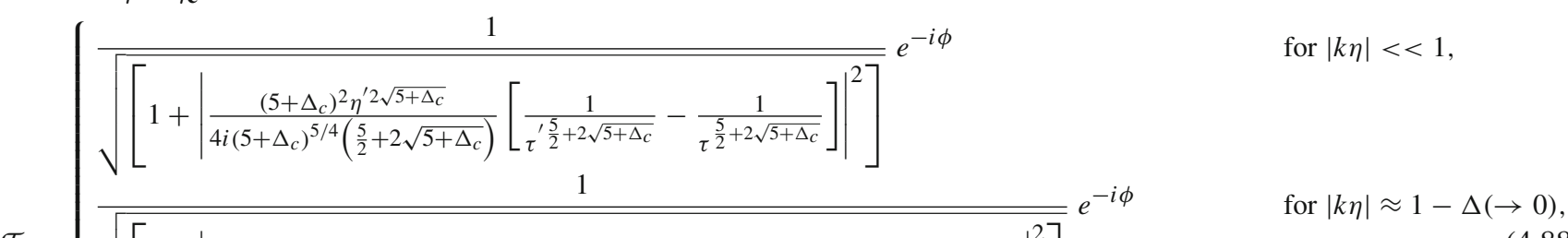

$$
\begin{aligned}
& \mathcal{T}= \begin{cases}\sqrt{\left[1+\mid \frac{\left(5+\Delta_{c}\right)^{2} \eta^{\prime 2} \sqrt{2 \Delta+4+\Delta_{c}}}{4\left(2 \Delta+4+\Delta_{c}\right)^{5 / 4}\left(\frac{5}{2}+2 \sqrt{2 \Delta+4+\Delta_{c}}\right)}\left[\frac{1}{\tau^{\frac{5}{2}+2 \sqrt{2 \Delta+4+\Delta_{c}}}-\frac{1}{\left.\left.\tau^{\frac{5}{2}+2 \sqrt{2 \Delta+4+\Delta_{c}}}\right]\left.\right|^{2}\right]}} e^{-i \phi}\right.\right.} \quad \text { for }|k \eta| \approx 1-\Delta(\rightarrow 0), & (4.88)\end{cases} \\
& \frac{1}{\left.\sqrt{\left[1+\mid\left[5+\Delta_{c}\right]^{2}\left[i \frac{\operatorname{Ei}(2 i k \eta) e^{-2 i k \eta^{\prime}}}{15}-\frac{e^{2 i k\left(\eta-\eta^{\prime}\right)}}{120 k^{5} \eta^{5}}\left(4 k^{4} \eta^{4}-2 i k^{3} \eta^{3}-2 k^{2} \eta^{2}+3 i k \eta+6\right)\right]_{\tau^{\prime}}^{\tau}\right.}\right]^{2}} e^{-i \phi} \text { for }|k \eta|>>1 .
\end{aligned}
$$




\section{For $\eta \sim \eta_{\mathbf{c}}$, early \& late $\eta$ :}

$\mathcal{R}_{\operatorname{diag}}\left(k ; \tau, \tau^{\prime}\right)= \begin{cases}\frac{\frac{\sqrt{5}}{2(2 \sqrt{5}-1)}\left[\tau^{2 \sqrt{5}-1}-\tau^{2 \sqrt{5}-1}\right]}{\sqrt{\left[1+\left|\frac{\sqrt{5}}{2(2 \sqrt{5}-1)}\left[\tau^{\prime 2 \sqrt{5}-1}-\tau^{2 \sqrt{5}-1}\right]\right|^{2}\right]} e^{-i \phi}} & \text { for }|k \eta|<<1, \\ \frac{5}{\left.\sqrt{\left[1+\mid \frac{5}{2 \sqrt{2 \Delta+4}(2 \sqrt{2 \Delta+4}-1)}\left[\tau^{\prime} 2 \sqrt{2 \Delta+4}-1\right.\right.}-\tau^{2 \sqrt{2 \Delta+4}-1}\right]} & e^{-i \phi} \text { for }|k \eta| \approx 1-\Delta(\rightarrow 0), \\ \frac{\frac{5}{2}\left[\frac{e^{-2 i k \eta}(2 i k \eta-1)}{4 k^{2} \eta^{2}}-\operatorname{Ei}(-2 i k \eta)\right]_{\tau^{\prime}}^{\tau}}{\left.\left.\sqrt{\left[1+\mid \frac{5}{2}\left[\frac{e^{-2 i k \eta}(2 i k \eta-1)}{4 k^{2} \eta^{2}}-\operatorname{Ei}(-2 i k \eta)\right]^{\tau}\right]^{\prime}}\right|^{2}\right]} e^{-i \phi} & \text { for }|k \eta|>>1 .\end{cases}$

For $\eta \sim \eta_{\mathbf{c}}$, early \& late $\eta$ :

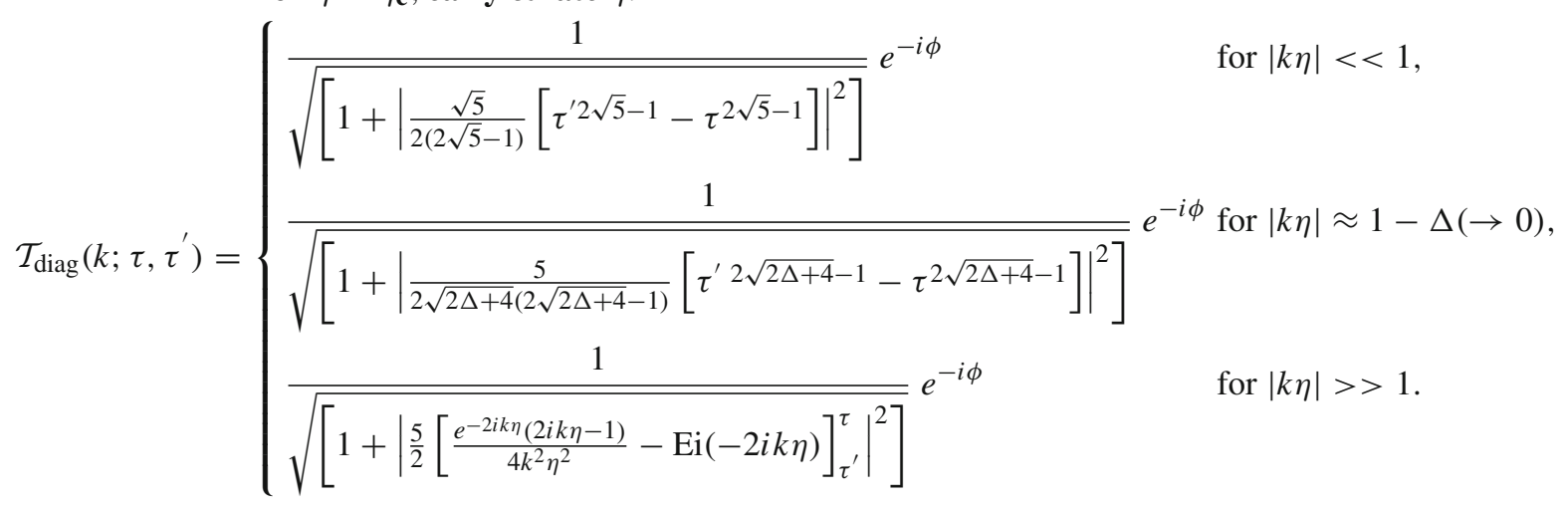

For $\eta<\eta_{\mathbf{c}}$ :

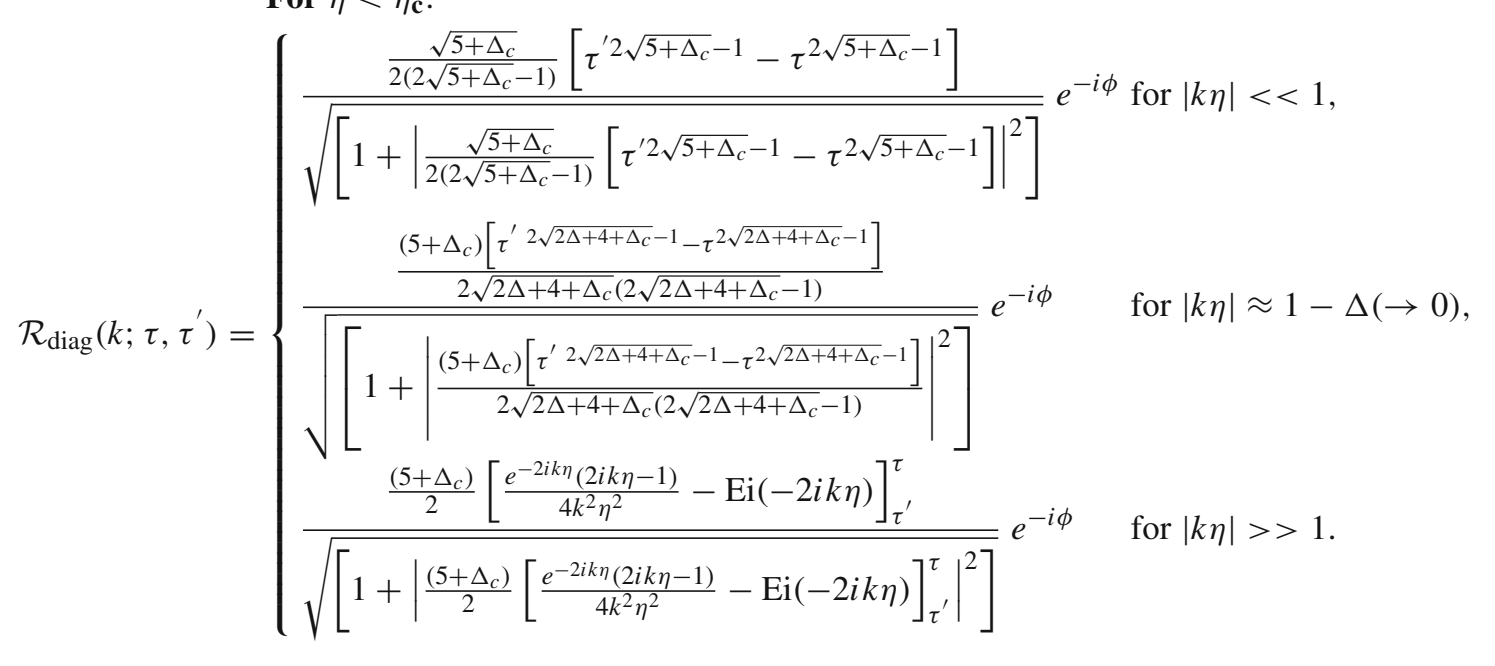


For $\eta<\eta_{\mathbf{c}}$ :

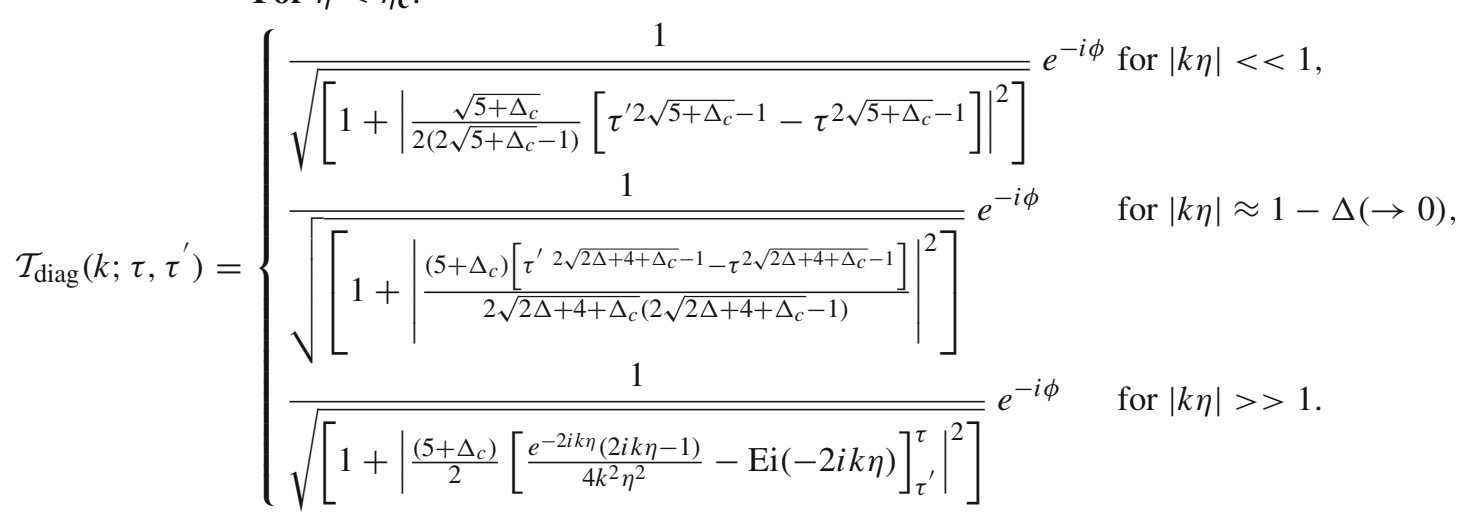

Next the expression for the number of produced particles at time $\tau$ can be expressed in the two representations as

$$
\begin{gathered}
\mathcal{N}\left(\tau, \tau^{\prime}, \eta^{\prime}\right)=\frac{1}{(2 \pi a)^{3}} \int \mathrm{d}^{3} \mathbf{k}\left\{\begin{array}{l}
\left|\int_{\tau^{\prime}}^{\tau} \mathrm{d} \eta \frac{25 \exp \left[2 i \int_{\eta^{\prime}}^{\eta} \mathrm{d} \eta^{\prime \prime} \sqrt{k^{2}-\frac{5}{\eta^{\prime \prime 2}}}\right]}{4 \eta^{6}\left(k^{2}-\frac{5}{\eta^{2}}\right)^{\frac{5}{2}}}\right|^{2} \\
\left|\int_{\tau^{\prime}}^{\tau} \mathrm{d} \eta \frac{\left[5+\Delta_{c}\right]^{2} \exp \left[2 i \int_{\eta^{\prime}}^{\eta} \mathrm{d} \eta^{\prime \prime} \sqrt{\left.k^{2}-\frac{\left[5+\Delta_{c}\right]}{\eta^{\prime \prime 2}}\right]}\right.}{4 \eta^{6}\left(k^{2}-\frac{\left[5+\Delta_{c}\right]}{\eta^{2}}\right)^{\frac{5}{2}}}\right|^{2}
\end{array} \text { for } \eta \sim \eta_{c}, \text { early \& late } \eta,\right.
\end{gathered}
$$

which are not exactly analytically computable. To study the behaviour of this integral we consider here three consecutive physical situations: $|k \eta|<<1,|k \eta| \approx 1-\Delta(\rightarrow 0)$ and $|k \eta|>>1$ for the de Sitter and the quasi de Sitter case. In the three cases we have

$$
\begin{aligned}
& \text { For } \eta \sim \eta_{\mathbf{c}} \text {, early \& late } \eta \text { : } \\
& \mathcal{N}\left(\tau, \tau^{\prime}, \eta^{\prime}\right)= \begin{cases}\frac{V}{(2 \pi a)^{3}} \frac{5^{3 / 2} \eta^{\prime 4 \sqrt{5}}}{16\left(\frac{5}{2}+2 \sqrt{5}\right)^{2}}\left[\frac{1}{\tau^{\prime \frac{5}{2}+2 \sqrt{5}}}-\frac{1}{\tau^{\frac{5}{2}+2 \sqrt{5}}}\right]^{2} & \text { for }|k \eta|<<1 \\
\frac{V}{(2 \pi a)^{3}} \frac{625 \eta^{\prime 4 \sqrt{2 \Delta+4}}}{16(2 \Delta+4)^{5 / 2}\left(\frac{5}{2}+2 \sqrt{2 \Delta+4}\right)^{2}}\left[\frac{1}{\tau^{\prime \frac{5}{2}+2 \sqrt{2 \Delta+4}}}-\frac{1}{\tau^{\frac{5}{2}+2 \sqrt{2 \Delta+4}}}\right]^{2} \text { for }|k \eta| \approx 1-\Delta(\rightarrow 0) \\
\frac{100 \pi}{(2 \pi a)^{3}} \int_{0}^{\infty} \mathrm{d} k k^{2} \mid\left\{\frac{i e^{-2 i k \eta^{\prime}}}{15}\left\{\operatorname{Ei}(2 i k \tau)-\operatorname{Ei}\left(2 i k \tau^{\prime}\right)\right\}\right. \\
-\frac{e^{-2 i k \eta^{\prime}}}{120 k^{5}}\left[\frac{e^{2 i k \tau}\left(4 k^{4} \tau^{4}-2 i k^{3} \tau^{3}-2 k^{2} \tau^{2}+3 i k \tau+6\right)}{\tau^{5}}\right. & \text { for }|k \eta|>>1 . \\
\left.\left.-\frac{e^{2 k \tau}\left(4 k^{4} \tau^{\prime 4}-2 i k^{3} \tau^{\prime 3}-2 k^{2} \tau^{\prime 2}+3 i k \tau^{\prime}+6\right)}{\tau^{\prime 5}}\right]\right\}\left.\right|^{2} & \end{cases}
\end{aligned}
$$


For $\eta<\eta_{\mathbf{c}}$ :

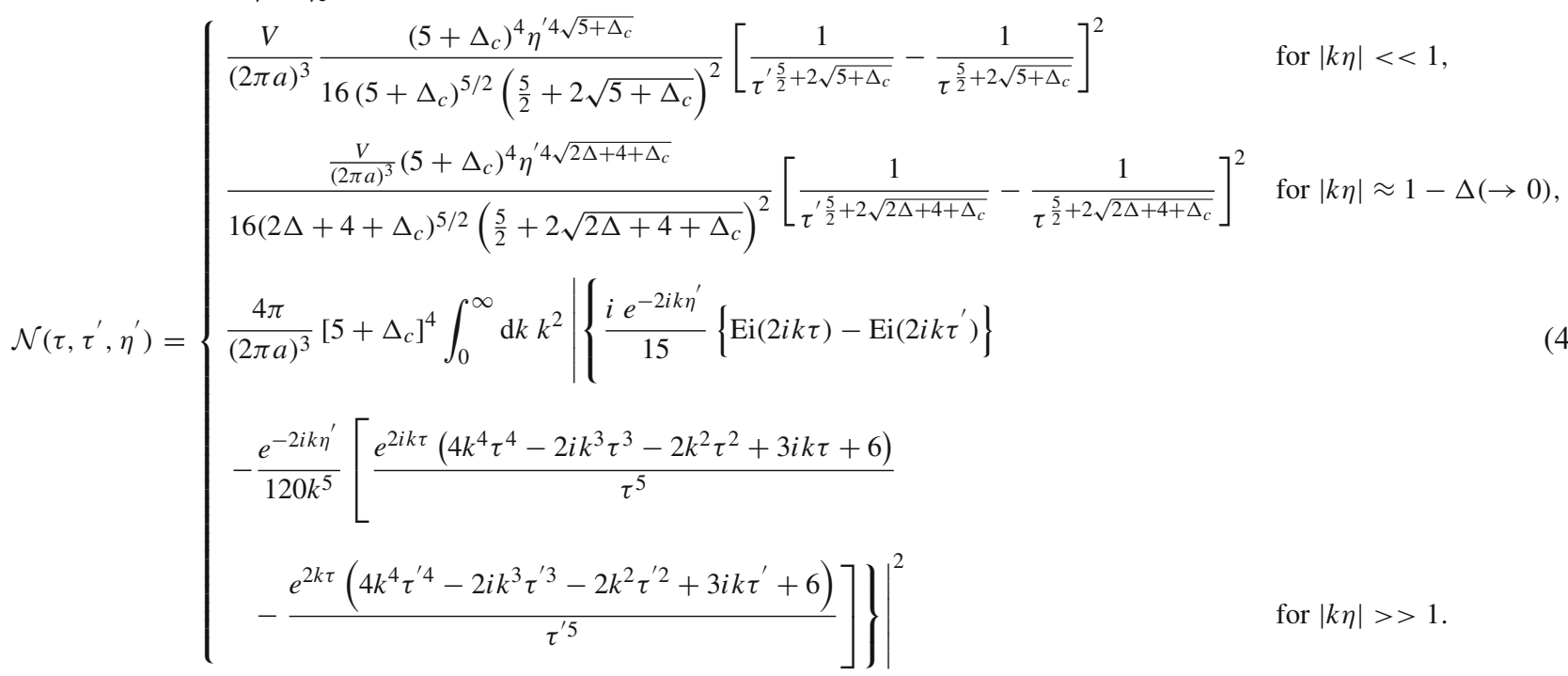

For $\eta \sim \eta_{\mathbf{c}}$, early \& late $\eta$ :

$\mathcal{N}_{\text {diag }}\left(\tau, \tau^{\prime}\right)= \begin{cases}\frac{V}{(2 \pi a)^{3}} \frac{5}{4(2 \sqrt{5}-1)^{2}}\left[\tau^{\prime 2 \sqrt{5}-1}-\tau^{2 \sqrt{5}-1}\right]^{2} & \text { for }|k \eta|<<1, \\ \frac{V}{(2 \pi a)^{3}} \frac{25}{4(2 \Delta+4)(2 \sqrt{2 \Delta+4}-1)^{2}}\left[\tau^{\prime 2 \sqrt{2 \Delta+4}-1}-\tau^{2 \sqrt{2 \Delta+4}-1}\right]^{2} & \text { for }|k \eta| \approx 1-\Delta(\rightarrow 0), \\ \frac{25 \pi}{(2 \pi a)^{3}} \int_{0}^{\infty} \mathrm{d} k k^{2} \mid\left[\left\{\frac{e^{-2 i k \tau}(2 i k \tau-1)}{4 k^{2} \tau^{2}}-\frac{e^{-2 i k \tau^{\prime}}\left(2 i k \tau^{\prime}-1\right)}{4 k^{2} \tau^{\prime 2}}\right\}\right. & \text { for }|k \eta|>>1 .\end{cases}$

For $\eta<\eta_{\mathbf{c}}$ :

$\mathcal{N}_{\text {diag }}\left(\tau, \tau^{\prime}\right)= \begin{cases}\frac{V}{(2 \pi a)^{3}} \frac{\left(5+\Delta_{c}\right)}{4\left(2 \sqrt{5+\Delta_{c}}-1\right)^{2}}\left[\tau^{\prime 2 \sqrt{5+\Delta_{c}}-1}-\tau^{2 \sqrt{5+\Delta_{c}}-1}\right]^{2} & \text { for }|k \eta|<<1, \\ \frac{V}{(2 \pi a)^{3}} \frac{\left(5+\Delta_{c}\right)^{2}\left[\tau^{\prime} 2 \sqrt{2 \Delta+4+\Delta_{c}}-1\right.}{4\left(2 \Delta+4+\tau_{c}^{2 \sqrt{2 \Delta+4+\Delta_{c}}-1}\right]^{2}} & \text { for }|k \eta| \approx 1-\Delta(\rightarrow), \\ \frac{\left(5+\Delta_{c}\right)^{2} \pi}{(2 \pi a)^{3}} \int_{0}^{\infty} \mathrm{d} k k^{2} \mid\left[\left\{\frac{e^{-2 i k \tau}(2 i k \tau-1)}{4 k^{2} \tau^{2}}-\frac{e^{-2 i k \tau^{\prime}}\left(2 i k \tau^{\prime}-1\right)}{4 k^{2} \tau^{\prime 2}}\right\}\right. & \text { for }|k \eta|>>1,\end{cases}$

where the symbol $V$ was defined earlier. 
Finally one can define the total energy density of the produced particles using the following expression:

$$
\begin{aligned}
& \rho\left(\tau, \tau^{\prime}, \eta^{\prime}\right)=\frac{1}{(2 \pi a)^{3} a} \int \mathrm{d}^{3} \mathbf{k}\left\{\begin{array}{l}
\sqrt{k^{2}-\frac{5}{\tau^{2}}}\left|\int_{\tau^{\prime}}^{\tau} \mathrm{d} \eta \frac{25 \exp \left[2 i \int_{\eta^{\prime}}^{\eta} \mathrm{d} \eta^{\prime \prime} \sqrt{k^{2}-\frac{5}{\eta^{\prime \prime 2}}}\right]}{4 \eta^{6}\left(c_{S}^{2} k^{2}-\frac{5}{\eta^{2}}\right)^{\frac{5}{2}}}\right|^{2} \quad \text { for } \eta \sim \eta_{c}, \text { early \& late } \eta, \\
\sqrt{k^{2}-\frac{\left[5+\Delta_{c}\right]}{\tau^{2}}}\left|\int_{\tau^{\prime}}^{\tau} \mathrm{d} \eta \frac{\left[5+\Delta_{c}\right]^{2} \exp \left[2 i \int_{\eta^{\prime},}^{\eta} \eta^{\prime \prime} \sqrt{k^{2}-\frac{\left[5+\Delta_{c}\right]}{\eta^{\prime \prime 2}}}\right]}{4 \eta^{6}\left(k^{2}-\frac{\left[5+\Delta_{c}\right]}{\eta^{2}}\right)^{\frac{5}{2}}}\right|^{2} \text { for } \eta<\eta_{c},
\end{array}\right. \\
& 1 \int \sqrt{k^{2}-\frac{5}{\tau^{2}}}\left|\int_{\tau^{\prime}}^{\tau} \mathrm{d} \eta \frac{5 \exp \left[-2 i \int^{\eta} \mathrm{d} \eta^{\prime} \sqrt{k^{2}-\frac{5}{\eta^{\prime 2}}}\right]}{2 \eta^{3}\left(k^{2}-\frac{5}{\eta^{2}}\right)}\right|^{2} \quad \text { for } \eta \sim \eta_{c} \text {, early \& late } \eta, \\
& \rho_{\text {diag }}\left(\tau, \tau^{\prime}, \eta^{\prime}\right)=\frac{1}{(2 \pi a)^{3} a} \int \mathrm{d}^{3} \mathbf{k}\left\{\begin{array}{l}
\mid \\
\sqrt{k^{2}-\frac{\left[5+\Delta_{c}\right]}{\tau^{2}}}\left|\int_{\tau^{\prime}}^{\tau} \mathrm{d} \eta \frac{\left[5+\Delta_{c}\right] \exp \left[-2 i \int^{\eta} \mathrm{d} \eta^{\prime} \sqrt{\left.k^{2}-\frac{\left[5+\Delta_{c}\right]}{\eta^{\prime 2}}\right]}\right.}{2 \eta^{2}\left(k^{2}-\frac{\left[5+\Delta_{c}\right]}{\eta^{2}}\right)}\right|^{2} \text { for } \eta<\eta_{c},
\end{array}\right.
\end{aligned}
$$

which is not exactly analytically computable. To study the behaviour of this integral we consider here three consecutive physical situations: $|k \eta|<<1,|k \eta| \approx 1-\Delta(\rightarrow 0)$ and $|k \eta|>>1$ for the de Sitter case and the quasi de Sitter case. In the three cases we have

$$
\begin{aligned}
& \text { For } \eta \sim \eta_{\mathbf{c}} \text {, early \& late } \eta \text { : } \\
& \rho\left(\tau, \tau^{\prime}, \eta^{\prime}\right)= \begin{cases}\frac{J}{(2 \pi a)^{3} a} \frac{5^{3 / 2} \eta^{\prime} 4 \sqrt{5}}{16\left(\frac{5}{2}+2 \sqrt{5}\right)^{2}}\left[\frac{1}{\tau^{\prime \frac{5}{2}+2 \sqrt{5}}}-\frac{1}{\tau^{\frac{5}{2}+2 \sqrt{5}}}\right]^{2} & \text { for }|k \eta|<<1, \\
\frac{J}{(2 \pi a)^{3} a} \frac{625 \eta^{\prime 4 \sqrt{2 \Delta+4}}}{16(2 \Delta+4)^{5 / 2}\left(\frac{5}{2}+2 \sqrt{2 \Delta+4}\right)^{2}}\left[\frac{1}{\tau^{\prime \frac{5}{2}+2 \sqrt{2 \Delta+4}}}-\frac{1}{\tau^{\frac{5}{2}+2 \sqrt{2 \Delta+4}}}\right]^{2} & \text { for }|k \eta| \approx 1-\Delta(\rightarrow 0), \\
\frac{100 \pi}{(2 \pi a)^{3} a} \int_{0}^{\infty} \mathrm{d} k k^{3} \mid\left\{\frac{i e^{-2 i k \eta^{\prime}}}{15}\left\{\operatorname{Ei}(2 i k \tau)-\operatorname{Ei}\left(2 i k \tau^{\prime}\right)\right\}\right. & \\
-\frac{e^{-2 i k \eta^{\prime}}}{120 k^{5}}\left[\frac{e^{2 i k \tau}\left(4 k^{4} \tau^{4}-2 i k^{3} \tau^{3}-2 k^{2} \tau^{2}+3 i k \tau+6\right)}{\tau^{5}}\right. & \text { for }|k \eta|>>1 .\end{cases} \\
& \text { For } \eta<\eta_{\mathbf{c}} \text { : }
\end{aligned}
$$

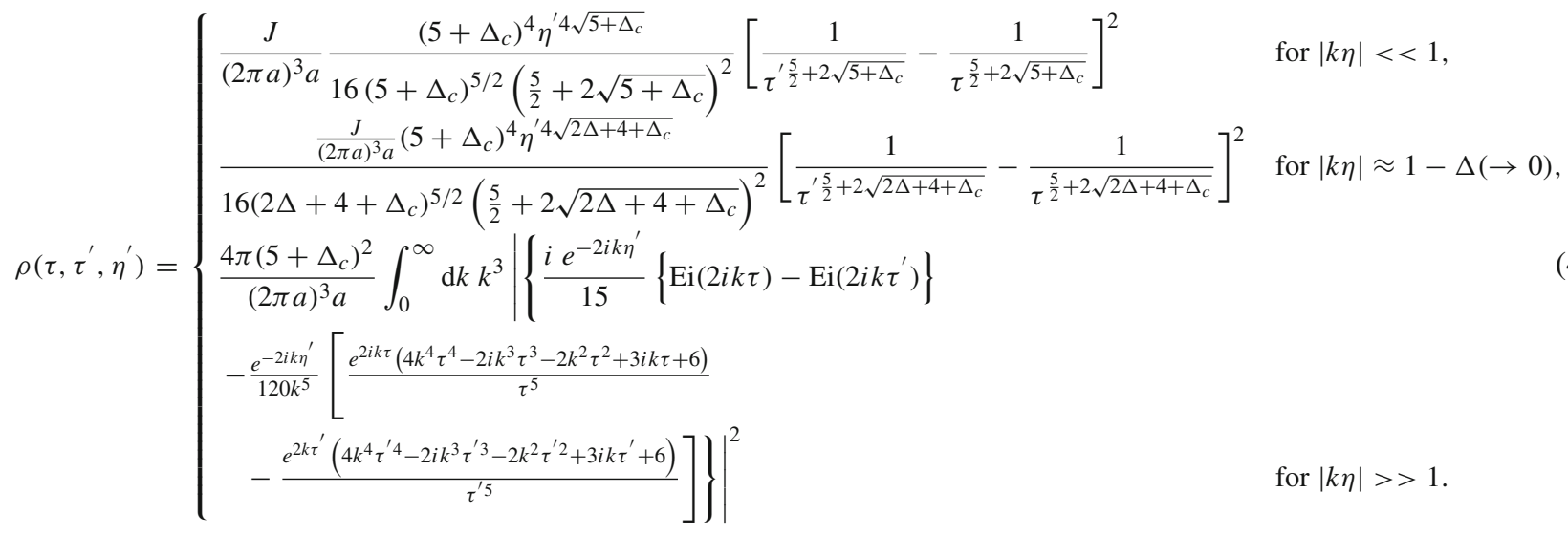


and

$$
\begin{aligned}
& \text { For } \eta \sim \eta_{\mathbf{c}}, \text { early \& late } \eta: \\
& \rho_{\text {diag }}\left(\tau, \tau^{\prime}\right)= \begin{cases}\frac{J}{(2 \pi a)^{3} a} \frac{5}{4(2 \sqrt{5}-1)^{2}}\left[\tau^{\prime 2 \sqrt{5}-1}-\tau^{2 \sqrt{5}-1}\right]^{2} & \text { for }|k \eta|<<1, \\
\frac{J}{(2 \pi a)^{3} a} \frac{25}{4(2 \Delta+4)(2 \sqrt{2 \Delta+4}-1)^{2}}\left[\tau^{\prime 2 \sqrt{2 \Delta+4}-1}-\tau^{2 \sqrt{2 \Delta+4}-1}\right]^{2} & \text { for }|k \eta| \approx 1-\Delta(\rightarrow 0), \\
\frac{25 \pi}{(2 \pi a)^{3} a} \int_{0}^{\infty} \mathrm{d} k k^{3} \mid \frac{e^{-2 i k \tau}(2 i k \tau-1)}{4(k \tau)^{2}}-\frac{e^{-2 i k \tau^{\prime}}\left(2 i k \tau^{\prime}-1\right)}{4\left(k \tau^{\prime}\right)^{2}} & \\
+\left.\left\{\operatorname{Ei}(-2 i k \tau)-\operatorname{Ei}\left(-2 i k \tau^{\prime}\right)\right\}\right|^{2} & \text { for }|k \eta|>>1 .\end{cases}
\end{aligned}
$$

For $\eta<\eta_{\mathbf{c}}$ :

$$
\rho_{\text {diag }}\left(\tau, \tau^{\prime}, \eta^{\prime}\right)= \begin{cases}\frac{J}{(2 \pi a)^{3} a} \frac{\left(5+\Delta_{c}\right)}{4\left(2 \sqrt{5+\Delta_{c}}-1\right)^{2}}\left[\tau^{\prime 2 \sqrt{5+\Delta_{c}}-1}-\tau^{2 \sqrt{5+\Delta_{c}}-1}\right]^{2} & \text { for }|k \eta|<<1, \\ \left.\frac{J}{(2 \pi a)^{3} a} \frac{\left(5+\Delta_{c}\right)^{2}\left[\tau^{\prime} 2 \sqrt{2 \Delta+4+\Delta_{c}}-1\right.}{4\left(2 \Delta+4+\Delta_{c}\right)\left(2 \sqrt{2 \Delta+4+\Delta_{c}}-1\right.}\right]^{2} & \text { for }|k \eta| \approx 1-\Delta(\rightarrow 0), \\ \frac{\left(5+\Delta_{c}\right)^{2} \pi}{(2 \pi a)^{3} a} \int_{0}^{\infty} \mathrm{d} k k^{3} \mid \frac{e^{-2 i k \tau}\left(2 i k c_{S} \tau-1\right)}{4(k \tau)^{2}}-\frac{e^{-2 i k \tau^{\prime}}\left(2 i k \tau^{\prime}-1\right)}{4\left(k \tau^{\prime}\right)^{2}} & \text { for }|k \eta|>>1 . \\ +\left.\left\{\operatorname{Ei}(-2 i k \tau)-\operatorname{Ei}\left(-2 i k \tau^{\prime}\right)\right\}\right|^{2} & \text { in }\left.\right|^{2}\end{cases}
$$

Throughout the discussion of the total energy density of the produced particles we have introduced a symbol $J$ defined as

$$
J=\int \mathrm{d}^{3} \mathbf{k} p(\tau)= \begin{cases}\int \mathrm{d}^{3} \mathbf{k} \sqrt{k^{2}-\frac{5}{\tau^{2}}} & \text { for } \mathrm{dS}, \\ \int \mathrm{d}^{3} \mathbf{k} \sqrt{k^{2}-\frac{\left[5+\Delta_{c}\right]}{\tau^{2}}} & \text { for qdS, }\end{cases}
$$

which physically signifies the total finite volume weighted by $p(\eta)$ in momentum space within which the produced particles are occupied. To study the behaviour of this integral we consider here three consecutive physical situations: $|k \eta|<<1$, $|k \eta| \approx 1-\Delta(\rightarrow 0)$ and $|k \eta|>>1$ for the de Sitter and the quasi de Sitter case. In the three cases we have

For $\eta \sim \eta_{\mathbf{c}}$, early \& late $\eta$ :

$J= \begin{cases}\int \mathrm{d}^{3} \mathbf{k} \frac{5}{\tau}=\frac{5 V}{\tau} & \text { for }|k \eta|<<1, \\ \int \mathrm{d}^{3} \mathbf{k} \frac{\sqrt{2 \Delta+4}}{\tau}=\frac{\sqrt{2 \Delta+4} V}{\tau} & \text { for }|k \eta| \approx 1-\Delta(\rightarrow 0), \\ \int \mathrm{d}^{3} \mathbf{k} k & \text { for }|k \eta|>>1 .\end{cases}$

\section{For $\eta<\eta_{\mathbf{c}}$ :}

$J= \begin{cases}\int \mathrm{d}^{3} \mathbf{k} \frac{\sqrt{\left[5+\Delta_{c}\right]}}{\tau}=\frac{V \sqrt{\left[5+\Delta_{c}\right]}}{\tau} & \text { for }|k \eta|<<1, \\ \int \mathrm{d}^{3} \mathbf{k} \frac{\sqrt{2 \Delta+4+\Delta_{c}}}{\tau}=\frac{\sqrt{2 \Delta+4+\Delta_{c}} V}{\tau} & \text { for }|k \eta| \approx 1-\Delta(\rightarrow 0), \\ \int \mathrm{d}^{3} \mathbf{k} k & \text { for }|k \eta|>>1 .\end{cases}$ 


\subsubsection{Case II: $m_{\mathrm{axion}} / f_{a}<<H$}

The equation of motion for the axion field for $m_{\text {axion }} / f_{a} \approx H$ is given by

$$
\begin{aligned}
& \vartheta_{k}^{\prime \prime}+\left\{k^{2}-\frac{6}{\eta^{2}}\right\} \vartheta_{k}=0 \quad \text { for } \eta \sim \eta_{\mathbf{c}}, \text { early \& late } \eta \\
& \vartheta_{k}^{\prime \prime}+\left\{k^{2}-\left[6+\Delta_{c}\right] \frac{1}{\eta^{2}}\right\} \vartheta_{k}=0 \quad \text { for } \eta<\eta_{\mathbf{c}} .
\end{aligned}
$$

The solution for the mode function for the de Sitter and the quasi de Sitter space can be expressed as

$$
\vartheta_{k}(\eta)= \begin{cases}\sqrt{-\eta}\left[C_{1} H_{5 / 2}^{(1)}(-k \eta)+C_{2} H_{5 / 2}^{(2)}(-k \eta)\right] & \text { for } \eta \sim \eta_{c}, \text { early \& late } \eta, \\ \sqrt{-\eta}\left[C_{1} H_{\sqrt{\frac{25}{4}+\Delta_{c}}}^{(1)}(-k \eta)+C_{2} H_{\sqrt{\frac{25}{4}+\Delta_{c}}}^{(2)}(-k \eta)\right] & \text { for } \eta<\eta_{c},\end{cases}
$$

where $C_{1}$ and $C_{2}$ are two arbitrary integration constants, which depend on the choice of the initial condition.

After taking the $k \eta \rightarrow-\infty, k \eta \rightarrow 0$ and $|k \eta| \approx 1-\Delta(\rightarrow 0)$ limits the most general solution as stated in Eq. (4.110) can be recast as

$$
\begin{aligned}
& \vartheta_{k}(\eta) \stackrel{|k \eta| \rightarrow-\infty}{=} \begin{cases}\sqrt{\frac{2}{\pi k}}\left[C_{1} e^{-i k \eta} e^{-\frac{3 i \pi}{2}}+C_{2} e^{i k \eta} e^{\frac{3 i \pi}{2}}\right] & \text { for } \eta \sim \eta_{c}, \text { early \& late } \eta, \\
\sqrt{\frac{2}{\pi k}}\left[C_{1} e^{-i k \eta} e^{-\frac{i \pi}{2}\left(\sqrt{\frac{25}{4}+\Delta_{c}}+\frac{1}{2}\right)}+C_{2} e^{i k \eta} e^{\frac{i \pi}{2}\left(\sqrt{\frac{25}{4}+\Delta_{c}}+\frac{1}{2}\right)}\right] & \text { for } \eta<\eta_{c},\end{cases} \\
& \vartheta_{k}(\eta) \stackrel{|k \eta| \rightarrow 0}{=} \begin{cases}\frac{i \sqrt{-\eta}}{\pi} \Gamma\left(\frac{5}{2}\right)\left(-\frac{k \eta}{2}\right)^{-\frac{5}{2}}\left[C_{1}-C_{2}\right] & \text { for } \eta \sim \eta_{c}, \text { early \& late } \eta, \\
\frac{i \sqrt{-\eta}}{\pi} \Gamma\left(\sqrt{\frac{25}{4}+\Delta_{c}}\right)\left(-\frac{k \eta}{2}\right)^{-\sqrt{\frac{25}{4}+\Delta_{c}}}\left[C_{1}-C_{2}\right] & \text { for } \eta<\eta_{c},\end{cases} \\
& \vartheta_{k}(\eta) \stackrel{i k \eta \approx 1-\Delta(\rightarrow 0)}{=}\left\{\begin{aligned}
\frac{i}{\pi} \sqrt{-\eta} & {\left[\frac{2}{5}-\gamma+\frac{5}{4}\left(\gamma^{2}+\frac{\pi^{2}}{6}\right)\right.} \\
& \left.-\frac{7}{8}\left(\gamma^{3}+\frac{\gamma \pi^{2}}{2}+2 \zeta(3)\right)+\cdots\right] \\
\times & \left(\frac{1+\Delta}{2}\right)^{-\frac{5}{2}}\left[C_{1}-C_{2}\right] \\
\frac{i}{\pi} \sqrt{-\eta} & {\left[\frac{1}{\left\{\sqrt{\frac{25}{4}+\Delta_{c}}\right\}}-\gamma+\frac{1}{2}\left(\gamma^{2}+\frac{\pi^{2}}{6}\right)\left\{\sqrt{\left.\frac{25}{4}+\Delta_{c}\right\}}\right\}\right.} \\
& \left.-\frac{1}{6}\left(\gamma^{3}+\frac{\gamma \pi^{2}}{2}+2 \zeta(3)\right)\left\{\sqrt{\frac{25}{4}+\Delta_{c}}\right\}^{2}+\cdots\right] \\
\times & \text { for } \eta \sim \eta_{c},
\end{aligned}\right.
\end{aligned}
$$

Next we assume that the WKB approximation is approximately valid for all times for the solution for the mode function $\vartheta_{k}$. In the standard WKB approximation the total solution can be recast in the following form:

$\vartheta_{k}(\eta)=\left[D_{1} u_{k}(\eta)+D_{2} \bar{u}_{k}(\eta)\right]$, 
where $D_{1}$ and $D_{2}$ are two arbitrary integration constants, which depend on the choice of the initial condition during WKB approximation at early and late time scale. In the present context $u_{k}(\eta)$ and $\bar{u}_{k}(\eta)$ are defined as

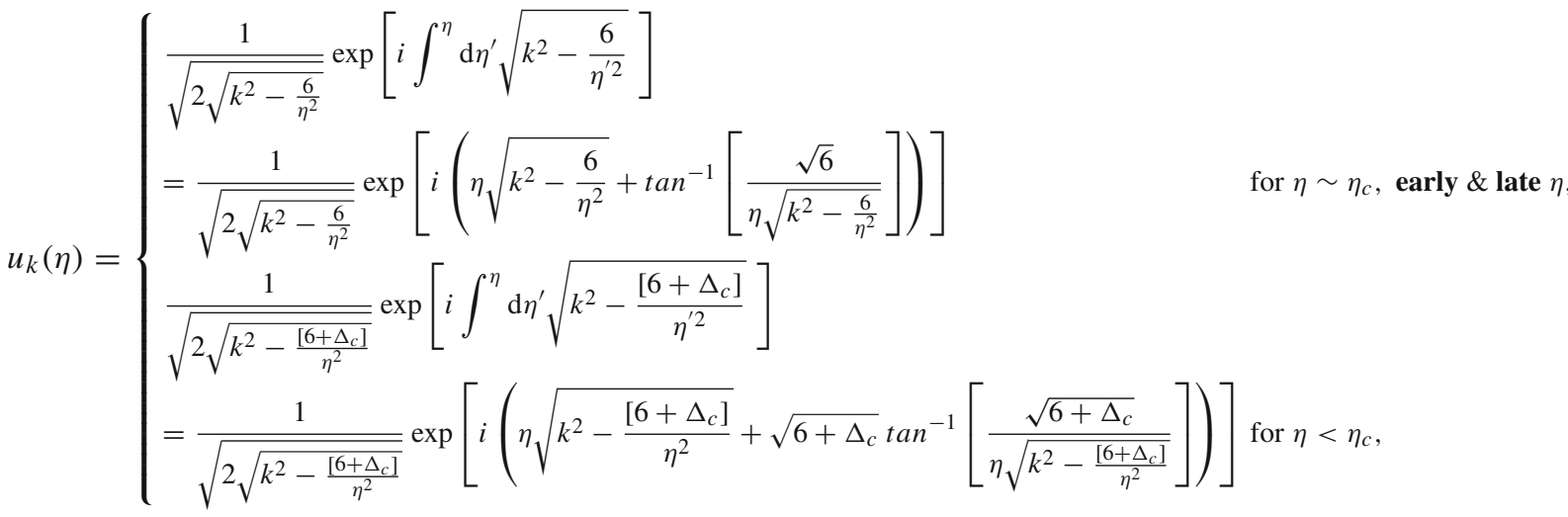

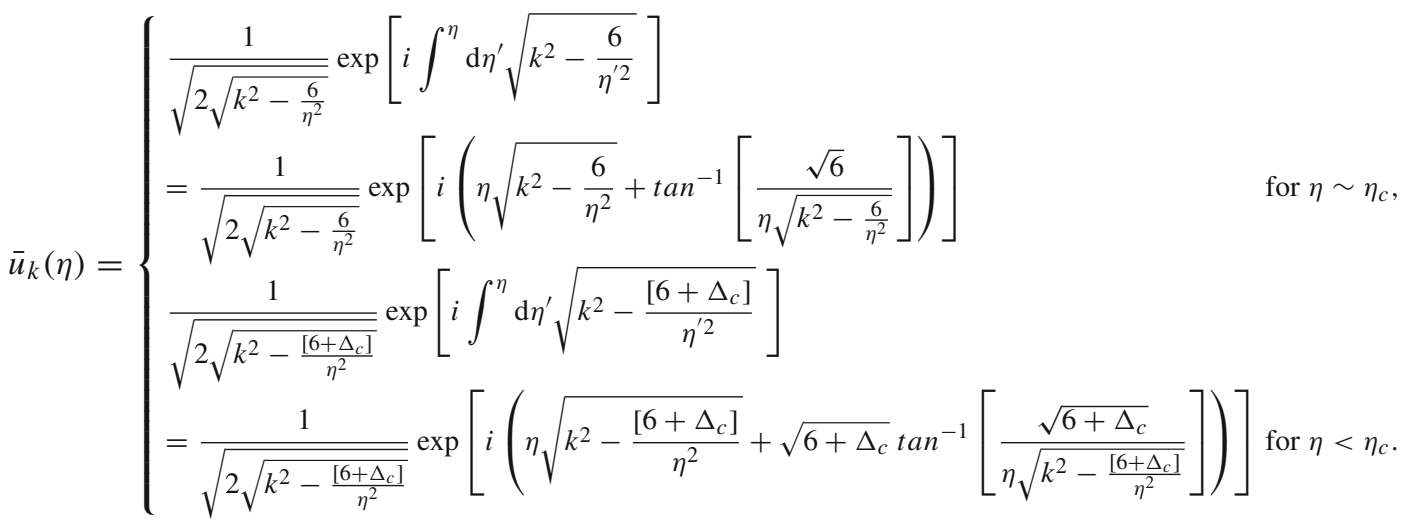

In the present context the Bogoliubov coefficient $\beta(k)$ in Fourier space can be computed approximately using the following expression:

$\beta\left(k, \tau, \tau^{\prime}, \eta^{\prime}\right)= \begin{cases}\int_{\tau^{\prime}}^{\tau} \mathrm{d} \eta \frac{36}{4 \eta^{6}\left(k^{2}-\frac{6}{\eta^{2}}\right)^{\frac{5}{2}}} \exp \left[2 i \int_{\eta^{\prime}}^{\eta} \mathrm{d} \eta^{\prime \prime} \sqrt{k^{2}-\frac{6}{\eta^{\prime \prime 2}}}\right] \quad \text { for } \eta \sim \eta_{c}, \text { early \& late } \eta, \\ \int_{\tau^{\prime}}^{\tau} \mathrm{d} \eta \frac{\left[6+\Delta_{c}\right]^{2}}{4 \eta^{6}\left(k^{2}-\frac{\left[6+\Delta_{c}\right]}{\eta^{2}}\right)^{\frac{5}{2}}} \exp \left[2 i \int_{\eta^{\prime}}^{\eta} \mathrm{d} \eta^{\prime \prime} \sqrt{k^{2}-\frac{\left[6+\Delta_{c}\right]}{\eta^{\prime \prime 2}}}\right] \text { for } \eta<\eta_{c},\end{cases}$

which is not exactly analytically computable. To study the behaviour of this integral we consider here three consecutive physical situations: $|k \eta|<<1,|k \eta| \approx 1-\Delta(\rightarrow 0)$ and $|k \eta|>>1$ for the de Sitter case and the quasi de Sitter case. In the three cases we have

For $\eta \sim \eta_{\mathbf{c}}$, early \& late $\eta: \quad \sqrt{\left\{k^{2}-\frac{6}{\eta^{2}}\right\}} \approx \begin{cases}\frac{i \sqrt{6}}{\eta} & \text { for }|k \eta|<<1, \\ \frac{i \sqrt{2 \Delta+5}}{\eta} & \text { for }|k \eta| \approx 1-\Delta(\rightarrow 0), \\ k & \text { for }|k \eta|>>1 .\end{cases}$

$$
\text { For } \eta<\eta_{\mathbf{c}}: \quad \sqrt{\left\{k^{2}-\left[6+\Delta_{c}\right] \frac{1}{\eta^{2}}\right\}} \approx \begin{cases}\frac{i \sqrt{\left[6+\Delta_{c}\right]}}{\eta \frac{\eta}{2 \Delta+5+\Delta_{c}}} & \text { for }|k \eta|<<1, \\ \frac{i \sqrt{k}}{k} & \text { for }|k \eta| \approx 1-\Delta(\rightarrow 0),\end{cases}
$$

and further using this result the Bogoliubov coefficient $\beta(k)$ in Fourier space can be expressed as 
For $\eta \sim \eta_{\mathbf{c}}$, early \& late $\eta$ :

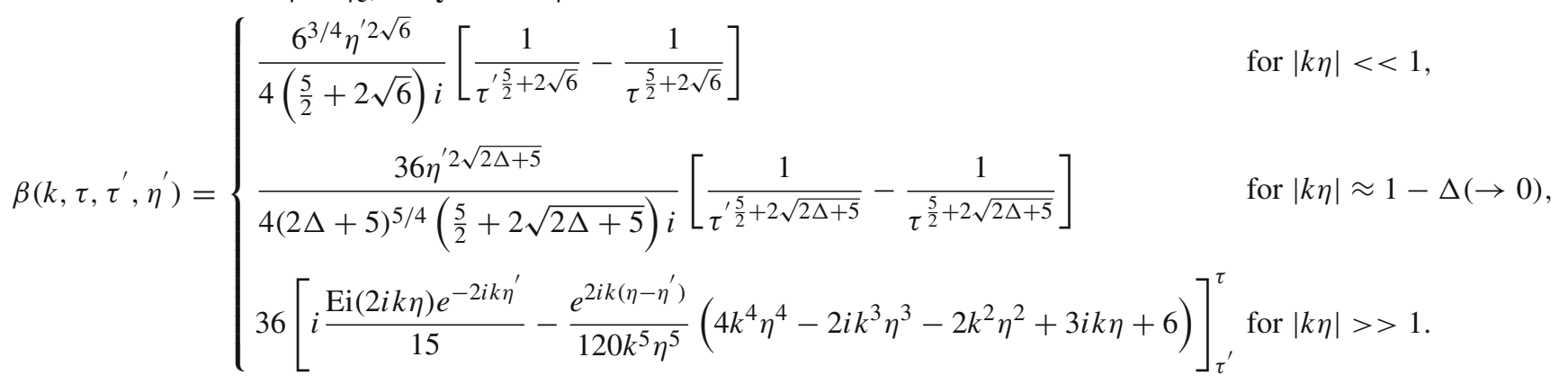

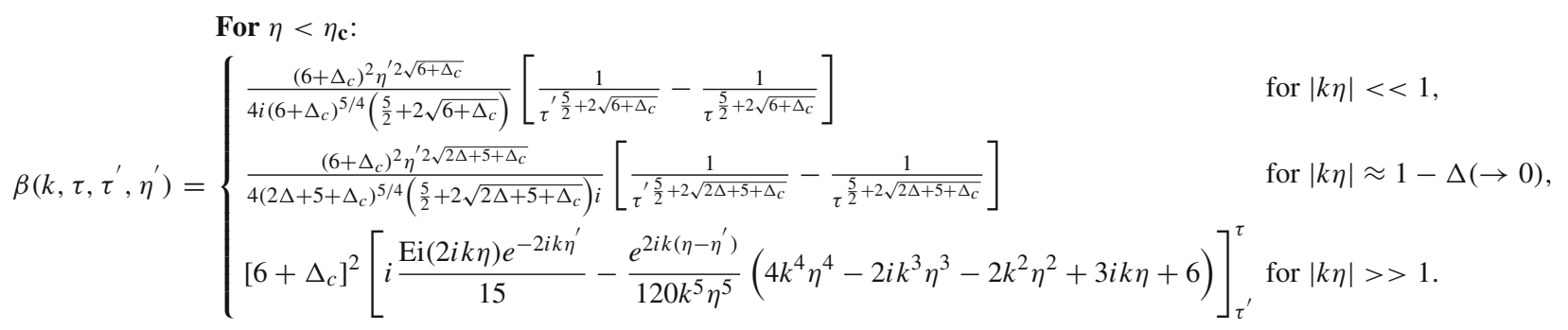

As mentioned earlier here one can use another equivalent way to define the Bogoliubov coefficient $\beta$ in Fourier space by implementing instantaneous Hamiltonian diagonalization method to interpret the results. Using this diagonalized representation the regularized Bogoliubov coefficient $\beta$ in Fourier space can be written as

$\beta_{\text {diag }}\left(k ; \tau, \tau^{\prime}\right)= \begin{cases}\int_{\tau^{\prime}}^{\tau} \mathrm{d} \eta \frac{6 \exp \left[-2 i \int^{\eta} \mathrm{d} \eta^{\prime} \sqrt{k^{2}-\frac{6}{\eta^{\prime 2}}}\right]}{2 \eta^{3}\left(k^{2}-\frac{6}{\eta^{2}}\right)} & \text { for } \eta \sim \eta_{c}, \text { early \& late } \eta, \\ \int_{\tau^{\prime}}^{\tau} \mathrm{d} \eta \frac{\left[6+\Delta_{c}\right] \exp \left[-2 i \int^{\eta} \mathrm{d} \eta^{\prime} \sqrt{k^{2}-\frac{\left[6+\Delta_{c}\right]}{\eta^{\prime 2}}}\right]}{2 \eta^{3}\left(k^{2}-\frac{\left[6+\Delta_{c}\right]}{\eta^{2}}\right)} & \text { for } \eta<\eta_{c},\end{cases}$

where $\tau$ and $\tau^{\prime}$ introduced as the conformal time regulator in the present context. In this case as well the Bogoliubov coefficient is not exactly analytically computable. To study the behaviour of this integral we consider here three similar consecutive physical situations for the de Sitter and the quasi de Sitter case as discussed earlier.

For $\eta \sim \eta_{\mathbf{c}}$, early \& late $\eta:$
$\beta_{\text {diag }}\left(k ; \tau, \tau^{\prime}\right)=\left\{\begin{array}{lc}\frac{\sqrt{6}}{2(2 \sqrt{6}-1)}\left[\tau^{\prime 2 \sqrt{6}-1}-\tau^{2 \sqrt{6}-1}\right] & \text { for }|k \eta|<<1, \\ \frac{6}{2 \sqrt{2 \Delta+5}(2 \sqrt{2 \Delta+5}-1)}\left[\tau^{\prime 2 \sqrt{2 \Delta+5}-1}-\tau^{2 \sqrt{2 \Delta+5}-1}\right] & \text { for }|k \eta| \approx 1-\Delta(\rightarrow 0), \\ \frac{6}{2}\left[\frac{e^{-2 i k \eta}(2 i k \eta-1)}{4 k^{2} \eta^{2}}-\operatorname{Ei}(-2 i k \eta)\right]_{\tau^{\prime}}^{\tau} & \text { for }|k \eta|>>1 .\end{array}\right.$

For $\eta<\eta_{\mathbf{c}}$ :

$\beta_{\text {diag }}\left(k ; \tau, \tau^{\prime}\right)=\left\{\begin{array}{l}\frac{\sqrt{6+\Delta_{c}}}{2\left(2 \sqrt{6+\Delta_{c}}-1\right)}\left[\tau^{\prime 2 \sqrt{6+\Delta_{c}}-1}-\tau^{2 \sqrt{6+\Delta_{c}}-1}\right] \text { for }|k \eta|<<1, \\ \frac{\left(6+\Delta_{c}\right)\left[\tau^{\prime 2 \sqrt{2 \Delta+5+\Delta_{c}}-1}-\tau^{2 \sqrt{2 \Delta+5+\Delta_{c}}-1}\right]}{2 \sqrt{2 \Delta+5+\Delta_{c}}\left(2 \sqrt{2 \Delta+5+\Delta_{c}}-1\right)} \text { for }|k \eta| \approx 1-\Delta(\rightarrow 0), \\ \frac{\left(6+\Delta_{c}\right)}{2}\left[\frac{e^{-2 i k \eta}(2 i k \eta-1)}{4 k^{2} \eta^{2}}-\operatorname{Ei}(-2 i k \eta)\right]_{\tau^{\prime}}^{\tau} \text { for }|k \eta|>>1 .\end{array}\right.$ 
Further using the regularized expressions for the Bogoliubov coefficient $\beta$ in two different representations as mentioned in Eq. (4.117) and Eq. (4.121), and substituting them in Eq. (3.57) we get the following regularized expressions for the Bogoliubov coefficient $\alpha$ in two different representations as given by

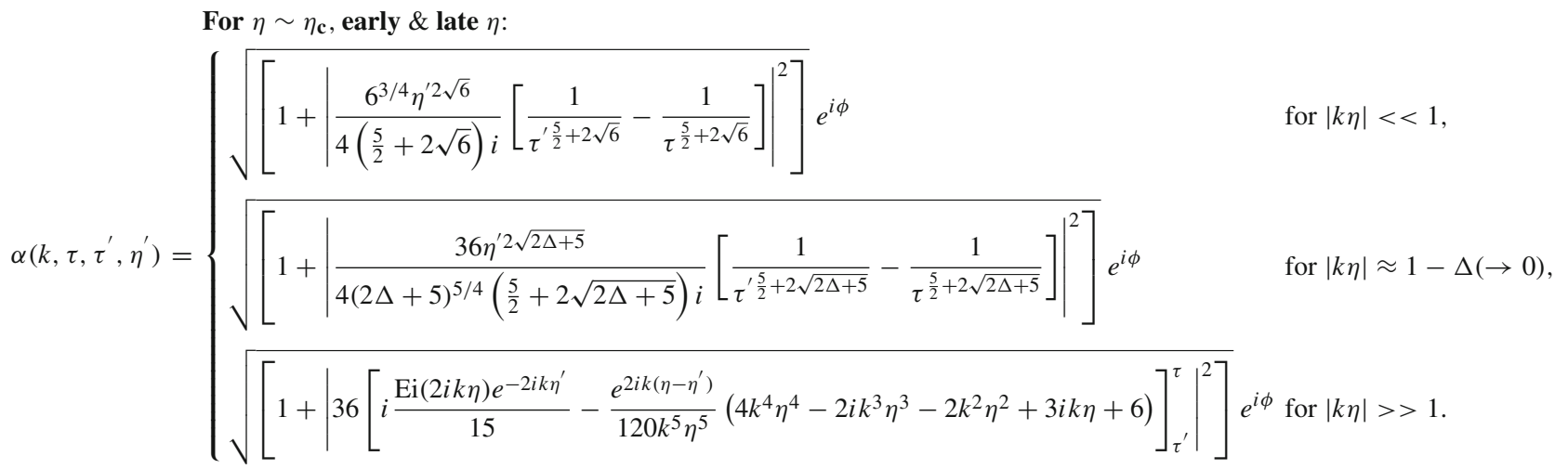

For $\eta<\eta_{\mathbf{c}}$ :

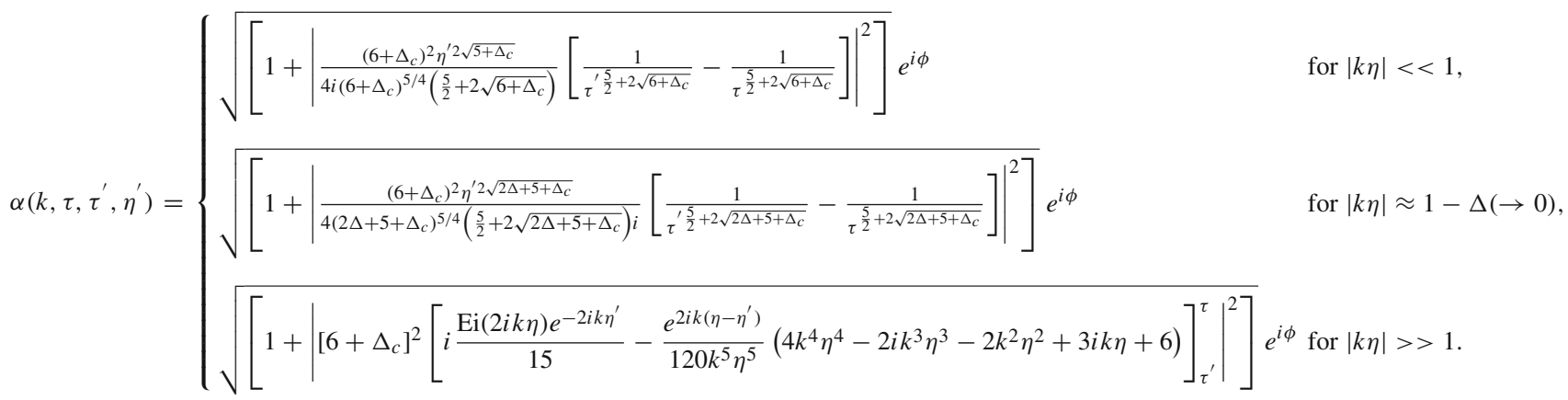

and

For $\eta \sim \eta_{\mathbf{c}}$, early \& late $\eta$ :

$\alpha_{\text {diag }}\left(k ; \tau, \tau^{\prime}\right)= \begin{cases}\sqrt{\left[1+\left|\frac{\sqrt{6}}{2(2 \sqrt{6}-1)}\left[\tau^{\prime 2 \sqrt{6}-1}-\tau^{2 \sqrt{6}-1}\right]\right|^{2}\right]} e^{i \phi_{\text {diag }}} & \text { for }|k \eta|<<1, \\ \sqrt{\left[1+\left|\frac{6}{2 \sqrt{2 \Delta+5}(2 \sqrt{2 \Delta+5}-1)}\left[\tau^{\prime 2 \sqrt{2 \Delta+5}-1}-\tau^{2 \sqrt{2 \Delta+5}-1}\right]\right|^{2}\right]} e^{i \phi_{\text {diag }} \text { for }|k \eta| \approx 1-\Delta(\rightarrow 0),} & \text { for }|k \eta|>>1 .\end{cases}$

For $\eta<\eta_{\mathbf{c}}$ :

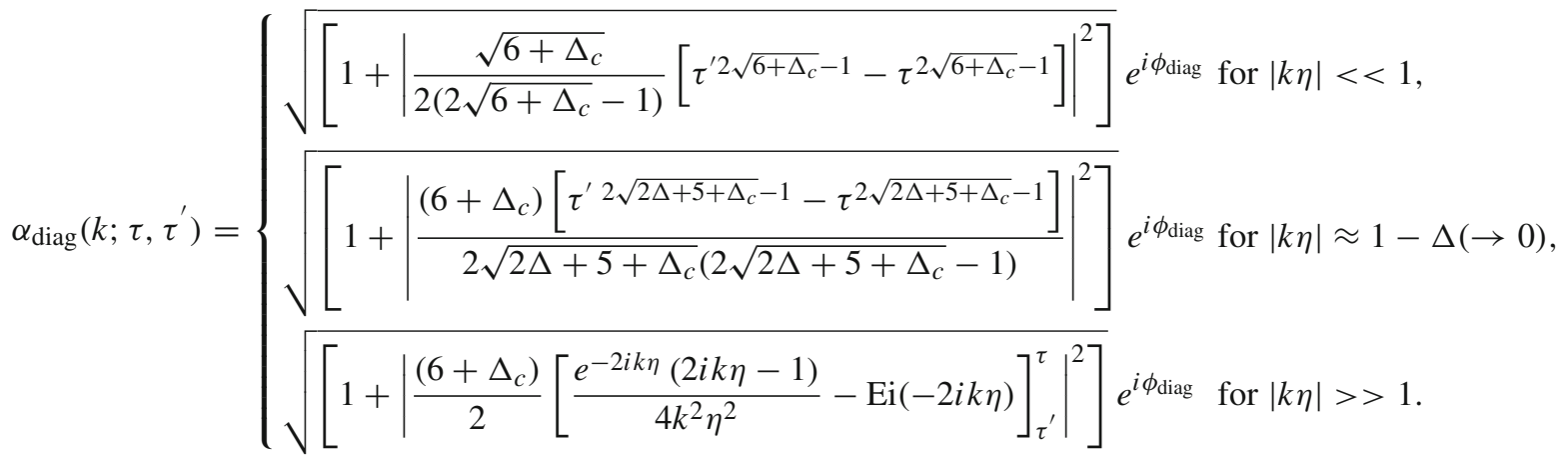


where $\phi$ and $\phi_{\text {diag }}$ are the associated phase factors in two different representations.

Further using the expressions for the Bogoliubov coefficient $\alpha$ in two different representations we get the following expressions for the reflection and transmission coefficient in two different representations for three consecutive physical situations: $|k \eta|<<1,|k \eta| \approx 1-\Delta(\rightarrow 0)$ and $|k \eta|>>1$ for the de Sitter case and the quasi de Sitter case as given by

For $\eta \sim \eta_{\mathbf{c}}$, early \& late $\eta$ :

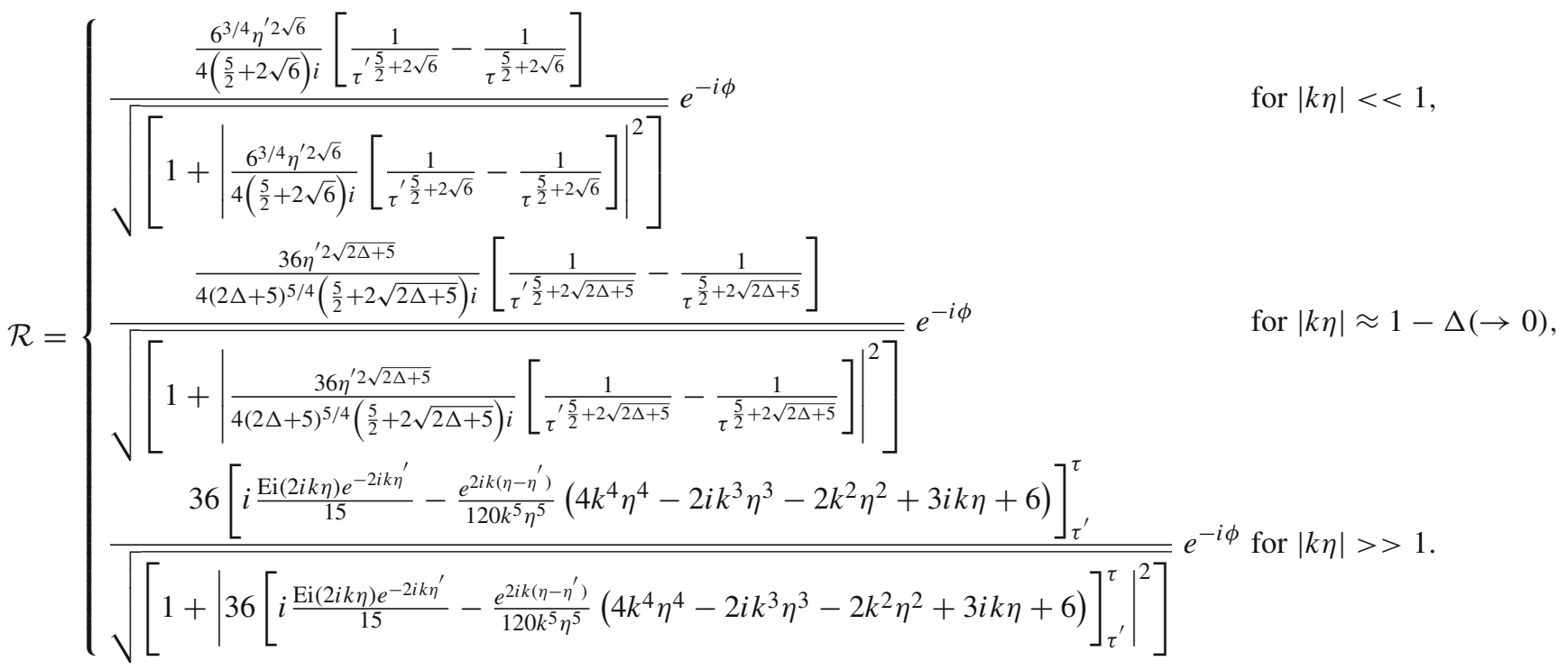

For $\eta \sim \eta_{\mathbf{c}}$, early \& late $\eta$ :

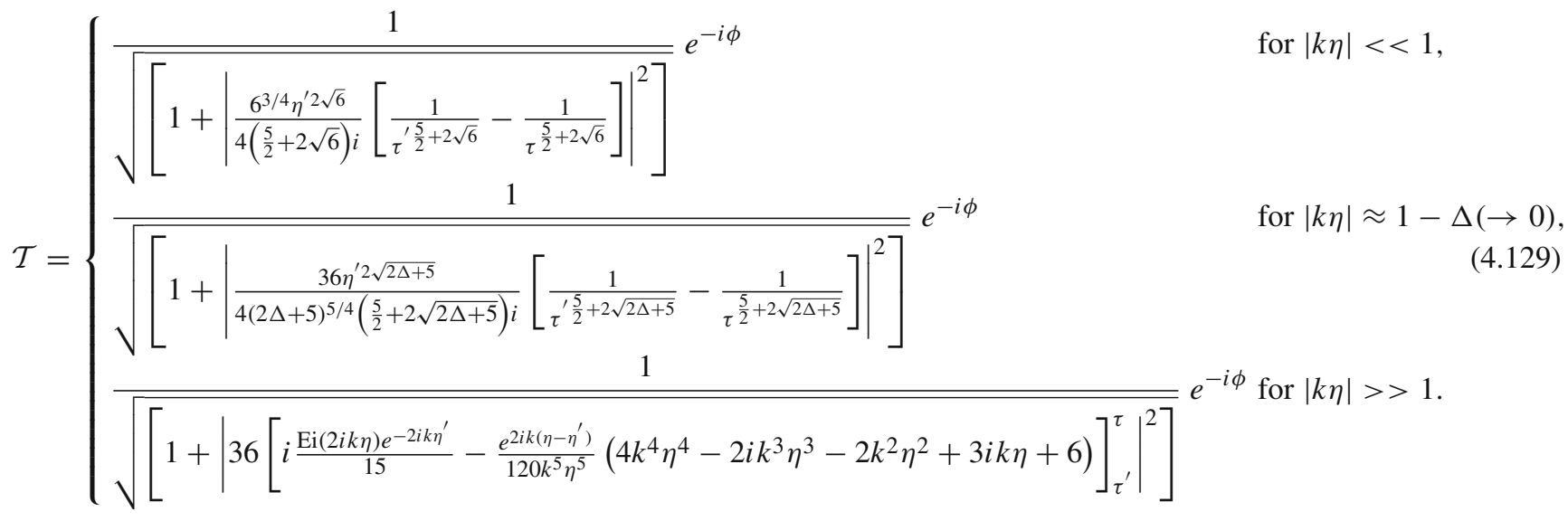

For $\eta<\eta_{\mathbf{c}}$ :

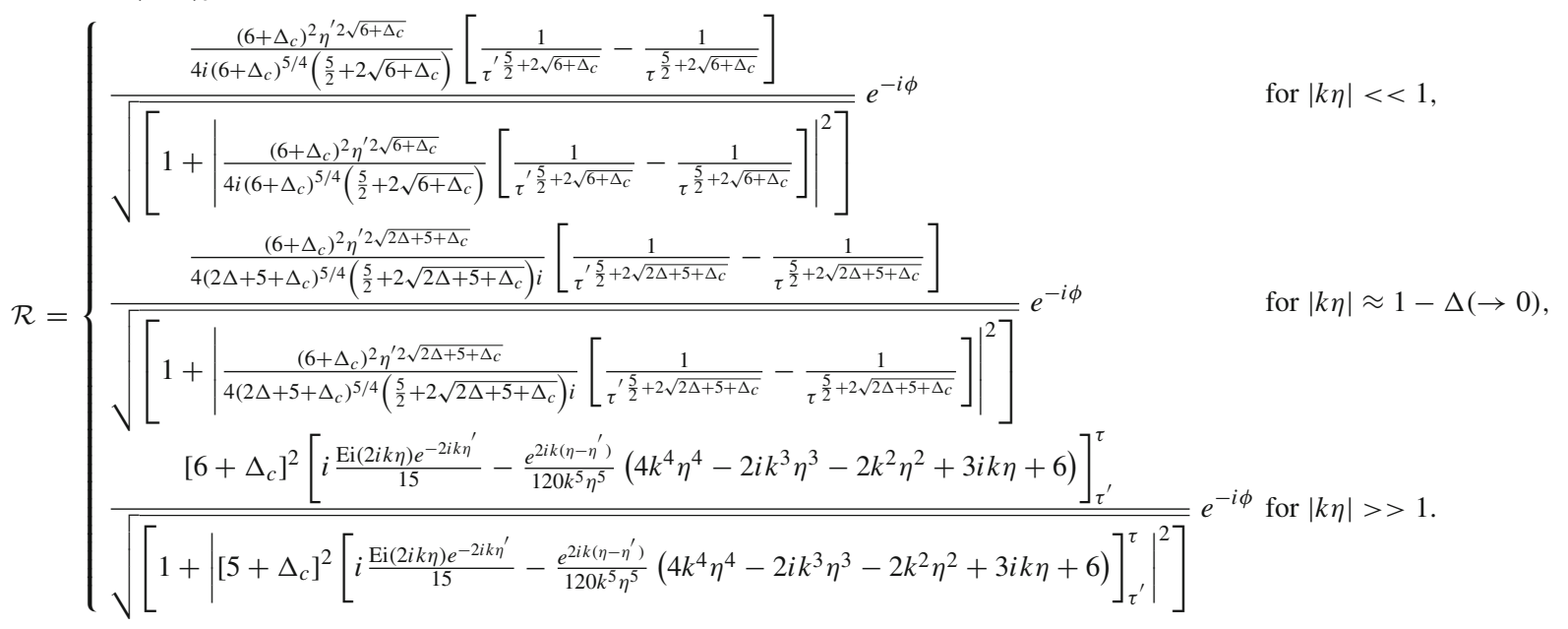


For $\eta<\eta_{\mathbf{c}}$ :

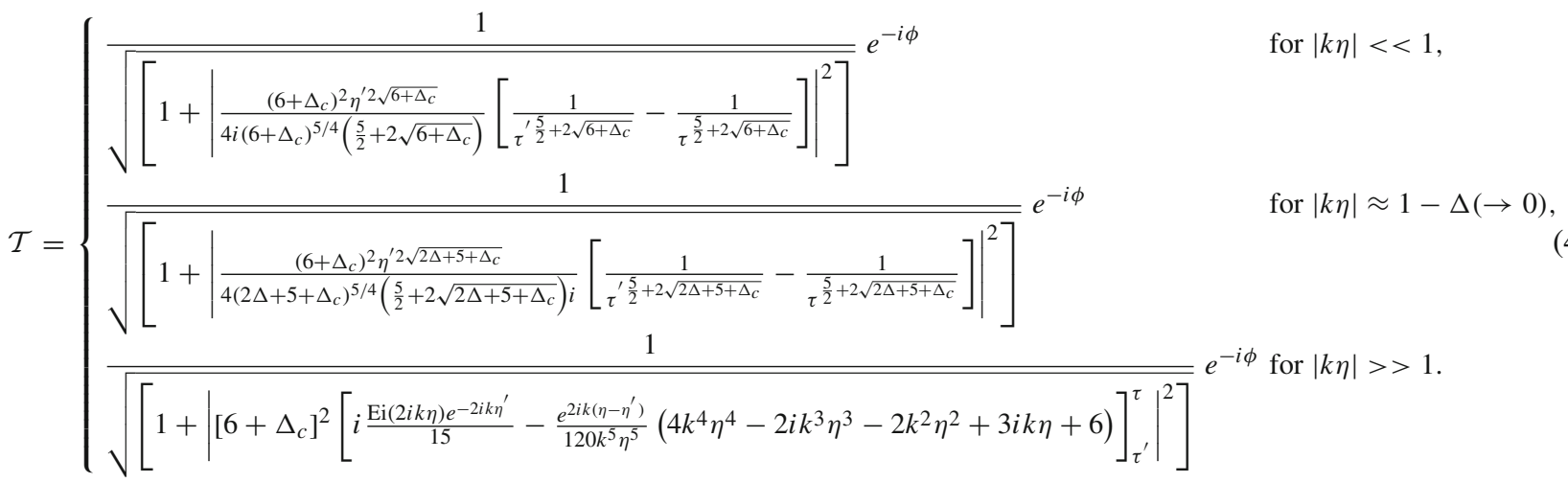

and

\section{For $\eta \sim \eta_{\mathbf{c}}$, early \& late $\eta$ :}

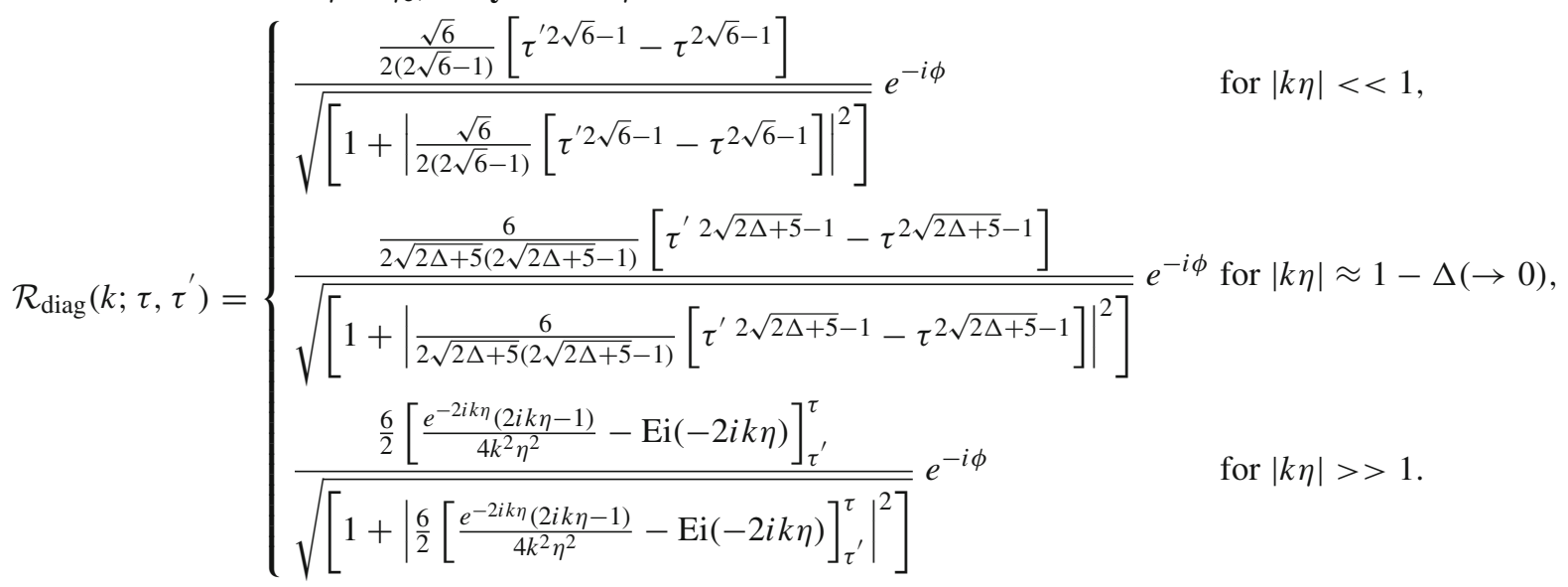

\section{For $\eta \sim \eta_{\mathbf{c}}$, early \& late $\eta$ :}

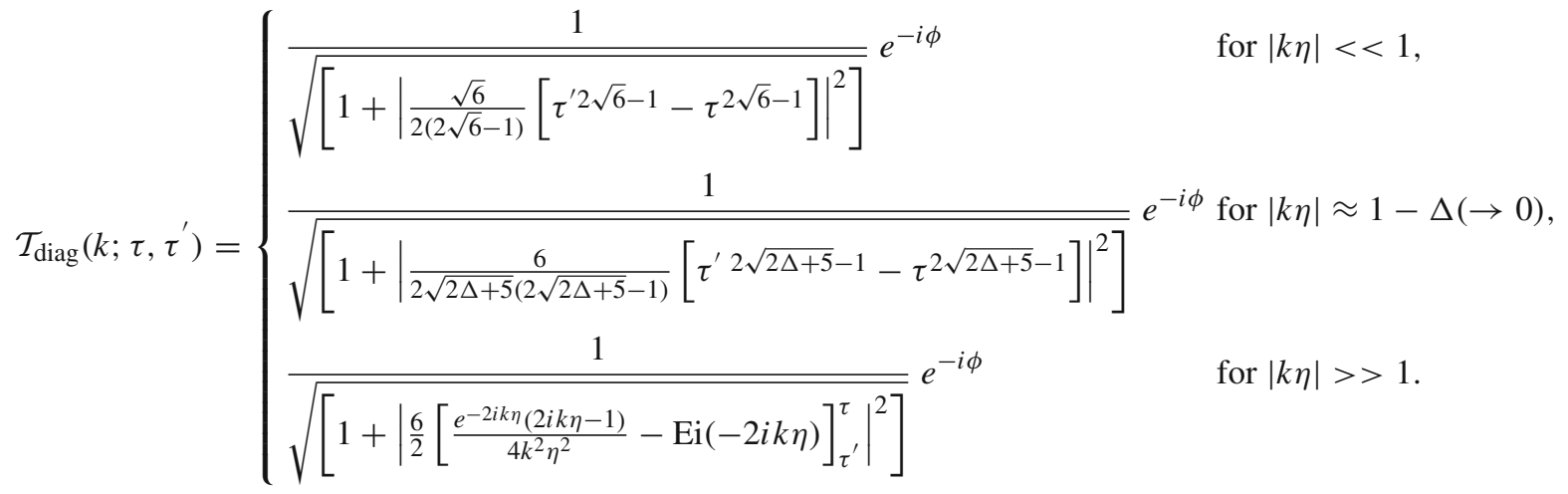




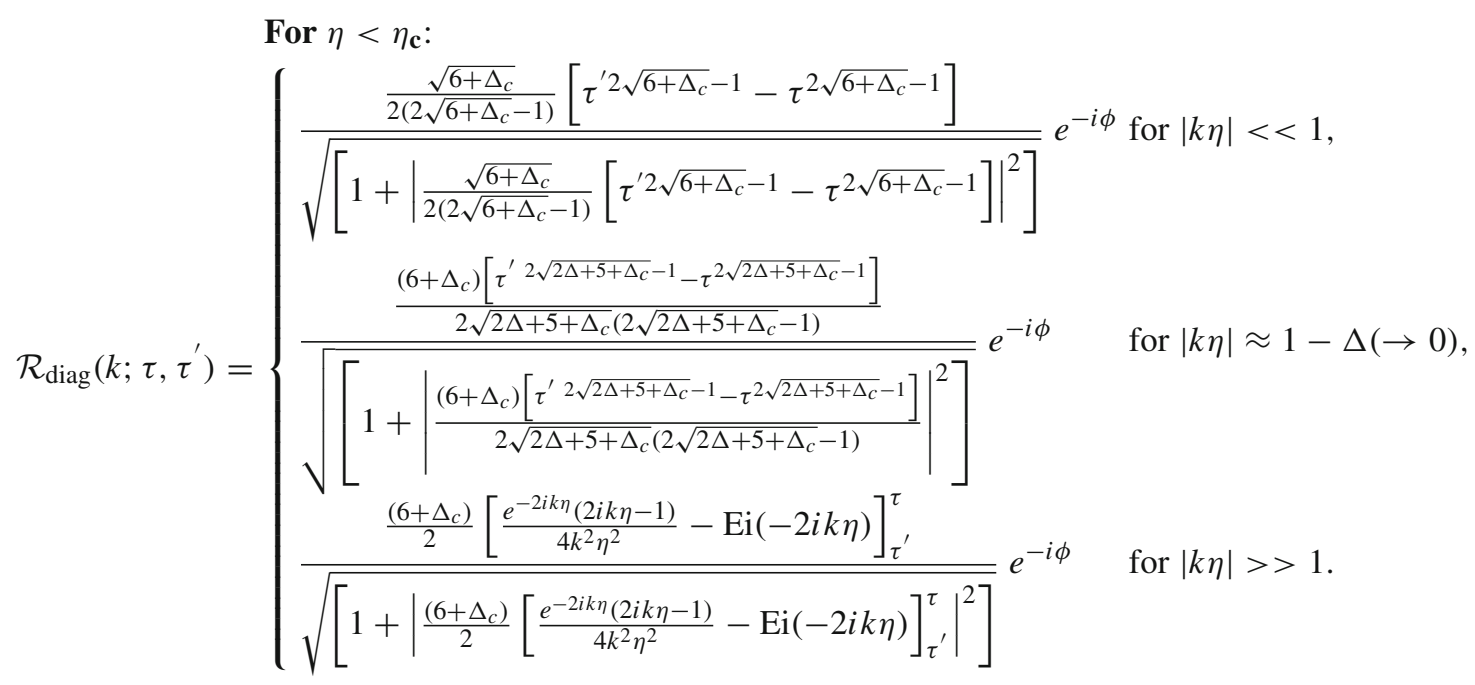

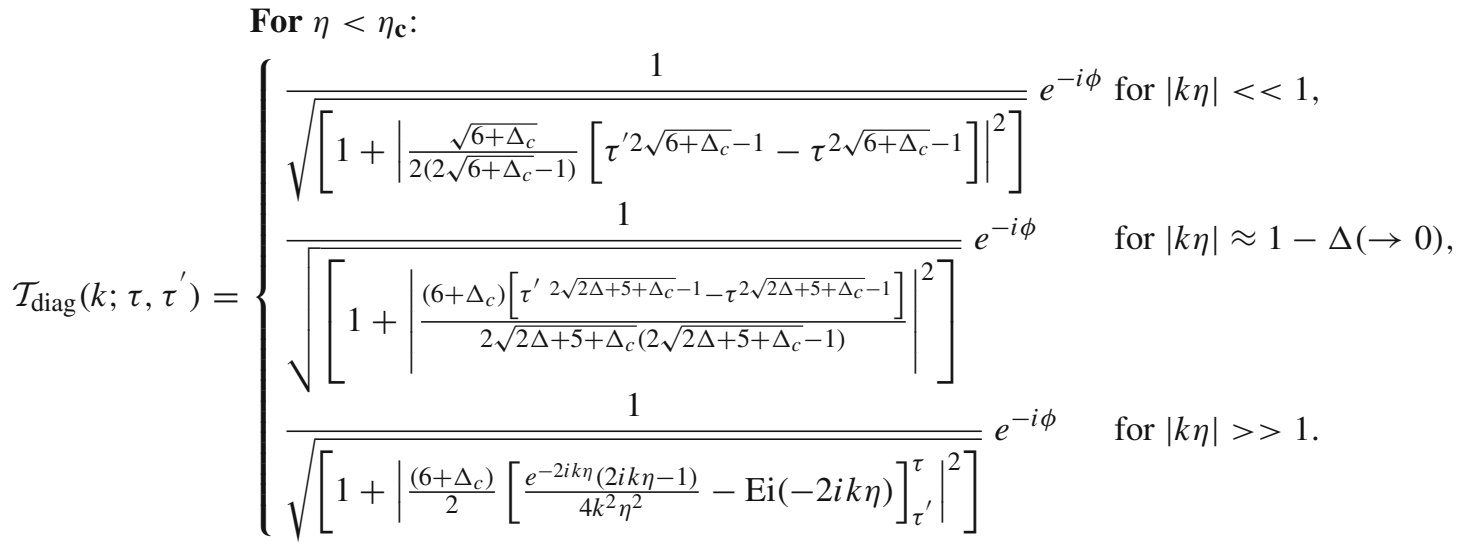

Next the expression for the number of produced particles at time $\tau$ can be expressed in the two representations as

$\mathcal{N}\left(\tau, \tau^{\prime}, \eta^{\prime}\right)=\frac{1}{(2 \pi a)^{3}} \int \mathrm{d}^{3} \mathbf{k}\left\{\begin{array}{l}\left|\int_{\tau^{\prime}}^{\tau} \mathrm{d} \eta \frac{36 \exp \left[2 i \int_{\eta^{\prime}}^{\eta} \mathrm{d} \eta^{\prime \prime} \sqrt{k^{2}-\frac{6}{\eta^{\prime \prime 2}}}\right]}{4 \eta^{6}\left(k^{2}-\frac{6}{\eta^{2}}\right)^{\frac{5}{2}}}\right|^{2} \quad \text { for } \eta \sim \eta_{c}, \text { early \& late } \eta, \\ \left|\int_{\tau^{\prime}}^{\tau} \mathrm{d} \eta \frac{\left[6+\Delta_{c}\right]^{2} \exp \left[2 i \int_{\eta^{\prime}}^{\eta} \mathrm{d} \eta^{\prime \prime} \sqrt{k^{2}-\frac{\left[6+\Delta_{c}\right]}{\eta^{\prime \prime 2}}}\right]}{4 \eta^{6}\left(k^{2}-\frac{\left[6+\Delta_{c}\right]}{\eta^{2}}\right)^{\frac{5}{2}}}\right|^{2} \text { for } \eta<\eta_{c},\end{array}\right.$

$\mathcal{N}_{\text {diag }}\left(\tau, \tau^{\prime}\right)=\frac{1}{(2 \pi a)^{3}} \int \mathrm{d}^{3} \mathbf{k} \begin{cases}\left|\frac{6 \exp \left[-2 i \int^{\eta} \mathrm{d} \eta^{\prime} \sqrt{k^{2}-\frac{6}{\eta^{\prime 2}}}\right]}{2 \eta^{3}\left(k^{2}-\frac{6}{\eta^{2}}\right)}\right|^{2} & \text { for } \eta \sim \eta_{c}, \text { early \& late } \eta, \\ \left|\frac{\left[6+\Delta_{c}\right] \exp \left[-2 i \int^{\eta} \mathrm{d} \eta^{\prime} \sqrt{k^{2}-\frac{\left[6+\Delta_{c}\right]}{\eta^{\prime 2}}}\right]}{2 \eta^{3}\left(k^{2}-\frac{\left[6+\Delta_{c}\right]}{\eta^{2}}\right)}\right|^{2} & \text { for } \eta<\eta_{c} .\end{cases}$

which are not exactly analytically computable. To study the behaviour of this integral we consider here three consecutive physical situations: $|k \eta|<<1,|k \eta| \approx 1-\Delta(\rightarrow 0)$ and $|k \eta|>>1$ for the de Sitter and the quasi de Sitter case. In the three cases we have 
For $\eta \sim \eta_{\mathbf{c}}$, early \& late $\eta$ :

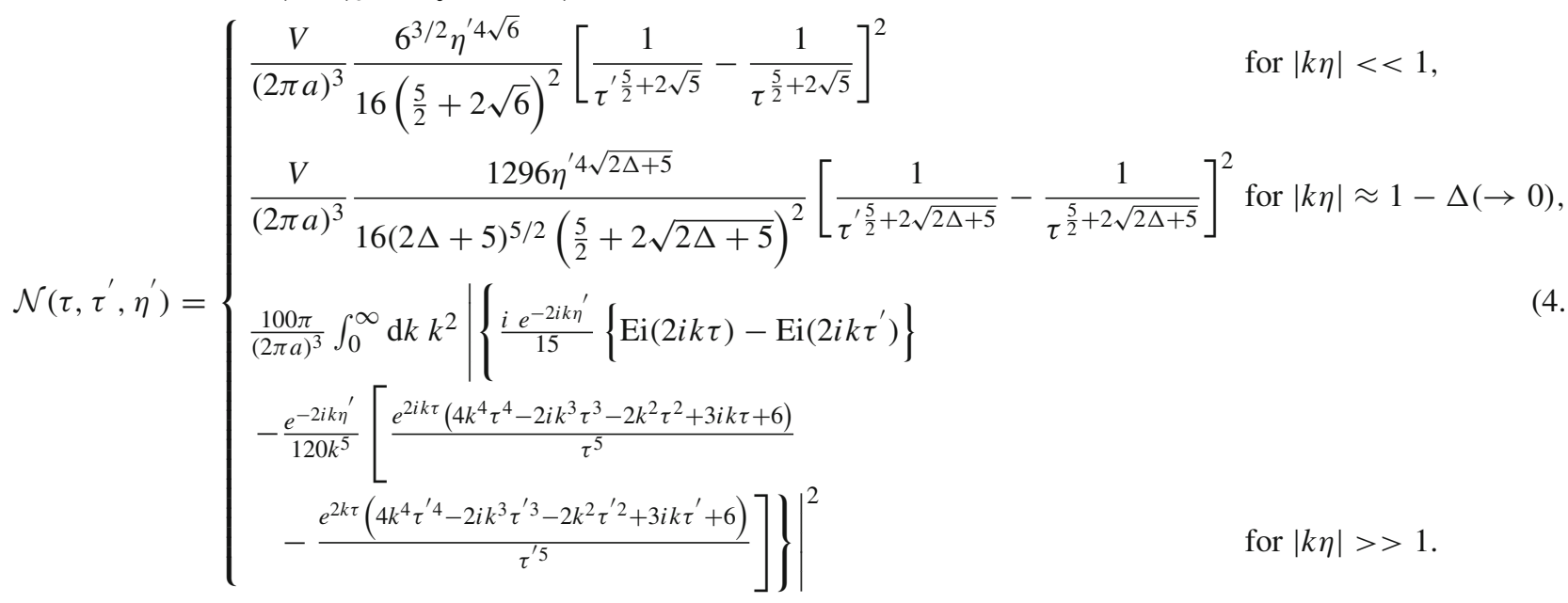

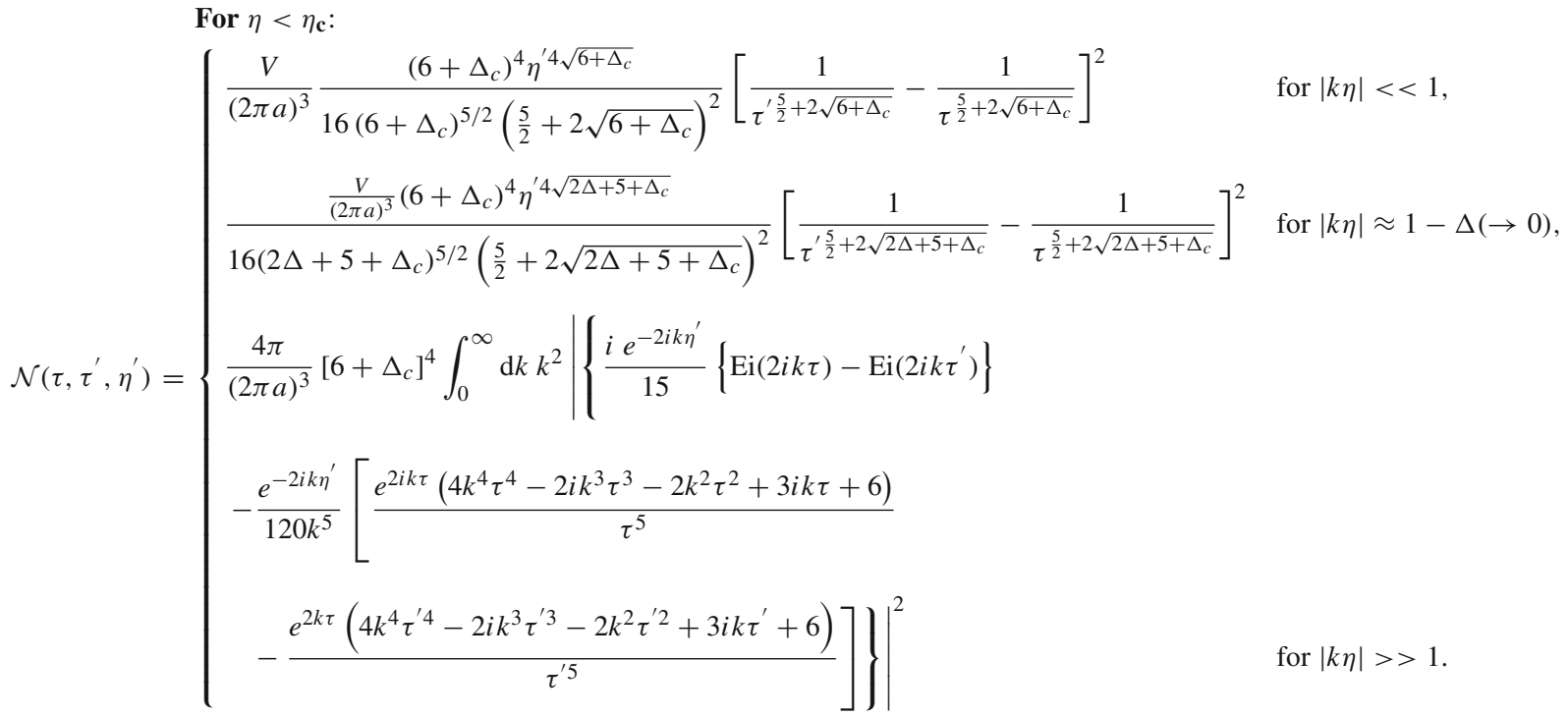

and

\section{For $\eta \sim \eta_{\mathbf{c}}$, early \& late $\eta$ :}

$$
\mathcal{N}_{\operatorname{diag}}\left(\tau, \tau^{\prime}\right)= \begin{cases}\frac{V}{(2 \pi a)^{3}} \frac{6}{4(2 \sqrt{6}-1)^{2}}\left[\tau^{\prime 2 \sqrt{6}-1}-\tau^{2 \sqrt{6}-1}\right]^{2} & \text { for }|k \eta|<<1, \\ \frac{V}{(2 \pi a)^{3}} \frac{36}{4(2 \Delta+5)(2 \sqrt{2 \Delta+5}-1)^{2}}\left[\tau^{\prime 2 \sqrt{2 \Delta+5}-1}-\tau^{2 \sqrt{2 \Delta+5}-1}\right]^{2} & \text { for }|k \eta| \approx 1-\Delta(\rightarrow 0), \\ \frac{36 \pi}{(2 \pi a)^{3}} \int_{0}^{\infty} \mathrm{d} k k^{2} \mid\left[\left\{\frac{e^{-2 i k \tau}(2 i k \tau-1)}{4 k^{2} \tau^{2}}-\frac{e^{-2 i k \tau^{\prime}}\left(2 i k \tau^{\prime}-1\right)}{4 k^{2} \tau^{\prime 2}}\right\}\right. & \text { for }|k \eta|>>1 .\end{cases}
$$


For $\eta<\eta_{\mathbf{c}}$ :

$$
\mathcal{N}_{\text {diag }}\left(\tau, \tau^{\prime}\right)= \begin{cases}\frac{V}{(2 \pi a)^{3}} \frac{\left(6+\Delta_{c}\right)}{4\left(2 \sqrt{6+\Delta_{c}}-1\right)^{2}}\left[\tau^{\prime 2 \sqrt{6+\Delta_{c}}-1}-\tau^{2 \sqrt{6+\Delta_{c}}-1}\right]^{2} & \text { for }|k \eta|<<1, \\ \left.\frac{V}{(2 \pi a)^{3}} \frac{\left(6+\Delta_{c}\right)^{2}\left[\tau^{\prime} 2 \sqrt{2 \Delta+5+\Delta_{c}}-1\right.}{4\left(2 \Delta+5+\tau_{c}\right)\left(2 \sqrt{2 \Delta+5+\Delta_{c}}-1\right.}\right]^{2} & \text { for }|k \eta| \approx 1-\Delta(\rightarrow 0), \\ \frac{\left(6+\Delta_{c}\right)^{2} \pi}{(2 \pi a)^{3}} \int_{0}^{\infty} \mathrm{d} k k^{2} \mid\left[\left\{\frac{e^{-2 i k \tau}(2 i k \tau-1)}{4 k^{2} \tau^{2}}-\frac{e^{-2 i k \tau^{\prime}}\left(2 i k \tau^{\prime}-1\right)}{4 k^{2} \tau^{\prime 2}}\right\}\right. & \text { for }|k \eta|>>1 .\end{cases}
$$

where the symbol $V$ is defined earlier.

Finally one can define the total energy density of the produced particles using the following expression:

$$
\begin{aligned}
& \rho\left(\tau, \tau^{\prime}, \eta^{\prime}\right)=\frac{1}{(2 \pi a)^{3} a} \int \mathrm{d}^{3} \mathbf{k}\left\{\begin{array}{l}
\sqrt{k^{2}-\frac{6}{\tau^{2}}}\left|\int_{\tau^{\prime}}^{\tau} \mathrm{d} \eta \frac{36 \exp \left[2 i \int_{\eta^{\prime}}^{\eta} \mathrm{d} \eta^{\prime \prime} \sqrt{k^{2}-\frac{6}{\eta^{\prime \prime 2}}}\right]}{4 \eta^{6}\left(k^{2}-\frac{6}{\eta^{2}}\right)^{\frac{5}{2}}}\right|^{2} \quad \text { for } \eta \sim \eta_{c}, \text { early \& late } \eta, \\
\sqrt{k^{2}-\frac{\left[6+\Delta_{c}\right]}{\tau^{2}}}\left|\int_{\tau^{\prime}}^{\tau} \mathrm{d} \eta \frac{\left[6+\Delta_{c}\right]^{2} \exp \left[2 i \int_{\eta^{\prime}}^{\eta} \mathrm{d} \eta^{\prime \prime} \sqrt{k^{2}-\frac{\left[6+\Delta_{c}\right]}{\eta^{\prime \prime 2}}}\right]}{4 \eta^{6}\left(k^{2}-\frac{\left[6+\Delta_{c}\right]}{\eta^{2}}\right)^{\frac{5}{2}}}\right|^{2} \text { for } \eta<\eta_{c} .
\end{array}\right. \\
& \rho_{\text {diag }}\left(\tau, \tau^{\prime}, \eta^{\prime}\right)=\frac{1}{(2 \pi a)^{3} a} \int \mathrm{d}^{3} \mathbf{k}\left\{\begin{array}{l}
\sqrt{k^{2}-\frac{6}{\tau^{2}}}\left|\int_{\tau^{\prime}}^{\tau} \mathrm{d} \eta \frac{6 \exp \left[-2 i \int^{\eta} \mathrm{d} \eta^{\prime} \sqrt{k^{2}-\frac{6}{\eta^{\prime 2}}}\right]}{2 \eta^{3}\left(k^{2}-\frac{6}{\eta^{2}}\right)}\right|^{2} \quad \text { for } \eta \sim \eta_{c}, \text { early \& late } \eta, \\
\sqrt{k^{2}-\frac{\left[6+\Delta_{c}\right]}{\tau^{2}}}\left|\int_{\tau^{\prime}}^{\tau} \mathrm{d} \eta \frac{\left[6+\Delta_{c}\right] \exp \left[-2 i \int^{\eta} \mathrm{d} \eta^{\prime} \sqrt{k^{2}-\frac{\left[6+\Delta_{c}\right]}{\eta^{\prime 2}}}\right]}{2 \eta^{2}\left(k^{2}-\frac{\left[6+\Delta_{c}\right]}{\eta^{2}}\right)}\right|^{2} \text { for } \eta<\eta_{c} .
\end{array}\right.
\end{aligned}
$$

which is not exactly analytically computable. To study the behaviour of this integral we consider here three consecutive physical situations: $|k \eta|<<1,|k \eta| \approx 1-\Delta(\rightarrow 0)$ and $|k \eta|>>1$ for the de Sitter case and the quasi de Sitter case. In the three cases we have

$$
\begin{aligned}
& \text { For } \eta \sim \eta_{\mathbf{c}} \text {, early \& late } \eta \text { : }
\end{aligned}
$$

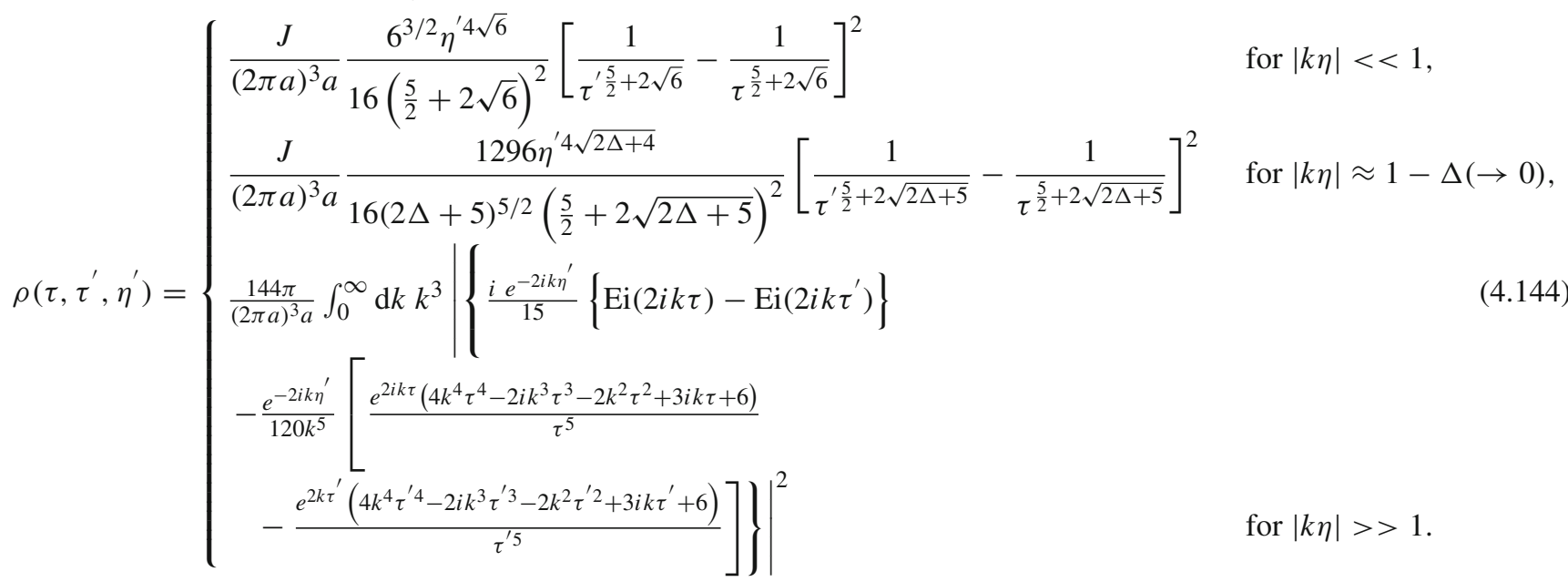




$$
\begin{aligned}
& \text { For } \eta<\eta_{\mathbf{c}} \text { : }
\end{aligned}
$$

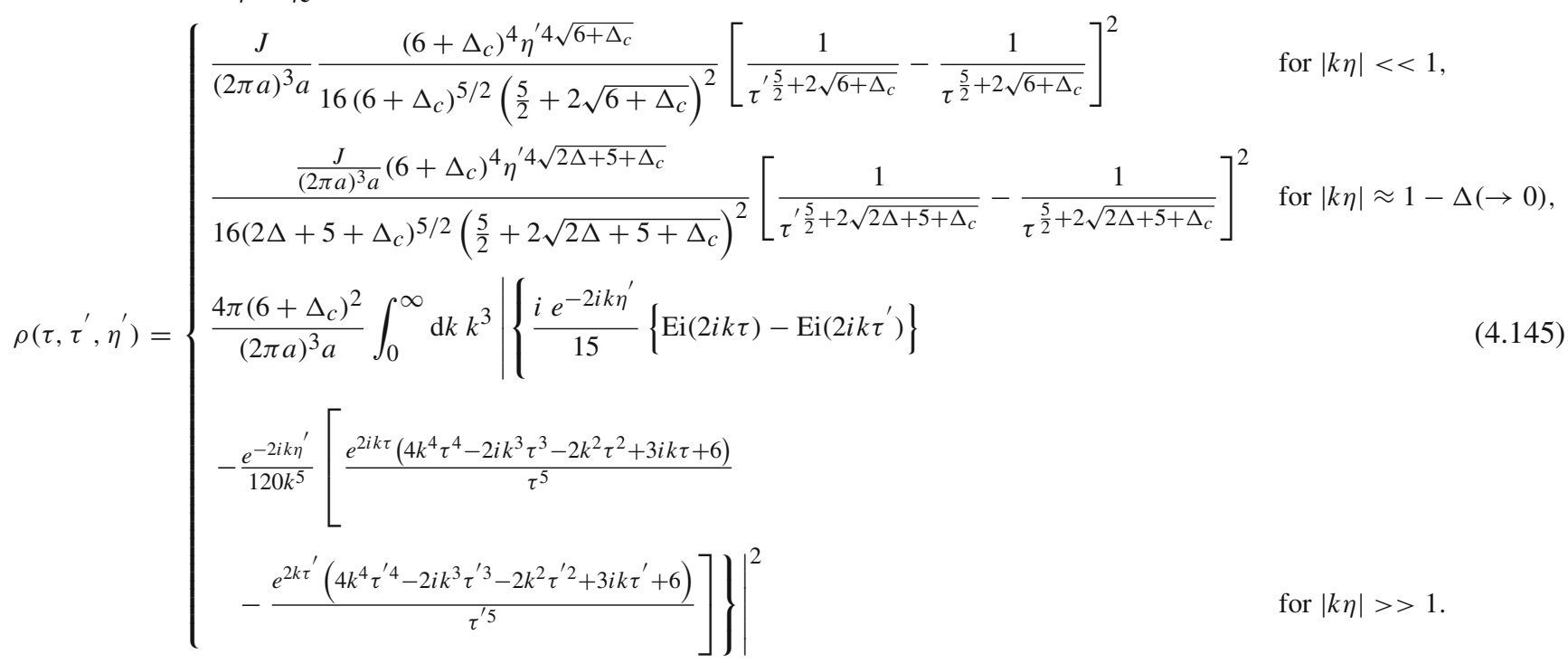

and

For $\eta \sim \eta_{\mathbf{c}}$, early \& late $\eta$ :

$$
\rho_{\text {diag }}\left(\tau, \tau^{\prime}\right)= \begin{cases}\frac{J}{(2 \pi a)^{3} a} \frac{6}{4(2 \sqrt{6}-1)^{2}}\left[\tau^{\prime 2 \sqrt{6}-1}-\tau^{2 \sqrt{6}-1}\right]^{2} & \text { for }|k \eta|<<1, \\ \frac{J}{(2 \pi a)^{3} a} \frac{36}{4(2 \Delta+5)(2 \sqrt{2 \Delta+5}-1)^{2}}\left[\tau^{\prime 2 \sqrt{2 \Delta+5}-1}-\tau^{2 \sqrt{2 \Delta+5}-1}\right]^{2} & \text { for }|k \eta| \approx 1-\Delta(\rightarrow 0), \\ \frac{36 \pi}{(2 \pi a)^{3} a} \int_{0}^{\infty} \mathrm{d} k k^{3} \mid \frac{e^{-2 i k \tau}(2 i k \tau-1)}{4(k \tau)^{2}}-\frac{e^{-2 i k \tau^{\prime}}\left(2 i k \tau^{\prime}-1\right)}{4\left(k \tau^{\prime}\right)^{2}} & \text { for }|k \eta|>>1 . \\ +\left.\left\{\operatorname{Ei}(-2 i k \tau)-\operatorname{Ei}\left(-2 i k \tau^{\prime}\right)\right\}\right|^{2} & \end{cases}
$$

For $\eta<\eta_{\mathbf{c}}$ :

$$
\rho_{\text {diag }}\left(\tau, \tau^{\prime}, \eta^{\prime}\right)= \begin{cases}\frac{J}{(2 \pi a)^{3} a} \frac{\left(6+\Delta_{c}\right)}{4\left(2 \sqrt{6+\Delta_{c}}-1\right)^{2}}\left[\tau^{\prime 2 \sqrt{6+\Delta_{c}}-1}-\tau^{2 \sqrt{6+\Delta_{c}}-1}\right]^{2} & \text { for }|k \eta|<<1, \\ \frac{J}{(2 \pi a)^{3} a} \frac{\left(6+\Delta_{c}\right)^{2}\left[\tau^{\prime} 2 \sqrt{2 \Delta+5+\Delta_{c}}-1\right.}{4\left(2 \Delta+5+\tau_{c}^{2 \sqrt{2 \Delta+5+\Delta_{c}}-1}\right]^{2}} & \text { for }|k \eta| \approx 1-\Delta\left(\rightarrow \sqrt{2 \Delta+5+\Delta_{c}}-1\right)^{2} \\ \frac{\left(6+\Delta_{c}\right)^{2} \pi}{(2 \pi a)^{3} a} \int_{0}^{\infty} \mathrm{d} k k^{3} \mid \frac{e^{-2 i k \tau}\left(2 i k c_{S} \tau-1\right)}{4(k \tau)^{2}}-\frac{e^{-2 i k \tau^{\prime}}\left(2 i k \tau^{\prime}-1\right)}{4\left(k \tau^{\prime}\right)^{2}} & \text { for }|k \eta|>>1 . \\ +\left.\left\{\operatorname{Ei}(-2 i k \tau)-\operatorname{Ei}\left(-2 i k \tau^{\prime}\right)\right\}\right|^{2} & \end{cases}
$$

Throughout the discussion of the total energy density of the produced particles we have introduced a symbol $J$ defined as

$$
J=\int \mathrm{d}^{3} \mathbf{k} p(\tau)= \begin{cases}\int \mathrm{d}^{3} \mathbf{k} \sqrt{k^{2}-\frac{6}{\tau^{2}}} & \text { for } \mathrm{dS}, \\ \int \mathrm{d}^{3} \mathbf{k} \sqrt{k^{2}-\frac{\left[6+\Delta_{c}\right]}{\tau^{2}}} & \text { for qdS, }\end{cases}
$$


which physically signifies the total finite volume weighted by $p(\eta)$ in momentum space within which the produced particles are located. To study the behaviour of this integral we consider here three consecutive physical situations: $|k \eta|<<1$, $|k \eta| \approx 1-\Delta(\rightarrow 0)$ and $|k \eta|>>1$ for the de Sitter and the quasi de Sitter case. In the three cases we have

For $\eta \sim \eta_{\mathbf{c}}$, early \& late $\eta$ :

$J= \begin{cases}\int \mathrm{d}^{3} \mathbf{k} \frac{6}{\tau}=\frac{6 V}{\tau} & \text { for }|k \eta|<<1, \\ \int \mathrm{d}^{3} \mathbf{k} \frac{\sqrt{2 \Delta+5}}{\tau}=\frac{\sqrt{2 \Delta+5} V}{\tau} & \text { for }|k \eta| \approx 1-\Delta(\rightarrow 0), \\ \int \mathrm{d}^{3} \mathbf{k} k & \text { for }|k \eta|>>1 .\end{cases}$

For $\eta<\eta_{\mathbf{c}}$ :

$J= \begin{cases}\int \mathrm{d}^{3} \mathbf{k} \frac{\sqrt{\left[6+\Delta_{c}\right]}}{\tau}=\frac{V \sqrt{\left[6+\Delta_{c}\right]}}{\tau} & \text { for }|k \eta|<<1, \\ \int \mathrm{d}^{3} \mathbf{k} \frac{\sqrt{2 \Delta+5+\Delta_{c}}}{\tau}=\frac{\sqrt{2 \Delta+5+\Delta_{c}} V}{\tau} & \text { for }|k \eta| \approx 1-\Delta(\rightarrow 0), \\ \int \mathrm{d}^{3} \mathbf{k} k & \text { for }|k \eta|>>1 .\end{cases}$

\subsubsection{Case III: $m_{\text {axion }} / f_{a}>>H$}

The equation of motion for the axion field for $m_{\mathrm{axion}} / f_{a} \approx H$ is given by

$$
\begin{aligned}
& \vartheta_{k}^{\prime \prime}+\left\{k^{2}+\frac{\lambda^{2}-6}{\eta^{2}}\right\} \vartheta_{k}=0 \quad \text { for } \eta \sim \eta_{\mathbf{c}}, \text { early \& late } \eta \\
& \vartheta_{k}^{\prime \prime}+\left\{k^{2}+\left[\lambda^{2}-6-\Delta_{c}\right] \frac{1}{\eta^{2}}\right\} \vartheta_{k}=0 \quad \text { for } \eta<\eta_{\mathbf{c}} .
\end{aligned}
$$

The solution for the mode function for the de Sitter and the quasi de Sitter space can be expressed as

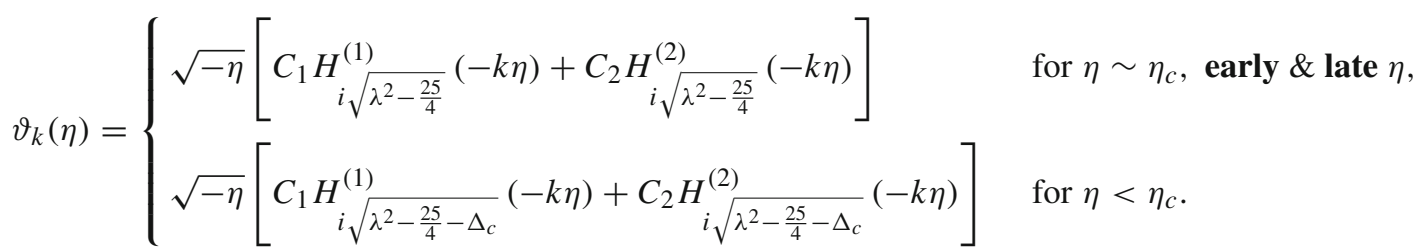

where $C_{1}$ and $C_{2}$ are two arbitrary integration constants, which depend on the choice of the initial condition.

After taking the $k \eta \rightarrow-\infty, k \eta \rightarrow 0$ and $|k \eta| \approx 1-\Delta(\rightarrow 0)$ limits the most general solution as stated in Eq. (4.153) can be recast as

$$
\begin{gathered}
\vartheta_{k}(\eta) \stackrel{|k \eta| \rightarrow-\infty}{=}\left\{\begin{array}{ll}
\sqrt{\frac{2}{\pi k}}\left[C_{1} e^{-i k \eta} e^{-\frac{i \pi}{2}\left(i \sqrt{\lambda^{2}-\frac{25}{4}}+\frac{1}{2}\right)}+C_{2} e^{i k \eta} e^{\frac{i \pi}{2}\left(i \sqrt{\lambda^{2}-\frac{25}{4}}+\frac{1}{2}\right)}\right] & \text { for } \eta \sim \eta_{c}, \text { early } \\
\sqrt{\frac{2}{\pi k}}\left[C_{1} e^{-i k \eta} e^{-\frac{i \pi}{2}\left(i \sqrt{\lambda^{2}-\frac{25}{4}-\Delta_{c}}+\frac{1}{2}\right)}+C_{2} e^{i k \eta} e^{\frac{i \pi}{2}\left(i \sqrt{\lambda^{2}-\frac{25}{4}-\Delta_{c}}+\frac{1}{2}\right)}\right] & \text { for } \eta<\eta_{c},
\end{array}\right] \text { for } \eta \sim \eta_{c}, \text { early \& late } \eta, \\
\vartheta_{k}(\eta) \stackrel{|k \eta| \rightarrow 0}{=} \begin{cases}\frac{i \sqrt{-\eta}}{\pi} \Gamma\left(i \sqrt{\lambda^{2}-\frac{25}{4}}\right)\left(-\frac{k \eta}{2}\right)^{-i \sqrt{\lambda^{2}-\frac{25}{4}}}\left[C_{1}-C_{2}\right] & \text { for } \eta<\eta_{c}, \\
\frac{i \sqrt{-\eta}}{\pi} \Gamma\left(i \sqrt{\lambda^{2}-\frac{25}{4}-\Delta_{c}}\right)\left(-\frac{k \eta}{2}\right)^{-i \sqrt{\lambda^{2}-\frac{25}{4}-\Delta_{c}}} & {\left[C_{1}-C_{2}\right.}\end{cases}
\end{gathered}
$$




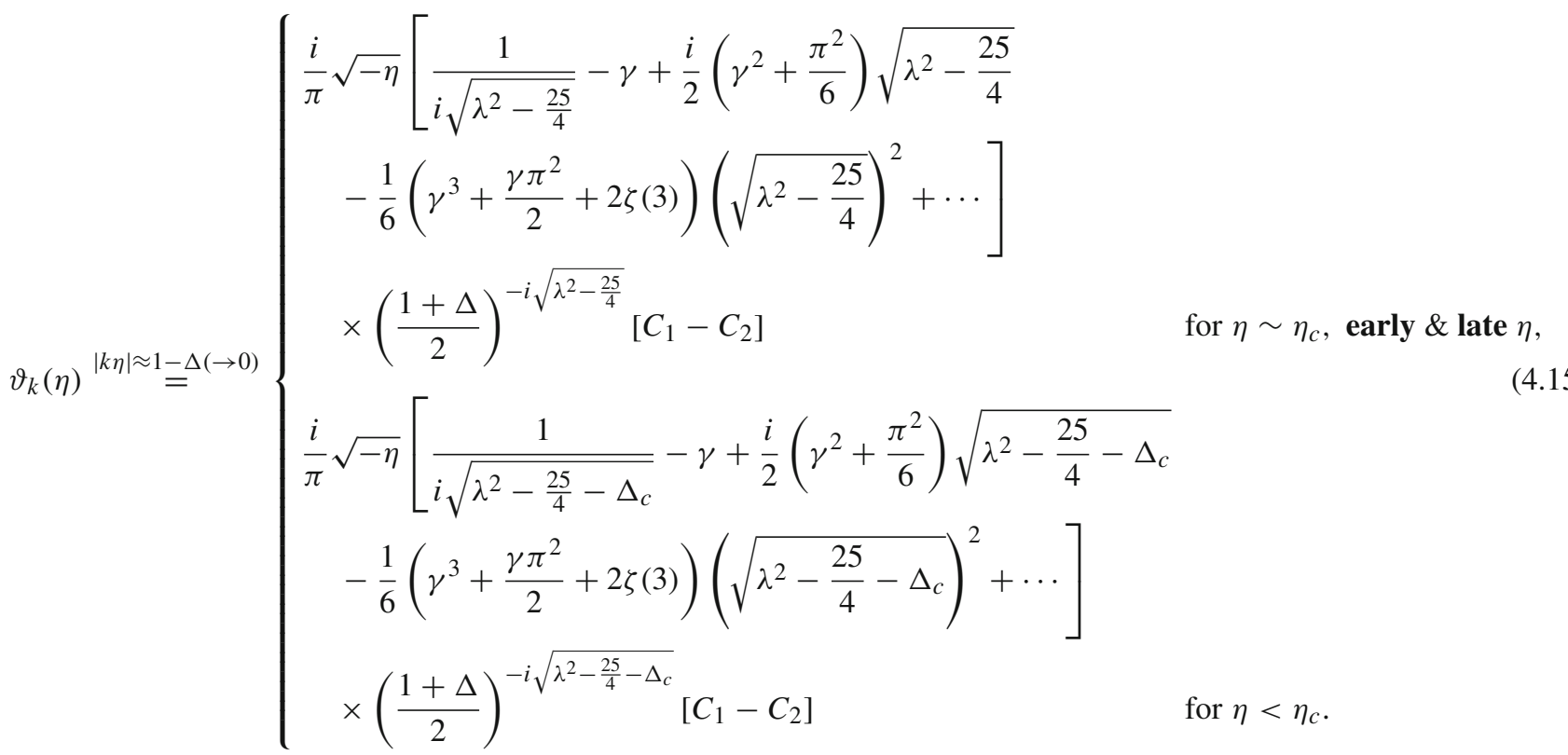

Next we assume that the WKB approximation is approximately valid for all times for the solution for the mode function $\vartheta_{k}$. In the standard WKB approximation the total solution can be recast in the following form:

$\vartheta_{k}(\eta)=\left[D_{1} u_{k}(\eta)+D_{2} \bar{u}_{k}(\eta)\right]$,

where $D_{1}$ and $D_{2}$ are two arbitrary integration constants, which depend on the choice of the initial condition during WKB approximation at early and late time scale. In the present context $u_{k}(\eta)$ and $\bar{u}_{k}(\eta)$ are defined as

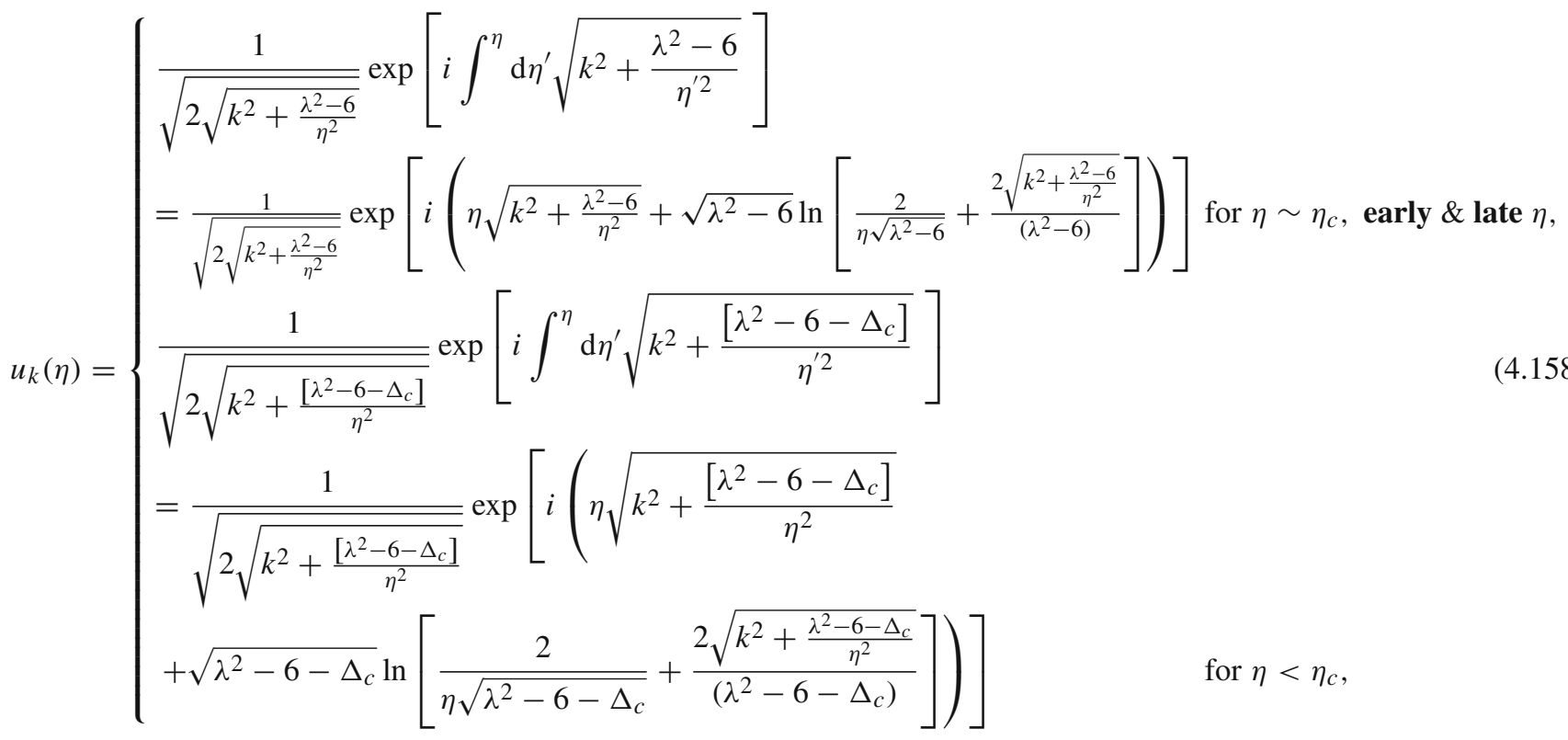




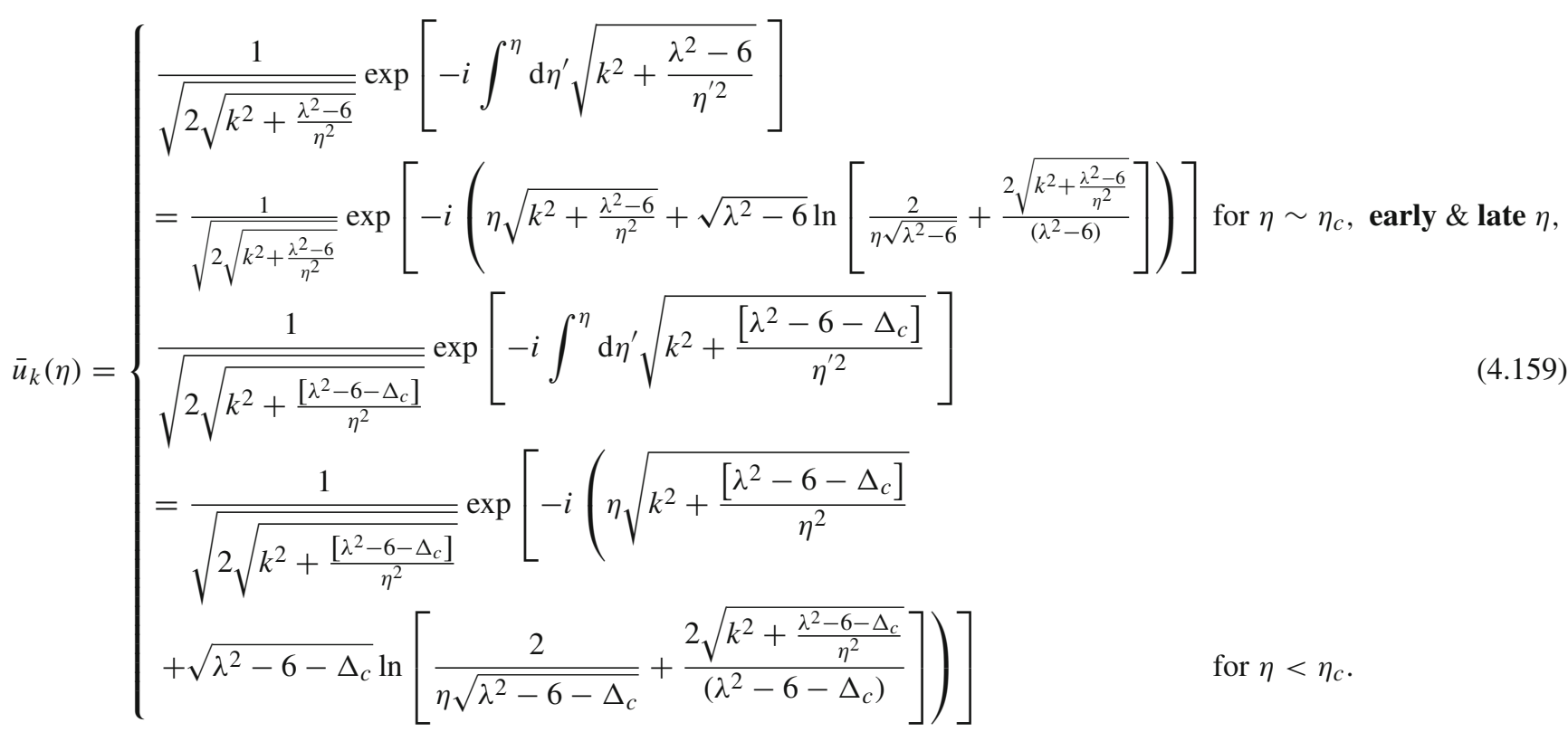

In the present context the Bogoliubov coefficient $\beta(k)$ in Fourier space can be computed approximately using the following expression:

$$
\beta\left(k, \tau, \tau^{\prime}, \eta^{\prime}\right)=\left\{\begin{array}{l}
\int_{\tau^{\prime}}^{\tau} \mathrm{d} \eta \frac{\left(\lambda^{2}-6\right)^{2}}{4 \eta^{6}\left(k^{2}+\frac{\left(\lambda^{2}-6\right)}{\eta^{2}}\right)^{\frac{5}{2}}} \exp \left[2 i \int_{\eta^{\prime}}^{\eta} \mathrm{d} \eta^{\prime \prime} \sqrt{\left.k^{2}+\frac{\left(\lambda^{2}-6\right)}{\eta^{\prime \prime 2}}\right]} \quad \text { for } \eta \sim \eta_{c}, \text { early \& late } \eta,\right. \\
\int_{\tau^{\prime}}^{\tau} \mathrm{d} \eta \frac{\left(\lambda^{2}-6-\Delta_{c}\right)^{2}}{4 \eta^{6}\left(k^{2}+\frac{\left(\lambda^{2}-6-\Delta_{c}\right)}{\eta^{2}}\right)^{\frac{5}{2}}} \exp \left[2 i \int_{\eta^{\prime}}^{\eta} \mathrm{d} \eta^{\prime \prime} \sqrt{k^{2}+\frac{\left(\lambda^{2}-6-\Delta_{c}\right)}{\eta^{\prime \prime 2}}}\right] \text { for } \eta<\eta_{c},
\end{array}\right.
$$

which is not exactly analytically computable. To study the behaviour of this integral we consider here three consecutive physical situations: $|k \eta|<<1,|k \eta| \approx 1-\Delta(\rightarrow 0)$ and $|k \eta|>>1$ for the de Sitter case and the quasi de Sitter case. In the three cases we have

For $\eta \sim \eta_{\mathbf{c}}$, early \& late $\eta: \quad \sqrt{\left\{k^{2}+\frac{\lambda^{2}-6}{\eta^{2}}\right\}} \approx \begin{cases}\frac{\sqrt{\lambda^{2}-6}}{\eta} & \text { for }|k \eta|<<1, \\ \frac{\sqrt{\lambda^{2}-2 \Delta-5}}{\eta} & \text { for }|k \eta| \approx 1-\Delta(\rightarrow 0), \\ k & \text { for }|k \eta|>>1 .\end{cases}$
For $\eta<\eta_{\mathbf{c}}: \quad \sqrt{\left\{k^{2}+\left[\lambda^{2}-6-\Delta_{c}\right] \frac{1}{\left.\eta^{2}\right\}}\right.} \approx \begin{cases}\frac{\sqrt{\lambda^{2}-6-\Delta_{c}}}{\eta} & \text { for }|k \eta|<<1, \\ \frac{\sqrt{\lambda^{2}-2 \Delta-5-\Delta_{c}}}{\eta} & \text { for }|k \eta| \approx 1-\Delta(\rightarrow 0), \\ k & \text { for }|k \eta|>>1 .\end{cases}$

and further using this result the Bogoliubov coefficient $\beta(k)$ in Fourier space can be expressed as 
For $\eta \sim \eta_{\mathbf{c}}$, early \& late $\eta$ :
$\beta\left(k, \tau, \tau^{\prime}, \eta^{\prime}\right)= \begin{cases}\frac{\left[\tau^{\left.2 i \sqrt{\lambda^{2}-6}-\tau^{\prime 2 i} \sqrt{\lambda^{2}-6}\right]}\right.}{8 i\left(\lambda^{2}-6\right) \eta^{\prime 2 i \sqrt{\lambda^{2}-6}}} & \text { for }|k \eta|<<1, \\ \frac{\left(\lambda^{2}-6\right)^{2}\left[\tau^{2 i \sqrt{\lambda^{2}-2 \Delta-5}}-\tau^{\left.\prime 2 i \sqrt{\lambda^{2}-2 \Delta-5}\right]}\right.}{8 i\left(\lambda^{2}-2 \Delta-5\right)^{3} \eta^{\prime 2} 2 i \sqrt{\lambda^{2}-2 \Delta-5}} & \text { for }|k \eta| \approx 1-\Delta(\rightarrow 0), \\ \left(\lambda^{2}-6\right)^{2}\left[i \frac{\operatorname{Ei}(2 i k \eta) e^{-2 i k \eta^{\prime}}}{15}-\frac{e^{2 i k\left(\eta-\eta^{\prime}\right)}}{120 k^{5} \eta^{5}}\left(4 k^{4} \eta^{4}-2 i k^{3} \eta^{3}-2 k^{2} \eta^{2}+3 i k \eta+6\right)\right]_{\tau^{\prime}}^{\tau} & \text { for }|k \eta|>>1 .\end{cases}$

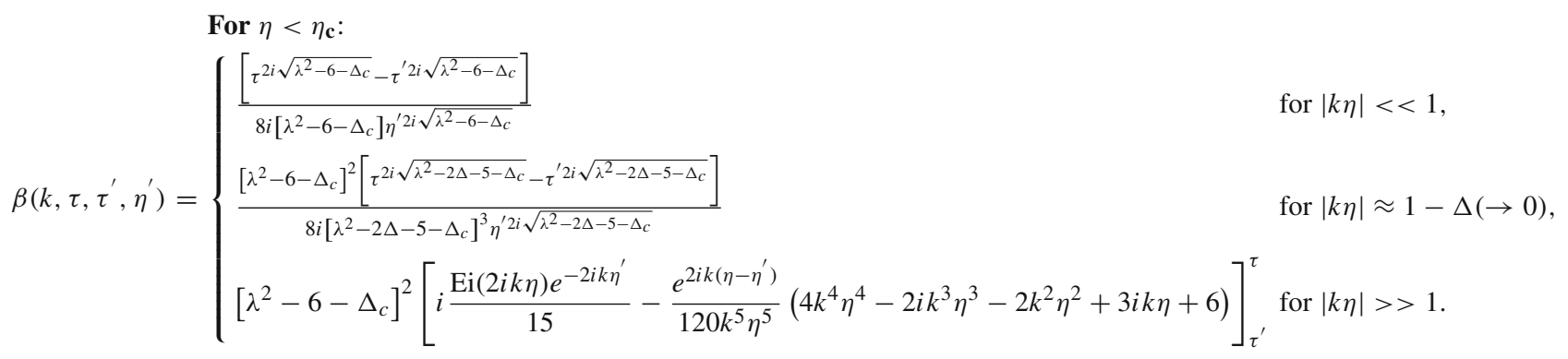

As mentioned earlier here one can use another equivalent way to define the Bogoliubov coefficient $\beta$ in Fourier space by implementing instantaneous Hamiltonian diagonalization method to interpret the results. Using this diagonalized representation the regularized Bogoliubov coefficient $\beta$ in Fourier space can be written as

$\beta_{\text {diag }}\left(k ; \tau, \tau^{\prime}\right)= \begin{cases}\int_{\tau^{\prime}}^{\tau} \mathrm{d} \eta \frac{\left(\lambda^{2}-6\right) \exp \left[-2 i \int^{\eta} \mathrm{d} \eta^{\prime} \sqrt{k^{2}+\frac{\lambda^{2}-6}{\eta^{\prime 2}}}\right]}{2 \eta^{3}\left(k^{2}+\frac{\lambda^{2}-6}{\eta^{2}}\right)} & \text { for } \eta \sim \eta_{c}, \text { early \& late } \eta, \\ \int_{\tau^{\prime}}^{\tau} \mathrm{d} \eta \frac{\left[\lambda^{2}-6-\Delta_{c}\right] \exp \left[-2 i \int^{\eta} \mathrm{d} \eta^{\prime} \sqrt{\left.k^{2}+\frac{\left[\lambda^{2}-6-\Delta_{c}\right]}{\eta^{\prime 2}}\right]}\right.}{2 \eta^{3}\left(k^{2}+\frac{\left[\lambda^{2}-6-\Delta_{c}\right]}{\eta^{2}}\right)} & \text { for } \eta<\eta_{c},\end{cases}$

where $\tau$ and $\tau^{\prime}$ introduced as the conformal time regulator in the present context. In this case as well the Bogoliubov coefficient is not exactly analytically computable. To study the behaviour of this integral we consider here three similar consecutive physical situations for the de Sitter and the quasi de Sitter case as discussed earlier.

\section{For $\eta \sim \eta_{\mathfrak{c}}$, early \& late $\eta$ :}

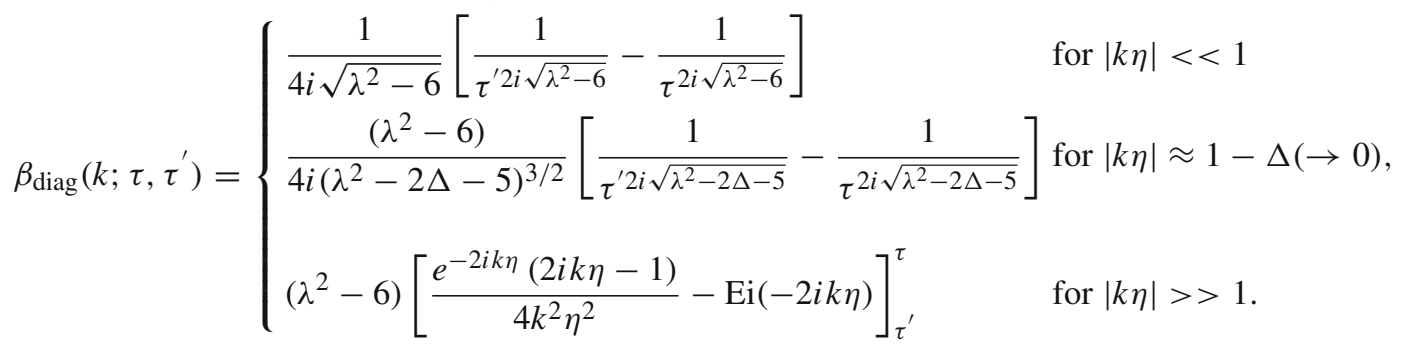


For $\eta<\eta_{\mathbf{c}}$ :

$\beta_{\text {diag }}\left(k ; \tau, \tau^{\prime}\right)= \begin{cases}\frac{1}{4 i \sqrt{\lambda^{2}-6-\Delta_{c}}}\left[\frac{1}{\tau^{\prime 2 i \sqrt{\lambda^{2}-6-\Delta_{c}}}}-\frac{1}{\tau^{2 i \sqrt{\lambda^{2}-6-\Delta_{c}}}}\right] & \text { for }|k \eta|<<1, \\ \frac{\left[\lambda^{2}-6-\Delta_{c}\right]}{4 i\left[\lambda^{2}-2 \Delta-5-\Delta_{c}\right]^{3 / 2}}\left[\frac{1}{\tau^{\prime 2 i} \sqrt{\lambda^{2}-2 \Delta-5-\Delta_{c}}}-\frac{1}{\tau^{2 i \sqrt{\lambda^{2}-2 \Delta-5-\Delta_{c}}}}\right] & \text { for }|k \eta| \approx 1-\Delta(\rightarrow 0), \\ {\left[\lambda^{2}-6-\Delta_{c}\right]\left[\frac{e^{-2 i k \eta}(2 i k \eta-1)}{4 k^{2} \eta^{2}}-\operatorname{Ei}(-2 i k \eta)\right]_{\tau^{\prime}}^{\tau}} & \text { for }|k \eta|>>1 .\end{cases}$

Further using the regularized expressions for the Bogoliubov coefficient $\beta$ in two different representations as mentioned in Eq. (4.160) and Eq. (4.164), and substituting them in Eq. (3.57) we get the following regularized expressions for the Bogoliubov coefficient $\alpha$ in two different representations:

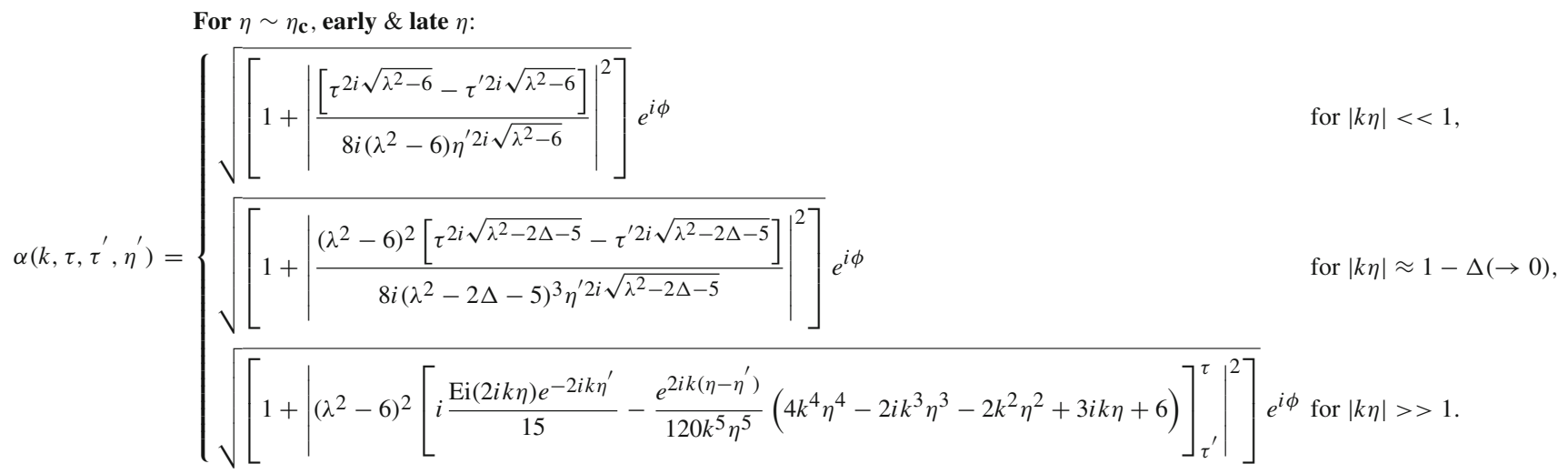

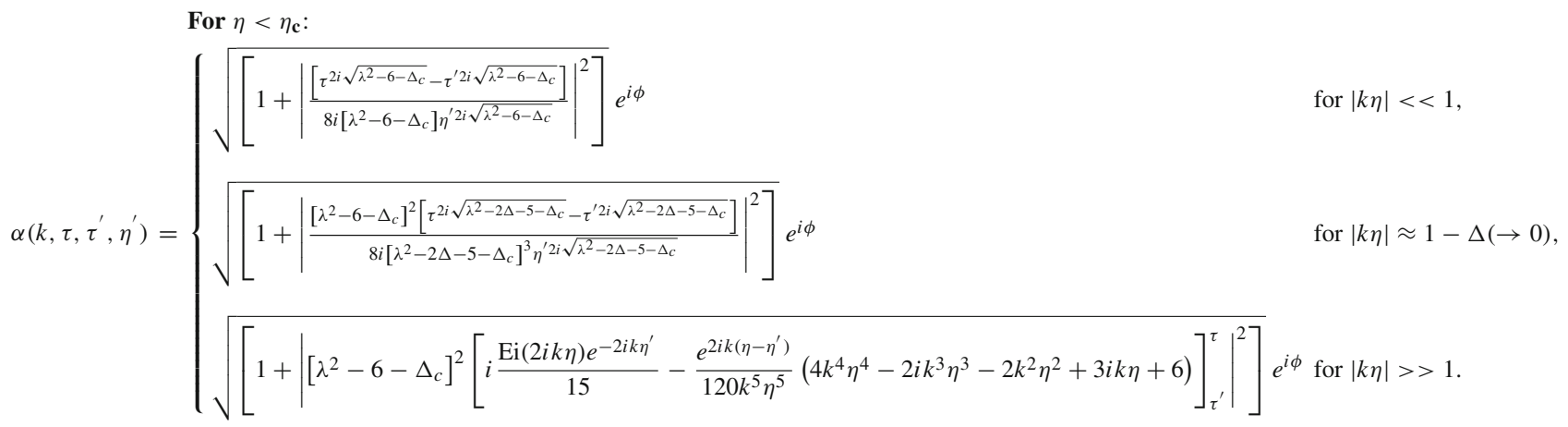

For $\eta \sim \eta_{\mathbf{c}}$, early \& late $\eta$ :

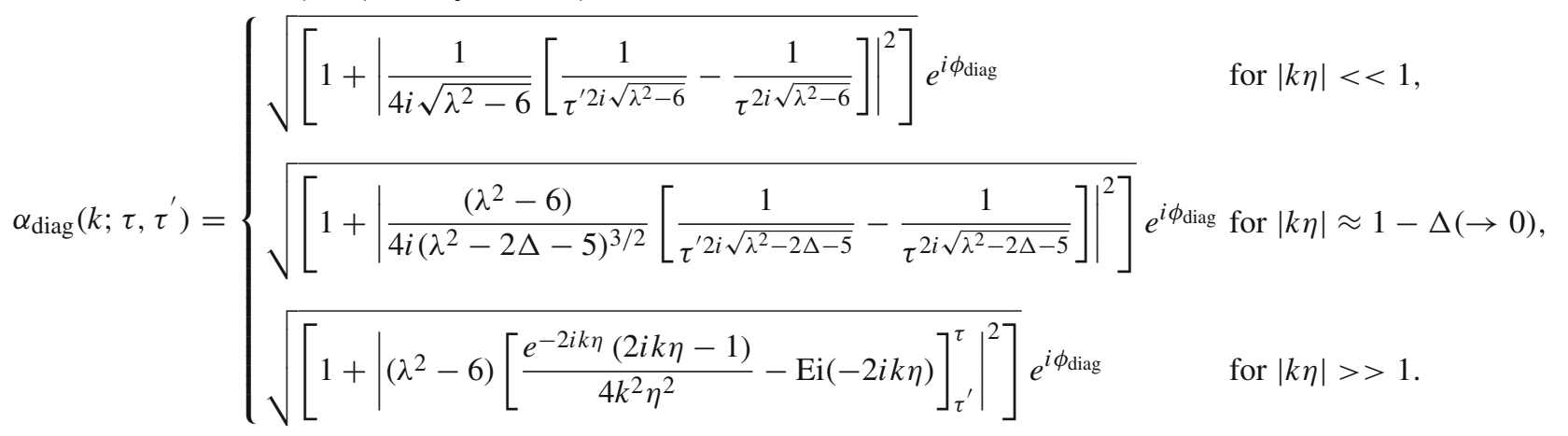




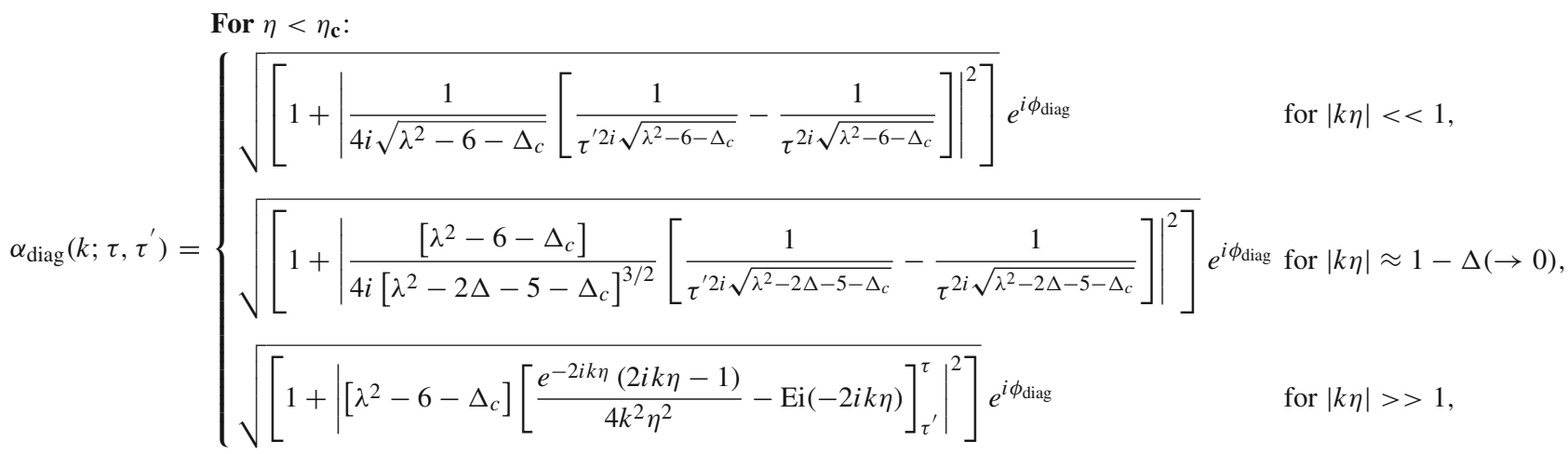

where $\phi$ and $\phi_{\text {diag }}$ are the associated phase factors in two different representations.

Further using the expressions for the Bogoliubov coefficient $\alpha$ in two different representations we get the following expressions for the reflection and transmission coefficient in two different representations for three consecutive physical situations: $|k \eta|<<1,|k \eta| \approx 1-\Delta(\rightarrow 0)$ and $|k \eta|>>1$ for the de Sitter case and the quasi de Sitter case as given by

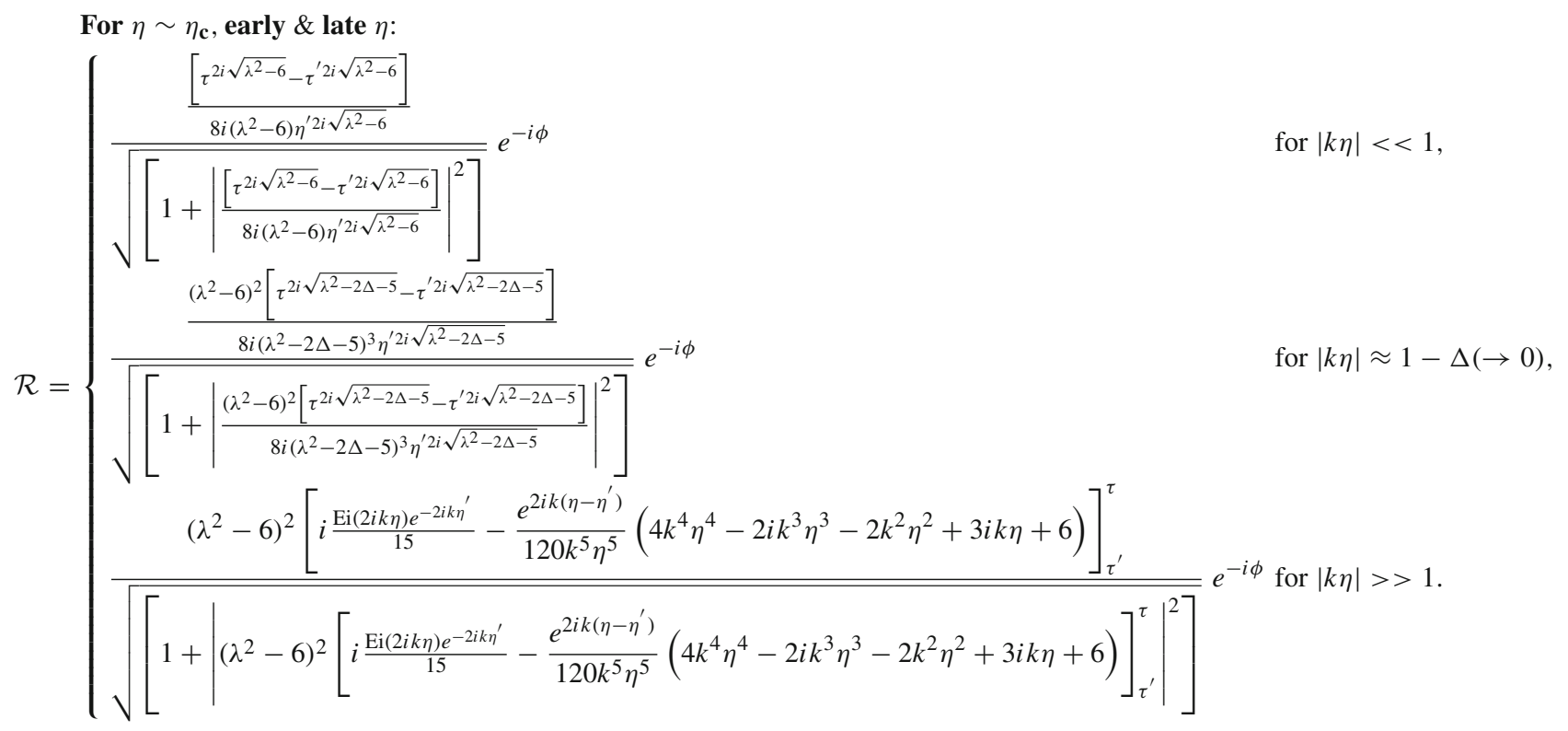

For $\eta \sim \eta_{\mathbf{c}}$, early \& late $\eta$ :

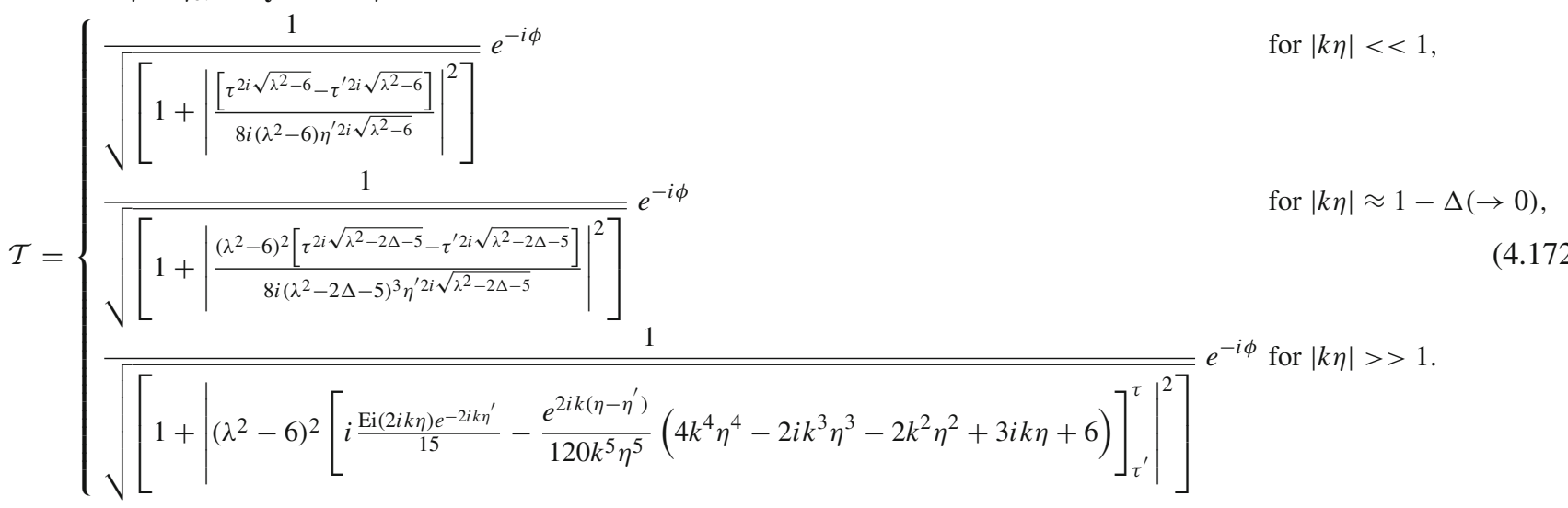


For $\eta<\eta_{\mathbf{c}}$ :

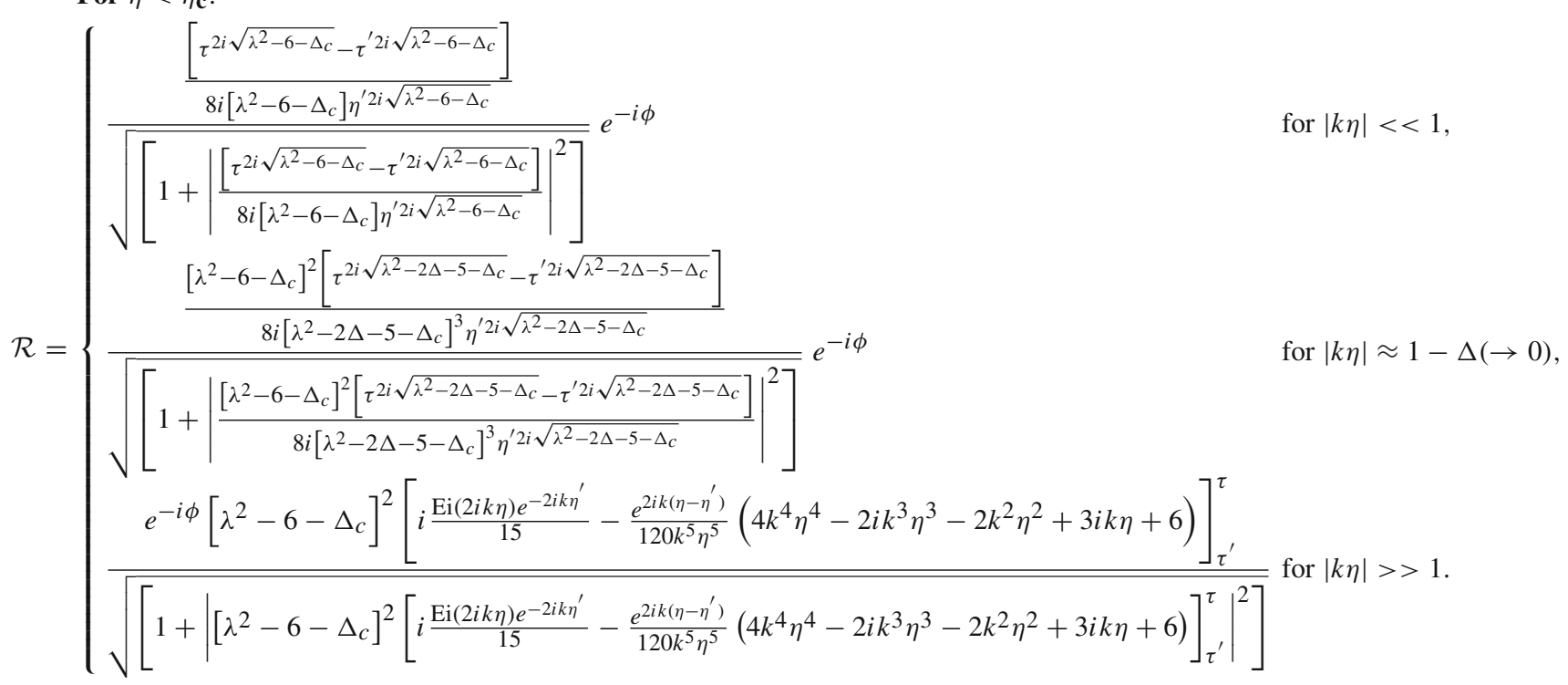

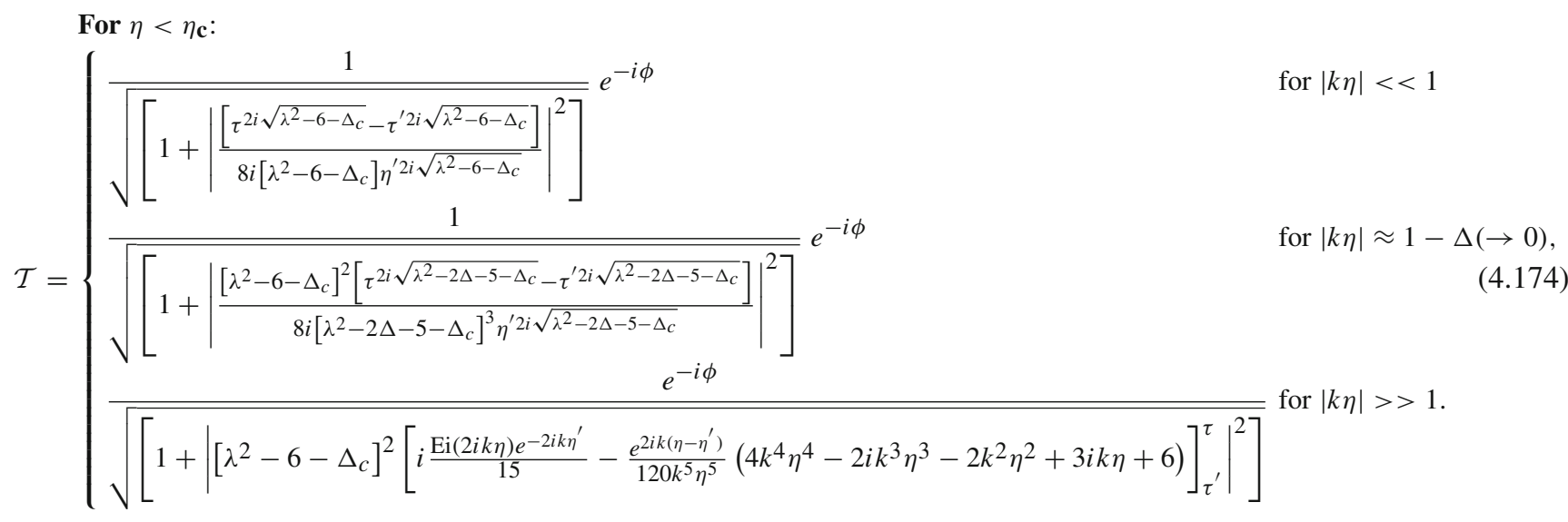

and

For $\eta \sim \eta_{\mathbf{c}}$, early \& late $\eta$ :

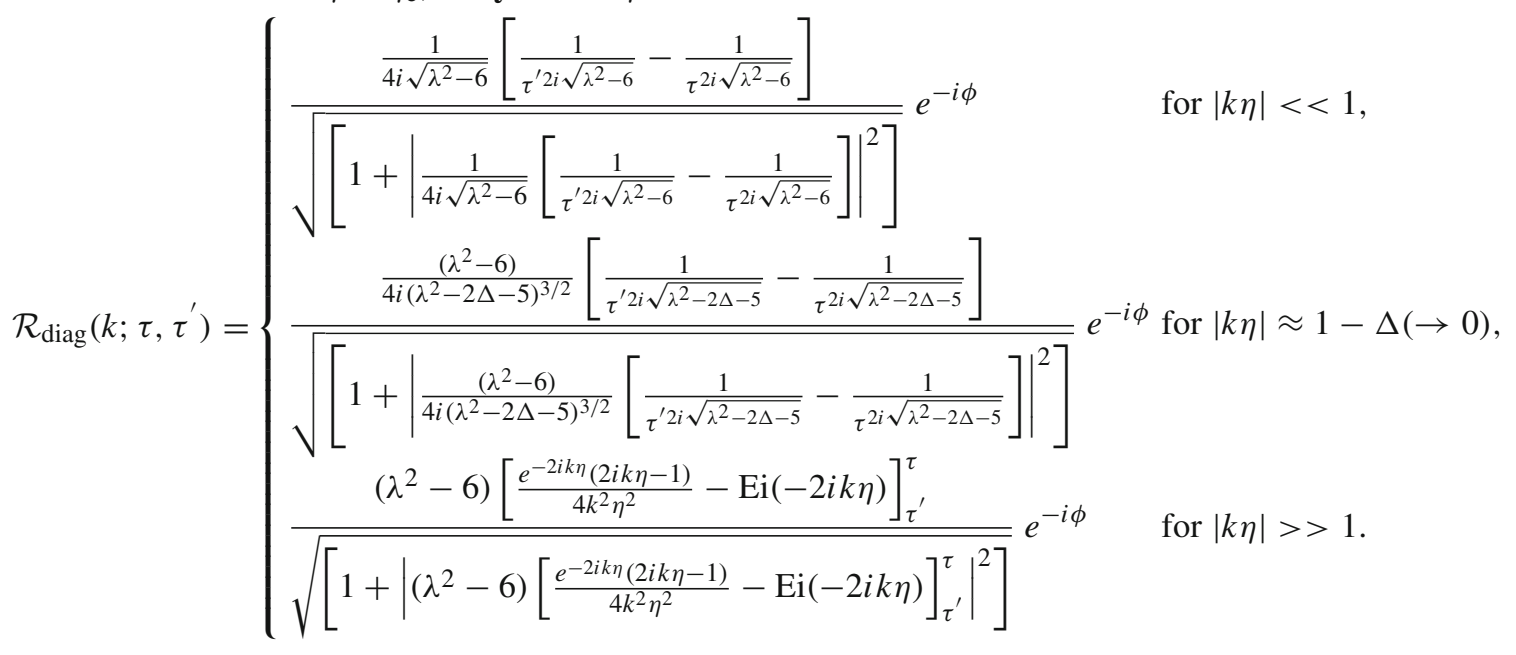


For $\eta \sim \eta_{\mathbf{c}}$, early \& late $\eta$ :

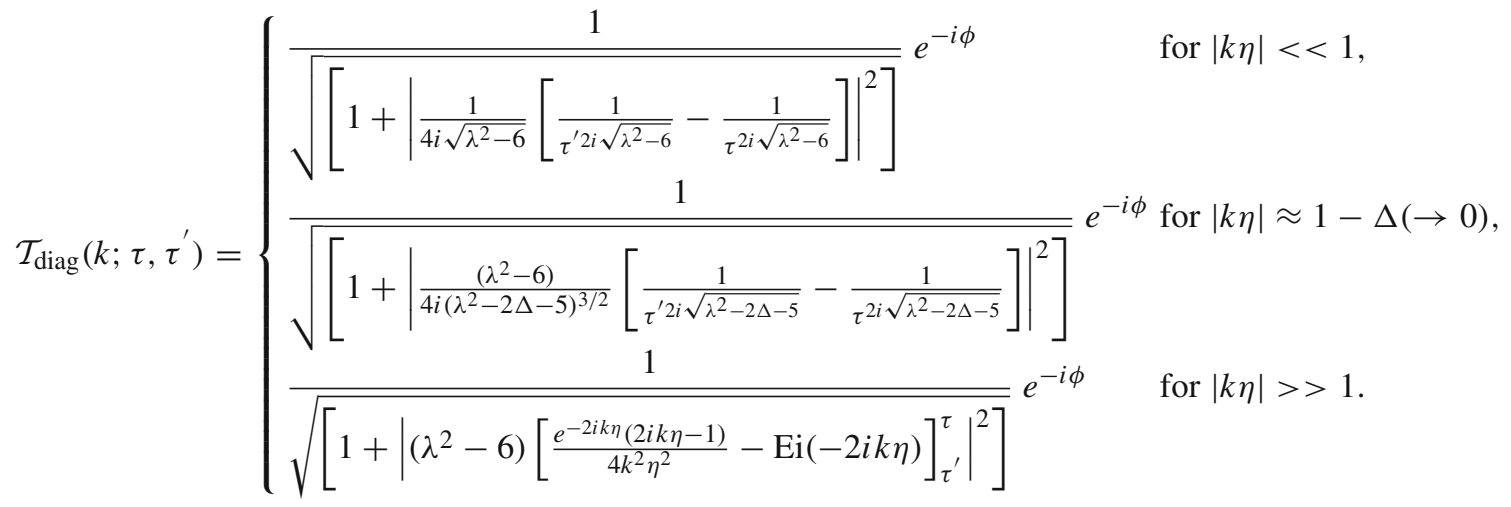

For $\eta<\eta_{\mathbf{c}}$ :

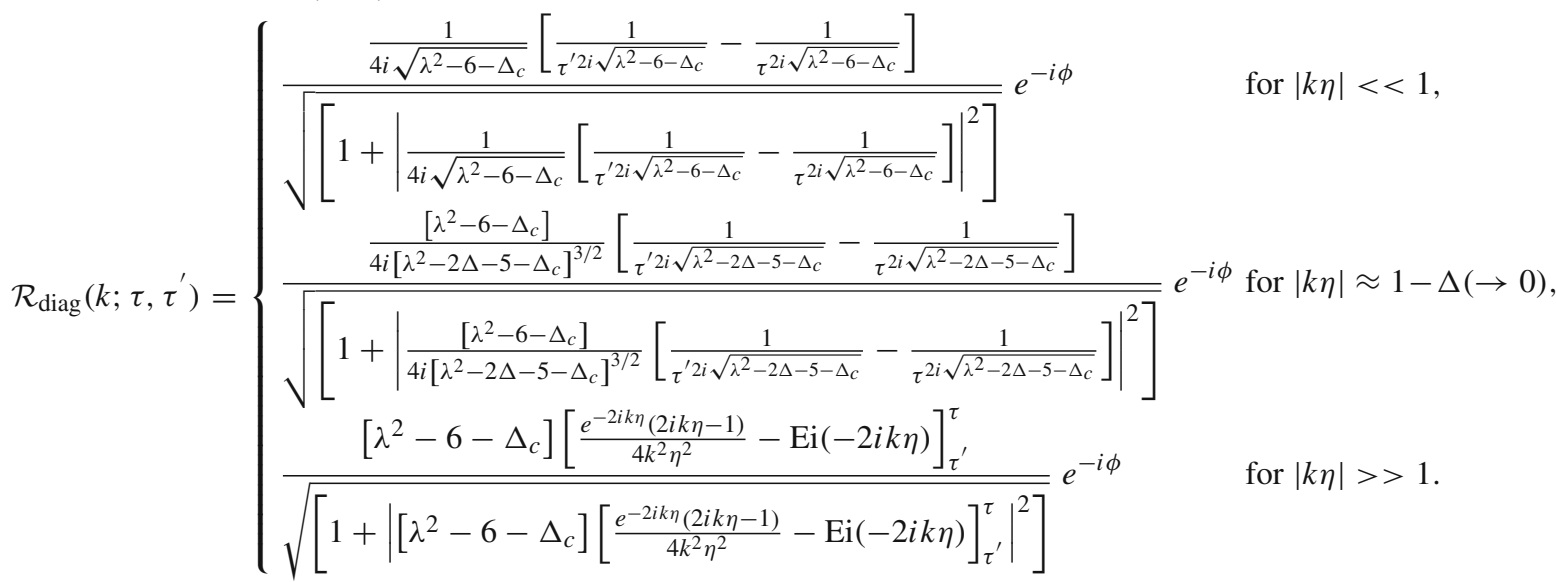

For $\eta<\eta_{\mathbf{c}}$ :

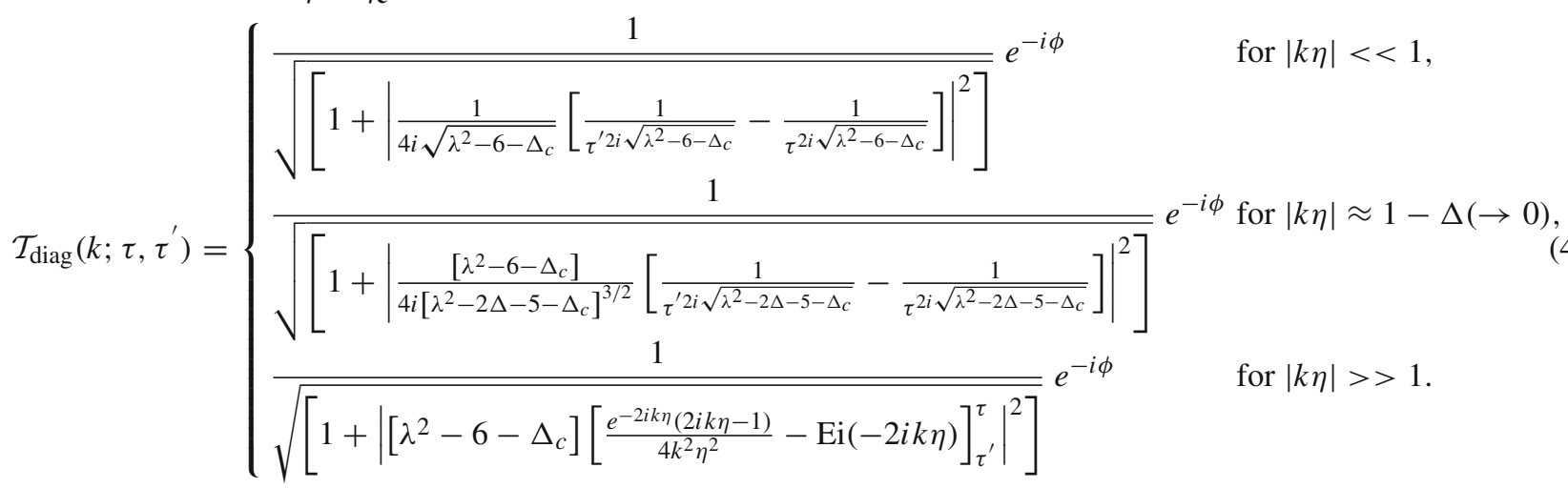

Next the expression for the number of produced particles at time $\tau$ can be expressed in the two representations as

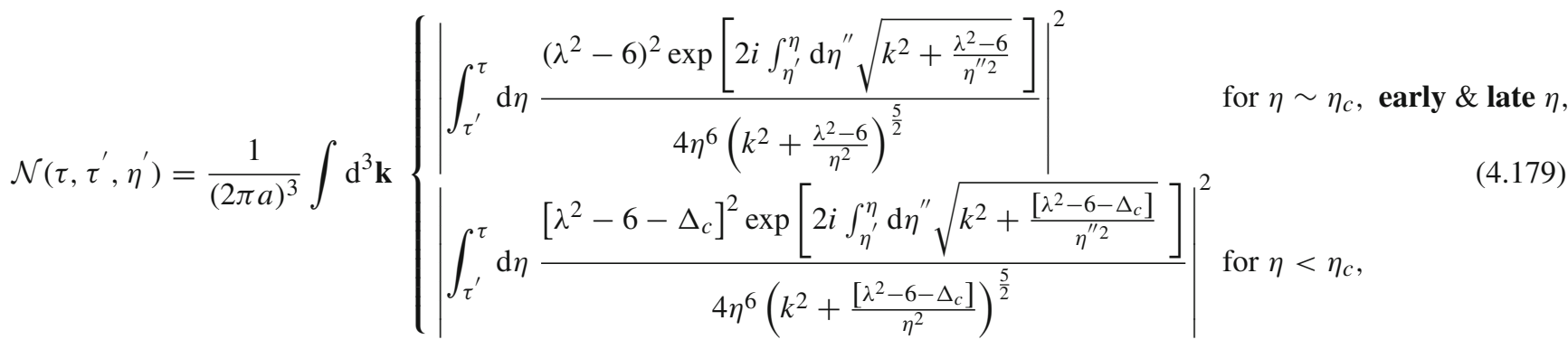




$$
\mathcal{N}_{\text {diag }}\left(\tau, \tau^{\prime}\right)=\frac{1}{(2 \pi a)^{3}} \int \mathrm{d}^{3} \mathbf{k}\left\{\begin{array}{l}
\left|\frac{\left(\lambda^{2}-6\right) \exp \left[-2 i \int \mathrm{d}^{\prime} \sqrt{k^{2}+\frac{\lambda^{2}-6}{\eta^{\prime 2}}}\right]}{2 \eta^{3}\left(k^{2}+\frac{\lambda^{2}-6}{\eta^{2}}\right)}\right|^{2} \quad \text { for } \eta \sim \eta_{c}, \text { early \& late } \eta, \\
\left|\frac{\left[\lambda^{2}-6-\Delta_{c}\right] \exp \left[-2 i \iint^{\eta} \mathrm{d} \eta^{\prime} \sqrt{k^{2}+\frac{\left[\lambda^{2}-6-\Delta_{c}\right]}{\eta^{\prime 2}}}\right]}{2 \eta^{3}\left(k^{2}+\frac{\left[\lambda^{2}-6-\Delta_{c}\right]}{\eta^{2}}\right)}\right|^{2} \text { for } \eta<\eta_{c},
\end{array}\right.
$$

which are not exactly analytically computable. To study the behaviour of this integral we consider here three consecutive physical situations: $|k \eta|<<1,|k \eta| \approx 1-\Delta(\rightarrow 0)$ and $|k \eta|>>1$ for the de Sitter and the quasi de Sitter case. In the three cases we have the following.

$$
\begin{aligned}
& \text { For } \eta \sim \eta_{\mathbf{c}} \text {, early \& late } \eta \text { : } \\
& \mathcal{N}\left(\tau, \tau^{\prime}, \eta^{\prime}\right)= \begin{cases}\frac{V}{(2 \pi a)^{3}} \frac{\left[\tau^{2 i \sqrt{\lambda^{2}-6}}-\tau^{\left.\prime 2 i \sqrt{\lambda^{2}-6}\right]^{2}}\right.}{64\left(\lambda^{2}-6\right)^{2} \eta^{\prime 4 i \sqrt{\lambda^{2}-6}}} & \text { for }|k \eta|<<1, \\
\frac{V}{(2 \pi a)^{3}} \frac{\left(\lambda^{2}-6\right)^{4}\left[\tau^{2 i \sqrt{\lambda^{2}-2 \Delta-5}}-\tau^{\left.\prime 2 i \sqrt{\lambda^{2}-2 \Delta-5}\right]^{2}}\right.}{64\left(\lambda^{2}-2 \Delta-5\right)^{6} \eta^{\prime} 4 i \sqrt{\lambda^{2}-2 \Delta-5}} & \text { for }|k \eta| \approx 1-\Delta(\rightarrow 0), \\
\frac{4 \pi\left(\lambda^{2}-6\right)^{4}}{(2 \pi a)^{3}} \int_{0}^{\infty} \mathrm{d} k k^{2} \mid\left\{\frac{i e^{-2 i k \eta^{\prime}}}{15}\left\{\operatorname{Ei}(2 i k \tau)-\operatorname{Ei}\left(2 i k \tau^{\prime}\right)\right\}\right. & \\
-\frac{e^{-2 i k \eta^{\prime}}}{120 k^{5}}\left[\frac{e^{2 i k \tau}\left(4 k^{4} \tau^{4}-2 i k^{3} \tau^{3}-2 k^{2} \tau^{2}+3 i k \tau+6\right)}{\tau^{5}}\right. & \\
\left.\left.-\frac{e^{2 k \tau}\left(4 k^{4} \tau^{\prime} 4-2 i k^{3} \tau^{\prime 3}-2 k^{2} \tau^{\prime 2}+3 i k \tau^{\prime}+6\right)}{\tau^{\prime 5}}\right]\right\}\left.\right|^{2} & \text { for }|k \eta|>>1 .\end{cases}
\end{aligned}
$$

For $\eta<\eta_{\mathbf{c}}$ :

$$
\mathcal{N}\left(\tau, \tau^{\prime}, \eta^{\prime}\right)= \begin{cases}\frac{V}{(2 \pi a)^{3}} \frac{\left[\tau^{2 i \sqrt{\lambda^{2}-6-\Delta_{c}}}-\tau^{\prime 2 i \sqrt{\lambda^{2}-6-\Delta_{c}}}\right]^{2}}{64\left[\lambda^{2}-6-\Delta_{c}\right]^{2} \eta^{\prime 4 i \sqrt{\lambda^{2}-6-\Delta_{c}}}} & \text { for }|k \eta|<<1, \\ \frac{V}{(2 \pi a)^{3}} \frac{\left[\lambda^{2}-6-\Delta_{c}\right]^{4}\left[\tau^{2 i \sqrt{\lambda^{2}-2 \Delta-5-\Delta_{c}}}-\tau^{\prime 2 i \sqrt{\lambda^{2}-2 \Delta-5-\Delta_{c}}}\right]^{2}}{64\left[\lambda^{2}-2 \Delta-5-\Delta_{c}\right]^{6} \eta^{\prime 4} \sqrt{\lambda^{2}-2 \Delta-5-\Delta_{c}}} & \text { for }|k \eta| \approx 1-\Delta(\rightarrow 0), \\ \frac{4 \pi\left(\lambda^{2}-6-\Delta_{c}\right)^{4}}{(2 \pi a)^{3}} \int_{0}^{\infty} \mathrm{d} k k^{2} \mid\left\{\frac{i e^{-2 i k \eta^{\prime}}}{15}\left\{\operatorname{Ei}(2 i k \tau)-\operatorname{Ei}\left(2 i k \tau^{\prime}\right)\right\}\right. & \\ \left.-\frac{e^{-2 i k \eta^{\prime}}}{120 k^{5}}\left[\frac{e^{2 i k \tau}\left(4 k^{4} \tau^{4}-2 i k^{3} \tau^{3}-2 k^{2} \tau^{2}+3 i k \tau+6\right)}{\tau^{5}}\right]\right\} & \text { for }|k \eta|>>1 . \\ \left.-\frac{e^{2 k \tau}\left(4 k^{4} \tau^{\prime 4}-2 i k^{3} \tau^{\prime 3}-2 k^{2} \tau^{\prime 2}+3 i k \tau^{\prime}+6\right)}{\tau^{\prime 5}}\right]\left.\right|^{2} & \end{cases}
$$

and 
For $\eta \sim \eta_{\mathbf{c}}$, early \& late $\eta$ :

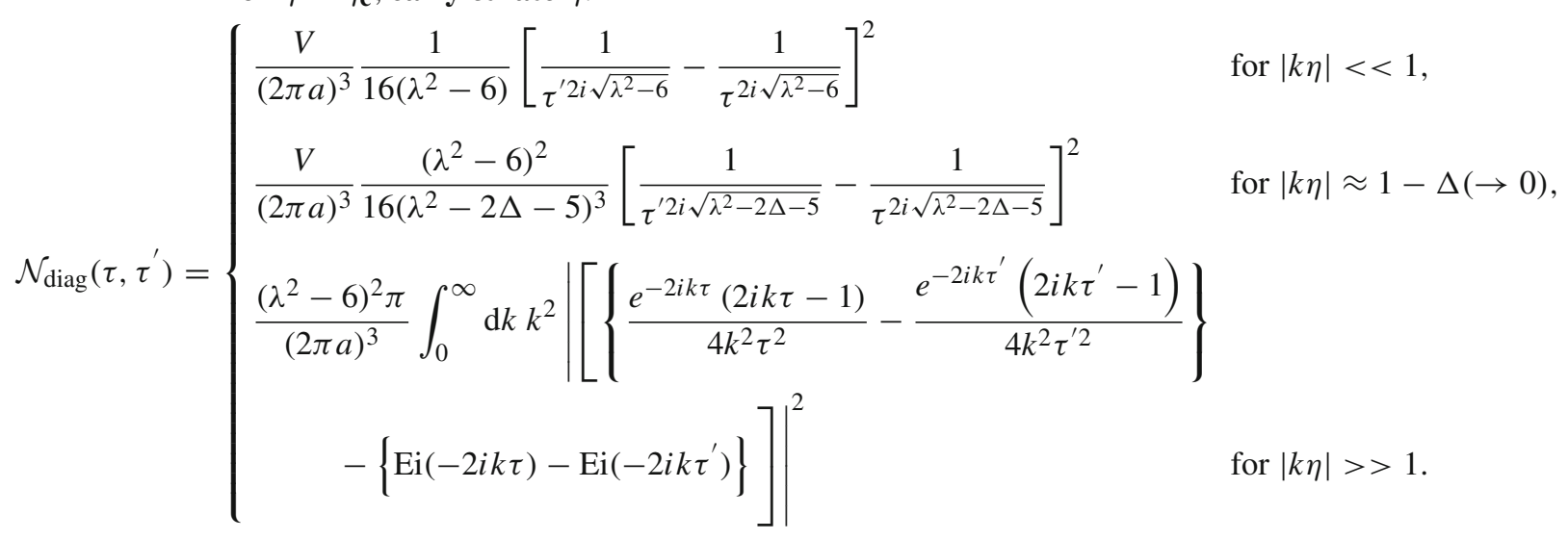

For $\eta<\eta_{\mathbf{c}}$ :

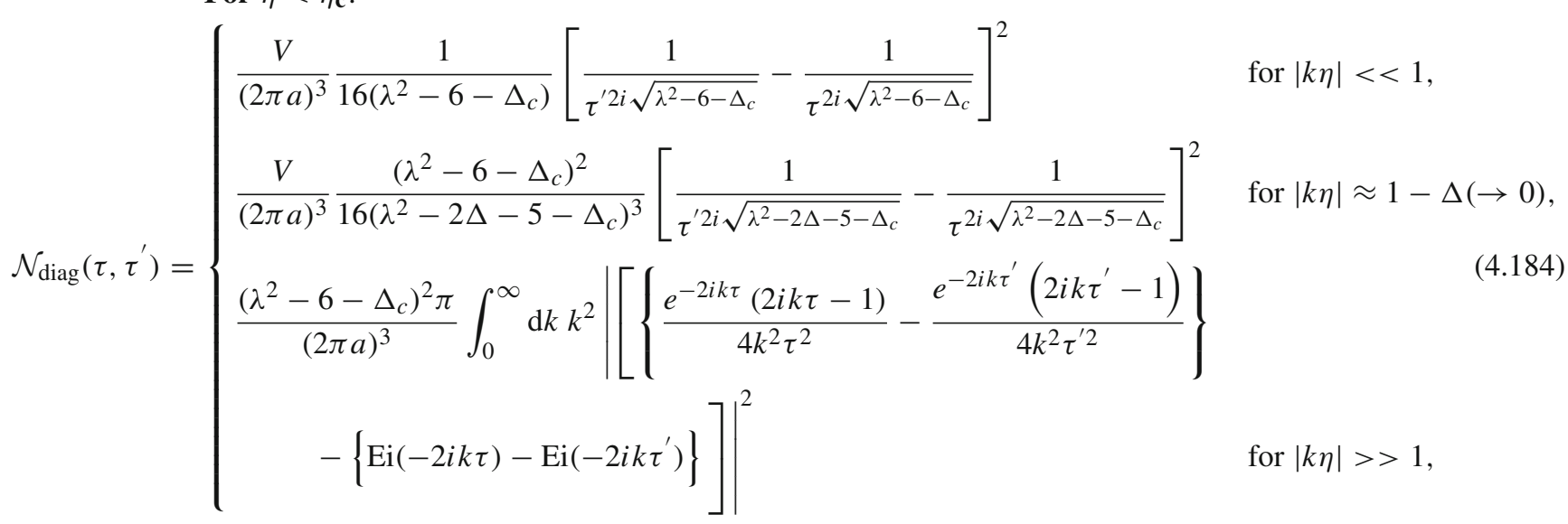

where the symbol $V$ is defined earlier.

Finally one can define the total energy density of the produced particles using the following expression:

$$
\begin{aligned}
& \rho\left(\tau, \tau^{\prime}, \eta^{\prime}\right)=\frac{1}{(2 \pi a)^{3} a} \int \mathrm{d}^{3} \mathbf{k}\left\{\begin{array}{l}
\sqrt{k^{2}+\frac{\lambda^{2}-6}{\tau^{2}}}\left|\int_{\tau^{\prime}}^{\tau} d \eta \frac{\left(\lambda^{2}-6\right)^{2} \exp \left[2 i \int_{\eta^{\prime}}^{\eta} \mathrm{d} \eta^{\prime \prime} \sqrt{k^{2}+\frac{\lambda^{2}-6}{\eta^{\prime \prime 2}}}\right]}{4 \eta^{6}\left(k^{2}+\frac{\lambda^{2}-6}{\eta^{2}}\right)^{\frac{5}{2}}}\right|^{2} \quad \text { for } \eta \sim \eta_{c}, \text { early \& late } \eta, \\
\sqrt{k^{2}+\frac{\left[\lambda^{2}-6-\Delta_{c}\right]}{\tau^{2}}}\left|\int_{\tau^{\prime}}^{\tau} \mathrm{d} \eta \frac{\left[\lambda^{2}-6-\Delta_{c}\right]^{2} \exp \left[2 i \int_{\eta^{\prime}}^{\eta} \mathrm{d} \eta^{\prime \prime} \sqrt{k^{2}+\frac{\left[\lambda^{2}-6-\Delta_{c}\right]}{\eta^{\prime \prime 2}}}\right]}{4 \eta^{6}\left(k^{2}+\frac{\left[\lambda^{2}-6-\Delta_{c}\right]}{\eta^{2}}\right)^{\frac{5}{2}}}\right|^{2} \text { for } \eta<\eta_{c},
\end{array}\right.
\end{aligned}
$$

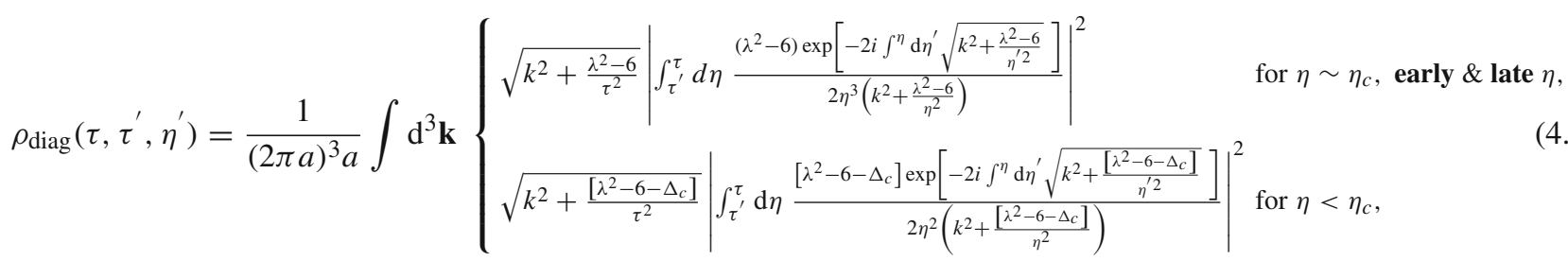

which is not exactly analytically computable. To study the behaviour of this integral we consider here three consecutive physical situations: $|k \eta|<<1,|k \eta| \approx 1-\Delta(\rightarrow 0)$ and $|k \eta|>>1$ for the de Sitter case and the quasi de Sitter case. In the three cases we have 
For $\eta \sim \eta_{\mathbf{c}}$, early \& late $\eta$ :

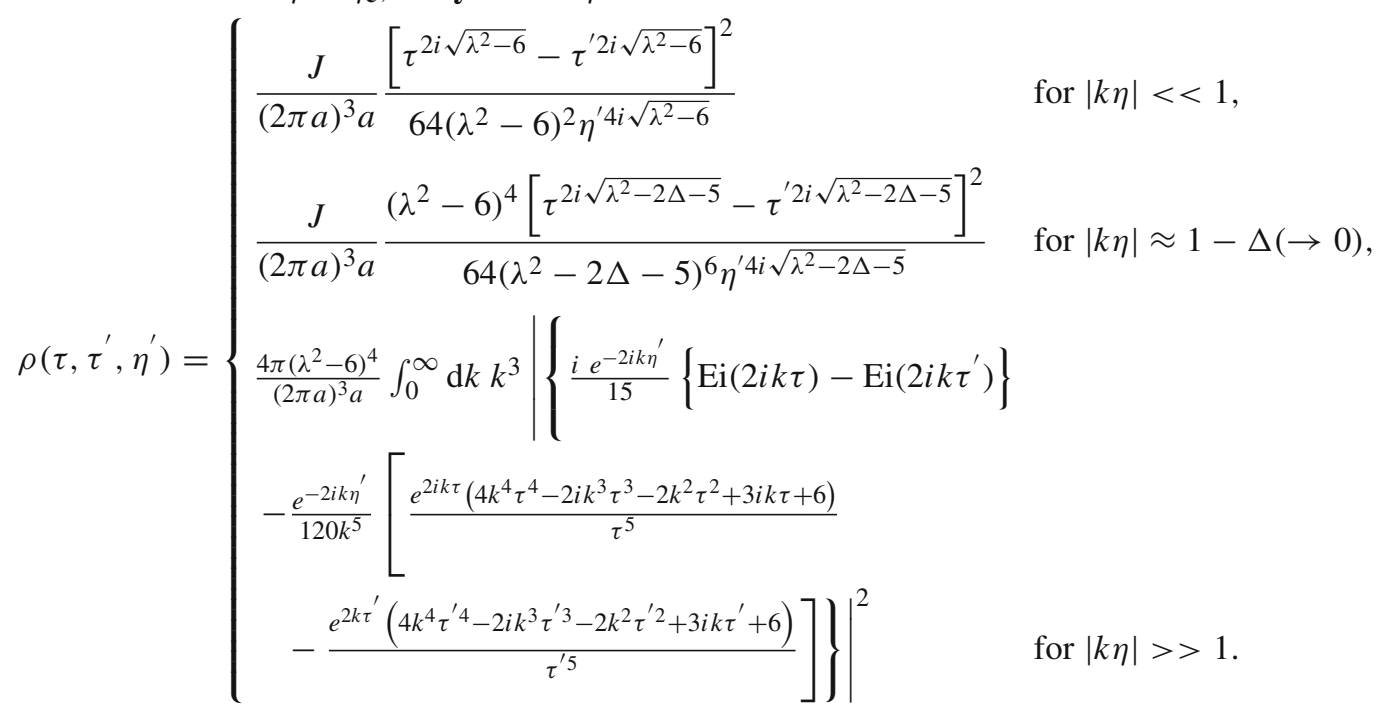

For $\eta<\eta_{\mathbf{c}}$ :

$\rho\left(\tau, \tau^{\prime}, \eta^{\prime}\right)= \begin{cases}\frac{J}{(2 \pi a)^{3} a} \frac{\left[\tau^{2 i \sqrt{\lambda^{2}-6-\Delta_{c}}}-\tau^{\left.\prime 2 i \sqrt{\lambda^{2}-6-\Delta_{c}}\right]^{2}}\right.}{64\left[\lambda^{2}-6-\Delta_{c}\right]^{2} \eta^{\prime 4 i \sqrt{\lambda^{2}-6-\Delta_{c}}}} & \text { for }|k \eta|<<1, \\ \frac{J}{(2 \pi a)^{3} a} \frac{\left[\lambda^{2}-6-\Delta_{c}\right]^{4}\left[\tau^{2 i \sqrt{\lambda^{2}-2 \Delta-5-\Delta_{c}}}-\tau^{\left.\prime 2 i \sqrt{\lambda^{2}-2 \Delta-5-\Delta_{c}}\right]^{2}}\right.}{64\left[\lambda^{2}-2 \Delta-5-\Delta_{c}\right]^{6} \eta^{\prime} 4 i \sqrt{\lambda^{2}-2 \Delta-5-\Delta_{c}}} & \text { for }|k \eta| \approx 1-\Delta(\rightarrow 0), \\ \frac{4 \pi\left(\lambda^{2}-6-\Delta_{c}\right)^{4}}{(2 \pi a)^{3} a} \int_{0}^{\infty} \mathrm{d} k k^{3} \mid\left\{\frac{i e^{-2 i k \eta^{\prime}}}{15}\left\{\operatorname{Ei}(2 i k \tau)-\operatorname{Ei}\left(2 i k \tau^{\prime}\right)\right\}\right. & \\ -\frac{e^{-2 i k \eta^{\prime}}}{120 k^{5}}\left[\frac{e^{2 i k \tau}\left(4 k^{4} \tau^{4}-2 i k^{3} \tau^{3}-2 k^{2} \tau^{2}+3 i k \tau+6\right)}{\tau^{5}}\right. & \text { for }|k \eta|>>1 . \\ \left.\left.-\frac{e^{2 k \tau^{\prime}}\left(4 k^{4} \tau^{\prime 4}-2 i k^{3} \tau^{\prime 3}-2 k^{2} \tau^{\prime 2}+3 i k \tau^{\prime}+6\right)}{\tau^{\prime 5}}\right]\right\}\left.\right|^{2} & \end{cases}$

and

For $\eta \sim \eta_{\mathbf{c}}$, early \& late $\eta$ :

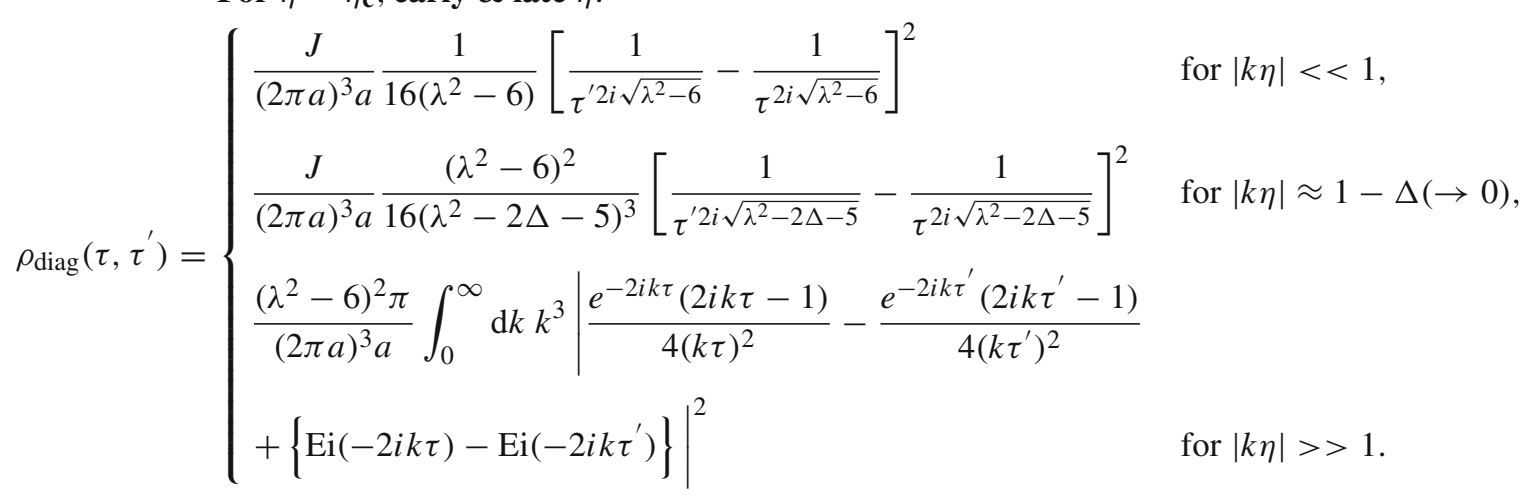




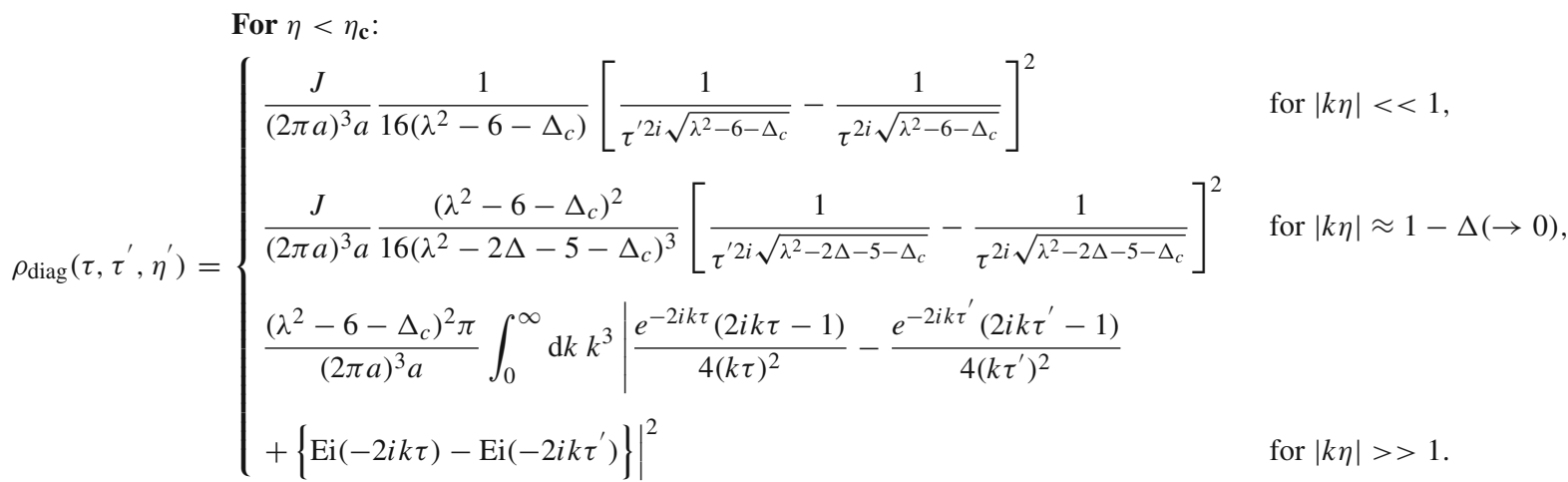

Throughout the discussion of the total energy density of the produced particles we have introduced a symbol $J$ defined as

$$
\begin{aligned}
J & =\int \mathrm{d}^{3} \mathbf{k} p(\tau) \\
& = \begin{cases}\int \mathrm{d}^{3} \mathbf{k} \sqrt{k^{2}+\frac{\lambda^{2}-6}{\tau^{2}}} & \text { for } \mathrm{dS}, \\
\int \mathrm{d}^{3} \mathbf{k} \sqrt{k^{2}+\frac{\left[\lambda^{2}-6-\Delta_{c}\right]}{\tau^{2}}} & \text { for } \mathrm{qdS},\end{cases}
\end{aligned}
$$

which physically signifies the total finite volume weighted by $p(\eta)$ in momentum space within which the produced particles are occupied. To study the behaviour of this integral we consider here three consecutive physical situations: $|k \eta|<<1,|k \eta| \approx 1-\Delta(\rightarrow 0)$ and $|k \eta|>>1$ for the de Sitter case and the quasi de Sitter case. In the three cases we have

For $\eta \sim \eta_{\mathfrak{c}}$, early \& late $\eta:$
$J= \begin{cases}\int \mathrm{d}^{3} \mathbf{k} \frac{\lambda^{2}-6}{\tau}=\frac{\left(\lambda^{2}-6\right) V}{\tau} & \text { for }|k \eta|<<1, \\ \int \mathrm{d}^{3} \mathbf{k} \frac{\sqrt{\lambda^{2}-2 \Delta-5}}{\tau}=\frac{\sqrt{\lambda^{2}-2 \Delta-5} V}{\tau} & \text { for }|k \eta| \approx 1-\Delta(\rightarrow 0), \\ \int \mathrm{d}^{3} \mathbf{k} k & \text { for }|k \eta|>>1 .\end{cases}$

$J= \begin{cases}\int \mathrm{d}^{3} \mathbf{k} \frac{\sqrt{\left[\lambda^{2}-6-\Delta_{c}\right]}}{\tau}=\frac{V \sqrt{\left[\lambda^{2}-6-\Delta_{c}\right]}}{\tau} & \text { for }|k \eta|<<1, \\ \int \mathrm{d}^{3} \mathbf{k} \frac{\sqrt{\lambda^{2}-2 \Delta-5-\Delta_{c}}}{\tau}=\frac{\sqrt{\lambda^{2}-2 \Delta-5-\Delta_{c}} V}{\tau} & \text { for }|k \eta| \approx 1-\Delta(\rightarrow 0), \\ \int \mathrm{d}^{3} \mathbf{k} k & \text { for }|k \eta|>>1 .\end{cases}$

In Fig. 15, we have explicitly shown the particle creation profile for Case I, Case II and Case III (Table 2).

\subsection{Axion-massive particle correspondence}

Now if here we identify the above axion fluctuation equations with the scalar fluctuations originating from the new particles as mentioned in the earlier section then one can write the following.

For $\eta \sim \eta_{\mathbf{c}}$, early \& late $\eta$

$$
\frac{m_{\text {axion }}^{2}}{f_{a}^{2} H^{2}}-6 \equiv \begin{cases}\frac{m^{2}}{H^{2}}-2 & \text { for } \mathrm{dS}, \\ \frac{m^{2}}{H^{2}}-\left(v^{2}-\frac{1}{4}\right) & \text { for qdS }\end{cases}
$$

$$
\begin{aligned}
p(\eta) & :=\sqrt{k^{2}+\left(\frac{m_{\text {axion }}^{2}}{f_{a}^{2} H^{2}}-6\right) \frac{1}{\eta^{2}}} \\
& \equiv \begin{cases}\sqrt{k^{2}+\left(\frac{m^{2}}{H^{2}}-2\right) \frac{1}{\eta^{2}}} & \text { for dS } \\
\sqrt{k^{2}+\left(\frac{m^{2}}{H^{2}}-\left(v^{2}-\frac{1}{4}\right)\right) \frac{1}{\eta^{2}}} & \text { for qdS }\end{cases}
\end{aligned}
$$




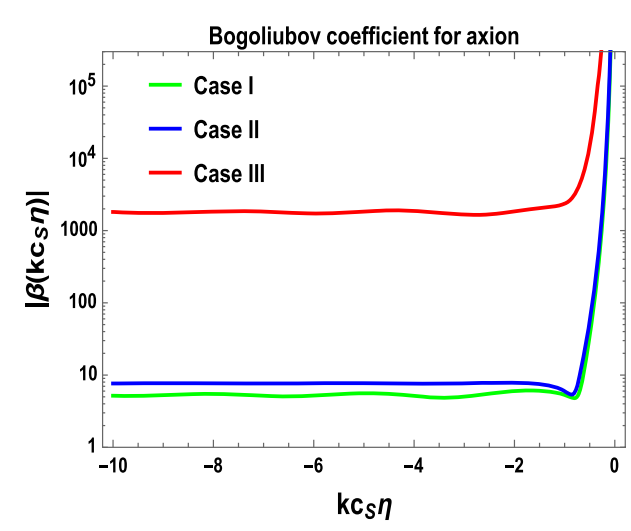

(a)

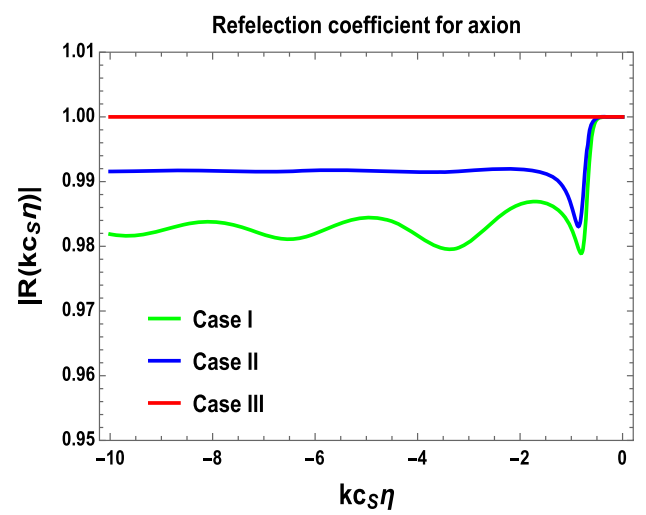

(c)

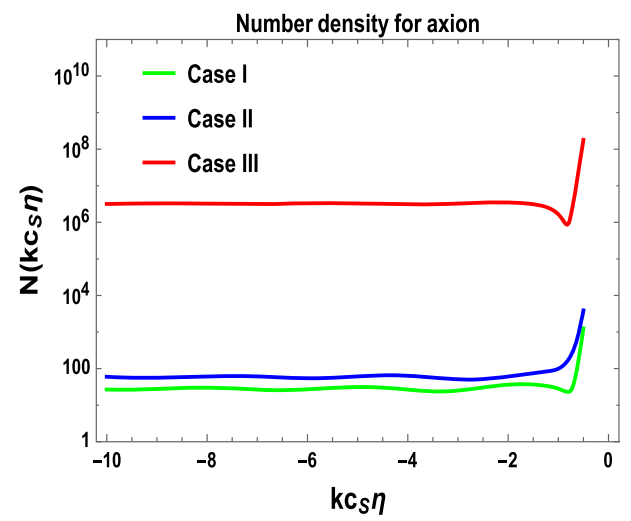

(e)

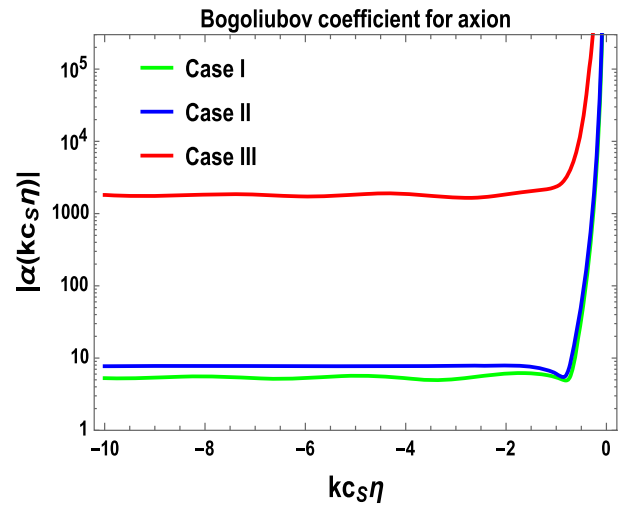

(b)

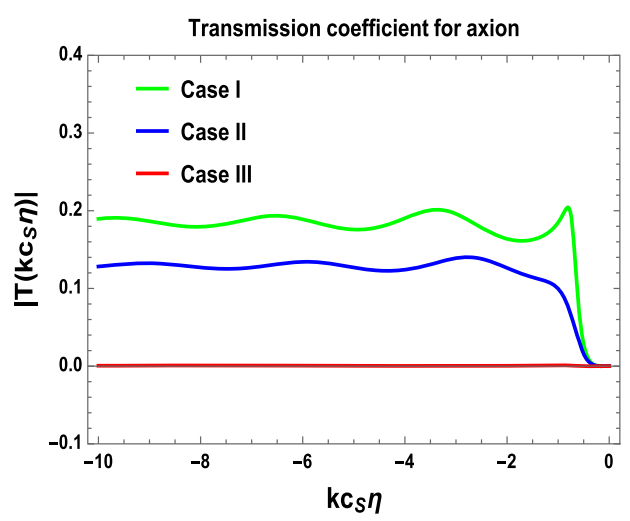

(d)

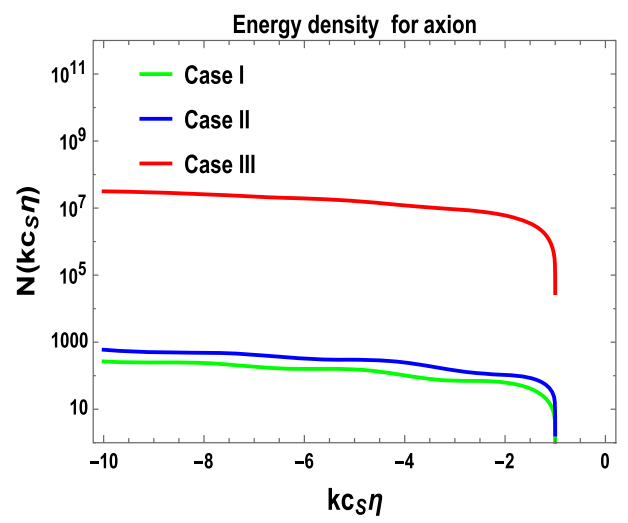

(f)

Fig. 15 Particle creation profile for Case I, Case II and Case III

$$
\begin{gathered}
\text { For } \eta<\eta_{\mathrm{c}} \\
\frac{m_{\text {axion }}^{2}-6-\Delta_{c}}{f_{a}^{2} H^{2}} \equiv \begin{cases}\frac{m^{2}}{H^{2}}-2 & \text { for dS, } \\
\frac{m^{2}}{H^{2}}-\left(v^{2}-\frac{1}{4}\right) & \text { for qdS, }\end{cases} \\
p(\eta):=\sqrt{k^{2}+\left(\frac{m_{\text {axion }}^{2}}{f_{a}^{2} H^{2}}-6-\Delta_{c}\right) \frac{1}{\eta^{2}}} \equiv \begin{cases}\sqrt{k^{2}+\left(\frac{m^{2}}{H^{2}}-2\right) \frac{1}{\eta^{2}}} & \text { for dS, } \\
\sqrt{k^{2}+\left(\frac{m^{2}}{H^{2}}-\left(v^{2}-\frac{1}{4}\right)\right) \frac{1}{\eta^{2}}} & \text { for qdS. }\end{cases}
\end{gathered}
$$




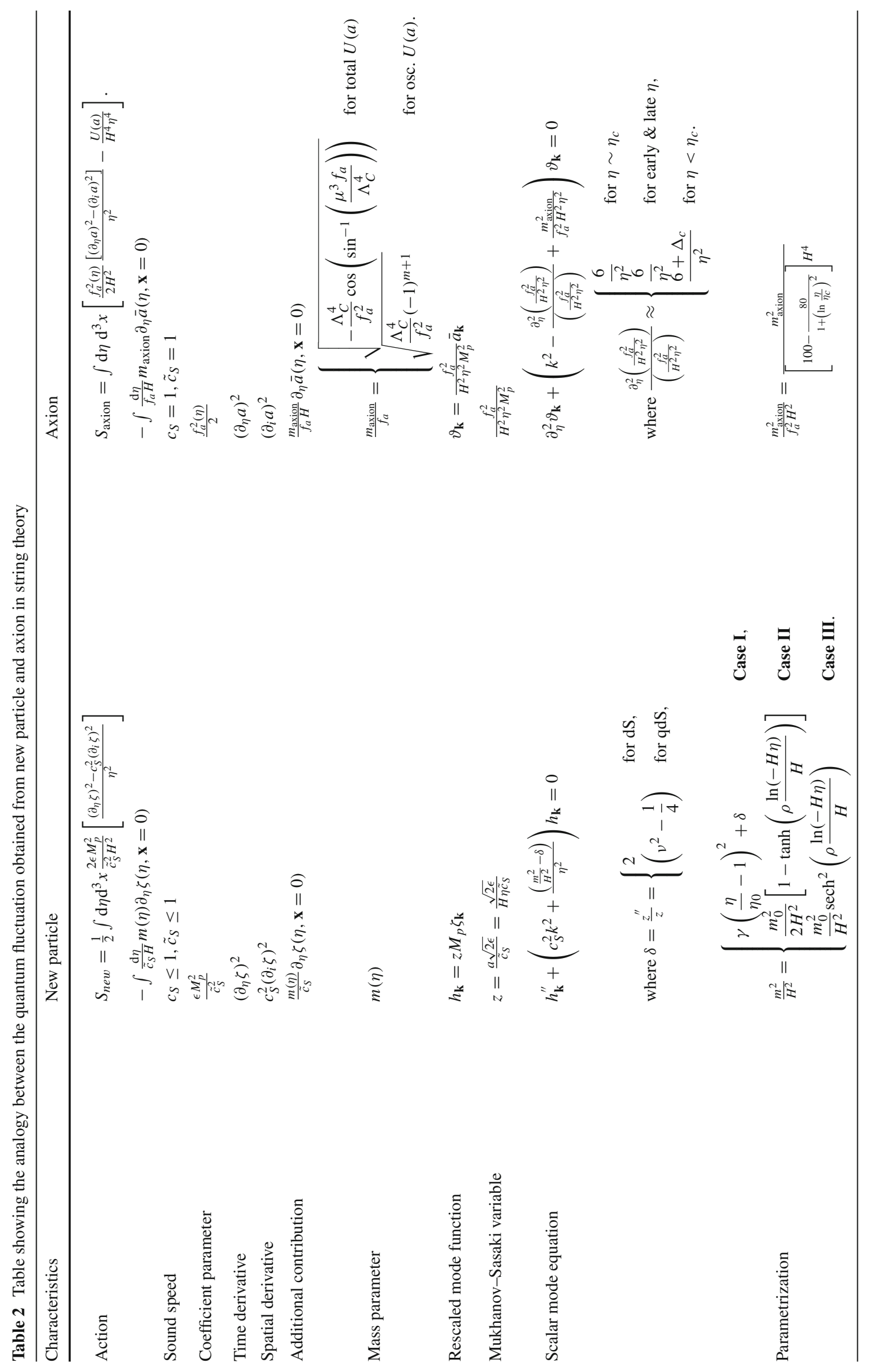


Consequently the actual solution and WKB solution for axion fluctuation can be recast for $\eta \sim \eta_{c}$, early \& late $\eta$ and $\eta<\eta_{c}$ as

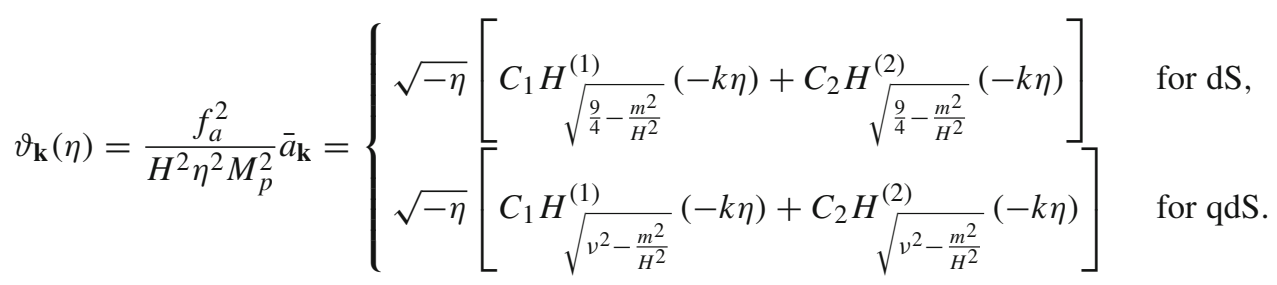

In the standard WKB approximation the total solution can be recast in the following form:

$\vartheta_{\mathbf{k}}(\eta)=\frac{f_{a}^{2}}{H^{2} \eta^{2} M_{p}^{2}} \bar{a}_{\mathbf{k}}=\left[D_{1} u_{k}(\eta)+D_{2} \bar{u}_{k}(\eta)\right]$,

where $u_{k}(\eta)$ and $\bar{u}_{k}(\eta)$ are identified as

For $\eta \sim \eta_{\mathbf{c}}$, early \& late $\eta$,

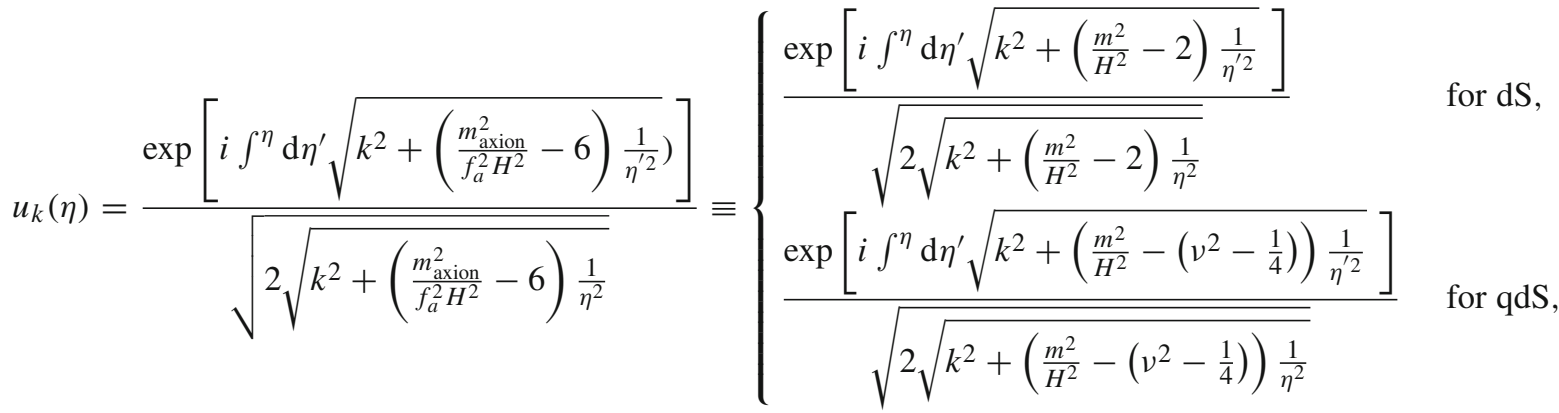

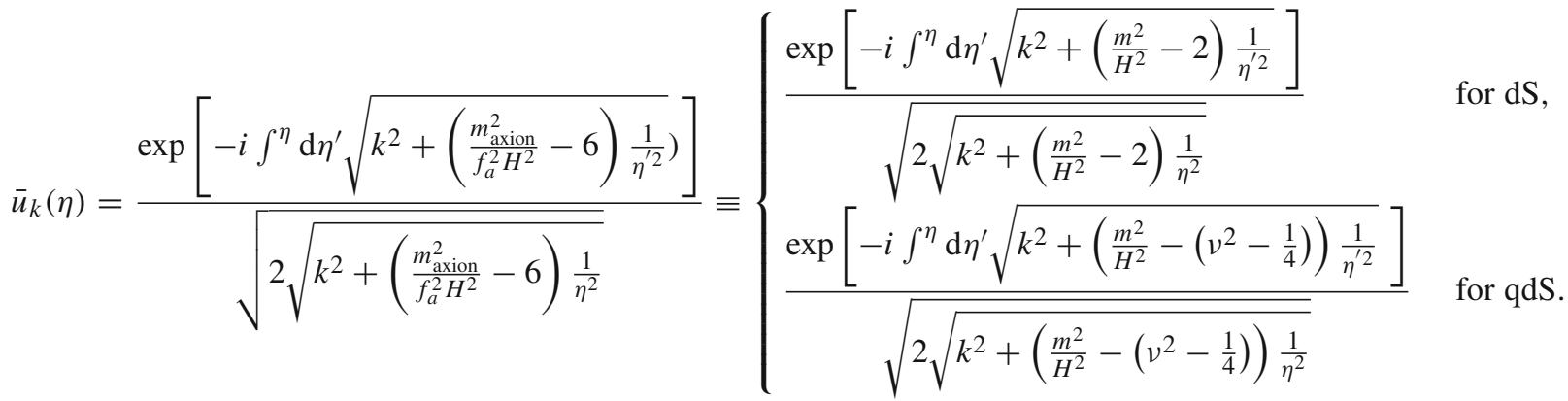

For $\eta<\eta_{\mathbf{c}}$

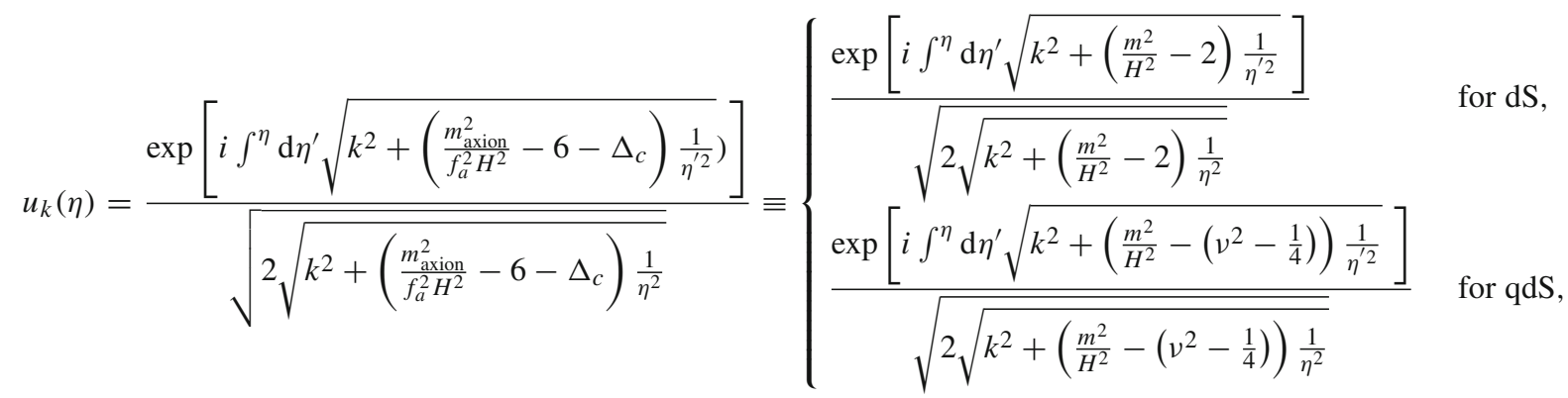




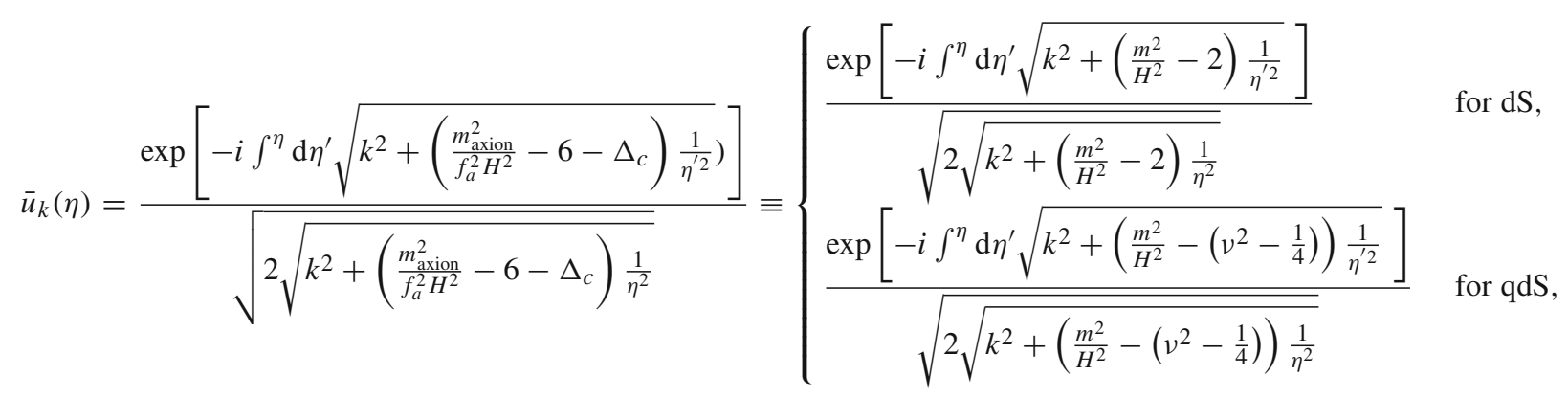

which is exactly the same as the mode function obtained for the new particles in earlier section with effective sound speed $c_{S}=1$. So one can naturally expect that even we use WKB approximation method, as we have properly identified the exact connection between new particle and axion through various parametrization of new particle mass parameter $m(\eta)$ as mentioned earlier, the final results obtained for the Bogoliubov coefficients, reflection and transmission coefficients for two different representation can also be reproduced, if we set effective sound speed $c_{S}=1$. But things will change once we consider the interaction term or more precisely the effective potential term as appearing in the context of axion. As we have already pointed out the mathematical structure of the interaction terms are different for the new particle and for the axion in the present context. But we are interested in this possibility as in both theories the mass parameters are conformal time-dependent in general. As we have already pointed out in the last section, such a contribution is solely responsible for the violation of Bell inequality in the cosmological experimental setup. In this section now we will explicitly investigate the cosmological consequences from the axion effective potential term in violating Bell inequality. Before going to the further details to check the consistency of this statement let us explicitly address four cases in the following where we give the exact estimate of the axion mass parameter for a given structure of new particle mass parameter:

1. Case $\mathbf{I}: m \approx H$

$$
\begin{gathered}
\text { For } \eta \sim \eta_{\mathbf{c}}, \text { early \& late } \eta \\
\frac{m_{\text {axion }}}{f_{a} H} \equiv \begin{cases}\sqrt{5} & \text { for } \mathbf{d S} \\
\sqrt{\frac{29}{4}-v^{2}} & \text { for } \mathbf{q d S},\end{cases} \\
p(\eta):=\sqrt{k^{2}+\left(\frac{m_{\text {axion }}^{2}}{f_{a}^{2} H^{2}}-6\right) \frac{1}{\eta^{2}}} \equiv \begin{cases}\sqrt{k^{2}-\frac{1}{\eta^{2}}} & \text { for } \mathbf{d S} \\
\sqrt{k^{2}-\left(v^{2}-\frac{5}{4}\right) \frac{1}{\eta^{2}}} & \text { for qdS. }\end{cases} \\
\text { For } \eta<\eta_{\mathbf{c}}
\end{gathered}
$$$$
\frac{m_{\text {axion }}}{f_{a} H} \equiv \begin{cases}\sqrt{5+\Delta_{c}} & \text { for } \mathbf{d S} \\ \sqrt{\frac{29}{4}+\Delta_{c}-v^{2}} & \text { for } \mathbf{q d S}\end{cases}
$$$$
p(\eta):=\sqrt{k^{2}+\left(\frac{m_{\text {axion }}^{2}}{f_{a}^{2} H^{2}}-6-\Delta_{c}\right) \frac{1}{\eta^{2}}} \equiv \begin{cases}\sqrt{k^{2}-\frac{1}{\eta^{2}}} & \text { for } \mathbf{d S}, \\ \sqrt{k^{2}-\left(v^{2}-\frac{5}{4}\right) \frac{1}{\eta^{2}}} & \text { for qdS. }\end{cases}
$$

Consequently the solution for axion fluctuation can be recast for the dS case and the qdS case as

$$
\vartheta_{\mathbf{k}}(\eta)=\frac{f_{a}^{2}}{H^{2} \eta^{2} M_{p}^{2}} \bar{a}_{\mathbf{k}}= \begin{cases}\sqrt{-\eta}\left[C_{1} H_{\sqrt{5} / 2}^{(1)}(-k \eta)+C_{2} H_{\sqrt{5} / 2}^{(2)}(-k \eta)\right] & \text { for dS } \\ \sqrt{-\eta}\left[C_{1} H_{\sqrt{v^{2}-1}}^{(1)}(-k \eta)+C_{2} H_{\sqrt{v^{2}-1}}^{(2)}(-k \eta)\right] & \text { for qdS. }\end{cases}
$$


In the standard WKB approximation the total solution can be recast in the following form:

$\vartheta_{\mathbf{k}}(\eta)=\frac{f_{a}^{2}}{H^{2} \eta^{2} M_{p}^{2}} \bar{a}_{\mathbf{k}}=\left[D_{1} u_{k}(\eta)+D_{2} \bar{u}_{k}(\eta)\right]$,

where $D_{1}$ and $D_{2}$ are two arbitrary integration constants, which depend on the choice of the initial condition on making the WKB approximation at early and late time scale. In the present context $u_{k}(\eta)$ and $\bar{u}_{k}(\eta)$ are defined as

$$
\begin{aligned}
& \text { For } \eta \lesssim \eta_{\mathbf{c}} \text {, early \& late } \eta \\
& u_{k}(\eta)= \begin{cases}\frac{\exp \left[i \int^{\eta} \mathrm{d} \eta^{\prime} \sqrt{k^{2}-\frac{1}{\eta^{\prime 2}}}\right]}{\sqrt{2 \sqrt{k^{2}-\frac{1}{\eta^{2}}}}} & \text { for } \mathrm{dS}, \\
\frac{\exp \left[i \int^{\eta} \mathrm{d} \eta^{\prime} \sqrt{k^{2}-\left(v^{2}-\frac{5}{4}\right) \frac{1}{\eta^{\prime 2}}}\right]}{\sqrt{2 \sqrt{k^{2}-\left(v^{2}-\frac{5}{4}\right) \frac{1}{\eta^{2}}}}} & \text { for qdS, }\end{cases} \\
& \bar{u}_{k}(\eta)= \begin{cases}\frac{\exp \left[-i \int^{\eta} \mathrm{d} \eta^{\prime} \sqrt{k^{2}-\frac{1}{\eta^{\prime 2}}}\right]}{\sqrt{2 \sqrt{k^{2}-\frac{1}{\eta^{2}}}}} & \text { for dS, } \\
\frac{\exp \left[-i \int^{\eta} \mathrm{d} \eta^{\prime} \sqrt{k^{2}-\left(v^{2}-\frac{5}{4}\right) \frac{1}{\eta^{\prime 2}}}\right]}{\sqrt{2 \sqrt{k^{2}-\left(v^{2}-\frac{5}{4}\right) \frac{1}{\eta^{2}}}}} & \text { for qdS. }\end{cases}
\end{aligned}
$$

2. Case II: $m \approx \sqrt{\gamma\left(\frac{\eta}{\eta_{0}}-1\right)^{2}+\delta} H$

For $\eta \sim \eta_{\mathbf{c}}$, early \& late $\eta$

$$
\begin{aligned}
& \frac{m_{\text {axion }}}{f_{a} H} \equiv \begin{cases}\sqrt{4+\gamma\left(\frac{\eta}{\eta_{0}}-1\right)^{2}+\delta} & \text { for dS } \\
\sqrt{\frac{25}{4}-v^{2}+\gamma\left(\frac{\eta}{\eta_{0}}-1\right)^{2}+\delta} & \text { for qdS }\end{cases}
\end{aligned}
$$

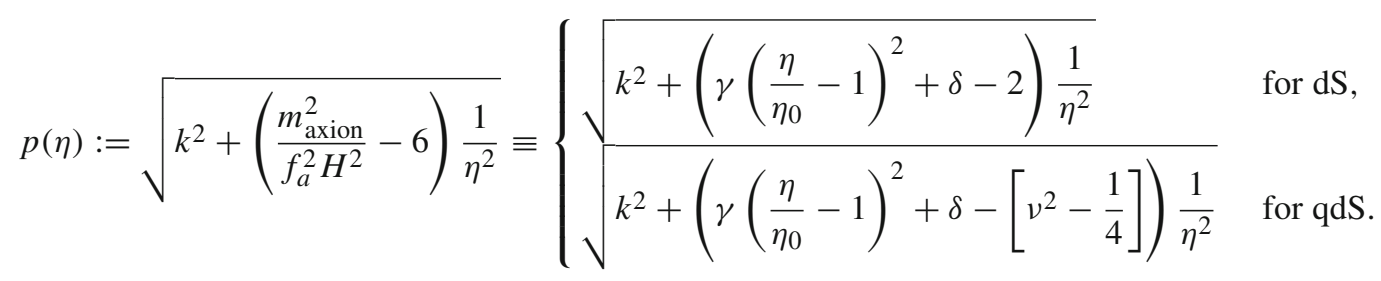




$$
\begin{gathered}
\text { For } \eta<\eta_{\mathbf{c}} \\
\frac{m_{\text {axion }}}{f_{a} H} \equiv \begin{cases}\sqrt{4+\gamma\left(\frac{\eta}{\eta_{0}}-1\right)^{2}+\delta+\Delta_{c}} & \text { for dS, } \\
\sqrt{\frac{25}{4}-v^{2}+\gamma\left(\frac{\eta}{\eta_{0}}-1\right)^{2}+\delta+\Delta_{c}} & \text { for qdS },\end{cases} \\
p(\eta):=\sqrt{k^{2}+\left(\frac{m_{\text {axion }}^{2}}{f_{a}^{2} H^{2}}-6-\Delta_{c}\right) \frac{1}{\eta^{2}}} \equiv \begin{cases}\sqrt{k^{2}+\left(\gamma\left(\frac{\eta}{\eta_{0}}-1\right)^{2}+\delta-2\right) \frac{1}{\eta^{2}}} & \text { for dS, } \\
\sqrt{k^{2}+\left(\gamma\left(\frac{\eta}{\eta_{0}}-1\right)^{2}+\delta-\left[v^{2}-\frac{1}{4}\right]\right) \frac{1}{\eta^{2}}} & \text { for qdS. }\end{cases}
\end{gathered}
$$

Consequently the solution for axion fluctuation can be recast for $\mathrm{dS}$ and the qdS case as

$$
\vartheta_{\mathbf{k}}(\eta)=\frac{f_{a}^{2}}{H^{2} \eta^{2} M_{p}^{2}} \bar{a}_{\mathbf{k}}=(-\eta)^{\frac{3}{2}} e^{-\frac{P \eta}{2}}(P \eta)^{A+\frac{d}{P}}\left[G_{11} F_{1}(A ; B ; P \eta)+G_{2} U(A ; B ; P \eta)\right]
$$

where $A, B$ and $P$ are defined as

$$
\begin{aligned}
& A= \begin{cases}-\frac{d}{2 i \sqrt{k^{2}+C}}+i \sqrt{(\gamma+\delta)-\frac{9}{4}}+\frac{1}{2} & \text { for dS }, \\
-\frac{d}{2 i \sqrt{k^{2}+C}}+i \sqrt{(\gamma+\delta)-v^{2}}+\frac{1}{2} & \text { for qdS }\end{cases} \\
& B= \begin{cases}2 i \sqrt{(\gamma+\delta)-\frac{9}{4}}+1 & \text { for dS } \\
2 i \sqrt{(\gamma+\delta)-v^{2}}+1 & \text { for qdS }\end{cases} \\
& P=2 i \sqrt{k^{2}+C}
\end{aligned}
$$

In the standard WKB approximation the total solution can be recast in the following form:

$$
\vartheta_{\mathbf{k}}(\eta)=\frac{f_{a}^{2}}{H^{2} \eta^{2} M_{p}^{2}} \bar{a}_{\mathbf{k}}=\left[D_{1} u_{k}(\eta)+D_{2} \bar{u}_{k}(\eta)\right],
$$

where $D_{1}$ and $D_{2}$ are two arbitrary integration constants, which depend on the choice of the initial condition on making the WKB approximation at early and late time scale. In the present context $u_{k}(\eta)$ and $\bar{u}_{k}(\eta)$ are defined as

For $\eta \lesssim \eta_{\mathbf{c}}$, early \& late $\eta$,

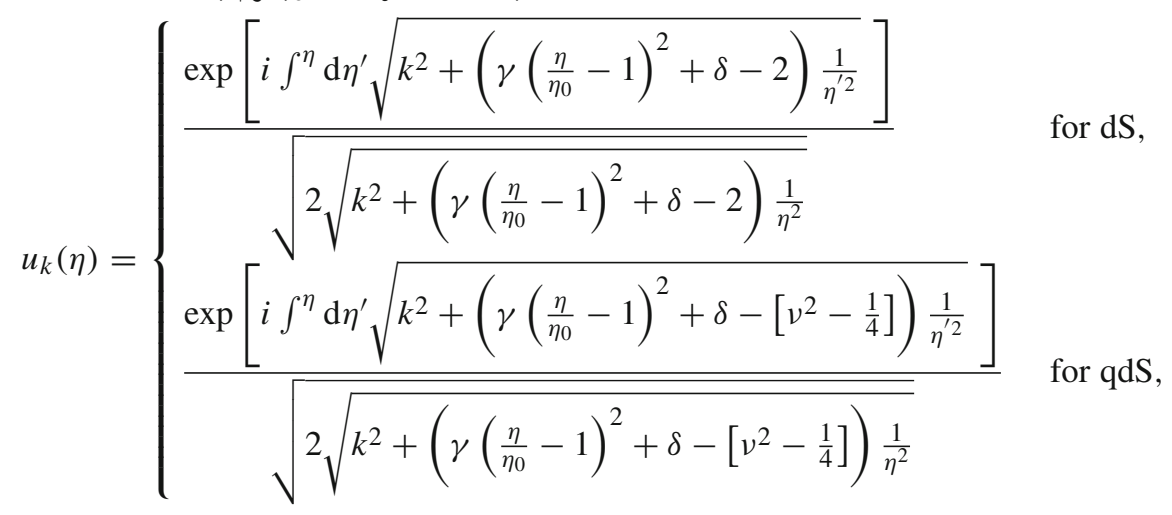




$$
\bar{u}_{k}(\eta)= \begin{cases}\frac{\exp \left[-i \int^{\eta} \mathrm{d} \eta^{\prime} \sqrt{k^{2}+\left(\gamma\left(\frac{\eta}{\eta_{0}}-1\right)^{2}+\delta-2\right) \frac{1}{\eta^{\prime 2}}}\right]}{\sqrt{2 \sqrt{k^{2}+\left(\gamma\left(\frac{\eta}{\eta_{0}}-1\right)^{2}+\delta-2\right) \frac{1}{\eta^{2}}}}} & \text { for } \mathrm{dS}, \\ \frac{\exp \left[-i \int^{\eta} \mathrm{d} \eta^{\prime} \sqrt{k^{2}+\left(\gamma\left(\frac{\eta}{\eta_{0}}-1\right)^{2}+\delta-\left[v^{2}-\frac{1}{4}\right]\right)} \frac{1}{\eta^{\prime 2}}\right.}{\sqrt{2 \sqrt{k^{2}+\left(\gamma\left(\frac{\eta}{\eta_{0}}-1\right)^{2}+\delta-\left[v^{2}-\frac{1}{4}\right]\right) \frac{1}{\eta^{2}}}}} & \text { for qdS. }\end{cases}
$$

3. Case III: $m>>H$

For $\eta \sim \eta_{c}$, early \& late $\eta$

$$
\begin{aligned}
& \frac{m_{\text {axion }}}{f_{a} H} \equiv \begin{cases}\sqrt{4+\Upsilon^{2}} & \text { for } \mathrm{dS}, \\
\sqrt{\frac{25}{4}-v^{2}+\Upsilon^{2}} & \text { for } \mathrm{qdS},\end{cases}
\end{aligned}
$$

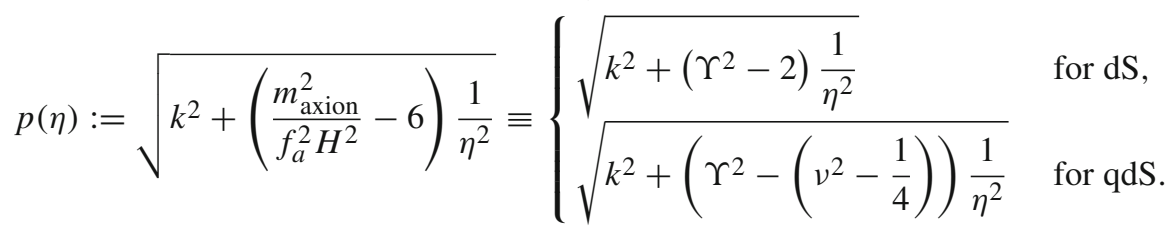

$$
\begin{aligned}
& \text { For } \eta<\eta_{\mathbf{c}} \\
& \frac{m_{\text {axion }}}{f_{a} H} \equiv \begin{cases}\sqrt{4+\Upsilon^{2}+\Delta_{c}} & \text { for } \mathrm{d} S \\
\sqrt{\frac{25}{4}-v^{2}+\Upsilon^{2}+\Delta_{c}} & \text { for } \mathrm{qdS},\end{cases} \\
& p(\eta):=\sqrt{k^{2}+\left(\frac{m_{\text {axion }}^{2}}{f_{a}^{2} H^{2}}-6-\Delta_{c}\right) \frac{1}{\eta^{2}}} \equiv \begin{cases}\sqrt{k^{2}+\left(\Upsilon^{2}-2\right) \frac{1}{\eta^{2}}} & \text { for } \mathrm{dS}, \\
\sqrt{k^{2}+\left(\Upsilon^{2}-\left(v^{2}-\frac{1}{4}\right)\right) \frac{1}{\eta^{2}}} & \text { for qdS. }\end{cases}
\end{aligned}
$$

Consequently the solution for axion fluctuation can be recast for the dS case and the qdS case as

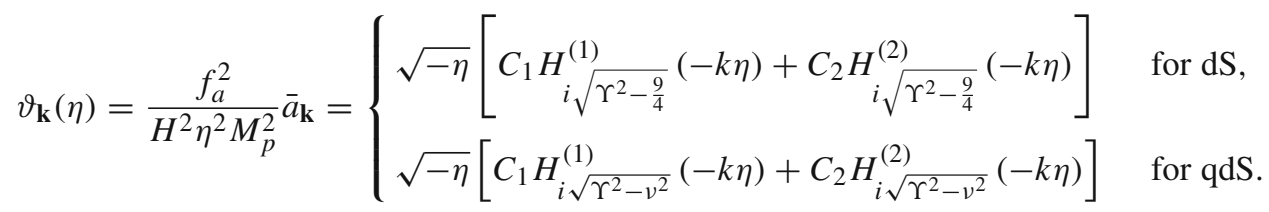

In the standard WKB approximation the total solution can be recast in the following form:

$\vartheta_{\mathbf{k}}(\eta)=\frac{f_{a}^{2}}{H^{2} \eta^{2} M_{p}^{2}} \bar{a}_{\mathbf{k}}=\left[D_{1} u_{k}(\eta)+D_{2} \bar{u}_{k}(\eta)\right]$

where $D_{1}$ and $D_{2}$ are two arbitrary integration constants, which depend on the choice of the initial condition on making the WKB approximation at early and late time scale. In the present context $u_{k}(\eta)$ and $\bar{u}_{k}(\eta)$ are defined as 
For $\eta \lesssim \eta_{\mathfrak{c}}$, early \& late $\eta$,

$$
\begin{aligned}
& u_{k}(\eta)= \begin{cases}\frac{\exp \left[i \int^{\eta} \mathrm{d} \eta^{\prime} \sqrt{k^{2}+\frac{\Upsilon^{2}-2}{\eta^{\prime 2}}}\right]}{\sqrt{2 \sqrt{k^{2}+\frac{\Upsilon^{2}-2}{\eta^{2}}}}} & \text { for dS, } \\
\frac{\exp \left[i \int^{\eta} \mathrm{d} \eta^{\prime} \sqrt{k^{2}+\left[\Upsilon^{2}-\left(v^{2}-\frac{1}{4}\right)\right] \frac{1}{\eta^{\prime 2}}}\right]}{\sqrt{2 \sqrt{k^{2}+\left[\Upsilon^{2}-\left(v^{2}-\frac{1}{4}\right)\right] \frac{1}{\eta^{2}}}}} & \text { for qdS, }\end{cases} \\
& \bar{u}_{k}(\eta)= \begin{cases}\frac{\exp \left[-i \int^{\eta} \mathrm{d} \eta^{\prime} \sqrt{k^{2}+\frac{\Upsilon^{2}-2}{\eta^{\prime 2}}}\right]}{\sqrt{2 \sqrt{k^{2}+\frac{\Upsilon^{2}-2}{\eta^{2}}}}} & \text { for } \mathrm{dS}, \\
\frac{\exp \left[-i \int^{\eta} \mathrm{d} \eta^{\prime} \sqrt{k^{2}-\left(v^{2}-\frac{5}{4}\right)} \frac{1}{\eta^{\prime 2}}\right]}{\sqrt{2 \sqrt{k^{2}-\left(v^{2}-\frac{5}{4}\right) \frac{1}{\eta^{2}}}}} & \text { for qdS. }\end{cases}
\end{aligned}
$$

4. Case IV: $m<<H$

$$
\begin{aligned}
& \text { For } \eta \sim \eta_{\mathbf{c}} \text {, early \& late } \eta \\
& \frac{m_{\text {axion }}}{f_{a} H} \equiv \begin{cases}2 & \text { for } \mathrm{dS}, \\
\sqrt{\frac{25}{4}-v^{2}} & \text { for qdS },\end{cases} \\
& p(\eta):=\sqrt{k^{2}+\left(\frac{m_{\text {axion }}^{2}}{f_{a}^{2} H^{2}}-6\right) \frac{1}{\eta^{2}}} \equiv \begin{cases}\sqrt{k^{2}-\frac{2}{\eta^{2}}} & \text { for dS, } \\
\sqrt{k^{2}-\left(v^{2}-\frac{1}{4}\right) \frac{1}{\eta^{2}}} & \text { for qdS. }\end{cases} \\
& \text { For } \eta<\eta_{\mathbf{c}} \\
& \frac{m_{\mathrm{axion}}}{f_{a} H} \equiv \begin{cases}\sqrt{4+\Delta_{c}} & \text { for } \mathrm{dS} \\
\sqrt{\frac{25}{4}-v^{2}+\Delta_{c}} & \text { for qdS }\end{cases} \\
& p(\eta):=\sqrt{k^{2}+\left(\frac{m_{\text {axion }}^{2}}{f_{a}^{2} H^{2}}-6-\Delta_{c}\right) \frac{1}{\eta^{2}}} \equiv \begin{cases}\sqrt{k^{2}-\frac{2}{\eta^{2}}} & \text { for dS, } \\
\sqrt{k^{2}-\left(v^{2}-\frac{1}{4}\right) \frac{1}{\eta^{2}}} & \text { for qdS. }\end{cases}
\end{aligned}
$$

Consequently the solution for axion fluctuation can be recast for $\mathrm{dS}$ and the qdS case as

$$
\vartheta_{\mathbf{k}}(\eta)=\frac{f_{a}^{2}}{H^{2} \eta^{2} M_{p}^{2}} \bar{a}_{\mathbf{k}}= \begin{cases}\sqrt{-\eta}\left[C_{1} H_{3 / 2}^{(1)}(-k \eta)+C_{2} H_{3 / 2}^{(2)}(-k \eta)\right] & \text { for dS, } \\ \sqrt{-\eta}\left[C_{1} H_{v}^{(1)}(-k \eta)+C_{2} H_{v}^{(2)}(-k \eta)\right] & \text { for qdS. }\end{cases}
$$

In the standard WKB approximation the total solution can be recast in the following form: 


$$
\vartheta_{\mathbf{k}}(\eta)=\frac{f_{a}^{2}}{H^{2} \eta^{2} M_{p}^{2}} \bar{a}_{\mathbf{k}}=\left[D_{1} u_{k}(\eta)+D_{2} \bar{u}_{k}(\eta)\right],
$$

where $D_{1}$ and $D_{2}$ are two arbitrary integration constants, which depend on the choice of the initial condition on making the WKB approximation at early and late time scale. In the present context $u_{k}(\eta)$ and $\bar{u}_{k}(\eta)$ are defined as

$$
\begin{aligned}
& \text { For } \eta \lesssim \eta_{\mathbf{c}} \text {, early \& late } \eta \text {, } \\
& u_{k}(\eta)= \begin{cases}\frac{\exp \left[i \int^{\eta} \mathrm{d} \eta^{\prime} \sqrt{k^{2}-\frac{2}{\eta^{\prime 2}}}\right]}{\sqrt{2 \sqrt{k^{2}-\frac{2}{\eta^{2}}}}} & \text { for } \mathrm{dS} \\
\frac{\exp \left[i \int^{\eta} \mathrm{d} \eta^{\prime} \sqrt{k^{2}-\left(v^{2}-\frac{1}{4}\right) \frac{1}{\eta^{\prime 2}}}\right]}{\sqrt{2 \sqrt{k^{2}-\left(v^{2}-\frac{1}{4}\right) \frac{1}{\eta^{2}}}}} & \text { for qdS, }\end{cases} \\
& \bar{u}_{k}(\eta)= \begin{cases}\frac{\exp \left[-i \int^{\eta} \mathrm{d} \eta^{\prime} \sqrt{k^{2}-\frac{2}{\eta^{\prime 2}}}\right]}{\sqrt{2 \sqrt{k^{2}-\frac{2}{\eta^{2}}}}} & \text { for } \mathrm{dS}, \\
\frac{\exp \left[-i \int^{\eta} \mathrm{d} \eta^{\prime} \sqrt{k^{2}-\left(v^{2}-\frac{1}{4}\right) \frac{1}{\eta^{\prime 2}}}\right]}{\sqrt{2 \sqrt{k^{2}-\left(v^{2}-\frac{1}{4}\right) \frac{1}{\eta^{2}}}}} & \text { for qdS. }\end{cases}
\end{aligned}
$$

As we have seen that the structure of the WKB solution for the axion fluctuation is exactly the same as the scalar mode fluctuation for different mass parametrization for the new particle as appearing in the previous section, the final results obtained for Bogoliubov coefficients, reflection and transmission coefficients for two different representation can also be the same, if we set the effective sound speed $c_{S}=1$ in the previously obtained results.

\subsection{Cosmological implication from axion fluctuation}

Here things will change once we consider the interaction term or more precisely the effective potential term as appearing in the context of axion. This is because of the fact that the mathematical structure of the interaction terms are different for the new particle and for the axion in the present context. Apart from this fact we are here interested in this possibility as in both theories the mass parameters are conformally time-dependent in general and this is the key point of this work. As we have already pointed out in the last section that such a type of contribution in the effective action is solely responsible for the violation of Bell inequality in the cosmological experimental setup. In this section we will explicitly investigate this possibility for axion fluctuation.

Here our prime objective is to explicitly compute the expression for VEV or one point function of the axion fluctuation in momentum space in the presence of the axion mass parameter present in the axion interaction term with respect to the arbitrary choice of vacuum, which leads to important contribution to the Bell inequalities or violation in the context of primordial cosmology. Before computing this effect one has to remember the fact that initially we have assumed that effect of back-reaction are very small and so that can easily be neglected from our analysis. Because of this we will only concentrate on the axion mass contribution in the effective interaction or more precisely in the axion potential. Using the interaction picture in a curved background in the present context the one point function of the axion fluctuation in momentum space can be expressed as

$$
\left\langle\bar{a}_{k}(\eta=0)\right\rangle=-i \int_{-\infty}^{0} \mathrm{~d} \eta a(\eta)\left\langle 0\left|\left[\bar{a}_{\mathbf{k}}(0), H_{i n t}^{\text {axion }}(\eta)\right]\right| 0\right\rangle,
$$

where $a(\eta)$ is the scale factor defined in the earlier section in terms of the Hubble parameter $H$ and the conformal time scale $\eta$. In the interaction picture the Hamiltonian can written as

$H_{\mathrm{int}}^{\text {axion }}=-\frac{m_{\text {axion }}}{f_{a} H} \partial_{\eta} \bar{a}(\eta, \mathbf{x}=0)$.

After applying the Fourier transform in Eq. (4.228) we get the following expression:

$$
\begin{aligned}
& H_{\mathrm{int}}^{\text {axion }}=-\int \frac{\mathrm{d}^{3} k}{(2 \pi)^{3}} \frac{M_{p}^{2} H^{2} \eta^{2}}{f_{a}^{2}} \frac{m_{\text {axion }}}{f_{a} H} \\
& \quad \times\left[\vartheta^{\prime}(\eta, \mathbf{k}) a(\mathbf{k})+\vartheta^{\dagger^{\prime}}(\eta,-\mathbf{k}) a^{\dagger}(-\mathbf{k})\right]
\end{aligned}
$$

and further substituting Eq. (4.229) in Eq. (4.227) finally we get

$$
\begin{aligned}
& \left\langle\bar{a}_{\mathbf{k}}(\eta=0)\right\rangle=-i \int_{-\infty}^{0} \mathrm{~d} \eta \frac{M_{p}^{4} H^{4} \eta^{4} a(\eta)}{f_{a}^{4}} \frac{m_{\text {axion }}}{f_{a}} \\
& \times\left(\vartheta_{\mathbf{k}}(0) \vartheta_{\mathbf{k}}^{\dagger^{\prime}}(\eta)-\vartheta^{\dagger}-\mathbf{k}(0) \vartheta_{-\mathbf{k}}^{\prime}(\eta)\right)
\end{aligned}
$$

where $h_{\mathbf{k}}(\eta)$ is the exact solution or the WKB solution of the mode function as explicitly computed in the earlier section of this paper. Now sometimes it happens that the exact solution of mode function is not exactly defined at $\eta=0$ point. To avoid such complexity in the present computation for the sake of clarity here we introduce a Infra-Red (IR) cut-off regulator $\xi$ in the conformal time integral and consequently Eq. (3.220) can be recast as

$$
\begin{aligned}
& \left\langle\bar{a}_{\mathbf{k}}(\eta=\xi \rightarrow 0)\right\rangle=-i \lim _{k c_{S} \xi \rightarrow 0} \int_{-\infty}^{\xi} \mathrm{d} \eta \frac{M_{p}^{4} H^{4} \eta^{4} a(\eta)}{f_{a}^{4}} \frac{m_{\text {axion }}}{f_{a}} \\
& \times\left(\vartheta_{\mathbf{k}}(0) \vartheta_{\mathbf{k}}^{\dagger^{\prime}}(\eta)-\vartheta^{\dagger}-\mathbf{k}(0) \vartheta_{-\mathbf{k}}^{\prime}(\eta)\right) .
\end{aligned}
$$


Further substituting the explicit form of the scalar mode functions computed from the exact solution or from the WKB approximated solution we get the following simplified expression for the VEV of the curvature fluctuation in momentum space:

$$
\left\langle\bar{a}_{\mathbf{k}}(\eta=\xi \rightarrow 0)\right\rangle=-i \lim _{k \xi \rightarrow 0} \int_{-\infty}^{\xi} \mathrm{d} \eta \frac{M_{p}^{4} H^{4} \eta^{4} a(\eta)}{f_{a}^{4}} \frac{m_{\mathrm{axion}}}{f_{a}} \sum_{i=1}^{2} \sum_{j=1}^{2} C_{i}^{*} C_{j}\left[\mathcal{A}_{i j}\right]_{C_{S}=1}
$$

where the conformal time-dependent functions $\mathcal{A}_{i j} \forall i, j=1,2$ in momentum space are defined in the earlier section.

For further simplification we consider here the two limiting cases $|k \eta| \rightarrow-\infty,|k \eta| \rightarrow 0$ and $|k \eta| \approx 1$, which are physically acceptable in the present context. First of we consider the results for $|k \eta| \rightarrow-\infty$. In this case we get

$$
\begin{aligned}
& \left\langle\bar{a}_{\mathbf{k}}(\eta=0)\right\rangle_{|k \eta| \rightarrow-\infty}=\frac{4}{M_{p}^{2} \pi} \int_{-\infty}^{0} \mathrm{~d} \eta \frac{H}{a(\eta) k} \frac{M_{p}^{4} H^{2} \eta^{2}}{f_{a}^{4}} \frac{m_{\mathrm{axion}}}{f_{a} H}\left[\left|C_{2}\right|^{2} e^{-i k \eta}-\left|C_{1}\right|^{2} e^{i k \eta}\right. \\
& \left.-i\left(C_{1}^{*} C_{2} e^{i \pi\left(\Lambda+\frac{1}{2}\right)}+C_{1} C_{2}^{*} e^{-i \pi\left(\Lambda+\frac{1}{2}\right)}\right) \sin k \eta\right] \\
& \begin{cases}-\frac{2}{M_{p}^{2}} \int_{-\infty}^{0} \mathrm{~d} \eta \frac{H}{a(\eta) k} \frac{M_{p}^{4} H^{2} \eta^{2}}{f_{a}^{4}} \frac{m_{\mathrm{axion}}}{f_{a} H} e^{i k \eta} & \text { for BD vacua } \\
\frac{4}{M_{p}^{2} \pi} \int_{-\infty}^{0} \mathrm{~d} \eta \frac{H}{a(\eta) k} \frac{M_{p}^{4} H^{2} \eta^{2}}{f_{a}^{4}} \frac{m_{\text {axion }}}{f_{a} H}\left[\sinh ^{2} \alpha e^{i k \eta}-\cosh ^{2} \alpha e^{-i k \eta}\right. & \end{cases} \\
& =\left\{\begin{array}{cc}
\left.+i \sinh 2 \alpha \cos \left(\pi\left(\Lambda+\frac{1}{2}\right)+\delta\right) \sin k \eta\right] & \text { for } \alpha \text { vacua Type-I, } \\
\frac{4\left|N_{\alpha}\right|^{2}}{M_{p}^{2} \pi} \int_{-\infty}^{0} \mathrm{~d} \eta \frac{H}{a(\eta) k} \frac{M_{p}^{4} H^{2} \eta^{2}}{f_{a}^{4}} \frac{m_{\mathrm{axion}}}{f_{a} H}\left[e^{\alpha+\alpha^{*}} e^{i k \eta}-e^{-i k \eta}\right. &
\end{array}\right. \\
& \left.+i\left(e^{\alpha} e^{i \pi\left(\Lambda+\frac{1}{2}\right)}+e^{\alpha^{*}} e^{-i \pi\left(\Lambda+\frac{1}{2}\right)}\right) \sin k \eta\right] \\
& \frac{16 i|C|^{2}}{M_{p}^{2} \pi} \int_{-\infty}^{0} \mathrm{~d} \eta \frac{H}{a(\eta) k} \frac{M_{p}^{4} H^{2} \eta^{2}}{f_{a}^{4}} \frac{m_{\text {axion }}}{f_{a} H} \sin k \eta \cos ^{2} \frac{\pi}{2}\left(\Lambda+\frac{1}{2}\right) \quad \text { for special vacua. }
\end{aligned}
$$

On the other hand for $|k \eta| \rightarrow 0$ we get the following simplified expression:

$$
\begin{aligned}
\left\langle\bar{a}_{\mathbf{k}}(\eta=\xi \rightarrow 0)\right\rangle_{|k \eta| \rightarrow 0}= & \frac{4 \pi \sqrt{\xi}}{M_{p}^{2}}\left(C_{1}^{*} C_{2}+C_{1} C_{2}^{*}-\left|C_{1}\right|^{2}-\left|C_{2}\right|^{2}\right) \int_{-\infty}^{\xi} \mathrm{d} \eta \frac{H}{a(\eta)(2 \pi)^{3} \sqrt{-\eta}} \frac{M_{p}^{4} H^{2} \eta^{2}}{f_{a}^{4}} \frac{m_{\mathrm{axion}}}{f_{a} H} \\
& \times\left(\Lambda-\frac{1}{2}\right)\left[\left(-\frac{k \eta}{2}\right)^{-\Lambda}\left(-\frac{k \xi}{2}\right)^{-\Lambda}+\left(\frac{k \eta}{2}\right)^{-\Lambda}\left(\frac{k c_{S} \xi}{2}\right)^{-\Lambda}\right] \\
= & I_{\xi} \times \begin{cases}\frac{-2 \pi^{2} \sqrt{\xi}}{M_{p}^{2}} & \text { for Bunch Davies vacua, } \\
\frac{4 \pi \sqrt{\xi}}{M_{p}^{2}}\left(\cos \delta \sinh 2 \alpha-\cosh ^{2} \alpha-\sinh ^{2} \alpha\right) & \text { for } \alpha \text { vacua Type-I, } \\
\frac{4 \pi \sqrt{\xi}\left|N_{\alpha}\right|^{2}}{M_{p}^{2}}\left(e^{\alpha}+e^{\alpha^{*}}-e^{\alpha+\alpha^{*}}-1\right) & \text { for } \alpha \text { vacua Type-II, } \\
0 & \text { for special vacua, }\end{cases}
\end{aligned}
$$


where the integral $I_{\xi}$ is defined as

$I_{\xi}=\int_{-\infty}^{\xi} \mathrm{d} \eta \frac{H}{a(\eta)(2 \pi)^{3} \sqrt{-\eta}} \frac{M_{p}^{4} H^{2} \eta^{2}}{f_{a}^{4}} \frac{m_{\text {axion }}}{f_{a} H}\left(\Lambda-\frac{1}{2}\right)\left[\left(-\frac{k \eta}{2}\right)^{-\Lambda}\left(-\frac{k \xi}{2}\right)^{-\Lambda}+\left(\frac{k \eta}{2}\right)^{-\Lambda}\left(\frac{k \xi}{2}\right)^{-\Lambda}\right]$.

Finally on the other hand for $|k \eta| \approx 1$ we get the following simplified expression:

$$
\begin{aligned}
&\left\langle\bar{a}_{\mathbf{k}}(\eta=\xi \rightarrow 0)\right\rangle_{|k \eta| \approx 1}= \frac{4 \pi \sqrt{\xi}}{M_{p}^{2}}\left(C_{1}^{*} C_{2}+C_{1} C_{2}^{*}-\left|C_{1}\right|^{2}-\left|C_{2}\right|^{2}\right) \int_{-\infty}^{\xi} \mathrm{d} \eta \frac{H}{a(\eta)(2 \pi)^{3} \sqrt{-\eta}} \frac{M_{p}^{4} H^{2} \eta^{2}}{f_{a}^{4}} \frac{m_{\text {axion }}}{f_{a} H} \\
&\left(\Lambda-\frac{1}{2}\right)\left(\frac{1}{2}\right)^{-\Lambda}\left[\left(-\frac{k \xi}{2}\right)^{-\Lambda}+(-1)^{-\Lambda}\left(\frac{k \xi}{2}\right)^{-\Lambda}\right] \\
&= \text { for Bunch Davies vacua, } \\
& J_{\xi} \times \begin{cases}-\frac{2 \pi^{2} \sqrt{\xi}}{M_{p}^{2}} & \text { for } \alpha \text { vacua Type-I, } \\
\frac{4 \pi \sqrt{\xi}}{M_{p}^{2}}\left(\cos \delta \sinh 2 \alpha-\cosh ^{2} \alpha-\sinh ^{2} \alpha\right) & \text { for } \alpha \text { vacua Type-II, } \\
\frac{4 \pi \sqrt{\xi}\left|N_{\alpha}\right|^{2}}{M_{p}^{2}}\left(e^{\alpha}+e^{\alpha^{*}}-e^{\alpha+\alpha^{*}}-1\right) & \text { for special vacua, }, \\
0 \quad & \end{cases}
\end{aligned}
$$

where the integral $J_{\xi}$ is defined as

$$
J_{\xi}=\int_{-\infty}^{\xi} \mathrm{d} \eta \frac{H}{a(\eta)(2 \pi)^{3} \sqrt{-\eta}} \frac{M_{p}^{4} H^{2} \eta^{2}}{f_{a}^{4}} \frac{m_{\text {axion }}}{f_{a} H}\left(\Lambda-\frac{1}{2}\right)\left(\frac{1}{2}\right)^{-\Lambda}\left[\left(-\frac{k \xi}{2}\right)^{-\Lambda}+(-1)^{-\Lambda}\left(\frac{k \xi}{2}\right)^{-\Lambda}\right] .
$$

Now to analyze the behaviour of the expectation value of scalar curvature perturbation in position space we need to take the Fourier transform of the expectation value of the scalar curvature perturbation already computed in momentum space. For the most general solution we get the following result:

$$
\langle\bar{a}(\mathbf{x}, \eta=0)\rangle=-i \int \frac{\mathrm{d}^{3} k}{(2 \pi)^{3}} \int_{-\infty}^{0} \mathrm{~d} \eta \frac{M_{p}^{4} H^{4} \eta^{4} a(\eta)}{f_{a}^{4}} \frac{m_{\mathrm{axion}}}{f_{a}}\left(\vartheta_{\mathbf{k}}(0) \vartheta_{\mathbf{k}}^{\dagger_{\mathbf{k}}^{\prime}}(\eta)-\vartheta_{-\mathbf{k}}^{\dagger}(0) \vartheta_{-\mathbf{k}}^{\prime}(\eta)\right)
$$

where $h_{\mathbf{k}}(\eta)$ is the exact solution or the WKB solution of the mode function as explicitly computed in the earlier section of this paper. Following the previous methodology here we also introduce a Infra-Red (IR) cut-off regulator $\xi$ in the conformal time integral and consequently Eq. (4.238) can be recast in the following form:

$$
\langle\bar{a}(\mathbf{x}, \eta=0)\rangle=-i \lim _{k \xi \rightarrow 0} \int \frac{\mathrm{d}^{3} k}{(2 \pi)^{3}} \int_{-\infty}^{\xi} \mathrm{d} \eta \frac{M_{p}^{4} H^{4} \eta^{4} a(\eta)}{f_{a}^{4}} \frac{m_{\text {axion }}}{f_{a}}\left(\vartheta_{\mathbf{k}}(0) \vartheta_{\mathbf{k}}^{\dagger^{\prime}}(\eta)-\vartheta_{-\mathbf{k}}^{\dagger}(0) \vartheta_{-\mathbf{k}}^{\prime}(\eta)\right) .
$$

Further substituting the explicit form of the scalar mode functions computed from the exact solution or from the WKB approximated solution we get the following simplified expression for the VEV of the curvature fluctuation in position space:

$$
\langle\bar{a}(\mathbf{x}, \eta=0)\rangle=-i \lim _{k \xi \rightarrow 0} \int \frac{\mathrm{d}^{3} k}{(2 \pi)^{3}} \int_{-\infty}^{\xi} \mathrm{d} \eta \frac{M_{p}^{4} H^{4} \eta^{4} a(\eta)}{f_{a}^{4}} \frac{m_{\mathrm{axion}}}{f_{a}} \sum_{i=1}^{2} \sum_{j=1}^{2} C_{i}^{*} C_{j} \mathcal{A}_{i j} e^{i \mathbf{k} . \mathbf{x}},
$$

where the conformal time-dependent functions $\mathcal{A}_{i j} \forall i, j=1,2$ in momentum space have already been defined earlier.

Similarly in position space the representative expressions for the expectation value of the scalar curvature perturbation along with the three limiting cases $|k \eta| \rightarrow-\infty,|k \eta| \rightarrow 0$ and $|k \eta| \approx 1$ are given by 


$$
\begin{aligned}
\langle\bar{a}(\mathbf{x}, \eta=0)\rangle_{|k \eta| \rightarrow-\infty} \approx & -\frac{4 H^{3} M_{p}^{2}}{\pi f_{a}^{4}}\left[\left|C_{2}\right|^{2} O_{1}-\left|C_{1}\right|^{2} O_{2}\right. \\
& \left.-i\left(C_{1}^{*} C_{2} e^{i \pi\left(\Lambda+\frac{1}{2}\right)}+C_{1} C_{2}^{*} e^{-i \pi\left(\Lambda+\frac{1}{2}\right)}\right) O_{3}\right] \\
= & \begin{cases}\frac{2 H^{3} M_{p}^{2}}{f_{a}^{4} O_{2}} & \text { for Bunch Davies, } \\
- & \frac{4 H^{3} M_{p}^{2}}{\pi f_{a}^{4}}\left[\sinh ^{2} \alpha O_{1}-\cosh ^{2} \alpha O_{2}\right. \\
\left.-i \sinh ^{2} \alpha \cos \left(\pi\left(\Lambda+\frac{1}{2}\right)+\delta\right) O_{3}\right] & \text { for } \alpha \text { vacua Type-I, } \\
-\frac{4 H^{3} M_{p}^{2}\left|N_{\alpha}\right|^{2}}{\pi f_{a}^{4}}\left[e^{\alpha+\alpha^{*}} O_{1}-O_{2}\right. & \\
-i\left(e^{\alpha} e^{i \pi\left(\Lambda+\frac{1}{2}\right.}\right) & \\
-\frac{16 i|C|^{2} H^{3} M_{p}^{2}}{\pi f_{a}^{4}} \cos ^{2} \frac{\pi}{2}\left(\Lambda+\frac{1}{2}\right) O_{3} & \text { for special vacua, }\end{cases}
\end{aligned}
$$

$\langle\bar{a}(\mathbf{x}, \eta=\xi \rightarrow 0)\rangle_{|k \eta| \rightarrow 0}=\frac{H^{3} M_{p}^{2} \sqrt{\xi}}{2 f_{a}^{4}}\left(C_{1}^{*} C_{2}+C_{1} C_{2}^{*}-\left|C_{1}\right|^{2}-\left|C_{2}\right|^{2}\right) O_{4}^{\xi \theta}$

$$
=O_{4}^{\xi \theta} \times \begin{cases}-\frac{2 H^{3} M_{p}^{2} \pi^{2} \sqrt{\xi}}{f_{a}^{4}} & \text { for Bunch Davies vacua } \\ \frac{H^{3} M_{p}^{2} \sqrt{\xi}}{2 f_{a}^{4}}\left(\cos \delta \sinh 2 \alpha-\cosh ^{2} \alpha-\sinh ^{2} \alpha\right) & \text { for } \alpha \text { vacua Type-I, } \\ \frac{H^{3} M_{p}^{2} \sqrt{\xi}\left|N_{\alpha}\right|^{2}}{2 f_{a}^{4}}\left(e^{\alpha}+e^{\alpha^{*}}-e^{\alpha+\alpha^{*}}-1\right) & \text { for } \alpha \text { vacua Type-II } \\ 0 & \text { for special vacua }\end{cases}
$$

$$
\begin{aligned}
\langle\bar{a}(\mathbf{x}, \eta=\xi \rightarrow 0)\rangle_{\left|k c_{S} \eta\right| \approx 1} & =\frac{H^{3} M_{p}^{2} \sqrt{\xi}}{2 f_{a}^{4}}\left(C_{1}^{*} C_{2}+C_{1} C_{2}^{*}-\left|C_{1}\right|^{2}-\left|C_{2}\right|^{2}\right) O_{5}^{\xi \theta} \\
& =O_{5}^{\xi \theta} \times\left\{\begin{array}{l}
-\frac{2 H^{3} M_{p}^{2} \pi^{2} \sqrt{\xi}}{f_{a}^{4}} \\
\frac{H^{3} M_{p}^{2} \sqrt{\xi}}{2 f_{a}^{4}}\left(\cos \delta \sinh 2 \alpha-\cosh ^{2} \alpha-\sinh ^{2} \alpha\right) \\
\frac{H^{3} M_{p}^{2} \sqrt{\xi}\left|N_{\alpha}\right|^{2}}{2 f_{a}^{4}}\left(e^{\alpha}+e^{\alpha^{*}}-e^{\alpha+\alpha^{*}}-1\right) \\
0
\end{array}\right.
\end{aligned}
$$

for Bunch Davies vacua,

for $\alpha$ vacua Type-I,

for $\alpha$ vacua Type-II

for special vacua,

where we introduce the integrals $O_{1}, O_{2}, O_{3}, O_{4}^{\xi \theta}, O_{5}^{\xi \theta}$ given by 


$$
\begin{aligned}
O_{1}= & \int \frac{\mathrm{d}^{3} k}{(2 \pi)^{3}} e^{i \mathbf{k} \cdot \mathbf{x}} \int_{-\infty}^{0} \mathrm{~d} \eta \frac{\eta^{3}}{k} \frac{m_{\mathrm{axion}}}{f_{a}} e^{-i k \eta} \\
O_{2}= & \int \frac{\mathrm{d}^{3} k}{(2 \pi)^{3}} e^{i \mathbf{k} \cdot \mathbf{x}} \int_{-\infty}^{0} \mathrm{~d} \eta \frac{\eta^{3}}{k} \frac{m_{\mathrm{axion}}}{f_{a}} e^{i k \eta} \\
O_{3}= & \int \frac{\mathrm{d}^{3} k}{(2 \pi)^{3}} e^{i \mathbf{k} \cdot \mathbf{x}} \int_{-\infty}^{0} \mathrm{~d} \eta \frac{\eta^{3}}{k} \frac{m_{\mathrm{axion}}}{f_{a}} \sin k \eta \\
O_{4}^{\xi \theta}= & \int \frac{\mathrm{d}^{3} k}{(2 \pi)^{3}} e^{i \mathbf{k} \cdot \mathbf{x}} \int_{-\infty}^{\xi} \mathrm{d} \eta \frac{\left(\Lambda-\frac{1}{2}\right) \eta^{2}}{a(\eta) \sqrt{-\eta}} \frac{m_{\mathrm{axion}}}{f_{a} H} \\
& \times\left[\left(-\frac{k \eta}{2}\right)^{-\Lambda}\left(-\frac{k \xi}{2}\right)^{-\Lambda}+\left(\frac{k \eta}{2}\right)^{-\Lambda}\left(\frac{k \xi}{2}\right)^{-\Lambda}\right] \\
O_{5}^{\xi \theta}= & \int \frac{\mathrm{d}^{3} k}{(2 \pi)^{3}} e^{i \mathbf{k} \cdot \mathbf{x}} \int_{-\infty}^{\xi} \mathrm{d} \eta \frac{\left(\Lambda-\frac{1}{2}\right) \eta^{2}}{a(\eta) \sqrt{-\eta}} \frac{m_{\mathrm{axion}}}{f_{a} H}\left(\frac{1}{2}\right)^{-\Lambda} \\
& \times\left[\left(-\frac{k \xi}{2}\right)^{-\Lambda}+(-1)^{-\Lambda}\left(\frac{k \xi}{2}\right)^{-\Lambda}\right]
\end{aligned}
$$

Now to compute these momentum integrals we follow a few sets of assumptions that we have mentioned for the new massive particle in the last section. Two situations where $\mathbf{k}$ and $\mathbf{x}$ are parallel and having an angle $\Theta$ in between them. For the first case $\mathbf{k} \cdot \mathbf{x}=k x$ and for the second case we have $\mathbf{k} . \mathbf{x}=k x \cos \Theta$, where the range of the angular parameter is lying within the window $\Theta_{1} \leq \Theta \leq \Theta_{2}$, where $\Theta_{1}$ and $\Theta_{2}$ are two cut-off in the angular coordinate which are introduced to regularize the momentum integrals in the present context. For Case I and Case II we get the following results:

For Case I:

$$
\begin{aligned}
O_{1} & =\frac{1}{2 \pi^{2}} \int_{0}^{\infty} \mathrm{d} k \int_{-\infty}^{0} \mathrm{~d} \eta \eta^{3} k \frac{m_{\text {axion }}}{f_{a}} e^{i k(x-\eta)} \\
& =-\frac{i|\mathbf{x}|^{2}}{2 \pi^{2}}\left(\frac{m_{\mathrm{axion}}}{f_{a}}\right)_{\eta=-|\mathbf{x}|} \\
O_{2}= & \frac{1}{2 \pi^{2}} \int_{0}^{\infty} \mathrm{d} k \int_{-\infty}^{0} \mathrm{~d} \eta \eta^{3} k \frac{m_{\mathrm{axion}}}{f_{a}} e^{i k(x+\eta)} \\
= & \frac{i|\mathbf{x}|^{2}}{2 \pi^{2}}\left(\frac{m_{\mathrm{axion}}}{f_{a}}\right)_{\eta=-|\mathbf{x}|}, \\
O_{3}= & \frac{1}{2 \pi^{2}} \int_{0}^{\infty} \mathrm{d} k \int_{-\infty}^{0} \mathrm{~d} \eta \eta^{3} k \frac{m_{\mathrm{axion}}}{f_{a}} e^{i k x} \sin k \eta \\
= & \frac{|\mathbf{x}|^{2}}{2 \pi^{2}}\left(\frac{m_{\mathrm{axion}}}{f_{a}}\right)_{\eta=-|\mathbf{x}|}, \\
O_{4}^{\xi \theta}= & \frac{1}{2 \pi^{2}} \int_{0}^{\infty} \mathrm{d} k k^{2} e^{i k x} \int_{-\infty}^{\xi} \mathrm{d} \eta \frac{\left(\Lambda-\frac{1}{2}\right) \eta^{2}}{a(\eta) \sqrt{-\eta}} \frac{m_{\mathrm{axion}}}{f_{a} H} \\
& \times\left[\left(-\frac{k \eta}{2}\right)^{-\Lambda}\left(-\frac{k \xi}{2}\right)^{-\Lambda}+\left(\frac{k \eta}{2}\right)^{-\Lambda}\left(\frac{k \xi}{2}\right)^{-\Lambda}\right]
\end{aligned}
$$

$$
\begin{aligned}
O_{5}^{\xi \theta}= & \frac{1}{2 \pi^{2}} \int_{0}^{\infty} \mathrm{d} k e^{i k x} k^{2-\Lambda} \int_{-\infty}^{\xi} \mathrm{d} \eta \frac{\left(\Lambda-\frac{1}{2}\right) \eta^{2}}{a(\eta) \sqrt{-\eta}} \frac{m_{\text {axion }}}{f_{a} H}\left(\frac{1}{2}\right)^{-\Lambda} \\
& \times\left[\left(-\frac{\xi}{2}\right)^{-\Lambda}+(-1)^{-\Lambda}\left(\frac{\xi}{2}\right)^{-\Lambda}\right]
\end{aligned}
$$

\section{For Case II:}

$$
\begin{aligned}
& O_{1}=\frac{1}{4 \pi^{2}} \int_{0}^{\infty} \mathrm{d} k \int_{-\infty}^{0} \mathrm{~d} \eta \int_{\Theta_{1}}^{\Theta_{2}} \mathrm{~d} \Theta \eta^{3} k \frac{m_{\text {axion }}}{f_{a}} e^{i k(x \cos \Theta-\eta)} \\
& =-i \int_{\Theta_{1}}^{\Theta_{2}} \mathrm{~d} \Theta \frac{|\mathbf{x}|^{2}\left(\frac{m_{\text {axion }}}{f_{a}}\right)_{\eta=-|\mathbf{x}| \cos \Theta}}{4 \pi^{2} c_{S}^{2}} \cos ^{2} \Theta, \\
& O_{2}=\frac{1}{4 \pi^{2}} \int_{0}^{\infty} \mathrm{d} k \int_{-\infty}^{0} \mathrm{~d} \eta \int_{\Theta_{1}}^{\Theta_{2}} \mathrm{~d} \Theta \eta^{3} k \frac{m_{\text {axion }}}{f_{a}} e^{i k(x \cos \Theta+\eta)} \\
& =i \int_{\Theta_{1}}^{\Theta_{2}} \mathrm{~d} \Theta \frac{|\mathbf{x}|^{2}\left(\frac{m_{\text {axion }}}{f_{a}}\right)_{\eta=-|\mathbf{x}| \cos \Theta}}{4 \pi^{2} c_{S}^{2}} \cos ^{2} \Theta, \\
& O_{3}=\frac{1}{4 \pi^{2}} \int_{0}^{\infty} \mathrm{d} k \int_{-\infty}^{0} \mathrm{~d} \eta \int_{\Theta_{1}}^{\Theta_{2}} \mathrm{~d} \Theta \eta^{3} k \frac{m_{\text {axion }}}{f_{a}} e^{i k x \cos \Theta} \sin k \eta \\
& =\int_{\Theta_{1}}^{\Theta_{2}} \mathrm{~d} \Theta \frac{|\mathbf{x}|^{2}\left(\frac{m_{\text {axion }}}{f_{a}}\right)_{\eta=-|\mathbf{x}| \cos \Theta}}{4 \pi^{2} c_{S}^{2}} \cos ^{2} \Theta, \\
& O_{4}^{\xi \theta}=\frac{1}{4 \pi^{2}} \int_{0}^{\infty} \mathrm{d} k \int_{-\infty}^{\xi} \mathrm{d} \eta \int_{\Theta_{1}}^{\Theta_{2}} \mathrm{~d} \Theta k^{2} e^{i k x \cos \Theta} \frac{\eta^{2}\left(\Lambda-\frac{1}{2}\right)}{a(\eta) \sqrt{-\eta}} \frac{m_{\text {axion }}}{f_{a} H} \\
& \times\left[\left(-\frac{k \eta}{2}\right)^{-\Lambda}\left(-\frac{k \xi}{2}\right)^{-\Lambda}+\left(\frac{k \eta}{2}\right)^{-\Lambda}\left(\frac{k \xi}{2}\right)^{-\Lambda}\right],
\end{aligned}
$$

$$
\begin{aligned}
O_{5}^{\xi \theta}= & \frac{1}{4 \pi^{2}} \int_{0}^{\infty} \mathrm{d} k \int_{\Theta_{1}}^{\Theta_{2}} \mathrm{~d} \Theta e^{i k x \cos \Theta} k^{2-\Lambda} \int_{-\infty}^{\xi} \mathrm{d} \eta \\
& \times \frac{\eta^{2}\left(\Lambda-\frac{1}{2}\right)}{a(\eta) \sqrt{-\eta}} \frac{m_{\mathrm{axion}}}{f_{a} H}\left(\frac{1}{2}\right)^{-\Lambda} \\
& \times\left[\left(-\frac{\xi}{2}\right)^{-\Lambda}+(-1)^{-\Lambda}\left(\frac{\xi}{2}\right)^{-\Lambda}\right]
\end{aligned}
$$

where $\Theta_{1}$ and $\Theta_{2}$ play the role of angular regulator in the present context.

Now our objective is to compute the expression for the two point correlation function from scalar curvature perturbation. Following the previously mentioned computational technique of in-in formalism, which is commonly known as the Swinger-Keyldish formalism here we compute the expression for the two point correlation function from scalar curvature perturbation. Using the interaction picture the two point correlation function of the curvature fluctuation in momentum space can be expressed as

$$
\left\langle\bar{a}_{\mathbf{k}}(\eta) \bar{a}_{\mathbf{q}}(\eta)\right\rangle=(2 \pi)^{3} \delta^{3}(\mathbf{k}+\mathbf{q}) \frac{2 \pi^{2}}{k^{3}} \Delta_{\bar{a}}(k),
$$


where the primordial power spectrum for the scalar mode at any arbitrary momentum scale can be written as

$\Delta_{\bar{a}}(k)=\frac{k^{3}\left|\vartheta_{\mathbf{k}}\right|^{2}}{2 \pi^{2}\left(f_{a}^{2} / H^{2} \eta^{2}\right)}=\frac{(-k \eta)^{3} H^{2}}{2 \pi^{2} f_{a}^{2}} \sum_{i=1}^{2} \sum_{j=1}^{2} C_{i}^{*} C_{j}\left[U_{i j}\right]_{C_{S}=1}$,

where $U_{i j} \forall i, j=1,2$ are defined for new massive particles in the earlier section.

For further simplification we consider here the three limiting cases $|k \eta| \rightarrow-\infty,|k \eta| \rightarrow 0$ and $|k \eta| \approx 1$, which are physically acceptable in the present context. First of we consider the results for $|k \eta| \rightarrow-\infty$. In this case we get

$\left\langle\bar{a}_{\mathbf{k}}(\eta) \bar{a}_{\mathbf{q}}(\eta)\right\rangle_{|k \eta| \rightarrow-\infty}=(2 \pi)^{3} \delta^{3}(\mathbf{k}+\mathbf{q}) \frac{2 \pi^{2}}{k^{3}}\left[\Delta_{\bar{a}}(k)\right]_{|k \eta| \rightarrow-\infty}$,

where the primordial power spectrum for the scalar mode at $|k \eta| \rightarrow-\infty$ can be written as

$$
\begin{aligned}
{\left[\Delta_{\bar{a}}(k)\right]_{|k \eta| \rightarrow-\infty} \approx } & \frac{H^{2}}{f_{a}^{2}} \frac{k^{2} \eta^{2}}{\pi^{3}}\left[\left|C_{2}\right|^{2}+\left|C_{1}\right|^{2}\right. \\
& \left.+\left(C_{1}^{*} C_{2} e^{2 i k c s \eta} e^{i \pi\left(\Lambda+\frac{1}{2}\right)}+C_{1} C_{2}^{*} e^{-2 i k c s \eta} e^{-i \pi\left(\Lambda+\frac{1}{2}\right)}\right)\right] \\
= & \begin{cases}\frac{H^{2}}{f_{a}^{2}} \frac{(-k \eta)^{2}}{2 \pi^{2}} & \text { for Bunch Davies, } \\
\frac{H^{2}}{f_{a}^{2}} \frac{(-k \eta)^{2}}{\pi^{3}}\left[\sinh ^{2} \alpha+\cosh ^{2} \alpha\right. & \text { for special vacua. } \\
\frac{H^{2}}{f_{a}^{2}} \frac{4(-k \eta)^{2}\left|N_{\alpha}\right|^{2}}{\pi^{3}} \cos ^{2}\left(k c_{S} \eta+\frac{\pi}{2}\left(\Lambda+\frac{1}{2}\right)-i \frac{\alpha}{2}\right) & \text { for } \alpha \text { vacua Type-II, } \\
\frac{H^{2}}{f_{a}^{2}} \frac{4(-k \eta)^{2}}{\pi^{3}} \cos ^{2}\left(k c_{S} \eta+\frac{\pi}{2}\left(\Lambda+\frac{1}{2}\right)\right) & \text { for } \alpha \text { vacua Type-I, }\end{cases}
\end{aligned}
$$

Next we consider the results for $|k \eta| \rightarrow 0$. In this case we get

$\left\langle\bar{a}_{\mathbf{k}}(\eta) \bar{a}_{\mathbf{q}}(\eta)\right\rangle_{|k \eta| \rightarrow 0}=(2 \pi)^{3} \delta^{3}(\mathbf{k}+\mathbf{q}) \frac{2 \pi^{2}}{k^{3}}\left[\Delta_{\bar{a}}(k)\right]_{|k \eta| \rightarrow 0}$,

where the primordial power spectrum for the scalar mode at $|k \eta| \rightarrow 0$ can be written as

$\left[\Delta_{\bar{a}}(k)\right]_{|k \eta| \rightarrow 0} \approx \frac{H^{2}}{f_{a}^{2}} \frac{(-k \eta)^{3}}{2 \pi^{4}} \Gamma^{2}(\Lambda)\left(-\frac{k \eta}{2}\right)^{-2 \Lambda}\left[\left|C_{2}\right|^{2}+\left|C_{1}\right|^{2}-\left(C_{1}^{*} C_{2}+C_{1} C_{2}^{*}\right)\right]$

$$
= \begin{cases}\frac{H^{2}}{f_{a}^{2}} \frac{(-k \eta)^{3-2 \Lambda}}{2^{2(2-\Lambda)} \pi^{2}}\left|\frac{\Gamma(\Lambda)}{\Gamma\left(\frac{3}{2}\right)}\right|^{2} & \text { for Bunch Davies, } \\ \frac{H^{2}}{f_{a}^{2}} \frac{(-k \eta)^{3-2 \Lambda}}{2^{(3-2 \Lambda)} \pi^{3}}\left|\frac{\Gamma(\Lambda)}{\Gamma\left(\frac{3}{2}\right)}\right|^{2}\left[\sinh ^{2} \alpha+\cosh ^{2} \alpha-\sinh 2 \alpha \cos \delta\right] & \text { for } \alpha \text { vacua Type-I, } \\ \frac{H^{2}}{f_{a}^{2}} \frac{(-k \eta)^{3-2 \Lambda}\left|N_{\alpha}\right|^{2}}{2^{(1-2 \Lambda)} \pi^{3}}\left|\frac{\Gamma(\Lambda)}{\Gamma\left(\frac{3}{2}\right)}\right|^{2} \sin ^{2} \frac{\alpha}{2} & \text { for } \alpha \text { vacua Type-II, } \\ 0 & \text { for special vacua. }\end{cases}
$$


Finally we consider the results for $|k \eta| \approx 1$. In this case we get

$\left\langle\bar{a}_{\mathbf{k}}(\eta=0) \bar{a}_{\mathbf{q}}(\eta=0)\right\rangle_{|k \eta| \approx 1}=(2 \pi)^{3} \delta^{3}(\mathbf{k}+\mathbf{q}) \frac{2 \pi^{2}}{k^{3}}\left[\Delta_{\bar{a}}(k)\right]_{|k \eta| \approx 1}$,

where the primordial power spectrum for the scalar mode at $|k \eta| \approx 1$ can be written as

$$
\begin{aligned}
{\left[\Delta_{\bar{a}}(k)\right]_{|k \eta| \approx 1} \approx } & \frac{H^{2}}{f_{a}^{2}} \frac{1}{2^{2(1-\Lambda)} \pi^{3}}\left|\frac{\Gamma(\Lambda)}{\Gamma\left(\frac{3}{2}\right)}\right|^{2}\left[\left|C_{2}\right|^{2}+\left|C_{1}\right|^{2}-\left(C_{1}^{*} C_{2}+C_{1} C_{2}^{*}\right)\right] \\
& = \begin{cases}\frac{H^{2}}{f_{a}^{2}} \frac{1}{2^{2(2-\Lambda)} \pi^{2}}\left|\frac{\Gamma(\Lambda)}{\Gamma\left(\frac{3}{2}\right)}\right|^{2} & \text { for Bunch Davies, } \\
\frac{H^{2}}{f_{a}^{2}} \frac{1}{2^{(3-2 \Lambda)} \pi^{3}}\left|\frac{\Gamma(\Lambda)}{\Gamma\left(\frac{3}{2}\right)}\right|^{2}\left[\sinh ^{2} \alpha+\cosh ^{2} \alpha-\sinh 2 \alpha \cos \delta\right] & \text { for } \alpha \text { vacua Type-I, } \\
\frac{H^{2}}{f_{a}^{2}} \frac{\left|N_{\alpha}\right|^{2}}{2^{(1-2 \Lambda)} \pi^{3}}\left|\frac{\Gamma(\Lambda)}{\Gamma\left(\frac{3}{2}\right)}\right|^{2} \sin ^{2} \frac{\alpha}{2} & \text { for } \alpha \text { vacua Type-II, } \\
0 & \text { for special vacua. }\end{cases}
\end{aligned}
$$

Now to analyze the behaviour of the two point correlation function of the scalar curvature perturbation in position space we need to take the Fourier transform of the two point correlation function of scalar curvature perturbation already computed in momentum space. For the most general solution we get the following result:

$\langle\bar{a}(\mathbf{x}, \eta) \bar{a}(\mathbf{y}, \eta)\rangle=\int \frac{\mathrm{d}^{3} k}{(2 \pi)^{3}} e^{i \mathbf{k} .(\mathbf{x}-\mathbf{y})} \frac{(-\eta)^{3} H^{2}}{f_{a}^{2}} \sum_{i=1}^{2} \sum_{j=1}^{2} C_{i}^{*} C_{j}\left[U_{i j}\right]_{C_{S}=1}$,

where the conformal time-dependent functions $U_{i j} \forall i, j=1,2$ in momentum space have already been defined earlier.

Similarly in position space the representative expressions for the expectation value of the scalar curvature perturbation along with limiting case $|k \eta| \rightarrow-\infty$ is given by

$$
\begin{aligned}
\langle\bar{a}(\mathbf{x}, \eta) \bar{a}(\mathbf{y}, \eta)\rangle_{|k \eta| \rightarrow-\infty} \approx & \frac{1}{4 \pi^{4}} \frac{H^{2}}{f_{a}^{2}} \eta^{2}\left[\left(\left|C_{2}\right|^{2}+\left|C_{1}\right|^{2}\right) J_{1}\right. \\
& \left.+\left(C_{1}^{*} C_{2} e^{i \pi\left(\Lambda+\frac{1}{2}\right)} J_{2}+C_{1} C_{2}^{*} e^{-i \pi\left(\Lambda+\frac{1}{2}\right)} J_{3}\right)\right] \\
& = \begin{cases}\frac{H^{2}}{f_{a}^{2}} \frac{\eta^{2}}{(2 \pi)^{3}} J_{1} & \text { for Bunch Davies, } \\
\frac{H^{2}}{f_{a}^{2}} \frac{\eta^{2}}{4 \pi^{4}}\left[\left(\sinh ^{2} \alpha+\cosh ^{2} \alpha\right) J_{1}\right. & \text { for } \alpha \text { vacua Type-II, } \\
& \left.+\frac{1}{2} \sinh 2 \alpha\left(e^{i\left(\pi\left(\Lambda+\frac{1}{2}\right)+\delta\right)} J_{2}+e^{-i\left(\pi\left(\Lambda+\frac{1}{2}\right)+\delta\right)}\right) J_{3}\right] \\
\frac{H^{2}}{f_{a}^{2}} \frac{\eta^{2}\left|N_{\alpha}\right|^{2}}{2 \pi^{4}}\left[J_{1}+\left(e^{i \pi\left(\Lambda+\frac{1}{2}\right)} e^{\alpha} J_{2}+e^{-i \pi\left(\Lambda+\frac{1}{2}\right)} e^{-\alpha} J_{3}\right)\right] & \text { for vacua Type-I, } \\
\frac{H^{2}}{f_{a}^{2}} \frac{\eta^{2}}{2 \pi^{4}}\left[J_{1}+\left(e^{i \pi\left(\Lambda+\frac{1}{2}\right)} e^{\alpha} J_{2}+e^{-i \pi\left(\Lambda+\frac{1}{2}\right)} e^{-\alpha} J_{3}\right)\right] & \text { for special vacua, }\end{cases}
\end{aligned}
$$

where the momentum integrals $J_{1}, J_{2}$ and $J_{3}$ are defined in the previous section. Here by setting $\mathbf{y}=0$ one can derive the results for $\langle\bar{a}(\mathbf{x}, \eta) \bar{a}(0, \eta)\rangle$ at conformal time scale $\eta$ with $|k \eta| \rightarrow-\infty$. 
Next we consider the results for $|k \eta| \rightarrow 0$. In this case we get

$\langle\bar{a}(\mathbf{x}, \eta) \bar{a}(\mathbf{y}, \eta)\rangle_{|k \eta| \rightarrow 0} \approx \frac{H^{2}}{f_{a}^{2}} \frac{(-\eta)^{3-2 \Lambda}}{2^{2(2-\Lambda)} \pi^{4}}\left|\frac{\Gamma(\Lambda)}{\Gamma\left(\frac{3}{2}\right)}\right|^{2}\left[\left(\left|C_{2}\right|^{2}+\left|C_{1}\right|^{2}\right)-\left(C_{1}^{*} C_{2}+C_{1} C_{2}^{*}\right)\right] K_{I}$

$$
=K_{I} \times \begin{cases}\frac{H^{2}}{f_{a}^{2}} \frac{(-\eta)^{3-2 \Lambda}}{2^{2(3-\Lambda)} \pi^{3}}\left|\frac{\Gamma(\Lambda)}{\Gamma\left(\frac{3}{2}\right)}\right|^{2} & \text { for Bunch Davies, } \\ \frac{H^{2}}{f_{a}^{2}} \frac{(-\eta)^{3-2 \Lambda}}{2^{(5-2 \Lambda)} \pi^{4}}\left|\frac{\Gamma(\Lambda)}{\Gamma\left(\frac{3}{2}\right)}\right|^{2}\left[\sinh ^{2} \alpha+\cosh ^{2} \alpha\right. & \\ -\sinh 2 \alpha \cos \delta] & \text { for } \alpha \text { vacua Type-I, } \\ \frac{H^{2}}{f_{a}^{2}} \frac{(-\eta)^{3-2 \Lambda}\left|N_{\alpha}\right|^{2}}{2^{(3-2 \Lambda)} \pi^{4}}\left|\frac{\Gamma(\Lambda)}{\Gamma\left(\frac{3}{2}\right)}\right|^{2} \sin ^{2} \frac{\alpha}{2} & \text { for } \alpha \text { vacua Type-II, } \\ 0 & \text { for special vacua, }\end{cases}
$$

where the momentum integrals $K_{I}$ are defined in the previous section. Here by setting $\mathbf{y}=0$ one can derive the results for $\langle\bar{a}(\mathbf{x}, \eta) \bar{a}(0, \eta)\rangle$ at conformal time scale $\eta$ with $|k \eta| \rightarrow 0$.

Finally we consider the results for $|k \eta| \approx 1$. In this case we get

$$
\begin{aligned}
& \langle\bar{a}(\mathbf{x}, \eta=0) \bar{a}(\mathbf{y}, \eta=0)\rangle_{|k \eta| \approx 1} \approx \frac{H^{2}}{f_{a}^{2}} \frac{1}{2^{2(2-\Lambda)} \pi^{4}}\left|\frac{\Gamma(\Lambda)}{\Gamma\left(\frac{3}{2}\right)}\right|^{2}\left[\left(\left|C_{2}\right|^{2}+\left|C_{1}\right|^{2}\right)-\left(C_{1}^{*} C_{2}+C_{1} C_{2}^{*}\right)\right] Z_{I}
\end{aligned}
$$

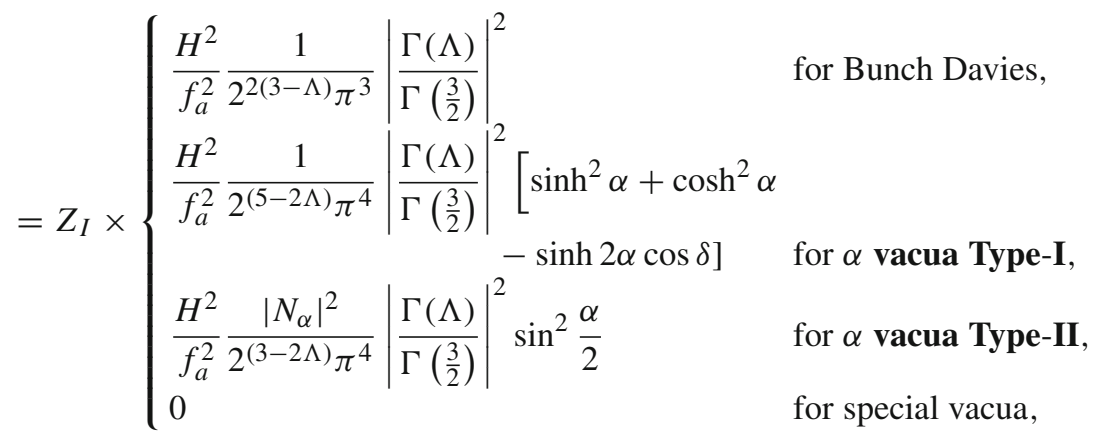

where the momentum integrals $Z_{I}$ are defined in the previous section. Here by setting $\mathbf{y}=0$ one can derive the results for $\langle\bar{a}(\mathbf{x}, \eta=0) \bar{a}(0, \eta=0)\rangle$ at conformal time scale $\eta$ with $\left|k c_{S} \eta\right| \rightarrow-\infty$.

Now to derive an exact connection between the computed VEV and two point function of the scalar curvature perturbation in the presence of the axion fluctuation in momentum space we write down the following sets of new consistency relations in primordial cosmology for the three limiting cases $|k \eta| \rightarrow-\infty,|k \eta| \rightarrow 0$ and $|k \eta| \approx 1$ :

$$
\begin{aligned}
\left\langle\bar{a}_{\mathbf{k}}(\eta=0)\right\rangle_{|k \eta| \rightarrow-\infty}=\left\langle\bar{a}_{\mathbf{k}}(\eta=0) \bar{a}_{\mathbf{q}}(\eta=0)\right\rangle_{\left|k c_{S} \eta\right| \rightarrow-\infty}^{\prime} \times \frac{2 k}{\eta^{2} H} \mathcal{I}_{1}, \\
\left\langle\bar{a}_{\mathbf{k}}(\eta=\xi \rightarrow 0)\right\rangle_{|k c s \eta| \rightarrow 0}=\left\langle\bar{a}_{\mathbf{k}}(\eta=\xi \rightarrow 0) \bar{a}_{\mathbf{q}}(\eta=\xi \rightarrow 0)\right\rangle_{|k \eta| \rightarrow 0}^{\prime} \\
\times \frac{2^{2-2 \Lambda} k^{2 \Lambda}}{(-\eta)^{3-2 \Lambda} H \pi}\left|\frac{\Gamma\left(\frac{3}{2}\right)}{\Gamma(\Lambda)}\right|^{2} \mathcal{I}_{2}, \\
\left\langle\bar{a}_{\mathbf{k}}(\eta=0)\right\rangle_{|k \eta| \approx 1}=\left\langle\bar{a}_{\mathbf{k}}(\eta=0) \bar{a}_{\mathbf{q}}(\eta=0)\right\rangle_{|k \eta| \approx 1}^{\prime} \\
\times \frac{2^{2-2 \Lambda} k^{3}}{H \pi}\left|\frac{\Gamma\left(\frac{3}{2}\right)}{\Gamma(\Lambda)}\right|^{2} \mathcal{I}_{3},
\end{aligned}
$$

where $\left\langle\bar{a}_{\mathbf{k}}(\eta=0) \bar{a}_{\mathbf{q}}(\eta=0)\right\rangle_{|k \eta| \rightarrow-\infty}^{\prime},\left\langle\bar{a}_{\mathbf{k}}(\eta=0) \bar{a}_{\mathbf{q}}(\eta=0)\right\rangle_{|k \eta| \rightarrow 0}^{\prime}$ and $\left\langle\bar{a}_{\mathbf{k}}(\eta=0) \bar{a}_{\mathbf{q}}(\eta=0)\right\rangle_{|k \eta| \approx 1}^{\prime}$ are defined as

$\left\langle\bar{a}_{\mathbf{k}}(\eta=0) \bar{a}_{\mathbf{q}}(\eta=0)\right\rangle_{|k \eta| \rightarrow-\infty}^{\prime}=\frac{\left\langle\bar{a}_{\mathbf{k}}(\eta=0) \bar{a}_{\mathbf{q}}(\eta=0)\right\rangle_{|k \eta| \rightarrow-\infty}}{(2 \pi)^{3} \delta^{3}(\mathbf{k}+\mathbf{q})}$, 


$$
\begin{aligned}
& \left\langle\bar{a}_{\mathbf{k}}(\eta=0) \bar{a}_{\mathbf{q}}(\eta=0)\right\rangle_{|k \eta| \rightarrow 0}^{\prime}=\frac{\left\langle\bar{a}_{\mathbf{k}}(\eta=0) \bar{a}_{\mathbf{q}}(\eta=0)\right\rangle_{|k \eta| \rightarrow 0}}{(2 \pi)^{3} \delta^{3}(\mathbf{k}+\mathbf{q})}, \\
& \left\langle\bar{a}_{\mathbf{k}}(\eta=0) \bar{a}_{\mathbf{q}}(\eta=0)\right\rangle_{|k \eta| \approx 1}^{\prime}=\frac{\left\langle\bar{a}_{\mathbf{k}}(\eta=0) \bar{a}_{\mathbf{q}}(\eta=0)\right\rangle_{|k \eta| \approx 1}}{(2 \pi)^{3} \delta^{3}(\mathbf{k}+\mathbf{q})} .
\end{aligned}
$$

Let us now define a new cosmological observable $\hat{O}_{\text {obs }}$ in the three limiting cases $|k \eta| \rightarrow-\infty,|k \eta| \rightarrow 0$ and $|k \eta| \approx 1$ as

$$
\begin{aligned}
& \hat{O}_{\text {obs }} \stackrel{|k \eta| \rightarrow-\infty}{=} \frac{2}{(-k \eta)^{2} H} \mathcal{I}_{1}, \\
& \hat{O}_{\text {obs }} \stackrel{|k \eta| \rightarrow 0}{=} \frac{2^{3-2 \Lambda} \pi}{(-k \eta)^{3-2 \Lambda} H}\left|\frac{\Gamma\left(\frac{3}{2}\right)}{\Gamma(\Lambda)}\right|^{2} \mathcal{I}_{2}, \\
& \hat{O}_{\text {obs }} \stackrel{|k \eta| \approx 1}{=} \frac{2^{3-2 \Lambda} \pi}{H}\left|\frac{\Gamma\left(\frac{3}{2}\right)}{\Gamma(\Lambda)}\right|^{2} \mathcal{I}_{3},
\end{aligned}
$$

where $\mathcal{I}_{1}, \mathcal{I}_{2}$ and $\mathcal{I}_{3}$ are defined as

$$
\mathcal{I}_{1}=-\frac{\int_{-\infty}^{0} \mathrm{~d} \eta \frac{\eta}{k} \frac{m_{\text {axion }}}{f_{a}}\left[\left|C_{2}\right|^{2} e^{-i k \eta}-\left|C_{1}\right|^{2} e^{i k \eta}-i\left(C_{1}^{*} C_{2} e^{i \pi\left(\Lambda+\frac{1}{2}\right)}+C_{1} C_{2}^{*} e^{-i \pi\left(\Lambda+\frac{1}{2}\right)}\right) \sin k \eta\right]}{\left[\left|C_{2}\right|^{2}+\left|C_{1}\right|^{2}+\left(C_{1}^{*} C_{2} e^{2 i k \eta} e^{i \pi\left(\Lambda+\frac{1}{2}\right)}+C_{1} C_{2}^{*} e^{-2 i k \eta} e^{-i \pi\left(\Lambda+\frac{1}{2}\right)}\right)\right]}
$$

$$
= \begin{cases}\int_{-\infty}^{0} \mathrm{~d} \eta \frac{\eta}{k} \frac{m_{\text {axion }}}{f_{a}} e^{i k \eta} & \text { for Bunch Davies, } \\ -\frac{\int_{-\infty}^{0} \mathrm{~d} \eta \frac{\eta}{k} \frac{m_{\text {axion }}}{f_{a}}\left[\sinh ^{2} \alpha e^{i k \eta}-\cosh ^{2} \alpha e^{-i k \eta}+i \sinh 2 \alpha \cos \left(\pi\left(\Lambda+\frac{1}{2}\right)+\delta\right) \sin k \eta\right]}{\left[\sinh ^{2} \alpha+\cosh ^{2} \alpha+\sinh 2 \alpha \cos \left(2 k \eta+\pi\left(\Lambda+\frac{1}{2}\right)+\delta\right)\right]} & \text { for } \alpha \text { vacua Type-I, } \\ -\frac{\int_{-\infty}^{0} \mathrm{~d} \eta \frac{\eta}{k} \frac{m_{\text {axion }}}{f_{a}}\left[e^{\alpha+\alpha^{*}} e^{i k \eta}-e^{-i k \eta}+i\left(e^{\alpha} e^{i \pi\left(\Lambda+\frac{1}{2}\right)}+e^{\alpha^{*}} e^{-i \pi\left(\Lambda+\frac{1}{2}\right)}\right) \sin k \eta\right]}{4 \cos ^{2}\left(k \eta+\frac{\pi}{2}\left(\Lambda+\frac{1}{2}\right)-i \frac{\alpha}{2}\right)} & \text { for } \alpha \text { vacua Type-II, } \\ -\frac{i \pi^{2} \int_{-\infty}^{0} \mathrm{~d} \eta \frac{\eta}{k} \frac{m_{\mathrm{axion}}}{f_{a}} \sin k \eta \cos ^{2} \frac{\pi}{2}\left(\Lambda+\frac{1}{2}\right)}{\cos ^{2}\left(k \eta+\frac{\pi}{2}\left(\Lambda+\frac{1}{2}\right)\right)} & \text { for special vacua, }\end{cases}
$$

$$
\begin{aligned}
& \mathcal{I}_{2}=\sqrt{\xi} \int_{-\infty}^{\xi} \mathrm{d} \eta \sqrt{-\eta} \frac{m_{\text {axion }}}{f_{a}}\left(\Lambda-\frac{1}{2}\right)\left[\left(-\frac{k \eta}{2}\right)^{-\Lambda}\left(-\frac{k \xi}{2}\right)^{-\Lambda}+\left(\frac{k \eta}{2}\right)^{-\Lambda}\left(\frac{k \xi}{2}\right)^{-\Lambda}\right], \\
& \mathcal{I}_{3}=\sqrt{\xi} \int_{-\infty}^{\xi} \mathrm{d} \eta \sqrt{-\eta} \frac{m_{\text {axion }}}{f_{a}}\left(\Lambda-\frac{1}{2}\right) 2^{1+\Lambda}\left(\frac{k \xi}{2}\right)^{-\Lambda}(-1)^{-\Lambda} .
\end{aligned}
$$

Next we write down the expression for new cosmological observable $\hat{O}_{\text {obs }}$ in terms of all other known cosmological

$$
\hat{O}_{\mathrm{obs}} \stackrel{k \eta \mid \approx 1}{=} \frac{2^{n_{\bar{a}}-1} \pi}{H}\left|\frac{\Gamma\left(\frac{3}{2}\right)}{\Gamma\left(\frac{4-n_{\bar{a}}}{2}\right)}\right|^{2} \mathcal{I}_{3}\left(\Lambda=\frac{4-n_{\bar{a}}}{2}\right),
$$

where the spectral tilt for scalar fluctuations is given by 


$$
\begin{aligned}
& n_{\bar{a}}-1 \equiv\left(\frac{\mathrm{d} \ln \Delta_{\bar{a}}(k)}{\mathrm{d} \ln k}\right)_{|k \eta| \approx 1} \approx 3-2 \Lambda= \\
& \left\{\begin{array}{l}
3-2 \sqrt{\frac{9}{4}-\frac{m_{\text {axion }}^{2}}{f_{a}^{2} H^{2}}} \text { for de Sitter } \\
3-2 \sqrt{v^{2}-\frac{m_{\text {axion }}^{2}}{f_{a}^{2} H^{2}}} \text { for quasi de Sitter, }
\end{array}\right.
\end{aligned}
$$

and substituting the explicit expression for the axion mass parameter we get

\section{For total U(a)}

\section{A. de Sitter}

$$
n_{\bar{a}}-1 \equiv\left(\frac{\mathrm{d} \ln \Delta_{\bar{a}}(k)}{\mathrm{d} \ln k}\right)_{|k \eta| \approx 1} \approx \begin{cases}3-2 \sqrt{\frac{9}{4}+\frac{\Lambda_{C}^{4} \times \cos \left(\sin ^{-1}\left(\frac{10 \mu^{3} H}{\Lambda_{C}^{4}}\right)\right)}{10000 H^{4}}} & \text { for early \& late } \eta, \\ 3-2 \sqrt{\frac{9}{4}+\frac{\Lambda_{C}^{4} \times \cos \left(\sin ^{-1}\left(\frac{\mu^{3} H}{\Lambda_{C}^{4}} \sqrt{\left.100-\frac{80}{1+\omega_{C}}\right)}\right)\right.}{\left[100-\frac{80}{1+\omega_{C}}\right]^{2} H^{4}}} & \text { for } \eta<\eta_{c} \\ 3-2 \sqrt{\frac{9}{4}+\frac{\Lambda_{C}^{4} \times \cos \left(\sin ^{-1}\left(\frac{2 \sqrt{5} \mu^{3} H}{\Lambda_{C}^{4}}\right)\right)}{400 H^{4}}} & \text { for } \eta \sim \eta_{c} .\end{cases}
$$

\section{B. Quasi de Sitter}

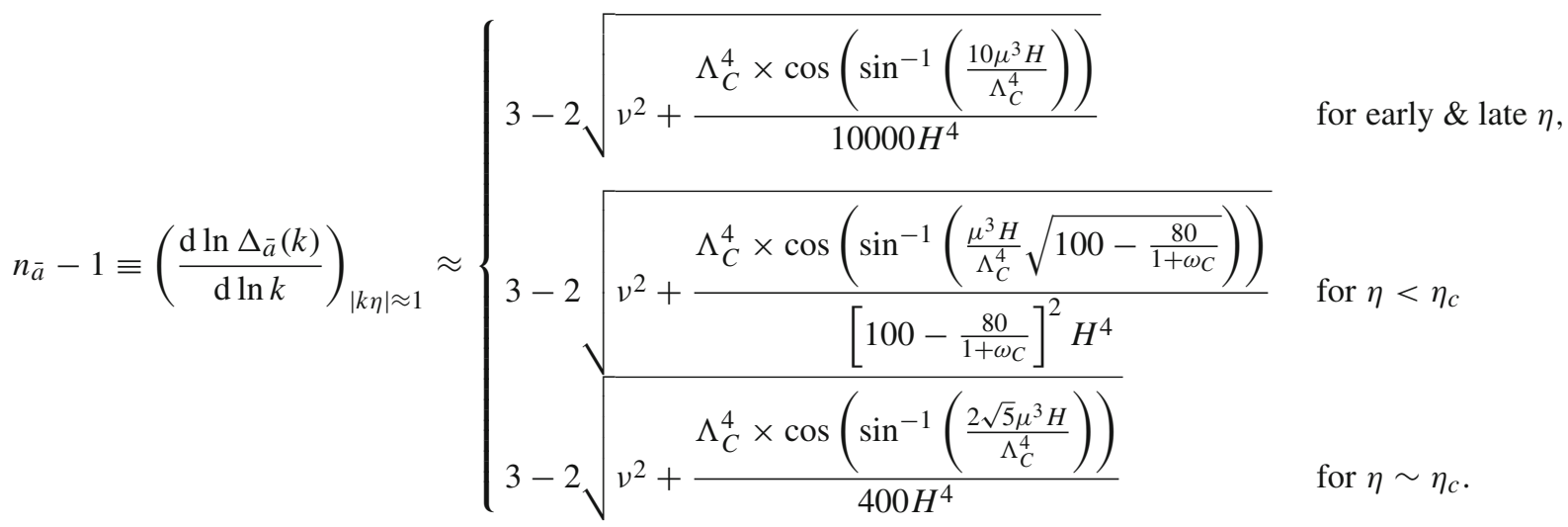

For osc. U(a)

\section{A. de Sitter}

$$
n_{\bar{a}}-1 \equiv\left(\frac{\mathrm{d} \ln \Delta_{\bar{a}}(k)}{\mathrm{d} \ln k}\right)_{|k \eta| \approx 1} \approx \begin{cases}3-2 \sqrt{\frac{9}{4}+\frac{\Lambda_{C}^{4}}{10000 H^{4}} \times(-1)^{m}} & \text { for early \& late } \eta, \\ 3-2 \sqrt{\frac{9}{4}+\frac{\Lambda_{C}^{4}}{\left[100-\frac{80}{1+\omega_{C}}\right]^{2} H^{4}} \times(-1)^{m}} & \text { for } \eta<\eta_{c} \\ 3-2 \sqrt{\frac{9}{4}+\frac{\Lambda_{C}^{4}}{400 H^{4}} \times(-1)^{m}} & \text { for } \eta \sim \eta_{c} .\end{cases}
$$




\section{B. Quasi de Sitter}

$$
n_{\bar{a}}-1 \equiv\left(\frac{\mathrm{d} \ln \Delta_{\bar{a}}(k)}{\mathrm{d} \ln k}\right)_{|k \eta| \approx 1} \approx \begin{cases}3-2 \sqrt{v^{2}+\frac{\Lambda_{C}^{4}}{10000 H^{4}} \times(-1)^{m}} & \text { for early \& late } \eta, \\ 3-2 \sqrt{v^{2}+\frac{\Lambda_{C}^{4}}{\left[100-\frac{80}{1+\omega_{C}}\right]^{2} H^{4}} \times(-1)^{m}} & \text { for } \eta<\eta_{c} \\ 3-2 \sqrt{v^{2}+\frac{\Lambda_{C}^{4}}{400 H^{4}} \times(-1)^{m}} & \text { for } \eta \sim \eta_{c} .\end{cases}
$$

where $\omega_{C}$ is a small contribution as pointed earlier.

It is important to mention here that if we use the constraint on the scalar spectral tilt as obtained from the Planck 2015 data we get the following $2 \sigma$ bound on the magnitude of the axion mass parameter:

\section{For dS:}

$0.23<\left|\frac{m_{\text {axion }}}{f_{a} H}\right|_{k c_{S} \eta \approx 1}<0.28$,

For qdS:

$$
\begin{aligned}
& 0.23 \times\left|\sqrt{1-56.18\left(\epsilon+\frac{\eta}{2}+\frac{s}{2}\right)}\right|<\left|\frac{m_{\text {axion }}}{f_{a} H}\right|_{k c_{S} \eta \approx 1} \\
& <0.28 \times\left|\sqrt{1-39.06\left(\epsilon+\frac{\eta}{2}+\frac{s}{2}\right)}\right| .
\end{aligned}
$$

Using this bound on the axion mass parameter one can restrict the value of $\Lambda_{C} / H$ as well from this calculation.

\subsection{Role of isospin breaking interaction}

To construct a cosmological Bell violating experimental setup specific role of isospin is very significant. See Ref. [3] for details on this important issue. To set up such a cosmological experiment we need here isospin breaking interactions in the present context, which can be implemented in a phenomenological way. Here one can start with any type of isospin breaking interactions which involves the mass term of the heavy field. To start with let us consider the following situation where the effective Lagrangian for the inflaton and heavy scalar field interaction is given by

$$
\begin{aligned}
S & =\int \mathrm{d}^{4} x \sqrt{-g}\left[\frac{1}{2} g^{\mu \nu}\left(\partial_{\mu} \mathcal{H}\right)^{\dagger}\left(\partial_{\nu} H\right)+\frac{1}{2} g^{\mu \nu}\left(\partial_{\mu} \phi\right)\left(\partial_{\nu} \phi\right)\right. \\
& \left.-\mathcal{H}^{\dagger}\left(\sum_{n=0}^{\infty} \mathcal{M}_{n}^{2}(\phi)(\sigma . \mathbf{n})^{n}\right) \mathcal{H}+\cdots\right],
\end{aligned}
$$

where $\cdots$ contains all other possible isospin breaking interactions in the effective Lagrangian. Here $\phi$ is inflaton field and $\mathcal{H}$ is the heavy field, which is an isospin $S U(2)$ doublet and structure of $\mathcal{H}$ is given by $\mathcal{H}=\left(\mathcal{H}_{1}, \mathcal{H}_{2}\right)$. Here
$\mathcal{M}_{n}^{2}(\phi)$ represents the mass term for every quadratic operator labeled by $n$. In the above mentioned interaction for $n=0$ it is an isospin preserving interaction and physically represents the mass term of the heavy scalar field $H$, provided the other interactions are absent or sub dominant in the effective Lagrangian. One can also identify this specific type of interaction as the leading order effect in the effective Lagrangian. Here terms for $n \geq 1$ take care of all the isospin breaking interaction in the present discussion. We here study the effect of these type of isospin breaking interactions explicitly. Here it is important to mention that $\sigma$ is the Pauli spin matrices and $\mathbf{n}$ is the unit vector along which we are taking isospin projection of the heavy field $H$. Before going into the further details it is important to note that in our computation we can use the following results:

$$
\begin{aligned}
& (\sigma . \mathbf{n})^{n}=\left(\sigma_{x} \cos m \theta+\sigma_{y} \sin m \theta\right)^{n} \\
& \quad= \begin{cases}I, & \text { for even } n \\
(\sigma . \mathbf{n}), & \text { for odd } n,\end{cases}
\end{aligned}
$$

where $I$ is an identity matrix, $m$ is an integer and $\theta$ is the angular dependence of isospin projection along the axis of measurement. Specially the role of the integer $m$ is very important for the present discussion as it amplifies the effect of quantum fluctuations. Now to understand the behaviour of the interactions in a more comprehensive manner let us investigate explicitly the first few terms in the above mentioned series:

- For $n=0$, the interaction term is given by the following expression:

$$
\mathcal{M}_{0}^{2}(\phi) \mathcal{H}^{\dagger} \mathcal{H}=\mathcal{M}_{0}^{2}(\phi)\left[\left|\mathcal{H}_{1}\right|^{2}+\left|\mathcal{H}_{2}\right|^{2}\right]
$$

In the case of axion one can identify $\mathcal{M}_{0}^{2}(\phi)=m_{\text {axion }}^{2}$.

- For $n=1$, the interaction term is given by the following expression:

$$
\begin{aligned}
\mathcal{M}_{1}^{2}(\phi) \mathcal{H}^{\dagger}(\sigma . \mathbf{n}) \mathcal{H}= & \mathcal{M}_{1}^{2}(\phi)\left[\exp (-i m \theta) \mathcal{H}_{1}^{*} \mathcal{H}_{2}\right. \\
& \left.+\exp (i m \theta) \mathcal{H}_{2}^{*} \mathcal{H}_{1}\right] .
\end{aligned}
$$


- For $n=2$, the interaction term is given by the following expression:

$$
\begin{aligned}
\mathcal{M}_{2}^{2}(\phi) \mathcal{H}^{\dagger}(\sigma . \mathbf{n})^{2} \mathcal{H} & =\mathcal{M}_{2}^{2}(\phi) \mathcal{H}^{\dagger} \mathcal{H} \\
& =\mathcal{M}_{2}^{2}(\phi)\left[\left|\mathcal{H}_{1}\right|^{2}+\left|\mathcal{H}_{2}\right|^{2}\right]
\end{aligned}
$$

- For $n=3$, the interaction term is given by the following expression:

$$
\begin{aligned}
\mathcal{M}_{3}^{2}(\phi) \mathcal{H}^{\dagger}(\sigma . \mathbf{n})^{3} \mathcal{H}= & \mathcal{M}_{3}^{2}(\phi) \mathcal{H}^{\dagger}(\sigma . \mathbf{n}) \mathcal{H} \\
= & \mathcal{M}_{3}^{2}(\phi)\left[\exp (-i m \theta) \mathcal{H}_{1}^{*} \mathcal{H}_{2}\right. \\
& \left.+\exp (i m \theta) \mathcal{H}_{2}^{*} \mathcal{H}_{1}\right]
\end{aligned}
$$

This implies that

$\mathcal{H}^{\dagger}(\sigma . \mathbf{n})^{n} \mathcal{H}=$
$\begin{cases}\mathcal{H}^{\dagger} \mathcal{H}=\left[\left|\mathcal{H}_{1}\right|^{2}+\left|\mathcal{H}_{2}\right|^{2}\right], & \text { for even } n \\ \mathcal{H}^{\dagger}(\sigma . \mathbf{n}) \mathcal{H}=\left[\exp (-i m \theta) \mathcal{H}_{1}^{*} \mathcal{H}_{2}+\exp (\operatorname{im} \theta) \mathcal{H}_{2}^{*} \mathcal{H}_{1}\right], & \text { for odd } n .\end{cases}$

Consequently the effective Lagrangian as stated in Eq. (4.291) can be recast as

$$
\begin{aligned}
& S=\int \mathrm{d}^{4} x \sqrt{-g}\left[\frac{1}{2} g^{\mu \nu}\left(\partial_{\mu} \mathcal{H}\right)^{\dagger}\left(\partial_{\nu} \mathcal{H}\right)+\frac{1}{2} g^{\mu \nu}\left(\partial_{\mu} \phi\right)\left(\partial_{\nu} \phi\right)\right. \\
& -\sum_{n=0,2,4}^{\infty} \mathcal{H}^{\dagger}\left(\mathcal{M}_{n}^{2}(\phi)(\sigma . \mathbf{n})^{n}\right) \mathcal{H} \\
& \left.-\sum_{n=1,3,5}^{\infty} \mathcal{H}^{\dagger}\left(\mathcal{M}_{n}^{2}(\phi)(\sigma . \mathbf{n})^{n}\right) \mathcal{H}+\cdots\right] \\
& =\int \mathrm{d}^{4} x \sqrt{-g}\left[\frac{1}{2} g^{\mu \nu}\left(\partial_{\mu} \mathcal{H}_{1}\right)^{*}\left(\partial_{\nu} \mathcal{H}_{1}\right)\right. \\
& +\frac{1}{2} g^{\mu \nu}\left(\partial_{\mu} \mathcal{H}_{2}\right)^{*}\left(\partial_{\nu} \mathcal{H}_{2}\right)+\frac{1}{2} g^{\mu \nu}\left(\partial_{\mu} \phi\right)\left(\partial_{\nu} \phi\right) \\
& -\left[\left|\mathcal{H}_{1}\right|^{2}+\left|\mathcal{H}_{2}\right|^{2}\right] \sum_{n=0,2,4}^{\infty} \mathcal{M}_{n}^{2}(\phi) \\
& \left.-\left[\exp (-i m \theta) \mathcal{H}_{1}^{*} \mathcal{H}_{2}+\exp (i m \theta) \mathcal{H}_{2}^{*} \mathcal{H}_{1}\right] \sum_{n=1,3,5}^{\infty} \mathcal{M}_{n}^{2}(\phi)+\cdots\right] \\
& =\int \mathrm{d}^{4} x \sqrt{-g}\left[\frac{1}{2} g^{\mu v}\left(\partial_{\mu} \mathcal{H}_{1}\right)^{*}\left(\partial_{\nu} \mathcal{H}_{1}\right)\right. \\
& +\frac{1}{2} g^{\mu \nu}\left(\partial_{\mu} \mathcal{H}_{2}\right)^{*}\left(\partial_{\nu} \mathcal{H}_{2}\right)+\frac{1}{2} g^{\mu \nu}\left(\partial_{\mu} \phi\right)\left(\partial_{\nu} \phi\right) \\
& -\left[\left|\mathcal{H}_{1}\right|^{2}+\left|\mathcal{H}_{2}\right|^{2}\right] \mathcal{M}_{\text {even }}^{2}(\phi) \\
& \left.-\left[\exp (-i m \theta) \mathcal{H}_{1}^{*} \mathcal{H}_{2}+\exp (i m \theta) \mathcal{H}_{2}^{*} H_{1}\right] \mathcal{M}_{\mathbf{o d d}}^{2}(\phi)+\cdots\right],
\end{aligned}
$$

where the mass terms $\mathcal{M}_{\text {even }}^{2}(\phi)$ and $\mathcal{M}_{\text {odd }}^{2}(\phi)$ are defined as

$$
\begin{aligned}
& \mathcal{M}_{\text {even }}^{2}(\phi)=\sum_{n=0,2,4}^{\infty} \mathcal{M}_{n}^{2}(\phi), \\
& \mathcal{M}_{\text {odd }}^{2}(\phi)=\sum_{n=1,3,5}^{\infty} \mathcal{M}_{n}^{2}(\phi) .
\end{aligned}
$$

From Eq. (4.298), one can construct a mass matrix as given by 16

$$
\begin{aligned}
& \mathcal{M}^{2}(\phi)=\left(\begin{array}{cc}
\mathcal{M}_{\text {even }}^{2}(\phi) & \exp (-i m \theta) \mathcal{M}_{\text {odd }}^{2}(\phi) \\
\exp (i m \theta) \mathcal{M}_{\text {odd }}^{2}(\phi) & \mathcal{M}_{\text {even }}^{2}(\phi)
\end{array}\right)
\end{aligned}
$$

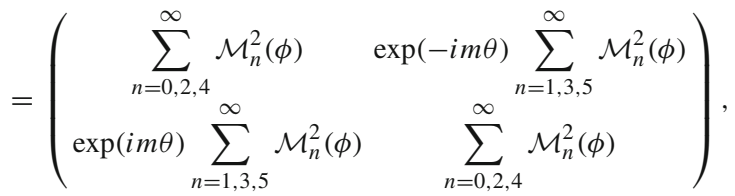

and one can construct a physical mass eigenbasis from the following eigenvalues:

$$
\begin{aligned}
\lambda_{ \pm}(\phi) & =\sqrt{\mathcal{M}_{\text {even }}^{2}(\phi) \pm \mathcal{M}_{\text {odd }}^{2}(\phi)} \\
& =\sqrt{\sum_{n=0,2,4}^{\infty} \mathcal{M}_{n}^{2}(\phi) \pm \sum_{n=1,3,5}^{\infty} \mathcal{M}_{n}^{2}(\phi)} .
\end{aligned}
$$

One can also express the eigenvalues presented in Eq. (4.304) as

$$
\begin{aligned}
\lambda_{ \pm}(\phi) & =\sqrt{\mathcal{M}_{\text {even }}^{2}(\phi)+\operatorname{sign}(\sigma . \mathbf{n}) \mathcal{M}_{\text {odd }}^{2}(\phi)} \\
& =\sqrt{\sum_{n=0,2,4}^{\infty} \mathcal{M}_{n}^{2}(\phi)+\operatorname{sign}(\sigma . n) \sum_{n=1,3,5}^{\infty} \mathcal{M}_{n}^{2}(\phi)}
\end{aligned}
$$

where $\operatorname{sign}(\sigma \cdot \mathbf{n})= \pm 1$. As the inflaton field $\phi$ is a function of the conformal time $\eta$, one can express the eigenvalues in terms of $\eta$ as well. Consequently the physical mass parameter for these two eigenstates can be written as

$$
\begin{aligned}
& { }^{16} \text { Here the determinant and trace of the mass matrix is given by } \\
& \operatorname{Det}\left[\mathcal{M}_{\text {even }}^{2}(\phi)\right]=\lambda_{ \pm}^{2}(\phi)\left(\lambda_{ \pm}(\phi)-\sqrt{2} \mathcal{M}_{\text {odd }}(\phi)\right) \\
& \left(\lambda_{ \pm}(\phi)+\sqrt{2} \mathcal{M}_{\text {odd }}(\phi)\right) \\
& =\lambda_{ \pm}^{2}(\phi)\left(\sqrt{2} \mathcal{M}_{\text {even }}(\phi)-\lambda_{ \pm}(\phi)\right) \\
& \left(\sqrt{2} \mathcal{M}_{\text {even }}(\phi)+\lambda_{ \pm}(\phi)\right), \\
& \operatorname{Tr}\left[\mathcal{M}_{\text {even }}^{2}(\phi)\right]=\mathcal{M}_{\text {even }}^{2}(\phi)+\mathcal{M}_{\text {odd }}^{2}(\phi)=\lambda_{ \pm}^{2}(\phi),
\end{aligned}
$$




$$
\begin{aligned}
\frac{\lambda_{ \pm}(\eta)}{H} & =\sqrt{\left(\frac{\mathcal{M}_{\text {even }}(\eta)}{H}\right)^{2} \pm\left(\frac{\mathcal{M}_{\text {odd }}^{2}(\eta)}{H}\right)^{2}} \\
& =\sqrt{\left(\frac{\sum_{n=0,2,4}^{\infty} \mathcal{M}_{n}^{2}(\eta)}{H}\right)^{2} \pm\left(\frac{\sum_{n=1,3,5}^{\infty} \mathcal{M}_{n}^{2}(\eta)}{H}\right)^{2}}
\end{aligned}
$$

Similarly in terms of the sign function the physical mass parameter can be expressed as

$$
\begin{aligned}
\frac{\lambda_{ \pm}(\eta)}{H} & =\sqrt{\left(\frac{\mathcal{M}_{\text {even }}(\eta)}{H}\right)^{2}+\operatorname{sign}(\sigma \cdot \mathbf{n})\left(\frac{\mathcal{M}_{\text {odd }}^{2}(\eta)}{H}\right)^{2}} \\
& =\sqrt{\left(\frac{\sum_{n=0,2,4}^{\infty} \mathcal{M}_{n}^{2}(\eta)}{H}\right)^{2}+\operatorname{sign}(\sigma . \mathbf{n})\left(\frac{\sum_{n=1,3,5}^{\infty} \mathcal{M}_{n}^{2}(\eta)}{H}\right)^{2}} .
\end{aligned}
$$

Now to show the time-dependent behaviour of the mass parameters constructed out of the even and odd contributions in the interaction picture here we now and then choose some time-dependent mass profile. For example,

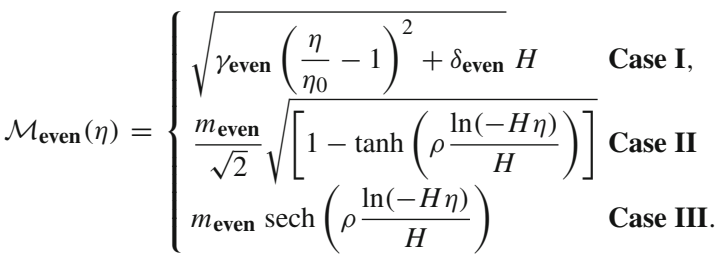

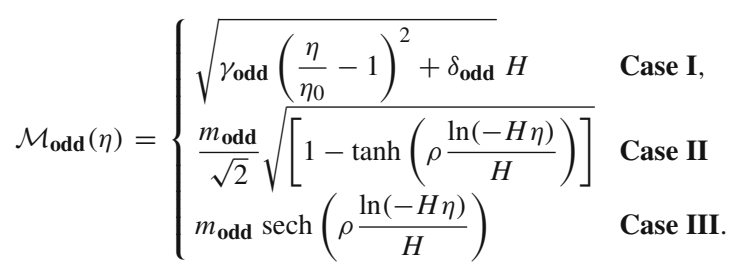

Consequently the physical mass parameter for these two eigenstates can be written as

$$
\begin{aligned}
\frac{\lambda_{ \pm}(\eta)}{H} & =\sqrt{\left(\frac{\mathcal{M}_{\text {even }}(\eta)}{H}\right)^{2} \pm\left(\frac{\mathcal{M}_{\text {odd }}^{2}(\eta)}{H}\right)^{2}} \\
& = \begin{cases}\sqrt{\left(\gamma_{\text {even }} \pm \gamma_{\text {odd }}\right)\left(\frac{\eta}{\eta_{0}}-1\right)^{2}+\left(\delta_{\text {even }} \pm \delta_{\text {odd }}\right)} & \text { Case I, } \\
\frac{1}{\sqrt{2} H} \sqrt{\left\{m_{\text {even }}^{2} \pm m_{\text {even }}^{2}\right\}\left[1-\tanh \left(\rho \frac{\ln (-H \eta)}{H}\right)\right]} & \text { Case II } \\
\frac{1}{H} \sqrt{\left\{m_{\text {even }}^{2} \pm m_{\text {even }}^{2}\right\}} \operatorname{sech}\left(\rho \frac{\ln (-H \eta)}{H}\right) & \text { Case III. }\end{cases}
\end{aligned}
$$

Similarly in terms of the sign function the physical mass parameter for these two eigenstates can be expressed as

$$
\begin{aligned}
& \frac{\lambda_{ \pm}(\eta)}{H}=\sqrt{\left(\frac{\mathcal{M}_{\text {even }}(\eta)}{H}\right)^{2} \pm\left(\frac{\mathcal{M}_{\text {odd }}^{2}(\eta)}{H}\right)^{2}} \\
& = \begin{cases}\sqrt{\left(\gamma_{\text {even }}+\operatorname{sign}(\sigma \cdot \mathbf{n}) \gamma_{\text {odd }}\right)\left(\frac{\eta}{\eta_{0}}-1\right)^{2}+\left(\delta_{\text {even }}+\operatorname{sign}(\sigma \cdot \mathbf{n}) \delta_{\text {odd }}\right)} & \text { Case I, } \\
\frac{1}{\sqrt{2} H} \sqrt{\left\{m_{\text {even }}^{2}+\operatorname{sign}(\sigma \cdot \mathbf{n}) m_{\text {even }}^{2}\right\}\left[1-\tanh \left(\rho \frac{\ln (-H \eta)}{H}\right)\right]} & \text { Case II } \\
\frac{1}{H} \sqrt{\left\{m_{\text {even }}^{2}+\operatorname{sign}(\sigma \cdot \mathbf{n}) m_{\text {even }}^{2}\right\}} \operatorname{sech}\left(\rho \frac{\ln (-H \eta)}{H}\right) & \text { Case III. }\end{cases}
\end{aligned}
$$

For the sake of simplicity here we introduce new parameters defined as

$$
\begin{aligned}
\gamma_{ \pm} & =\gamma_{\text {even }} \pm \gamma_{\text {odd }}=\gamma_{\text {even }}+\operatorname{sign}(\sigma . n) \gamma_{\text {odd }} \\
\delta_{ \pm} & =\delta_{\text {even }} \pm \delta_{\text {odd }}=\delta_{\text {even }}+\operatorname{sign}(\sigma . n) \delta_{\text {odd }} \\
m_{0 \pm} & =\sqrt{m_{\text {even }}^{2} \pm m_{\text {even }}^{2}}=\sqrt{m_{\text {even }}^{2}+\operatorname{sign}(\sigma . n) m_{\text {even }}^{2}}
\end{aligned}
$$

Then in terms of these new parameters the physical mass parameter for these two eigenstates can be recast as

$$
\begin{aligned}
\frac{\lambda_{ \pm}(\eta)}{H} & =\sqrt{\left(\frac{\mathcal{M}_{\text {even }}(\eta)}{H}\right)^{2} \pm\left(\frac{\mathcal{M}_{\text {odd }}^{2}(\eta)}{H}\right)^{2}} \\
& = \begin{cases}\sqrt{\gamma_{ \pm}\left(\frac{\eta}{\eta_{0}}-1\right)^{2}+\delta_{ \pm}} & \text {Case I } \\
\frac{m_{0 \pm}}{\sqrt{2} H} \sqrt{\left[1-\tanh \left(\rho \frac{\ln (-H \eta)}{H}\right)\right]} & \text { Case II } \\
\frac{m_{0 \pm}}{H} \operatorname{sech}\left(\rho \frac{\ln (-H \eta)}{H}\right) & \text { Case III. }\end{cases}
\end{aligned}
$$

In Fig. 16 we explicitly show the conformal time scale dependent behaviour of heavy particle mass profile for two eigenstates.

Let us give the physical interpretation of both eigenvalues obtained in this context. According to the phenomenological construction of the above mentioned effective Lagrangian $\mathcal{H}$ is a complex $S U(2)$ isospin doublet. During the particle production $\mathcal{H}=\left(\mathcal{H}_{1}, \mathcal{H}_{2}\right)$ and its complex conjugate part $\mathcal{H}^{*}=\left(\mathcal{H}_{1}^{*}, \mathcal{H}_{2}^{*}\right)=\left(\tilde{\mathcal{H}}_{1},-\tilde{\mathcal{H}}_{2}\right)$ both of them participate in the creation of particle and antiparticle production in the present context.

Here we need the following requirements:

- To avoid any instability in the eigenbasis, eigenvalues of the mass matrix are always positive definite. Consequently we get the following constraint condition:

$$
\begin{aligned}
\mathcal{M}_{\text {even }}^{2}(\phi) & \geq \mathcal{M}_{\text {odd }}^{2}(\phi) \Rightarrow \sum_{n=0,2,4}^{\infty} \mathcal{M}_{n}^{2}(\phi) \\
& \geq \sum_{n=1,3,5}^{\infty} \mathcal{M}_{n}^{2}(\phi) .
\end{aligned}
$$




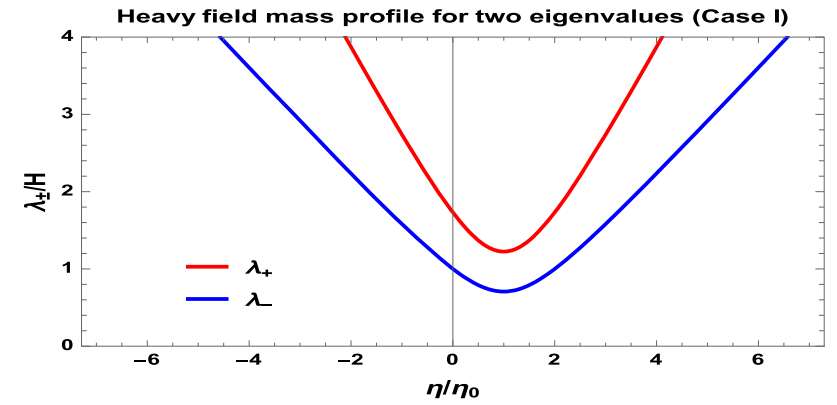

(a) Here we set $\gamma_{\text {even }}=1=\delta_{\text {even }}, \gamma_{\text {odd }}=0.5=\delta_{\text {odd }}$.

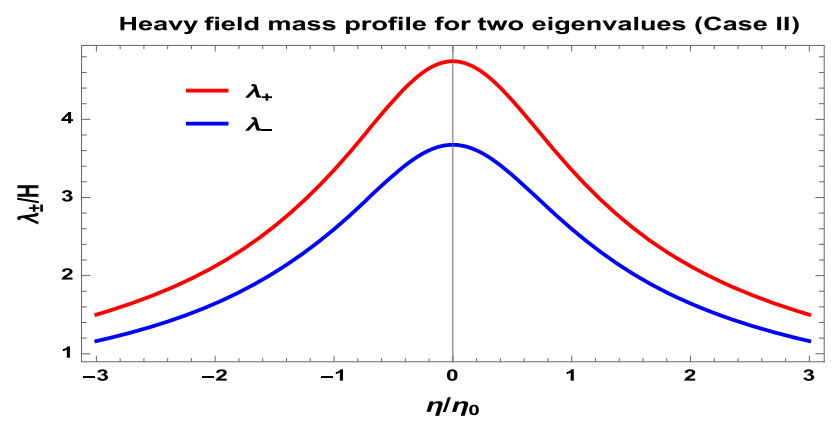

(b) Here we set $m_{\text {even }}=3, m_{\text {odd }}=1.5$ and $\rho / H=1$.

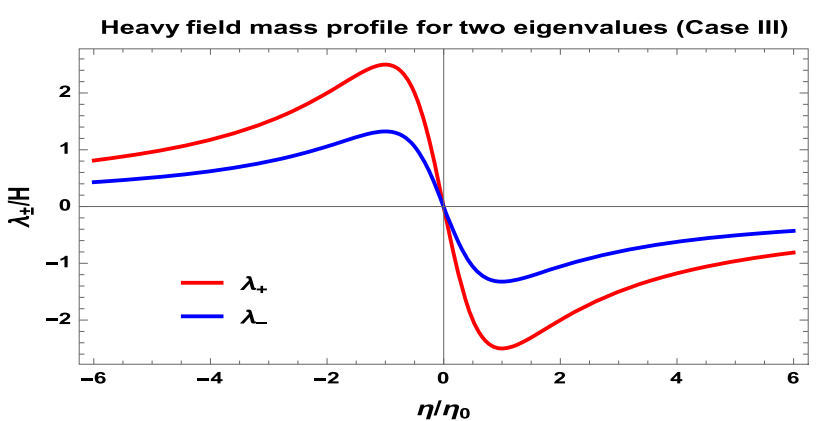

(c) Here we set $m_{\text {even }}=2, m_{\text {odd }}=1.5$ and $\rho / H=1$.

Fig. 16 Conformal time scale dependent behaviour of heavy particle mass profile for two eigenstates

For the specific three mass profile one can rewrite this constraint condition as

$0 \leq \begin{cases}{\left[\gamma_{-}\left(\frac{\eta}{\eta_{0}}-1\right)^{2}+\delta_{-}\right] H^{2}} & \text { Case II } \\ \frac{m_{0-}^{2}\left[1-\tanh \left(\rho \frac{\ln (-H \eta)}{2}\right)\right]}{H} & \text { Case II } \\ m_{0-}^{2} \operatorname{sech}^{2}\left(\rho \frac{\ln (-H \eta)}{H}\right) & \text { Case III. }\end{cases}$

- At late time scales:

$$
\begin{aligned}
& \mathcal{M}_{\text {even }}^{2}(\phi) \sim \mathcal{M}_{\text {odd }}^{2}(\phi) \Rightarrow \sum_{n=0,2,4}^{\infty} \mathcal{M}_{n}^{2}(\phi) \\
& \sim \sum_{n=1,3,5}^{\infty} \mathcal{M}_{n}^{2}(\phi) .
\end{aligned}
$$

Here if the value of $\mathcal{M}_{\text {even }}(\phi)$ increases then the magnitude of $\mathcal{M}_{\text {odd }}(\phi)$ also increases as they are of the same order. Consequently, the eigenvalue of the mass matrix also increases. For the specific three mass profile one can rewrite this constraint condition as

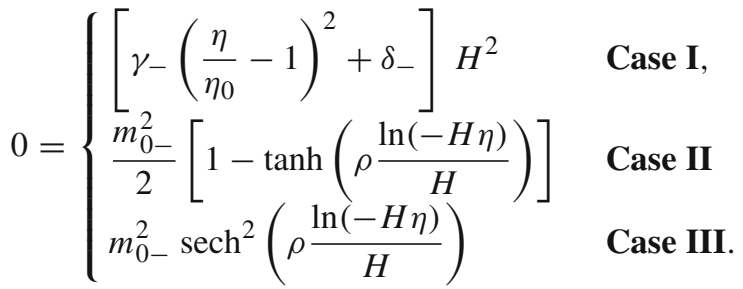

- Another crucial requirement is that the eigenvalue of the mass matrix is of the order of UV cut-off of the EFT $\Lambda_{U V}$. In our purpose one can choose $\Lambda_{U V} \sim M_{p}=$ $2.43 \times 10^{18} \mathrm{GeV}$. This implies that

$$
\begin{aligned}
& \lambda_{ \pm}^{2} \sim \mathcal{M}_{\text {even }}^{2}(\phi) \pm \mathcal{M}_{\text {odd }}^{2}(\phi) \\
& =\left[\sum_{n=0,2,4}^{\infty} \mathcal{M}_{n}^{2}(\phi) \pm \sum_{n=1,3,5}^{\infty} \mathcal{M}_{n}^{2}(\phi)\right] \approx \Lambda_{U V}^{2} \sim M_{p}^{2} .
\end{aligned}
$$

This is necessarily required to measure the eigenvalues with the given Bell violating cosmological setup. For the specific three mass profiles one can rewrite this constraint condition as

$\lambda_{ \pm}^{2} \sim \Lambda_{U V}^{2}= \begin{cases}{\left[\gamma_{ \pm}\left(\frac{\eta}{\eta_{0}}-1\right)^{2}+\delta_{ \pm}\right] H^{2}} & \text { Case I, } \\ \frac{m_{0 \pm}^{2}}{2}\left[1-\tanh \left(\rho \frac{\ln (-H \eta)}{H}\right)\right] & \text { Case II } \\ m_{0 \pm}^{2} \operatorname{sech}^{2}\left(\rho \frac{\ln (-H \eta)}{H}\right) & \text { Case III. }\end{cases}$

- When all the isospin breaking interactions are absent from the effective Lagrangian in that case the eigenvalue of the mass matrix is given by

$\lambda(\phi) \sim \mathcal{M}_{\text {even }}(\phi)$.

This is another very crucial criterion, relevant for observation during the time of heavy mass particle creation with $S U(2)$ isospin singlet state. For the specific three mass profile one can rewrite this constraint condition as 


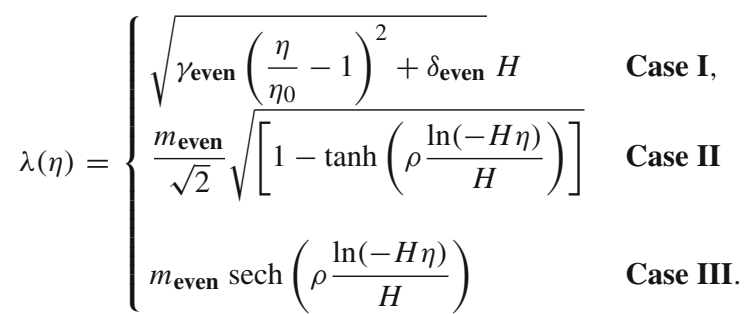

- Here signature of the angular parameter $\theta$ and its functional dependence on the background plays very crucial role to setup the Bell violating setup in the context of cosmology. For a very simplified case one can assume that $\theta$ is a constant. In this case if we identify the particle mass eigenvalue as $\lambda_{ \pm}(\phi)$, then the antiparticle mass eigenvalues also characterized by $\lambda_{ \pm}(\phi)$. A sign flip in the eigenvalue of the antiparticle mass eigenstate may happen if the angular parameter $\theta$ is background dependent and not a constant quantity.

Now we will include additional quartic contributions in the phenomenological effective Lagrangian to describe isospin breaking interactions through self integrations between the heavy fields. In that case Eq. (4.291) can be modified as

$$
\begin{aligned}
S= & \int \mathrm{d}^{4} x \sqrt{-g}\left[\frac{1}{2} g^{\mu v}\left(\partial_{\mu} \mathcal{H}\right)^{\dagger}\left(\partial_{\nu} \mathcal{H}\right)+\frac{1}{2} g^{\mu \nu}\left(\partial_{\mu} \phi\right)\left(\partial_{\nu} \phi\right)\right. \\
& -\mathcal{H}^{\dagger}\left(\sum_{n=0}^{\infty} \mathcal{M}_{n}^{2}(\phi)(\sigma . \mathbf{n})^{n}\right) \\
& \left.\mathcal{H}-\left(\mathcal{H}^{\dagger}\left(\sum_{n=0}^{\infty} G_{n}(\phi)(\sigma . \mathbf{n})^{n}\right) \mathcal{H}\right)^{2}+\cdots\right]
\end{aligned}
$$

where $G_{n}(\phi)$ is the quartic coupling for each values of $n$. Let us analyze the effect of only last term first and then we will comment on the total combined effect. Here in this context one can write down only the quartic contribution as

$$
\begin{aligned}
S_{\text {quartic }}= & -\int \mathrm{d}^{4} x \sqrt{-g}\left(\mathcal{H}^{\dagger}\left(\sum_{n=0}^{\infty} G_{n}(\phi)(\sigma . \mathbf{n})^{n}\right) \mathcal{H}\right)^{2}, \\
= & -\int \mathrm{d}^{4} x \sqrt{-g}\left[\mathcal{H}^{\dagger}\left(\sum_{n=0,2,4}^{\infty} G_{n}(\phi)(\sigma . \mathbf{n})^{n}\right)\right. \\
& \left.\times \mathcal{H}+\mathcal{H}^{\dagger}\left(\sum_{n=1,3,5}^{\infty} G_{n}(\phi)(\sigma . \mathbf{n})^{n}\right) \mathcal{H}\right]^{2}, \\
= & -\int \mathrm{d}^{4} x \sqrt{-g}\left[\left(\left|\mathcal{H}_{1}\right|^{2}+\left|\mathcal{H}_{2}\right|^{2}\right) \sum_{n=0,2,4}^{\infty} G_{n}(\phi)\right. \\
& \left.+\left[\exp (-i m \theta) \mathcal{H}_{1}^{*} \mathcal{H}_{2}+\exp (i m \theta) \mathcal{H}_{2}^{*} \mathcal{H}_{1}\right] \sum_{n=1,3,5}^{\infty} G_{n}(\phi)\right]^{2},
\end{aligned}
$$

$$
\begin{aligned}
= & -\int \mathrm{d}^{4} x \sqrt{-g}\left[\left(\left|\mathcal{H}_{1}\right|^{2}+\left|\mathcal{H}_{2}\right|^{2}\right) G_{\text {even }}(\phi)\right. \\
& \left.+\left[\exp (-i m \theta) \mathcal{H}_{1}^{*} \mathcal{H}_{2}+\exp (i m \theta) \mathcal{H}_{2}^{*} \mathcal{H}_{1}\right] G_{\text {odd }}(\phi)\right]^{2},
\end{aligned}
$$

where the couplings $G_{\text {even }}(\phi)$ and $G_{\text {odd }}(\phi)$ are defined by the following expressions:

$$
\begin{aligned}
& G_{\text {even }}(\phi)=\sum_{n=0,2,4}^{\infty} G_{n}(\phi), \\
& G_{\text {odd }}(\phi)=\sum_{n=1,3,5}^{\infty} G_{n}(\phi) .
\end{aligned}
$$

Here further we assume that all the higher mass dimensional non-renormalizable operators of the structure

$\mathcal{O}_{N R}=\sum_{d>2}^{\infty} \frac{1}{\Lambda_{U V}^{2 d-4}}\left[\mathcal{H}^{\dagger}\left(\sum_{n=0}^{\infty} Z_{n}(\phi)(\sigma . \mathbf{n})^{n}\right) \mathcal{H}\right]^{d}$

are highly suppressed by the EFT cut-off scale $\Lambda_{U V}$, so that one may neglect all such contributions in the EFT Lagrangian.

Further combining the effect of quartic and quadratic interaction the renormalizable part of the total effective potential can be written as

$$
V\left(\mathcal{H}_{1}, \mathcal{H}_{2}, \theta\right)=V_{\mathbf{I P}}\left(\mathcal{H}_{1}, \mathcal{H}_{2}\right)+V_{\mathbf{I B}}\left(\mathcal{H}_{1}, \mathcal{H}_{2}, \theta\right),
$$

where the isospin preserving and isospin breaking renormalizable contributions are given by

$$
\begin{aligned}
V_{\mathbf{I P}}\left(\mathcal{H}_{1}, \mathcal{H}_{2}\right)= & \mathcal{M}_{\text {even }}^{2}(\phi)\left(\left|\mathcal{H}_{1}\right|^{2}+\left|\mathcal{H}_{2}\right|^{2}\right) \\
& +G_{\text {even }}^{2}(\phi)\left(\left|\mathcal{H}_{1}\right|^{2}+\left|\mathcal{H}_{2}\right|^{2}\right)^{2}, \\
V_{\mathbf{I B}}\left(\mathcal{H}_{1}, \mathcal{H}_{2}, \theta\right)= & \mathcal{M}_{\text {odd }}^{2}(\phi)\left[\exp (-i m \theta) \mathcal{H}_{1}^{*} \mathcal{H}_{2}+\exp (i m \theta) \mathcal{H}_{2}^{*} \mathcal{H}_{1}\right] \\
& +G_{\text {odd }}^{2}(\phi)\left[\exp (-2 i m \theta)\left(\mathcal{H}_{1}^{*} \mathcal{H}_{2}\right)^{2}\right. \\
& \left.++\exp (2 i m \theta)\left(\mathcal{H}_{2}^{*} \mathcal{H}_{1}\right)^{2}\left|\mathcal{H}_{1}\right|^{2}\left|\mathcal{H}_{2}\right|^{2}\right] \\
& +G_{\text {even }}(\phi) G_{\text {odd }}(\phi)\left(\left|\mathcal{H}_{1}\right|^{2}+\left|\mathcal{H}_{2}\right|^{2}\right) \\
& \times\left[\exp (-i m \theta) \mathcal{H}_{1}^{*} \mathcal{H}_{2}+\exp (i m \theta) \mathcal{H}_{2}^{*} \mathcal{H}_{1}\right] .
\end{aligned}
$$

Now exactly identifying the specific role of self interactions in the present context one can consider the following simplest but special physical situation where the self coupling can be connected with the mass parameter for the even type of interactions as

$G_{\text {even }}(\phi)=\mathcal{M}_{\text {even }}^{2}(\phi)$.

According to our three previous choice of mass parameter here one can re-express the self coupling parameter for the even type of contributions in the isospin preserving interactions as 
$G_{\text {even }}(\eta)=\mathcal{M}_{\text {even }}^{2}(\eta)= \begin{cases}{\left[\gamma_{\text {even }}\left(\frac{\eta}{\eta_{0}}-1\right)^{2}+\delta_{\text {even }}\right] H^{2}} & \text { Case I, } \\ \frac{m_{\text {even }}^{2}\left[1-\tanh \left(\rho \frac{\ln (-H \eta)}{H}\right)\right]}{2} & \text { Case II } \\ m_{\text {even }}^{2} \operatorname{sech}^{2}\left(\rho \frac{\ln (-H \eta)}{H}\right) & \text { Case III. }\end{cases}$

Similarly following the same argument one can also express the connection between self coupling and mass parameter for the odd type of interactions as

$G_{\text {odd }}(\phi)=\mathcal{M}_{\text {odd }}^{2}(\phi)$.

According to our three previous choice of mass parameter here one can re-express the self coupling parameter for the odd type of contributions in the isospin violating interactions as

$$
\begin{aligned}
G_{\text {odd }}(\eta) & =\mathcal{M}_{\text {odd }}^{2}(\eta) \\
& = \begin{cases}{\left[\gamma_{\text {odd }}\left(\frac{\eta}{\eta_{0}}-1\right)^{2}+\delta_{\text {odd }}\right] H^{2}} & \text { Case I } \\
\frac{m_{\text {odd }}^{2}}{2}\left[1-\tanh \left(\rho \frac{\ln (-H \eta)}{H}\right)\right] & \text { Case II } \\
m_{\text {odd }}^{2} \operatorname{sech}^{2}\left(\rho \frac{\ln (-H \eta)}{H}\right) & \text { Case III. }\end{cases}
\end{aligned}
$$

Because of this identification one can also express the two eigenvalues of the mass eigenstates in terms of the self coupling parameters as

$$
\begin{aligned}
\lambda_{ \pm} & =\sqrt{G_{\text {even }} \pm G_{\text {odd }}}=\sqrt{G_{\text {even }}+\operatorname{sign}(\sigma \cdot \mathbf{n}) G_{\text {odd }}} \\
& =\mathcal{G}_{ \pm}(\eta),
\end{aligned}
$$

where $\mathcal{G}_{ \pm}(\eta)$ are defined as

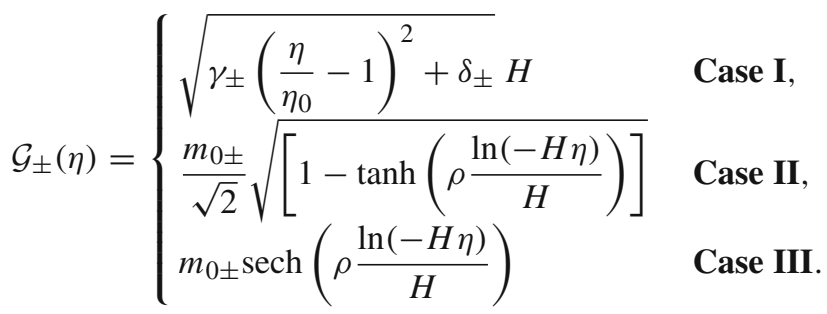

Here it is important to mention here that the isospin preserving renormalizable interaction can be identified with the axion interaction as discussed in the earlier section. In the case of axion interaction we have neglected the quartic interaction due to the very small back-reaction. But in the case of an axion also one can include the effects of isospin breaking interaction to measure the mass eigenvalues in the eigenstate as discussed here for any heavy fields. During the post inflationary era to avoid the problem of formation of domain wall one can set the axion potential to be zero at that period. As the signatures of these heavy fields or the axion have not been observed yet through any observational probes, one can treat such heavy fields or the axions correspond to a component of dark matter and the corresponding density fluctuations can be treated as isocurvature fluctuations.

\section{Conclusion}

To summarize, in the present article, we have addressed the following points:

- Firstly we have briefly reviewed Bell's inequality in quantum mechanics and its implications. For this we reviewed the proof of Bell's inequality. Further we have discussed the violation of Bell's inequality in the context of quantum mechanics. Also we have given the explanation for such a violation, which finally gives rise to new physical concepts and phenomena.

- Next we have briefly discussed about the setup for Bell's inequality violating test experiment in the context of primordial cosmology. Further we have studied the creation of new massive particles as introduced in the context of inflationary paradigm for various choice of timedependent mass profile. We have also presented the calculation for the three limiting situations: (1) $m \approx H,(2)$ $m>>H$ and (3) $m<<H$. To describe a very small fraction of particle creation after inflation we have computed the expression for the Bogoliubov coefficient $\beta$ in FLRW space-time, which characterizes the amount of mixing between the two types of WKB solutions. Next using the results for the Bogoliubov coefficients we have further calculated reflection and transmission coefficients, number density and energy density of the created particles for various mass profiles. Here we have provided the results for three specific cases:

1. $\left|k c_{S} \eta\right|=c_{S} k / a H<<1$ (super horizon),

2. $\left|k c_{S} \eta\right|=c_{S} k / a H \approx 1$ (horizon crossing),

3. $\left|k c_{S} \eta\right|=c_{S} k / a H>>1$ (sub horizon).

Further we have studied cosmological scalar curvature fluctuations in the presence of new massive particles for arbitrary choice of initial condition and also for any arbitrary mass profile. Here we have explicitly derived the expression for one point and two point correlation function using the in-in formalism. Then we have quoted the results for the three limiting situations: (1) $m \approx H$, (2) $m>>H$ and (3) $m<<H$ in super horizon, sub horizon and horizon crossing. Here in our computation we have introduced a new cosmological observable which captures the effect of Bell's inequality violation in cosmology. Further we have expressed the scale of inflation in terms of the amount of Bell's inequality violation in cosmology experimental setup using model independent 
prescription like EFT. Additionally we have derived a model-independent expression for the first Hubble slowroll parameter $\epsilon=-\dot{H} / H^{2}$ and the tensor-to-scalar ratio in terms of the Bell inequality violating observable within the framework of EFT. Additionally, we have given an estimate of heavy field mass parameter $m / H$ to violate Bell's inequality within the cosmological setup.

- It is important to note that when all the EFT interactions are absent in that case both $c_{S} \sim \tilde{c}_{S}=1$ and one can get back the results for canonical slow-roll models. On the other hand when the EFT interactions are switched on within the present description, this can enable one to accommodate the non-canonical as well as non-minimal interactions within this framework. In that case both $c_{S}$ and $\tilde{c}_{S}$ are less than unity and in such a situation one can always constrain the sound speed parameter as well as the strength of the EFT interactions using observational probes (Planck 2015 data). One can easily compare the present setup with the effective time varying mass parameter with the axions with a time varying decay constant. For $m<<H$ the last term in the effective action is absent and in that case the reduced form of the action will enable one to explain the EFT of inflation in the presence of previously mentioned non-trivial effective interactions. Once we switch off all such interactions the above action mimics the case for single field slow-roll inflation.

- Further we have given an example of an axion model with time-dependent decay constant as appearing in the context of string theory. Hence we have mentioned the effective axion interaction of axion fields. Now to give an analogy between the newly introduced massive particle and the axion we have further discussed the creation of axion in early universe. Next we have established the one to one correspondence between heavy field and axion by comparing the particle creation mechanism, one and two point correlation functions. Additionally, we have given an estimate of axion mass parameter $m_{\text {axion }} / f_{a} H$ to violate Bell's inequality within cosmological setup. Finally, we have discussed the specific role of isospin breaking interaction for axion type of heavy fields to measure the effect of Bell's inequality violation in primordial cosmology.

- Next we have explicitly shown the role of quantum decoherence in cosmological setup to violate Bell's inequality. Additionally here we have also mentioned a possibility to enhance the value of primordial non-Gaussianity from Bell's inequality violating setup in presence of massive time-dependent field profile. Further we have discussed the role of three specific time-dependent mass profiles for producing massive particles and to generate quantum fluctuations. Finally, we have discussed the role of arbitrary spin heavy field to violate Bell's inequality. Here we have provided a bound on the mass parameter for massive scalar with spin $\mathcal{S}=0$, axion with $\operatorname{spin} \mathcal{S}=0$, graviton with $\operatorname{spin} \mathcal{S}=2$ and for particles with high $\operatorname{spin} \mathcal{S}>2$ in horizon crossing, super horizon and sub horizon regime.

The future prospects of our work are appended below:

- In this work we have not explored in detail the possibility of enhancing the primordial non-Gaussianity from the violation of Bell's inequality and the exact role of timedependent mass profile for heavy fields. In appendix we have pointed on some of the issues but not given detailed calculation on this issue. In near future we are planning to address this important issue.

- One can also comment on the dependence on timedependent mass profile for the heavy field to derive the consistency relation in the context of inflationary cosmology. Due to the enhancement of the primordial nonGaussian amplitude it is expected from the basic understanding that due to the presence of such non-negligible Bell violating contribution, all the inflationary consistency relations will modify significantly. In future we are also planning to derive all such modified consistency relations from this work.

- In this work we have implemented the idea of Bell violation in the context of inflationary cosmology. But the explicit role of an alternative idea of inflation to design a Bell inequality violating experiment in cosmology is not studied yet. One can check whether this can be done or not. If this is possible, then one can also study the consequences from this, including non-Gaussianities.

- The explicit role of entanglement entropy is very important to understand the underlying physical principles in the present context for the de Sitter case and the quasi de Sitter case. In this paper we have not addressed this issue in detail, which one might address in the future.

- One can also carry forward our analysis in the context of a higher derivative gravity set-up which nobody has addressed yet.

- To give a model-independent bound on the scale of inflation and primordial gravitational waves we have defined a new cosmological observable which explicitly captures the effect of violation of Bell's inequality in cosmology. But for this we need prior knowledge of such observables. We have only mentioned the explicit role of isospin breaking interactions in this context. But in this work we have not studied the exact connection between such isospin breaking interactions for heavy fields and newly defined Bell violating cosmological observable. One can also address this issue to comment on the measurement of such observables through various observational probes.

Acknowledgements SC would like to thank the Department of Theoretical Physics, Tata Institute of Fundamental Research, Mumbai, for 
providing me Visiting (Post-Doctoral) Research Fellowship. SC would like to thank Gautam Mandal and Guruprasad Kar for useful discussions and suggestions. SC takes this opportunity to thank sincerely Sandip P. Trivedi and Shiraz Minwalla for their constant support and inspiration. SC additionally takes this opportunity to thank the organizers of STRINGS, 2015, International Centre for Theoretical Science, Tata Institute of Fundamental Research (ICTS,TIFR), Indian Institute of Science (IISC) and specially Shiraz Minwalla for giving the opportunity to participate in STRINGS, 2015 and also providing the local hospitality during the work. SC also thanks the organizers of National String Meet 2015 and the International Conference on Gravitation and Cosmology, IISER, Mohali and COSMOASTRO 2015, Institute of Physics, Bhubaneswar, for providing local hospitality during the work. SC also thanks the organizers of the School and Workshop on Large Scale Structure: From Galaxies to Cosmic Web, The Inter-University Centre for Astronomy and Astrophysics (IUCAA), Pune, India, and specially Aseem Paranjape and Varun Sahni for providing the academic visit during the work. A very special thanks to The Inter-University Centre for Astronomy and Astrophysics (IUCAA), Pune, India, where the problem was formulated, and the Institute of Physics, Bhubaneswar, where part of the work was done. SC also would like to thank the Department of Theoretical Physics, Indian Association for the Cultivation of Science, Kolkata, and specially Somitra SenGupta for providing the academic visit during the work. RS would like to thank the Department of Theoretical Physics, Tata Institute of Fundamental Research and the Department of Physics, Savitribai Phule Pune University, for providing a platform to work on this topic and for their constant support and resources. Last but not least, we would all like to acknowledge our debt to the people of India for their generous and steady support for research in the natural sciences, especially for theoretical high energy physics, string theory and cosmology.

Open Access This article is distributed under the terms of the Creative Commons Attribution 4.0 International License (http://creativecomm ons.org/licenses/by/4.0/), which permits unrestricted use, distribution, and reproduction in any medium, provided you give appropriate credit to the original author(s) and the source, provide a link to the Creative Commons license, and indicate if changes were made. Funded by SCOAP . $^{3}$

\section{Appendix}

6.1 Role of quantum decoherence in Bell violating cosmological setup

Before going to the next section let us briefly discuss the some more issues related to the cosmological setup in which we want to study the violation of Bell inequality. In this context the basis field eigenstates can be identified with $|\zeta(\mathbf{x})\rangle$, which satisfies the following eigenvalue equation:

$\hat{\zeta}(\mathbf{x})|\zeta(\mathbf{x})\rangle=\zeta(\mathbf{x})|\zeta(\mathbf{x})\rangle$

where the quantum operator $\hat{\zeta}(\mathbf{x})$ is specified in any point in space-time and the eigenvalue $\zeta(\mathbf{x})$ represents the classical configuration in the present context. Here for our discussion we start with a Gaussian arbitrary vacuum state which can be expressed in terms of the coherent superposition of scalar curvature fluctuation field eigenstates:
$\left|\Psi_{\zeta}\right\rangle \equiv|\Psi[\zeta(\mathbf{x})]\rangle=\sum_{\zeta} \mathcal{A}_{\zeta(\mathbf{x})}|\zeta(\mathbf{x})\rangle$

where the Gaussian functional coefficient $\mathcal{A}_{\zeta(\mathbf{x})}$ can be defined as

$\mathcal{A}_{\zeta(\mathbf{x})}=\left\langle\zeta(\mathbf{x}) \mid \Psi_{\zeta}\right\rangle \equiv\langle\zeta(\mathbf{x}) \mid \Psi[\zeta(\mathbf{x})]\rangle$.

Within this theoretical setup to accommodate quantum decoherence phenomena we consider a general environment source functional $G(\mathbf{x})$ as we have already introduced earlier. In general, this may account any type of non-linear interaction with the environment. But the specific structure of the interaction actually determines the behaviour of the response to the classical configuration characterized by $\zeta(\mathbf{x})$ and consequently the response of the interaction with environment can be characterized by the following quantum entanglement:

$|\Psi[G(\mathbf{x})]\rangle \otimes|\zeta(\mathbf{x})\rangle=|\Psi[G(\mathbf{x})]\rangle_{\zeta(\mathbf{x})} \otimes|\zeta(\mathbf{x})\rangle$,

where one can physically interpret $|\Psi[G(\mathbf{x})]\rangle_{\zeta(\mathbf{x})}$ as the conditional state or pointer state in the present context which satisfies the following condition:

$\langle\Psi[G(\mathbf{x}) \mid \Psi[G(\mathbf{x})\rangle=1$.

Further using this expression one can express the configuration space joint state vector as

$$
\begin{aligned}
\mid \Psi & {[G(\mathbf{x})]\rangle \otimes|\Psi[\zeta(\mathbf{x})]\rangle } \\
& =|\Psi[G(\mathbf{x})]\rangle \otimes\left\{\sum_{\zeta} \mathcal{A}_{\zeta(\mathbf{x})}|\zeta(\mathbf{x})\rangle\right\} \\
& =\sum_{\zeta} \mathcal{A}_{\zeta(\mathbf{x})}(|\Psi[G(\mathbf{x})]\rangle \otimes|\zeta(\mathbf{x})\rangle) \\
& =\sum_{\zeta} \mathcal{A}_{\zeta(\mathbf{x})}\left(|\Psi[G(\mathbf{x})]\rangle_{\zeta(\mathbf{x})} \otimes|\zeta(\mathbf{x})\rangle\right),
\end{aligned}
$$

which in turn destroys the effect of a coherent superposition of the eigenstates $|\zeta(\mathbf{x})\rangle$. Additionally it is important to mention here that the reduced density matrix for the system can be represented by

$$
\begin{aligned}
& \rho_{\text {Reduced }}[\zeta(\mathbf{x}), \alpha(\mathbf{x})] \\
& =\underbrace{\Psi[\zeta(\mathbf{x})](\Psi[\alpha(\mathbf{x})])^{\dagger}}_{\text {Interference term }} \\
& \quad \times\left\{\int \mathcal { D } G \Psi [ G ( \mathbf { x } ) ] _ { \zeta ( \mathbf { x } ) } \left(\Psi[G(\mathbf{x})]_{\left.\zeta(\mathbf{x}))^{\dagger}\right\}}\right.\right. \\
& =\underbrace{\Psi[\zeta(\mathbf{x})](\Psi[\alpha(\mathbf{x})])^{\dagger}}_{\text {Interference term }}\left\langle\Psi[G(\mathbf{x})]_{\zeta(\mathbf{x})} \mid \Psi[G(\mathbf{x})]_{\zeta(\mathbf{x})}\right\rangle,
\end{aligned}
$$


where we use

$$
\begin{aligned}
\int & \mathcal{D} G \Psi[G(\mathbf{x})]_{\zeta(\mathbf{x})} \Psi[G(\mathbf{x})]_{\zeta(\mathbf{x})}^{\dagger} \\
= & \left\langle\Psi[G(\mathbf{x})]_{\zeta(\mathbf{x})} \mid \Psi[G(\mathbf{x})]_{\zeta(\mathbf{x})}\right\rangle .
\end{aligned}
$$

Here the most general structure of the conditional or pointer wave function for the environment can be expressed as

$$
\begin{aligned}
\Psi[G(\mathbf{x})]_{\zeta(\mathbf{x})}= & \mathcal{N}_{G \zeta} \exp [\zeta(\mathbf{x}) \star G(\mathbf{x}) \star G(\mathbf{x}) \star(\operatorname{Re} \mathcal{M}(\mathbf{x}) \\
& +i \operatorname{Im} \mathcal{M}(\mathbf{x}))]
\end{aligned}
$$

where $\mathcal{N}_{G \zeta}$ represents the normalization constant for conditional or pointer wave function and $\star$ characterize the convolution operation in the present context. From this specific structure of the conditional or pointer wave function it is clearly observed that:

- $\operatorname{Re} \mathcal{M}(\mathbf{x})$ and $\operatorname{Im} \mathcal{M}(\mathbf{x})$ is the unknown function in the present context which one needs to compute for a specified structure of the interaction between system and environment.

- Here contribution from $\operatorname{Im} \mathcal{M}(\mathbf{x})$ is large compared to $\operatorname{Re} \mathcal{M}(\mathbf{x})$.

- The phase factor is rapidly oscillating in conditional or pointer wave function and consequently the following integral vanishes:

$$
\begin{aligned}
& \int \mathcal{D} G \Psi[G(\mathbf{x})]_{\zeta(\mathbf{x})} \Psi[G(\mathbf{x})]_{\zeta(\mathbf{x})}^{\dagger} \\
& \quad=\left\langle\Psi[G(\mathbf{x})]_{\zeta(\mathbf{x})} \mid \Psi[G(\mathbf{x})]_{\zeta(\mathbf{x})}\right\rangle \sim 0 .
\end{aligned}
$$

- Finally the off diagonal component of the reduced density matrix vanishes.

Now in the Schrödinger picture of quantum mechanics we define a configuration space eigenstate by $|G(\mathbf{x}), \zeta(\mathbf{x})\rangle$, satisfying the following set of eigenvalue equations:

$$
\begin{gathered}
\hat{\zeta}(\mathbf{x})|G(\mathbf{x}), \zeta(\mathbf{x})\rangle=\zeta(\mathbf{x})|G(\mathbf{x}), \zeta(\mathbf{x})\rangle, \\
\hat{\zeta}(\mathbf{x})|G(\mathbf{x}), \zeta(\mathbf{x})\rangle=\zeta(\mathbf{x})|G(\mathbf{x}), \zeta(\mathbf{x})\rangle .
\end{gathered}
$$

Further using configuration space eigenstate $|G(\mathbf{x}), \zeta(\mathbf{x})\rangle$ one can also define the wave functional of the joint system and environment at conformal time $\eta$ as

$$
\langle G(\mathbf{x}), \zeta(\mathbf{x}) \mid \Psi(\eta)\rangle=\Psi[G(\mathbf{x}), \zeta(\mathbf{x})](\eta) .
$$

Also one can express the wave functional of the joint system and environment at time $\eta$ in the following convenient product form:

$$
\begin{aligned}
\Psi & {[G(\mathbf{x}), \zeta(\mathbf{x})](\eta)=\underbrace{\Psi_{\text {Gaussian }}[\zeta(\mathbf{x})](\eta) \Psi_{\text {Gaussian }}[G(\mathbf{x})](\eta)}_{\text {Gaussian component }} } \\
& \times \underbrace{\Psi_{\text {Non-Gaussian }}[G(\mathbf{x}), \zeta(\mathbf{x})](\eta)}_{\text {Non-Gaussian component }},
\end{aligned}
$$

where each of the components of the wave functional can be written as

$$
\begin{aligned}
& \Psi_{\text {Gaussian }}[\zeta(\mathbf{x})](\eta) \\
&= \mathcal{N}_{\zeta}(\eta) \exp \left[-\int \frac{\mathrm{d}^{3} \mathbf{k}}{(2 \pi)^{3}} \zeta_{\mathbf{k}} \zeta_{\mathbf{k}}^{\dagger} \Theta_{\zeta}(k, \eta)\right], \\
& \Psi_{\text {Gaussian }}[G(\mathbf{x})](\eta) \\
&=\mathcal{N}_{G}(\eta) \exp \left[-\int \frac{\mathrm{d}^{3} \mathbf{k}}{(2 \pi)^{3}} G_{\mathbf{k}} G_{\mathbf{k}}^{\dagger} \Theta_{G}(k, \eta)\right], \\
& \Psi_{\text {Non-Gaussian }[G(\mathbf{x}), \zeta(\mathbf{x})](\eta)} \\
&=\mathcal{N}_{G \zeta}(\eta) \exp \left[\int_{\mathbf{k}} \int_{\mathbf{p}} \int_{\mathbf{q}} \frac{\mathrm{d}^{3} \mathbf{k}}{(2 \pi)^{3}} \frac{\mathrm{d}^{3} \mathbf{p}}{(2 \pi)^{3}} \frac{\mathrm{d}^{3} \mathbf{q}}{(2 \pi)^{3}}\right. \\
&\left.\quad \times(2 \pi)^{3} \delta^{3}(\mathbf{k}+\mathbf{p}+\mathbf{q}) G_{\mathbf{k}} G_{\mathbf{p}} \zeta_{\mathbf{q}} \mathcal{M}_{\mathbf{k}, \mathbf{p}, \mathbf{q}}(\eta)\right]
\end{aligned}
$$

Here $\mathcal{N}_{\zeta}(\eta), \mathcal{N}_{G}(\eta)$ and $\mathcal{N}_{G \zeta}(\eta)$ characterize the conformal time-dependent normalization constant for system, environment and the joint system environment in the present context. Hence by explicitly studying the time evolution using the master equation from the generic cubic type of interaction one can write down the model independent expressions for the complex functions $\Theta_{\zeta}(k, \eta), \Theta_{G}(k, \eta)$ and $\mathcal{M}_{\mathbf{k}, \mathbf{p}, \mathbf{q}}(\eta)$ in the present context. By knowing such structures for arbitrary vacuum state one can further study various issues related to theoretical and observational part of cosmology.

Further the reduced density matrix can be written as

$\rho_{\text {Reduced }} \equiv \operatorname{Tr}_{G}\left(\rho_{\text {Global }}\right)=\int \mathcal{D} G\left\langle G\left|\rho_{\text {Global }}\right| G\right\rangle$,

where we have integrated or traced over the environment $G$ here. Additionally, it is important to note that $\rho_{\text {Global }}$ is the global density matrix, which is defined as

$\rho_{\text {Global }} \equiv|\Psi\rangle\langle\Psi|$

In the field basis the reduced density matrix can be reexpressed as 
$\rho_{\text {Reduced }}[\zeta(\mathbf{x}), \alpha(\mathbf{x})]=\left\langle\zeta(\mathbf{x})\left|\rho_{\text {Reduced }}\right| \alpha(\mathbf{x})\right\rangle$

$$
\begin{aligned}
& =\underbrace{\Psi[\zeta(\mathbf{x})](\Psi[\alpha(\mathbf{x})])^{\dagger}}_{\text {Interference term }}\left\{\int \mathcal{D} G \Psi[G(\mathbf{x})]_{\zeta(\mathbf{x})}\left(\Psi[G(\mathbf{x})]_{\zeta(\mathbf{x})}\right)^{\dagger}\right\} \\
& =\int \mathcal{D} G \Psi[\zeta(\mathbf{x}), G(\mathbf{x})]_{\zeta(\mathbf{x})}\left(\Psi[\alpha(\mathbf{x}), G(\mathbf{x})]_{\zeta(\mathbf{x})}\right)^{\dagger} \\
& =\int \mathcal{D} G \underbrace{\Psi_{\text {Gaussian }}[\zeta(\mathbf{x})](\eta) \Psi_{\text {Gaussian }}[G(\mathbf{x})](\eta)}_{\text {Gaussian component }} \underbrace{\Psi_{\text {Non-Gaussian }}[G(\mathbf{x}), \zeta(\mathbf{x})](\eta)}_{\text {Non-Gaussian component }} \\
& \times(\underbrace{\Psi_{\text {Gaussian }}[\alpha(\mathbf{x})](\eta) \Psi_{\text {Gaussian }}[G(\mathbf{x})](\eta)}_{\text {Gaussian component }} \underbrace{\Psi_{\text {Non-Gaussian }}[G(\mathbf{x}), \zeta(\mathbf{x})](\eta)}_{\text {Non-Gaussian component }})^{\dagger} \\
& =\Psi_{\text {Gaussian }}[\zeta(\mathbf{x})](\eta)\left(\Psi_{\text {Gaussian }}[\alpha(\mathbf{x})](\eta)\right)^{\dagger} \underbrace{\mathbf{D}_{\text {Decoherence factor }}}_{\text {Decoherence }[\zeta(\mathbf{x}), \alpha(\mathbf{x})]},
\end{aligned}
$$

where the decoherence factor is defined as

$$
\begin{aligned}
& \mathbf{D}_{\text {Decoherence }}[\zeta(\mathbf{x}), \alpha(\mathbf{x})]=\frac{\left|\rho_{\text {Reduced }}[\zeta(\mathbf{x}), \alpha(\mathbf{x})]\right|}{\sqrt{\rho_{\text {Reduced }}[\zeta(\mathbf{x}), \zeta(\mathbf{x})] \rho_{\text {Reduced }}[\alpha(\mathbf{x}), \alpha(\mathbf{x})]}} \\
& =\int \mathcal{D} G\left|\Psi_{\text {Gaussian }}[G(\mathbf{x})](\eta)\right|^{2}\left|\Psi_{\text {Non-Gaussian }}[G(\mathbf{x}), \zeta(\mathbf{x})](\eta)\right|^{2} \\
& =\left|\mathcal{N}_{G \zeta}(\eta)\right|^{2} \int \mathcal{D} G\left|\Psi_{\text {Gaussian }}[G(\mathbf{x})](\eta)\right|^{2} \\
& \times \exp \left[\int_{\mathbf{k}} \int_{\mathbf{p}} \int_{\mathbf{q}} \frac{\mathrm{d}^{3} \mathbf{k}}{(2 \pi)^{3}} \frac{\mathrm{d}^{3} \mathbf{p}}{(2 \pi)^{3}} \frac{\mathrm{d}^{3} \mathbf{q}}{(2 \pi)^{3}}\right. \\
& \left.\times(2 \pi)^{3} \delta^{3}(\mathbf{k}+\mathbf{p}+\mathbf{q}) G_{\mathbf{k}} G_{\mathbf{p}}\left\{\zeta_{\mathbf{q}} \mathcal{M}_{\mathbf{k}, \mathbf{p}, \mathbf{q}}(\eta)+\alpha_{\mathbf{q}} \mathcal{M}_{\mathbf{k}, \mathbf{p}, \mathbf{q}}^{\dagger}(\eta)\right\}\right] \\
& =\left|\mathcal{N}_{G \zeta}(\eta)\right|^{2}\left|\mathcal{N}_{G}(\eta)\right|^{2} \int \mathcal{D} G \exp \left[-\int \frac{\mathrm{d}^{3} \mathbf{k}}{(2 \pi)^{3}} G_{\mathbf{k}} G_{\mathbf{k}}^{\dagger}\left(\Theta_{G}(k, \eta)+\Theta_{G}^{\dagger}(k, \eta)\right)\right] \\
& \times \exp \left[\int_{\mathbf{k}} \int_{\mathbf{p}} \int_{\mathbf{q}} \frac{\mathrm{d}^{3} \mathbf{k}}{(2 \pi)^{3}} \frac{\mathrm{d}^{3} \mathbf{p}}{(2 \pi)^{3}} \frac{\mathrm{d}^{3} \mathbf{q}}{(2 \pi)^{3}}\right. \\
& \left.\times(2 \pi)^{3} \delta^{3}(\mathbf{k}+\mathbf{p}+\mathbf{q}) G_{\mathbf{k}} G_{\mathbf{p}}\left\{\zeta_{\mathbf{q}} \mathcal{M}_{\mathbf{k}, \mathbf{p}, \mathbf{q}}(\eta)+\alpha_{\mathbf{q}} \mathcal{M}_{\mathbf{k}, \mathbf{p}, \mathbf{q}}^{\dagger}(\eta)\right\}\right] \\
& =\left|\mathcal{N}_{G \zeta}(\eta)\right|^{2}\left|\mathcal{N}_{G}(\eta)\right|^{2} \int \mathcal{D} G \exp \left[-2 \int \frac{\mathrm{d}^{3} \mathbf{k}}{(2 \pi)^{3}} G_{\mathbf{k}} G_{\mathbf{k}}^{\dagger} \operatorname{Re}\left[\Theta_{G}(k, \eta)\right]\right] \\
& \times \exp \left[\int_{\mathbf{k}} \int_{\mathbf{p}} \int_{\mathbf{q}} \frac{\mathrm{d}^{3} \mathbf{k}}{(2 \pi)^{3}} \frac{\mathrm{d}^{3} \mathbf{p}}{(2 \pi)^{3}} \frac{\mathrm{d}^{3} \mathbf{q}}{(2 \pi)^{3}}\right. \\
& \left.\times(2 \pi)^{3} \delta^{3}(\mathbf{k}+\mathbf{p}+\mathbf{q}) G_{\mathbf{k}} G_{\mathbf{p}}\left\{\Delta_{q+} \operatorname{Re}\left[\mathcal{M}_{\mathbf{k}, \mathbf{p}, \mathbf{q}}(\eta)\right]+i \Delta_{q-} \operatorname{Im}\left[\mathcal{M}_{\mathbf{k}, \mathbf{p}, \mathbf{q}}(\eta)\right]\right\}\right],
\end{aligned}
$$

which takes care of off diagonal amplitudes as well as non-Gaussian contributions in the present context. Here the newly defined functions $\Delta_{q+}$ and $\Delta_{q-}$ are defined as

$$
\begin{aligned}
& \Delta_{q+}=\zeta_{\mathbf{q}}+\alpha_{\mathbf{q}}, \\
& \Delta_{q-}=\zeta_{\mathbf{q}}-\alpha_{\mathbf{q}} .
\end{aligned}
$$

Further absorbing the normalization factors in the definition of the coherence factor one can write 


$$
\begin{aligned}
& \mathcal{D}_{\text {Decoherence }}[\zeta(\mathbf{x}), \alpha(\mathbf{x})] \\
& =\frac{\mathbf{D}_{\text {Decoherence }}[\zeta(\mathbf{x}), \alpha(\mathbf{x})]}{\left|\mathcal{N}_{G \zeta}(\eta)\right|^{2}\left|\mathcal{N}_{G}(\eta)\right|^{2}} \\
& =\int \mathcal{D} G \exp \left[-2 \int \frac{\mathrm{d}^{3} \mathbf{k}}{(2 \pi)^{3}} G_{\mathbf{k}} G_{\mathbf{k}}^{\dagger} \operatorname{Re}\left[\Theta_{G}(k, \eta)\right]\right. \\
& +\int_{\mathbf{k}} \int_{\mathbf{p}} \int_{\mathbf{q}} \frac{\mathrm{d}^{3} \mathbf{k}}{(2 \pi)^{3}} \frac{\mathrm{d}^{3} \mathbf{p}}{(2 \pi)^{3}} \frac{\mathrm{d}^{3} \mathbf{q}}{(2 \pi)^{3}} \\
& \times(2 \pi)^{3} \delta^{3}(\mathbf{k}+\mathbf{p}+\mathbf{q}) G_{\mathbf{k}} G_{\mathbf{p}}\left\{\Delta_{q+} \operatorname{Re}\left[\mathcal{M}_{\mathbf{k}, \mathbf{p}, \mathbf{q}}(\eta)\right]\right. \\
& \left.\left.+i \Delta_{q-} \operatorname{Im}\left[\mathcal{M}_{\mathbf{k}, \mathbf{p}, \mathbf{q}}(\eta)\right]\right\}\right] \\
& =\langle\exp [\mathcal{Z}]\rangle \\
& =\exp [\underbrace{\sum_{n=1}^{\infty} \frac{1}{(2 n) !}\left\langle\mathcal{Z}^{2 n}\right\rangle_{\mathbf{C}}}_{\text {Connected even cumulants }}+\underbrace{\sum_{m=0}^{\infty} \frac{1}{(2 m+1) !}\left\langle\mathcal{Z}^{2 m+1}\right\rangle_{\mathbf{D C}}}_{\text {Disconnected odd cumulants }}],
\end{aligned}
$$

where the factor $\mathcal{Z}$ is defined as

$$
\begin{aligned}
\mathcal{Z}= & -2 \int \frac{\mathrm{d}^{3} \mathbf{k}}{(2 \pi)^{3}} G_{\mathbf{k}} G_{\mathbf{k}}^{\dagger} \operatorname{Re}\left[\Theta_{G}(k, \eta)\right] \\
& +\int_{\mathbf{k}} \int_{\mathbf{p}} \int_{\mathbf{q}} \frac{\mathrm{d}^{3} \mathbf{k}}{(2 \pi)^{3}} \frac{\mathrm{d}^{3} \mathbf{p}}{(2 \pi)^{3}} \frac{\mathrm{d}^{3} \mathbf{q}}{(2 \pi)^{3}}(2 \pi)^{3} \delta^{3}(\mathbf{k}+\mathbf{p}+\mathbf{q}) \\
& \times G_{\mathbf{k}} G_{\mathbf{p}}\left\{\Delta_{q+} \operatorname{Re}\left[\mathcal{M}_{\mathbf{k}, \mathbf{p}, \mathbf{q}}(\eta)\right]+i \Delta_{q-} \operatorname{Im}\left[\mathcal{M}_{\mathbf{k}, \mathbf{p}, \mathbf{q}}(\eta)\right]\right\}
\end{aligned}
$$

and the subscript $\mathbf{C}$ and $\mathbf{D C}$ indicates the connected and disconnected contribution of the correlation function. To further simplify the form of the rescaled decoherence factor $\mathcal{D}_{\text {Decoherence }}[\zeta(\mathbf{x}), \alpha(\mathbf{x})]$ one can introduce the environment two point correlation function as

$\left\langle G_{\mathbf{k}} G_{\mathbf{q}}\right\rangle_{\eta}=(2 \pi)^{2} \delta^{3}(\mathbf{k}+\mathbf{q}) P_{G}(k, \eta)$,

where $P_{G}(k, \eta)$ is the power spectrum of the environment and can be expressed in terms of the variance of the wave function. Here due to the presence of the additional nonGaussian part in the wave function it is expected that decoherence effect cannot be negligible in the present context, provided the structure of complex functions $\Theta_{\zeta}(k, \eta), \Theta_{G}(k, \eta)$ and $\mathcal{M}_{\mathbf{k}, \mathbf{p}, \mathbf{q}}(\eta)$ involves the time-dependent mass parameter used for violating Bell's inequality in the cosmological setup. Due to the presence of a time-dependent mass parameter in the interaction part of the Hamiltonian one can expect that the contribution from the disconnected odd cumulants in the correlation function is non-negligible. Specifically due to Bell violation $\langle\mathcal{Z}\rangle$ contributes to the rescaled decoherence factor $\mathcal{D}_{\text {Decoherence }}[\zeta(\mathbf{x}), \alpha(\mathbf{x})]$ and in the expression for reduced density matrix $\rho_{\text {Reduced }}[\zeta(\mathbf{x}), \alpha(\mathbf{x})]$. Also $\left\langle\mathcal{Z}^{2}\right\rangle$, $\left\langle\mathcal{Z}^{3}\right\rangle$ and $\left\langle\mathcal{Z}^{4}\right\rangle$ captures the effect of power spectrum, nonGaussian bi-spectrum and tri-spectrum in the present context. See Refs. [10,11] for more details. After finding the specific structure of the reduced density matrix one can define the Wigner function as

$$
\begin{aligned}
W= & \frac{1}{(2 \pi)^{2}} \int \mathrm{d} x \int \mathrm{d} y \exp \left[-i\left(\pi_{\mathbf{k}} x+\pi_{-\mathbf{k}} y\right)\right] \\
& \times\left\langle q_{\mathbf{k}}+x / 2, q_{-\mathbf{k}}+y / 2\left|\rho_{\mathbf{R e d u c e d}}\right| q_{\mathbf{k}}-x / 2, q_{-\mathbf{k}}-y / 2\right\rangle \\
= & \frac{1}{(2 \pi)^{2}} \int \mathrm{d} x \int \mathrm{d} y \exp \left[-i\left(\pi_{\mathbf{k}} x+\pi_{-\mathbf{k}} y\right)\right] \\
& \times \Psi_{\text {Gaussian }}\left[\zeta\left(q_{\mathbf{k}}+x / 2, q_{-\mathbf{k}}+y / 2\right)\right] \\
& \times\left(\Psi_{\text {Gaussian }}\left[\alpha\left(q_{\mathbf{k}}-x / 2, q_{-\mathbf{k}}-y / 2\right)\right]\right)^{\dagger} \\
& \times \underbrace{\mathbf{D}_{\text {Decoherence }}\left[\zeta\left(q_{\mathbf{k}}+x / 2, q_{-\mathbf{k}}+y / 2\right), \alpha\left(q_{\mathbf{k}}-x / 2, q_{-\mathbf{k}}-y / 2\right)\right]}_{\text {Decoherence factor }} .
\end{aligned}
$$

To understand the structure of the Wigner function we need to substitute the specific form of the wave functions as mentioned earlier. Here after substitution the wave function and absorbing the normalization constants the Wigner function can be recast in the following rescaled form:

$$
\begin{aligned}
\mathcal{W}= & \frac{W}{\left|\mathcal{N}_{G \zeta}(\eta)\right|^{2}\left|\mathcal{N}_{G}(\eta)\right|^{2}} \\
= & \frac{1}{(2 \pi)^{2}} \int \mathrm{d} x \int \mathrm{d} y \exp \left[-i\left(\pi_{\mathbf{k}} x+\pi_{-\mathbf{k}} y\right)\right] \\
& \times \Psi_{\text {Gaussian }}\left[\zeta\left(q_{\mathbf{k}}+x / 2, q_{-\mathbf{k}}+y / 2\right)\right] \\
& \times\left(\Psi_{\mathbf{G a u s s i a n}}\left[\alpha\left(q_{\mathbf{k}}-x / 2, q_{-\mathbf{k}}-y / 2\right)\right]\right)^{\dagger} \\
& \times\left\langle\exp \left(\mathcal{Z}\left[\zeta\left(q_{\mathbf{k}}+x / 2, q_{-\mathbf{k}}+y / 2\right), \alpha\left(q_{\mathbf{k}}-x / 2, q_{-\mathbf{k}}-y / 2\right)\right]\right)\right\rangle .
\end{aligned}
$$

As our motivation of this paper is restricted to set up the cosmological experiment where Bell's inequality violation can be tested, we have not computed further results in this paper from an arbitrary vacuum state. But we will report soon on such a computation in the continuation part of this paper, where we will also comment on the primordial nonGaussianity for this cosmological setup as well.

6.2 Time dependent mass profile for heavy field

6.2.1 Profile $A: m=\sqrt{\gamma\left(\frac{\eta}{\eta_{0}}-1\right)^{2}+\delta} H$

The equation of motion for the massive field is

$$
\begin{aligned}
& h_{k}^{\prime \prime}+\left(c_{S}^{2} k^{2}+C+\frac{(\gamma+\delta-2)}{\eta^{2}}-\frac{\mathrm{d}}{\eta}\right) h_{k}=0 \quad \text { for } \mathrm{dS} \\
& h_{k}^{\prime \prime}+\left(c_{S}^{2} k^{2}+C+\frac{\left(\gamma+\delta-\left[v^{2}-\frac{1}{4}\right]\right)}{\eta^{2}}-\frac{\mathrm{d}}{\eta}\right) h_{k}=0 \quad \text { for qdS }
\end{aligned}
$$

where we introduce two new parameters:

$C=\frac{\gamma}{\eta_{0}^{2}}, \quad d=\frac{2 \gamma}{\eta_{0}}$.

The solution for the mode function for the de Sitter and the quasi de Sitter space can be expressed as 
$h_{k}(\eta)=\left\{\begin{array}{l}G_{1} M_{-\frac{\mathrm{d}}{2 i \sqrt{c_{S}^{2} k^{2}+C}}, i \sqrt{(\gamma+\delta)-\frac{9}{4}}}\left[2 i \sqrt{c_{S}^{2} k^{2}+C} \eta\right]+G_{2} W_{-\frac{\mathrm{d}}{2 i \sqrt{c_{S}^{2} k^{2}+C}}, i \sqrt{(\gamma+\delta)-\frac{9}{4}}}\left[2 i \sqrt{k^{2}+C} \eta\right] \text { for dS, } \\ G_{1} M_{-\frac{\mathrm{d}}{2 i \sqrt{c_{S}^{2} k^{2}+C}}, i \sqrt{\gamma+\delta-v^{2}}}\left[2 i \sqrt{c_{S}^{2} k^{2}+C} \eta\right]+G_{2} W_{-\frac{\mathrm{d}}{2 i \sqrt{c_{S}^{2} k^{2}+C}}, i \sqrt{\gamma+\delta-\nu^{2}}}\left[2 i \sqrt{c_{S}^{2} k^{2}+C \eta}\right] \text { for qdS, }\end{array}\right.$

where $G_{1}$ and $G_{2}$ are two arbitrary integration constants, which depend on the choice of the initial condition. For the sake of simplicity one can recast these solution as

$h_{k}(\eta)=(-\eta)^{\frac{3}{2}} e^{-\frac{P \eta}{2}}(P \eta)^{A+\frac{\mathrm{d}}{P}}\left[G_{11} F_{1}(A ; B ; P \eta)+G_{2} U(A ; B ; P \eta)\right]$

where $A, B$ and $P$ are defined as

$A= \begin{cases}-\frac{\mathrm{d}}{2 i \sqrt{c_{S}^{2} k^{2}+C}}+i \sqrt{(\gamma+\delta)-\frac{9}{4}}+\frac{1}{2} & \text { for } \mathrm{dS}, \\ -\frac{\mathrm{d}}{2 i \sqrt{c_{S}^{2} k^{2}+C}}+i \sqrt{(\gamma+\delta)-v^{2}}+\frac{1}{2} & \text { for qdS },\end{cases}$

$B= \begin{cases}2 i \sqrt{(\gamma+\delta)-\frac{9}{4}}+1 & \text { for dS, } \\ 2 i \sqrt{(\gamma+\delta)-v^{2}}+1 & \text { for qdS, }\end{cases}$

$P=2 i \sqrt{c_{S}^{2} k^{2}+C}$.

After taking the $k c_{S} \eta \rightarrow-\infty$ and $k c_{S} \eta \rightarrow 0$ limits for the arbitrary choice of the initial condition or vacuum we get the following results:

$$
\begin{aligned}
& \lim _{k c_{S} \eta \rightarrow-\infty} U\left(A ; B ; Q k c_{S} \eta\right) \approx\left(Q k c_{S} \eta\right)^{-A}, \\
& \lim _{k c_{S} \eta \rightarrow-\infty}{ }_{1} F_{1}\left(A ; B ; Q k c_{S} \eta\right) \approx \frac{\Gamma(B)\left(-Q k c_{S} \eta\right)^{-A}}{\Gamma(B-A)}+\frac{\Gamma(B) e^{Q k c_{S} \eta}\left(Q k c_{S} \eta\right)^{A-B}}{\Gamma(A)}, \\
& \lim _{k c_{S} \eta \rightarrow 0} U\left(A ; B ; Q k c_{S} \eta\right) \approx \frac{\Gamma(1-B)}{\Gamma(1+A-B)}\left(1+\frac{A Q k c_{S} \eta}{B}\right)+\frac{\left(Q k c_{S} \eta\right)^{1-B} \Gamma(B-1)}{\Gamma(A)}, \\
& \lim _{k c_{S} \eta \rightarrow 0}{ }_{1} F_{1}\left(A ; B ; Q k c_{S} \eta\right) \approx\left(1+\frac{A Q k c_{S} \eta}{B}\right),
\end{aligned}
$$

where the parameter $Q$ is defined as

$Q=\frac{P}{k c_{S}}$.

One can also consider the following approximations to simplify the final derived form of the solution for arbitrary vacuum with $\left|k c_{S} \eta\right|=1$ or equivalently $\left|k c_{S} \eta\right| \approx 1-\Delta$ with $\Delta \rightarrow 0$ :

1. We start with the Laurent expansion of the Gamma function:

$$
\begin{aligned}
& \Gamma(X)=\frac{1}{X}-\gamma+\frac{1}{2}\left(\gamma^{2}+\frac{\pi^{2}}{6}\right) X \\
& -\frac{1}{6}\left(\gamma^{3}+\frac{\gamma \pi^{2}}{2}+2 \zeta(3)\right) X^{2}+\mathcal{O}\left(X^{3}\right)
\end{aligned}
$$

where $\gamma$ is the Euler-Mascheroni constant and $\zeta(3)$ characterizing the Riemann zeta function of order 3 originating in the expansion of the gamma function. Here the parameter $X$ is defined as

$$
X=A, B-1,1-B, A-B+1,
$$


where for the de Sitter case and the quasi de Sitter case we get

$$
\begin{aligned}
& A= \begin{cases}-\frac{\mathrm{d}}{2 i \sqrt{c_{S}^{2} k^{2}+C}}+i \sqrt{(\gamma+\delta)-\frac{9}{4}}+\frac{1}{2} & \text { for } \mathrm{dS}, \\
-\frac{\mathrm{d}}{2 i \sqrt{c_{S}^{2} k^{2}+C}}+i \sqrt{(\gamma+\delta)-v^{2}}+\frac{1}{2} & \text { for qdS }\end{cases} \\
& B-1= \begin{cases}2 i \sqrt{(\gamma+\delta)-\frac{9}{4}} & \text { for dS, } \\
2 i \sqrt{(\gamma+\delta)-v^{2}} & \text { for qdS, }\end{cases} \\
& 1-B= \begin{cases}-2 i \sqrt{(\gamma+\delta)-\frac{9}{4}} & \text { for dS, } \\
2 i \sqrt{(\gamma+\delta)-v^{2}} & \text { for qdS, }\end{cases} \\
& A-B+1= \begin{cases}-\frac{\mathrm{d}}{2 i \sqrt{c_{S}^{2} k^{2}+C}}-i \sqrt{(\gamma+\delta)-\frac{9}{4}}+\frac{1}{2} & \text { for dS } \\
-\frac{\mathrm{d}}{2 i \sqrt{c_{S}^{2} k^{2}+C}}-i \sqrt{(\gamma+\delta)-v^{2}}+\frac{1}{2} & \text { for qdS }\end{cases}
\end{aligned}
$$

2. In this case the solution's hyper-geometric functions of the first and second kind can be re-expressed in the following simplified form:

$$
\begin{aligned}
& \lim _{\left|k c_{S} \eta\right| \approx 1-\Delta(\rightarrow 0)} U\left(A ; B ; Q k c_{S} \eta\right) \approx f_{1}(A, B)\left(1-\frac{A Q(1+\Delta)}{B}\right) \\
& +f_{2}(A, B)(-Q(1+\Delta))^{1-B} \\
& \lim _{\left|k c_{S} \eta\right| \approx 1-\Delta(\rightarrow 0)}{ }_{1} F_{1}\left(A ; B ; Q k c_{S} \eta\right) \approx\left(1-\frac{A Q(1+\Delta)}{B}\right)
\end{aligned}
$$

where $f_{1}(A, B)$ and $f_{2}(A, B)$ are defined as

$$
\begin{aligned}
f_{1}(A, B)= & \frac{\Gamma(1-B)}{\Gamma(1+A-B)} \approx\left[\frac{(1+A-B)}{(1-B)}\right. \\
& \left.-\gamma+\frac{1}{2}\left(\gamma^{2}+\frac{\pi^{2}}{6}\right)(1-B)(1+A-B)+\cdots\right], \\
f_{2}(A, B)= & \frac{\Gamma(B-1)}{\Gamma(A)} \approx\left[\frac{A}{(B-1)}\right. \\
& \left.-\gamma+\frac{1}{2}\left(\gamma^{2}+\frac{\pi^{2}}{6}\right)(B-1) A+\cdots\right] .
\end{aligned}
$$

After taking the $k c_{S} \eta \rightarrow-\infty, k c_{S} \eta \rightarrow 0$ and $\left|k c_{S} \eta\right| \approx 1-\Delta(\rightarrow 0)$ limits the most general solution as stated in Eq. (6.33) can be recast as

$$
\begin{aligned}
h_{k}(\eta) \stackrel{|k c S \eta| \rightarrow-\infty}{=}(-\eta)^{\frac{3}{2}} e^{-\frac{P \eta}{2}}(P \eta)^{A+\frac{\mathrm{d}}{P}}\left[G _ { 1 } \left\{\frac{\Gamma(B)\left(-Q k c_{S} \eta\right)^{-A}}{\Gamma(B-A)}\right.\right. \\
\left.\left.+\frac{\Gamma(B) e^{Q k c_{S} \eta}\left(Q k c_{S} \eta\right)^{A-B}}{\Gamma(A)}\right\}+G_{2}\left(Q k c_{S} \eta\right)^{-A}\right]
\end{aligned}
$$




$$
\begin{aligned}
& \left\{\begin{array}{l}
{[-\eta)^{\frac{3}{2}} e^{-i \sqrt{c_{S}^{2} k^{2}+C} \eta}\left(2 i \sqrt{c_{S}^{2} k^{2}+C} \eta\right)^{i \sqrt{(\gamma+\delta)-\frac{9}{4}}+\frac{1}{2}}} \\
{\left[\frac{\Gamma\left(2 i \sqrt{(\gamma+\delta)-\frac{9}{4}}+1\right)}{\Gamma\left(i \sqrt{(\gamma+\delta)-\frac{9}{4}}+\frac{1}{2}+\frac{\mathrm{d}}{2 i \sqrt{c_{S}^{2} k^{2}+C}}\right)}\right.}
\end{array}\right. \\
& \times\left(-2 i \sqrt{c_{S}^{2} k^{2}+C \eta}\right)^{\frac{\mathrm{d}}{2 i \sqrt{c_{S}^{2} k^{2}+C}}-i \sqrt{(\gamma+\delta)-\frac{9}{4}}-\frac{1}{2}} \\
& +\frac{\Gamma\left(2 i \sqrt{(\gamma+\delta)-\frac{9}{4}}+1\right)}{\Gamma\left(-\frac{\mathrm{d}}{2 i \sqrt{c_{S}^{2} k^{2}+C}}+i \sqrt{(\gamma+\delta)-\frac{9}{4}}+\frac{1}{2}\right)} \\
& \left.\times \frac{e^{2 i \sqrt{c_{S}^{2} k^{2}+C} \eta}}{\left(2 i \sqrt{c_{S}^{2} k^{2}+C} \eta\right)^{i \sqrt{(\gamma+\delta)-\frac{9}{4}}+\frac{1}{2}+\frac{\mathrm{d}}{2 i \sqrt{c_{S}^{2} k^{2}+C}}}}\right\} \\
& \left.+G_{2}\left(2 i \sqrt{c_{S}^{2} k^{2}+C \eta}\right)^{\frac{\mathrm{d}}{2 i \sqrt{c_{S}^{2} k^{2}+C}}-i \sqrt{(\gamma+\delta)-\frac{9}{4}}-\frac{1}{2}}\right] \quad \text { for } \mathrm{dS} \text {, } \\
& (-\eta)^{\frac{3}{2}} e^{-i \sqrt{c_{S}^{2} k^{2}+C} \eta}\left(2 i \sqrt{c_{S}^{2} k^{2}+C} \eta\right)^{i \sqrt{(\gamma+\delta)-v^{2}}+\frac{1}{2}} \\
& G_{1}\left\{\frac{\Gamma\left(2 i \sqrt{(\gamma+\delta)-v^{2}}+1\right)}{\Gamma\left(i \sqrt{(\gamma+\delta)-v^{2}}+\frac{1}{2}+\frac{\mathrm{d}}{2 i \sqrt{c_{S}^{2} k^{2}+C}}\right)}\right. \\
& \times\left(-2 i \sqrt{c_{S}^{2} k^{2}+C} \eta\right)^{\frac{\mathrm{d}}{2 i \sqrt{c_{S}^{2} k^{2}+C}}-i \sqrt{(\gamma+\delta)-v^{2}}-\frac{1}{2}} \\
& +\frac{\Gamma\left(2 i \sqrt{(\gamma+\delta)-v^{2}}+1\right)}{\Gamma\left(-\frac{\mathrm{d}}{2 i \sqrt{c_{S}^{2} k^{2}+C}}+i \sqrt{(\gamma+\delta)-v^{2}}+\frac{1}{2}\right)} \\
& \left.\times \frac{e^{2 i \sqrt{c_{S}^{2} k^{2}+C} \eta}}{\left(2 i \sqrt{c_{S}^{2} k^{2}+C} \eta\right)^{i \sqrt{(\gamma+\delta)-\nu^{2}}+\frac{1}{2}+\frac{\mathrm{d}}{2 i \sqrt{c_{S}^{2} k^{2}+C}}}}\right\} \\
& \left.+G_{2}\left(2 i \sqrt{c_{S}^{2} k^{2}+C \eta}\right)^{\frac{\mathrm{d}}{2 i \sqrt{c_{S}^{2} k^{2}+C}}-i \sqrt{(\gamma+\delta)-v^{2}}-\frac{1}{2}}\right] \text { for qdS, }
\end{aligned}
$$

$$
\begin{aligned}
h_{k}(\eta) \stackrel{|k c S \eta| \rightarrow 0}{=} & (-\eta)^{\frac{3}{2}} e^{-\frac{P \eta}{2}}(P \eta)^{A+\frac{\mathrm{d}}{P}}\left[G_{1}\left(1+\frac{A Q k c_{S} \eta}{B}\right)\right. \\
& \left.+G_{2}\left\{\frac{\Gamma(1-B)}{\Gamma(1+A-B)}\left(1+\frac{A Q k c_{S} \eta}{B}\right)+\frac{\left(Q k c_{S} \eta\right)^{1-B} \Gamma(B-1)}{\Gamma(A)}\right\}\right]
\end{aligned}
$$


Eur. Phys. J. C (2017) 77:60

Page 169 of $181 \quad 60$

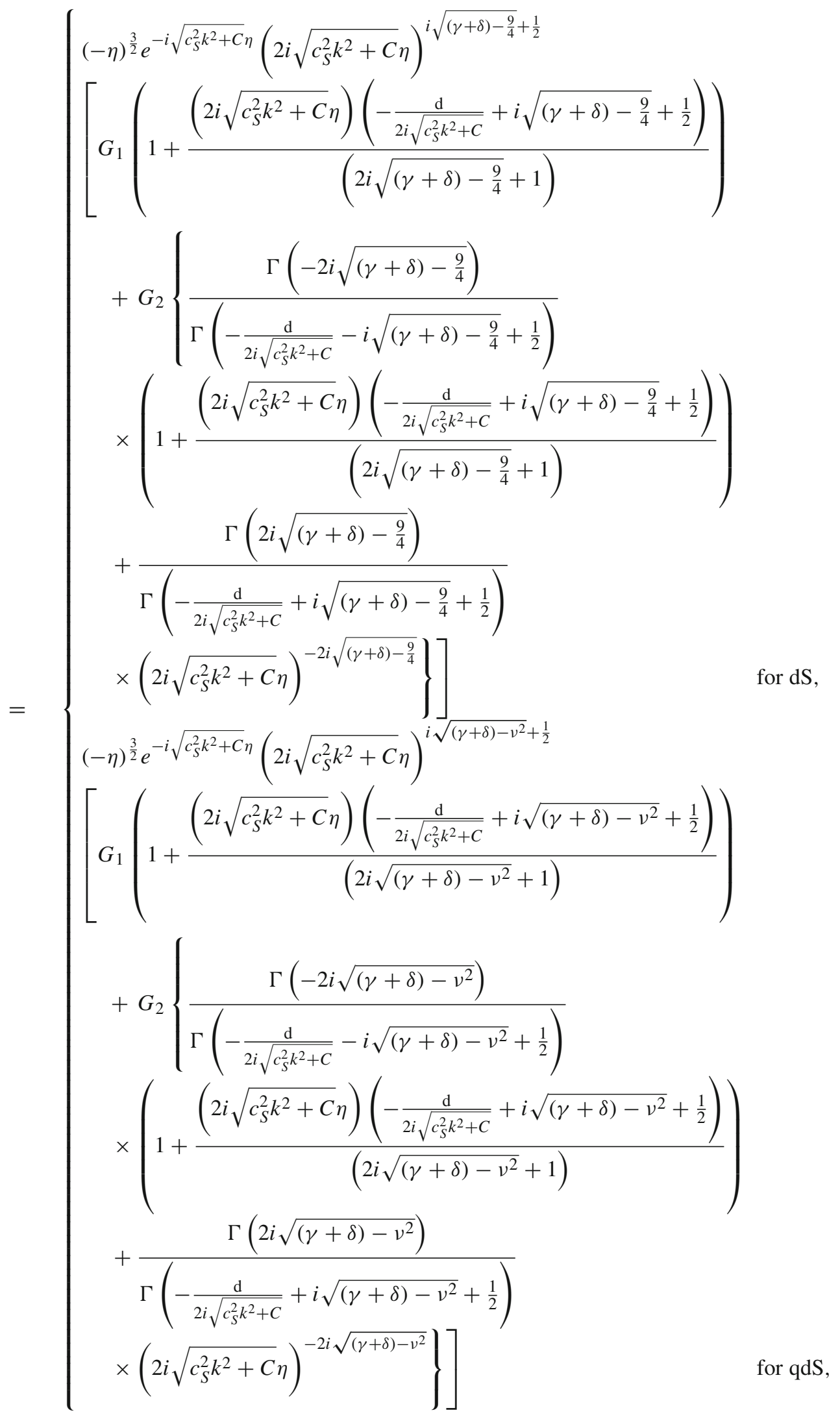

Springer 


$$
\begin{aligned}
& h_{k}(\eta) \stackrel{\left|k c_{S} \eta\right| \approx 1-\Delta(\rightarrow 0)}{=}(-\eta)^{\frac{3}{2}} e^{-\frac{P \eta}{2}}(P \eta)^{A+\frac{\mathrm{d}}{P}}\left[G_{1}\left(1-\frac{A Q(1+\Delta)}{B}\right)\right. \\
& \left.+G_{2}\left\{f_{1}(A, B)\left(1-\frac{A Q(1+\Delta)}{B}\right)+f_{2}(A, B)(-Q(1+\Delta))^{1-B}\right\}\right] \\
& {\left[\begin{array}{l}
(-\eta)^{\frac{3}{2}} e^{-i \sqrt{c_{S}^{2} k^{2}+C} \eta}\left(2 i \sqrt{c_{S}^{2} k^{2}+C \eta}\right)^{i \sqrt{(\gamma+\delta)-\frac{9}{4}}+\frac{1}{2}} \\
G_{1}\left(1-\frac{\left(\frac{2 i}{k c_{S}} \sqrt{c_{S}^{2} k^{2}+C}(1+\Delta)\right)\left(-\frac{\mathrm{d}}{2 i \sqrt{c_{S}^{2} k^{2}+C}}+i \sqrt{(\gamma+\delta)-\frac{9}{4}}+\frac{1}{2}\right)}{\left(2 i \sqrt{(\gamma+\delta)-\frac{9}{4}}+1\right)}\right)
\end{array}\right.} \\
& +G_{2}\left\{\left[\frac{\left(-\frac{\mathrm{d}}{2 i \sqrt{c_{S}^{2} k^{2}+C}}-i \sqrt{(\gamma+\delta)-\frac{9}{4}}+\frac{1}{2}\right)}{\left(-2 i \sqrt{(\gamma+\delta)-\frac{9}{4}}\right)}-\gamma+\cdots\right]\right. \\
& \times\left(1-\frac{\left(\frac{2 i}{k c_{S}} \sqrt{c_{S}^{2} k^{2}+C}(1+\Delta)\right)\left(-\frac{\mathrm{d}}{2 i \sqrt{c_{S}^{2} k^{2}+C}}+i \sqrt{(\gamma+\delta)-\frac{9}{4}}+\frac{1}{2}\right)}{\left(2 i \sqrt{(\gamma+\delta)-\frac{9}{4}}+1\right)}\right) \\
& +\left[\frac{\left(-\frac{\mathrm{d}}{2 i \sqrt{c_{S}^{2} k^{2}+C}}+i \sqrt{(\gamma+\delta)-\frac{9}{4}}+\frac{1}{2}\right)}{\left(2 i \sqrt{(\gamma+\delta)-\frac{9}{4}}\right)}-\gamma+\cdots\right] \\
& \begin{array}{c}
\left.\left.\times\left(2 i \sqrt{c_{S}^{2} k^{2}+C \eta}\right)^{-2 i \sqrt{(\gamma+\delta)-\frac{9}{4}}}\right\}\right] \text { for } \mathrm{dS} \\
\eta)^{\frac{3}{2}} e^{-i \sqrt{c_{S}^{2} k^{2}+C} \eta}\left(2 i \sqrt{c_{S}^{2} k^{2}+C \eta}\right)^{i \sqrt{(\gamma+\delta)-v^{2}}+\frac{1}{2}}
\end{array} \\
& {\left[G_{1}\left(1-\frac{\left(\frac{2 i}{k c_{S}} \sqrt{c_{S}^{2} k^{2}+C}(1+\Delta)\right)\left(-\frac{\mathrm{d}}{2 i \sqrt{c_{S}^{2} k^{2}+C}}+i \sqrt{(\gamma+\delta)-v^{2}}+\frac{1}{2}\right)}{\left(2 i \sqrt{(\gamma+\delta)-v^{2}}+1\right)}\right)\right.} \\
& +G_{2}\left\{\left[\frac{\left(-\frac{\mathrm{d}}{2 i \sqrt{c_{S}^{2} k^{2}+C}}-i \sqrt{(\gamma+\delta)-v^{2}}+\frac{1}{2}\right)}{\left(-2 i \sqrt{(\gamma+\delta)-v^{2}}\right)}-\gamma+\cdots\right]\right. \\
& \times\left(1-\frac{\left(\frac{2 i}{k c_{S}} \sqrt{c_{S}^{2} k^{2}+C}(1+\Delta)\right)\left(-\frac{\mathrm{d}}{2 i \sqrt{c_{S}^{2} k^{2}+C}}+i \sqrt{(\gamma+\delta)-v^{2}}+\frac{1}{2}\right)}{\left(2 i \sqrt{(\gamma+\delta)-v^{2}}+1\right)}\right) \\
& +\left[\frac{\left(-\frac{\mathrm{d}}{2 i \sqrt{c_{S}^{2} k^{2}+C}}+i \sqrt{(\gamma+\delta)-v^{2}}+\frac{1}{2}\right)}{\left(2 i \sqrt{(\gamma+\delta)-v^{2}}\right)}-\gamma+\cdots\right] \\
& \left.\times\left(2 i \sqrt{c_{S}^{2} k^{2}+C \eta}\right)^{-2 i \sqrt{(\gamma+\delta)-v^{2}}}\right\} \text { for qdS. }
\end{aligned}
$$


Next we assume that the WKB approximation is approximately valid for all times for the solution for the mode function $h_{k}$. In the standard WKB approximation the total solution can be recast in the following form:

$h_{k}(\eta)=\left[D_{1} u_{k}(\eta)+D_{2} \bar{u}_{k}(\eta)\right]$,

where $D_{1}$ and $D_{2}$ are two arbitrary integration constants, which depend on the choice of the initial condition during WKB approximation at early and late time scale. In the present context $u_{k}(\eta)$ and $\bar{u}_{k}(\eta)$ are defined as

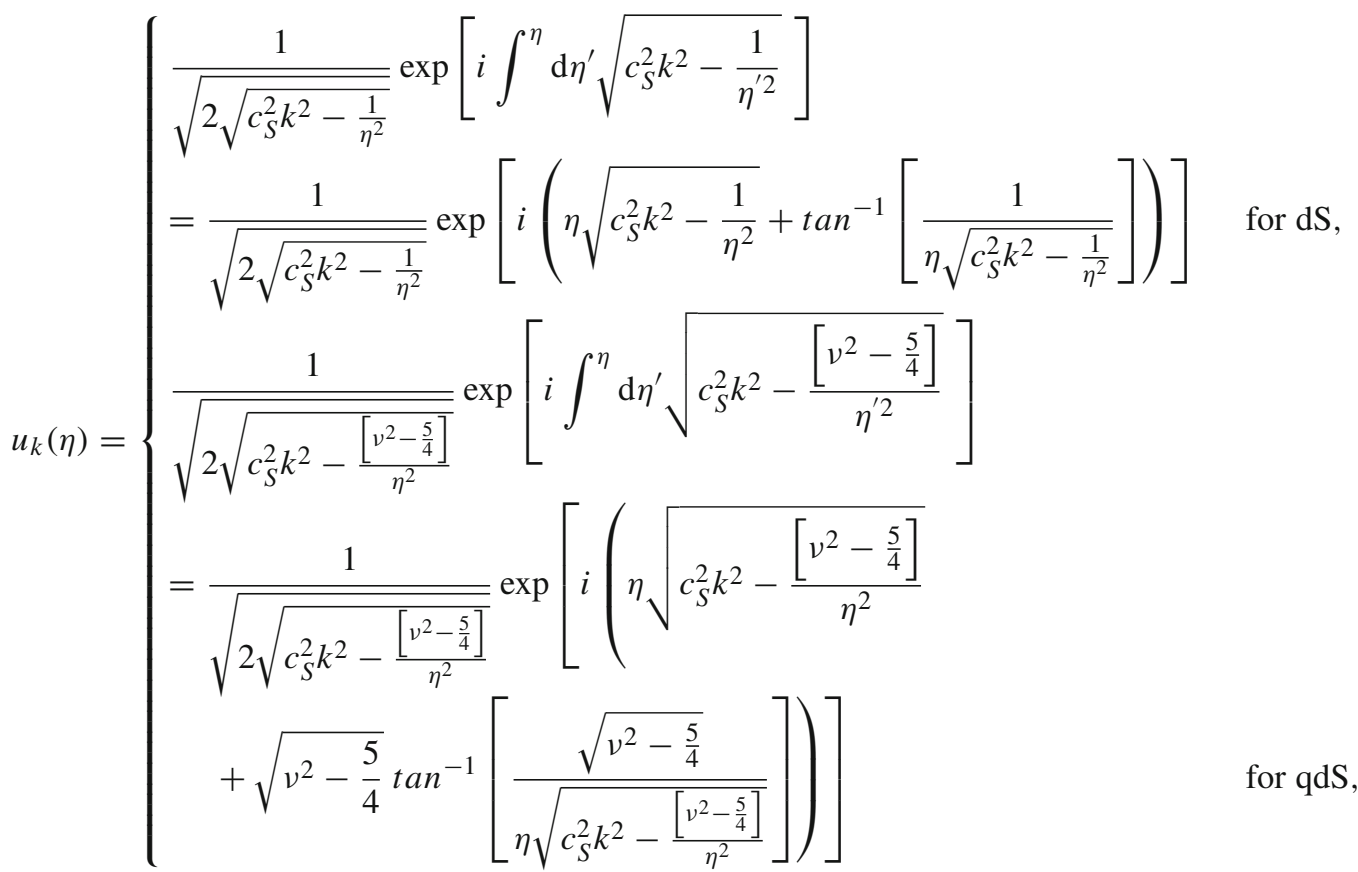

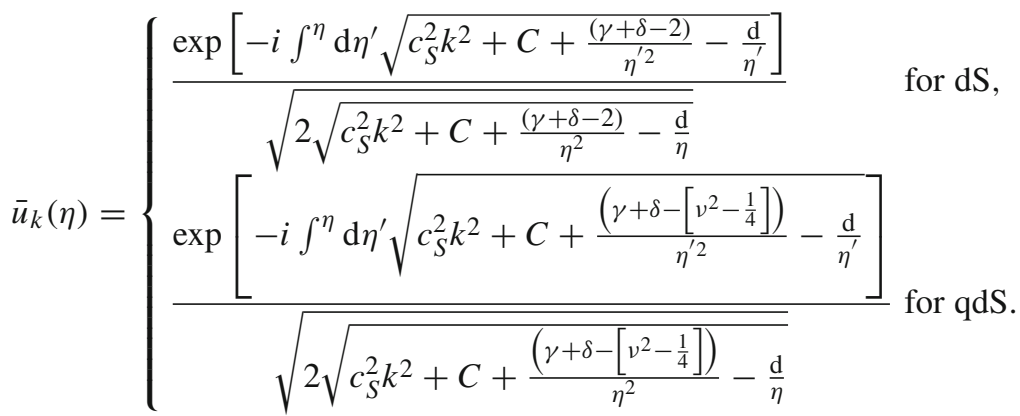

For dS:

$$
\begin{aligned}
& \int^{\eta} \mathrm{d} \eta^{\prime} \sqrt{c_{S}^{2} k^{2}+C+\frac{(\gamma+\delta-2)}{\eta^{\prime 2}}-\frac{\mathrm{d}}{\eta^{\prime}}} \\
= & \frac{1}{2 \sqrt{C+c_{S}^{2} k^{2}}}\left\{2 \sqrt{C+c_{S}^{2} k^{2}} \sqrt{\gamma+C \eta^{2}-d \eta+\delta+\eta^{2} c_{S}^{2} k^{2}-2}\right. \\
& -d \ln \left[2 \sqrt{C+c_{S}^{2} k^{2}} \sqrt{\gamma+C \eta^{2}-d \eta+\delta+\eta^{2} c_{S}^{2} k^{2}-2}+2 C \eta-d+2 \eta c_{S}^{2} k^{2}\right] \\
& \left.-2 \sqrt{\gamma+\delta-2} \sqrt{C+c_{S}^{2} k^{2}} \ln \left[\frac{2 \sqrt{\gamma+C \eta^{2}-d \eta+\delta+\eta^{2} c_{S}^{2} k^{2}-2}+\frac{i(2 \gamma-d \eta+2 \delta-4)}{\sqrt{-\gamma-\delta+2}}}{\eta(\gamma+\delta-2)}\right]\right\} .
\end{aligned}
$$


For qdS:

$$
\begin{aligned}
& \int^{\eta} \mathrm{d} \eta^{\prime} \sqrt{c_{S}^{2} k^{2}+C+\frac{\left(\gamma+\delta-\left[v^{2}-\frac{1}{4}\right]\right)}{\eta^{\prime 2}}-\frac{\mathrm{d}}{\eta^{\prime}}} \\
& =\frac{1}{2 \sqrt{C+c_{S}^{2} k^{2}}}\left\{2 \sqrt{C+k^{2}} \sqrt{\gamma+C \eta^{2}-d \eta+\delta+\eta^{2} c_{S}^{2} k^{2}-\left[v^{2}-\frac{1}{4}\right]}\right. \\
& -d \ln \left[2 \sqrt{C+k^{2}} \sqrt{\gamma+C \eta^{2}-d \eta+\delta+\eta^{2} c_{S}^{2} k^{2}-\left[v^{2}-\frac{1}{4}\right]}+2 C \eta-d+2 \eta c_{S}^{2} k^{2}\right] \\
& -2 \sqrt{\gamma+\delta-\left[v^{2}-\frac{1}{4}\right]} \\
& \left.\times \sqrt{C+c_{S}^{2} k^{2}} \ln \left[\frac{2 \sqrt{\gamma+C \eta^{2}-d \eta+\delta+\eta^{2} c_{S}^{2} k^{2}-\left[v^{2}-\frac{1}{4}\right]}+\frac{i\left(2 \gamma-d \eta+2 \delta-2\left[v^{2}-\frac{1}{4}\right]\right)}{\sqrt{-\gamma-\delta+\left[v^{2}-\frac{1}{4}\right]}}}{\eta\left(\gamma+\delta-\left[v^{2}-\frac{1}{4}\right]\right)}\right]\right\},
\end{aligned}
$$

where we have written the total solution for the mode $h_{k}$ in terms of two linearly independent solutions. Here it is important to note that the two solutions are hermitian conjugates of each other. If in the present context the exact solution of the mode $h_{k}$ is expanded with respect to these two linearly independent solutions then particle creation is absent in our EFT setup. In the present context correctness of the WKB approximation is guaranteed at very early and very late time scales. In this discussion $u_{k}(\eta)$ is valid at very early time scale and $\bar{u}_{k}(\eta)$ perfectly works in the late time scale.

\subsubsection{Profile $B: m=\frac{m_{0}}{\sqrt{2}} \sqrt{\left[1-\tanh \left(\frac{\rho}{H} \ln (-H \eta)\right)\right]}$}

The equation of motion for the massive field is

$$
\begin{aligned}
& h_{k}^{\prime \prime}+\left(c_{S}^{2} k^{2}+\left\{\frac{m_{0}^{2}}{2 H^{2}}\left[1-\tanh \left(\frac{\rho}{H} \ln (-H \eta)\right)\right]-2\right\} \frac{1}{\eta^{2}}\right) h_{k}=0 \quad \text { for dS } \\
& h_{k}^{\prime \prime}+\left(c_{S}^{2} k^{2}+\left\{\frac{m_{0}^{2}}{2 H^{2}}\left[1-\tanh \left(\frac{\rho}{H} \ln (-H \eta)\right)\right]-\left[v^{2}-\frac{1}{4}\right]\right\} \frac{1}{\eta^{2}}\right) h_{k}=0 \quad \text { for qdS. }
\end{aligned}
$$

This is not at all solvable exactly. But if we assume that

$$
\rho<<\frac{H}{\ln (-H \eta)}
$$

then in that limiting situation one can recast the equation of motions as

$$
\begin{aligned}
h_{k}^{\prime \prime}+\left(c_{S}^{2} k^{2}+\left\{\frac{m_{0}^{2}}{H^{2}}-2\right\} \frac{1}{\eta^{2}}\right) h_{k}=0 & \text { for dS } \\
h_{k}^{\prime \prime}+\left(c_{S}^{2} k^{2}+\left\{\frac{m_{0}^{2}}{H^{2}}-\left[v^{2}-\frac{1}{4}\right]\right\} \frac{1}{\eta^{2}}\right) h_{k}=0 & \text { for qdS }
\end{aligned}
$$

which is exactly similar to the equation of motions obtained for the cases where the conformal time-dependent mass function is varying slowly. The solution for the mode function for the de Sitter and the quasi de Sitter space in these limiting cases can 
be expressed as

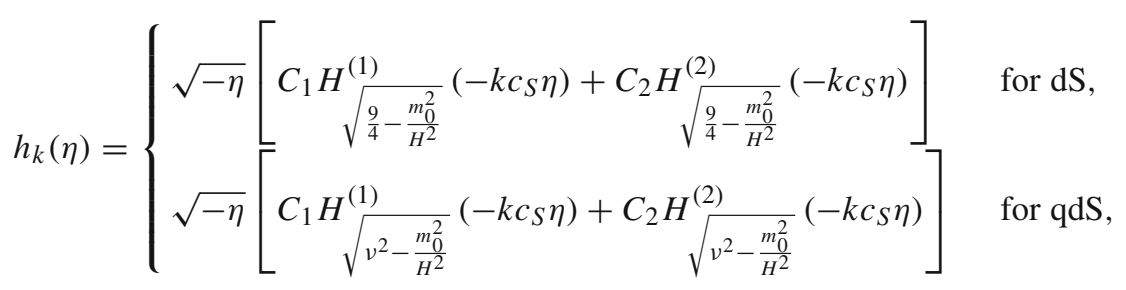

where $C_{1}$ and $C_{2}$ are two arbitrary integration constants, which depend on the choice of the initial condition. From this solution one can study $m_{0} \approx H, m_{0}>>H$ and $m_{0}<<H$ physical situations as studied before.

To solve this we assume that the WKB approximation is approximately valid for all times for the solution for the mode function $h_{k}$. In the standard WKB approximation the total solution can be recast in the following form:

$h_{k}(\eta)=\left[D_{1} u_{k}(\eta)+D_{2} \bar{u}_{k}(\eta)\right]$,

where $D_{1}$ and $D_{2}$ are two arbitrary integration constants, which depend on the choice of the initial condition during WKB approximation at early and late time scale. In the present context $u_{k}(\eta)$ and $\bar{u}_{k}(\eta)$ are defined as

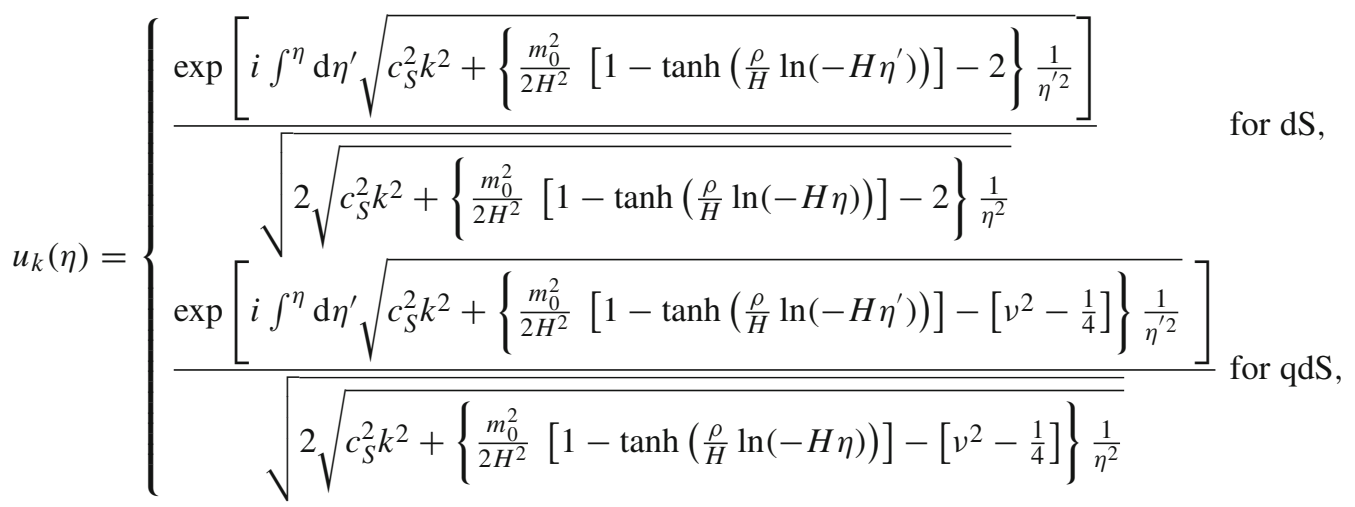

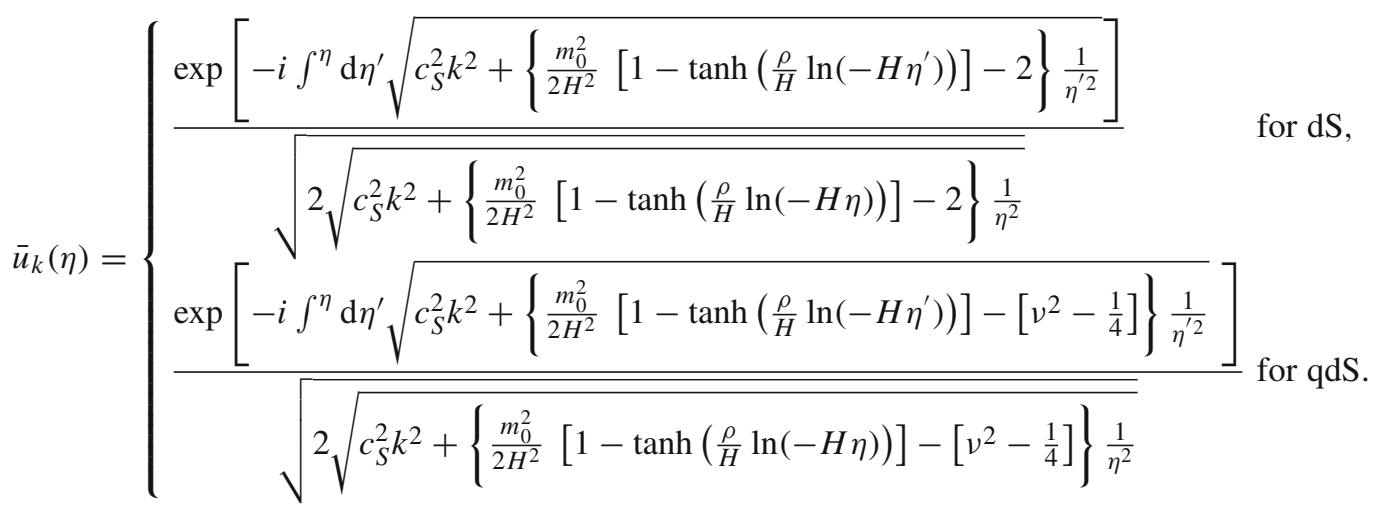

Again if we assume $\rho<<\frac{H}{\ln (-H \eta)}$ then these WKB solutions can be recast as 


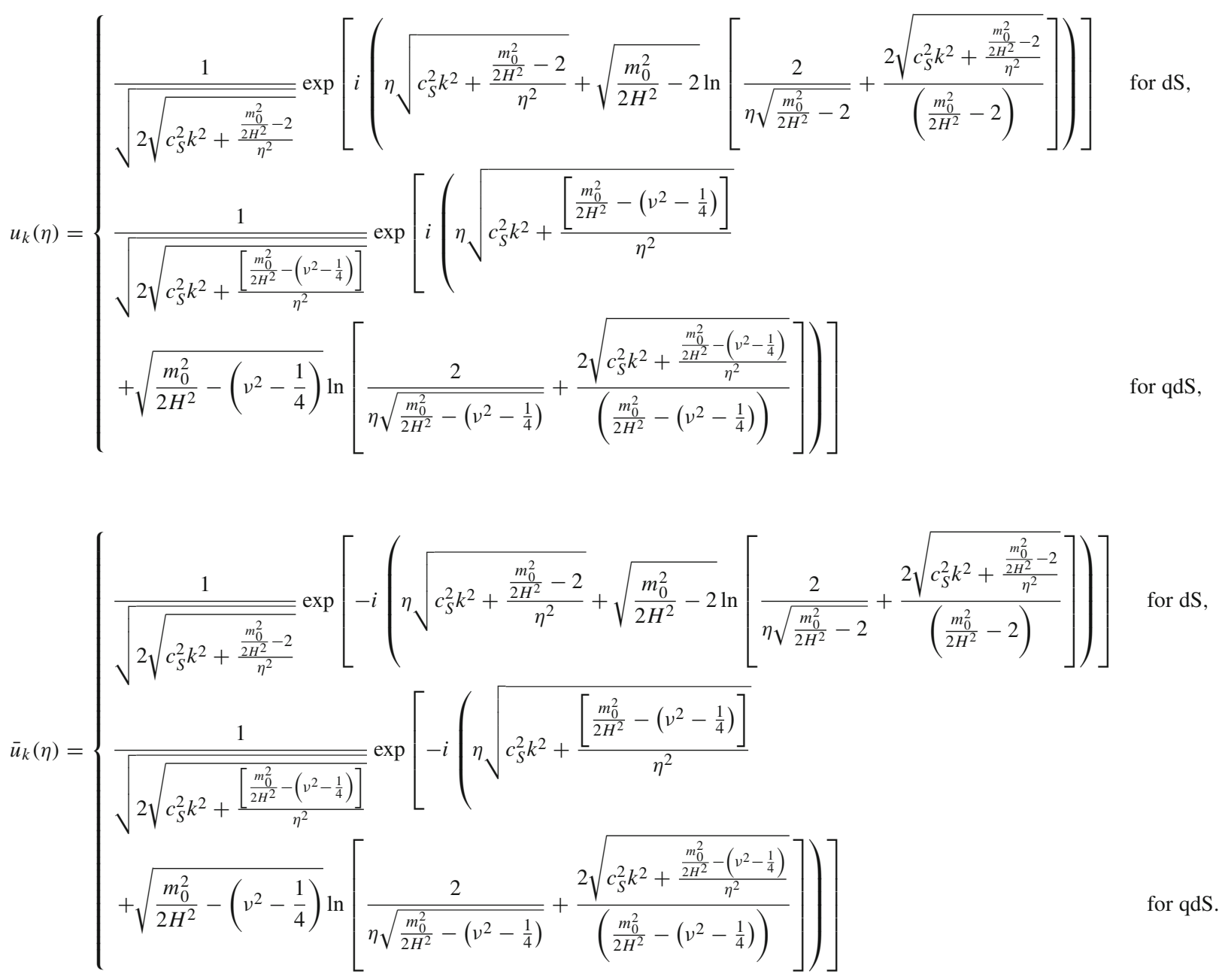

6.2.3 Profile $C: m=m_{0} \operatorname{sech}\left(\frac{\rho}{H} \ln (-H \eta)\right)$

The equation of motion for the massive field is

$$
\begin{aligned}
& h_{k}^{\prime \prime}+\left(c_{S}^{2} k^{2}+\left\{\frac{m_{0}^{2}}{H^{2}} \operatorname{sech}^{2}\left(\frac{\rho}{H} \ln (-H \eta)\right)-2\right\} \frac{1}{\eta^{2}}\right) h_{k}=0 \quad \text { for dS } \\
& h_{k}^{\prime \prime}+\left(c_{S}^{2} k^{2}+\left\{\frac{m_{0}^{2}}{H^{2}} \operatorname{sech}^{2}\left(\frac{\rho}{H} \ln (-H \eta)\right)-\left[v^{2}-\frac{1}{4}\right]\right\} \frac{1}{\eta^{2}}\right) h_{k}=0 \quad \text { for qdS. }
\end{aligned}
$$

This is not at all solvable exactly. But if we assume that

$\rho<<\frac{H}{\ln (-H \eta)}$,

then in that limiting situation the solutions are exactly the same as appearing for Profile B. Only here we have to change $m_{0}^{2} / 2 H^{2} \rightarrow m_{0}^{2} / H^{2}$.

To solve this we assume that the WKB approximation is approximately valid for all times for the solution for the mode function $h_{k}$. In the standard WKB approximation the total solution can be recast in the following form:

$h_{k}(\eta)=\left[D_{1} u_{k}(\eta)+D_{2} \bar{u}_{k}(\eta)\right]$, 
where $D_{1}$ and $D_{2}$ are two arbitrary integration constants, which depend on the choice of the initial condition during WKB approximation at early and late time scale. In the present context $u_{k}(\eta)$ and $\bar{u}_{k}(\eta)$ are defined as

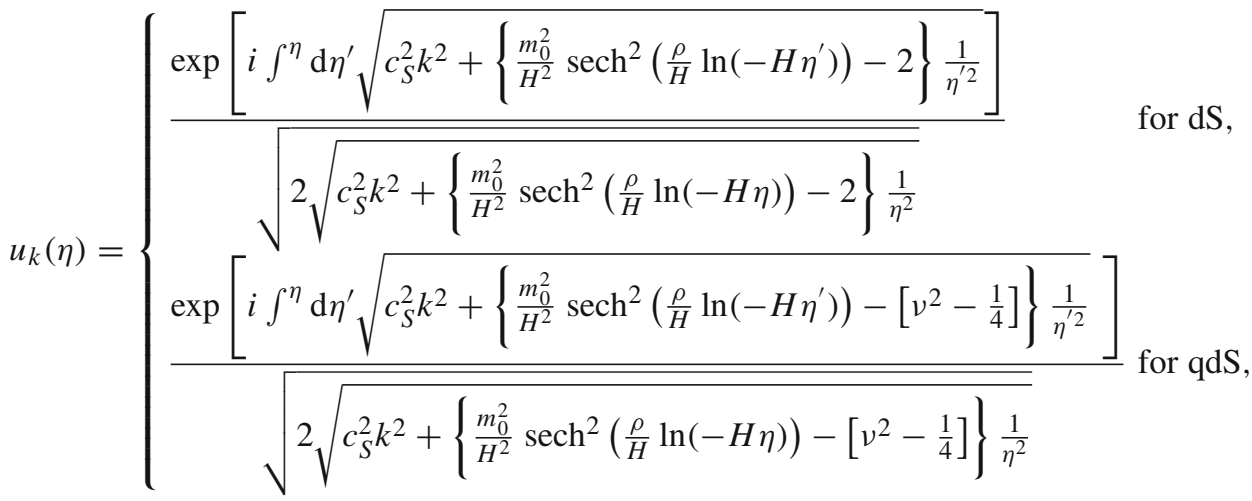

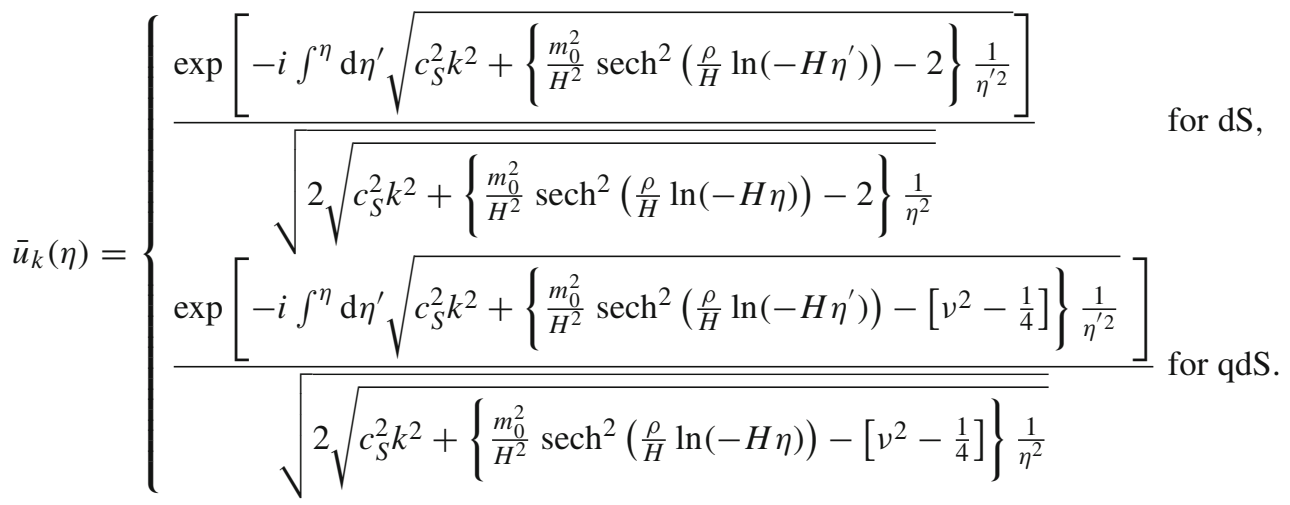

Again if we assume $\rho<<\frac{H}{\ln (-H \eta)}$ then these WKB solutions are exactly the same as appearing for Profile B. Only here we have to change $m_{0}^{2} / 2 H^{2} \rightarrow m_{0}^{2} / H^{2}$.

\subsection{Role of spin for heavy field}

Let us consider the situation for a dynamical massive field with arbitrary spin $\mathcal{S}$. In this case we assume that the dynamics of all such arbitrary spin fields with spin $\mathcal{S}>2$ is similar to the graviton. For this case the classical time dependence of the high spin modes leads to a time-dependent mass $m_{\mathcal{S}}(\eta)$ for the spin field. The equation of motion for the massive field with arbitrary spin $\mathcal{S}$ is given by

$h_{k}^{\prime \prime}+\left\{c_{S}^{2} k^{2}+\left(\frac{m_{\mathcal{S}}^{2}}{H^{2}}-\left[v_{\mathcal{S}}^{2}-\frac{1}{4}\right]\right) \frac{1}{\eta^{2}}\right\} h_{k}=0$,

where in the de Sitter case and the quasi de Sitter case the parameter $v_{\mathcal{S}}$ can be written as

$v_{\mathcal{S}}= \begin{cases}\left(\mathcal{S}-\frac{1}{2}\right) & \text { for } \mathrm{d} \mathrm{S}, \\ \left(\mathcal{S}-\frac{1}{2}\right)+\epsilon+\frac{\eta}{2}+\frac{s}{2} & \text { for qdS. }\end{cases}$

The most general solution of the mode function for the de Sitter case and the quasi de Sitter case can be written as

$h_{k}(\eta)=\sqrt{-\eta}\left[C_{1} H_{\sqrt{v_{\mathcal{S}}^{2}-\frac{m_{\mathcal{S}}^{2}}{H^{2}}}}^{(1)}\left(-k c_{S} \eta\right)+C_{2} H_{\sqrt{v_{\mathcal{S}}^{2}-\frac{m_{\mathcal{S}}^{2}}{H^{2}}}}^{(2)}\left(-k c_{S} \eta\right)\right]$.

Here $C_{1}$ and $C_{2}$ are the arbitrary integration constants and the numerical value depend on the choice of the initial condition or more precisely the vacuum. It is important to note that the solution for spin $\mathcal{S}=2$ exactly matches with our previously 
obtained results for a massive scalar fields. This implies that tensor fluctuations for the massive graviton field mimic the role of the massive scalar field with $\operatorname{spin} \mathcal{S}=0$ at the level of the equation of motion if we identify

$\frac{m_{\mathcal{S}}^{2}}{H^{2}}= \begin{cases}\frac{m^{2}}{H^{2}}-2 & \text { for } \mathrm{dS} \\ \frac{m^{2}}{H^{2}}-\left[v^{2}-\frac{1}{4}\right] & \text { for qdS. }\end{cases}$

But here for arbitrary spin (mostly for high spin with spin $\mathcal{S}>2$ ) we get a generic result which may be different from the previously mentioned massive scalar field as well as useful to study the effects of other high spin massive fields in the present context.

Here it is important to mention that the argument in the Hankel function for the solution of the $h_{k}$ takes the following values in a different regime:

For dS: $\quad \sqrt{v_{\mathcal{S}}^{2}-\frac{m_{\mathcal{S}}^{2}}{H^{2}}} \approx \begin{cases}\sqrt{\left(\mathcal{S}-\frac{1}{2}\right)^{2}-1} & \text { for } m_{\mathcal{S}} \approx H \\ i \sqrt{\Upsilon_{\mathcal{S}}^{2}-\left(\mathcal{S}-\frac{1}{2}\right)} & \text { for } m_{\mathcal{S}}<<H\end{cases}$

For qdS: $\quad \sqrt{v_{\mathcal{S}}^{2}-\frac{m_{\mathcal{S}}^{2}}{H^{2}}} \approx \begin{cases}\sqrt{\left(\left(\mathcal{S}-\frac{1}{2}\right)+\epsilon+\frac{\eta}{2}+\frac{s}{2}\right)^{2}-1} & \text { for } m_{\mathcal{S}} \approx H \\ i \sqrt{\left.\Upsilon_{\mathcal{S}}^{2}-\left(\left(\mathcal{S}-\frac{1}{2}\right)+\epsilon+\frac{\eta}{2}+\frac{s}{2}\right)+\epsilon+\frac{\eta}{2}+\frac{s}{2}\right)^{2}} & \text { for } m_{\mathcal{S}}<<H\end{cases}$

Here we set $m_{\mathcal{S}}=\Upsilon_{\mathcal{S}} H$, where the parameter $\Upsilon_{\mathcal{S}}>>1$ for $m_{\mathcal{S}}>>H$.

After taking the $k c_{S} \eta \rightarrow-\infty, k c_{S} \eta \rightarrow 0$ and $\left|k c_{S} \eta\right| \approx 1-\Delta(\rightarrow 0)$ limits the most general solution as stated in Eq. (6.85) can be recast as

$$
\begin{aligned}
& h_{k}(\eta) \stackrel{\left|k c_{S} \eta\right| \rightarrow-\infty}{=}\left\{\begin{array}{l}
\sqrt{\frac{2}{\pi k c_{S}}}\left[C_{1} e^{-i k c_{S} \eta} e^{-\frac{i \pi}{2}\left(\sqrt{\left(\mathcal{S}-\frac{1}{2}\right)^{2}-\frac{m_{\mathcal{S}}^{2}}{H^{2}}+\frac{1}{2}}\right)}+C_{2} e^{i k c_{S} \eta} e^{\frac{i \pi}{2}\left(\sqrt{\left.\left(\mathcal{S}-\frac{1}{2}\right)^{2}-\frac{m_{\mathcal{S}}^{2}}{H^{2}}+\frac{1}{2}\right)}\right.}\right] \\
\sqrt{\frac{2}{\pi k c_{S}}}\left[C_{1} e^{-i k c_{S} \eta} e^{-\frac{i \pi}{2}\left(\sqrt{\left(\left(\mathcal{S}-\frac{1}{2}\right)+\epsilon+\frac{\eta}{2}+\frac{s}{2}\right)^{2}-\frac{m_{\mathcal{S}}^{2}}{H^{2}}}+\frac{1}{2}\right)} \mathrm{dS},\right. \\
\left.+C_{2} e^{i k c_{S} \eta} e^{\frac{i \pi}{2}\left(\sqrt{\left(\left(\mathcal{S}-\frac{1}{2}\right)+\epsilon+\frac{\eta}{2}+\frac{s}{2}\right)^{2}-\frac{m_{\mathcal{S}}^{2}}{H^{2}}+\frac{1}{2}}\right)}\right] \\
\text { for qdS, }
\end{array}\right. \\
& h_{k}(\eta) \stackrel{\left|k c_{S} \eta\right| \rightarrow 0}{=} \begin{cases}\frac{i \sqrt{-\eta}}{\pi} \Gamma\left(\sqrt{\left(\mathcal{S}-\frac{1}{2}\right)^{2}-\frac{m_{\mathcal{S}}^{2}}{H^{2}}}\right)\left(-\frac{k c_{S} \eta}{2}\right)^{-\sqrt{\left(\mathcal{S}-\frac{1}{2}\right)^{2}-\frac{m_{\mathcal{S}}^{2}}{H^{2}}}}\left[C_{1}-C_{2}\right] & \text { for dS, } \\
\frac{i \sqrt{-\eta}}{\pi} \Gamma\left(\sqrt{\left(\left(\mathcal{S}-\frac{1}{2}\right)+\epsilon+\frac{\eta}{2}+\frac{s}{2}\right)^{2}-\frac{m_{\mathcal{S}}^{2}}{H^{2}}}\right)\left(-\frac{k c_{S} \eta}{2}\right)^{-\sqrt{\left(\left(\mathcal{S}-\frac{1}{2}\right)+\epsilon+\frac{\eta}{2}+\frac{s}{2}\right)^{2}-\frac{m_{\mathcal{S}}^{2}}{H^{2}}}}\left[C_{1}-C_{2}\right] & \text { for } \mathrm{qdS},\end{cases}
\end{aligned}
$$




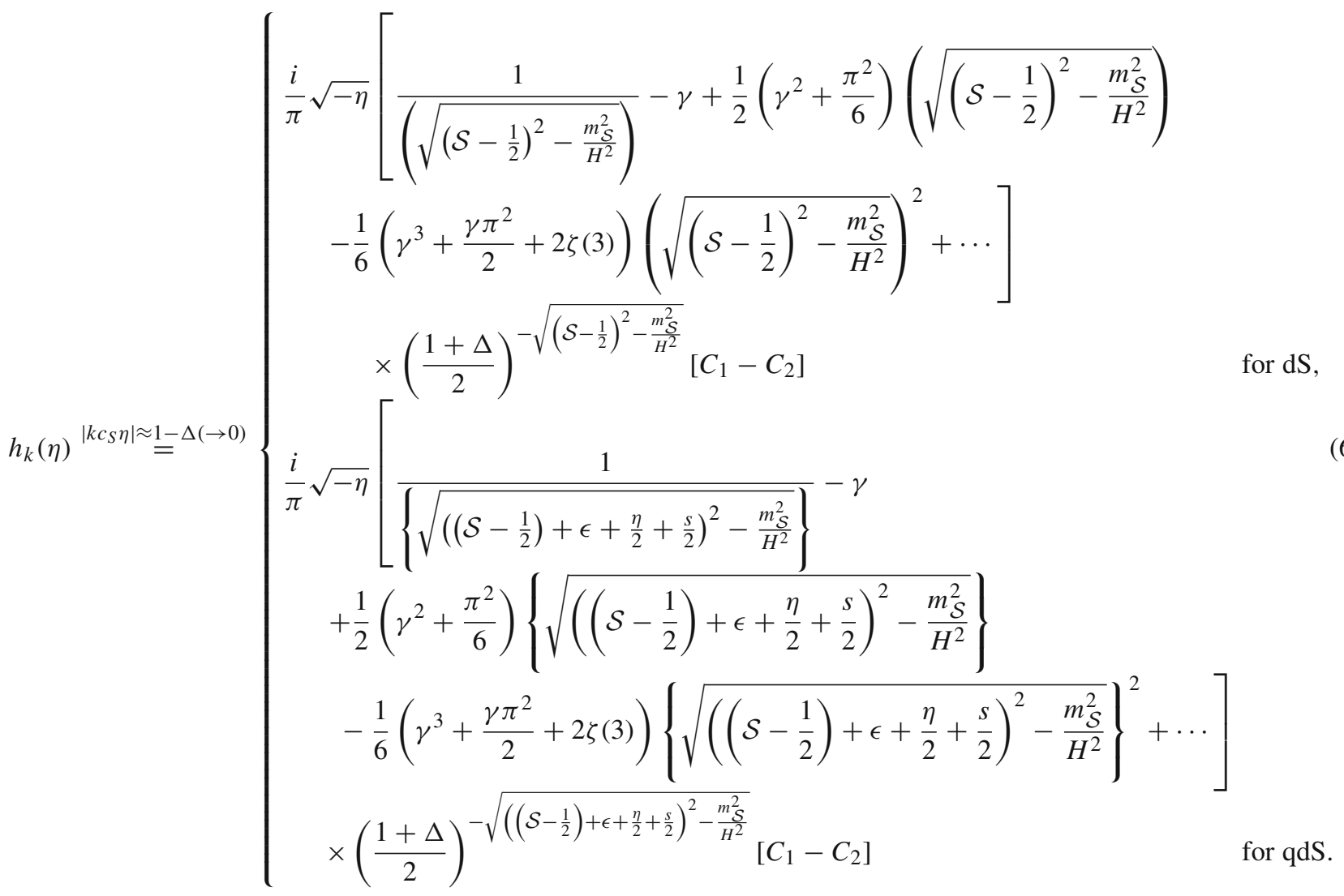

In the standard WKB approximation the total solution can be recast in the following form:

$h_{k}(\eta)=\left[D_{1} u_{k}(\eta)+D_{2} \bar{u}_{k}(\eta)\right]$,

where $D_{1}$ and $D_{2}$ are two arbitrary integration constants, which depend on the choice of the initial condition on making the WKB approximation at early and late time scale. In the present context $u_{k}(\eta)$ and $\bar{u}_{k}(\eta)$ are defined as

$$
\begin{aligned}
& u_{k}(\eta)=\frac{1}{\sqrt{2 p(\eta)}} \exp \left[i \int^{\eta} \mathrm{d} \eta^{\prime} p\left(\eta^{\prime}\right)\right] \\
& \bar{u}_{k}(\eta)=\frac{1}{\sqrt{2 p(\eta)}} \exp \left[-i \int^{\eta} \mathrm{d} \eta^{\prime} p\left(\eta^{\prime}\right)\right]
\end{aligned}
$$

where we have written the total solution for the mode $h_{k}$ in terms of two linearly independent solutions. Here in the most general situation the new conformal time-dependent factor $p(\eta)$ is defined as

$p(\eta)= \begin{cases}\sqrt{\left\{c_{S}^{2} k^{2}+\left(\frac{m_{\mathcal{S}}^{2}}{H^{2}}-\left\{\left(\mathcal{S}-\frac{1}{2}\right)^{2}-\frac{1}{4}\right\}\right) \frac{1}{\eta^{2}}\right\}} & \text { for dS, } \\ \sqrt{\left\{c_{S}^{2} k^{2}+\left(\frac{m_{\mathcal{S}}^{2}}{H^{2}}-\left\{\left(\left(\mathcal{S}-\frac{1}{2}\right)+\epsilon+\frac{\eta}{2}+\frac{s}{2}\right)^{2}-\frac{1}{4}\right\}\right) \frac{1}{\left.\eta^{2}\right\}}\right.} & \text { for qdS, }\end{cases}$

using which one can calculate the Bogoliubov coefficients and other components as we have computed for the other cases.

In this context one can compare the dynamical equations for scalar mode fluctuations with the well-known Schrödinger scattering problem in one spatial dimension then one can write down the following expression for the spin-dependent potential and energy: 


$$
\begin{aligned}
V(t) & = \begin{cases}-\frac{1}{2 m}\left(\frac{m_{\mathcal{S}}^{2}}{H^{2}}-\left[\left(\mathcal{S}-\frac{1}{2}\right)^{2}-\frac{1}{4}\right]\right) H^{2} e^{2 H t} & \text { for dS }, \\
-\frac{1}{2 m}\left(\frac{m_{\mathcal{S}}^{2}}{H^{2}}-\left[\left(\left(\mathcal{S}-\frac{1}{2}\right)+\epsilon+\frac{\eta}{2}+\frac{s}{2}\right)^{2}-\frac{1}{4}\right]\right) H^{2} e^{2 H t} & \text { for qdS },\end{cases} \\
E & =\frac{1}{2 m} c_{S}^{2} k^{2}
\end{aligned}
$$

Now we have already presented in the earlier section a detailed study of cosmological scalar curvature fluctuations from new massive particles, where we have computed the expression for the one point and two point functions in terms of the parameter $\Lambda$. Here in the presence of arbitrary spin (mostly for high spin with $\mathcal{S}>2$ ) this $\Lambda$ parameter is replaced by the new parameter $\Lambda_{\mathcal{S}}$, where $\Lambda_{\mathcal{S}}$ is defined as

$$
\Lambda_{\mathcal{S}}= \begin{cases}\sqrt{\left(\mathcal{S}-\frac{1}{2}\right)^{2}-\frac{m_{\mathcal{S}}^{2}}{H^{2}}} & \text { for dS } \\ \sqrt{\left(\left(\mathcal{S}-\frac{1}{2}\right)+\epsilon+\frac{\eta}{2}+\frac{s}{2}\right)^{2}-\frac{m_{\mathcal{S}}^{2}}{H^{2}}} & \text { for qdS. }\end{cases}
$$

For $\mathcal{S}=2$ only $\Lambda_{\mathcal{S}}=\Lambda$ both for the de Sitter case and the quasi de Sitter case. Otherwise the rest of the computation of the one point and the two point correlation functions is exactly the same. If we replace $\Lambda$ by $\Lambda_{\mathcal{S}}$ then the spectral tilt for scalar fluctuations with the horizon crossing $\left|k c_{S} \eta\right| \approx 1$ can be expressed in the presence of an arbitrary spin contribution as

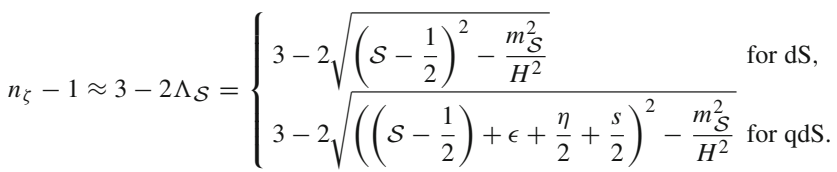

As the value of the scalar spectral tilt $n_{\zeta}$ is known from observation, one can easily give the estimate of the value of the ratio of the mass parameter $m$ with Hubble scale during inflation $H$ as

$$
\left|\frac{m_{\mathcal{S}}}{H}\right|= \begin{cases}\left|\sqrt{\left(\mathcal{S}-\frac{1}{2}\right)^{2}-\frac{\left(4-n_{\zeta}\right)^{2}}{4}}\right| & \text { for } \mathrm{dS} \\ \sqrt{\left(\left(\mathcal{S}-\frac{1}{2}\right)+\epsilon+\frac{\eta}{2}+\frac{s}{2}\right)^{2}-\frac{\left(4-n_{\zeta}\right)^{2}}{4}} & \text { for qdS. }\end{cases}
$$

It is important to mention here that if we use the constraint on scalar spectral tilt as obtained from the Planck 2015 data we get the following $2 \sigma$ bound on the magnitude of the mass parameter of the new heavy particles:
For dS:

$$
\begin{aligned}
& \left|\sqrt{\left(\mathcal{S}-\frac{1}{2}\right)^{2}-2.33}\right|<\left|\frac{m_{\mathcal{S}}}{H}\right|_{\left|k c_{S} \eta\right| \approx 1} \\
& <\left|\sqrt{\left(\mathcal{S}-\frac{1}{2}\right)^{2}-2.30}\right|,
\end{aligned}
$$

For qdS:

$$
\begin{aligned}
& \left|\sqrt{\left(\left(\mathcal{S}-\frac{1}{2}\right)+\epsilon+\frac{\eta}{2}+\frac{s}{2}\right)^{2}-2.33}\right|<\left|\frac{m_{\mathcal{S}}}{H}\right|_{\mid k c \operatorname{\eta } \eta \approx 1} \\
& <\left|\sqrt{\left(\left(\mathcal{S}-\frac{1}{2}\right)+\epsilon+\frac{\eta}{2}+\frac{s}{2}\right)^{2}-2.30}\right|
\end{aligned}
$$

and for $\left|k c_{S} \eta\right|<<1$ and $\left|k c_{S} \eta\right|>>1$ the allowed lower bound on the magnitude of the mass parameter of the new heavy particle is given by

$$
\begin{aligned}
& \left|\frac{m_{\mathcal{S}}}{H}\right|_{\left|k c_{S} \eta\right|<<1,\left|k c_{S} \eta\right|>>1} \geq \sqrt{v_{\mathcal{S}}^{2}-\frac{1}{4}} \\
& = \begin{cases}\sqrt{\left(\mathcal{S}-\frac{1}{2}\right)^{2}-\frac{1}{4}} & \text { for dS, } \\
\sqrt{\left(\left(\mathcal{S}-\frac{1}{2}\right)+\epsilon+\frac{\eta}{2}+\frac{s}{2}\right)^{2}-\frac{1}{4}} & \text { for qdS. }\end{cases}
\end{aligned}
$$

For graviton with $\operatorname{spin} \mathcal{S}=2$ the bound on the graviton mass parameter for $\left|k c_{S} \eta\right| \approx 1,\left|k c_{S} \eta\right|<<1$ and $\left|k c_{S} \eta\right|>>1$ is given by

For dS:

$0.23<\left|\frac{m_{\mathcal{S}=2}}{H}\right|_{k c_{S} \eta \approx 1}<0.28$,

For qdS:

$$
\begin{aligned}
& 0.23 \times\left|\sqrt{1-56.18\left(\epsilon+\frac{\eta}{2}+\frac{s}{2}\right)}\right|<\left|\frac{m_{\mathcal{S}=2}}{H}\right|_{k c_{S} \eta \approx 1} \\
& <0.28 \times\left|\sqrt{1-39.06\left(\epsilon+\frac{\eta}{2}+\frac{s}{2}\right)}\right|
\end{aligned}
$$




$$
\begin{aligned}
& \left|\frac{m_{\mathcal{S}=2}}{H}\right|_{\left|k c_{S} \eta\right|<<1,\left|k c_{S} \eta\right|>>1} \\
& \geq \begin{cases}\sqrt{2} & \text { for } \mathrm{dS}, \\
\sqrt{2} \sqrt{1+\frac{3}{2}\left(\epsilon+\frac{\eta}{2}+\frac{s}{2}\right)+\frac{1}{2}\left(\epsilon+\frac{\eta}{2}+\frac{s}{2}\right)^{2}} & \text { for qdS. }\end{cases}
\end{aligned}
$$

On the other hand for a massive scalar field with $\mathcal{S}=0$ we get the following lower bound on the mass parameter:

$$
\begin{aligned}
& \left|\frac{m}{H}\right|_{\left|k c_{S} \eta\right|<<1,\left|k c_{S} \eta\right|>>1} \\
& \geq\left\{\begin{array}{l}
\sqrt{2} \\
\sqrt{2} \sqrt{1+\frac{3}{2}\left(\epsilon+\frac{\eta}{2}+\frac{s}{2}\right)+\frac{1}{2}\left(\epsilon+\frac{\eta}{2}+\frac{s}{2}\right)^{2}} \text { for } \mathrm{dS},
\end{array}\right.
\end{aligned}
$$

which is exactly similar to the graviton. Similarly for the axion field with $\mathcal{S}=0$ we get the following lower bound on the mass parameter:

$$
\begin{aligned}
& \left|\frac{m_{\text {axion }}}{f_{a} H}\right|_{\left|k c_{S} \eta\right|<<1,|k c s \eta|>>1} \\
& \geq \begin{cases}\sqrt{6} & \text { for } \eta \sim \eta_{c}, \text { early \& late } \eta, \\
\sqrt{6+\Delta_{C}} & \text { for } \eta<\eta_{c},\end{cases}
\end{aligned}
$$

where $\Delta_{C}$ is a slowly varying quantity as introduced earlier in the context of the axion fluctuation.

\subsection{More on Bell inequalities}

\subsubsection{CHSH inequality}

The theory of locality of Einstein is based on another form of the Bell inequality, which applies to a situation in which Aace can measure either one of two observables $A_{1}$ and $A_{2}$, while Bace can measure either $B_{1}$ or $B_{2}$.

Now suppose that the observables $A_{1}, A_{2}, B_{1}$ and $B_{2}$ take values in \pm 1 , and that they are functions of hidden random variables. If $A_{1}, A_{2}= \pm 1$, therefore either $A_{1}+A_{2}=0$, in which case $A_{1} A_{2}= \pm 2$, or else $A_{1} A_{2}=0$, in which case $A_{1}+A_{2}= \pm 2$; therefore

$$
C=\left(A_{1}+A_{2}\right) B_{1}+\left(A_{1}-A_{2}\right) B_{2}= \pm 2 .
$$

This is where the local hidden-variable assumption comes in. Here it is assumed that values in \pm 1 can be assigned simultaneously to all four observables, even though it is impossible to measure both of $A_{1}$ and $A_{2}$, or both of $B_{1}$ and $b_{2}$. Therefore,

$$
\begin{array}{r}
|\langle C\rangle| \leq\langle|C|\rangle=2 \\
\left|\left\langle A_{1} B_{1}\right\rangle+\left\langle A_{1} B_{2}\right\rangle+\left\langle A_{2} B_{1}\right\rangle-\left\langle A_{2} B_{2}\right\rangle\right| \leq 2 .
\end{array}
$$

This inequality is called the CHSH (Clauser-Horne-ShimonyHolt) inequality. See ref [81-91] for more details. One can also test the future consequences for CHSH inequality violation in the cosmological setup. After Aspect's second experiment in 1982, many test experiments were done which used the CHSH inequality.

\subsubsection{Consequences of Bell inequality violation}

- Bell's inequalities violation due to entanglement gave solid evidence that the theory of quantum mechanics cannot be represented by any theory of classical physics.

- Elements which are compatible with classical theory are complementarity and wave function collapse.

- The 'Einstein, Rosen and Podolsky' paper pointed out some properties of entangled states which are unusual, which in turn is the fundamental foundation for the applications of quantum physics we use today in daily lives such as quantum cryptography and quantum nonlocality.

- The theorem of Bell proved that the quantum mechanical property entanglement has a degree of nonlocality which cannot be explained by any local theory.

- No phenomenon which is predicted by the theory of quantum mechanics can be reproduce by any combination of local deterministic and local random variables and this is also observed experimentally.

- There are various applications of entanglement in quantum information theory. Many impossible works can be done using the concept of entanglement theory.

- The best-known applications are superdense coding and quantum teleportation.

- Quantum computing and quantum cryptography [81-91] are very well-known disciplines of physics where entanglement theory is used. Entanglement-based quantum cryptography is very useful to detect the presence of any third party between two communication parties.

\section{References}

1. A. Einstein, B. Podolsky, N. Rosen, Can quantum-mechanical description of physical reality be considered complete? Phys. Rev. 47, 777 (1935)

2. J.S. Bell, On the Einstein-Podolsky-Rosen paradox. Physics 1, 195 (1964)

3. J. Maldacena, A model with cosmological Bell inequalities. Fortsch. Phys. 64, 10 (2016). arXiv:1508.01082 [hep-th]

4. V.F. Mukhanov, G.V. Chibisov, Quantum fluctuations and a nonsingular universe. JETP Lett. 33, 532 (1981)

5. V.F. Mukhanov, G.V. Chibisov, Pisma. Zh. Eksp. Teor. Fiz. 33, 549 (1981)

6. J.M. Bardeen, P.J. Steinhardt, M.S. Turner, Spontaneous creation of almost scale-free density perturbations in an inflationary universe. Phys. Rev. D 28, 679 (1983)

7. S.W. Hawking, The development of irregularities in a single bubble inflationary universe. Phys. Lett. B 115, 295 (1982) 
8. A.A. Starobinsky, Dynamics of phase transition in the new inflationary universe scenario and generation of perturbations. Phys. Lett. B 117, 175 (1982)

9. A.H. Guth, Inflationary universe: a possible solution to the horizon and flatness problems. Phys. Rev. D 23, 347 (1981)

10. C.P. Burgess, R. Holman, D. Hoover, Decoherence of inflationary primordial fluctuations. Phys. Rev. D 77, 063534 (2008). arXiv:astro-ph/0601646

11. E. Nelson, Quantum decoherence during inflation from gravitational nonlinearities. JCAP 1603, 022 (2016). arXiv:1601.03734 [gr-qc]

12. D. Polarski, A.A. Starobinsky, Semiclassicality and decoherence of cosmological perturbations. Class. Quant. Grav. 13, 377-392 (1996). arXiv:gr-qc/9504030

13. F.C. Lombardo, D. Lopez Nacir, Decoherence during inflation: The Generation of classical inhomogeneities. Phys. Rev. D 72, 063506 (2005). arXiv:gr-qc/0506051

14. P. Martineau, On the decoherence of primordial fluctuations during inflation. Class. Quant. Grav. 24, 5817-5834 (2007). arXiv:astro-ph/0601134

15. E. Calzetta, B.L. Hu, Quantum fluctuations, decoherence of the mean field, and structure formation in the early universe. Phys. Rev. D 52, 6770-6788 (1995). arXiv:gr-qc/9505046

16. R.H. Brandenberger, R. Laflamme, M. Mijic, Classical perturbations from decoherence of quantum fluctuations in the inflationary universe. Mod. Phys. Lett. A 5, 2311-2318 (1990)

17. T. Prokopec, G.I. Rigopoulos, Decoherence from isocurvature perturbations in inflation. JCAP 0711, 029 (2007). arXiv:astro-ph/0612067

18. J.W. Sharman, G.D. Moore, Decoherence due to the horizon after inflation. JCAP 0711, 020 (2007). arXiv:0708.3353

19. M. Franco, E. Calzetta, Decoherence in the cosmic background radiation. Class. Quant. Grav. 28, 145024 (2011). arXiv:1103.0188

20. D. Lopez Nacir, R.A. Porto, L. Senatore, M. Zaldarriaga, Dissipative effects in the effective field theory of inflation. JHEP 1201, 075 (2012). arXiv:1109.4192 [hep-th]

21. S.R. Behbahani, A. Dymarsky, M. Mirbabayi, L. Senatore, (Small) Resonant non-Gaussianities: signatures of a discrete shift symmetry in the effective field theory of inflation. JCAP 1212, 036 (2012). arXiv: 1111.3373 [hep-th]

22. S. Choudhury, A. Mazumdar, Sub-Planckian inflation and large tensor to scalar ratio with $r \geq 0.1$. arXiv:1404.3398 [hep-th]

23. S. Choudhury, A. Dasgupta, Effective field theory of dark matter from membrane inflationary paradigm. Phys. Dark Univ. 13, 35 (2016). arXiv:1510.08195 [hep-th]

24. S. Choudhury, Reconstructing inflationary paradigm within effective field theory framework. Phys. Dark Univ. 11, 16 (2016). arXiv:1508.00269 [astro-ph.CO]

25. C. Cheung, P. Creminelli, A.L. Fitzpatrick, J. Kaplan, L. Senatore, The effective field theory of inflation. JHEP 0803, 014 (2008). arXiv:0709.0293 [hep-th]

26. T. Noumi, M. Yamaguchi, D. Yokoyama, Effective field theory approach to quasi-single field inflation and effects of heavy fields. JHEP 1306, 051 (2013). arXiv:1211.1624 [hep-th]

27. A. Einstein, On the electrodynamics of moving bodies. Ann. Phys. 17, $891(1905)$

28. A. Einstein, Ann. Phys. 14, 194 (2005)

29. S.J. Freedman, J.F. Clauser, Experimental test of local hiddenvariable theories. Phys. Rev. Lett. 28, 938 (1972)

30. A. Aspect, P. Grangier, G. Roger, Experimental tests of realistic local theories via Bell's theorem. Phys. Rev. Lett. 47, 460 (1981)

31. A. Aspect, J. Dalibard, G. Roger, Experimental test of Bell's inequalities using time varying analyzers. Phys. Rev. Lett. 49, 1804 (1982)
32. W. Tittel, J. Brendel, H. Zbinden, N. Gisin, Violation of Bell inequalities by photons more than $10 \mathrm{~km}$ apart. Phys. Rev. Lett. 81, 3563 (1998). arXiv:quant-ph/9806043

33. M.A. Rowe et al., Experimental violation of a Bell's inequality with efficient detection. Nature 409(6822), 791-794 (2001)

34. S. Grblacher et al., An experimental test of non-local realism. Nature 446, 8715 (2006)

35. D. Salart et al, Spacelike Separation in a Bell Test Assuming Gravitationally Induced Collapses. Phys. Rev. Lett. 100(22), 220404. doi:10.1103/PhysRevLett.100.220404

36. M. Ansmann et al., Violation of Bell's inequality in Josephson phase qubits. Nature 461(504-6), 2009 (2009)

37. B.G. Christensen et al., Detection-loophole-free test of quantum nonlocality, and applications. Phys. Rev. Lett. 111(7448), 130406 (2013)

38. B. Hensen et al., Experimental loophole-free violation of a Bell inequality using entangled electron spins separated by $1.3 \mathrm{~km}$. Nature 526, 682 (2015). arXiv:1508.05949 [quant-ph]

39. M. Giustina et al., A significant-loophole-free test of Bell's theorem with entangled photons (2015). arXiv: 1511.03190

40. L.K. Shalm et al., A strong loophole-free test of local realism (2015). arXiv:1511.03189

41. A.D. Linde, A new inflationary universe scenario: a possible solution of the horizon, flatness, homogeneity, isotropy and primordial monopole problems. Phys. Lett. B 108, 389393 (1982)

42. A. Albrecht, P.J. Steinhardt, Cosmology for grand unified theories with radiatively induced symmetry breaking. Phys. Rev. Lett. 48, 12201223 (1982)

43. A.H. Guth, S.Y. Pi, Fluctuations in the new inflationary universe. Phys. Rev. Lett. 49, 11101113 (1982)

44. Planck Collaboration, R. Adam et al., Planck 2015 results. I. Overview of products and scientific results. arXiv:1502.01582

45. Planck Collaboration, P. Ade et al., Planck 2015 results. XIII. Cosmological parameters. arXiv: 1502.01589

46. C. Kiefer, D. Polarski, Why do cosmological perturbations look classical to us? Adv. Sci. Lett. 2, 164173 (2009). arXiv:0810.0087 [astro-ph]

47. A. Albrecht, P. Ferreira, M. Joyce, T. Prokopec, Inflation and squeezed quantum states. Phys. Rev. D 50, 48074820 (1994). arXiv:astro-ph/9303001

48. L.P. Grishchuk, YuV Sidorov, On the quantum state of relic gravitons. Class. Quant. Grav. 6, L161-L165 (1989)

49. L.P. Grishchuk, Y.V. Sidorov, Squeezed quantum states of relic gravitons and primordial density fluctuations. Phys. Rev. D 42, 34133421 (1990)

50. A.H. Guth, S.-Y. Pi, The quantum mechanics of the scalar field in the new inflationary universe. Phys. Rev. D 32, 18991920 (1985)

51. C. Kiefer, D. Polarski, A.A. Starobinsky, Quantum to classical transition for fluctuations in the early universe. Int. J. Mod. Phys. D 7, 455462 (1998). arXiv:gr-qc/9802003

52. M.-A. Sakagami, Evolution from pure states into mixed states in de Sitter space. Prog. Theor. Phys. 79, 442 (1988)

53. C.P. Burgess, R. Holman, G. Tasinato, M. Williams, EFT Beyond the horizon: stochastic inflation and how primordial quantum fluctuations go classical. JHEP 03, 090 (2015). arXiv: 1408.5002

54. R.H. Brandenberger, H. Feldman, V.F. Mukhanov, Classical and quantum theory of perturbations in inflationary universe models, in Evolution of the universe and its observational quest. Proceedings, 37th Yamada Conference, Tokyo, Japan, June 8-12, 1993, pp. 1930 (1993). arXiv:astro-ph/9307016

55. N. Itzhaki, E.D. Kovetz, Inflection point inflation and time dependent potentials in string theory. JHEP 0710, 054 (2007). arXiv:0708.2798 [hep-th] 
56. S. Panda, M. Sami, S. Tsujikawa, Prospects of inflation in delicate D-brane cosmology. Phys. Rev. D 76, 103512 (2007). arXiv:0707.2848 [hep-th]

57. D. Baumann, A. Dymarsky, I.R. Klebanov, L. McAllister, Towards an Explicit Model of D-brane Inflation. JCAP 0801, 024 (2008). arXiv:0706.0360 [hep-th]

58. D. Baumann, A. Dymarsky, I.R. Klebanov, L. McAllister, P.J. Steinhardt, A delicate universe. Phys. Rev. Lett. 99, 141601 (2007). arXiv:0705.3837 [hep-th]

59. G. Mandal, S. Paranjape, N. Sorokhaibam, Thermalization in 2D critical quench and UV/IR mixing. arXiv:1512.02187 [hep-th]

60. S.R. Das, D.A. Galante, R.C. Myers, Quantum quenches in free field theory: universal scaling at any rate. JHEP 1605, 164 (2016)

61. S.R. Das, D.A. Galante, R.C. Myers, Universality in fast quantum quenches. JHEP 1502, 167 (2015). arXiv: 1411.7710 [hep-th]

62. S.R. Das, D.A. Galante, R.C. Myers, Universal scaling in fast quantum quenches in conformal field theories. Phys. Rev. Lett. 112, 171601 (2014). arXiv:1401.0560 [hep-th]

63. S.R. Das, T. Morita, Kibble-Zurek scaling in holographic quantum quench: backreaction. JHEP 1501, 084 (2015). arXiv:1409.7361 [hep-th]

64. P. Basu, D. Das, S.R. Das, K. Sengupta, Quantum quench and double trace couplings. JHEP 1312, 070 (2013). arXiv:1308.4061 [hep-th]

65. P. Basu, D. Das, S.R. Das, T. Nishioka, Quantum quench across a zero temperature holographic superfluid transition. JHEP 1303, 146 (2013). arXiv:1211.7076 [hep-th]

66. G. Mandal, T. Morita, Quantum quench in matrix models: dynamical phase transitions, selective equilibration and the generalized Gibbs ensemble. JHEP 1310, 197 (2013). arXiv:1302.0859 [hepth]

67. E. Silverstein, A. Westphal, Monodromy in the CMB: gravity waves and string inflation. Phys. Rev. D 78, 106003 (2008). arXiv:0803.3085 [hep-th]

68. N. Iizuka, Trivedi an inflationary model in string theory. Phys. Rev. D 70, 043519 (2004)

69. E. Witten, The Feynman $i \epsilon$ in string theory. JHEP 1504, 055 (2015). arXiv:1307.5124 [hep-th]

70. P. Svrcek, E. Witten, Axions in string theory. JHEP 0606, 051 (2006). arXiv:hep-th/0605206

71. C. Beasley, E. Witten, New instanton effects in string theory. JHEP 0602, 060 (2006). arXiv:hep-th/0512039

72. C.E. Beasley, Three instanton computations in gauge theory and string theory. UMI-31-69786
73. E. Witten, Comments on string theory. arXiv:hep-th/0212247

74. E. Witten, String theory. eConf. C 010630, P337 (2001)

75. E. Witten, Small instantons in string theory. Nucl. Phys. B 460, 541 (1996). arXiv:hep-th/9511030

76. E. Witten, Some comments on string dynamics. In Los Angeles 1995, Future perspectives in string theory, pp. 501-523. arXiv:hep-th/9507121

77. E. Witten, String theory dynamics in various dimensions. Nucl. Phys. B 443, 85 (1995). arXiv:hep-th/9503124

78. E. Witten, Quantum background independence in string theory. Salamfest 1993, 0257-0275. arXiv:hep-th/9306122

79. S. Choudhury, S. Panda, COSMOS- $e^{\prime}-$ GTachyon from string theory. Eur. Phys. J. C 76(5), 278 (2016). arXiv:1511.05734 [hep-th]

80. S. Panda, Y. Sumitomo, S.P. Trivedi, Axions as quintessence in string theory. Phys. Rev. D 83, 083506 (2011). arXiv:1011.5877 [hep-th]

81. A.K. Ekert, Quantum cryptography based on Bell's theorem. Phys. Rev. Lett. 67, 661 (1991)

82. G. Adesso, T.R. Bromley, M. Cianciaruso, Measures and applications of quantum correlations. arXiv:1605.00806 [quant-ph]

83. N.J. Beaudry, Assumptions in quantum cryptography. arXiv:1505.02792 [quant-ph]

84. K. Fujikawa, Quantum discord, CHSH inequality and hidden variables - critical reassessment of hidden-variables models. arXiv:1302.0916 [quant-ph]

85. K. Fujikawa, Does CHSH inequality test the model of local hidden variables? Prog. Theor. Phys. 127, 975 (2012). arXiv:1204.3437 [quant-ph]

86. R. Horodecki, P. Horodecki, M. Horodecki, K. Horodecki, Quantum entanglement. Rev. Mod. Phys. 81, 865 (2009). arXiv:quant-ph/0702225

87. N. Gisin, G. Ribordy, W. Tittel, H. Zbinden, Quantum cryptography. Rev. Mod. Phys. 74, 145 (2002)

88. T. Jennewein, C. Simon, G. Weihs, H. Weinfurter, A. Zeilinger, Quantum cryptography with entangled photons. Phys. Rev. Lett. 84, 4729 (2000)

89. R.J. Hughes, G.L. Morgan, C.G. Peterson, Practical quantum key distribution over a 48-km optical fiber network. J. Mod. Opt. 47, 533 (2000). arXiv:quant-ph/9904038

90. J. Kempe, On multiparticle entanglement and its applications to cryptography. Phys. Rev. A 60, 910 (1999). arXiv:quant-ph/9902036

91. S.J. Lomonaco, Jr., A quick glance at quantum cryptography. [arXiv:quant-ph/9811056] 